

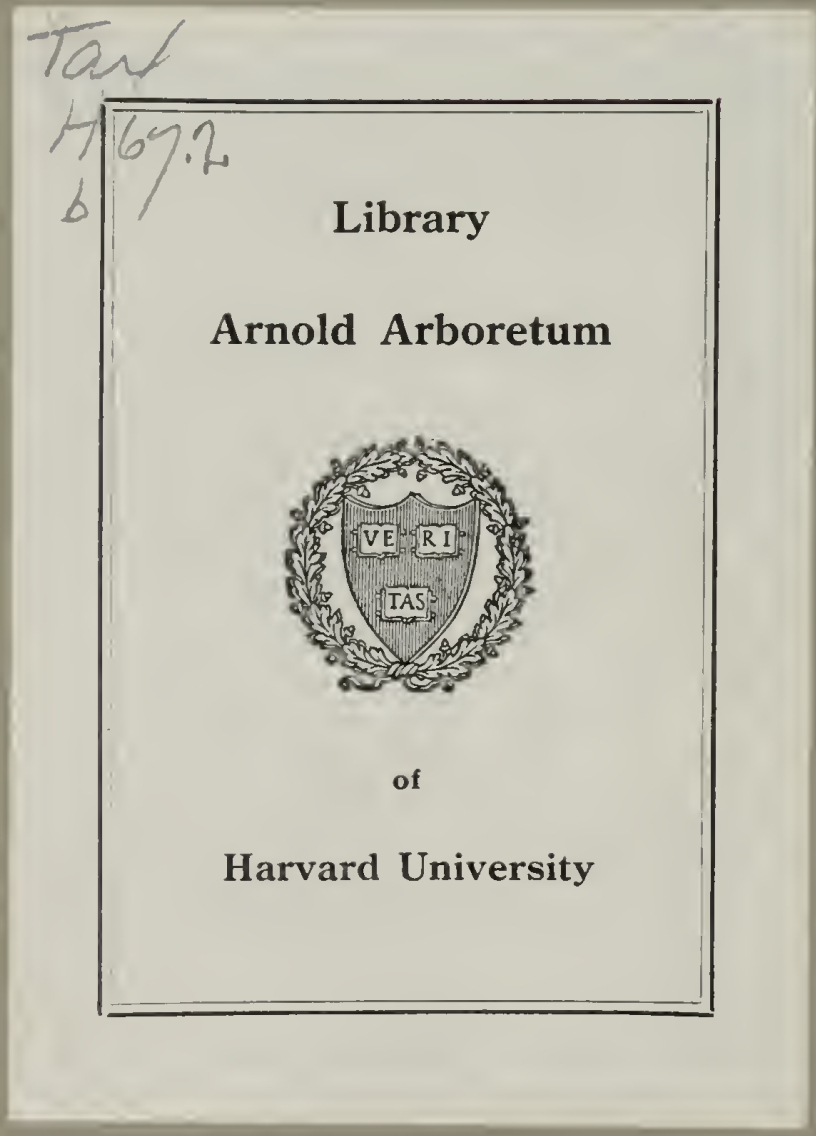


<smiles></smiles> 

Digitized by the Internet Archive in 2017 with funding from BHL-SIL-FEDLINK

https://archive.org/details/botanischerbilde00hoff 



\section{\# 2.ำ}

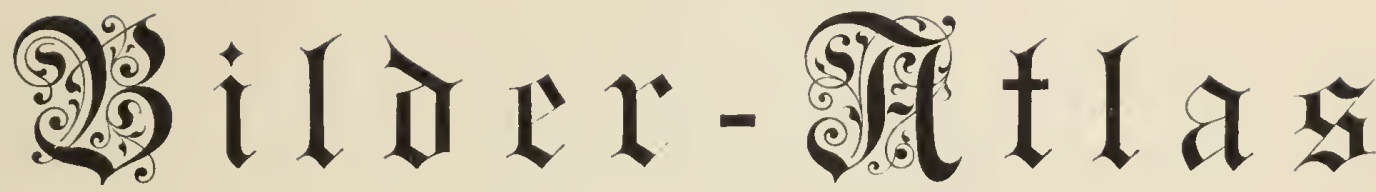

natj

Di

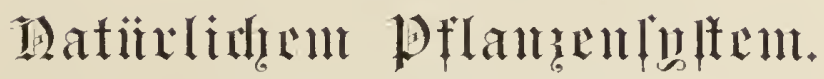

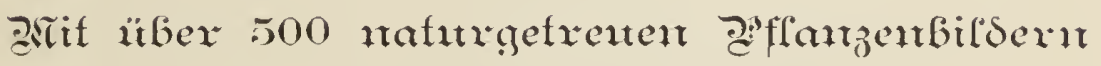

auf 85 frim Volutimente Tafulu

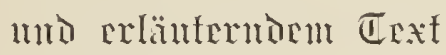

nnu

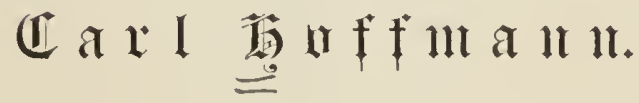

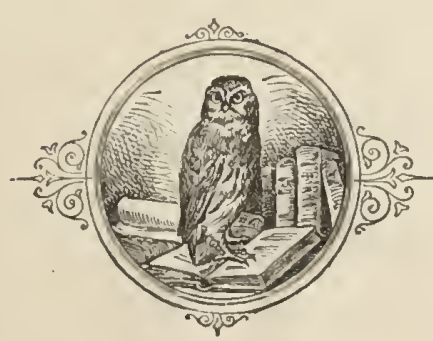

कृ $\mathfrak{f} \mathfrak{d} \mathfrak{f} \mathfrak{f} \mathfrak{g}$ a $\mathfrak{x} \mathfrak{f}$.

D.

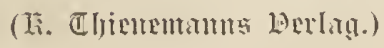

1884. 
Wllar. 22,1939

Drud ber soffmamidoen Bubbrucferei in Stutgatt. 


\section{Allacutcinc (rinlcitunta.}

Dis unz ungebende, burdi) unjere Simne mahrnehm= bare Rörperwelt, weldye wir in igren cimjelnen Teilen burdf bie Raturgcidjidfte fennen lernen, wiro von bicjer

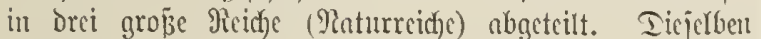
geipen:

1) Doa Etcin = oder Minernlreid),

2) bas

3) Dn: Tierreid.

Die Semutnis des Etcinreidg, Der Maineralien, nennt man Mineralogie, die dea Bflanjenreida: Botanif oder Phytologie, bie Dez gierrcid) Boologic.

Das Mineralreich enthält nur unbelebte, tote

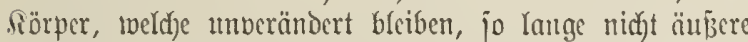

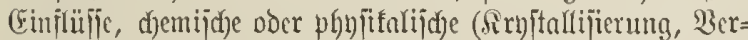
foljlung, Seldmeljung u.j. iv.), umgejtaltend auf jie cin= mirfen. Shr Dujein ijt semnad) an feinen Beitranm ge= bunden, fie ïborbauern, wenn jene Einmorfungen nidyt ftattimoen, Jalurtaujende. Eie beitizen seder (Empfin=

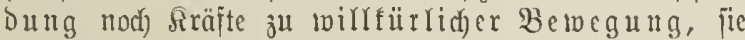

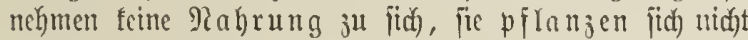

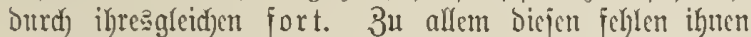
dic Drgalle ober $\mathfrak{B e r f j}$ euge.

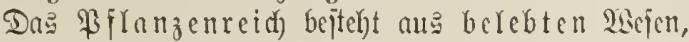
weldec, wenn aud) ohne (Empfindung und willfïr=

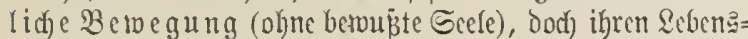

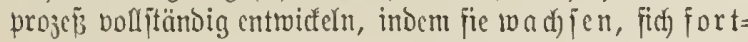
pflanzen und vergeben. Bermittelft getwifier $2 \mathfrak{B} e$

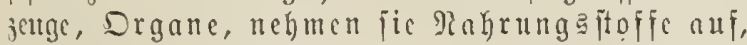
ideiben unbraudfare Etoffe auz, unb toadfil

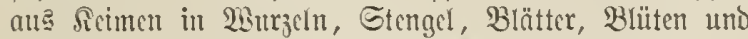

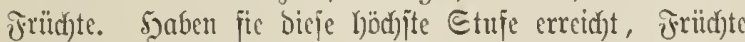

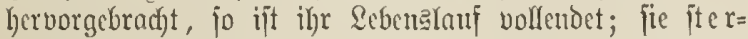
ben ab, Ginterlaffen aber zur Fortpflanjung cinjeltie, bcjonbere Srgane: $\mathfrak{B} u r z \mathrm{cln}$, Etengel, Samen u. ¡. w.

Das Iicrreidf beiteht gleidfalls aus belcbten (Bejdöpfen, meld)e Durdy Daju beitinmte, beiondere Srgane

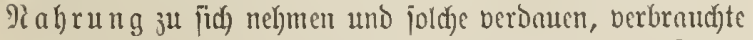

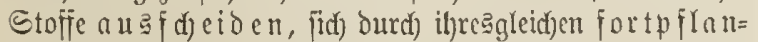

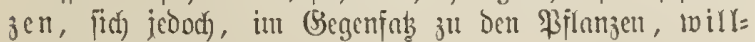
fürlid beregen und empfillden. Wirlfürlide Bebegung uno Empfindung nber fitno 2hasflifife ber Seele - bie Tiere jind demnnd bcjeclte (Bejdjppe

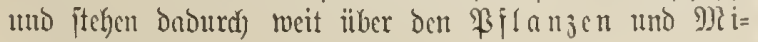
neralien.

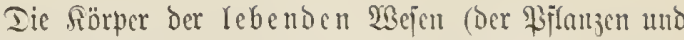

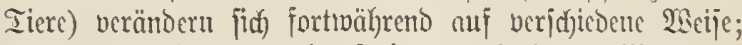

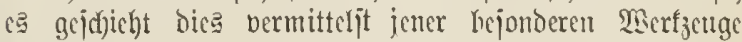
(Ergane), woeldye bicje $\mathfrak{B}$ erändorungen burd) eigene be=

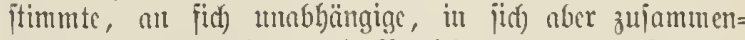
laängende Ihätigfeiten und Berridftungen Ketberfïtelligen. Soldye Drgante find bei den Tieren: die Sinnesmerf=

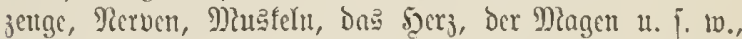
bei den Pilanzen: bic Bellen, 2Burzeln, der Stamm, bie Blüte, die Enmen.

Scocr lebende Sörper ift ein Ganjes, Defien einjelne Ieile (bie Drgane und Drganbeittnoteile) fid) gegenicitig besingen und zum 3roede bes Ganjen bereinigen. Eine joldse Bereinigung, die Gejumtheit verbundener Ergane, nent man cincu Erganismuz.

Dic mit Erganen beriegenen (Bejdjöpfe (BFfanzen und Tiere) heisen demnad) organijde, iene Rörver aber obue jolde Srgane (Dic Mincralien) unorga= nijule, anorganifde.

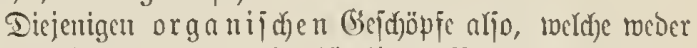
Empfindung nod) wirlfürlidye Bewegung beitsen, bitden das Bflanjenreid), mit deflen fiemutniannbue (ber Pffanzenfunde, Botanif) wir unz in biejem

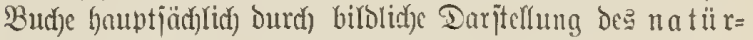

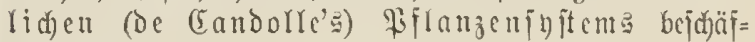
tigen wollen.

Die D.sfanjeullunde, D.:sofanik, wirb von ber

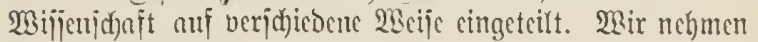
3wei Sampteinteilungen an: bie theoretijere und bie prattijede Biflamzentutio.

I. Tie theoretijd)e ober reine Botanif betradjet dic

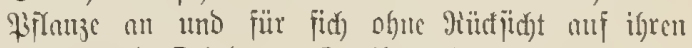
शutzen und Estrabert. $3 \mathrm{n}$ iffr gehören:

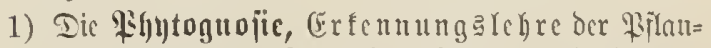
jell nad) ifareı äuß̉eren (Eigenijdaften uno Berbält= nifīin, unt

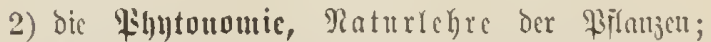
Erjorid)ung und Inrlegung der Befoge, meld)e in Der Entwifflumg, Gejtaltung und Dem Beftefen der Bïlanzen vorberrid)ent.

3ur Bhytognojic (Erfenungălehre) gehören folgende cinjelne 31weige: 
a) Iie botanifde finfiprad) (Ierminologie ober (Gloffologie), wald)e die bei Fiflangen=

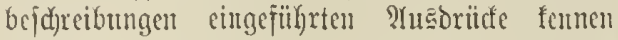
lelyrt.

b) Die Bejarteibungalehre (Phytographie) oder bie anleitung zur Bejdrcifung und $\mathfrak{B} e=$ nermutg Der einjelnen ßF̈tanjen doex PFlanjen= gruppen in शैusorücten Der finjtipracte.

c) Die Snftemfunde (Iaxonomie), melde die Regcln und (Srumbjätse barlegt, die bei ber

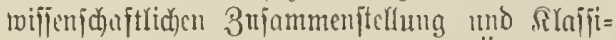
ftjierung der ihrem Bau u. j. w., zu bejolgen fint.

d) Die \$iflanzengeographie, Mngabe der Wer= brcitung ocr PFlanjen nad) Baterlano und Stano=

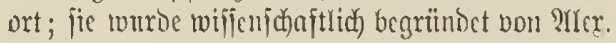
vou \$ृumboldt († 1859).

Die Phytonomie, die Paturlebre der Pflan= jen, fajit in fidu):

a) Die Giftaltlehre, Formenlehre (Morpho= Iogie), dic Betrad)tung der Drgaue der Prilan=

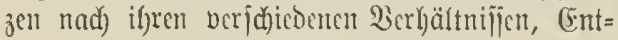
tridtelungen und IImtwanblungen.

b) Sie Bflanjenanatoute (\$lyntotomie, sjito= logic, bflanzenzergliebcrungalebre, (Ge= wcbelebre); jie criprjadt den inuern $\mathfrak{B} a$ der Brlanzen, ibrer Srgane im einjelnen und im 3ujammentange, potvie iffere d)emija)en Bejtand=

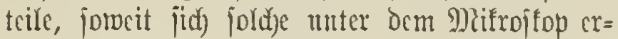
femuen \aijen.

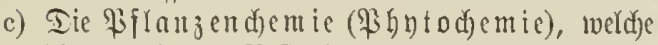
die demijajen Beftundtcile und Mijadungaber=

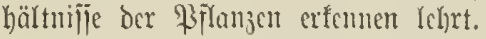

d) Dic Lebenzlehre (Biologie, łhyfiologie). Sie jucht die Berbältnifie der organifchen Iłätig= feiten iu \&eben Der \$iFlnujen iut gejunden $3 \mathfrak{u}=$ ftande, in ilyrem 23odjatum, ihrer ?fusbildung, Fortpflanjuttg u. i. w. ju crgründen.

e) Die frantheitalebre (Pflanjerpatho= rogie).

II. Die praftijde noer angewande Botanif Yebrt die

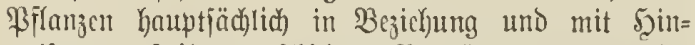
locijung auf ihre beridficome Benẗbung, atrf ijre

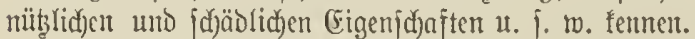
Sie beljandolt

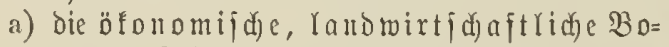

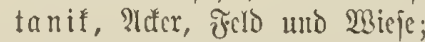

b) die Gartenbotanif, Dbit, Sïd)engenädje, 3icrpflanzen 2c.;

c) die Forjtbotanif, den Marb;

d) Die medizinijd =pharmaceutijde Bota= nif, Ir

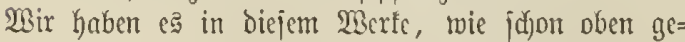

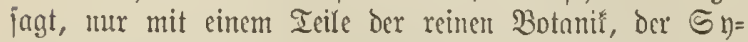
ftemfunde und ztoar pecicll mit ocr Darftellutg be natürliden Pilanzeningtems nad) de candorle ac. in Wort und Bild ju thu.

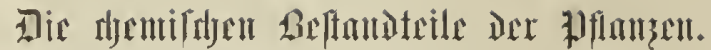

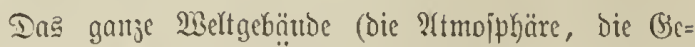

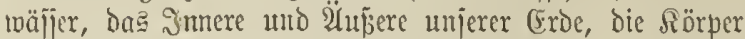

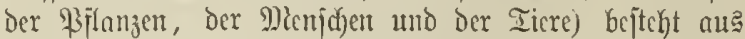
etma $70^{*}$ ) Utritoffen (einfad)en Stoffen, Ele = menten) mit ihren verjusdenen श2ijd)ungen und $\mathfrak{B} e r=$ binoungen; dieje (relemente teilt man in metallijd) (Metalle) und nidjtmetallijode (Midutmetalle).

Die gridutmetalle fino Saueritoff, diolylen= ftoff, Stiufitofi, SBafieritoff, (5hlor, joo, Brom, Flnor, Gdimefel, Gelen, Iellax, Phos= plyor, arjen, ficjel, Bor.

Die Metarle find 1) bie jog. (eid)ten Metnfle: falium, Ratrium, \&ithium, Barium, (salcium,

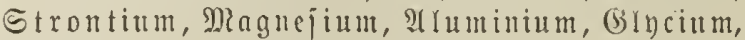
Birfonium, gttrium, Ihorium, cerium, san= that, Dionm, Erebium, Ierbium; 2) bie jog.

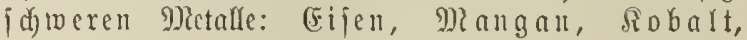

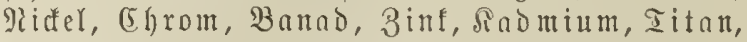

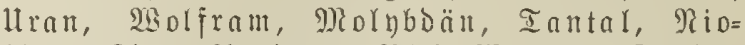
bium, 3inn, Intimon, Blei, 2Bismu, fupfer, Enedifber, Silber, Rhodium, Dsmium, Jri=

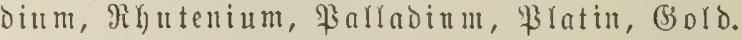

Die Eremente find demiju) unjerlegbar; fie foumen

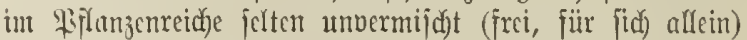
bor, es entitel)en aber burd) ihre veridjicoenen Berbin= bungen und 9lijidungen bicle neue, mit ganj anderen

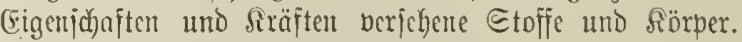

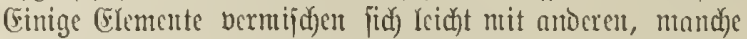
aber and fo jofwer, Dnis die betreffende Bcrcinigung mur ourd) fümitlide MRittel zo beiocrfjtefligen ift; man ucunt dieje (Figenjd)ajt ber verjdiedenen (Elemente: biele ober geringe (d)emijac) Berwandijaft, If ffinität. 3rei bercutigte (binär berbundene) (Elemente bil= ben die unorganijaen Beitambteile; orei= oder vier= fad)e Bereinigunga (teruäre mo quateruäre Ber= binoungen) die organifden ober uäheren Bejtandteile der Pilanjen. Eritere, Die binäre, Berbinountg madjt $90-99^{\circ} 0$, bie leşteren, Die tcrmäre und quaternäre, madjen je $1-10 \%$ bor Hiflarzentörper aus.

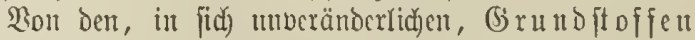
oder (Elementen fommen etra 15 in Den श)enjd)e uno Sicrtörpern, etwa 20 im Filamjenreidje vor.

Die im łffanjenreide vorfommenden find die folgenden uto fügen wir bei jcoem bcriclben bie wiffen=

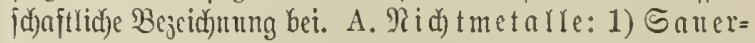
ft tofi, Oxygenium (O.); 2) Soblenft off, Carbonium (C.); 3) Mafjeritofi, Hydrogenium (H.); 4) Stiuf= itofi, Nitrogenium (N.); 5) (5hlor (Cl.); 6) jod (J.); 7) Brom (Br.); 8) Fluor (Fl.); 9) Sd) ruefel, Sulphur (S.); 10) Bhoâphor (P.); 11) Siejer, Silicium (Si.). - B. Metalle: 1) fialium (Kia.); 2) Ratrium (Na.); 3) Carcium (Ca.); 4) Mag=

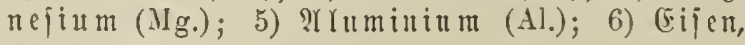

*) Yad) neueren Unterfudutrgen fino einige berfelben feine Grumb $=$, fonbern 3ufammengefezte Etofie. 
Ferrum (Fe.); 7) Mangan (Ma.); 8) 3int (Zn.); 9) Rupfer, Cuprum (Cu.).

Dic eben genannten metallijd) (anorganifden)

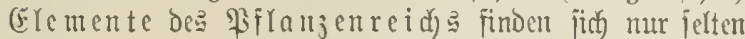
und in geringer Menge in ben \$ilanjen; fujt iumer aber in ber Geftalt bon Saljen, o. h. als Berbin= dungen ifrer Dryde mit organijden ober antorganijden Sä 11 ren.

Tie vidftigiten: $\mathscr{f} a \mathfrak{l}$ ium, $\Re$ atrium, (5arcium, fommen nid)t frei in ber Patur vor und aud ifre Ernde, Rali, $\Re$ atron uno $\mathfrak{R} a l f$, juto megen igrer grobent Bermandidjaft mit den Säuren intmer mur als Salje allutreffen: Der Ralt ala fohlen=, pho aphor= und ichme $c=$ Felfaurer Ralf; Daz Ratron als fohlenjaures (Soda) ober jalyjaures (fodjjalj) ober ale jofrwejeljaurez Ratron ((S)lauberjalj); dos $\Omega$ a li als foblenjaures (Plottajde) ober falpeterjaure Rali (Salpeter).

Tie nid)tmetallijden (organijden) (Elemente

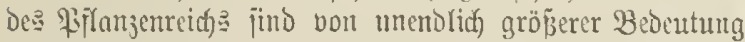
als bie metallijon; am meitten fommen von ihnen vor: Der Sanerjoff, Der Sioblenjtoff, Der $2 B a f j e r j t o f i$ und ber Stidftoff. Dieje vier finden firt in allen

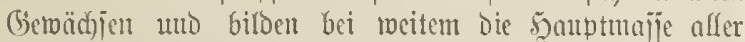
orgamijhen förper überbaupt; fie verbinoen fidf in un= begrenjter WBeije nid)t mur miteinanber, jondern aud mit

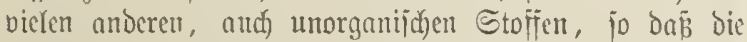
?fnjahl ifrer Miidjungen ins Unendliche geht. Man be= jeidfuet fie ifgrer gropen 2Bidtigfeit megen autd in all= gemeiten als: bie vier organijden (Elemente ober Drgandene.

1) Der Saueritoï, daz Saneritoffgas, Drugen (ङäureerjenger).

(Fin luftiörmiges, furb=, gerud)= und gejd)madtojes

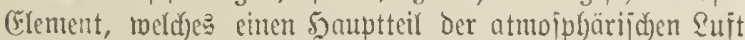

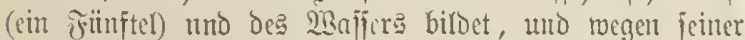

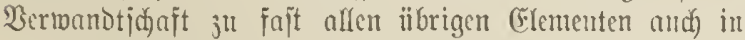

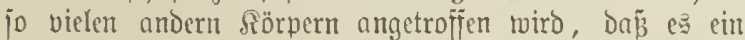
volfes Iritteil bes Materials jum 2ufbau unjer (Erbe,

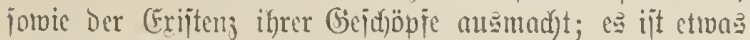
jd)werer ala die atmojphärijate \&uft.

Sein Gidfverbinden mit andern fïrpern (Eijen, Rupfer, Mangan, Binf) nemt man Drybieren (baher (Eijen=

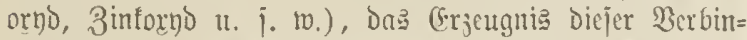
Dungen Dxyd. Die Dryde find von juecerlei IIrt, faure

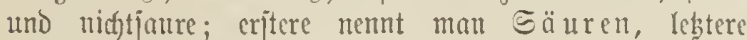
Bajen; beide juto nahe bermant und berbinden jid ju den jogenamiten Saljen.

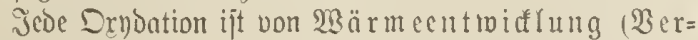
brenttug mit ober obne jidftbare [Sidftentwidfung] Flamme) begleitet; bie unvolljtändige Berbrenmung gejdicht ohne jidftbare Flanme und heifft Berfohlung. Beim rojtenden Eijen findet ebenjo, roie beim Berbremten

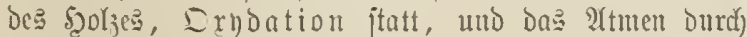
unjere Sungen ijt ebenfala ein $\mathfrak{B}$ erbrennen, weăbalb Denn aud Liebig ben menfoliden fïrper mit cinem EFen vergleidft.

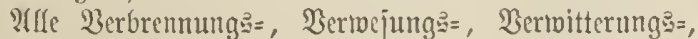

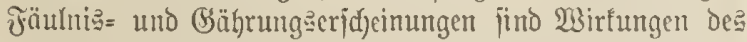
mit einem anberen (Elemente jid) berbindentben Gauerjtoff?.

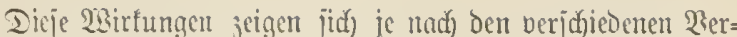
bältnifjen janeller ober langjamer: ganj diejelbe 2Bärme

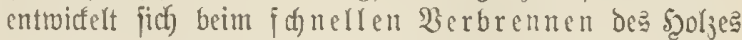
Durd) Fetter, wie bei dem langjamen Bermejen des Şoljes, im eriteren Falle jonell und voribergebend, in letsterem unmerflid) bei jabrelanger Iauer.

Der mit Sohlenit off verbundene Gauerjoif heiñt Soblemiäure, Der mit 26 ajjerjtofi verbumbene $\mathfrak{B a} j=$

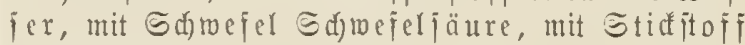
Salpeterjäure, mit (salcium $\mathfrak{R} a l \neq$, mit $\mathfrak{R a} g=$

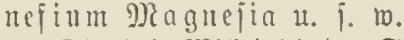

Die hohe Widjtigfeit Des Sauerjtoifß für dic Brlanzen= welt jowie für Menfiden und Iiere finden wir reiter unten gejưfildoert.

2) Der Siolfenitoif, Carbonium, carbogen (Foblenterjeuger),

ijt ein fejtez, gerud)= uno gejd)madflojez (element, meldhes am remiten (fryitallijert) ale Tiamant uno Graphit uno (amorph, gejtaltlos) in Der Soble, dem Ruj u. j. w. vorfommt, mit auberen (Elementen berbunden aber in allen organijoden Subitanjen ju finden ijt. Crr bildet Den 5ूaupt= bejtandeil Der fiolile, was ifm den ?kamen gab, wird aber audf) Phytogen (Bïlanjenjtofferjenger) gentannt,

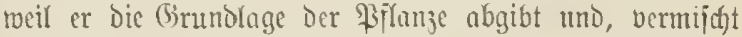
mit Gaueritoif unD Wajïerjtoï, Die meijten BFlanjentör=

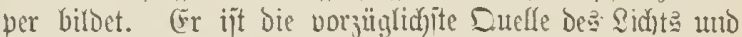
Der $\mathfrak{B a ̈ r m e , ~ j o t v i c ~ a u d ) ~ D e r ~ j o f m a r i e n ~ F a r b e . ~ S e i n e ~} \mathfrak{B e r}=$ binoung mit Saurertofi, Deren Probutt man fioblen= jä urc nennt, ijt für Menjujen, Sicre uno Brlanjen, be= jonder als Szauptnabrunganittel ber lebteren, jorvie als Bejtandteil Der jogenamten fioblenjäuerlinge, ala ein Ieil

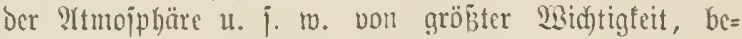

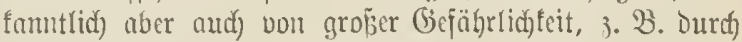

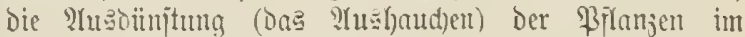
Dunfelt, die Etiffluft in fillern und Brumen น. ¡. w. Daz $\mathfrak{B a f f e r}$ von Euclen und Brunten, reldjes vicl foblemiauren fialf entyält, nemt man harte 2 Bajier. Berbrennt man sely im Freten, io verbindet fid. Der Subleujtofi mit dem Eauerjtofi Der \&uft und entid)mindet

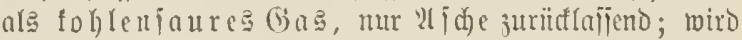
aber beim $\mathfrak{B}$ erbrenten Der 3utritt Der \&(tmojphäre berfin= Dert, jo fanm Die genannte 2 erbindung nidgt jtattintoen und es bleibt (beim liohlenbremen) die Pfranjento hle ala beintale reiner fiohlenitorif jurtid.

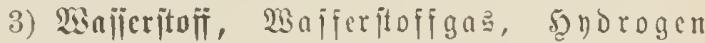
(2Baïererjeuger),

ijt cin leidhter, farb=, gerudf)= unto gejdmadtojer, ไuftjör= miger, bremtarer Grunditofi, weld)er in ber Matur nir= gends ungemijat (jrei), jondern ftets iu Bereinigung mit

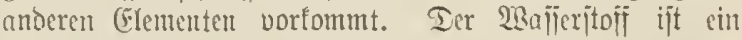
Sauptbejtandecil bes 2Bajier: uno in fajt aflen piflanj= lichen Enbjtanjen in grō̄en Mengen ju finden; Das

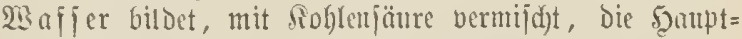

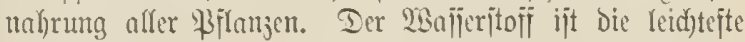
aller Enftarten und wird beähalb audf jur F̈̈lllung ber Sujtballonz angemendet. Mit Stidtoff verbunden gibt er 2rmmoniat; mit (E)lor, jod und Brom die

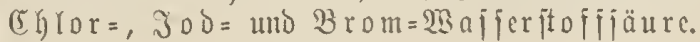


4) Etiffitufi, Stiffitoffgas, \{rot, Nitrogen (Salpetererjeuger), iit cbenip, roie bic borigen, cin luffförmiges, far $b=$, ge=

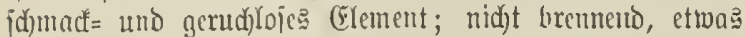

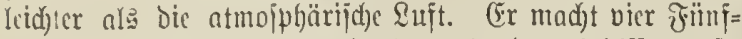
teile ber Iebteren aus und hat als bas indifferentejte

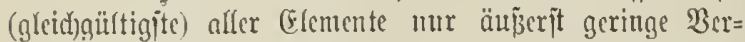
toandfidgaft ju Den übrigen (Erementen. Dod) billoet er (gebmben, unfrei) bie (Grumblage Der meiften terija)en $\widetilde{S} \mathfrak{b}=$ jtanjen, jowis namentlid) ber Getreibcjamen uno Şïljen= früd)te; bieje Sïrper gehen aber, eben wegen ber geringen Bermantijaft be Stifitoffe zu ambcren (ETententen, Durd) Berbindung Desjelbeu mit Dem Ma\|jeritofï, feidyt in 3er=

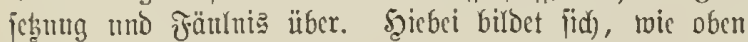

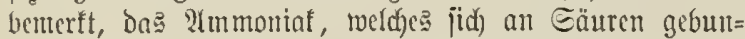

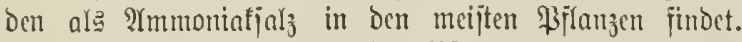
Die Ammontatjalje werben der \&iflanje atte dem Boden Durd) Berjeşung jticfitofiflaltiger, organijder fïrper juge= fithrt, weshalb Denn aud) junge Bellen megr Sticfitoif entfalten als alte, und junges Bras bem $\mathfrak{B}$ ich gebeif $=$ lidyer ijt ale altes. Den Ramen Sticfitofi ober 2(zot hat bicjer Grumbjtoif Daher, weif er für fid) arlein Das Seben ber Menjigen und Tiere, forwie and jede Flamme jum Berlöidyen bringt ober er it icf t.

Mit Rohlenftoff verbunden bildet ber Sticfitoif Das gifftige (5nall, mit Saueritoff berifiedene Srvontions= jtufen, jo bie Ealpeterjäure, toelde fid) weit ber= breitet im Galpeter findet und jur Bereitumg bon Sd)efeliäure, Bhoaphorjäure, Sdiep̧bam= tolle, 厄djęppuloer u. j. w. Dient.

श्uber bicjen 4 Drganogenen fitto nod) von eini= ger Bedeutung fïr bic $\mathfrak{B}$ flanjen folgenbe, cbenfallz nid)t=

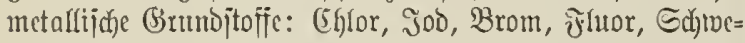
fel, Bhoaphor und Sitejer; fie finden jift) aber weniger häufig uno in geringerer Menge in Denjelben.

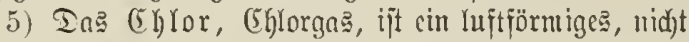
bremnbares Ersement voll grïnlidjer F̈̈rbung uno fted)en=

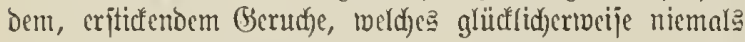
fret uno unbermijeft in Der Natur vorfommt, in Berbin= oung mit anderen EXementen aber äußerjt tvidytige, für

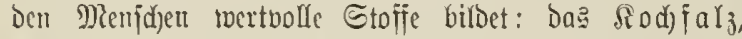
Egloroform, den Ehlorfalf, die Salziäure.

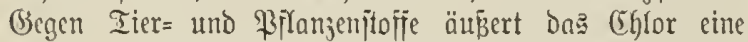
jd)nell zeritörente Wirfung, tweâthalb ę aud) zum $\mathfrak{B} e r=$

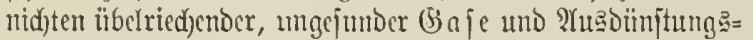

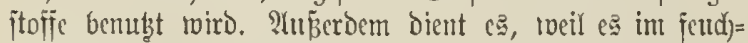
ten 3ujtanbe alle organijden $₹ a r b e n$ idjefl jerjtört, in Berbinoung mit Suly = ober Sdjweferfäure, zun Bleidjen des ßapiers und ber Berwebe aus Biflamjenfajern. (eflor finbet jid), immer bermija)t mit anbern Elcmenten, in allen im Saljibajier ober anf Ealjboden wadjjenden Brifanzen.

6) น. 7) Эob und $\mathfrak{B r o m}$ find cinfad)e, nit bem (5Glor verwande Grumbitofife, weld)e in allen Meer = uno Solijplanjen, namentlich) in Den Ianget, enthalten findo. Das Job twird in verjdjicocnen Berbindungen in Der Photograpfie und Mebijin gebrantat, and zur (Betvinnung

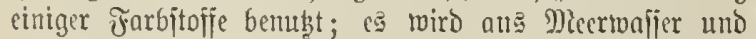
Micerpflanzen, Den jogenanuten fielppiflanjen, burd) Dejtil=
Yation 4. ₹. w. getromuen. Bon den daraus fabrijicrten Sirnjtallen probuljiert Edyottlano jährlid) tiber 75000 , Franf= reid) über $55000 \Omega i l_{0}-B 3$ rom triro weniger aus MReer= maijer, als alts Goolquellen getwomen. Seine Bertwen= bung ift bic des JoD, weldjes immermefhr bom Brom

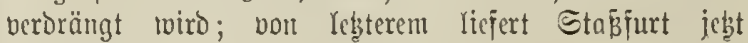
jährlid) iiber 7500, (Equrdjill (Eounty über 10000 Sito.

8) $\widetilde{J}$ I uor ift gosförmig, farblo?, nur in Berbinoung mit andoren Ercmentent vorfonmento. Mit $\mathfrak{B a f j e r}$ itofï ver=

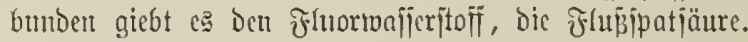

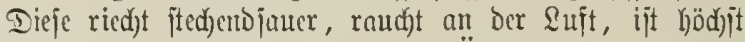

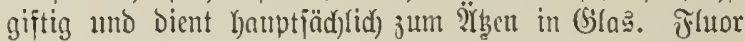
foum in geringer Menge in ben Pillanzen unt aud) ebenjo in Dem Edjuthe der 3ähne uno in Den Snodjen ber Menijen unt Tiere vor.

9) Dir $\Xi$ d) refel, Sulphur, ift cin ziemlid) ver= breitcte feites, gelbes, Yeidjt verbrennlidece (Element, wel= d)e gebiegen uto in Verbindung mit anbern Brunditofifen vorfommt. Mit $S_{a} u \in$ ritoff berbunden entricfelt er

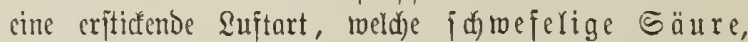

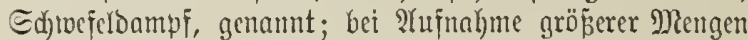
von Saucritofif entifteht $\Xi$ dueferjäure (Bitriolöl). Mit WBafjeritoff berbindet fidf ber Sdywefel zu Dem, wie faule Eier ricdemben Edinefelwafieritofigaz, bem d)arafteriftijajen Beitandeil ber heiffräftigen E(j)wefel= wajier. Der Sdjwefiel findet fidid in frorm iftivefelfaurer Snlje fuit überafl im Piflanjemreid), in Berbindung mit

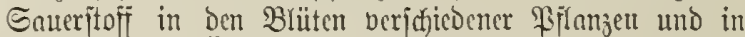
Den ätherifden B̈ren ber Sdjotengetwädje. Die zu finem Bulber verobifteten Edjwefeldämpfe fommen ala $S$ d w we $f$ e $=$ blumen, gejdymolzen und in Formen gegoifen alz $\subseteq_{t a n}=$ genjidwefel in ben Sambel. Der Enfinefel bient jur

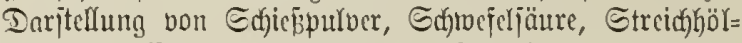

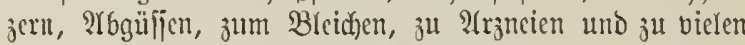
anderen tedjuifden 3rocefen. Italien لiefert jährlidy gegen 350 Miflionen, Epanien 4 Miflionen, Deutjulano mit B̈̈treid) etroa $1^{1}{ }_{4}$, dą ïbrige (Europa ${ }^{3 / 4}$ Millionen Sillo Extivefel.

10) Der łhoaphor (Sidfträger) ijt cin feîtes, getblid)e weldes ijid) jajon bei gewöhnlidyer Temperatur mit bem

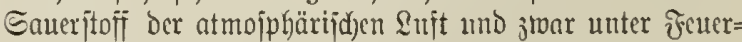
crid)cimung verbindot und beâhalb jeffitt im Dunfeen leuctetet.

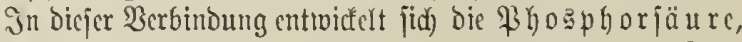

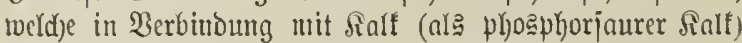
bie fejte Bruntalage Der Biflanjen=, Tier= uno Menjajen= förper bilbet, too er jid) Dann bejonders reidfidid in Den Sinoden und Dem Fleijd) Der Menjajen und Tiere, jorwie in ben Samenhyinflen fajt afler Broäjer findet. Er bient ju veridjedenen tedjuifden 3locefen, ju Büntwaren, als Irrjneimittel und als hejtig toirfendes Rattengift; bie Bhosphorjalze haben vielfadje Bertwendung aud in Der Sanbmirtidaft.

11) Siejel, Silicium, fommt in Berbinoung mit Saucritoff als sicfelerde fajt in allen Prianzen vor umb findet jidu in größeren Mengen bejonocrs bei ben Echaudtellgalmen uno Bräjern, Deren Salme der Ricjel= eroe ifre Saärte und Feftigfeit verbanten. Die Ealje 


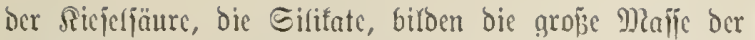

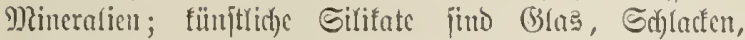
23aj̄ergla:

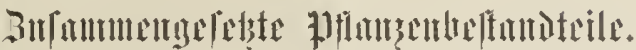

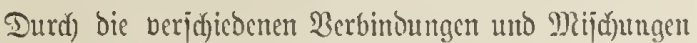
der oben behandelten Grundit offe (Eemente) entiteben Die zujammengejebten, die eigentliden vegeta= bilijacen Gnbitanjen, aljo alle flüjiigen unb fejten

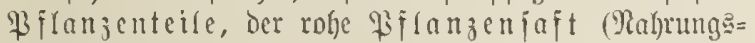
und $\mathfrak{B}$ iloungăfaft) mit jeinen jahlreichen Darang entjteben= den Subitumzen.

Dicje zujammengejebten Bejtandtcile Der Piflanjen teift

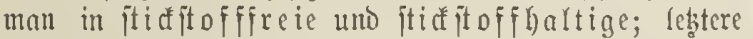
werden aud Proteinjofife genannt uno bejtehen in

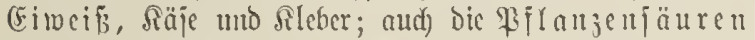
umb Pflanzenalfalicu fömen, da jie einigen $S$ tid it ofi enthalten, bazu gered)net weroen. Sic it id it ofi $\bar{i}=$

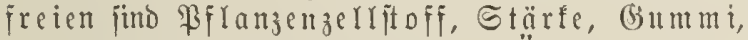
Buffer, Beingeift und dic fetten Ör

1) Die veridjiebenen $\mathfrak{B}$ fYanjenjäuren entiteben aus Berbindungen des Satreritofif mit Sohlen= uno

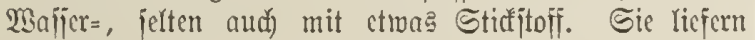

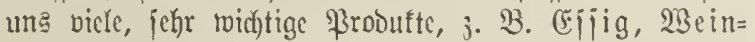

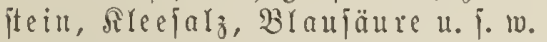

2) Die Pilanzenalfarien (arlfaloide, orga= nifa) $\mathfrak{B}$ (ajen), Berbinoungen von Sancr=, Sohlen=, 2Bajer = uno Sticfitoff. Sie enthalten bie fräftigiten Irrmeien, aber aud) bie hejtigiten Biite. Wir mennen

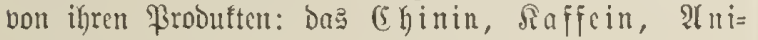
rin, Ritotin, Marphin, Solanin, Strnduin und Beratrin.

3) Die (5imcisptoffe, Mrbuminjoffe, Fro= tein jtofie (tierijd)=ucgetabifijace Stoffe). Sie jumb jtick=

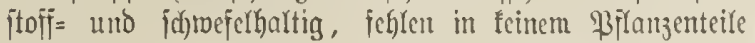
uno fino atd) in allen Tiertörpern vorberijchent. Sie

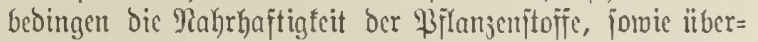

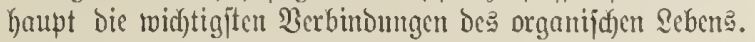

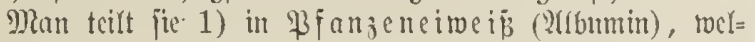

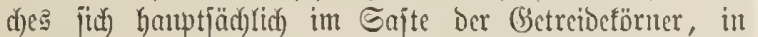

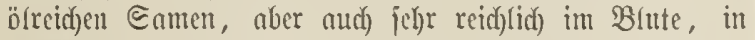
ben (Eiern u. T. 10. Finoct; in Räjejtoif (Sajein), haupt=

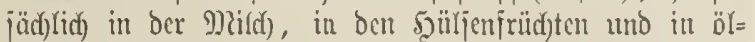
reid)en fiernen und Samen; 3) in Sileber (PJïlanjenlcim, Prifanzenfibrin), เoclder in jemer d)emijd)en 3ujammen= febsung fajt ganz dem (Eiveifs gleid)t, in alfen Yebenden 3ellen cutharten und cin Saauptbejtandeil Der Getreide= törner ijt. - Sas (ritwe ib ijt bemnad) hauptiächlid) Nahrungamittel; es roiro aber audd in grober Menge in

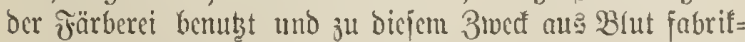

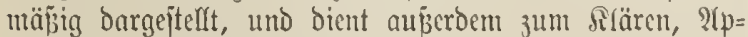
preticren, Grunbieren, アitten u. i. to.

Den Scautbeftandteil, das Gerippe alfer \$iflanzen, Die (Sruntolnge aller Pilanzengemebe, Die 2Bandmtgen

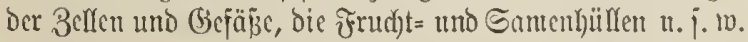
bilibet

4) der Bifanjenfajeritofi (Memoramitoif, 3cll=

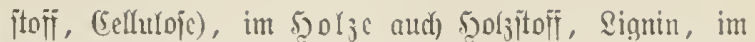
Marfe Medulfin, im Rorfe Suberin, in Den $\circlearrowleft$ d) เö $m=$

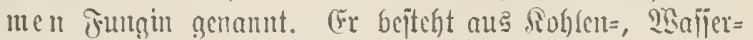
uno Sancritoif, ijt elajtiju), fïr alle s(iijitgfeiten ourd)=

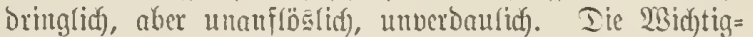
feit bicjes Gtoijes fïr bas praftijd) Seben ijt jebr $b c=$ beuteno; jeine Kauptiäd)liajten Prooufte jino Banmmolle, Flad)s, ฐुanf, Siorf น. โ. 10.

5) Tie Stärfe, dą Stärfe= docr Satzmeld, Amy-

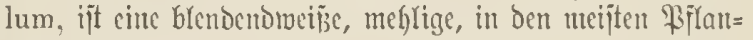

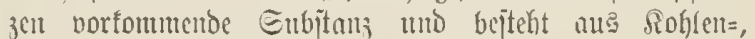

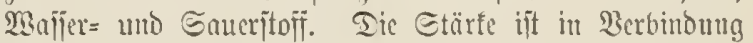
mit ben (Eimeiz̄itofifen Der nahrhaftejte Ieif ber Pflanzen utto findet jidt) bejonders in gropier Menge in ben $G e=$ treidejamen, Den Sjülienjritaten, Den fiartofieln, im Marf Der Eagopalme (dem edfen Eaģo), in Der Picilmurjel

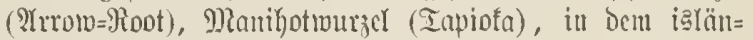
bijd)en Mooje und andern Rahrungapplanjen. Cie ijt in

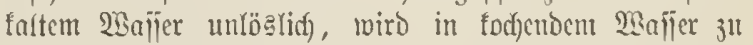
Deul befannten Sleijter mo Durd) weitere Bebandlung

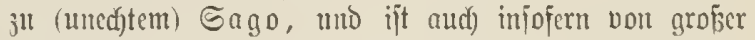
WGid)tigfeit, ala jie leid)t in (Gi um m i (Dertrin, Stärfe= gumui) vermanoclt merben faum, aue beldfem bam weiter Iraubenjuffer uno 23 einge i jt bereitet werden.

6) (Sum mi uno \$if anjenfdy)leim gleid)en in ihrer 3ujammenjebung Der Stärfe. Reine 65 um $\mathrm{i}$ ijt im trocfenen 3ujtande hart, jpröbe, ourdjildytig, leidjt in

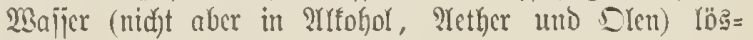

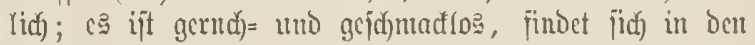
Gummigängen vieler ß̊̈lanjen, quilft getwöhnlid) ans Den acborjtenen Binden herbor uno erhärtet bamn an ber \&ujt. Dic verjojiedenen ?rten (arabijd)es Gummi, Tragant, Da:

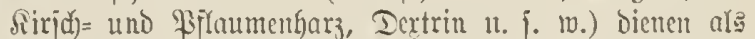

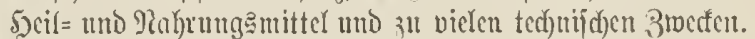
Bejonders vidytin ijt das Dextrin (i. Stärfe) als

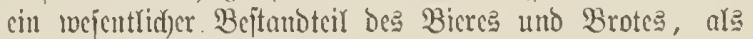
3ujab ju feinem Bactwerf und in jeiner llmwanblung

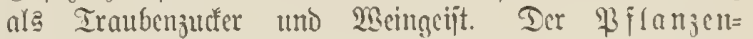
id) lcint (Iragantid)lcim, Salcpid)lcim, Ecinjamen= unb

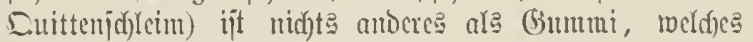

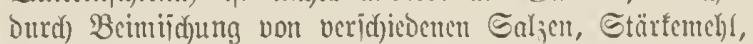

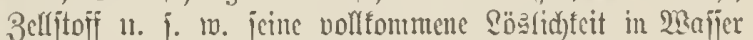
verforen hat. - Dą Gummi elastic u m (Riutid)uf,

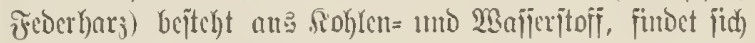
im Mild)jait vicler Bjilanjen und wiro bejonters aus ber iiibancrifanijd)en Siphonia elastica mo ber indijdyen Urceola elastica uno Ficus elastica getwomren.

7) $3 u$ exer (Saccharum) ijt ein im Pilanjenreidye jefre verbreiteter Stofif, weldyer in vericficoenen ?rten

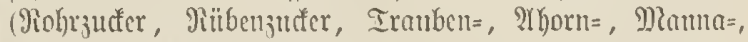

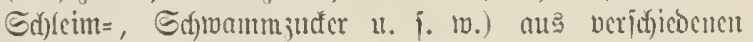

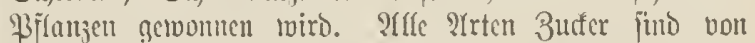

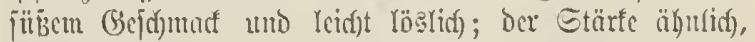
bejteben. jie aus fohlen=, 2 anfifr= uno Saucritoff. Die Bernenoung bes 3uffers ift befanntlich) äujerjt mannig= fad); er bient in jeinen verjojicoenen Prten uno Gejtal=

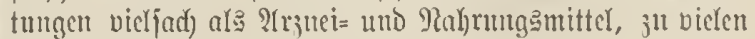

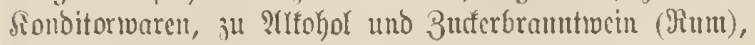

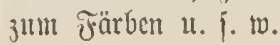


Die 3ucferfabrifation bejöäftigter melgrere Milfionen

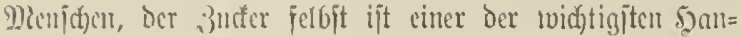

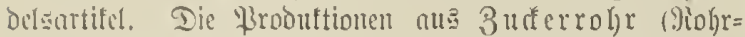

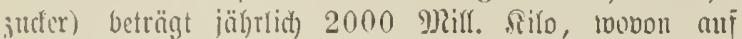

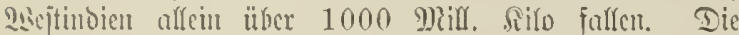

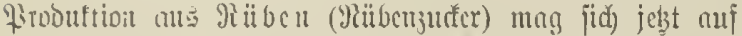

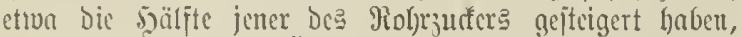
Ivovon Deutichland mit S̈ jtreid) etwa $300-350$, Frantreid)

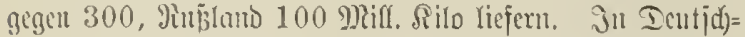

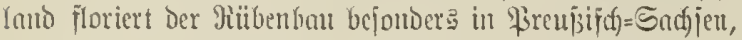

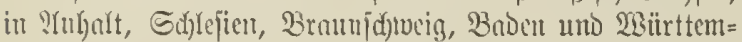
berg. - Die ?h grut=3ucterfabrifntion in ?orbamerifa licfert den ?Ifyornjuffer, weld)er ebenfallz fein unbebenten= der sjandelantifel ijt.

8) Die fetten D̈re aus dem \$iflanjenreide (bie Ziflanjenjette) fino, wie Der 3utfer uto die Etärfe, ftict=

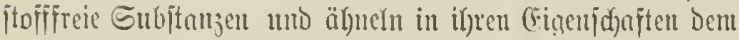

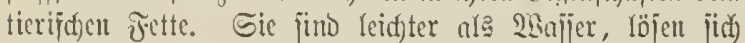
nidyt in Demjelben, wohl aber in Ylether und fodjendem

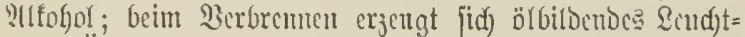
oder D̈rga. Fette Dle finden fitid bejonders in Samen (Mübjuat, 5zanf, 9)?ohn), Frudjtternen (Mandel=

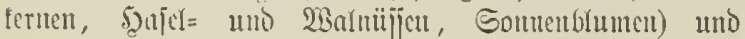
Friid)ten (Dliven).

Einige Diejer D̈re trodnen in Der \&njt jut einer Durdjidseinenden, feiten Manje unb tocroen ju Firnifjen umb Drfarben bemtbt; ju ifuen gebören dos Scin=,

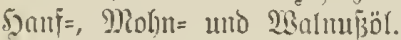

Yubere verdiden fich in ocr \&uyt uno breiben jaduierig uno

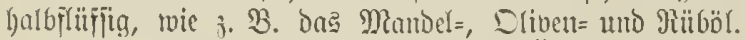

3u Den fejten, butterartigen D̈len! gebört Das

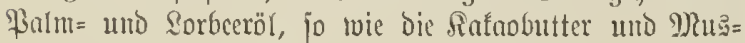
fatbutter.

Die fetten B̈le fülo für don Menjd)en von gröpjter

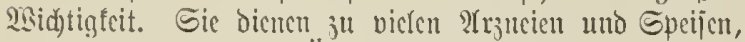

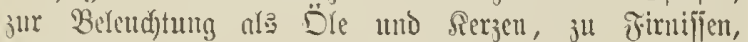
jarben Into vielen anocren ted)nijdjen 31weďen.

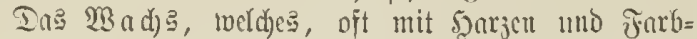
itofïen verbunben, in ben meif́ten Pillanjen, bejonders im Silüteritunbe vorfommt, glcid)t in jeinen (Eigenja)aften Den jetten D̈rru.

9) Die ätherijden (früd̆tigen D̈re, (Ej门enjen, twerben Durd) Depifflation obcr and) burd) \$refiung nu:

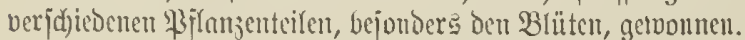

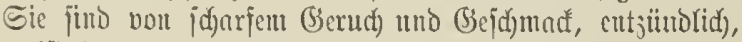
mcijt jorbloz, Ycidjter alz Bafjer, in biejem wentig, in ?rlfogol, ?ether und fetten D̈ren Yeid)t löslid). Gie jind frei von Sticjitoif unb bejtehen auz Jiohlen= und $\mathfrak{Z}$ a itoif, odcr aus fiolylen=, 2Bajper = uno Ganeritoij. 3u criteren (ogne Soncritofï) gehören Terpcutin=, 3itronelt=, Pomeranjenblïten=, Ritimmel=, Fend)d= und গelfenöl; ju ben Ickstern (mit Snucritoif) Pfefferminj=, franjeminj=,

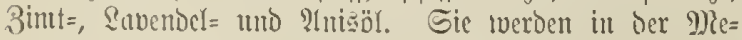

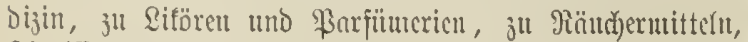
Fimifien u. j. 10. vermentot.

10) Die jarze jind cigentlid) nichte ale orydicrte,

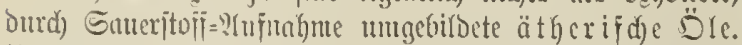

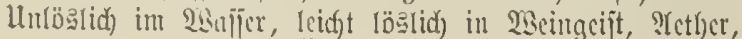
fetten uto ätherija) b̈r
Sic finden jidd nie ganz rein, mcipt mit Farbftofifen, ätherijd)en ذ̇ं

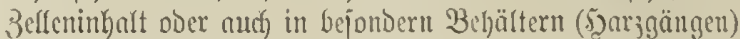
in veridjedenen Pilanjenteilen. Die tvid)tigiten jino Fidftenlyarz, (Elenti, Eanoaraf, 9)?aîtir, Danmar, Gummi= lacf, Sopal, Storar uno Benjoe. - Sie bienen ju Snden und Firnijien, ju Geip̄n und Ritten uno vielen auberı ted)nijden 3rocten.

Einige anore vegetabilijde Subitnnjen, bie Bitter= itoffe, Baljame, Färbjtoffe, tömen roir ljer nur itt Sïrye crmähnct.

3u den Bitteritoffen gefört bas 2rojinthin im Wermut, Irmygoalin in bitteren Manden, (5etrarin

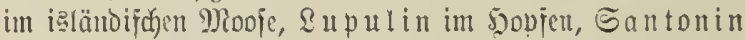

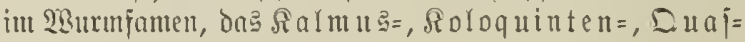
jia $=$ bitter, u. a. m.

3u don Barfauten (Sdyleim= oder (Summi=lyargen): Sdfllad, 2Beigraud), (Summigutt, Sopaivbal= jam, Mreftabaljam, Tloe t. a. m.

Dic mictstigiten färbjtoffe liejern: Der Srapp, Das firapprot umb firappgelb; der Saflor bas

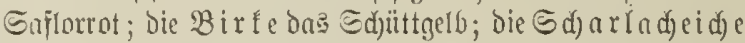

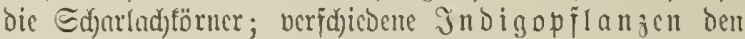

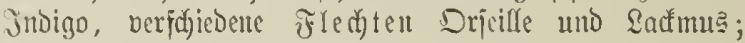

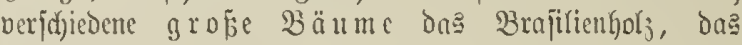
Gimmigutt น. โ. เา.

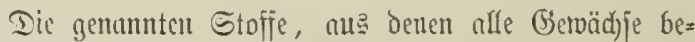
ftelyen, jino teile verbremulich, teils unverbrenulid). Die in Der Bflange enthaltenen 4 Drganogene verbremen in ifren verjdiedenen Berbimbungen ju fioljlenjäure, $\mathfrak{B a f j e r}$,

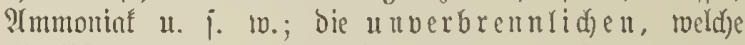
alle Piflanjenteile nad) Dem Berbremen (der 3 erjetzung,

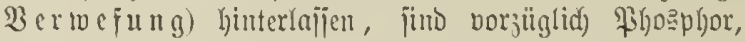

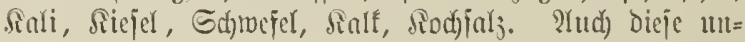
verbrennlid)en Seile find, twie bie verbrenliden, ivid)tige

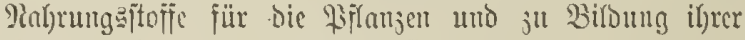
einjelnen Teile unenthelyr(id); fie bebingen, nadjoem jie

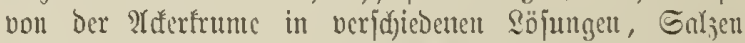

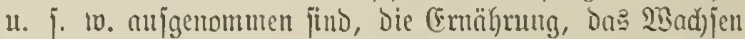
uno Gedeifen Der Prlanjen, und ez ijt Darum Die fennt=

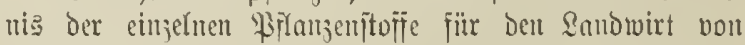

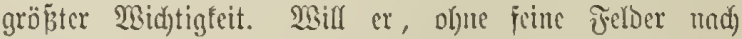

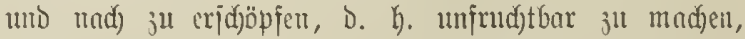

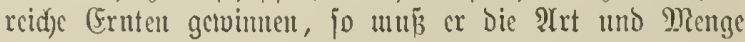
der iu Boben feiner Felder enthaltenen, die PFlanjen

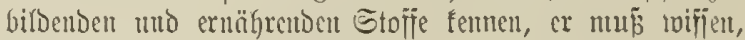
meldje (Bemö̈dje bem Boben biejen oder jencu Stofi methr obcr mintor cutjiel)en, unb cifrigit Sorge tragen, Dn

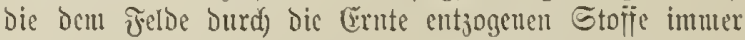

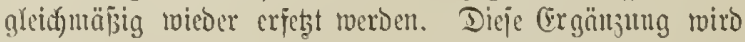

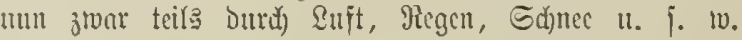

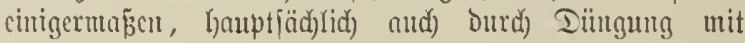

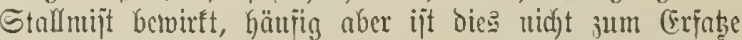

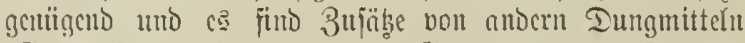
(Bip:, Sialf, Mergel, शlidjen, Guano, Snljen, finod)en=

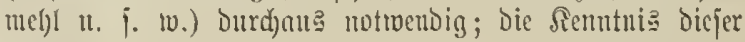
Stofie, in Dualität uno wirfung für die beridjiedenen 
Bobenartell, ijt für den Sanblvirt heutigen Iages cine Reben sirage.

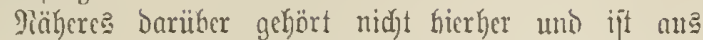
Den ipejiell landwirtidjajtlid)en Edyriften jull crīchen.

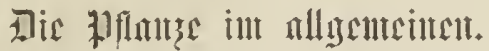

Die bortfommene Prfanze bepteht 1) aus der 20urzel, radix; 2) Dem Etengel, caulis, Doer Stamme, truncus; 3) Den îfiten, rami, แnd 3toeigen, ramuli; 4) Den Brättern, folia; 5 Der $\mathfrak{B} I$ ïte, flos; 6) Der Frudt, fructus. mit Den Sanen, semina.

Die Bflanzen, plantae, D. h. alle (Gemäd)je vom mäd)tigen Walbbaume bie jum flcinjten Moojc, von Der Gimmelanjtrebenden ßa!me bis zum faum fidtbaren Edjimmetpilze, jino, wie wir oben gejehen haben, orga= nijde, rebende 2hejen, wenn aud ohne (5mpfindung und toirlfürride Beıegung.

(E⿱ jind Landpflanjen, wenu fie in oer Eroe, auf dent Lande murzelu und madjjen, $\mathfrak{B}$ a jierpflanzen, tremn jie jidh im 2Bajjer entridfeln.

(E. jumb entudeder A. einfrüd)tige oder B. wieder= früct)tige Piflumen.

1. Lie cinfrïdtigen, pl. monocarpeae, jterben nad) cimmaliger Frud)terzeugung gämjlicf) $a b$. 3 in ifucn geljören a) bie eimjägrigen oder Eommer= (bemädje,

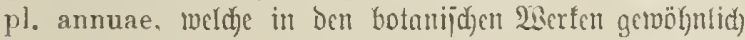
mit $\odot$, Dem falcuberzeidjen der Eonne, bezeidunet rerben; und b) Die zweijährigen, pl. biennes, gewölnnlid

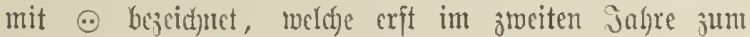
Blühen und frud)ttragen gelangen.

B. Lic wiederirüidytigen \$ĭlanjon, pl. polycarpeae. find Sräuter ober Etauden, Deren meijte Ieile nad) ber Eamenreife abjterben, Deren $2 B$ urzel jto af aber mit einem Isile der Wurzel Yebend jurït6́leibt und cine neue Piflanje crivadjen läpt. (5e jind a) Die jogenannton perennieren= den, auzoauernden (Gemädje, pl. perennes. mit 과

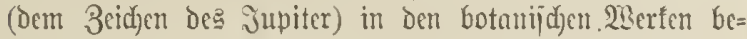
jeid)net; nder b) Sträud)er (frutices) mno 5ुalb= jträud)er (suffrutices). Deren berfolyte, ausoaucrnoe Etengel unb 3iveige jabrelang lebend bleiben, mit $\bar{b}$ (bom 3eiden des Gnturn) bezcidjnct; Docr c) Bäume (arbores), ineldye vicle Jahre lang leben uno in lyöheren,

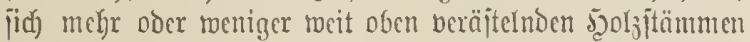
wad)jen. Sebtere meroen mit 青 bejeidunt.

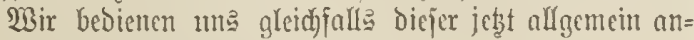
genommenen 3 cidjen, uno nehmen

C für einjährige \$rlanjen,

(.) " jmeijäfrige łílamjen,

7 "peremuicrende \$îlanzen,

b " itraudjartige fir̆lanjen, uno

夆 " Bäume.

\section{Dic Ergane ser \$if̆na}

Tie Ergane der Prfanjen merben singeteift in

A. Elementarorgane, einfadje Ergane, uno

B. 3 ujammengejesste, äuß̈ere Drganc.
Die (Elementarorgane find bie widtigiten, wem aud) cinfadjfen Srgane, indem ite ben 2lufang und bie (Srundlage fämtfid)er fejter Seife Der Piflanjen autsmadyen.

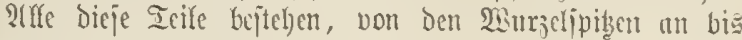
зน Den oberften 3rocigen, Blättern uno Blïten, aน ntentarorgantel, deren $2 a n$ und Jnfyalt nur ourd bas Mifroftop beutfid) ju erfemen und ju unterid)ciden fino. - Die jujammengejebten ober änberen Ergane merden Durd) Die Berbindung Der (Efenentarorgante gebildet.

\section{A. Dis Encmentarorgatie}

jimb bie Bellen, cellae, cellulae, in iffen verjoficoenen Cinjelheiten, Formen und Bejtaltungen.

Wenn wir cin bïnne Edjeibd)en irgend cines Piflanjen=

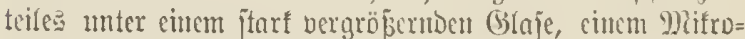
jfop, betradjten, jo jehen wir, Daj bieję Dümne Edjeibden aน: berjdieden geformten, fleinen fiörpern, ciner Bienen= twake nidht unähnlid, jujamntengejebt ijt. Dieje mifro= jopijd) fleinen förper jind gejd)lofiene, mit Fend)tigfeit

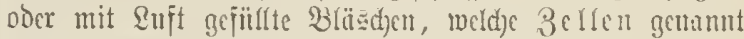
merden. Jn ber ganjen organije)en Vatur, aljo im Tier= und aud im Piflanjenreide, bilbet, wie fdjon oben ange= butet, die 3 elfe die (Grumblage aller anatomijden Ieile, Das eigentlid)e organiide (Element, Daz (Elementar = organ, weldes als joldes an fid) ober mit syilje ber

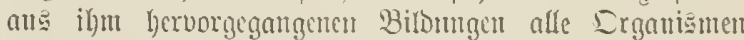
bon Der einfadjeten liflunje bis ginauf jum ricpigen Elephanten - in cinjeluen twie in ganjen grïndet und јuјammenjeșt.

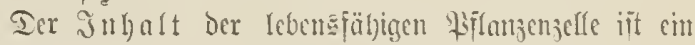
Durdjidtiger, farblojer, bie veridjicomiten demijden Etofie entfoltcnoer Gaft oocr Edylem: da Frotoplasma*), in weldgem fïd bištbeilen tleite fiörner von Etörfemebl birben, von Denen bie bidft unter ier Dberhaut lefinds= lid)en cine harjartige शatur mb grüne Färbung ange=

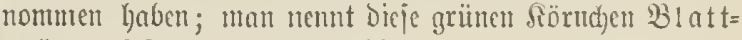
grïn, chlorophyllum**).

Die Den flitifigen Jnhalt ungebende Dïnnhäutige

*) Ias Rrotoplasma, ber Zebensitoij, urjuleim,

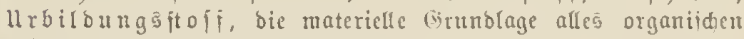
sebens, ber weientlidfte Bellenintyalt ber Siflanjen mo itere, soldher früher ba ift als bie Bellenmembran unb oit olute dicje anitritt; cin eitocijartiger Etolif, heleht, mit pontaner, b. h. aus jid) felbjt roirtenoer seroeglidfeit uno ber jüljigfeit, obne ent jpredento äusere 2 njtökie anbere jormen anjunchmen; trit rein und cheinbar ohne Erganijation idon in ben einfadjiten (Ge= idiopfen, ben 2(möben und ihren Berwanbten ant, uno eriült unter bem Ranen Bathybins it grojen Eträngen nnd Rlumpen mafienhait auj hunberte bon gieilen bin ben tiejeren झlectes boben.

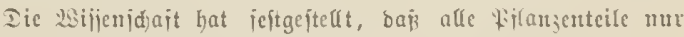
io lange leben, als biefer llridbleim in ihnen borljanben iit. Niur bie Beflen, welde Frotoplasma enthaiten, tönnen wadjen, ueue demijhe Serbindungen erzeugen unb ueue 3cllen bilben.

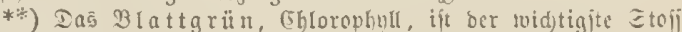
Des 3elleninhalts. (Es ijt an fid jarblos, ber barin enthalteue Eaueritoij entwidtelt aber in jeiner Berjestung butd) ben (Einiluis

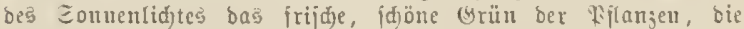
gelbe, braute und rote şerbjtiärbung ber Bä̈tter, jowic (waร ïbrigens nod) nidit jider jeftgeitellt ijt) ïberhanpt bic mannig= jadje jarbenprad)t ber \&lätter, Blïten unb 天̃üdjt:. 
3errentwand beiteht aus einer, ans froblen=, Wajper=

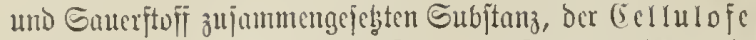
(łiflamzenjelltofi, soljfajer). Sie berändert mit Der 3unnhme, mit Dem 26ad)fen des Belleninhaltö die juerjt fajt lugelige form all vielfude $2 B$ eife; es bilden fidl) ftern=, haltmond =, fugcl=, fdeciben=, tofelförmige,

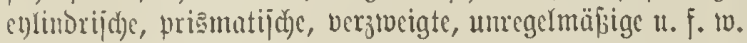
3eflen.

Meifenweije über einander jtegende Bellen billoen, indem Die 3mijd)entwände verjd)tvinden, Dur(t)gehende, innen hohle

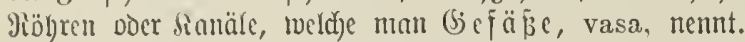

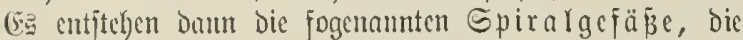

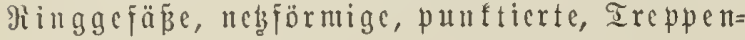

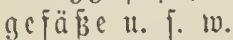

Die Bercinigung mehrerer in gleider Richtung ver=

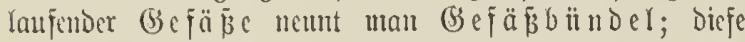

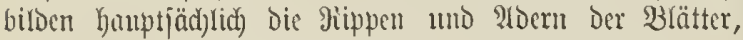
und machen greidjam das Sfelett Der Biflanje aนs.

Bo orei 3ellen jujammenjtojen, bilocn jiđa oreijeitige

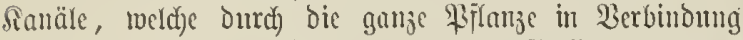

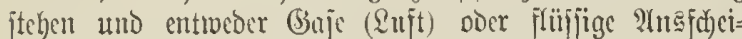

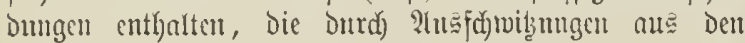
3ellen erzengt funb. Dicje fianäle heipen J̄ntereellu= largänge, meatus intercellulares.

Daz Berrengerobe, tela cellulosa, entitebt aus einer 9) ift eine Gefamtheit von 3eflen, Deren jede ein bejonderes Ganjes bildet; Den verfodjedenen (rigenjaduten und $\mathfrak{B} e=$

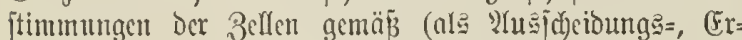

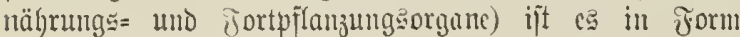

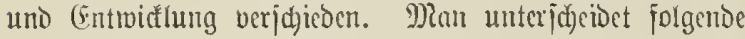
3ellgemebearten:

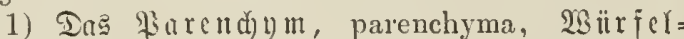
ober Füllgewebe. Diejes ijt das wiatigjte Geweóe;

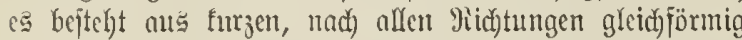
aügebildeten, runblichen ober ectigen 3ellen und findet

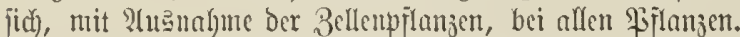

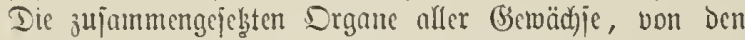

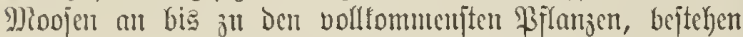

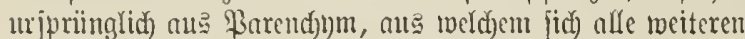

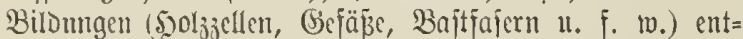
เoiffeln.

2) Daß Fajergetocbe, prosenchyma, eg bejteht aน: gejtrectiten, incinamber greifenden Bellen olne J̈nter= cellulargänge. Sie find im Bajtgcmebe die jähen, bieg= jamen Fiffern, im şolze bilden fic bas sjoljgetwebe.

3) Das Bildunggetwebe, cambium, jartwan= Dige, meijt geitrectte, reid) mit Snjt gefiillte Bellen, tweldi)e

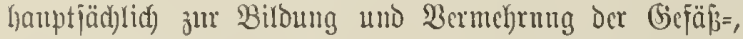

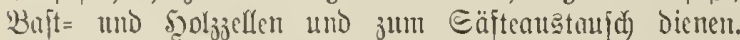

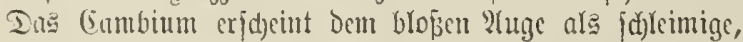
galfertartige 9lajie; jeder junge ßĩlanjenteil Der höberen (Getwäc)je beitcht aus ifyn uno jugleid) beruht auf igm

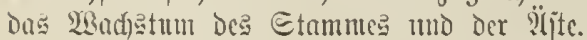

4) $\mathfrak{D a}^{3}$ Dbcr bantgelvebe, tela epidermoidalis, ijt cin feines, eigentïmlid) tonjtrniertes Gervebe, tocldese

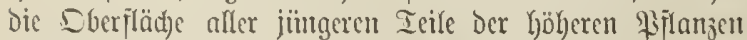
ïberjieht und jie gegen äuß̧ere (eimflïfje joüht. Diejer Ucberjug, bie Sberkaut, epidermis, bejtel)t getoöhnlid

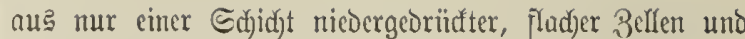
ift wieberum mit einem zarten, gleidjartigen, ablöz̧baren Soäutchen, cuticula, iiberzogen. Sjt Das Getwebe Der Dberbaut fofthaltig und zartwandig, twie z. B. auf der Sberfläd)e der Blumenblätter, fo nennt man es epitheliunl. Bei den in ber (Jirbe ober im Wafjer wardfenton PFlanzentcilen bleibt die Dberhaut geidylojien, bei ben jid in Der \&ujt entmidtelnden, grïn gefärbten aber jeigen fid viele mifrojtopijd) fleine \&uftlöd)er, Boren, Spalt= öfinungen (pori, stomata), deren locter verbundene Bellen anjebnliche S̈ntereeflulargänge bildoen, in tweldye jich überafl Suft eindrängt uno beren Meündungen die (5in= jangung und ?lusbünjtung der Brlanzen vermitteln. 2tuf biefe 2Beije baudjen Diefelben Durd) Die Epaltöfinungen bei Tage Enueritoff, bei গacht Rohleniäure aus, uno nehmen Dagegen bei Inge Sohlenfäure, bei Nacht Saucr= itoif au der \&uft ein.

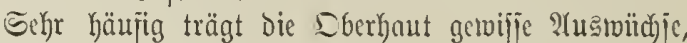
Yebenorgane, Die man mit Den bejonderen Mamen: Spaare,

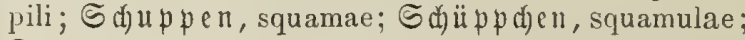

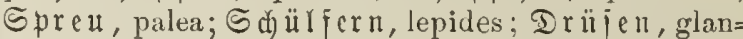
dulae; Stad) ern aculei, u. F. 10. bezeidnet. Sic be= Itehen jämtrid) aus veridjiedenen Bellen und bebingen in ihrer Gejamtheit Den Uleberzug verjediedener BFlanjenteile.

5) Das diorlgewebe, ober bie als Sort, suber, befaute Subjtunj, bejteht ans Flachen, tafelförmigen, \&nit enthaltenden Bellen, Deren elajtijde Wandungen Den

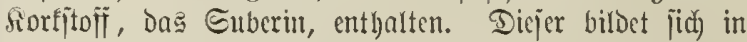

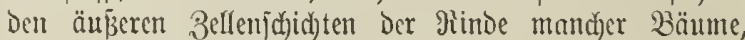

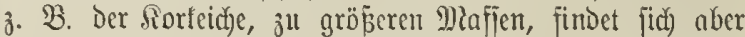
aud) in allen anderen Baumrinden, fo wie in ben Ginden der Sträudjer und ber jaftigen Rrautpilanzen. (5r ge= hört überkaupt zur Rindenbildung uno entjegt erjt in ober unter Der Dberhaut, weldfe bei Der fortbildung $a b=$ jtirbt. Bildet fid) Der fiort in Den tieferen Rimbenjujicften, fo löft fid die abjterbende rinde in ftärferen Sdunppen und Etreifen (B̈orfen) ab, 3. B. bei ben (Eichen und Radellölzern.

6) Das Biljgerwebe, das Gerwebe der ßifje,

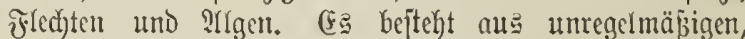
langen, bïmen, fuDenförmigen oder fugligen, joblundyartig

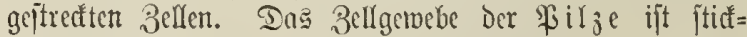
jtofireide), gallertartig uno jehr vergänglid); Daz ber Fledjten bürr und jäle, reidy an Etärfmelyl; Daz Der Ylgen bejteht aus oromungelofen, einfacten oder unter cinamber veräjtelten 3eflenreibe; es unterjd)eidet jick von Den altocren baburd), daj es etwae Blattgrün, (Ghlorophyll, enţält.

je nad) Der (Entroiftung der Elementarorgane teilt man die verfdjecomen (Gembädje ein in 3ellenp planjen uno Gefä $\bar{\beta} p$ fl a ze

1) Die Bellenpllanjen, plantae cellulares, be= jtchen nน $\mathrm{r}$ aน gleidfförmigen, cinzelnen ober cinfad) an= einander gereihten Bellen; bei innen ift jener, in Den

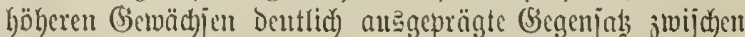
Der 2 d) fe (2Burjel mo Stamm) und Deren Seiten= organen, den B̉lättern, nicht vorbanoen uno man nent fie baher aud adjenlofe lifr., Igallophyten, Lagerpflanjen. (5z find die cigentliden Bellen= 


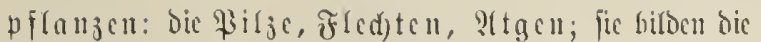
nicberite Silnjie be

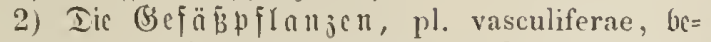

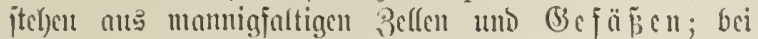
ignen jeigt fid) Der oben angefïl)rte (Begenjatz zloijd)en

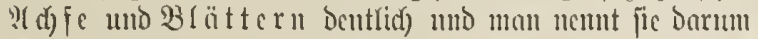
श( d) fenpfla ben Samenlappen (cotyledones) licgendon frimling

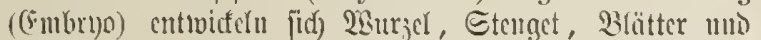

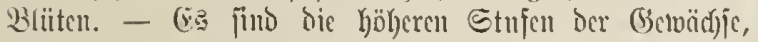

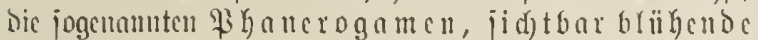
Pĭlanjen.

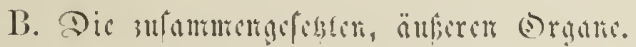

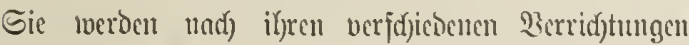

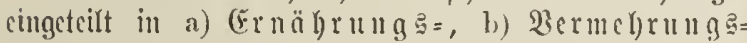
umb c) Fortpfranjung organc.

Sic (Erü̈brungeorganc werben and) Funba= mentalorgane genami mo bejteben ans 1) ber $\mathfrak{W} \Perp r-j e l, 2)$ Dem Stengel mit 3locigen unb 3) Blättern.

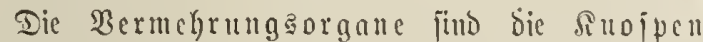

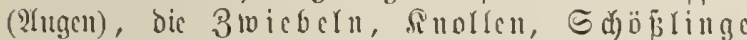
(Whir; cliprofien).

Die Fortpfanzung ang

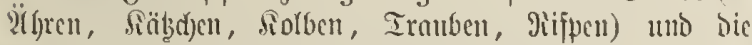

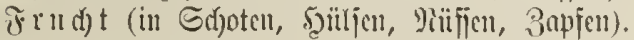

\section{a) Dic Ërñlirumgsorgane (Jundaแncutap- odcr Dิadjs fumsorganc).}

(2Burzeln, Etenger, 3tweige und $\mathfrak{B}$ !ätter.)

Die 4 organifden (Elemente (bic Drganngene): Der Soljenjtoff, Wajieritoff, Gaueritoff mo Etidf=

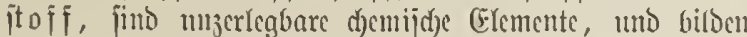

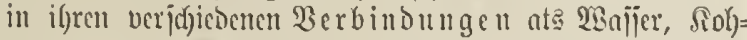

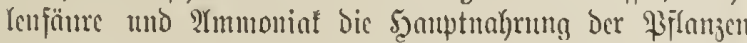
mo and) Die Sanutmafje dez \$itanjenförperz; nußerbem

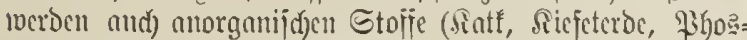
phor, Gduweficl, (Eijen, verjdjicone alfalien u. j. w.) in Form aufgelöjter Salje von Den Birlanjen aus ber 2Iffer= frmme (১ç Bobcus) jur (Ernährung mo namenttid) jur (Beriiftbitoung aufgenommen. Dis mit dicfen organij()en uno anorganija)en Stoifen crfülfte Frliifigteit wiro von ben

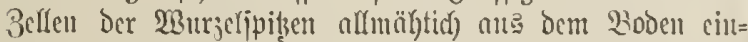
gejogen und von do bircft in bie Yilanje cingefïlfrt; fic

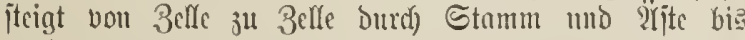

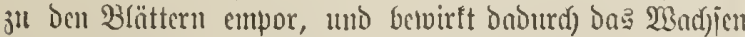

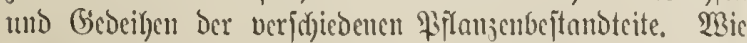
j(d)on oben bemerft, nehmen bic Blätter und bic anderı

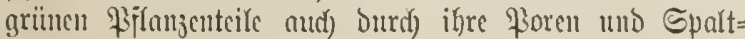

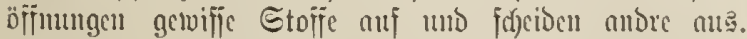
PYu Iage, mamentlidy im Eomenfody, atmen fic bic

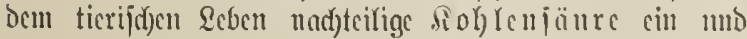
fdeciben jugleidy mijern unt ber Iiere \&ebenștoff, beu Sanerjtoff, anz; und)t dangegen mo im Dunfeln

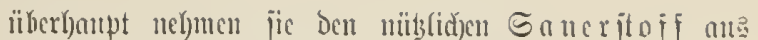

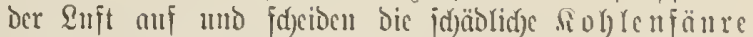

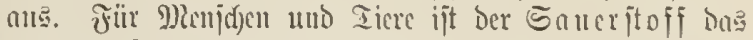

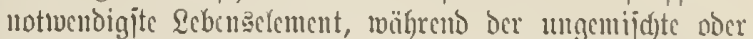

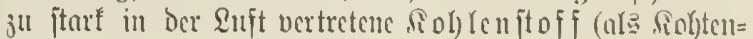
jünre) für Den lluterhalt des ticrifd)en Lebens ıntanglid),

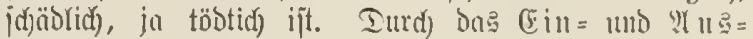

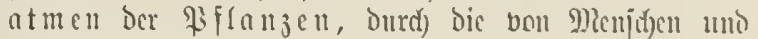

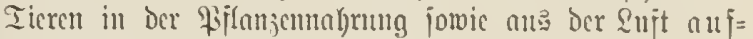
genommenen ut ansgefdicdenen Mengen von

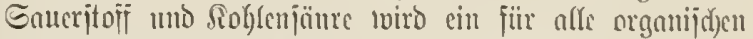
Bejen notwendiges Gilcidggelvidyt beider Stoffe bergejtellt, und Sioblenjäure und Sancrītofi crgänjen jić) gegenicitig

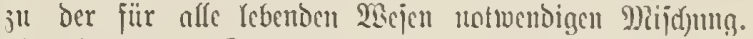

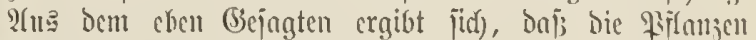
Durd) ihre (Eill= mo 2 (t)

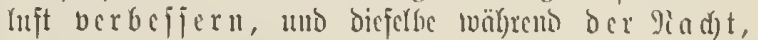

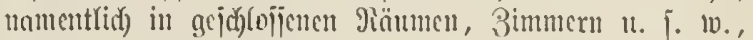
merffid) ver fdited tern. -

(Gel)en bir mun ju den cinjelnen (Ernäl)rug organen ïber.

\section{Die Wurz̧ॄl, radix.}

Die 2Burjel ijt ber untere, mad) unten wadjjende Teil des Stengels oder Stammes; alle ilfre Icile be= jtreben fid), in cince vom Sidjte abgetwanbten Midftumg in Den 30Den ju Dringen, um in Demjelben fid) ju be= feitigen uno auta iłm Rabrung eiuzujangen. Dis meijten

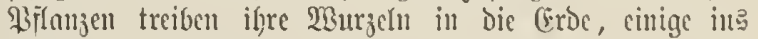

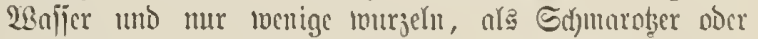

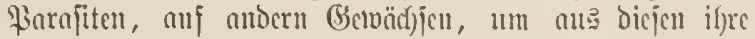
शabrung ju jichen. 21ber aud bic flempten פRajjer=

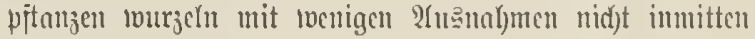
Des WBajicrs, jombern auf Dem (Srumbe Desjetben, Dem Bobell.

Dic 2:urjeln unterjojeiden fid vom Stamme nocr Stengel Dured) i(jren Plangel an B̧ättern uno buburd), donj fie mur bou ifgen Epilzen nus wadjjen.

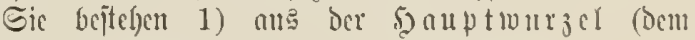

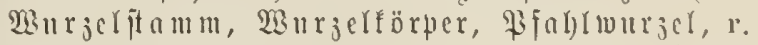
palaria odcr primaria, caudex descendens $I_{\text {. }}$ ); 2 ) an Dicfecen Berjweigungen, $\mathfrak{B} \Perp r_{j}$ eläften, rami; 3) Den feinen foderartigen, jum Eimjangen Der ?al)rung

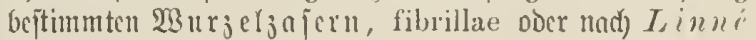

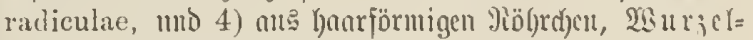

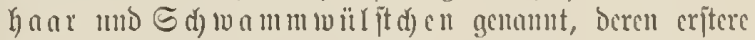
Dic ganje Wurjel Der MRooje, dic leţteren die jetrigen Verdidungen an den Wurjetjajern, wie j. 23. hei Den WBajier(injen, nusmadjen. Wurjetn, betd)e bireft an? bem Gtamme felbjt wadjicn mo jidf) von da mez jum

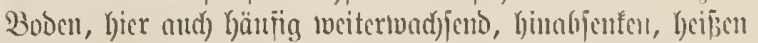
Quftwurjeln, radices ä̈reac; لiebcuturjeln, r. adventitiae, find bicjenigen, Iveld)e jeitlid) ans bem

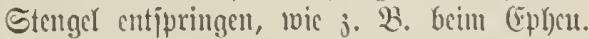

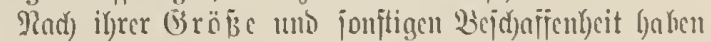

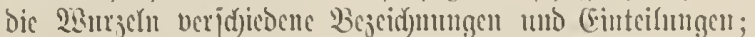

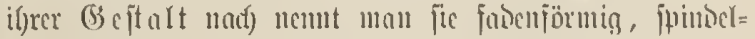

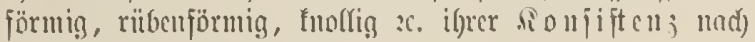
lyoljig, itcijobig, melylig, joftig :c. 


\section{Jer Steungl, caulis.}

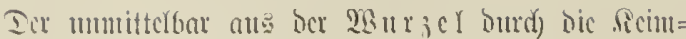

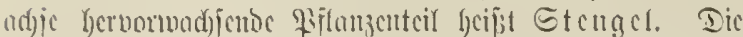

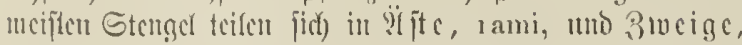

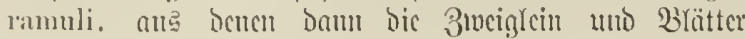
inardicti.

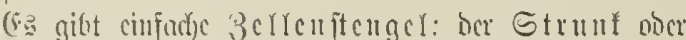
Gd) ait bei Den Hirjen, stipes; ferner et wa tom ucre Stenger: hei ben MRonicn mb Iangen,

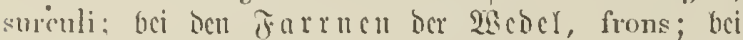

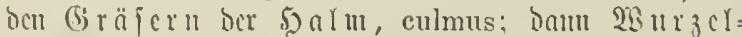

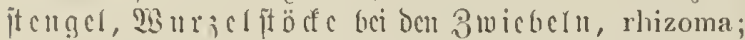

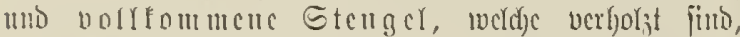
bicle Jafre bancrn, mo Etamm, trumcus, beifsen.

Der Etengel nimmt ben Tiafrumgajaft der Piflanze

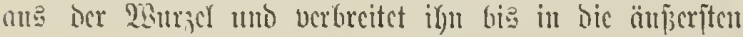

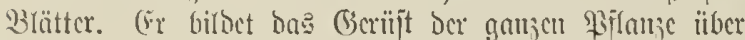

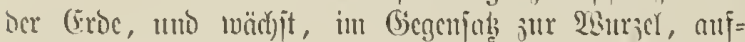
märtอ, !nad) obcu.

(Fin quer burd)idynittener Etengel, 3weig ober Etanm

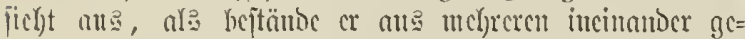

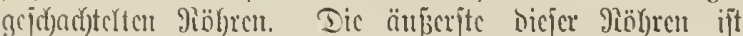
troden, mcijt sumfer gcfärbt mo im ?reter riffig; fic

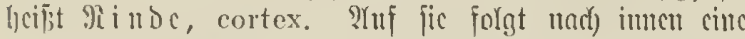

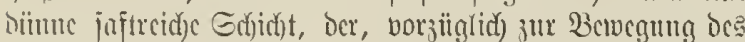
Eaftes Dicnemoc 23ajt, liber. Daranf jolgt bas Sjols

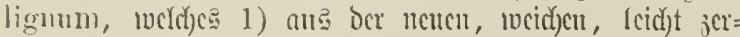

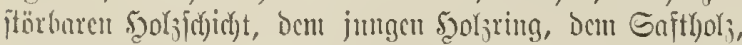

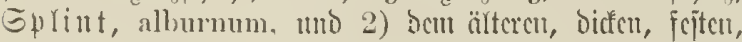
pajrigen Sautboly, ficulfoly, duramen, mit jeincn

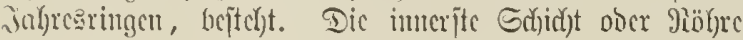

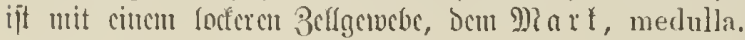
crfiilft.

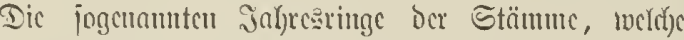

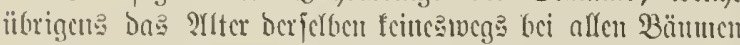

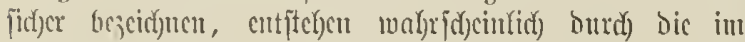
2isinter cintretento Gtodiung im Piflamicumudje, iverdec

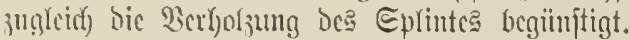

\section{Dir ßlätter, faubblïtter, folia.}

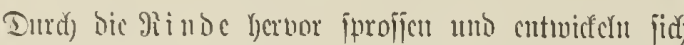
bic Gtcugel= obcr 3wcigfü̈jpdycn, anz bicjen bic

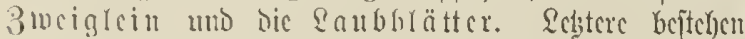

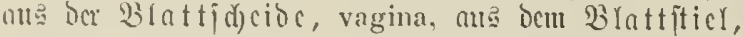
petiolus, mb ber Blattfläd)e ober blattipreite, lamina, mit ifren lieppen unto foren; oft forylt ber cinc ober antere bicjer brci Icife. Dic Sanbblätter, gemöl)nliç) nur

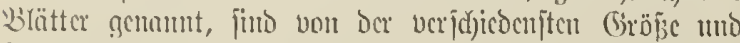

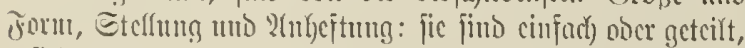

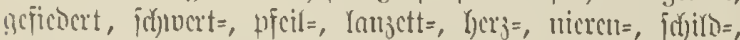
fcil=, Ycierfürmig, rmolic), oval, fitumpf, fpits, antegcran= bct, juftig, flcijc)ig, frautig, Icocrartig, am गianbs gc= зäl)nt, gciäģt, geferbt, gcichucift, gewimuert, bortig, rauth II. i. il.

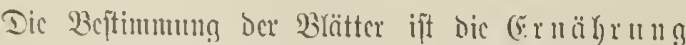

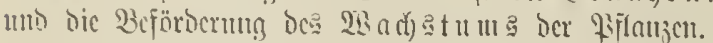

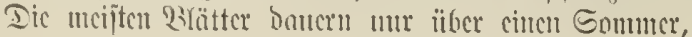

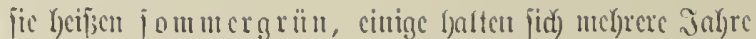

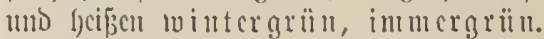

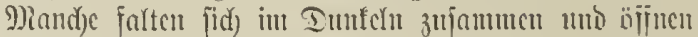

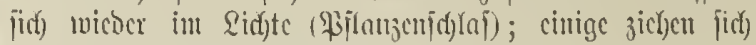

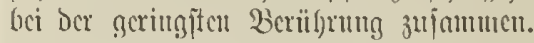

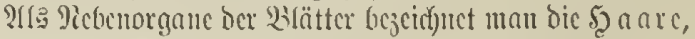
Grupucu, Drüicu, Gtadern mb dianfen.

2un den ficimblättern ( a menlappen, cotyledones) ponvic von ben Iectulätern (Brïten=

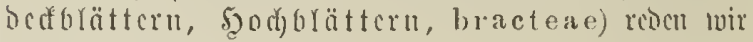
lociter nuten.

\section{h) Dic Dicructirnugs- nocr Sicgenerationsorgane.}

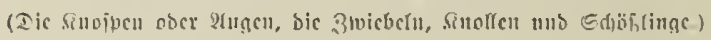

\section{Tite finnfte, Das Aung, gemma,}

ijt Dic ame cincm Etamme obcr 2lite now Bilatte locr=

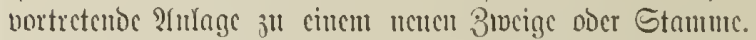

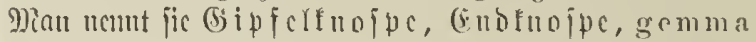
terminalis, menu fic bas jümgitc Endo des Etammez

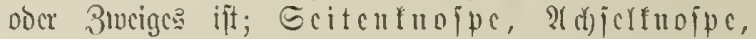
g. Iateralis, woun fic unterhalb doc (Gipfch ftcht

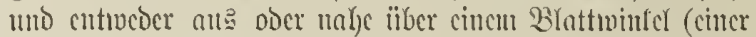

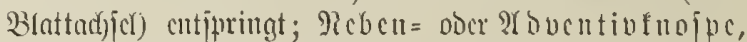
menn fic, oljue bou cincm Blattc geptittjt ju jein, an andern, mbejtimmten Stclicn Des Stengels herbortommit.

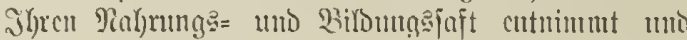
berarbeitet bie finoipe ans ben Mlaterial ber Dintter=

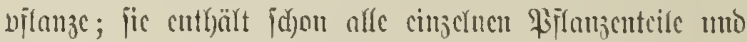
ijt baburd) befärjigt, wicber cinc gantje \$illanje glcidt)

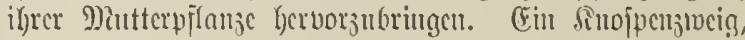
ju geljöriger 3eit abgejduitten, in bic pajjende (Erose ge=

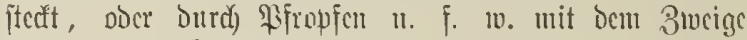

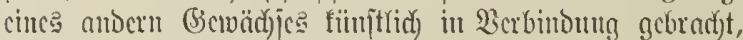

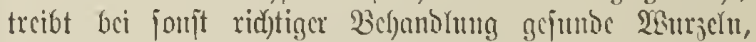

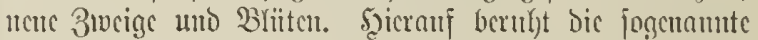

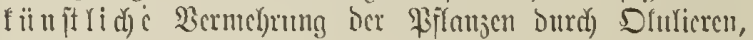

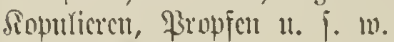

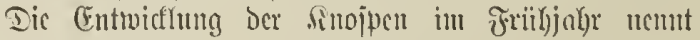

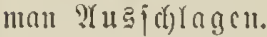

Rebcudrgauc Der finopipen finto, wic and hei ben Brättern, dic Sorncn, Sinuten, Gtad) cln it. i. iv.

\section{Dif Btvichel, bulbus.}

Dic 3̈vicbeln finb cigentlid) nid) ale Sinoipen auf

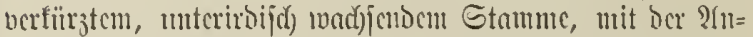

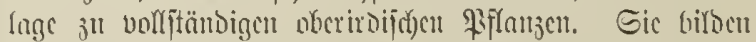

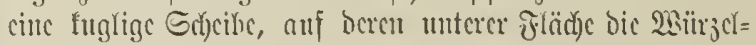
(t)en, auf ber oberen bic idjuppigen, yantigen Ed)cibcu=

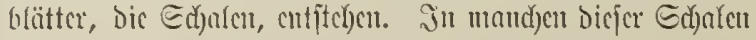

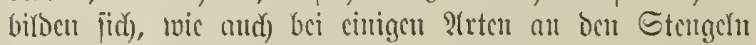
mo blïten, neue Rinojpen nocr junge 3wiclicln, joge=

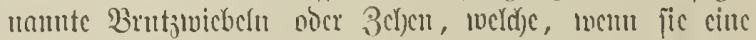

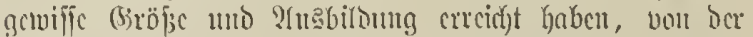

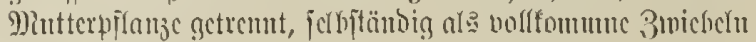
unciter bud 


\section{Die fînolle, tuber.}

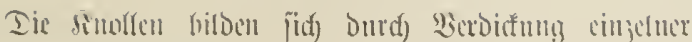

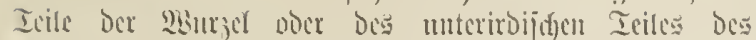

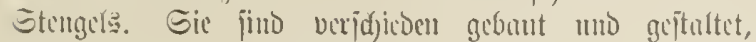

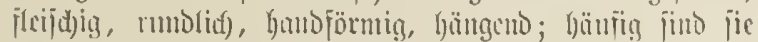
mit sintopen (siciment verjeben, wo bum jeder dicjux sirime wieder cinc ganje fiflanje entbält. Inté bieje

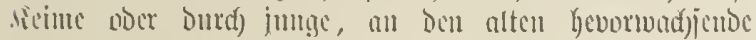

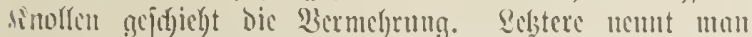

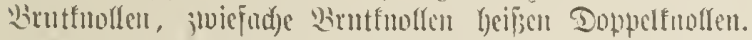

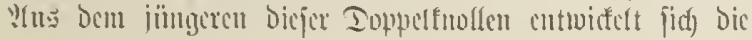
nene Piflunje.

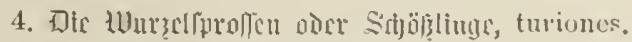

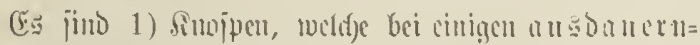

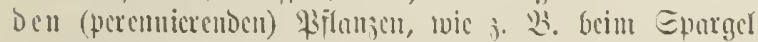

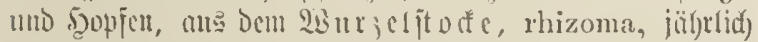

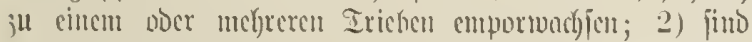

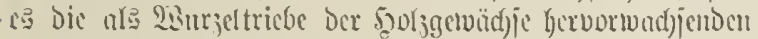

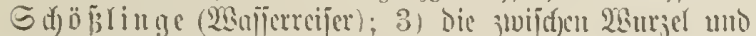
Stumm herausıadj)enden Iricbe, wekd)e, wic hein Şajel=

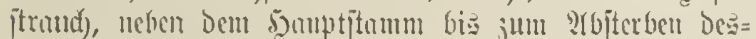
juben, mene frifitige Stengel ober Stämme bilben. Gie

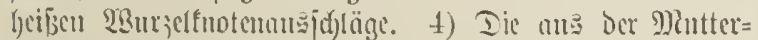
pilanje bervorjprieß̄endon, fadigen, j(t)wad)en @tenget mo Yiunfen, welde an Boben forthaufend, entweder an ifyrem

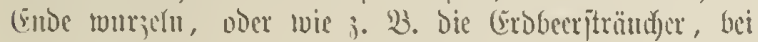
joor 'Blattbilonng men 2intjeln treiben.

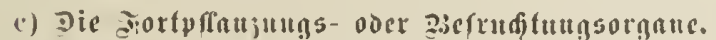

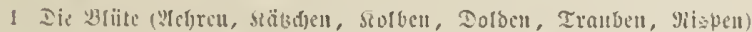

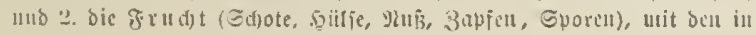
ibr cuthuteutu $e_{a u} u$.

\section{Die Blitite, flos.}

Iic Ergane der \$filamje, welde jur Erjengumg nemer

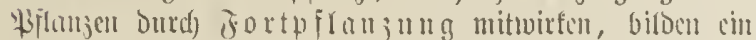

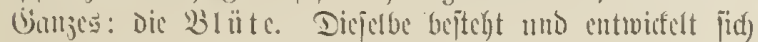

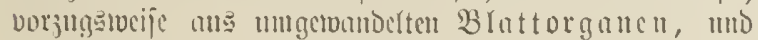

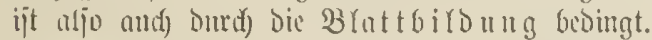

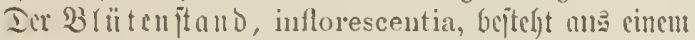

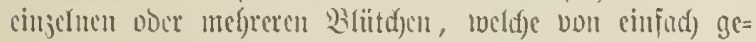

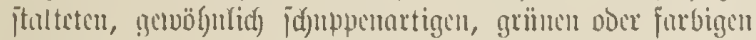

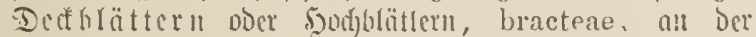
Stribe Des Etengela ungeben fint. Dicje Dedflütter

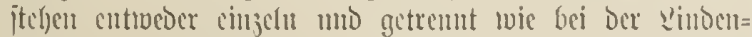

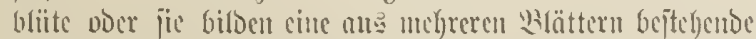

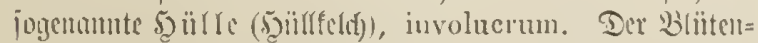
jtumo jeigt fehr verjejedene formen, die ge fere, spica, Daß fïlzd fu, amentum, Den díolben, spalix, Dic Dorbe, umbella, dic Irubc, racemus, dic dijpe, paricula.

Die cinjelnen Ieile tine vollitündigen $\mathcal{B}$ ! ït

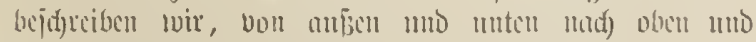

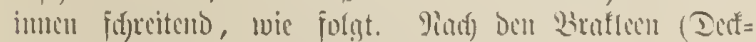
blätterii) ît Dor unterîte Icil Der Blïte
1) Ier steld), cialys.

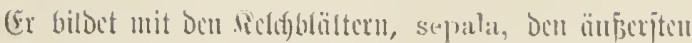

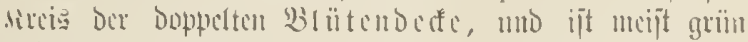

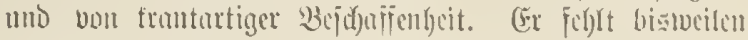

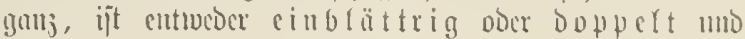

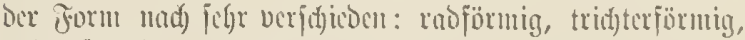

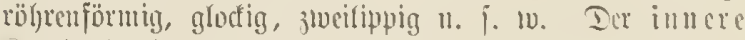

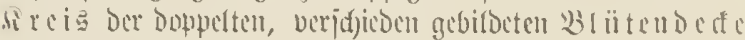
ijt bic $231 \mathrm{tl} m \mathrm{c}$.

2iso cin ficld ijt, ba ijt fojt immer and cine

\section{2) 3 lume, 28lumentrouc, struc, corolli.}

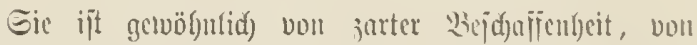
melgr voer toeniget joübuer Farbe mid fällt bulo mudf) Dor Weirnd)tung ab. (Einblätrig (monopetala) bcij;t jie: Blumentrone, corolla. in engern Eimne; wem fit mit mefreren getremuten Wlättern cinen Blattfreig bilbet, nem man fie viefblätrig, c. polypetala, uno Dicjen Sircis jelbjt: $\mathcal{B}$ ln menblätter, petala.

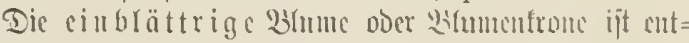
weber a) rölrig, ober b) glotig, c) trideterförmig, ober d) lippenförmig, mo juar cinlippig, mmo jweilippig, oder fenlig, tellerförmig, radjörmig 11. i. 10 .

Die viclblättrige goipt a) regelmäßig, b) rofenatig, c) uclfcuartig, oder aud ungleidf,

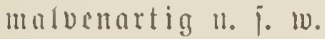

2Geniger widglige Irife, Micbenteife, Der Biliten find

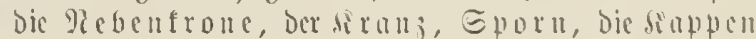
I. ¡. 10. Llugerumbute obur verfïmmerte Bfiitentcile

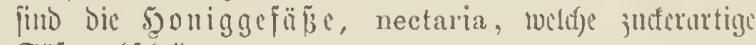

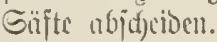

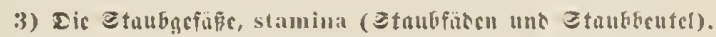

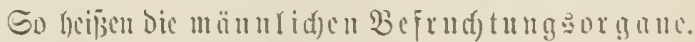
Iir Etunbjäbcu dow Irïger, filamenta, find bic fuben= förmigen Stenget, an Deren Epilge Dic Stanbucutel, antherae, itehen. 3uht und Rängle dox

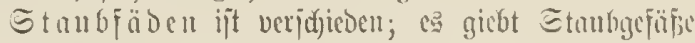
mit längeren mo fürjeren Etanbfïbon juglcid), mit fallen mid befourten, mit viclen mo lvenigen, mit cin=

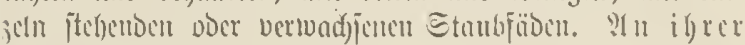
Spils jitsu bie

Stunbbentel, flcinere ober grö̈sere, häntige, jut= förmige Gebilde von ber verjoficonipten Gejtultumg, ge=

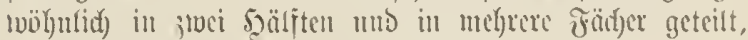

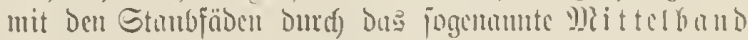
comnectivum. verfundon. Sic entraften ben hejrudfenden

Brïtenitanb, pollen. Iicjer ift cin fincs, gi=

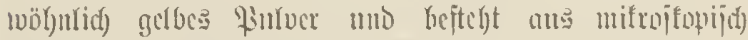
fleinen, veridjieden gejtafteten sï̈rnd)en, exiülft von cincr

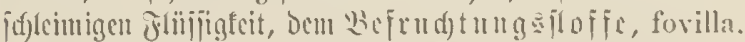

Den inner jten Blattereis Der Wititenorgune bil=

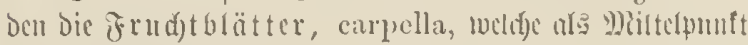
ber varlitïnoigen 'Slüte

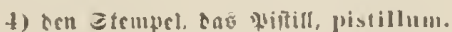

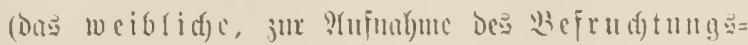

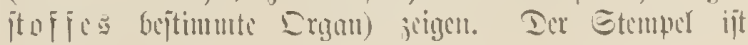




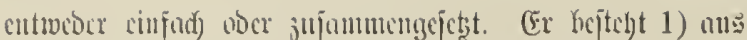
Dem frudffuoteu, Eicritod, germen Dber ovarium, Dem unterften Icile oce Etompcle. Dicfer ift gepïllt

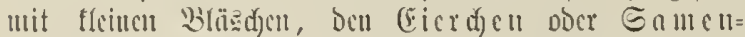

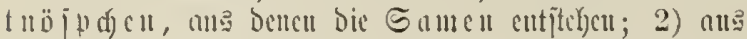
bem Giriffel, Stanbivege, stylus, cincul loctem,

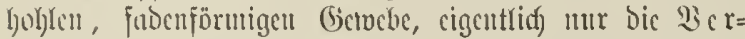

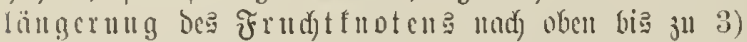
ber liarbe, stigma. Dieje ift ber oberjte Ieil bis

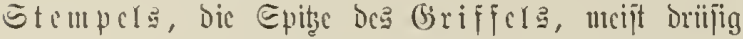

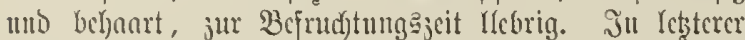

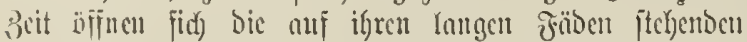

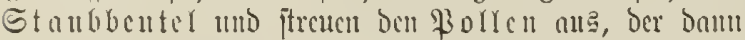

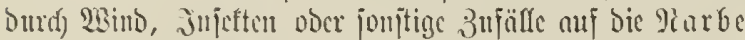
getragen wirt. Iurd) Den (6riffel gelangt Darnuf Der

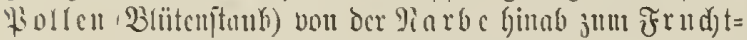
tuoten, in tocldfem fidf, twic jdjon oben bemerft, bic

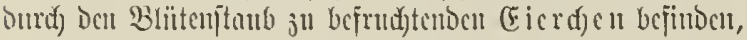

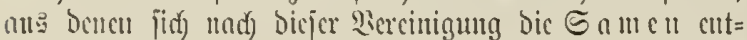
vidfeln. Dicje Sorgänge ncunt man Die B̉efrud)tung.

Die meiften \$frlanjen vercinigen in ifren wliiten bie

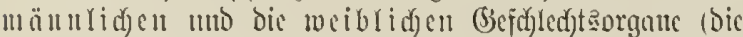

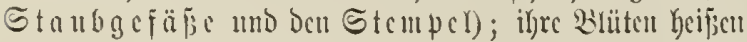
3witterblïteu, flores hermaphroditici. Ea gicbt

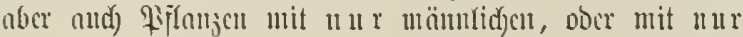

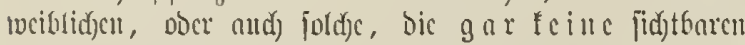

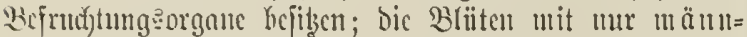

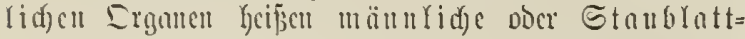
bliiten, fl. masculi; jene mit ur weiblid cu Drganeu wciblidac doer Stcmpelgräteu, fl. feminei; bie or)te

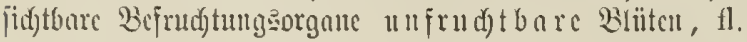
neutri.

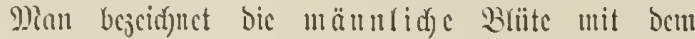

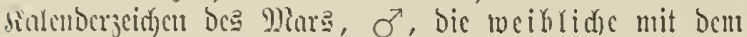
bar Ban

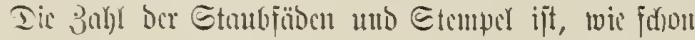
Dhen benertt, jegr verjadicoen; fie ijt aber fo jtreng und

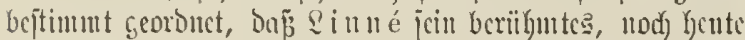

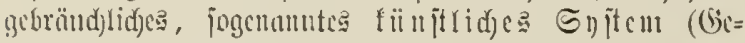

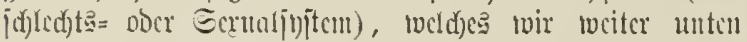
mitteilen, Gouptiäd)lid) Daranj gegrïmbet hat.

2Eir Kaben oben bic Bifrudfung crtlärt und Doburd)

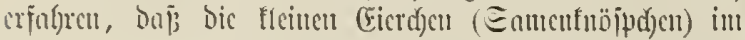

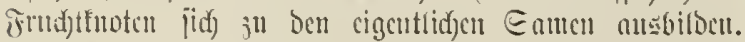

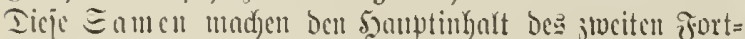

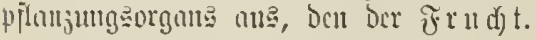

\section{Die Erudjt, fructus,}

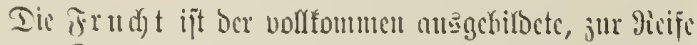
grlungte Frud)tunteu, Frudtbobcu, Der Sauc aber baz völlig mägchilbcte Gaucufuojpd) (Ei).

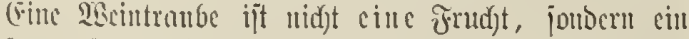
(⿻)

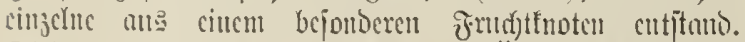

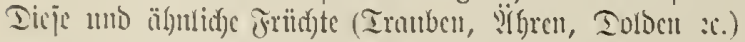

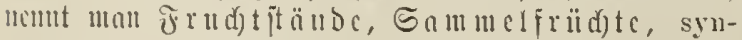

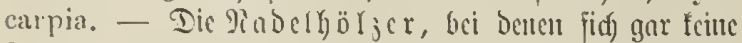

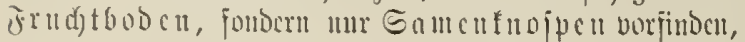

bejitsen leine cigentridfen Friid)te und ca jumb bie 3 apfen

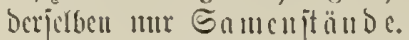

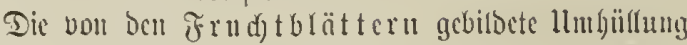

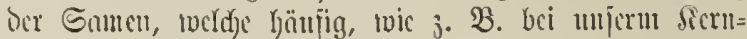
obit, Den widjtigiten Icil ber ganjen Jrud)t nusmad)t,

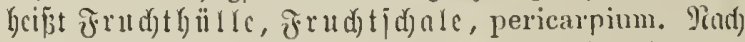

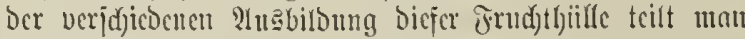
bic Fritd)te aud) cill in 1) Irodf cufrii d) te (Frudf)thülle

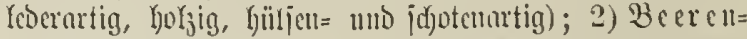

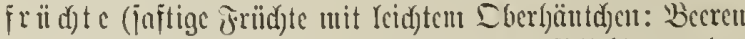

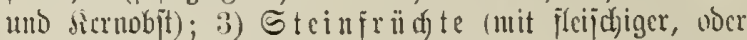

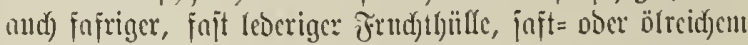
Fleijd)e umb meljr doer muber bartem, holjigen Stciue: MRandeh, Steinohyt, 2Balmußi).

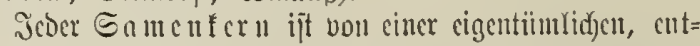

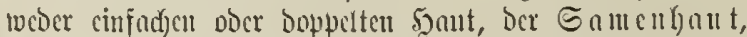

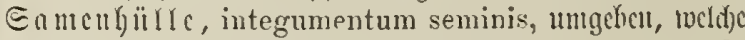

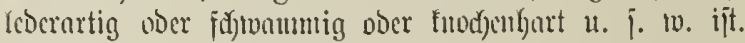

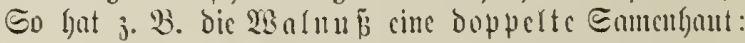

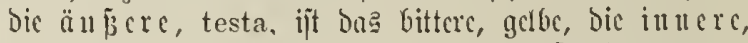

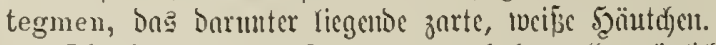

Die $S_{\text {ant }}$ u jelbjt, semina, baben alle mögridjen

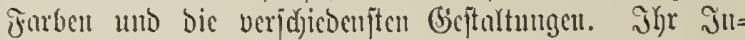
halt hefteht nus bem ficruc mit bem fieme, Dem Stcugeldfu und Den Samenlappen.

Dor fiern, Eiforn, Snmentern, nucleus, ift fleijujig, obcr meblig, ober fnorpclig und cnthalt (neben ober ofje Samencimei $\tilde{\beta}$, albumen) bcu $\Omega$ ei

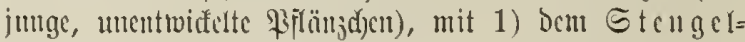

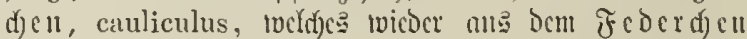

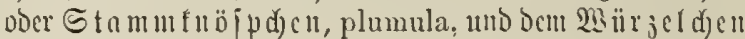

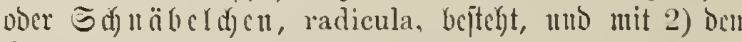
ऽamculappcu ober Sicimblättcru, cotyledones.

Iicji listerin, die Samcnlappen (cotyledones), find bald biinn und blattartig, balo bidt und preijotig, umb bleiben Kei ber ficmung in ber Picgel unter Dem Boben Sii bilben nad) Jujfien bic (Simubluge ber neturen unb neucjten jogenamute ut a

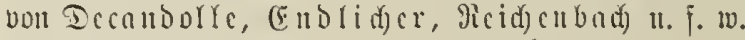

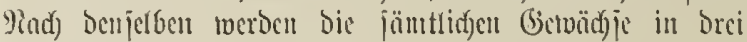
5emutgruppen eingeteilt, in

Acotyledones, firlanjen ohne Eamenlapha,

Monocotyledones, Pirlanju mit cincm Eamen= rappen,

Dicotyledones, Lifmisn mit joci (oocr mefreren) Enmenl(appen*).

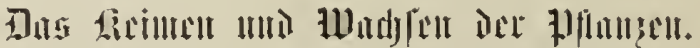

Syat bur Boben ben Enuten amfoctumnen, jo hegimnt

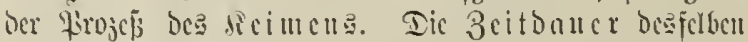

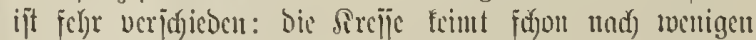

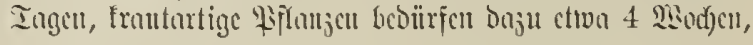

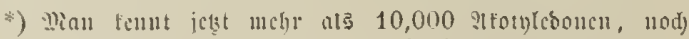

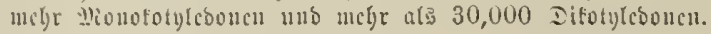




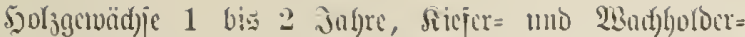
jamen feinen erit ium oritten Juhre. B̉i den uncijten

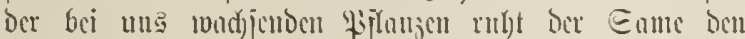
2Binter

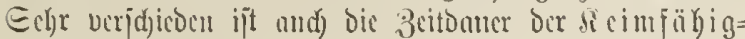

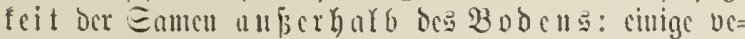
licren bicje Fïhigteit jehr justell, andere bebalten jic vicle Jabre, in Jagrtanjende binourd, woic j. B. Der

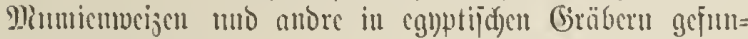
Dene €ämereicu.

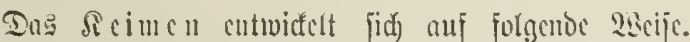

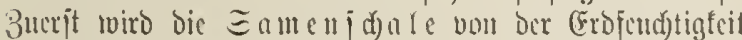

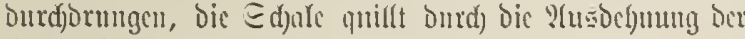

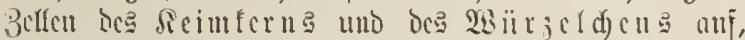
ber cigentlid)e feim oringt hervor uno jenft fid ju: 2Burzelbiloung in dic Eroe, bas 2siltjelden jelbit cut=

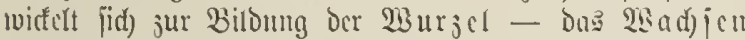

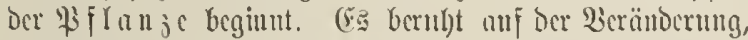
Bergrölscrung und Yienbildung ber berfeficoencu 3clfen,

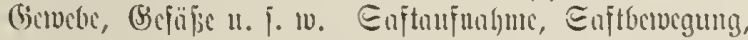
Enftucrmijdung verbmocn mit ?แ

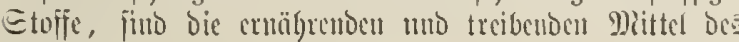
233 a d j 11 :

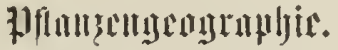

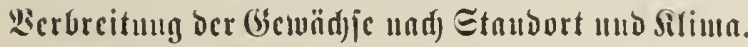

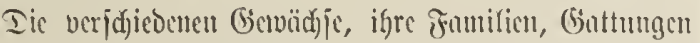
und ?(rten, find ungleid) ïber bic Erobberfläd) verteilt, uno Der Berbreitungäbejinf cincr \$Flange ift bulo grö̈̄er,

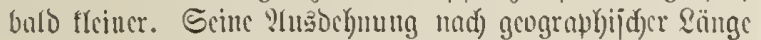
uno Breite (iu wagred)ter ritd)tung) neunt mun cinc

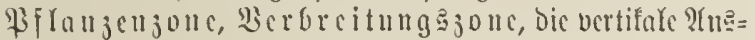
Defumng vou unten nad) oben (in jenfred)ter Piid)tumg) cine Hicgion*).

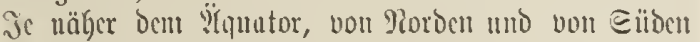
her, befto mannigfultiger uno gro 3 artiger entroidfelt jid)

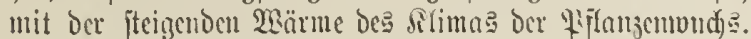

Dicje längit befounte Sfatjacte nuroe erjt in neucerer 3eit futärfer anfgefajit mo ju cincm ciguen 3weige ocr Wififujdaft: Der \$flanjengeographie, amsgebiloct.

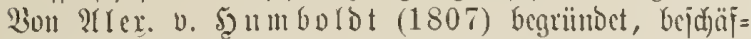
tigten jid) jeitbem vicle ausgejeiduncte (Gelebrte mit Der= filben, uno huben namentlicf)

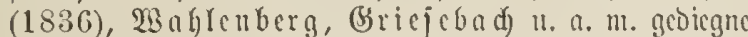
Forja)ungen darin unternomure und unfa[jende Bejtim= mumaen bariiber veröjijentfictyt.

Die gewölunlide, in Der phulifalipd)en (Geographic ge= bräud)liche Einteilung ber Eroberiläd)e iteflt folgende fï uf 3onen anf, beren Begrenjung Dic beiden 2 Bende= freije und bie Rolarfreife bilben. Gie beipen:

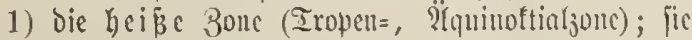

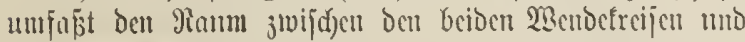

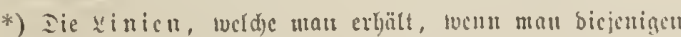

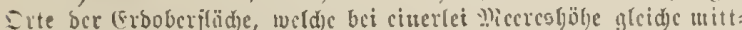
lere Salyestcuperatur bubcu, mit ciuanber verbinda, beijen jio= tyetucu oocr villica gleider wärue.

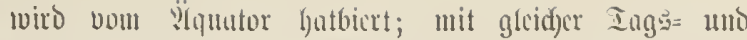

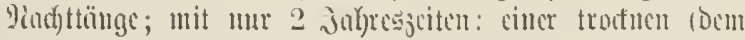

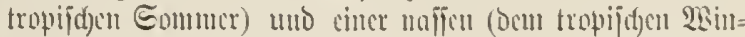
ter ober Dor Viegenjeit). Lic letitre fällt nibrolidf voul ?ilnator in unjer Sommer=, jïblid von dempelben in mujar 2Binterhalbjubr.

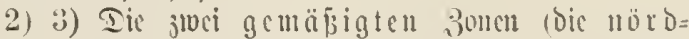
(id) mo bie jiiblid) gemajpigte jone), is juijd)en

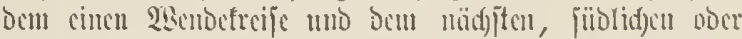
mörolidjen Folarfreije; mit verüntorlid)er Inges= mo Jiad)tlänge uno mit 4 jufresjoiten.

4) 5) Die jloci falten 3onten (dic nörolide uno bic jüblidge fulte 3one); fingelabj(f)nitte, Deren slittel=

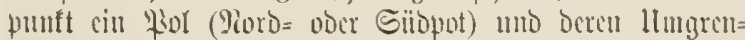

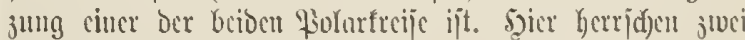

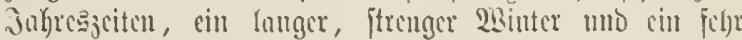
furjer Eommer; ocr längite Tag jow bie lïngite Jiad) Dumern fier ie 24 Etmulen.

J. $\Subset$ d) ettun genaner verfolgen uno fejtitellen ju fömen, dic

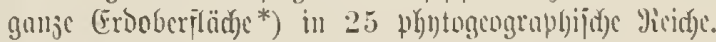

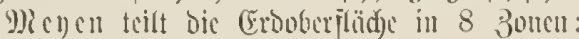

1) Dic ölunatorinljouc, beife 3 one, Bunc

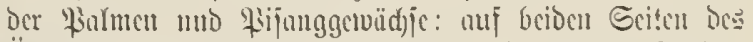

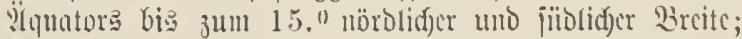

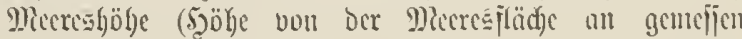
nocr gered)uct) bis jul $630 \mathrm{~m}$; mittlere Iemperntur + 21-230 Rcaumur $(26-280$ (5eljü). - Dic

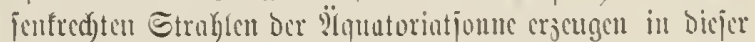

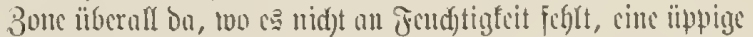
Entuvidflung Der manmigfud)iten mo gropartigiten Formen

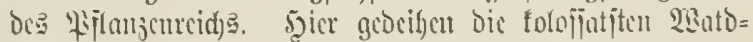

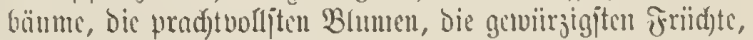

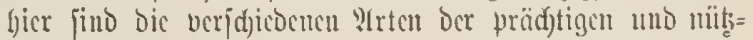

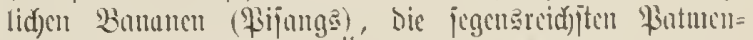

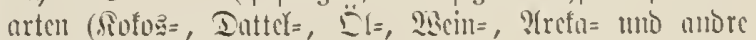

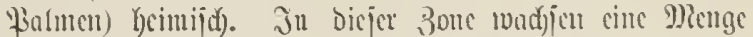

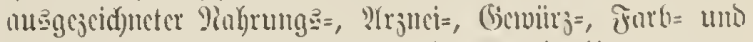
uno unorer Yintzpilunjen, bon Denen wir hier mur an=

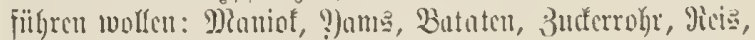
Sinfice, Rafuo, Banifle, Lifejer, Bimt, bic jog. Brotfrudt,

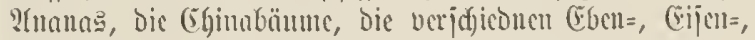

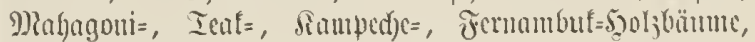
bann Baumwolle, bic bejten janf= und Flad)enten, Iabaf, Jubigo, dic feimpten Ëre, Baljame unb șarje.

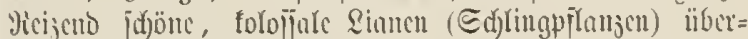
jpamen, vou 2 aum ju Baun fletterno, groje llruälocr, ricfige Farne und finftecn, inumberbar gejormte mo gc= färbte Erdjibecn und anbre präd)tige lłarajiten haben fier

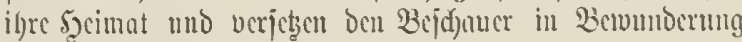
nII0 Entiütfen.

2) Tie juei tropijd) Zn 3onen, Tegion ber Frigen,

*) Sie gcjumte (Evbobcriläd)e ijt ani 9,261,000 Entornt

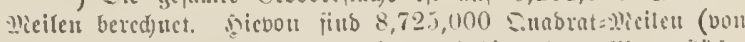

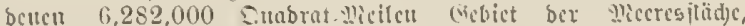

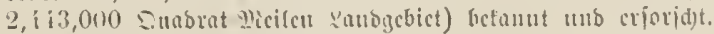

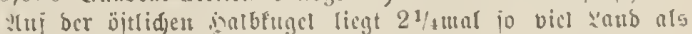
anj ber uncitliden, anf bex nörbliden 3 mal io vicl als alf ber jiiblidjett. 


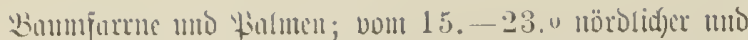

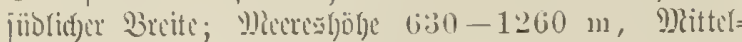
tompcrotur + $19-210$ R. $(23-261)$ C. $)$ - Dic

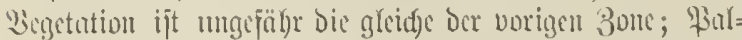

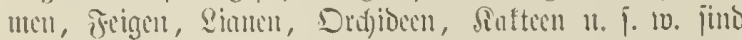

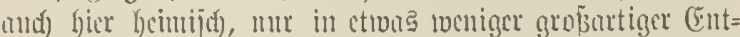

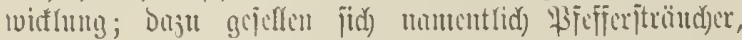

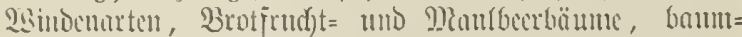

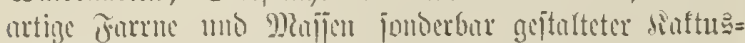

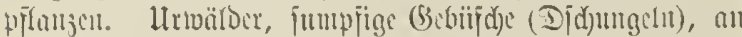

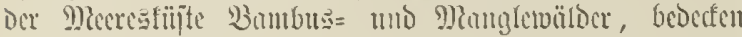
grofje Sanditrid)c.

3) Die jtoci jubtropijden 3onen, Veegion ber गु(y)rtu und Sorbecren: je bon Den Dembetreijen bis jum :4." nörblid)er mo jüblidfer B̀reite, von 1260 bie $1890 \mathrm{~m}$ 5öbe, 9littertemperatur $+1 \pm 0$ bis $190 \mathrm{R}$.

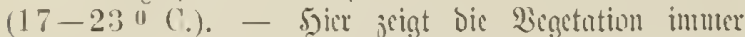

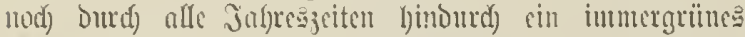
dileid, Damefen aber viel bentlidjer cinen llebergang ju

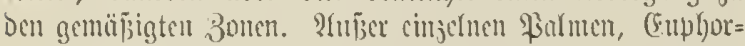

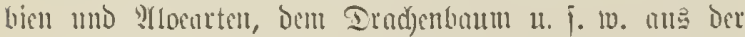
norigen Pegion jint hier daraterijtijd) bic immergrinen

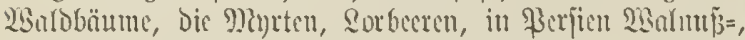

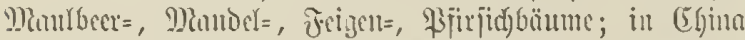
Der Theeptrund); Sciperiben (Drangegetoüdje), (Erifen,

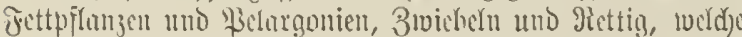
fier fujt ïbrall gedeiben; anj cinigen Etrufen jeigen pid) and) Die erjten 2Beiden, Psappe(n mo (5id)en, anf cinigen

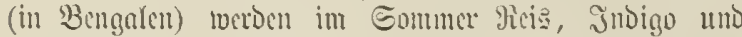

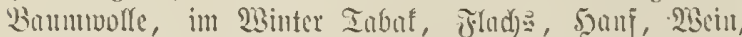

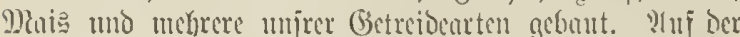
nörblid)en uno jïblid)en 5albtugel jino bie grojen Etep= peil, ßampa: d)arafteriitijid).

1) Die juci vörmeren acmäвigten Bonen, Jic= gion Der immergrïnen Sanblyöljer ; voun 34. -45.0 möro= fidjer uno jüblidjer Breite, 5̧öhe von $1890-2520 \mathrm{~m}$, mittfere Iemperatur $+10-140$ h. $(12-170$ C.).

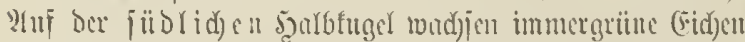
mแD ?horlyölser, itrand $=$ mo bammartige Gräjer mo

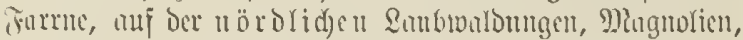
2iscinreben, Dormige Jojen, Etmomom mo Stründur mit

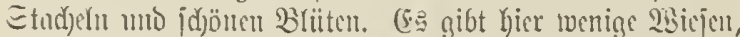
Dugegen (in Viorbumerifa uno ?[jien) an: ('irüricm).

5) Dic jwei färteren gemäpigten jonen, bic

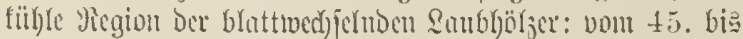
$58 .{ }^{0}$ nöroblidjer mo jïblid)er Breite, von $2520-3150 \mathrm{~m}$ 5öhe, 9leitteytenucrutur $+5-100$ R. $(6-120$ C.).

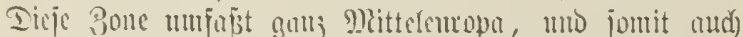

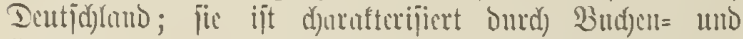

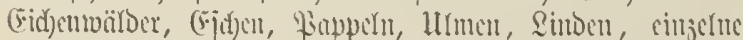

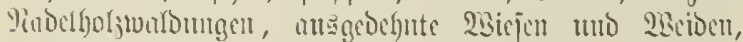

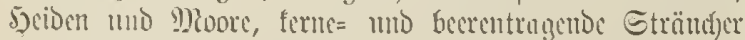

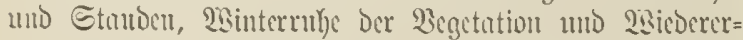

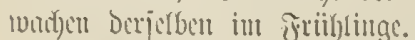

6) Iie juci inbarftijen oder talten Bonen, dic=

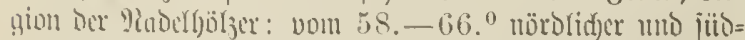
lid)er Breite, won :3150-3780 m 5jöhe, mittlere Iem= perutur + 3-50 li. $(4-60$ C.). Dicje ;onte un=

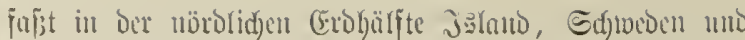

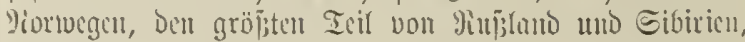

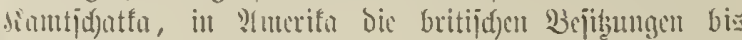

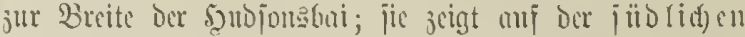

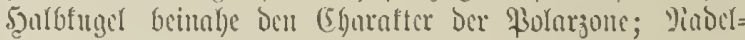

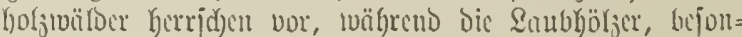
Dere Birfen, ErIen und Bitterpoppeln, mur cinjeln ju

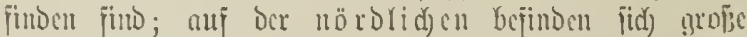

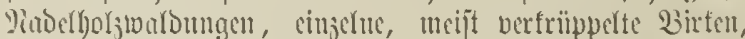

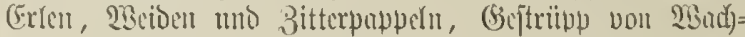

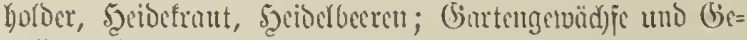

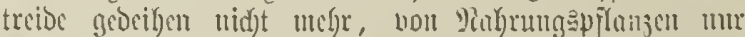

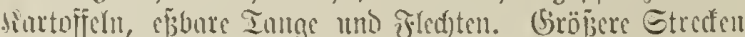
jint mit liteo= mo (Enpergräjern, Smmpfitreffen mit Sorf= moven bedectt.

7) Die jtoci arttijd) $n$ 3onen, untere Edjuceregion ber 2r(penftrüud)er: $66 .-72.0$ nörofider uno jiïblid)er Breite, Sö̈he voir $3780-4110 \mathrm{~m}$, 9Ritteltemperatur $+0-30$ R. $(0-40$ C.). Sin Dicjer Dicgion licgen Sapplunb, das nördlidjite Gibirien, Grönland un dic Sitijtenlänocr bee arftijdjen Djeuns. Baumartig findet

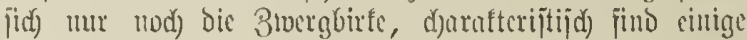

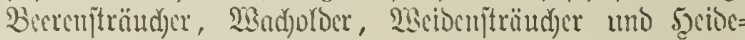

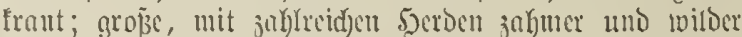
Jemuticre bevölferte Etrefen (Tumbraz), nuj Denen jajt gunj bie ärntlide Exijtenj Der Sappländer nno notojibi=

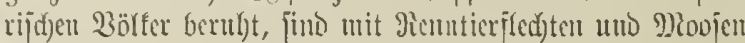
bidcutt.

8) Die juci \$olarjoutu, obere Edjnecregion, Jiegion Der ?flpenfünuter, vom $72 .-90.0$ mörblid)er mo jiiolidjer Breite, von $4+10-5040 \mathrm{~m}$ mo bariifor

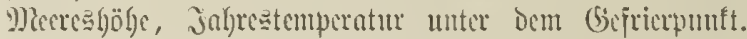

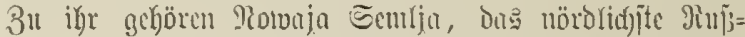

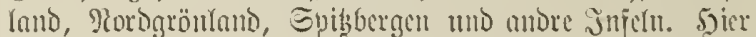

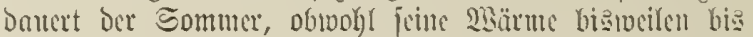

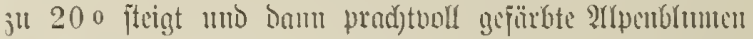
Gervorbringt, höd)jtens 3 1)?onat; von ?Yrfang 9lovember

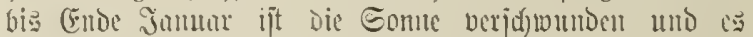

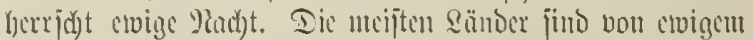
Eis bebedt vocr umpitart, es gebeigen mur nod) cinige

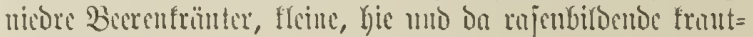
artige Piflanjen und diruptogamen.

\section{Ailitinlanlemitr.}

Mland

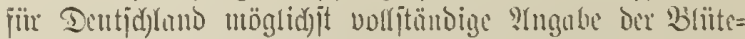

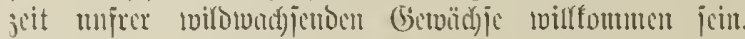

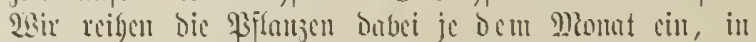
tocldem jis juerit bliigen.

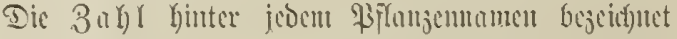

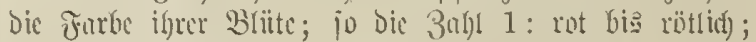
2: gelb bis getblid); 3: tuciis bis เociiplid); 4 : bram

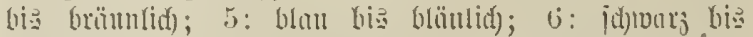

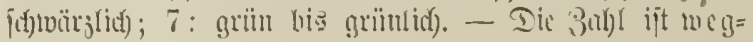

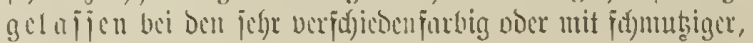




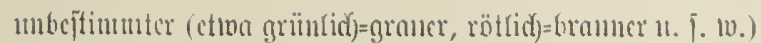

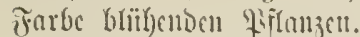

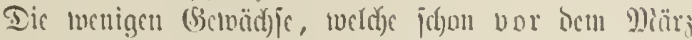

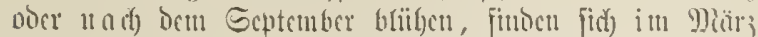

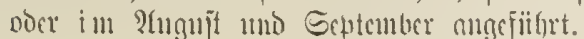

\section{jul श)ïri blïbcu:}

Adoxa moschatcllina, Mojdustrant 7 Alnus, (5ric.

Ancmone vernalis, 2iborösdicn

Asarum curop. 5ajelwurs i).

Bellis perennis, (G̈̈nieblïnd)en 3.

Buxus sempervirens, Wudlebaum.

(arex, giconras, veridjicoenc 'Irtin.

('elastium, Sortt rout is.

('lirysosplenum alternifol.. S)kil strout?.

('ornus masenla, fiormclfirid)e 2 .

Corylus, sojelftrand).

('rocus vernus, Eairan.

Daphne Merereum. Scioclbait 1.

Mraba rerna, 5omgerblïmd)e1 3.

Erodiun cicutarium. Rcilscriduttolol 1

Galanthus nivalis. Ed)ncenlö ffdell :3.

(ilechoma hederaceat, (ismbclrobe i)

(iramineas, verichicbenc (istosarten.

Ilellehorus viridis, grïnc glicfimur; 7 . bis Mï̈r.

Ilolost'um umbicllatum, ๔umre: 3.

Iamiun purpur., rote Taubucific 1.

Lencojun vernum, Sindetublume :3.

P'opulus alla, nigra, tremul:i. Linffitn.

Potentilla verna. Fingerfraut 2.

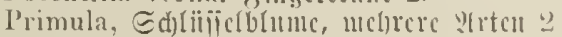

Prunus spinosa, Edhlche :

Pulmonaria olfic.. Eungcufrant 1.5.

P’nlsatilla vulg.. Sü̈dcuid)cllc \%).

Rosmarinus offic. Pownarill 1.

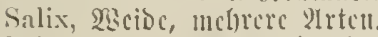

Scilla lifolia, y)iccrawicher

Senecio vuls. Sircustraut 2.

Stcllaria media, Bogcluticre 3.

Taxus baceata, (sibe.

Tussilago Farfara, Donflattich 2.

Ulinis, llumc.

P'etasites, 2icitwur' 1.

Veronica agrestis, hederactolia. arrensis, (rifrenpreis 5 .

Viola odorata et uliginosa, 2icildjen 5.

Viscum, Mijtel 2, (bliibt id)on in Jebruar).

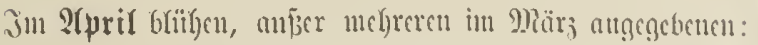

Actaca spicata. (Sbriftoplystrant:3.

$\Lambda$ juga reptans, (Giinjel ह5)

Alliaria offic., (2and)lycocrich) 3 .

Anemone nemorosa, Wucunouc 1.

Betula alla. Sirfe.

Capsclla bursa pastoris, Soirtentaid) 3.

Caltha pal., Dotterblume "2.

Cardamine pratensis. Edhamfraut.

('hacropliyllum sylv., Sörbel 3.

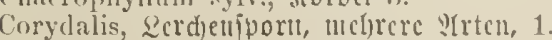

Crelamen europarum, Eanbrot 1.

I)raha aizoides, 5yutgcrbliimdl)cu 2.

Euphorbia Cyparissias, 2.bolfoumild 2.

Fraxinus excelsior, (5id).

(raleol)dolon luteum, (bolducifde.

(ientiana verna, (sinjion 5.

Gramineae, viclc Girobarten.

Ilippoplaë, ๔andodril 2.

Iris germanica, Edjucrtel :1.

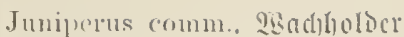

Laminm allmm, Taubnciicl 3 .

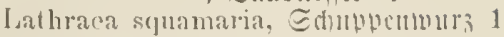

leontodon Taraxacun. Söucuial)! 2.

Menyantlirs trifoliata. Foborflet

Mnscuri, Traubenbuasintloc 5 .

(brnithogalum mmbellatum. Liogchuild) 3.

Orobus allous 3. 1. thalocrbic.

(1)alis Acetosolla, Eaucrffec.

l'irus commmunis, solizbirne 3 Malus, Troliagicl 3.

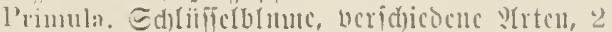

I'munus avium, Loycltirlde :3.

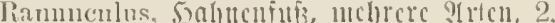

Salix, 2iscioc, dic meiften ?Iten.

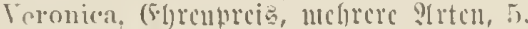

Vinea minor, Simnturin 5

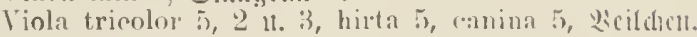

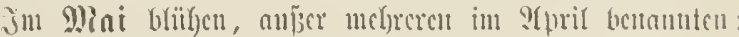

lcer, शrhoril.

Aesculus Hippoeastanum, diofifajtanic.

Alchemilla vilg.. Einan 2.

dlyssum calycinum. Etciufrefic :

Andromeda polifoli: Suldoroutcos 1.

Anemone syli., 2tucilunt 3

Anthericum Liliago, 3antinic 3.

Anthriscus rulg. et Cerefolimm, sictbel:3.

Antliyllis vulneraria, Disubflec 2.

Aquilegia vulo.. P(f́clct) 5.

Aretostaphylos I'va ursi. 23äroutranbe 3

Arenaria verna, Eamblraut?

Aristolochia Clematss, Eiterfuzci ?

Arum maculatum. YTronsmurs 1.

Asperula odorata, Walomciftcr :3.

liarbiuea volg., Disinterfieffe"

Berleris vulg., Eaterioril 2 .

Biscutclla laevigata, Wrillenidote

Camclina sativa, 2cindotter "2.

Carninus lictulus, soainbudic.

Carum Carvi, siinmutucl 3 .

Chelidonium majus, Ed)cllfraut 2

Cochlearia offic., ¿offeltraut.

Colutea arborescens, Blajcuitrand) ?2

Convallaria Majalis, Miaiblume 3 .

Cratacgus Oxyacantlia, wisifjdorll 3.

Crnanclum Vincetoxicum, Edıualbcunura: 3

Cynoolossum offic. Soundözunge 1.

Cypripedium Calceolus, Francuic()ulh 2, and 1 .

Cytisns laburnum. Bohnembanm 2.

Dianthus cacsius, Federuclte 1.

Dictammes, Iiptan 3 und 1.

Doronicum Pirdalianches, (iscutanul:

Invas octopetala, Gilbcrumtris 3.

Erica carnea. Socide 1.

Eumporbia lisula, gisolfamild) 2.

Evonynus curop. Epillbantm 2.

Fagus sylvatica, Dud c c

Fragaria resca, (5rbbecre 3.

Fnmaria offie, (5robaud) 1.

Galium glaucum, cruciatum, Mollugn. Eabfrout:3.

Genista tinetoria, pilosa, germanica), (iinterte.

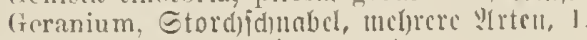

Filadiolus comm.. Eicnivura 1.

(ilohularia vulg.. Suggelolutuc 5.

(iramineac, bicle firngarten.

llicracium I'ilosella, sabid)tefrant ‘.

Ilex annifolinm, Stedipalnic 3 .

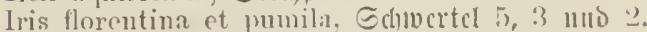

Isatis tinctoria. Qiaio 2.

Lathyous pratensis, Ifatterbic 2.

I.elum pal., Eumupporit ?. 
Lepidium eampestre, Feldfrefic 3.

Lithospermum offic., Eteinfane 3.

Lonicera Caprifolium et Xylosteum, (iicifblatt 3.

Lotus cornieulatus, Edhotulflec 2.

I unaria rediviva, Mlonbucild)en 5.

Lychnis flos cuculi. Studuffablunt 1.

$$
\text { Viscaria, Zectunelfe } 1 .
$$

Majanthemum bifolium, Edhatterblume 3.

Matricaria Chamomilla, ed)te Sianille 3.

Melittis grandiflora, Jmmenblatt 3.

Menyanthes trifoliata, Fieberflec 3.

Mespilus germanica, Mianel 3.

Morus alba, Mandbecrbann 7 .

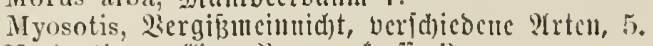

Nasturtium offic, Wrumuenfrefie 3.

Neslen, Rę̧це 2.

Onolyychis sativa, (sipariette 1.

Ophrys aranifera, fraucuthrüue 7 .

Orchis, Snabentrant, mef)rere P(rtell, 1.

Preonia offic., Ifingitroie 1.

Paris quadrifolia, (Finbeere 7 .

Pedicularis pal., \&änjeftrant 1.

Pimpinella magna. Limtuinelle 3.

Pinguicula vulg., Fettfraut 5.

l'inus, Fidłte, Ianuc, Föhre, \&ärd)e 1. 2. 7.

Pisum arvense, Felderbie 5.

Plantago lanceolata et media, Saegerid) 3 .

Platanus occident., Blatane.

Polygala amara 5, et vulg. 1, Sx renjblïmd)en.

Poterium Sanguisorba, Bedjerblume 1.

Prunus padus, Iranbentiridie 3.

Quercus Robur et pedunculata, (Eid)e 7 .

Ranunculus, 5ahnenfu[3, mehrere 9 (rtell, 2.

Rhamus catharticus, Sireuzdorn' 2. Frangula, Jaulbaum 3.

Rhinanthus villosus. 5abncufamm 2.

Ribes rubrum, nigrun, Johanuişbere 7 . Grossularia, Stadjelbecte 7.

Rubia tinctorum, $\Omega \mathfrak{r} a \mathfrak{p p} 2$

Rulus Ilaeus, 5imbere 3.

Rumex crispus et Acetosella, Shupfer.

Salvia pratensis, Salbei 5.

Sanicula europ., Sanifel 3.

Saxifraga granulata, Etcinbred) 3.

Sinapis arvensis, 2lderjen 2.

Soldanella alpina, Drottelblume 5 .

Sonchus oleraceus, Iiftelialat 2.

Sorlus Aucuparia, 20 getbecte 3. A ria, Melibcere 3.

torminalis, (ebereide 3.

Spartium Scoparium, Bejenftrand) 2.

Staphylea pinnata, Bimtucrunis 3.

Syringa vulg., Surituge 5.

Thalietrum aquilegifolinm, Esicjenrante 3.

Thlaspi arvense, Iafdeffrant 3 .

Tragopogon pratensis, Boffabart 2.

Trifolium, Silee, veridjiebenc 9rten.

Trollius europ., Trollblume 2.

Tulipa silvestris, $\mathfrak{B a r b t u l p e} 2$.

Vlex europaeus, Sectiantenitraud) 2

Vacrinium Myrtillus, vitis Idaca, uliginosum, Sacidel=, Iircifzel=, Smlutbecre 1.

Veronica, (5hrenpreiä, melurere 9frten, 5. 1.

Vilourum Opulus, Ed)ucebalf 3.

Vicia sativa, Futterwicte. sepium, Ballntidf.

Vinla arvensis, Ifferveil(t)en 2. palustris, Emmpfucildien 5.

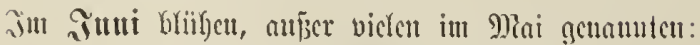

Aconitum (ammarum, geul. (sifenlunt 5.

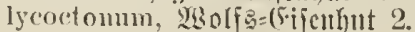

Acorus Calamus, Sidumut 7.
Actrea spicata, (5hriftoplastrant 3

Adonis aestivalis, $2 \mathcal{H}$ (uteströpfd)cu 1.

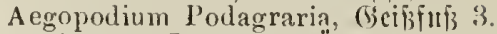

Agrimonia Eupatoria, Ëocrututing 2.

Alchemilla arvensis. Eirnat 7.

Althaca offic., (Eibijd) 1.

Anagallis arvensis, Gand) beil 1.

Anchusa offic., Ddjenzunge 5 .

Ancthum graveolens, Dili 2.

Anthemis nobilis 3 , tinctoria 2, sumbafantille

Anthericum ramosum, Baullilic 3.

Arabis arenosa 1. hirsuta 3 . Gäninefrant.

Armeria vulg., Girnsnclfe 1.

Aruica montana, $\mathfrak{B o h}$ lverlei 2.

Asperula cynanchica, Bräunctuır; 1.

Asperugo procumbens, Raulftraut 5.

Astragalus glyeyphyllos, Giißstce 2.

Atropa Belladonna, Iollfiridue 1.

Ballota foetida, Stiufnefiel 1. nigra, Sd)warzuefiel 7 .

Borago offic., Boretj() 5 .

Bryonia alba 3 und dioica 7,3 anurïbe.

Buphthalmum, Minbsauge 2.

Bupleurum rotundifolium, \$a aicuof)r 2.

Butomus umbellatus, BHInmenbinje 1.

Calendula arvensis, Riugelbfunc 2.

Calla palustris, $\Xi_{d)}$ langeninurz 3.

Campanula glomerata, patula, rotundifolia, Gilodell= blume 5 .

Cannalsis sativa, $5 a n j 7$.

Caucalis daucoides et latifolia, sajtoolde 3.

Centaurea Cyanus, Siormblnmic 5. nigra, Jacea, Scabiosa, Tlodenbrume 1

Centunculus minimus, $2 B$ icienfle cinliutg 3 .

Cerinthe major, $\mathfrak{B a d}$ )

Chenopodium allum. (sänj cinß 7 .

Chrysanthemum Leucanthemum, Radherblume 3.

Cochlearia Armoracia, Dieerrettig 3.

Convolvulus arvensis, Rimbe 1.

Cornus sanguinea et suecica, 5ुartricgd 3.

Coronilla minima, Srontwicfe 2.

Crambe maritima, Gectohy 3.

Cynanchum Vincetoxicum, Sdywalbenwura 3.

Delphinium Consolida, Ritteriport 5).

Dentaria bulbifera, 3ahutwurz 1

Dianthus caesius, Jedernelfe 1. Carthusianorum, Sartbäıjernelfe 1.

Digitalis purpurea 1, lutea 2, granditlora 2 , Jingerfut. Dracoceplialum Ruyschiana, Dradjentopf.

Echium vulg., Ratterwurz 5, aud) 1.3 .

Epilobium hirsutum, $\mathfrak{B}$ cibenrösche॥ 1.

Erigeron acris, Bernfigtrant 1.

Ervum Lens, hirsutum, tetraspermum, Linje 5.

Erysimum cheiranthoides, Sd)otendotter 2.

Galega offic., Beifflec 3.

Galium Aparine, Labfrant 3.

Geranium sanguineum 1, pratense 5. Ctordjidnabcl.

Gcum urbanum, Yeffeutunrz 2.

Gladiolus palustris, ธicqmu1 1

Glycyrrhiza glabra, Siifhola 3.

Gramineae, viele Brasartell.

Gratiola offic. Gincidentrant 3.

Ilahenaria, Stcndcliwnrz 3.

IIclianthemum vulg., Eouncurösd)en 2.

Heraclcum Sphondylium, särenflan 3

II icracium, Sabidhtatrant, vicle Strten, 2.

Hippuris vulg.. Tatueutwedel 7.

Hot tonia palustris, $\mathbb{B a j i}$ erieder 1.

llyoscyamus niger, Biffettrant

Ilyperieum humifusum, Sohnunisfrant 2.

Hypochocris maculata et ralicata, Ferfelfraut 2.

Iris sibirica 5, Pseud-Acorus 2, Ediwertel.

Lappago. Ggeljame 5.

Lapsana comm., Soaficulattidy 2. 
Lathyrus tuherosus, 代atterbie 1.

Lemna gibha et minor, Sisaijerlitien 7.

leonurus Cardiaca, عöıcuid)tveif 1

Ligustrum vulg., Railtweibe :

Lilium bulhiferum et Martagon, Silie 1.

Limodorum abortivum, Dingel 5.

linum, 2 eitt 1.

lirindendron tulipifera. Iulvenbaum.

Isolium temulentum, Inumellold) 3.

Innicera Pcriclymenum, (Beibblatt 1 ttits :3.

I.ychnis Githago, Sormrabe 1.

I. yeopsis arv. Srumulyal: 5.

I isymachia vulgaris, nemorum et nummularia, fifeutig= fraut 2.

IIalva sylv. et rotundifolia, Mlalve, sïjepapuel 1 .

Medicago sativa, 2Æzerne 5 .

Melampyrum arv. 1, prat. 2, sylv. 2, Stullucizeu.

Melilotus arv. et offic., Steittlee 2.

Muscari racemosum et comosum, Millathyaziutbe 5.

Myrrhis odorata, ofuigferbel 3.

Nigella arv., Sdhwarzfü̈utut 5 .

Nuphar lutea, Sumpiroie 2.

Nymphaea alba, Gecroje 3.

Oenanthe fistulosa, Rebentolde 3.

Onouis spinosa, 5rathedel 1.

Ophrys apifera, arachnites, fuciflora. Myndes, Fitulte thrätte.

Orchis maculata, Sitabenfrant 1. bifolia, Rucfutfablutue 3 .

Orobanche coerulea, minor, nudiflora. Rapum, Eotuture = tourz.

Papaver Rhoeas, Mohn 1.

Parietaria offic., GIaßtrnut 7

Parnassia pal., Serzblatt 3.

Pedicularis pal., ¿äujefraut 1.

Physalis Alkekengi, Jnbentiridje 3.

Pisum sat., Erbje 3.

Polemonium coeruleum, Sperrtrant 5 .

Polygonum viviparum et Bistorta, Snöterid) 1 .

Potentilla reptans, Jingerfraut 2.

l'renanthes muralis, Sojetijalat 2.

Prunella vulg. et grandiflora, firïnelle 5. 1. 3.

Prunus padus, Traubentiride 3.

Pyrola, Wintergrün, verichiedete 24rten, 3.

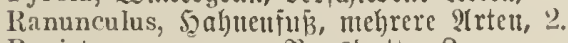

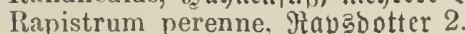

Reseda lutea, gelbe Piciede 2.

Rhodiola rosea, Plojentwitr 1 .

Rhus Toxicodendron, (Giitiutuad) 2.

Robinia Pseudo-Acacia, 2lfazie 3

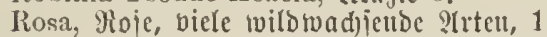

Rulous caesius, idaeus, vulgaris, Jimbeere :3 fruticosus, 2rombecre 3 .

Sagittaria sagittifolia, Iffciftrant 3.

Salvia offic., Salbei 5.

Sambucus nigra, 5oltuber 3 .

Ebulus, 2(ttid) 3 .

Sanicula europ., Secilfraut 3.

Saponaria offic. Eeifentrant 1.

Scabiosa arrensis, (Grindfrant 5. 1. 3.

Scheuchzeria palustris, Sdjeudjerit 7.

Scilla maritima, Meerziviebel 3.

Scirpus lacustris, Simie, Dinie 1.

Scleranthus perennis, ßutatel 3.7

Scropluularia nodosa, 23 rammum 4 oder 7 .

Sedum allum 3 , reflexum et are 2 . Tetthentuc.

Sicnecio Jacoladea, Irrellztrant 2.

Serapias rubra, $\mathfrak{B a l b v o ̈ g e l c i n ~} 1$.

Silene inflata et nutans, 2eiutfrant 3.

Sinapis alba, nigra, longifol., Ecuf 2.

Sisymbrium offic. et Sophia, 9tanfe 2.

Solanum Dulcamara, Sitteriiiß 5 .

Sonchus arv. et oleracens, Diitclialat 2.

Spergula arv. et saginoilles, Єparf 3.
Spiraea ulmaria, filipendula et Aruncus, Spieritrand) 3

Stachys german. et sylratica 1, palustris 5. 3iejt.

Symphytum offic. 3, patens. 1. Heituvell.

Tamarix germ., Inulariste 1.

Thalictrum minus, RBicieuratte 2.

Thymus rulg., Ilumian 1

Tilia grandiflora, Eommerlinte

Tormentilla erecta, Blutivur 2

Trientalis europ., Siebentrahl 3

Trifolium, slee, bie uciiten 9lrten, 1 oier 2 noer 3 .

Typha latifolia, Molyrfolbe 4.

Vaccinium Oxyecocos, Mloogbecte 1.

Valeriana offic., Halbrinı 1.

Ycronica offic., (E)reupreis 5

Vicia Cracea et tenuifolia, פBiffe 5.

Viola tricolor, seild)en 2. 3. 5.

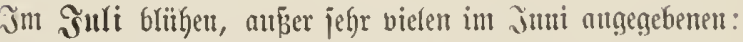

Achillea millefolium et nobilis, Sdjafgarhe 3.

Aethusa Cynapium, Sutto apeteriflic 3.

Alisma Plantago, Froid) (̈̈ffel 3.

Allium Cepa, sativum, olcraceum, 3wiebel, Ratth), Snob= lattd).

Anacyclus offic, Ringblume 3.

Anethum Foeniculum, Fettd)el.

Angelica sylvatica et Archangelica, (5ngelawwur3 3. 2.7.

Antirrbinum majus, ¿ömetumall.

Apium petroselinum. Beteriilie.

Aretium Lappa, Sllette 1.

Artemisia vulg., $\mathfrak{B}$ eifuß 2

Absinthium, WBernut ?

Atriplex patula et angustifolia, 多elise 7

Beta Cicla, vulgaris, Mangold 7 .

vulgaris rubra, rote fiibe 7 . altissima, Mutuelriibe 7

lietonica "sylvatica et offic., Wathengel 1.

Blitum capitatum et virgatum, (Frobecripinat 1 .

Cacalia alpina, 2frpetidoit 1.

Calamintha acinos 1 ,

$$
\text { mintha acinos } 1, \text { oftic. } 5 \text {, sialauinthe. }
$$

Campanula, Glocfenblume, mel)rere P(rten, 5)

Carduus benedictus, crispus, uutans utt antocre 2frtell, Diftel 1.

Carlina vulg., (5.berwurz 3

Carthamus tinctorius, Eafilor 2.

Caucalis grandiflora, saftoolde 3 .

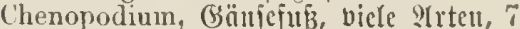

('hondrilla juncea, fitorpeljalat?

(ichorium Intybus, $\mathfrak{B}$ egivarte 5 .

('icuta virosa, Waîeridfierling 3.2.

Cireaea lutetiana, Sgcrentrant 3 .

Clematis Vitalla, $\mathfrak{B}$ ald rebe 3

Clinopodium vulg., $\mathfrak{\text { Birbeldoit } 1 .}$

('ochlearia Coronopus, Sdivcitzatrelie 3.

Conium maculatum, Sdierling 3.2

('onvolvulus arvensis et sepium, $23 i n d e .3$.

Coriandrum sat., froriantoer 3.

Crepis biennis, İippau 2.

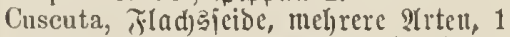

Datura Stramoniunl, Etediapfel 3.

Dianthus Armeria, Mielfe 1.

Dipsacus, Maubfarbe, eituige Prten, 1.

Drosera rotundifolia. Sommentan 3.

Flatine hexandra, $\mathfrak{T}$ áutul 1.

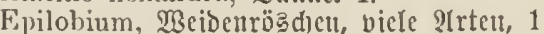

Firyngium camp., Mautistreu 3.

Grythraea Centaurium, Iaujendguldeuf raut 1.

Fuphrasia offic. 3 , lutea 2 , nlontides 1 . 9I ugentroit.

Foeniculum offic., Fettd)el 2.

(Galeopsis, 50 blzalyn, mcl)rere 9rten, 1.

Galium verum, 2, Aparine :3, sylvaticum 3. Sabtrant.

Gentiana lutea 2: rruciata 5), (5 
Geranium prat., Stordjiduabel 5.

Guaphalium arenarium und andre :trten, Pillgrfrant 2. Gypsophila muralis, (sipeftrant 1.

IIelianthus annuus, Souttcuroic 2.

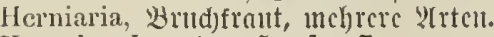

Ilumulus Lupulus, 5rovfen 7 .

IIydrochoris Morsus ranae, Frojd) bi 3. 3.

Hypericum, Jolnamistrant, bicle 24rten, 2.

Ilypochoeris, Ferfelftraut 2.

Ilyssopus offic., शipp 5.

Jasione montana, Syciltraut 5

Iberis amara, Baltcrlicuf 3.

Impatiens noli me tangere, Baljaninc 2.

Illecebrum verticillatum, Ruoruclfrant 3.

Imperatoria Ostruthium, Siaifertutr? 3 .

Inula Helenium, \{rant 2 .

Juncus effusus, Sintie 1.

Lactuca virosa, (sifitlattid) 2.

Lavandula Spica, Qabender 5 .

I avatera thuringiaca, Stambenuappel 1.

Laserpitium, Eajerfrant, mchrerc 24 ten, 3.

Levisticum offic., Ricbitödfl' 2 .

Linaria vulg. 2 , minor 5, Reill.

Lindernia, Bïd)jeufraut 1.

I inum usitatissimum, TI( $\mathrm{d}$ () 5 .

Lycopus, 2 olf

Lythrum salicaria, 2 eiderid) 1.

Maclia sativa, Elmabie 2.

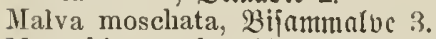

Mar'rubium vulg., aftuborm 3.

Matricaria Chamomilla, edfte Samille :3.

Medicago falcata, Sduncefenflec 2.

Melilotus alba, Steinflee 3.

Melissa offic., Melifịc 3 .

Mentha arvensis, aquat., sylv., Diiußc 1. piperita et crispa, Mlin3 1

Mercurialis annua, Bingelfrant 7 .

Neum athamanticum, 23 ärwıır 3.

Myriophyllum verticillatum et spicatum, Inujcubblatt 7 .

Narthecium ossifragum, Bcitbcil 2.

Nepeta Cataria et nuda, Siaßnumilzc 3.

Ocuanthe fistulosa, Mebcudolioc 3

Onopordon Acanthium, (siclabijtel 1.

Orchis pyramidalis. Sutabcufraut 1.

() riganum vulg., MBol)lgemut 1.

Majoralla, Mkajoralt :3.

Oxalis stricta, Gancrflec 2.

Oxytropis pilosa, Falutucuwiffe 2.

Petroselinum sat., \$Scteritilie 2.

Peucedanum offic., 5zaritrang 2.

Phellandrium aquaticum, 23ailerif cld jel 3.

Picris hieracioides, Bittcrfraut '2.

Pimpinclla saxifraga, Bimpinetre 3 . Anisum, $\mathfrak{A}$ III 3.

l'lantago major, $\mathfrak{R}_{\text {e egerid) } 3 .}$

Polyenemum arv., Sinorpelfrant 3.

l'olygonum, Stü̈terid), viele Irten, 1. 3. 7. Fingopyrum, Band)tvcizcil 3.

Portulaca olcracea, giortul of ?

l'otamogeton, Raid)trant, Leridicbenc :irtcu, 1.

Prenanthes purpurea, Sajicujalat 1.

lieseda luteola, $\mathfrak{B a u} 2$.

lihinanthus Crista galli, : Pderroicl 2.

Rumex, PImpier, vicle शrterı, †.

IRuta gravolens, Piante 2.

Sagina glandulosa, Majtfrant 3.

Salsola Kali, Galztrant.

Sanguisorla offic., Misicicnfnopf 1.

Saponaria offic., Ecifcutraut 1.

Satureja liort., \&iffferfraut 5.

Saxifraga $\Lambda$ izoon, Єtcinlurcd) 2.

Scaliosa columbaria et succisa, (Brindfrant 5.

Schoenus mariscus, Sduncidgras 2.

Scirpus pal., Ed)lammbinic.
Scleranthus annuus, Sitaucl $\%$.

Scutellaria galericulata, Sdjilbfrant 1

Sedum tectorum, 5ूats (nแb) 1.

Sempervivum, Saustourz, melfute 9 (rten, 1 แnd 2.

Senecio, Strelzfrant, ntebrerc $\mathscr{Y}$ trten, 2.

Serratula tinctoria, Färbcridartc 1 .

Sherardia arv., Sherarbic 1.

Sium latifol., Mert 3.

Solanum nigrum, $\Re(n d j$ tid)atten 3.

"tuberosum, Sartoficl.

Soliclago Virgaurea, Bolbrutc 2

Sparganium, Igelatolbe, ulchrerc $2(r t c t, 7$.

Stachys germanica, Bieit.

Stratiotes aloides, Waineraloc 3

Tanacetum vulg., Rainfarı 2.

Teucrium Chamacdrys et Scordium, (sintuntioer 1

Thalictrum flavum, Rnute 2.

Thymus Serpyllum, Thymian 1.

Tilia parvifolia, $\mathfrak{B}$ ituterlinde.

Trapa natans, Baffermin3 3.

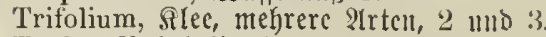

Typha, Rolinfolbe 4

Urtica urens et dioica, Bremuncifel 7.2

Utricularia vulg., $\mathfrak{W}$ afferid)(and) 2.

Valerianella Auricula et olitoria, 21dcrinlat 5.

Veratrum album, Bermel 3.

Verbascum Thapsus, $\mathfrak{B o l l b l u t m e} 2$.

Verbena offic. (sifcutrant 1.5.

Vicia Fala, श̂décibone 3.

Zannichellia pal., Seidengras 7.

Zea, MRaiz 2

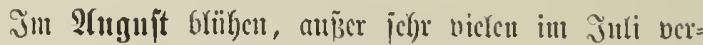
jeidjucten:

Aconitum Anthora, (5ijenlyut 2

Apium graveolens, Scllctic ?

Aster Amcllus, Stcrublumic 5

Astrantia major, Steritonlde 7 .

Bidens tripartita, 3weizalit 2.

('allitriche autumnalis, $\mathfrak{B a f i} c r i t c r n 7$.

Carlina acaulis et vulg., (Ebcriwura 3

Cervaria, Sinfd)ivnr 3 , mel)rerc ?(rtel 3 .

Chrysanthemum segetum, 2ind)crblumc ?

Dianthus superbus, $\mathfrak{R e l f e} 1$.

Erica vulg., Srcide 1.

Gentiana acaulis, (5nzian 5.

Gnaphalium uliginosum et sylvaticum, ?hlyttant 2

Hedera Ilelix, Spheu 3.

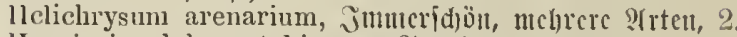

IIerniaria glabra et hirsuta, 23rudefrant 2 mid 7 .

Linaria, Reitffraut, mchrece $9(1 \mathrm{tclt}, 5$

Lycopus curop., 2Bolfaftui3 3 .

Parnassia pal., (5inblatt 3 .

Peucedanum offic, Soaritrang 2

I'hragmites, Sdjilfuolur 4.

Salicornia herbacea, (silagid)malz 7.

Scrophularia aquat., Brautuvur 1.2

Sescli bicnne, 9ioffitimmul 3.

Spiranthes autumnalis, Drchährc 3.

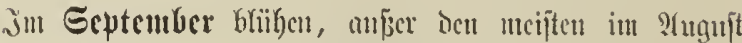
angegebchent:

Colchicum autumnale, Soctupitzeitloic 1

Crocus sativus, Gafroul 5.

Gentiana camp., ciliata et germanica, (5nzian 5. 


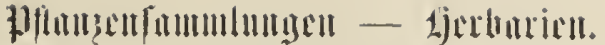

Dic Garmlofe mo licblidge (Gowohngeit, anf Epajicr=

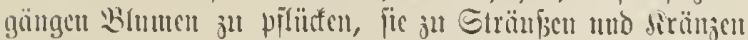

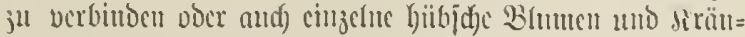

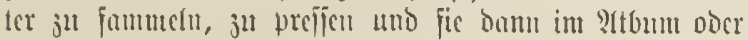

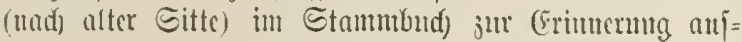
jubcubagren, ijt cinc jür bisunit und (bsjmbhcit an= genchme, in vieler Bejichung cunpeglenswerte llnter= boltuntg. Dunteben müd)ten wir aber bie jungen gintur=

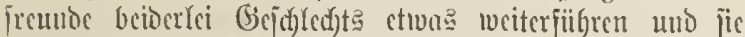

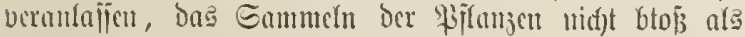
flitd)tigen Beituertreib, jonbern and) afs cine Bejdjüftignutg

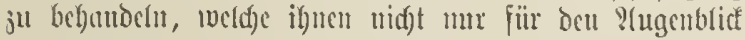

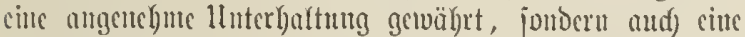

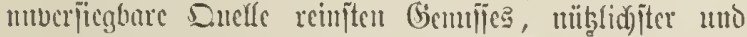

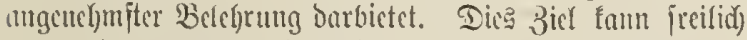

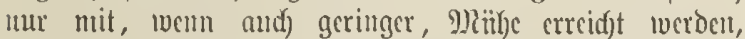

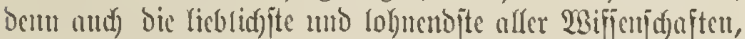
Dic Biflunjentumbe, verlaugt cinige Becharrlid)feit umb

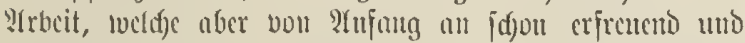
logueno ijt umo bic Edfulung Der Oscijteathätigfeiten,

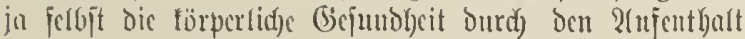

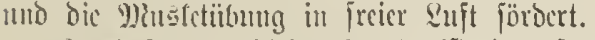

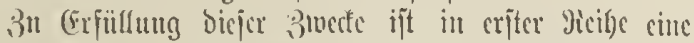

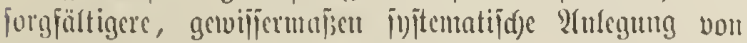

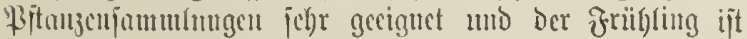
die 3eit, in weldyer mit beut Enmmeln ju begimen ift.

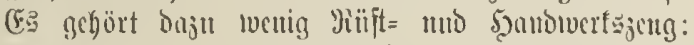

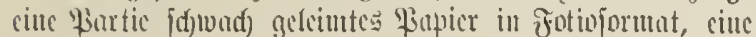

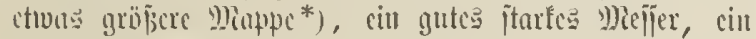

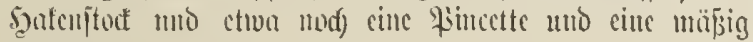
vergröbertuoe sinte.

Das Berjabren jelbjt ijt jugr cinfad). Dic in fräf=

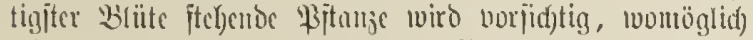

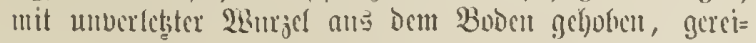

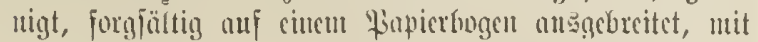
einem jucilen Bogen bedect unt jo in bic M)?appe gelegt; ebenjo wiro mit Den weiteren Pflanjen verfabren. -

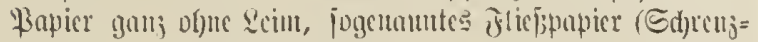

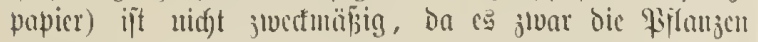

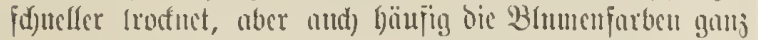

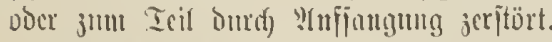

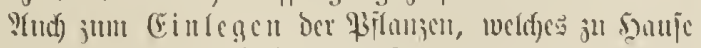

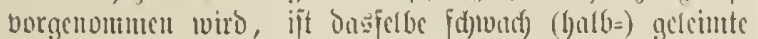

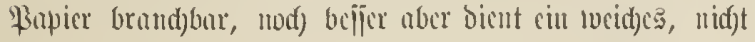

*) (Eine botanijierbüd) ie ift fïr bie (tinjammluty

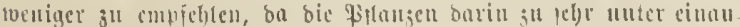
ber gemijd)t uto aus ifjer natiitlidjeu :age gebrad)t tverbes; bei

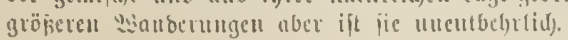

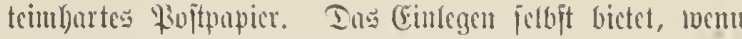

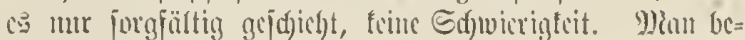
Durf baju $(;-10$ bïmer gtuttgehobelter Birettefen bou ber

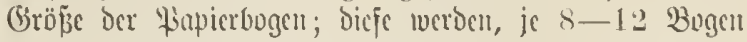
mit ben inficgenoen \$iflanjen jwijd)en 2 Brettern aนf=

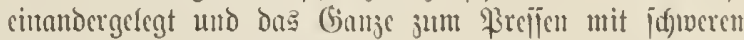
Steinen oocr Metallitüicent belegt. SJat man cinc cin=

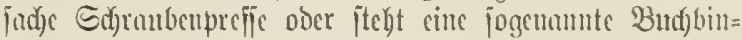

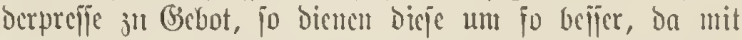

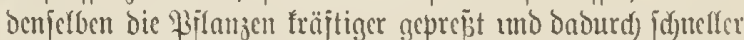
getroffuet werben. TYlle $2-3$ Iage Lerben num bic Yiflan= jen u ungelegt, b. K. aน马 bemt mehr ober minder feudj)

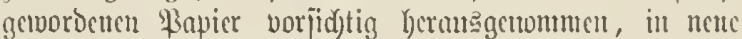

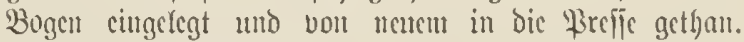
Dics geidjicht fo oft, bis bic Biflanjen vodftändig ge=

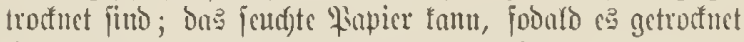
ift, red)t gut wicber bon nemem verwenoet werben.

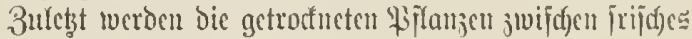
Wioft= ober Edjreinpapier getegt und mit (Bmumi leid)t aufgeflebt - fic birben bum jüamuten das \$gerba= rium, iveldyes am bejten in beforbern Mntappen mufju= bewalyren ift.

Bis bufhin ijt bie Sndfe inmer nod) cinc, wemu and)

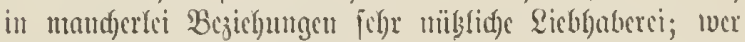
aber und) nur bicje bejwedt, wer jeine Eanumluntg mur

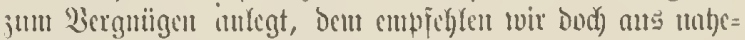

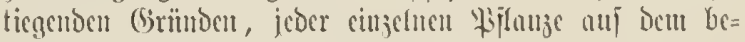

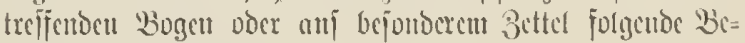
mertutgen heizufüget:

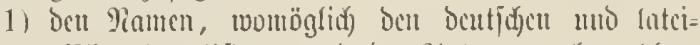

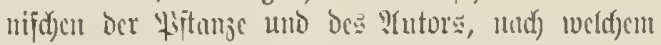
fic beremunt loorben ift;

2) bic Gegento, wo fic, fouvic

3) baz Tatum, ant weld)ent fic gefunton leurbe;

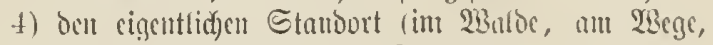
anf bor ․ㅗicje, in Sumpi u. j. w.).

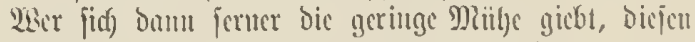

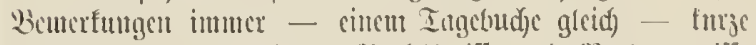
Yeotizen iiber die weileren Berbäftuiffe uno Bortoummiffe Des betreffendon Inges beijuffïgen, oer wirb all: feutem

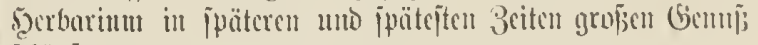

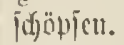

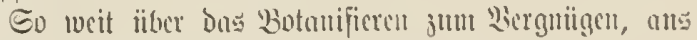

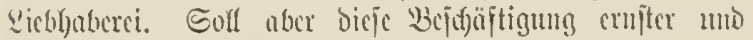

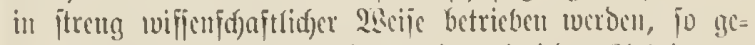

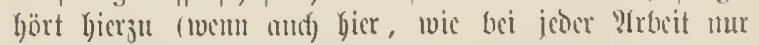

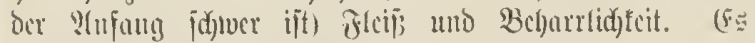

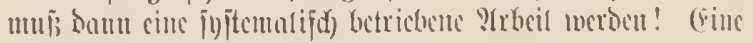

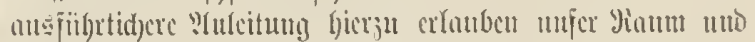

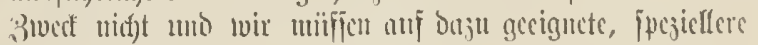
Mbirfe verwecifut. 


\section{Autuxu-Axmiltx.}

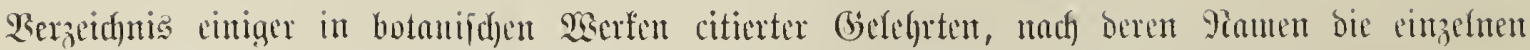

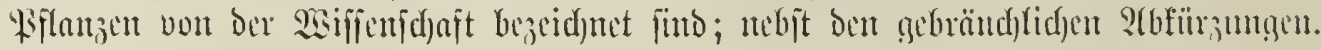

Ach. $=$ Acharius. 1757-1819. Frof. it Stoďholut.

Ad. ober Adns. = Adansoll. 1727-1806, geb. 311 2lir, † in Paris.

Afz. Afzelius, 1750-1837, geb. 3n zarf, + in llufara.

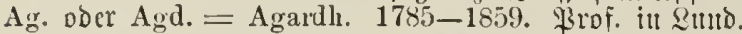
Agass. = Agassiz, geb. 1807 it Drbe, $† 1873$ in (Eatt= bribge, Prof. ber Raturgejodidte.

Ait. = Aiton, 1731-1793. Gartentanfeber in Sicto.

Alb. = Albertini. 1769=1831. Bifd)of in Serruhut.

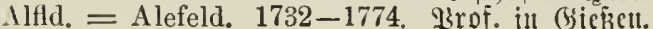

Andr. = Andrews, geb. 1813 in Belfait, Prof. Der (Eh)e= tuic baj.

Ard. = Arduino. Frof. Der Laubivirtidaft in \&abua; Berte 1764 it. fif.

Iudouin $=$ Audouin. 1797-1841. Raturf. itt Wari

Bal, = Babington. 1757-1833. 2rrzt itt Qonbolt.

Balb, = Balbis, Prof. in 2yon, $\uparrow 1831$.

Bald. = Baldinger. 1738-1804. Brof. it Marburg.

Bart. = Barton. 1766-1815. Prof. ill \&̧̧ilabelphia.

Bartl. = Bartling. 1798-1830. Wrof. in G̈öttittgen.

Batsch = Batsch. 1761-1802. Prof. itt Jetn.

Baul. = Bauhin. 1560-1624. Trof. in Zajel.

Baumg. = Baumgarten, 1765-1843. Arzt in Sdyäß burg.

Beauv. (Bv.) =- Beauvais, 1752-1820. 2(bv. il :Irrą, $\dagger$ ill Zaris.

Benth. = Bentham, engliffjer Botanifer; $\mathfrak{B}$ erfe: 1826 it. ff.

Bertol. = Bertoloni, Brof. it Bologuta; 2Berfe: 1803 u. if.

Besl. = Besler, 1561-1629. 2rpothefer in Rürmberg.

B1. = Bluft, 1805-1837. Irizt itl F(ad)ett.

Boengh. = Bönninghausen, Boritand bec botanifdeu Gartete in Mïtufter; 2 erke: 1824 u.

Boerh. = Boerhave. 1868-1738, Zrof. ill ¿etydt.

Boiss. = Boissier de Sauvages. 1706-1767. Trof́. ill Montpellier.

Bolt. = Bolton, ettgl. Botanifer; $\mathfrak{E}$ erfe: 1785 u. ff.

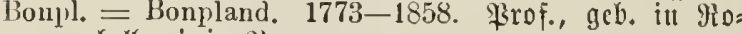
d)elle, $\dagger$ in Liaragua!.

Borkh. = Borkhausen. 1760-1806. Prijefior it Darm= itabt.

Brandt $=$ Brandt, geb. 1793 it Berliu, \$rof. ill \&eter butra.

Brign, = Brignoli, Prof. in Beroua; $\mathfrak{B e r f e : ~} 1810$ ॥. if. Brnl. = Bernhardi. 1774-1839. Wrof. ill Erfurt.

lirogn. = Brongniart, geb. 1801, Z3rof. it Zarig; † 1876. Brot. = Brotera, Gartendireftor ill Siffabon, $\rightarrow 1829$.

Buchan = Buchanan. Englëmber, Rcifellder in Dittubien.

Bung. = A. v. Bunge, geb. 1803 it Rieı,, Hrof. it Dorpat.

Burm. = Burmeister, geb. 1807 in Straljund, Prof. it joalle.

Camb. $\stackrel{\text { Cambessèdes, Frranzpic; } \text { : }}{=}$ crte vou 1828 u. fi.

Cass. = Cassini. 1781-1832. Pair vou Fraufreid).

Cav. = Cavanilles. 1745-1804. Direttor bes botatijdyen Gartene it Madrio.

Cliam. = Chamisso. 1781-1831. Didjter unt Viatur= Forfiber itt Merritt.

Corda = Corda. 1810-1849. Poot. ill Lirag.

Crut\%. = Crantz, geb. 1722, $† \mathfrak{a l g} \mathfrak{Q}$ razt in Jubenburg.

Cunugh. = Cunningham. 1793-1835. Bot. ilt Gioutet).
Curt. = Curtis. $1746-1799$. Wot. in Lonbon.

Cuss. = Cusson. $1727-1785$. Z3rof. ill Mlnntpellier.

D. C. und De Cand. = De Candolle. 1758-1841. Frof. ill bientí.

Desf. = Desfontaincs, 1752-1833. Wirof̃. Dur Botanif ill Iinriz.

Desv. = Desvaux. Fraıว. Botanifer. 23crfe: 18us bi 1827.

Dicrb. = Dierbach. 1788-1346. Irof. ill Sreibelberg.

Dietr. = Dietrich, geb. 1800 in Biegenthain, llıtucrfitäts gärtnter itl getta.

Dill. = Dillenius, geb. 1687 in Darmịtadt, itarb 1747 als Gartendireftor itl Drford

Dod. = Dodonaeus. 1518-1586. Zirof. itl Qcndent.

D. Don = Lavid Don, in Routoon † 1841.

Dougl. = Douglas. Sdjotte, reifte voll 1823 an in rifa, + anf bell Sandwidjufelt.

Drumm. = Drummond. Bercifte ?tuterita als liatur= forfd)er, † 1835 anf (Enba.

Duby = Duby. Framzöitider Botantiłer.

Duh. = Duhamel. 1700-1782. Fratz. Warinteinjpeftor.

Dum. = Dumortier, geb. 1797 in Tournal), 厄tatămau it betl शicberlamben.

Ehrbg. = Ehrenberg, geb. 1795 in Delitid), Firof. in Berfitt.

Ehrh. = Ehrhart. 1742-1795. Gartentupeftor in sou reuthatifet bei \$2amtuder.

Endl. = Endlicher. 1805-1849. Lirof. it 2isiell.

Eschsch. = Eschscholtz. 1793-1831. Wirof. itl Iorpat

Esp. = Esper. 1742-1810. Prof. ill Erlangen.

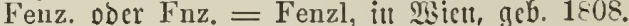

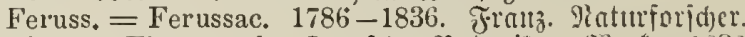

Fing, = Fingerhuth. Deutid)er Dotatifer. 28 erfe: 1822 it. if.

Flk. = Flörke. +1835 alb Iircttor bes botunifdent Giartents it Roitod.

Forsk. = Forskal. 1736-1763, t'rō. itl Sopculyagen.

Forst. $=$ Forster. 1754-1794, ill Manits, $\dagger$ in Harig. $^{2}$

Fr. = Fries. 1794-1865. Iirof. ill llpiala.

Fres. = Fresenius, D.reftor Der Settenberg'id)en Etif tumgen in Fratefurt; $26 \mathrm{erfe}: 1832-1853$

Freyc. = Freycinet. 1779-1842. Franz. Patmiprid)er. Froel. = Froelich. Medicitultat in (5lltuangen, $† 1811$ Fror $=$ Froriep. 1779-1847. Faturforid)er in iscimtar. Gaert. ober Gacrtn. = Gaertner. 1732-1791. Gieb. jull Calw, Brof. it Psetersbura, $\dagger$ ill Calw.

Grek. = Garcke, Z3rofeffor Der Botanif in Berlin.

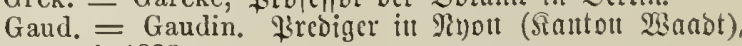
$+1883$.

Gesn. = Gesner. 1516-1565. Sd)tocizer 2iaturforid)er. Gilib. = Gilibert. 1741-1814. I'rof. int Qyolt.

Gm. obcr Gmel. = Gmelin, 1748-1803. 감ㄷ, geb. it Iitbittgelt, $\dagger$ in Göttittgett.

Gochn. = Gochnat. Framzö. Wotanifer. Werte: 1808, Goepp. = Goeppert, geb. 1800, Prof. it Dreălat. WSert"e 1827 it. fif.

Grab, = Grabowski, 2rpothefer in Dppelı.

Graum. = Graumüller. 1770-1825. Irof. ilt ienta.

Gray = Gray, amerif. Botnuifer; Mectf: $1836 \mathrm{ut}$. if.

Griess. = Griesselich. 1809-1848, 29rot in Siarlstul).

Grisb. = Grisebach, Zirof. it Göttittget, geb. 1814 it 5amtover. 
Haenk. = Haenke, geb. in Srcibic (2öl)men), † 1817 in Bolivin.

Hall. = Ilaller, 1708-1777. Hrof. Der \&otanif. Grojer Iidjter und Stantsmant in Wern.

Hamilt. = Hamilton, Bot. is Euglant); 2Berfo: 1825

Harim. = Ilartmann, 1790-1849. 91r3t it Gtoffholut.

Ilayn. = Ilayne. 1763-1832. Wirof. ill Berlin.

Irehst. = Hoehstetter, Etadtpfarrer in (5.jtitact, + 183!)

Ildg. ober IIedw. = Iledwig. 1730-1799, geb. in siton= itabt, † it Scipzig.

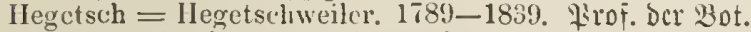
แnt) Yegicruttgrat it 3itrid).

Heist. = Heister. 1683-175s. Hirof. in solutitedt.

l'Ilerit. = l'Heritier. 1746-1800. \&intis. WBerfe: 1784 bis 1790.

St. IIil. $=$ St. IIilaire. 1799-1853. Matmiorid) in Drleans.

Huffin. = Ioffmann. 1760-1826. Istof. in Göttitgcu, +1826 itt Mogfall.

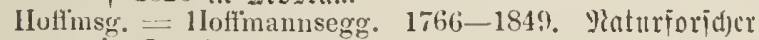
it Dresoctt.

Huok. = James Hooker, gcb. in Eriter 1785, Garten= bireftor its Sew, + 1865 .

Ifurnem.= Hornemanı. 1770-1811. Wrof. Der \&ুot. it Sioperthagen.

I1pp. = Hoppe. 1760-1846. Wirof. Der Bot. in Hegents= butig.

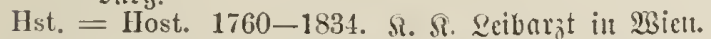

Iluds, = IIudson. 1730-1793. 2(untf)efer it 201toolt.

Irüg. = Hügel, geb. 1796 it Yicgeteburg, Heifender tmb Botanifer; 28 erfe $1837-1852$.

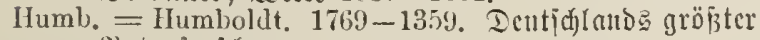
Minturforider.

I. B. K. = Humboldt, Bonpland et Kunth.

Jacks. = Jaekson. 1767-1815. Sirafibent ber 2erei= ninten Stanten vou 9?ordancrifa.

Jacr. = Jacquin. 1727-1817. (Sartellbirettor, † ill 2 situ.

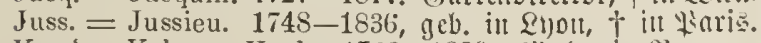
K. obcr Kch. = Koeh. 1768-1839. v) Uler it Mout.

Karw. = Karwinsky. Jlt Miïnd)en, bercifte Mexito.

Kielm. = Kielmeyer. 1765-1844. Wrof. it I Itbittget.

Kit. $^{\circ}=$ Kitaibel. 1759-1817. Brof. Der Bot. it Bubapeit.

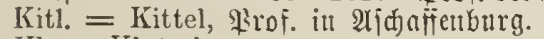

Kl. = Klotseh.

Klnggf. = Klinggraeff:

Knz. = Kunze. 1793-1851. Wrof. Dor Bot. it உcipzig.

Koch $=$ Koeh. 1771-1849. Q3rof. itt Ertanget.

Koel. = Koeler, Zrof. in Minain; Wert: 1802 u. ff.

Koelr. = Koelreuter. 1734-1806. Wrof. in Sar[Erttl)c.

Koert. $=$ Koerte. 1782-1845. Pirof. in Dlöglut.

Krombh. $=$ von Krumbholz. $1783-1843$. Itrof. in \&irag.

Kth. = Kunth. 1788-1851. Pirof., gch. ill Seipzig, † it Berlit.

Kütz. = Kützing, gev. 1807, grof. it Norohaujet.

L. oder Linn $=$ Limné. $1707-1778.2$ crïfuttejter Botantifer.

lamk. = Lamarek. 1744-1829. Lirof. itt Liaris.

Langsd. = Jangsdorff. 1794-1852. Beriilututer Mcilell= ber tuto Paturforiduer, $\dagger$ in Freiburg i. Br.

Lap. = Lapeyrouse. 1744-1818. Brof. it Ionlonic.

Ledeb. = Ledebour. 1785-1851. Lirof. Der BHot., $\dagger$ in miütdjent.

Lchm. $=$ Lehmann. Direttor Des botatijd)en (B)artenz it 5auburg; $23 e r f e: ~ 1817-1844$.

Less. = Lessing. Rotauter, bereifte 1832-1837 9uß $=$ Ialto, Morivegen น. j. to.

Lestib. = Lestihoudois. (2iater, Sol)แ แtb (EnfCl), Bot.

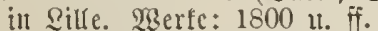

Lohte. = Lightfoot. 1735-1788. Lifarrer z̆ Gotham. Lichtst. = Iiehtenstein. 1780-1857. W'rof. in Bertitr. Lindl. = Lindley. 1790-1865. Ysrof. it Sollooll.

Lk. oder Lnk. = Link. 1786-1850. W'rof. in Berlin. Lmk. = Lamarek. 1744-1829. Brof. ill Wiariß.

Loud. = Loudon. 1783-1843. Botnutfer it 2oubolt. Lz. = Lenz. 1799-1870. QChrer it Gdutepfentbal.
M. et $K_{.}=$Mertens et Koeh. Mierteng, † 1831 แ Direftor Der sambelsidtule it $23 r e m c u t$.

Mart. = Martens, geb. it Winedig 1788, + in Stuttgart $187 \cdot 2$.

Marts. = Martius. 1794-1866. Wiroi. in Mituditu.

II. B. = Marschall ron Bieberstein.

Med. = Medieus. $1771-1850$. Wirof. in yiütd)en.

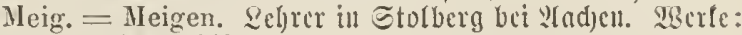
$1804-1842$.

L. Mey. = Ernst Meyer. Iireftor Dis bot. Gartus it Sïnigabcrg. 28crfe: 1822-1836.

G. Ney. = G. F. W. Meyer. 1782-1856. Hirof. Der Botanif in Göttintgets.

Michx. = Miehaux. 1716-1802.

Mik. = Mikan. 1769-1844. Brof. Der Bot. in Lirug.

Mill. $=$ Miller. 1691-1771. Gartendireftor it (Shelien.

Mnels. = Moench. Firof. it Mrarburg, + 1805 .

Moehr. = Moehring. QI $r_{3}$ t ats Danzia, + 1702 it jever.

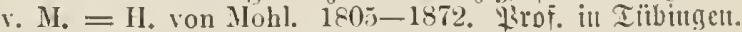

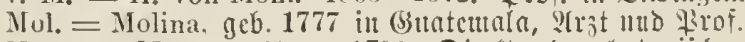

Murr. = Murray. 1740-1791. Dircftor Des botaliijdill Bartente in Göttingen.

I. oder N. v. E. = Nees von Esenbeck. 3twei Brïber, beide beriifhnte Botaliter. Ier ältere $† 1837$ it Bont, Der jïngere 1858 it 2 reslatt.

Naeg. = Naegeli. Botanifer ill 3̈trid). 26erte: 1841 11. ff.

Neek. $=$ Necker. 1729-1793. Bot. it Mautucim.

Nestl. = Nestler. Hrof., Gartatoireftor in Etrap̧burg.

Neuw. = Neuwied. 1782-18.. 2Berfe: 1815 II. if.

Nit. $=$ Nolte. Wrof. in Siel. 2iscrfe: 1826 it. if.

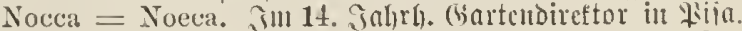

Nutt. = Nuttal. Wirof. in Yibiladelpljiu. 2kerte: 1818 II. If. $^{2}$

Pall. = Pallas, 1741-1811. Lircuije, lebte itt Vimjanto, + it Berlin.

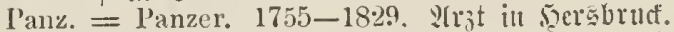

P. Br. = Patrik Browne. 1720-1790. Whijt mito Wot. iit irtalti.

Pers. = Persoon, geb. anf Deut Lorgebirge dor gltten 5offumt +1836 itt 13 ario.

Peterm. = Petermann, geb. 1806 in Slandan, (biograpl) in (Gotlina.

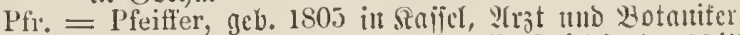

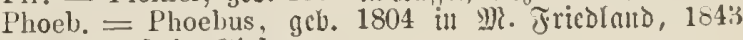
Brof. in Gieß̄en.

P. M. E. = Patze, Meyer et Elkan.

Poepp. = Poeppig. 1797-1868. 壮rof. in Rcipjig.

Puhl = Pohl. 1770-1850. Prof. tt Lcipjig.

Poir. = Poiret. Jranjöi. (Geiftlid)er. Tiserfe: 1789 bi 1866.

Poll. = Pollieh. 1740-1780. Sot. it Staiferglanturt.

Presl. $=$ Presl. Wot. it Wrag. WBarte: 1826-1811.

Pursh = Pursh. Bercijte 1799-1811 Viorbamerifa, † 1820 .

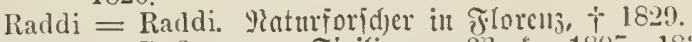

Rafin. = Rafinesque. Sijilianer. 23erfe: $1807-1830$.

Ram. = Ramond. Prof. Der Ylatmigeldidjte, geb. illt

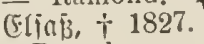

Ratz. = Ratzeburg, geb. 1801 in Berlin, Frof. in (Ebers= ivalbc.

R. Br. $=$ Robert Brown. 1781-1858. Hrüjiocnt bor

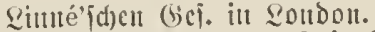

Rclıb. = Reiehenbach. Wirof. ill Dicabcu, geb. 1793, †

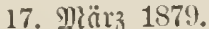

Rchb. fil. = G. Reiehenbach Eolıt. Firof. Der Wot. it Eeipoig.

Rchd. = Iieichard. 1685-1775. Matımī. in (5iut.

Red. = Redouté. 1759-1840. Dialer und Frof. itl Harie.

Iiegt. = Regel, gev. 1815, Direftor des botanijd)u (6ar= tens iit Beterouburg.

Reinw, = Reinwardt, geb. 1773, 1 :rof. il Sel)ben.

Retz. = Retzius. 1742-1821. Rirof. it \&mid. 
Rey. = Reynier. 1762-1821. Woitsirctior in Sumiaute. Rich. = Richard. 1754-182 I. Frof., † it Zaris.

Riv. = Rivinus, 1652-1722. Yrof. Dor \&ot. il Scipjig. hochl. = Rochling. 1726-1813. If arrer in Mefienthein.

Rocm. = Rocmer. 1763-1819. शी răt ilt 3ïrid).

liucss. $=$ Rocssig. 1752-1805. Frof. ill Reipzig

lottb. = Rottboell. 1727-1797, lirof. ill sopeulageu

loxb. = Roxburgh. Gartenbireftor 3̆l Mabrag, † 1814 . loz. = Rozicr. 1734-1793, † it 2ivou.

R. et Pav. = Ruiz et Pavon. Epanicr. Bot., Mcijuloc ill Südancrifa. Merte: 1794 แ.

R. et Schult. $=$ Roemer et Schultes.

Rith. $=$ Roth. 1757-1834. † alB 21r3t in 23 teutu.

Rumph $=$ Rumph., ans 5attall, gcb. $1637,+1706$.

Sad. = Sadler. Firof. bur Bot. ill Wubapejt. 23erte: 1825 4. $\overline{\text { f. }}$ \%.

Sal. = Salisbury. (5ıtglifuer Botanifer.

Sav. = Savi. Wirof. ill Wifia. 2̧erfe: 1798 u. if.

Schaeff. = Schaeffer. 1718-1790. Enperiutciloent in Hegetı̈burg.

S'chbl. et Mart. $=$ Schübler et Martens.

Schk. $=$ Schkuhr. 1741-1811. † ill Wittcuberg ala lluiveri.=Med)autifus.

Schldl. = Schlechtendal. † 1866 ale Frof. Dor Wot. ill Salle.

Schlcid, = Schleiden, gcb. 1804 in 5amuburg, jeit 1839 Hrof. ill Iona.

Schloth. = Schlotheim. 1761-1832. Ebergofunrialuall in Giotha.

Schlz. = Scbultz. 2rat ill Mlcubraubenburg, +1837.

Schmeh. $=$ Schumacher, geb. ill Solfteill, +1830 als Zirof. Der Ruatoutie ilt sioperthanell.

Schmp. = Schimper, geb. 1808 in Iofleulycin, icit 1839

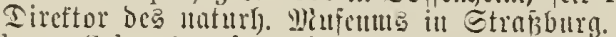

Schomb. = Schomlurger. geb. 1804 itt Freiburg a. 11 Ecit 1848 brit. Somjul in $\mathfrak{S B}_{\text {Bitimbicu. }}$

Schouw $=$ Schouw. 1789-1852. Weriifuter Wotanifer it Sropeultagen.

Schrd. = Schrader. 1761-1836, geb. in 2rafelo, tilvi., $\dagger$ in Giöttingelı.

Schreb. oocr Schb, = Schreber, geb. 1739 in פBciß̄ulc,

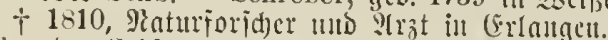

Schunk. ober Schk. = Schrank. 1747-1835. Gorten= Dircftor ill Mïuducu.

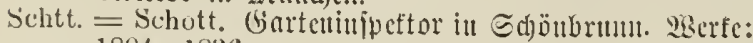
$1804-1836$

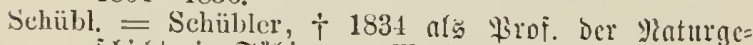

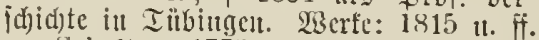

Schult. = Scbultes, 1773-1832. Wrof. ill פisicu, Sirafan, Sแแtsbrutf, Raแtoglyt.

Schum. = Schumacher. Geborner \$odfteitcr, $+18: 30$ als Firof. Der Fuatomic ill Slopenhagen.

Schw. = Schweigger. 1779-1857. Wrof. it Erlangen IIItD Salle.

Schwein. = Schweinitz, Botanifer in Lencrifa. WHerte: 180 \% $\%$. f.

Scop. = Scopoli. 1725-1788. Tiroler; 2irof. ill Wavia.

Sel, = Scbastiani. Stal. Bot. Merfe: $1813-181 \mathrm{~s}$.

Sibth. = Sibthorn. Botanifer ill Edforb. Reifte in Gric= d) culatib. +1796 .

siebold = Sicbold, geb. 1796 in 28 üraburg. Eberit itu nicocrländijejen Bincralitabe.

Sm. = James Sinith, $1759-1828,+$ it Routoon.

Suland. = Solander. 1736-1781. Traturf́. ill Soubon.

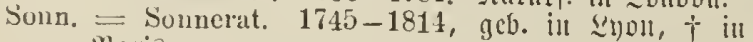
tiaria.

Soy. Will, = Soyer Willemet, 17£j 1805. Gartu= Dircftor ill Yianter.

Spenn. Spenner: 1799-184. Zisof. it freiburg itt Breiggan.

sprr. - Sprengel. 1766-1833 qrof. ill salle.
St. = Sturm. 1771-1818, Raturforider in Nürnberg St. Hil. = St. Hilairc. 1799-1853. Botauter in \&aris. 23erfe: 1824 11. if.

St. oder Sternb. = Sternberg. 1761-18:38. Gicheimcrat iil \$rag.

Stev. = Steven. Muijiijacr Etantarat, + 1820.

Suck. = Suckow. 1751-1813. \$rof. ill Jeibelberg.

Sw. = Swartz. 1790-1817. Frof. in ฐtoctjolut.

Swect $=$ Sweet. Sandelsgärtuct in Zonbon. Werfe 1818 \%. fif.

Tausch = Tauseh. Frof. ill \$rrag. 23 crfe: 1823 11. if.

Thom. = Thomas. 3twei 2 triiber in Ber, wclde socr: bariet vou Sdtwcizerpflanzen heransgaben.

Thor. = Thorc. Jranzöitid)er Botanifer 1803.

Thbg. = Thunberg. 1743-1828. Frof. Dcr B̉otanif it llujala.

Torr. et Gray = Torrey et Gray. Torr. = Torrey, Rirof. it Rerw=? orf. 93erfe: 1834 u. fi.

Tomm. = Tommasini. Mgagitratepräjibcut iu Trict.

Tourn. = Tournefort, 1656-1708, geb. in : Yix, $\dagger$ in Bariz.

Trev. = Trevirauus, geb. 1779 ill Brentel, Wrof. ber Botanif ilt Bolli.

Trin. = Trinius, geb. 1778 in Eiglebcu, + 1814 in Zeteraburg.

Trtt. = Trattinick, 1764-1849. Eujtos Der Viaturalielt Gamulung in wisien.

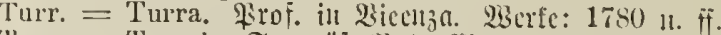

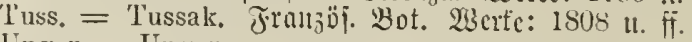

Unger $=$ Unger.

d'Ur\% $=$ d'Urville. $1790-1842$. Fraแล. 2lomiral.

Vahl = Vahl. 1749-1801. Fiof. Der Bot. it llpiala.

Vaill. = Vaillant. 1669-1722 Wrof. Der Hot. ill Larig.

Vent. = Ventenat. 1746-1808. Zirof, + ill Ziario.

Vhl. = Vahl. 1749-1804. Frof. ber bot. ill Siopen= l)ugetr.

Yis. = Visiani. Wrof. Der Bot. ull Pabua.

W. ct Grab. = Wimmer et Grabowski, Beibe Bot. in

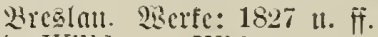

IV. mio Willd. = Willdenow. 1765-1812. Wirof. in Berlint.

Wahllux. - Waluberg, gev. 1800 in Gothenburg, Zirof. iil Gtodfioful.

Wahlubg. = Wahlenberg. 1780--1851. W'rō. iu llpjala.

W. K. = Waldstcin et Kitaibel. WBarbitcin 1759 bis 1823, bercipte mit sitaibel melyrere Jahere llugarn.

W. et M. = White et Maton.

IV. et $\mathrm{N} .=\mathrm{W}^{\text {ceilhe et Nees. }}$

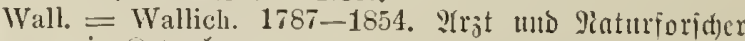
in siopenhagen.

Wall: = Wallroth. 1792-1857, † in Torbhaticu.

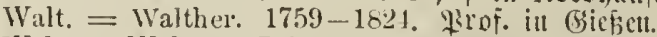

Web. = Welser. 1752-1823. (5tatgrat ill sicl.

Weig. = Wveigel. 1748-1831. Airof. ill (Srcifstwald.

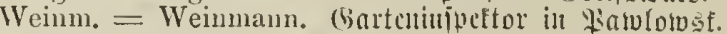

Wend. = Wendland. Smuftgärtuer in sanmover. Waerfe: 1798 It. ff.

Wendr, = IVenderoth. Brof. in Marburg. WBerfe: 1821 II. î̀.

Wickst1: $=$ Wickstrocm. 1789-1856. Bot. ill Etocf= holit.

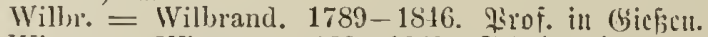

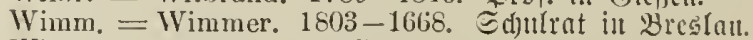

Wirts. = Wirtgen. +1870 .

Wulf. oder Wulff. = Wulfen obcr Wulffen. llut bie

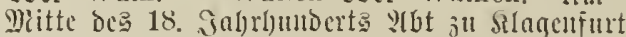

Zahlb. = Zahlloruckner. 1782-1851. Bot. ill (b́rïk.

Zenk. = Zenker. 1799-1837. Wrof. il Teun.

Zeyh. = Zeyher. Gartendireftor il Ed)tvejitgen, † 1813.

Zucc. = Zuccarini. 1798-1848. Frof. it lieiutucu. 


\section{Dit Pflamblultum.}

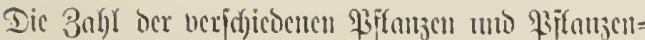

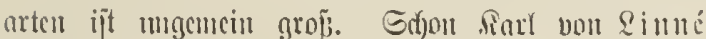
(gcb. 13/24. Mtai 1707 ju अaghult in Exfyuchen, gcit. 10. Jamtar 1778 in Upjala) famute mo flajīi-

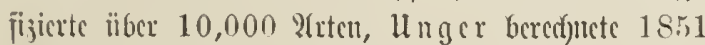

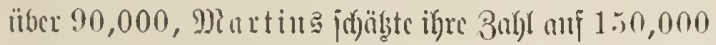

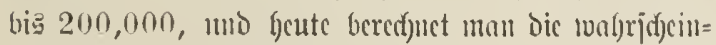

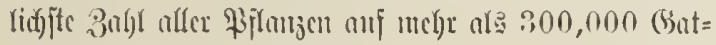
tumgen, 2letten mid Eppiclarten. Dic $3 a b l$ ber 311 be= jontoem 3ructen fultivierten ober in Den 52 and

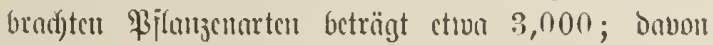

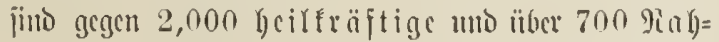
ruแg

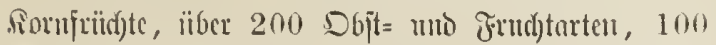

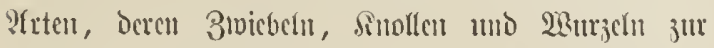

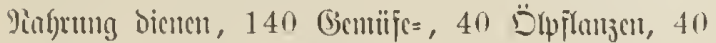

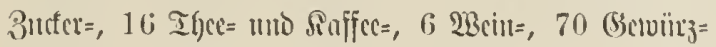
pilanzer. Heber 40 bicnen ju Bicgfutter, ï̈er

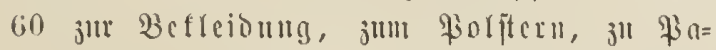
pice 11. i. เo., gegen 100 jum Fürben, ïber

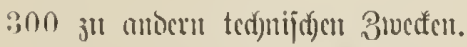

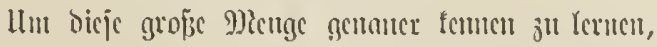

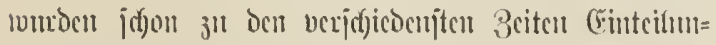
gen verjud)t; fo zucrit im Julfer 1588 von (5ajal= pill (grit. 1608), 1694 vou J. 2. Ioltucfort (geịt. 1798), I. Mt. a., beren feine aber bem wadd)= jenben bebïrfuis anf dic Duncr geningen founte.

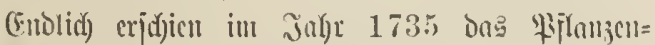

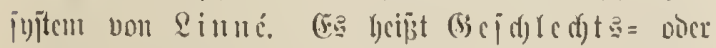

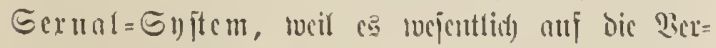

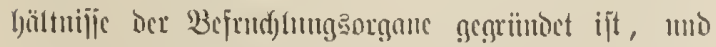

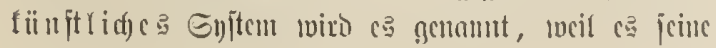

(Eintcillungaggrïmos chen mtr von wenigen 5)mutorganen

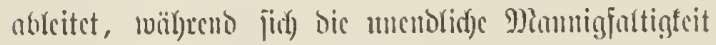

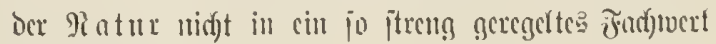
cimjujlicijen läpist.

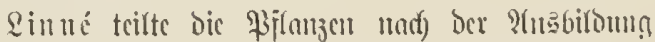
Der Stunbgefäjic in 24 Silujpen mis bicje nudid ber ocr Stempel in Drommugen cin. Ecine Dromungen jerfallen in Battungen umb dicje in ?f $\mathrm{x} t \mathrm{cu}$.

Dis eriten 2:: Slofjen unfonfon bic Blïten=

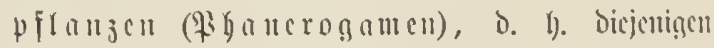

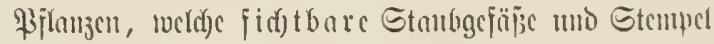
(jerten an Der Stefle Der Stempel mur Enmentuojpen)

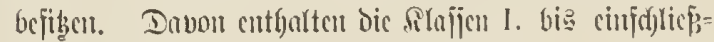

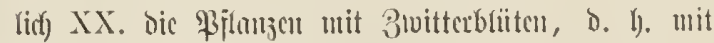

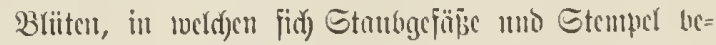

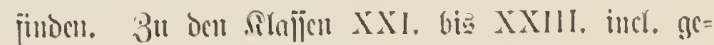

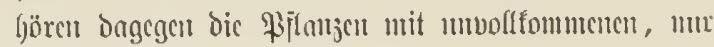
Stnutbageäpic oocr utur Etempel entfgattenoen 23titten (Etmogefä̈ $=$ oder Etentperblïtert). In ben 11 criten bifloct Dic Bugl Der freicu Stanbfäden Den (Fintci=

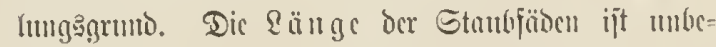

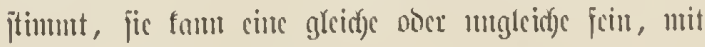

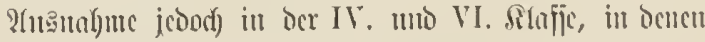

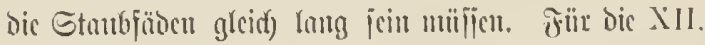

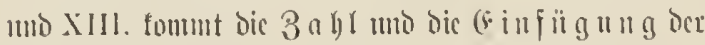

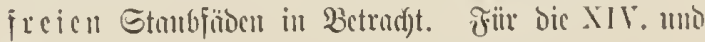

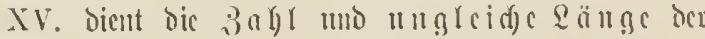

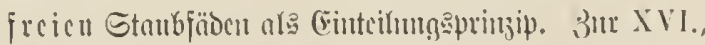

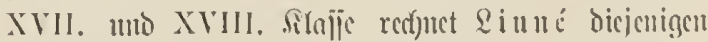

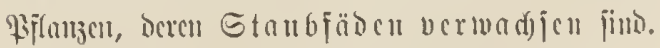

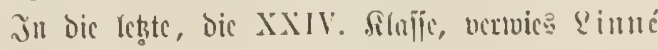
dic Rinptogamen, o. h. Dicjenigen Piflumjen, Decen 


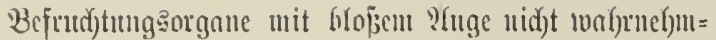

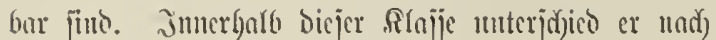
ber natïrfiden Beruanotfifyaft 4 Dromungen: Dic Farne, Mlopje, IIgen, Pilǰc. -

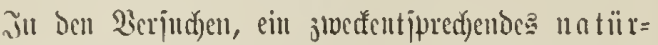

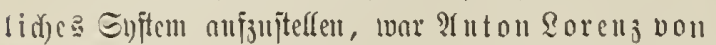

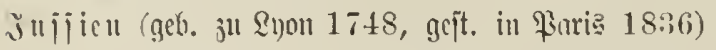
ber erite, wefdyer ein broutdoures, nod) bente den (i)rulu alfer untiirlidyeu Elytente bildentoe $n a=$ tiirriducs Sujtem erfanto und veröffentlidjt.

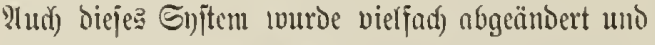
mit mefre ober locniger Ǵliid verbefjert; io 1tament=

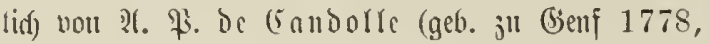
gejt. Dajecbitt 1841), ferner von (Endrider in Wien

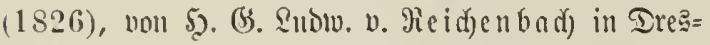
ben $(1828)$, jolvic von mchrerell studeren. Dic (Ei= gentiiulfidjfeiten biejer veridjicocuen untïrlidjen Enjteme tonflen toir in Sïrze mittcifen.

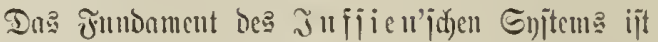

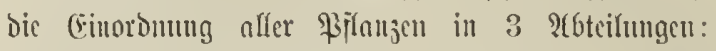
Biflambel ob he Samenlappen, Acotyledones, joldye mit einem, Monocotyledones, und joldye mit $310 \mathrm{ci}$ oder mefreren Gamentappen, Inicotyledones.

Dicje 3 stbteilungen jeigen in ifrer gejauten Dr= ganijation eine jo flare Grumbverjajebenkeit von ein= anber, Daj̄ jie ofme 3ıeeifel für tvirffid) iแ Der Эatur begründete 5zauptgruppen anjunchmen

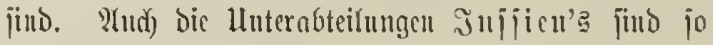
flar und jidfer anfigcitellt, Daj bic meijten Derjerben

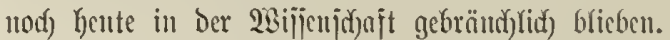

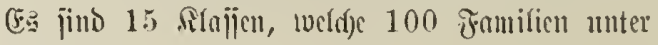
iiid) begreifen:

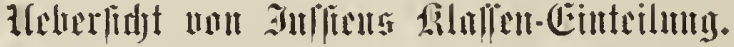

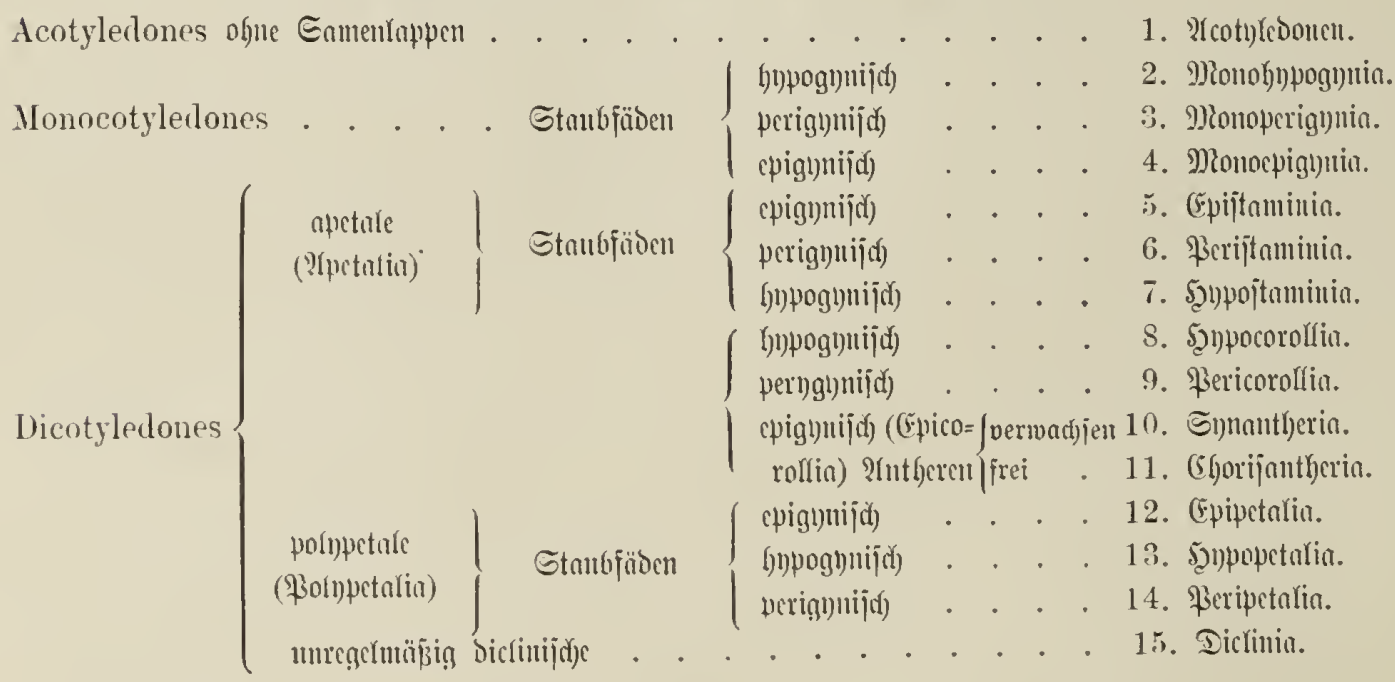

De (5 a no or e grünocte bic Şmuptabteilungen fei= ue natürlidfen Gyftemz anf ben ganjen inueru

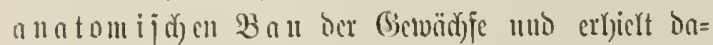

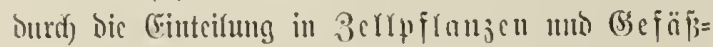
prfanjen. Sic Berrpfranjen unteridjicd or in

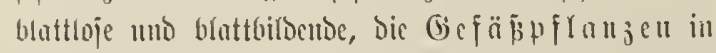

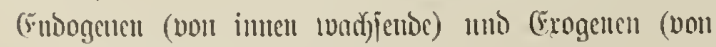

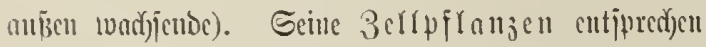

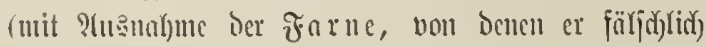
aminumt, onj jie mit cincu Gamentappen foimen) Den arcotylebonen Jujiene, jeine (Endogenen den 9)onocotnledonen, bie Exogenen gemun ben Di= cotnlcdone In Injiens.
Stephan (Endrider, Frofofipor Der Botanif in

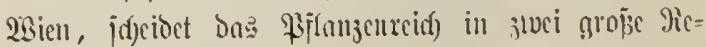
gionen, in $\&$ agerpfla

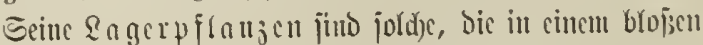

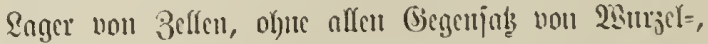

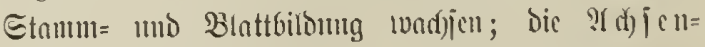
pflanjeu joldse, bei benen bie reb̧teren bilbungeu Denttid) auftreten.

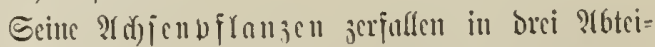

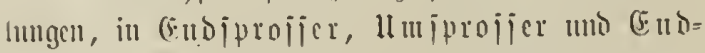

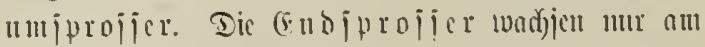
(Bipfer, bic แmjprojier mur im Unfange, bic (E) umiprojier am (bipfel utb llufange zugleid). (Eno) 
liders (5noumiprojier entipredfen ben Dicotyle= bonen Jujieus und ben Erogencu Decan= bollez; dic llmiprojier jimb bie MRonocotyle=

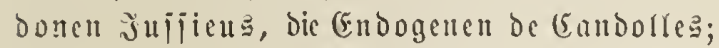
bie (Endiproffer begreifen einen Teil ber $\mathfrak{A}(\mathrm{cot})=$ I çonen J̌ujiicus (bie Mlopie uno Farne), jowic Dic blattbirbenden 3errpfranjen jamt ben en= Dogenen Rruptogamen be candorlez.

Die $\{$ a gerpfla nzen (End lider en entipreden den

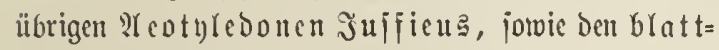
lojen 3ellpflanjen de candollez.

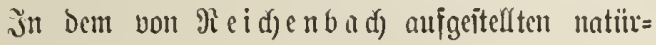
lidfen Syjteme iit bie Steigernng Der Drgani= jution ber \$flanjen in unjdanlidjer $\mathfrak{B}$ Beife burd) alle Alaijen butdgefiührt; ar teilt bie Bjlanzen in jwei gropie 2tbtcilungen, in 1) Protophyten (ur= ipriinglidye Bïlanjen, Denen mur ein Erbleben zu=

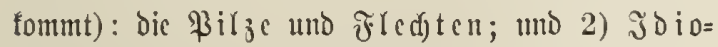

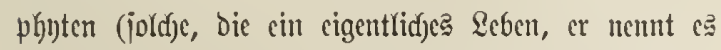

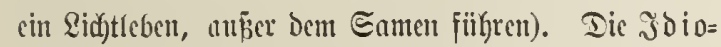
phyten teilt er in brei grope Gruppen, in $3 e \mathfrak{l l}=$ feimer, Spibteimer uno Blattfeimer. Die Brotophyten nebjt ben Berfecimern entipreden

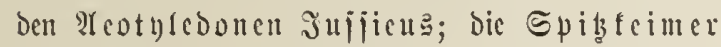
find befien Mondecotyledouen, und bis Bratt= feimer jeime Dicotiledonen.

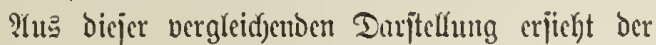
Sejer, Dus die genannten vier groß̈en Botanifer, ob= gleid) jie nidgt biejelben lețten (Einteilungägrïnoc an= nelymen, bod) in ber Jamutjadye unf basjelbe $\Re e=$ jultat tamen bei ihrer sinteilung bes Prlanzenteidfe

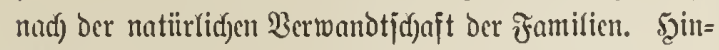

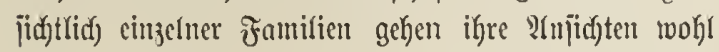
jutweilen unseinanber (mie 3. B. Reidenbud) ben RIgen cine böhcre Stufie antweijt, ala (Endlidfer), aber bie

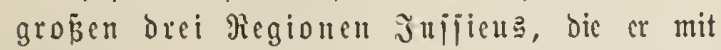
2(coty) edonen, Meonocoty) id)arf bejecidjnete, jteksen für alle vier gleid fejt.

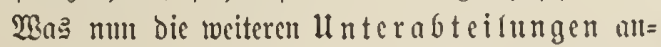
belangt, jo jinto aude) hier von ben vier Belchrten jo jiemllids) bicjectben Merfmale, meijt bie Berbältnifie ber Blïtenumfitiflungen, 3tu Grumbe gelegt wordent.

De Eambolles Ex ty genent bilden bic 2lbteilungen ber Mlonod)la mideen (ber PF̃lanzen, beren Blüten mit eince cinzigen 5̧ü̈lle umgeben jïto), Der (5ororri= floren (ber \$ilanjen mit cinblätteriger Blumenfrone),

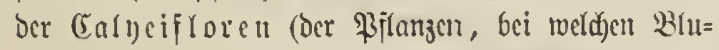

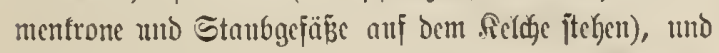
ber Ihalam ifloren (Der Pilanzen, beren Blumen= frome in mef)rere blätter geteilt iit, twelche anf Dem B(ïtentboden Itegent).

Sdjon Juipien loat aber peine Dicotyledonen jerfullen lajien in Blumentronenloje, in joldje mit einblätteriger und joldje mit melgrblätteriger Blumenfrone, unt jeoc bicjer brei Mbteilungen bc= greift algbann brei jogenamnte SIajien, bic er nadf Der (Einfügung Der Staubgefäijie ober nad) Der (Ein= fügung Der Błluntenfroure, voenu joldse cinblätterig ift, gebilbet hat, ie nuddosem bieje nämtidf) hylyo= gunijd) (unterneibig), periguniid) (umıveibig, mittel= ftänbig), obcr epignnija) (obcrtweibig) ijt, b. h. ie nudjoem jie anf bem Blïtenboben ober auf Dent in=

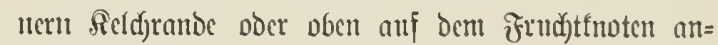
getvadjien ift.

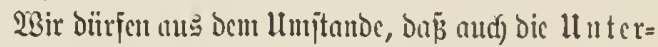
abteilungen von jeocm biejer vier Syjtematifer auf=

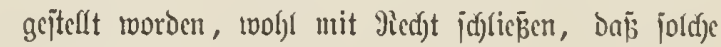
cbenfarle danaratterijtijd)e, in Der ganjen Drganijation Der barunter jujammengefap̄ten Familien begriindocte Unterjajede Darbicten. Innerhalb diejer lnterab= teilungen aber wird bie ?tneinanderreigung ber einjel= nen Famtilien bald mefhe bald weniger eine $f$ in 11 it $=$ Iidje in allen vier Enjtemen, von benen infrigens da? jenige de (5,andolfez heute fajt iiberall jur Gel= tumg gefommen ijt.

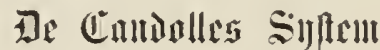

cutipridst wie alfe natür(id)en Sujteme in ben

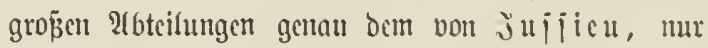
Find bie Dicotyledonen anjtatt in 11 , bloz in \pm Sluffen eingeteilt, nämlid) in Ihalamifloren, (Ealieifloren, Eorollifloren mo Monod)la= mindecti.

Dicje (Einteilung ijt jom nidft natïrlidjer at? bie von Jujpïen, (Endlidjer, Reidfenbad) u. a., abcr jie ijt treit bequemer, tweaghalb fie and in viclen lyet= vorragenden \$iflanjemwerten Eingang gefunben hat, j. $\mathfrak{B}$.

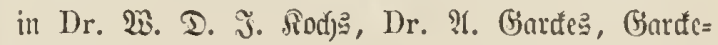
Wagners, Scunis' und fajt aflen ncucren Deutidfen Fadjwerfen; ferner in ben 5̧auptwerfen bon be (5an= bolle, Simblen, ßereira, Grenier, Sobron, Midjarb, 5jooter unb Bentham :c. :

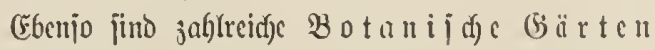
nad) bicjer Silajiififitation eingeridftet, numentlich) ber von Montpellier, igrem llrjprungeort, ber von (5enf mob vicle in Ientidfland mo Englano. 


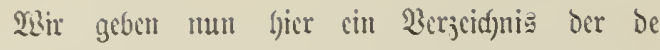

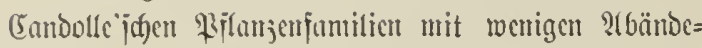
rungen nud) Der ?(ufitteflung von jeinem Solfne in ber neuen, 1844 mijfienenen \$luflage von Théorie élémentaire de la botanique.

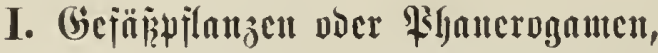

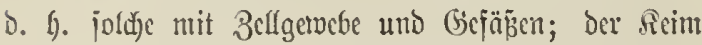

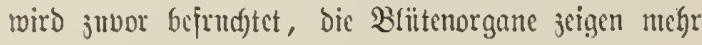
oder wentger Symmetric.

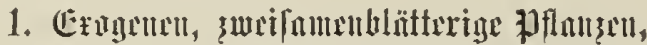

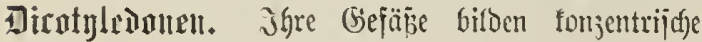
Edjidften, mobci jid bic jïrgiten ausen befimben; Der Reim hat entgegengejest ober wirtelförmig itthenbe Samenlappen.

I. Ilnterabteilung. - Thalamiflorae. Frudt bodenglütige \$iranjen.

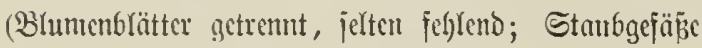
mcijt unteritänbig.)

$3 \mathfrak{u}$ ignem gejören unje Familien $1-53$.

II. Unterabtcilung. - Calyciflorae. Seldubrütige Pjlanjen.

(BBfumentblätter getrennt ober mefse ober toeniger ver= raadjen, immer perigynijd auj bem Reldf eingefügt.)

31t ifnen geffören unjer Æamilien 54-110.

III. Ilnterabteitung. - Corolliflorae. Rronblütige glfanjen.

(B̉lumenblätter in cine bom Neld getremte, mrijt vferittändige, bic Etmuhgefäß̄e bergende Srone ver= radjicn.)

$3 \mathfrak{3}$ ifnen gefören unjer foumilien $111-1+2$.
IV. Unterabteilung. - Monochlamydeae. Blumenblattloje Bjfanjen. (Aicld) uno Srone bilben nur cine cinjige Syitlle.) 3 in ifuen gchören unjre Familien $143-164$.

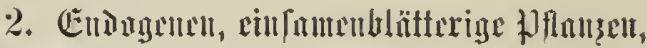

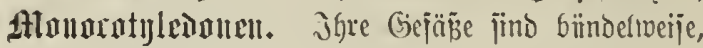
nidft in Sofjidften angeoronet.

$3 \mathfrak{3}$ ignen getgören unjre famtlien $165-187$.

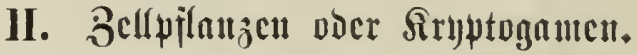

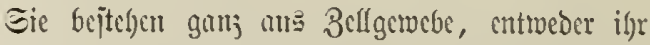

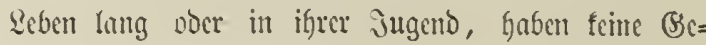

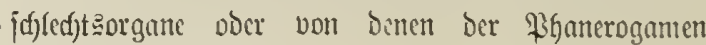
völfig abreidfende.

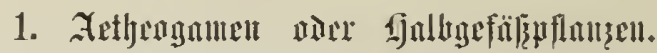

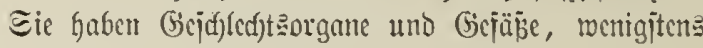
in irgeno cirrem Beitpunft ifges Sebens.

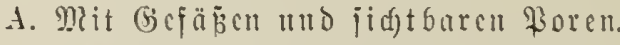
Siche unje Frumitien 188-191.

B. Mlit wenigen oder feinen Ğejäpen und of me toren.

IInire Familicn 192, 193.

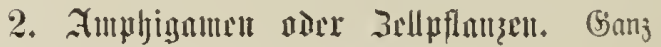

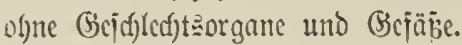

Unjere Familien $194-197$. 



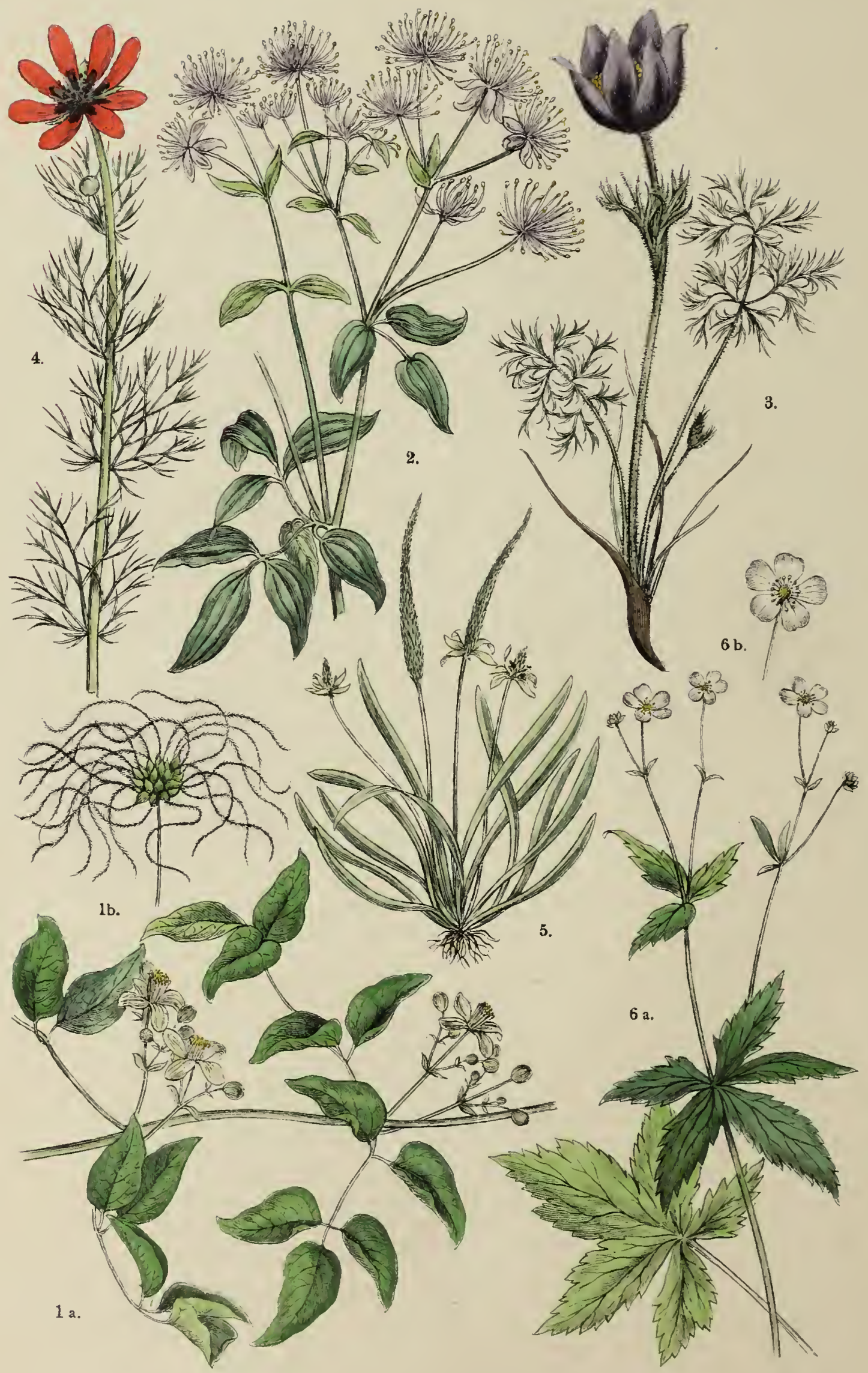




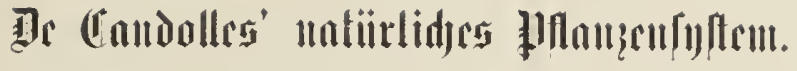

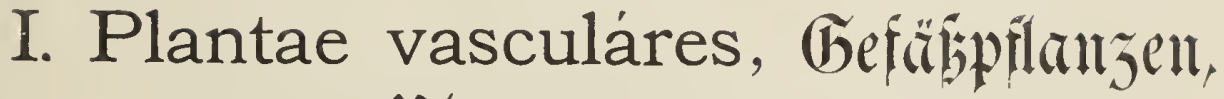 Dhanerogamen.}

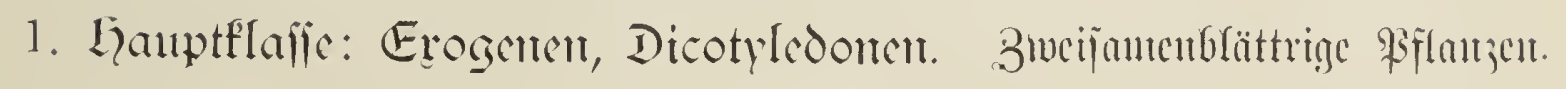

\section{Llnterflafje: Thalamiflor:ıe, fundtbodenblitige pilangen.}

Tai. 1.

1. Foutilic. Ranuneuláceae Juss.

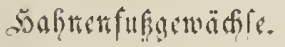

fitị. 1 a. b. Clématis Vitalba L., gemeine 2isalorcbe, Brenufraut, Teuferzzirn.

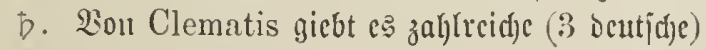
I(tten *) mit jajarfen, oft jefr giftigen $\Xi_{a f t c}$; oft mefyrere Micter lang; ১ie abgebil(sote 9rut twäd)jt

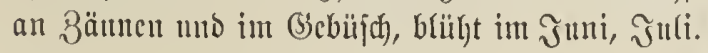

Eịn. 2. Thalictrum aquilegifolium L., iffeci

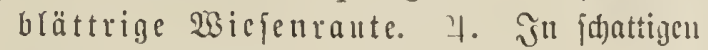

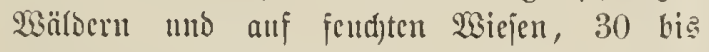
95 cm. hod), b(üht iun Miai uno ริmui. (3ift=

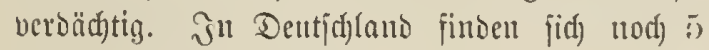
anoere Thalictrum=?trten.

f́tị. 3. Anemóne P'ulsatilla L., (Pulsatilla vulgaris Mill.), gemcine Sïdjenfdelle. 2\%.

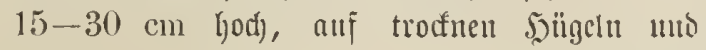

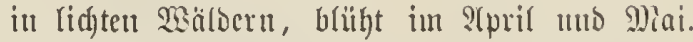

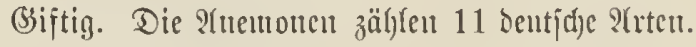

fiig. 4. Adónis aestivalis L., Commer $=900=$

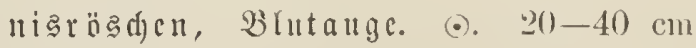

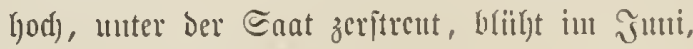
Intli. 4 dectutjd) ?(rtent.

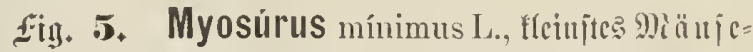

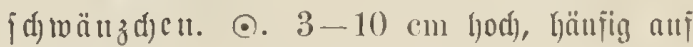

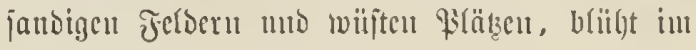

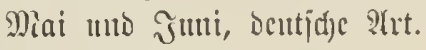

fig. 6 a. b. Ranúnculus aconitifolius L., furm! =

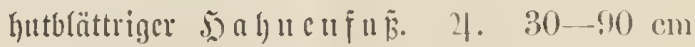

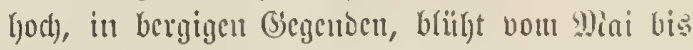

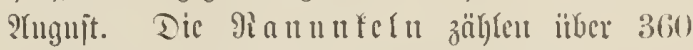
Irten, Davou etwa 30 beutfde); cintige :Itrten prad)twolfe Bicrpflanzen umjerer Gä̈rten.

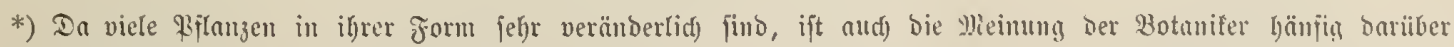
geteilt, ob biefe unb jene form als eigentlidje $21 \mathrm{t} t$, ober nls Spielart zu betradjten jei. Wo in unjerem $20 t a n i f d j e n$

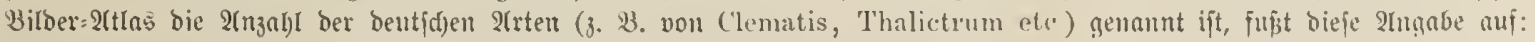

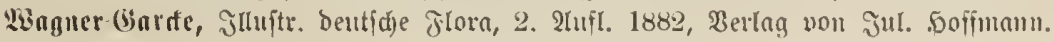




\section{Taf. 2.}

fiin. 7 a. b. Caltha palústris L., ভmup $=$ Dot

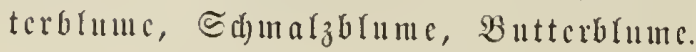
7. $10-40 \mathrm{~cm}$ hod), auf feudjen wiejen ge= unciu, briilyt vou ?(pril Jumi. 1 bentid)e ?(rt. Giftig.

fịj. 8. Tróllius europaeus L., curopäijd)c Troflblumc, Engclblumc. 21. 10 bie $40 \mathrm{~cm}$ hodh), auf feutfiten wiejen und $\mathfrak{B} \mathrm{crg}=$ weiben, blïht vout Miai bis Эulli. 1 bentjaje ?irt. ভcjöuc Bartenpflanze.

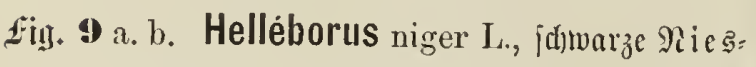

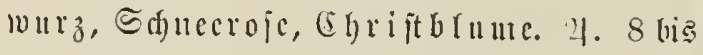
$30 \mathrm{~cm}$ hod), in j(h)attigen (Bebirgswälderu, b(iih)t

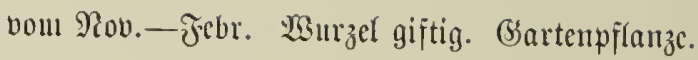
হion Helléborus giebt ç 3 Deutiche 2(rten.

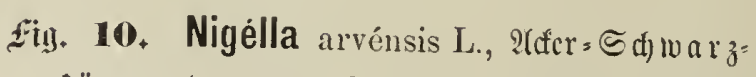
f ï ulu ucl. $\odot . \quad 10-20 \mathrm{~cm}$ ljodh, llutraut iu ben Eaatferoeru, bliilgt vou כuli- Septbr.

fiù. 11. Aquilégia vulgáris L., gemcine afterei,

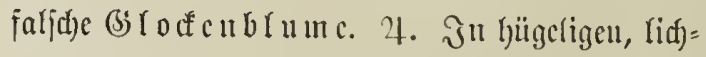

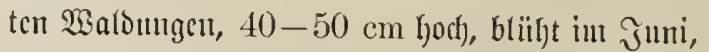
Jufi. Man unterjheidet melyrerc Epicfniten.

fị̂y. 12. Delphinium Consólida L., feclo= 9 i $i t=$ teriporu. $\odot .30-45 \mathrm{~cm}$. hodb. Renjtiges lln= frant in Bictrcibcfeloerı, blüht vom Эunt-?tug. Son Delphínium gieht es 2 icutidye 9rtent. 


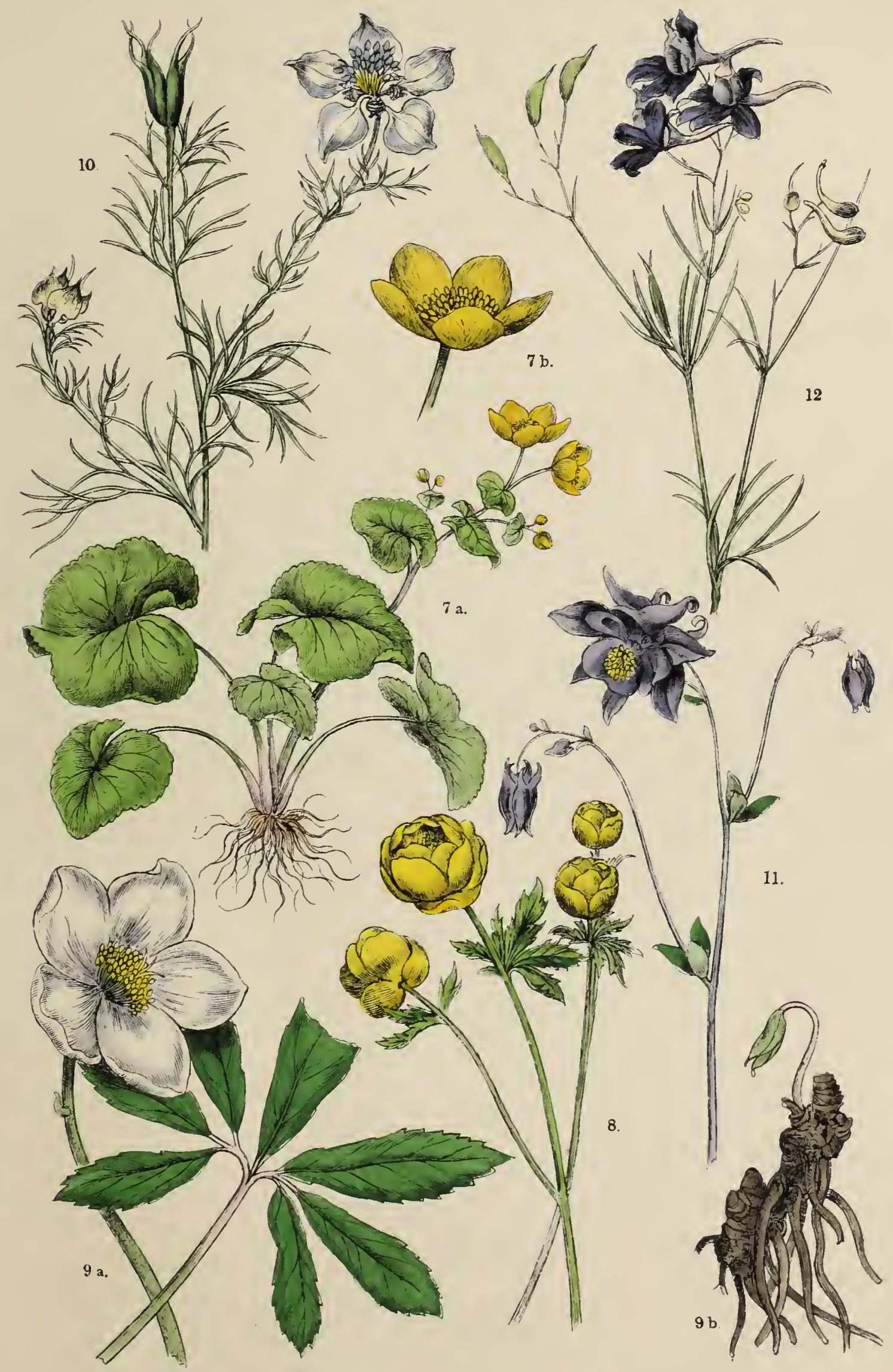





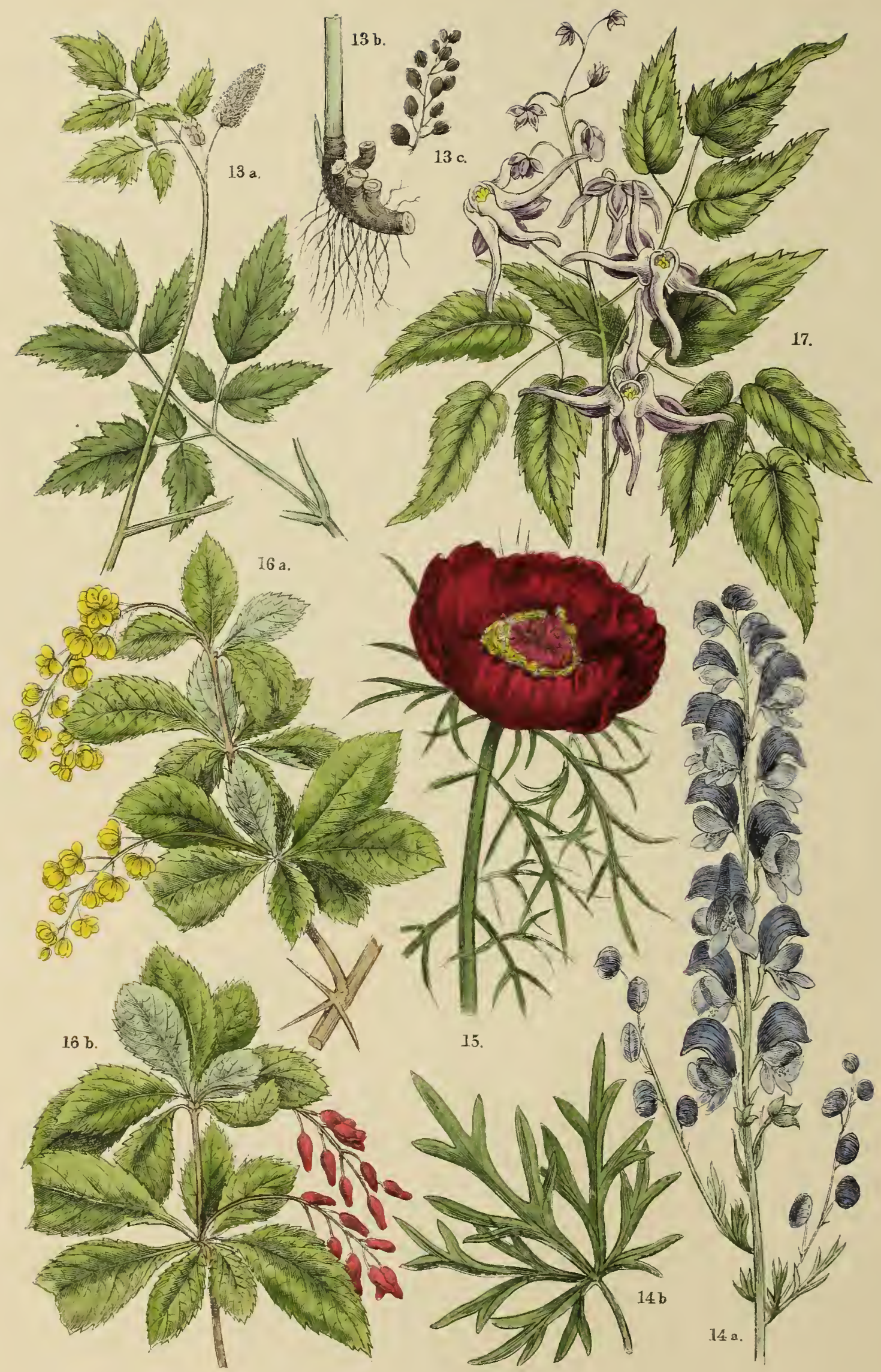




\section{๕ai. ๘.}

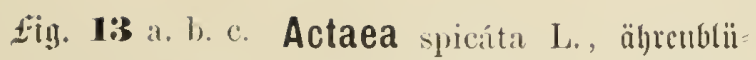

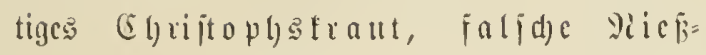

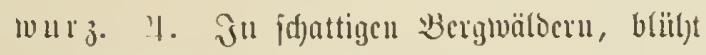

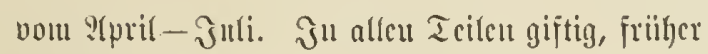
ofïizinell.

fiig. 11 a. b. Aconitum Naunellus 1., ecleter

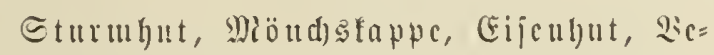

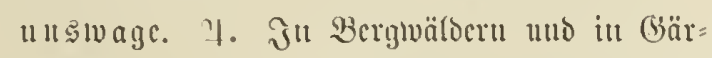

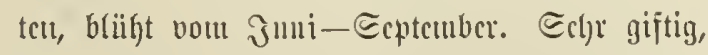
abcr aud) arzucifräftig. Sou Aconitum gicót $\mathfrak{c}$ 5 ocutide 2trten.

f́ig. 15. Paeónia tenuifúlia L., friublättrige Bjiugitroje, Bsidtroje 21. Esilo in Dit= curopa unto s(jïrn. 30 - 60 cul lod), b(ül)t vout Wini-Suli. Sicrpĩlauze uit ueb)reren Spicl= arten.

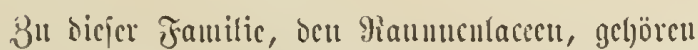
ferucr: Atrágene, altpcurebc; Hepática, ?́cbcrblüu=

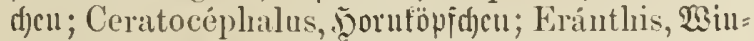

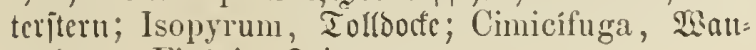
zenftaut; Ficária, テcigumr.

\section{Tramilic. Dilleniáceac Dce.}

Djiçı get)örcu Dillénia L., Banu Djtindicus; Delíma L., Strand) Dialnbarz; Tetrácera L., Sitimmjtraud) (Bntincas.

\section{Fantilic. Magnoliáceac Dec.}

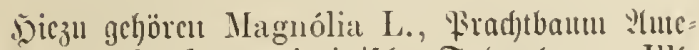
rifaڤ, Liriodéndron, birgiuijd)er 2 ulpeubaum; Illi- ciun, Etcruanisbaum in (Cbina, Japan; 1)rinys in Chili ullo Brajilicu.

\section{Fautic. Inouácene Juss.}

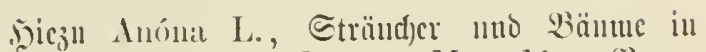

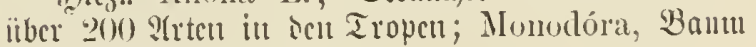
auf Jauaita, แ. a. แt.

\section{Fantilic. Menisprérmeat Juss.}

Enttl): Menispérmum, Mionojauc, Ed)liug= pF̄lauze ill Dịtafrita; Cocculus, Soltelstörucritrand) in Ditindicn; Cissímpelos, Gricsumuzel, Etrand) in 2icpitittoict.

\section{Faunilic. Berberídare Vent.} samiroormgendols.

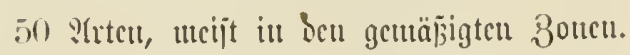

tiig. It a. b. Bérberis vulgaris L., genci= ucr Eaucrooru, Jucrbcrize, Eaurad. Jll ganz (Ëropa gciutijd. ち. Straud) von

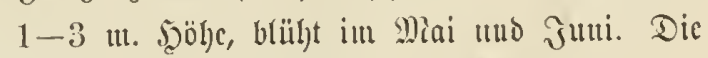
Becren bicucu zu Säpten uno Soupitureu.

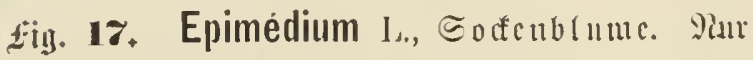

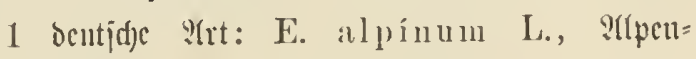
joctenblunte, iu jdjattigen B̉ergwälioern, 15 bis $30 \mathrm{~cm}$ bod), 24, b(ïl)t ill I(pril ullo Miai. Dic abgebiloete Ulrt, E. macríntlium, ijt ciuc juönc 3̇icrpflanze aı๖ כapan. 
Tẫ. 4.

7. Tautilie. Nymphacáceac Sulisl. corolomgemidils.

30) jajt ïber alfe weltteile verbreitete :irten.

17in. 18. Nymphaea alba L., weipe Eecroje. 1. Zierde mirer itschenden Goswäjier; blüht vom Juni-September. Die äguptijdye ?otos= blume, Nymphaea Lotus L., galt int ?fitertmm als cit こumbol oce lïberfluffes.

fiig. 19. Nuphar lúteum L., getbe Secroje, Ieidroje, ఇiz̨blume. \%. Wie die vorige

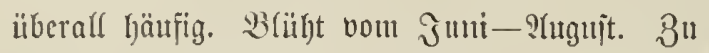

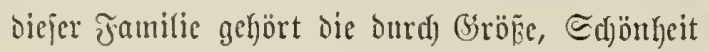
แno : Lindl., weit verbreitet in ben groşen @trömen ฐiisamerifas.

8. Faunilic. Papareráceae Juss.

añotroncmütfle.

70 : Yrten in Črtropa uto Rordajien.

fiy. 20. Papáver Rhoeas L., Slatid)roje, Felomohn. $\odot$. Säitiges llufraut in ben Eaatjeldoern, 30-60 cm lyod), blüht vont Maa bi Jult. Difizizell. Der Gartenunolnu, Pap. somniferum L., ijt in Eïbenropa heintija) uno bifot cine vielfad) variierenoc Bierpflanze unjerer Gär= ten. Dic Camen werien zur S̈lbereitung, dic

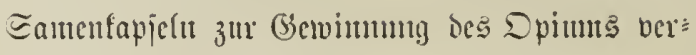
wentot.

fỉy. 21. Chelidónium majus L., gem. Sdjöll=

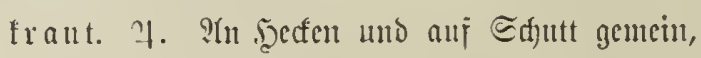
$30-90 \mathrm{~cm}$ hod), b(iift vou Maa-Sept. Der

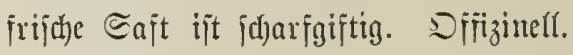

fign. 22. Glaucium líteum Scop., (Chelido-

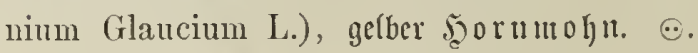
Ftuf jandigen Sïijten uno şügcln, $30-45 \mathrm{~cm}$ hod), blüht oom Эmui-2(ngujt.

8u ben Papaveraceen gefören feruer Esch-

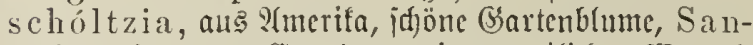
guinária, als Canaba, mit arzzneilidjer $\mathfrak{s u r z e l , ~}$

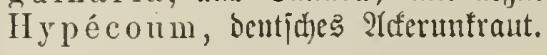

9. Familic. Fumariáceac Dec.

šrdrandgewäd)

PIn 60 Irten, bie meijten in ber nörblidjen ge= mäpinten 3one.

fiy. 2:3 a. b. Fumária officinalis L., geuteinev Eroraud), Fetoraute. ๑. Bemein in Bärten utto Feloern, 15 - 30 cm hod), blüht vom Mai-DHtober. Ilufraut, früher offizinell. 


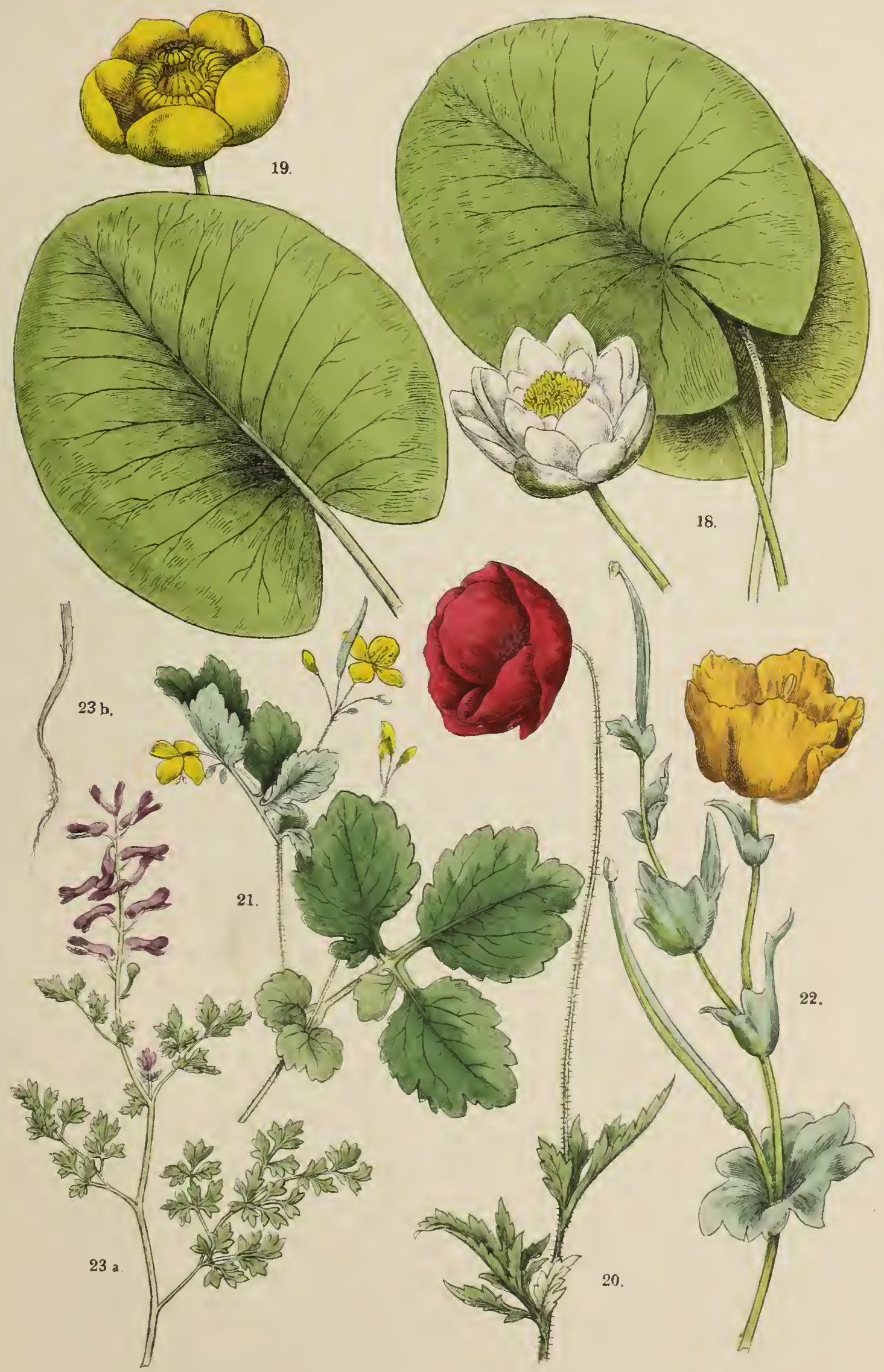





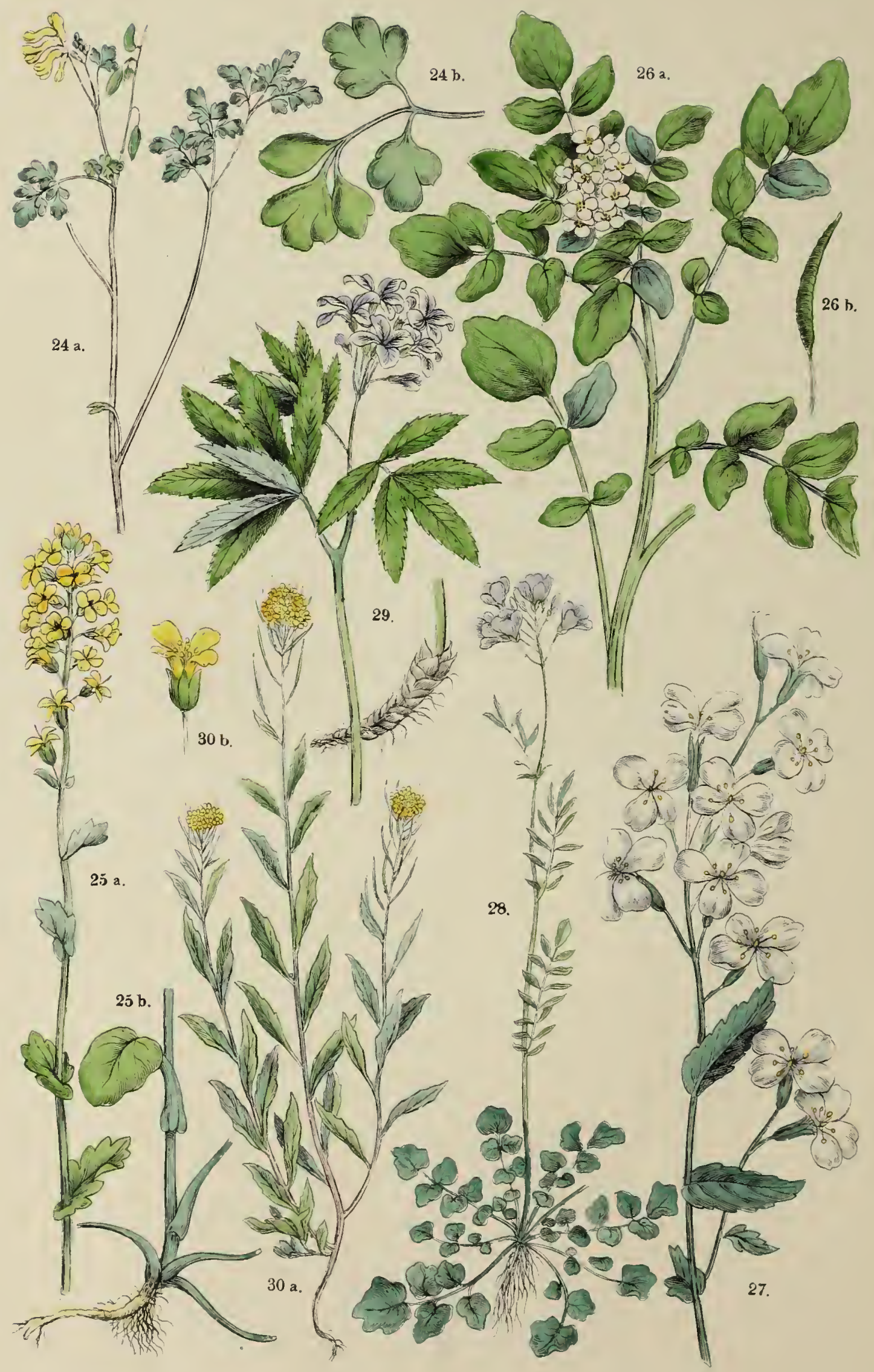




\section{Tậ. 5.}

fiin. 21 a. b. Corýdalis luteil (Fumaria lute: L.), gelber Eerdenjporn. \%. In Süteuropa l)eimija); in Dentid)lano als (Sartenpillanze 11110 ocrivilbert. $10-20 \mathrm{~cm}$ hod), blïgt nom Miai bis ङeptember.

Dic jejöne mo beliebte rotblïgente Garten= pFlanze Diclytra, Doppeliporu, gehört zul siejer Familic.

\section{Jumilic. Cencifierae Juss.

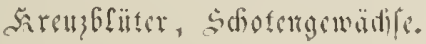

Über 1200) 2(rten in mehr als 120 Gáttungen, meijt in ber gemäpaigten uno falten Zone.

fiuj. 25 a. b. Barbaraea vulgáris R. B. (Erysinum barbaraea L.), gemeine $\mathfrak{B} \mathfrak{a} \mathbf{r}=$ barafraut, gemeine Erofrejie. ¿. 万öufig an jeudien Eteflen uno T(ferräntern in ganz Cேuropa, 30-60 cm hod), blüht rom Diai bis julti. Llıfiraut.

fig. 26 a. b. Nasturtium officinale R. Br. (Sisymbrium nastúrtium L.), gemeine $\mathfrak{B} r m n=$ nenfecje, wajierfiejie. 2\%. Fajt in allen

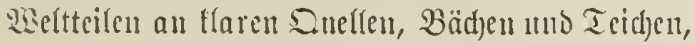

Stenget $15-60$ cul lang, bliil)t voul Mai bis Ecpt, Bsibt guttu Enlat, ift arguteiträftig unto wiro in Frantreid) uno Dentichlano (in Erfurt) iun grofen fultiviert. 2:on Nasturtium giebt es (i) bentidye 9 inten.

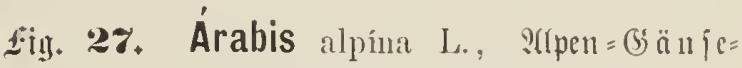

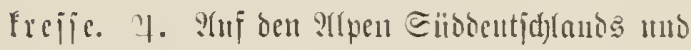
ber $\Subset d y e i z, 10-15 \mathrm{~cm}$ hod), blüht rom Diai bis

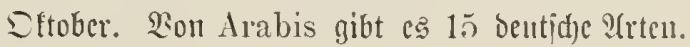

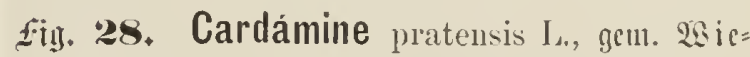

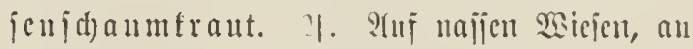

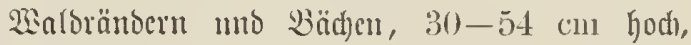
büht in ?pril, Miai. 2:on Cardamine gibt es 9) ientidje $\mathfrak{\text { Itrtent. }}$

fị\}. 29. Dentária pinnáta L., geficoerte $3 \mathfrak{a} \mathfrak{l} n=$

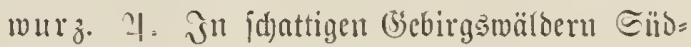
deutjdrands, 30-60 cm lyod), blüht im s:pril, Miai. 5 bentịc ?(rten.

fị. 30 a. b. Erýsimum cheiranthoüles L., Sacfartiger Edyotendotter, Deberid). $\odot$

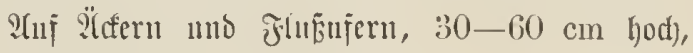
büht vom Jumi-Eeptember. 


\section{Taf. 6.}

fin. 31. Matthiola incámna R. Br., wisintcr.

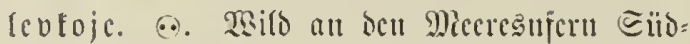
curopas, $30-120 \mathrm{~cm}$ hod), bitiht voul 9 (pril bie Dftober. Wist uns beliebte wohfriedentoe Bicr= piflanze in jaffreid)cn Epiclarten.

1in. 3:2. Cheiranthus Cheiri I., Golola di,

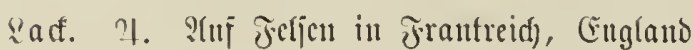

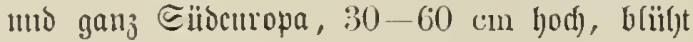

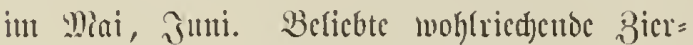
pĩlanze. Llujere 2 Lbbilonng zeigt cine tuttivierte Epiefart mit gefïilltcu BBfuncu. fin. 33 a. b. Brassica olerácea capitata L., sopftogl, sopftraut, siseiptraut, sap=

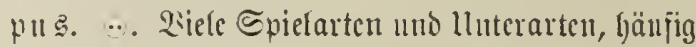
in Dentijfland gebaut nulo zul Ealat, Gicuiijie,

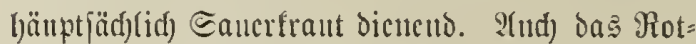
fraut ift cinc Epicfart bes sopftohfle.

Siig. 34 a. b. Brassica oleraceal sabauda L.,

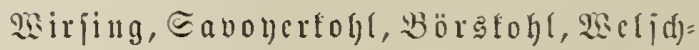

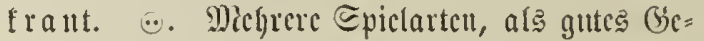
miije überall angebautt. 


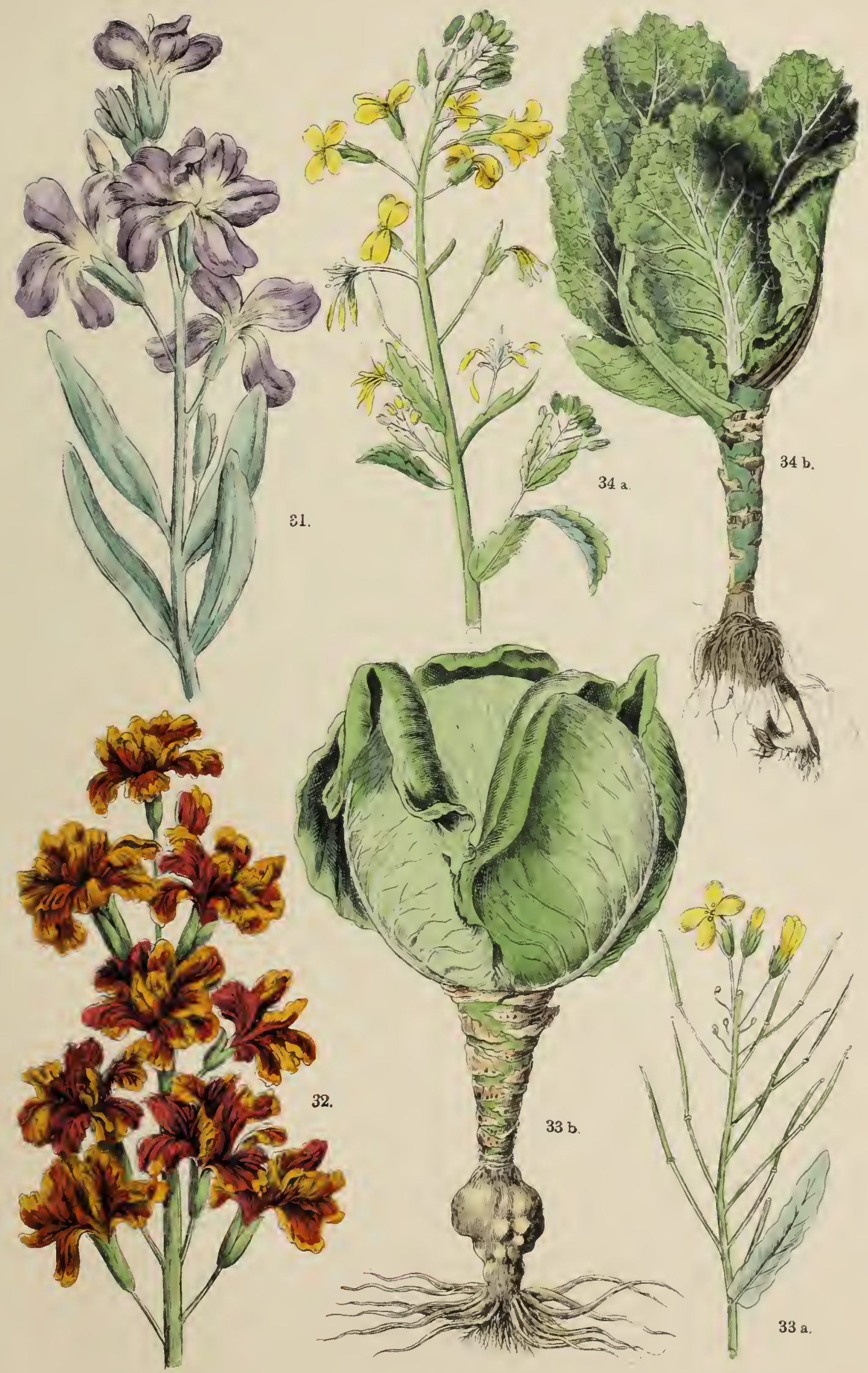






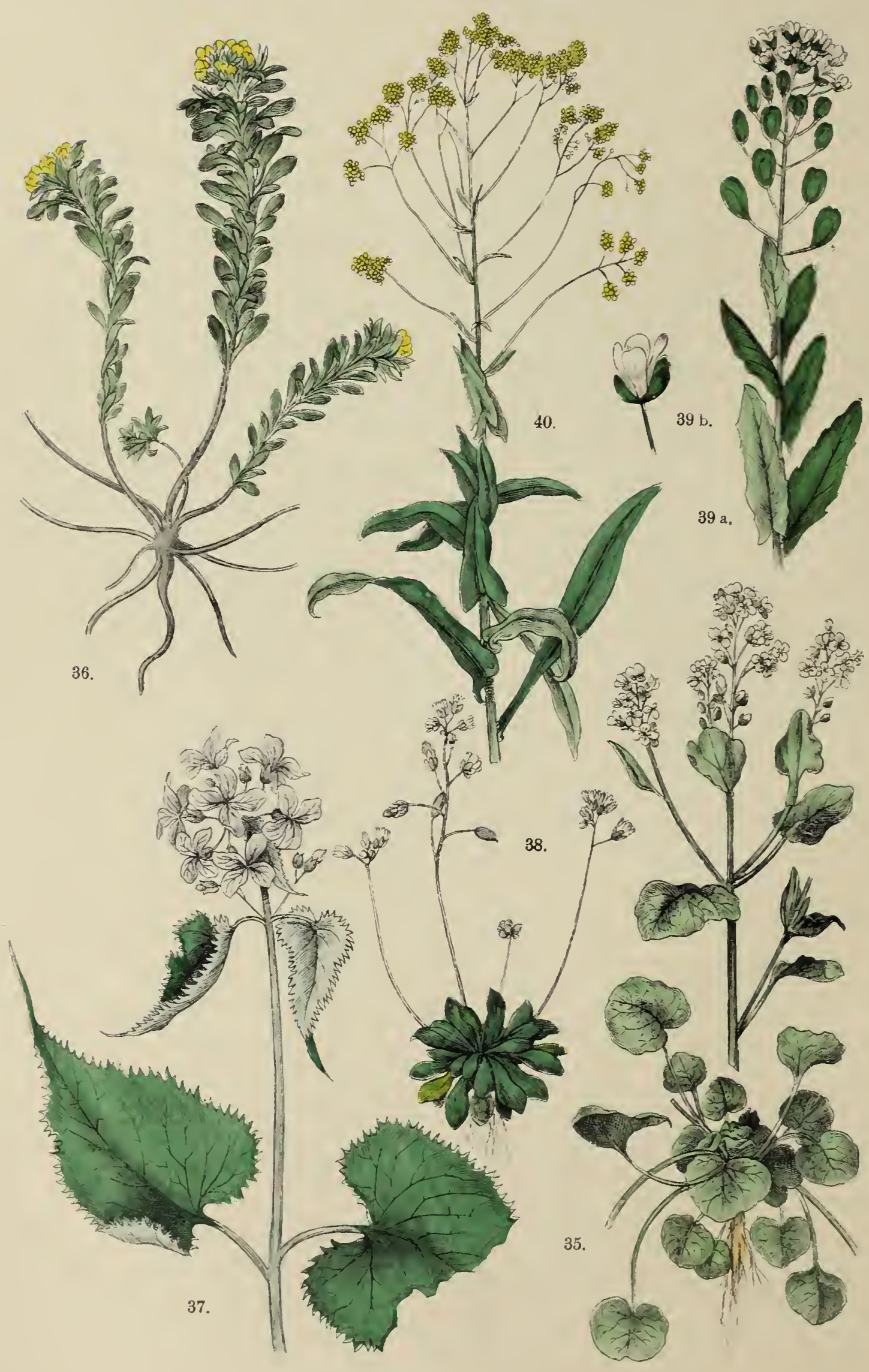




\section{Taf. 7 .}

4ị. 3.5. Cochleária officinális I., gentines,

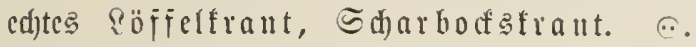
In Diceresutuern und Ealzquellen, $15-30 \mathrm{~cm}$ hod, blüht im Miai uno Jૈmi. Arguci= und 2utz̧pflanze. 2on Cochleára zählt man 4 scut= id)e 2(rten, barmuter ocr Mnecrrettig, Cochlearia Armoracia I.

fiig. 36. Alyssum calycinum $\mathrm{I}_{\text {.., }}$ feldjirüdtiges Stciufiaut. $\odot \cdot 10-30 \mathrm{~cm}$ hod), fintet fid) auf Feloern unb fonnigen sialthügefn, blïfyt vom atprif-September.

fig. 3\%. Lunaria rediviva L., anßoaucrnte Mronoviole. \%. Jn Fend)ten, idjattigen Berg= wäl(dern, 30-90 $\mathrm{cm}$ hod), h(ïht vom Miai bis

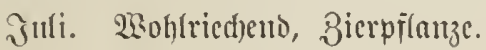

fị̂n. 38. Drába verna L. (Fróplita rulgáris

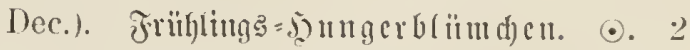
bis $10 \mathrm{~cm}$ ljod). Tsäd)it als llufraut alli ma= germ, fonnigem Bòct, h(üht vom N)iärz-S̄ni.

fing. 39) a. b. Thlaspi arrénse I., थder=

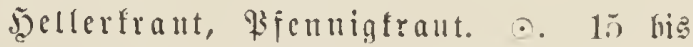
$30 \mathrm{~cm}$. lood), mäd)ît als läitiges llufrant anf šlefern แmo Giartenfändorn nno bliiht nom 2tpril-Ôttober.

fin. 40. Isatis tinctória $I_{\text {. }}$, Färbermais,

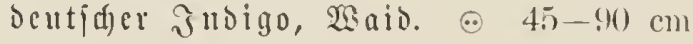

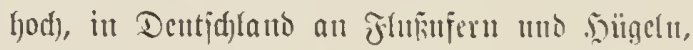

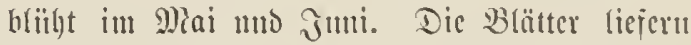

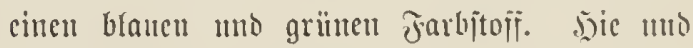

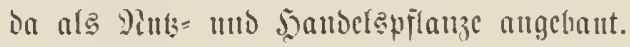




\section{Taf. 8.}

fir. 41 a. b. c. Ráphanus Raphanístrum L.,

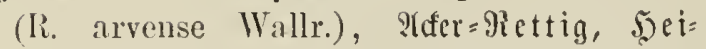
Denrettig. $\odot$ Etengel $30-60 \mathrm{~cm}$ lod); an rsegen uno auf sildern als llutraut, briiht vout slai-Septor.

fig. 42. Crambe marítima L., Niecrforl, Miecrienf, Secfoly. 24. Seöhe $30-130 \mathrm{~cm}$;

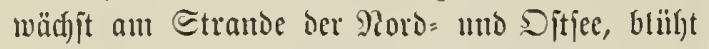
im Niai, Juni. Die jungen Slätter nul Sprojien geven eine gute Speije als Bemiije tmo Ealat.

Zu den Srenzblüter (Cruciferae), Fantilic 10, gefjören ferner: Anastática L., Jerichoroje aแț :ïgupten uno Paläitina uno die zur Dentidjen Flora gel)örenton Hésperis, Radatviole; Alliária, \{́and)= hederidf; Braya, Braye; Peltária, Edeibenfraut; P'etrocállis, Stciufdmiidéf; Camelina, Reintotter; Subulária, Bjiriementreffe; Teesdália, Banterufenf: Ibéris, Sdjleifenblume; Hutchínsia, J̦ttchimfic;

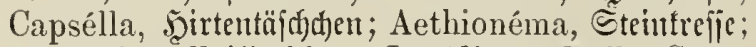
Biscutélla, Briffenfinote; Lepídium, firefie; Sene-

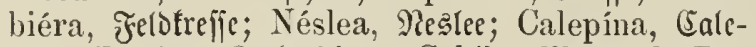
pine; Bunias, Zactenfijote; Cakile, Miecrjenf; Rapístrum, Hapsootter; Sinápis, Senf; Roripa, Wiafjerfreffe; Turrítis, Immfrant.

\section{Fonilie. Capparídeae Juss.}

sappertigewädje.

3u biejer Fautilie gefjören: Cápparis $\mathrm{L}_{\text {. }}$, Siap= permitraud) in Nordafrifa und Siidenropa; Cleóme L., Giflenbaum in Dittnoien; Crataeva L., Guato= baum in $\mathfrak{B e p t i n d i e n .}$

12. Fantlie. Resedáceate Der. asaugewädili. 30 :\{rten, meijt aun Mittelnteer.

fiig. 4:3. Reséda luteola $L_{\text {. }}$, Järber $=93 \mathfrak{3}$ ॥. $\odot$.

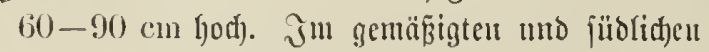
Emropa, in Dentidtano nidjt jelten, blïlyt vom Jutni-2) Jarbitofís angebaut.

\section{Fantilie. Flacourtiáceae Rirl.}

30 : (rten Etränder แnঠ flcine Bäume, แreift zmijden ben 2Bendefreijen wadjent, einige mit ef = baren, andere mit giftigen Frifhten.

\section{Fantilic. Bixíneae Kmith.}

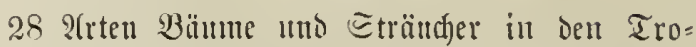
peu. lluter ihuen Bixa orellána L.. edfter Dr. leanbaum, auf ben ?attiffen und im tropijden ?tucrifa.

\section{Fanilie. Cistíneat Jler.

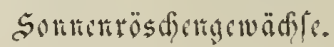

(Etwa 200 S(rten Sträud)er, Sjalbjträudder umo Bäunte; meiît aut Mittelmeer heimifa. Unter ihuen: Cistus créticus L., flebriges ciftrösden alf

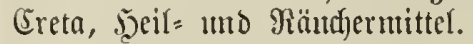

fị. 44. Heliánthemum vulgare Gärtn., gemeines Souncurösden. \%. Ŝl Den meiiten Begenden Dentidhlands heimija, $30 \mathrm{~cm}$ l)od), bliil)t vout Jumi-2(nguft. Bariiert mehr= jadf.

16. Fanilie. Violaríeae Dre. Dinifdergewädfe.

2(11 250, über alle Teile ber Groe berbreitete,

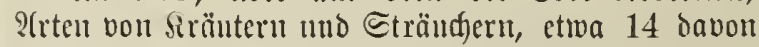

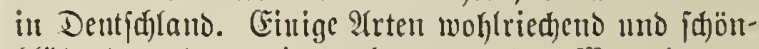
blithend, antere mit bred)enerregenden $\mathfrak{B}$ mzelı.

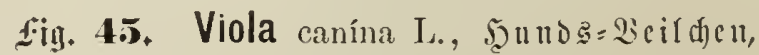

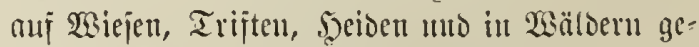

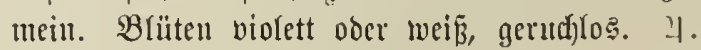
B̉ïlyt vom Miai-gnlti.

17. Fontilic. Droseráceae Der.

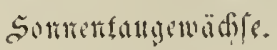

¿0 : (rten; ïber bie ganze Crobe verbreitet; 3 Dentidje Alrteu. Sumpipflauzen.

fịg. 46. Drósera rotundifólia L., runbbrättriger Souneutan. भ. $8-20$ cm hod, in Sïmpfen แno anf Torjuoden, namentlid)

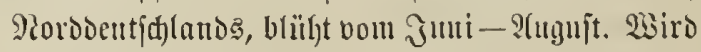
z" Den jogenaunten injeftenfrefienden Biflanzen gezäblt. 
8.

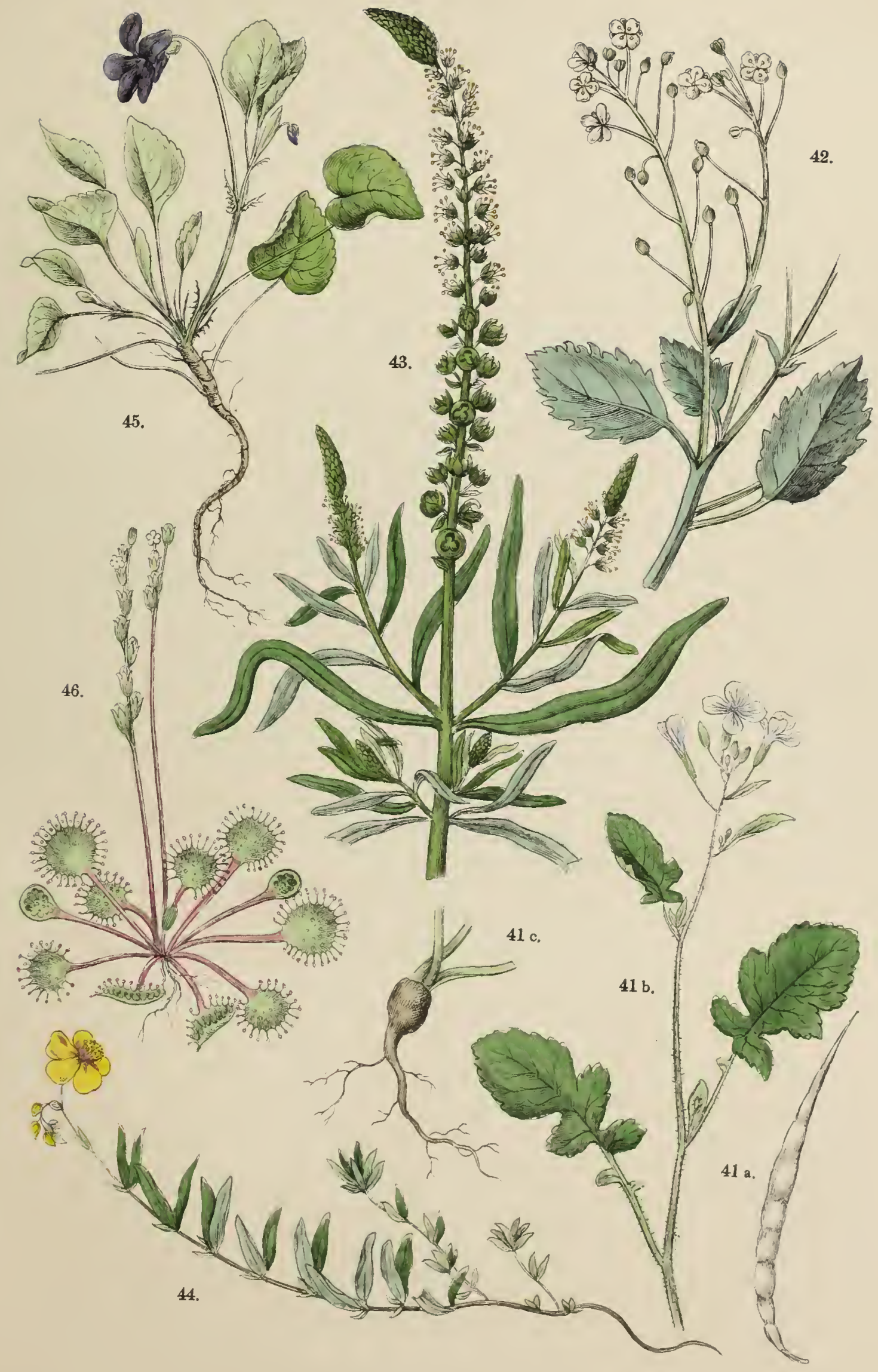





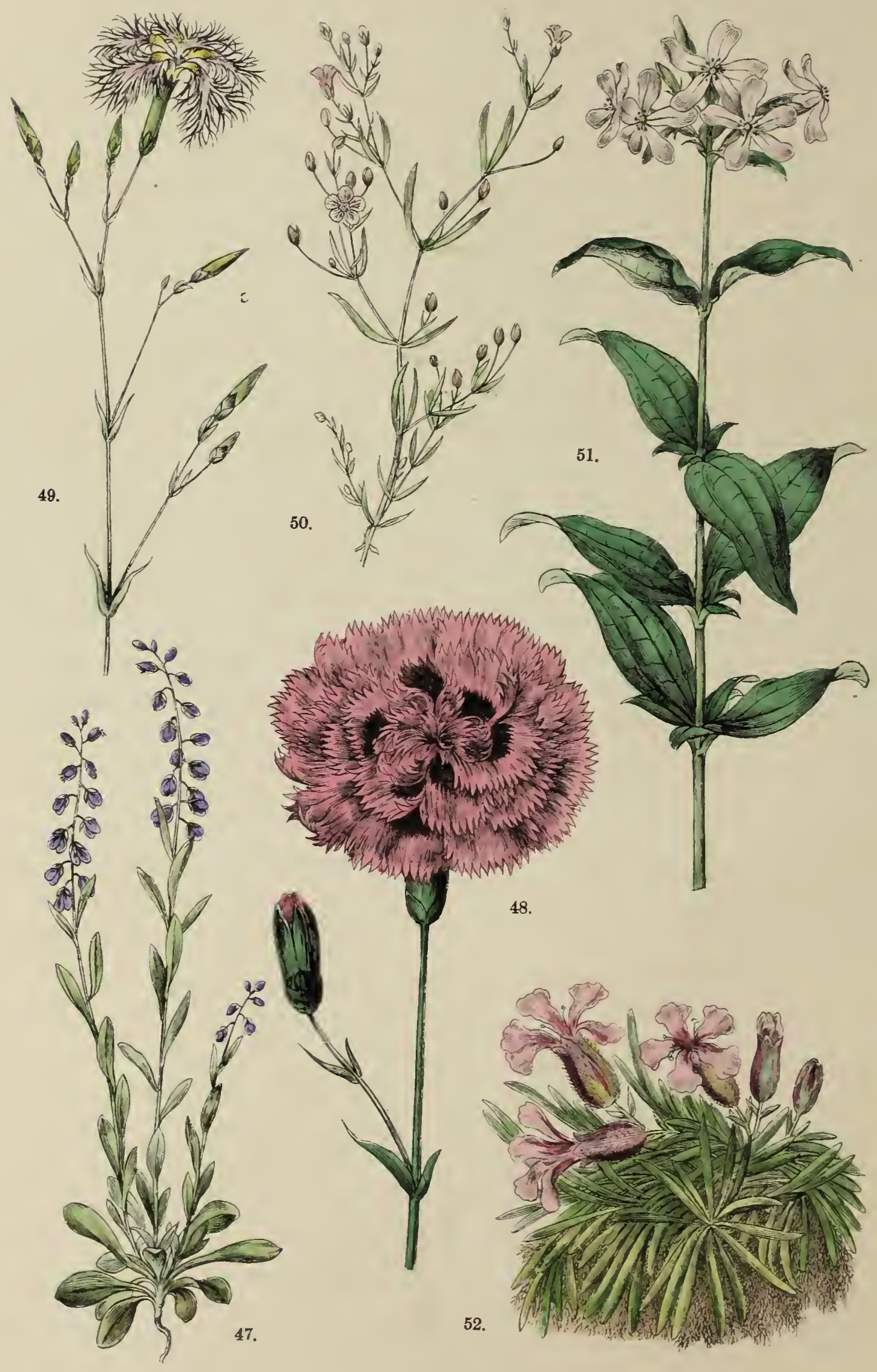




\section{厄af. 9.}

18. Fantilie. Polygaláceae Juss. ßBiffertinge. über 300 Irten Sräuter oder Sträıdfer, über die 3 mittleren 3 onen aller $\mathfrak{B e l t t e i l e ~ b e r b r e i t e t . ~}$

5iig. 4\%, Polygala amara L., bittere $\operatorname{Reu} z=$ blume, Ramjel. 2. Ridjt jelten anf feudfen Waldrwiejen, blüft rot oder blau, vom Эuni biæ :Inguĩt. 2Hzzneifräftig.

19. Familie. Tremandráceae $R, B r$.

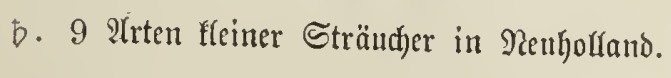

20. Fanilic. Pittospórene $R . B r$. ¿hleblametrgewädfle.

D. 韦. Etwa 30 Arten, meijt in Rentholfano.

21. Fanilie. Frankeniáceae St. Hil. 20 Arten, meijt an jübliden Mecresfüjten.

22. Fanitlie. Caryophýlleae Juss.

(Siléneae Bartling, Qręker uno Alsineae Bartling, aniterte.

Relfen 350 2Trten in den gemäp̈igten 3onen,

Micren über 300 2trten in Den fälteren Zonen, eriftere pradhtodle Zier= uno Gartenpflanzen.

5ịn. 48. Diánthus plumárius I., Feocr = Rerfe. 4. WSofrriechent, in vielen idjönen, gẹüllten Barietäten als Giartenpillanze beliebt; bie Stamm= art wäđjit wito auf felïigen Seitgeln S̈itreid)s, ift $10-30 \mathrm{~cm}$ hod uno blülyt im Mai, Juni.

fHig. 49. Diánthus superbus L., Rracht=Feder=

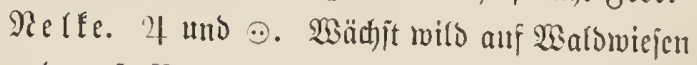
und auf Bergen, twird $30-60 \mathrm{~cm}$ hod) unto

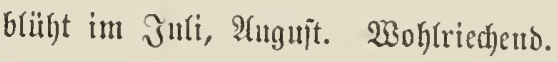

fig. 50. Gypsophila muralis I., Miauer= Bipsfraut. $\odot$. IInfraut auf Miaucru uno janbigen :̈̈(fern, $5-15 \mathrm{~cm}$ hod), b(ïht im Juli, Anguit.

5ity. 51. Saponária officinalis L., getucines Seifentrant. 4. $30-60 \mathrm{~cm}$ hodh, alli Sand= boben an ben Fłupujern bon fajt ganz Europa,

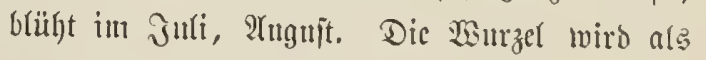
Majajmitter verwentet uno ijt offizizituell.

fịg. 52. Siléne pumilio Wulf., (Cucubalus L.), 3werg=eeinfraut, Slcbuclte. 4. Ju

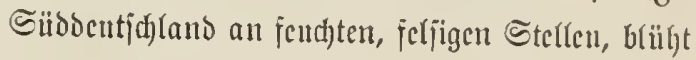
im Juni, Эuri. Ein niedcres, bidjte Riajen bil=

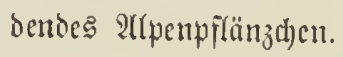


đẫ. 10.

fig. 5:3. Lychnis dim'na Sibth., rote Ridits

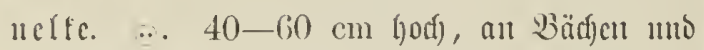

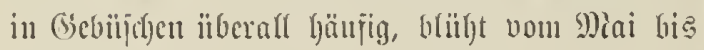
P(uguit. Bserud)los, Bicrpflanze.

fing. 54 a. b. Arenária rerna I. (Alsíne Bartl., Subulina Tichb.), früblings = $\subseteq$ an of $\mathfrak{x}$ ant. 1. 2ui îteinigen Bicbirgtweioen in ben meijten Qänocrn von Europa, bliilyt vom Maa-

fig. $\mathbf{5 . 5}$ a. b. Cerástium alpínum I., 9(lpen= Dorufraut. ?. Etengel friedjento, Blüten=

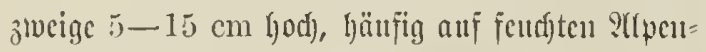

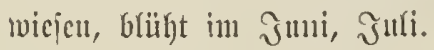

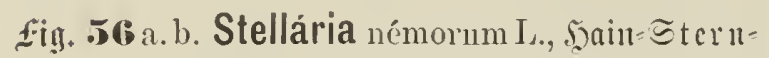
micre. 7. 30 - (30) cm hod), in (Bchuijdjen utto

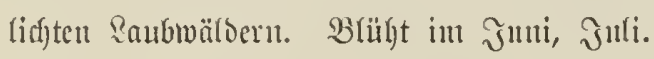

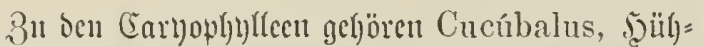
nertiziz; Sagína, Miaptrant; Cherléria, Eherleric; Mönchia, Miöndjic; Agrostémma, Jiàd; Alsíne, Mierc; Holósteum Epmrre; Portuláca, Portulaf; Elatine, Iäunel; Montia, Sucflenfraut; Levísta,

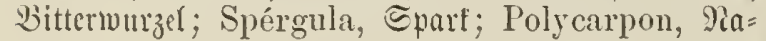
gelfraut.
23. Fmmific. Líneac de $C$. seirgewädfle.

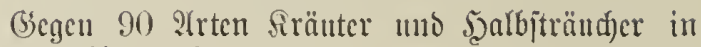
ben gemäßigten 3 onen.

fị̂. 5\%. Línum usitatíssimum I., gentciuer seiu, Flad)s. $\odot . \quad 30-60 \mathrm{~cm}$ hod), liberall angcbaut, blüht iut Juli, Ittguit. Dic Bait= fajern bes Stengele dienen zut Gejpinjten, bie

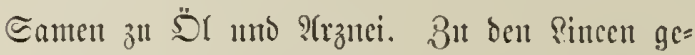
hört nodf Radíla, Stralffraut, 3 werg=

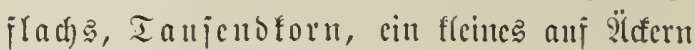
lüufitiges, gcjelliges siraut.

\section{Familic. Malváceac Barll.}

xiofvergemadfle, wappereletr.

PIn 1000 2roten, $2_{3}$ in ber heipen, $1 / 3$ in ten gemä̈igten 3onen aller 2isclteile.

fiin. 58. Malva silvéstris $L_{\text {. }}$, wilde ober $\Re$ of $=$ Mialoc, Sïjepappel. t. $30-90 \mathrm{~cm}$ hod),

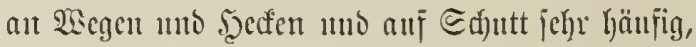

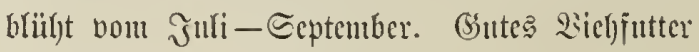
unto, wic mechrete antocre 9 itten, offizinelf. 


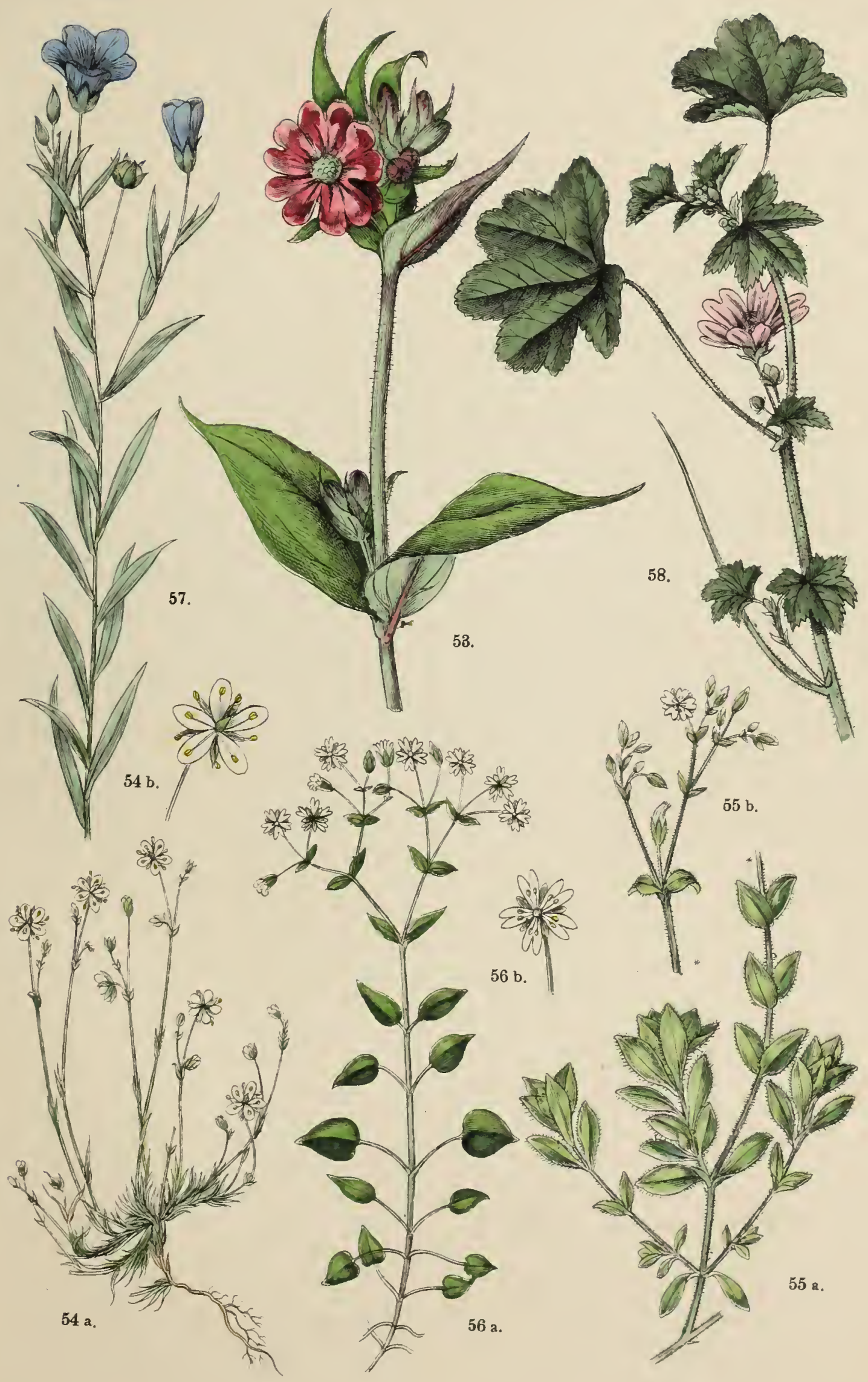





\section{.}




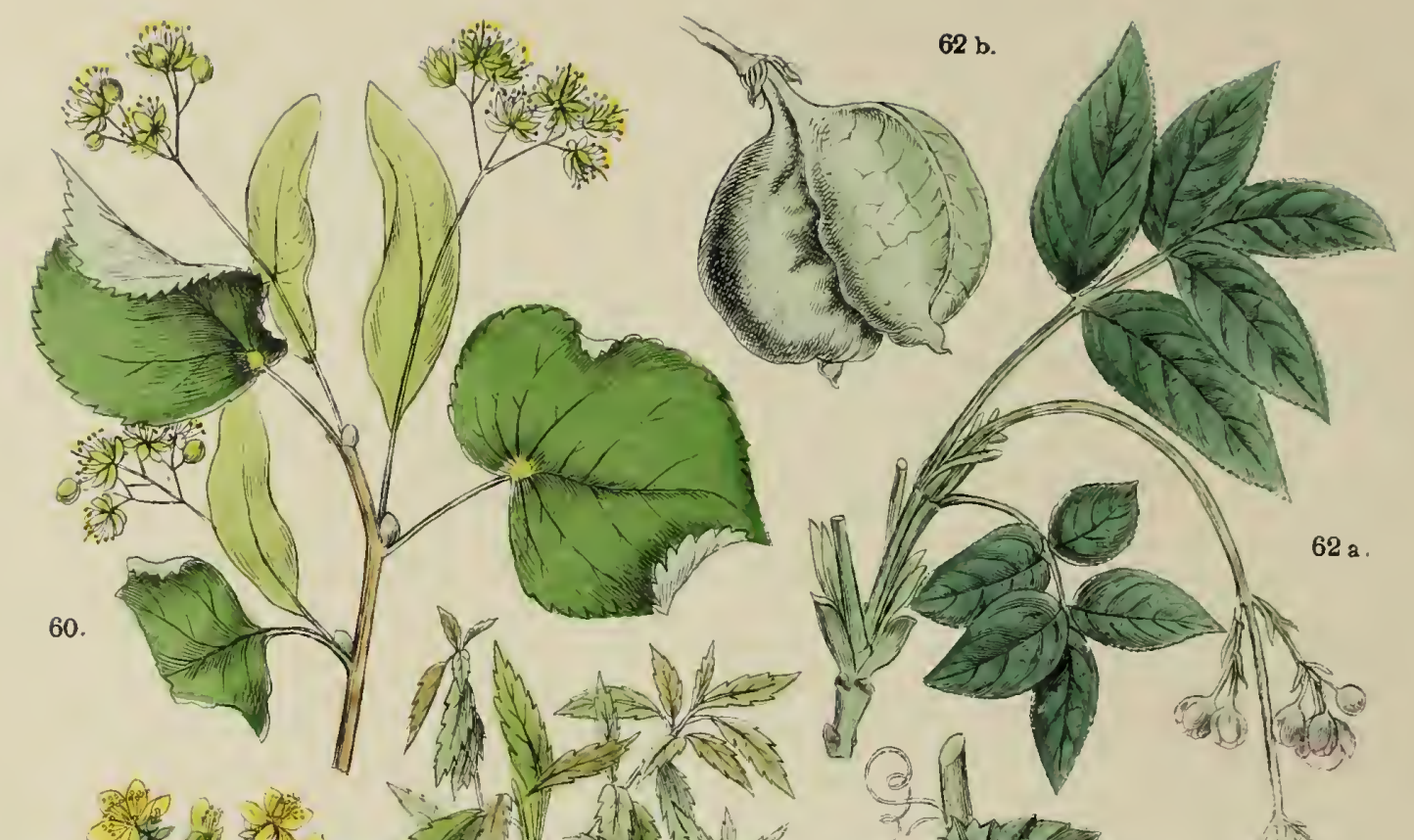

3) a
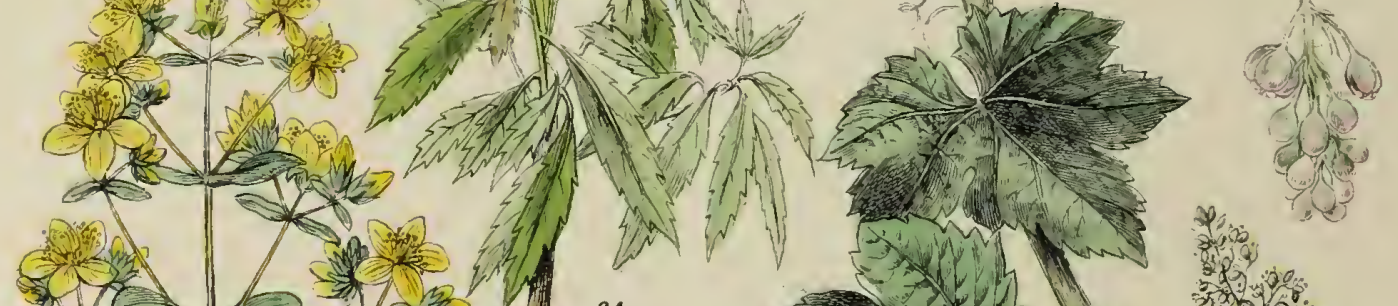

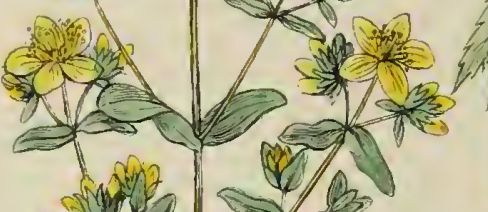
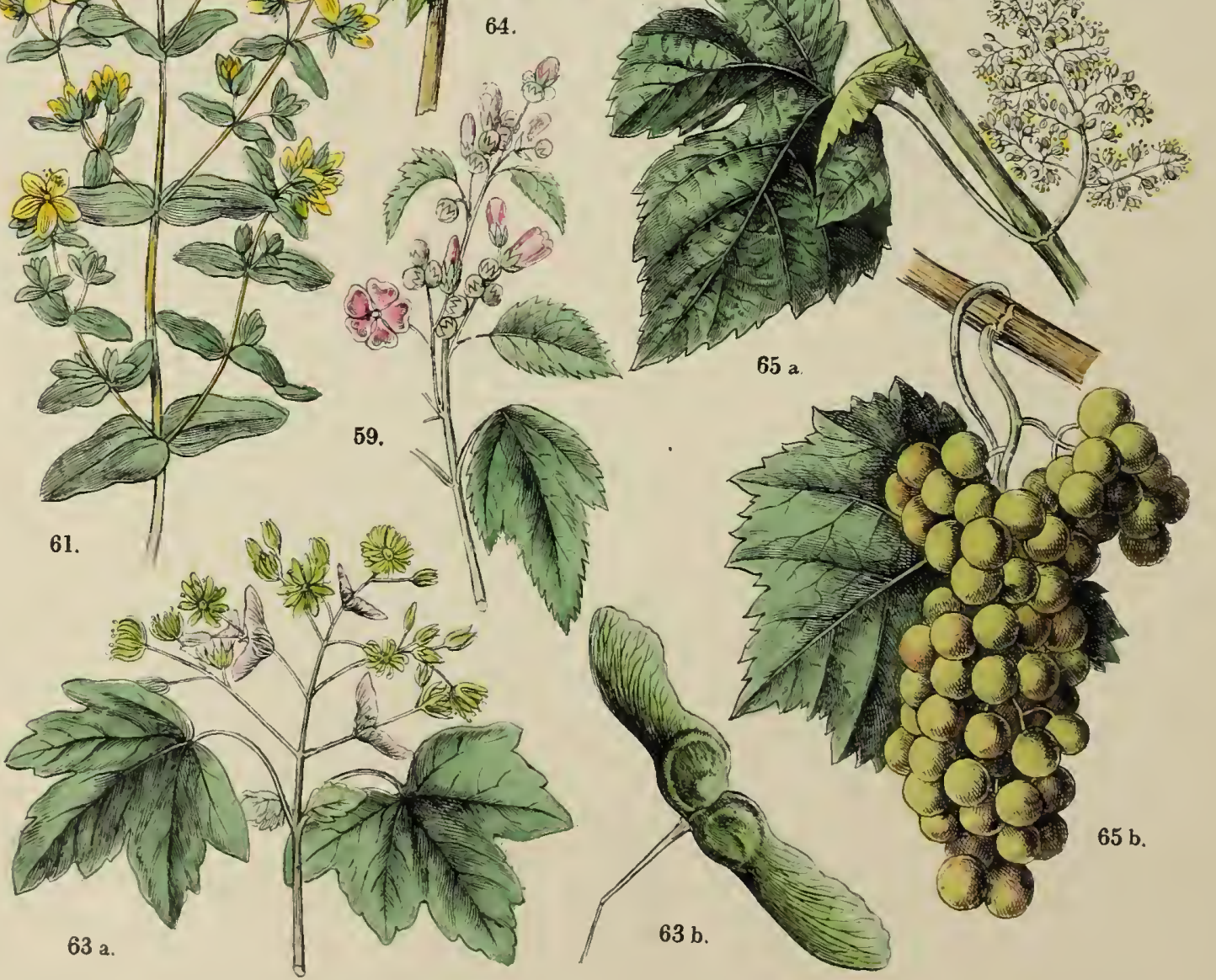


\section{『ấ. 11.}

fin. 59. Althaea ofticinalis $L$., getucinct $匚 i=$

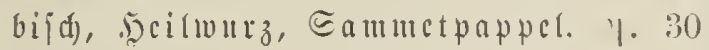

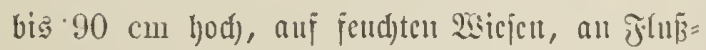

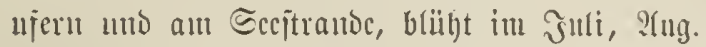
Dic $23 \lll z c l$ sicut vielfad) arzneifräjtịg. $3 u$ ben Mialvacecn zällt man: Hibiscus, Jbija); Gossypium, Banntwollc; Lavatéra, Savatcre; Mátlope, Mialope.

\section{Fnuilic. Bombáceae Kulill.}

Dejicrz̆l geljörcu: Bombax, 28ollbaun; Ochróma, ফ̉aljambaum; Adansónia, Yfffubrotbaum; Helic-

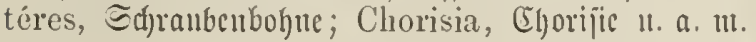

\section{Fnulicic. Biittueriáceate R. Br.}

Ettwa 70 अrten @träud)er obcr viäunte itu tro= prijd)u YYucrifa. Fajt alíc cutfalten in beu Gauten fette S̈le, bie wid)tige 5̧alloclsartifel bilben. 311 bicicr Fantilic gefören: Stercúlia, Etiufbaum; Theobróma Cacao; Büttnéria; Heritiera, Brcttbaum.

\section{Fanific. Tiliaceate. simosmgenäsfs.}

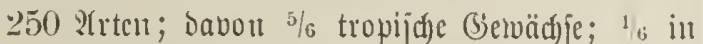
Deıt gemäß̈igteı 3oucu.

fin. 60. Tilia europaea L., sinoc. 言. \$ract)tuoffe $\mathfrak{B a l b}=4110$ ?llfecbännte, $18-40 \mathrm{~m}$ loud), 3 Epiclartcu, ïbcrall in Deutidflanto be=

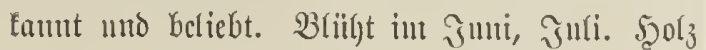
unto Soljle weroen benïkst, BHitten finto offizincll. 3" ben Sinten gehören: Córchorus, Эutoerpap= pel; Elaeocárpus, Gantitcrbaum.

\section{Trantilic. Dipterocárpeae Blume.}

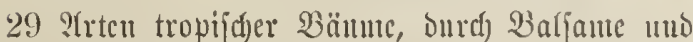

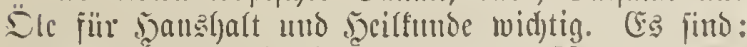

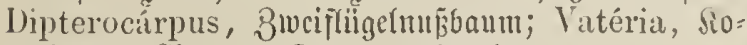
palbaum; Shórea, Dammıaraharzbaum.

\section{Fnuilic. Chlacnáceac Pet. Thouurs.}

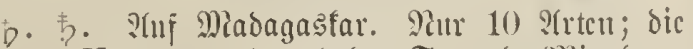
widjtigite Hugonia, $4 \mathrm{~m}$ lobjer Strand), Mittel ge= gen 巨d)laugeubiß.

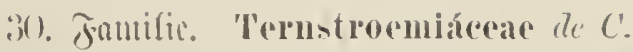

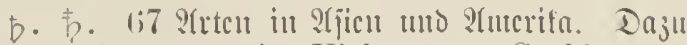
gefjörcu : Ternstroemia, Kiehneyera, Cochlospérmuบ1.

31. Jamilic. Camelliáceac de C.

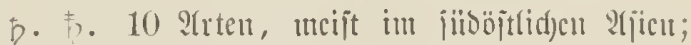

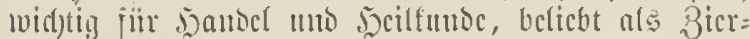
pflauzcn. Dic wiedtigiten ?trteı füb: Camétlia japonica L.; Thea chinensis Sins., Ifecitraudf.

32. Trantilic, Aurantiáceate Corr.

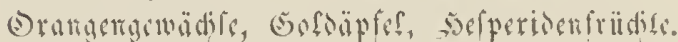

Эmuncrgrüuc Bänme แn๖ Eträndcr, in ctma

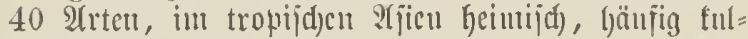
tivicrt in Eiideuropa. Bevorzugt burd idjönc $\mathcal{B}_{\mathrm{c}}=$ laubung, wolylricd)ente $\mathfrak{B}$ (iiten unto vortreffllid)e Frtiid)tc. Befantut jumb: bic Ri mo ne (Citrus Limonimm Risso), Dic Eitronc (Citrus merlica Risso), bie Drange (Citrus aurantium L.).

33. Fnnilic. Iyperieíneate Juss. sัarthengemäds!c.

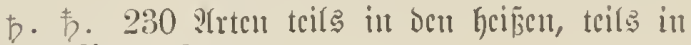
deแ gemäpigten 30ucu.

fiij. (51. Hypericum perforatum L. Bemeics

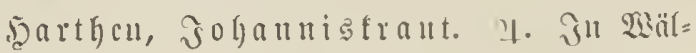
scru tund alt 2 segräubcru, bis $910 \mathrm{~cm}$ lod), blitht

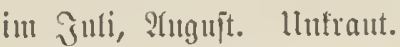

\section{Fomilic. Guttiferae Juss.

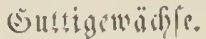

韦. Ettua 100 ?(rten tropija)cr Bäunc, ocreı

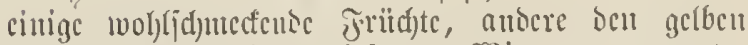

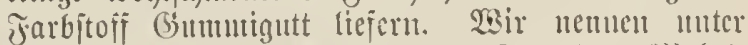
ifucu: Garcínia Mangostána L., ber \$lodul=

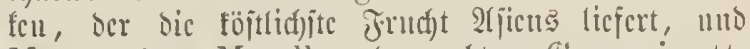
Mangostána Morella, ocn ed)ten (b) un migutt=

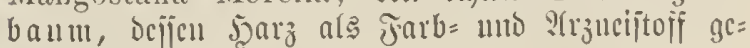
idjäkst ijt.

\section{Fmuilic. Marcerraviácene Juss.}

5. Etıva 20) nidgt genau befaute Eträud)c

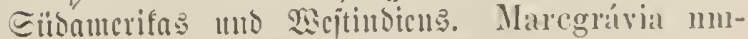
bellata L., ein Siletteriftrand) Eiionuterifas, scijen ciuzclue Teile bort ala necilutittel bicueu. 


\section{Fanilie. Hippocastáneae de $C$. arofikaftamien.}

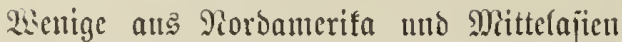
jtamuentoe Yrtet. Diezu gehört: A esculus hip-

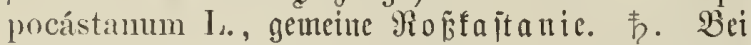

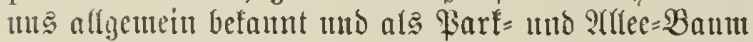
beliebt. Das meidie und fdumanumige $50 l_{z}$ wird

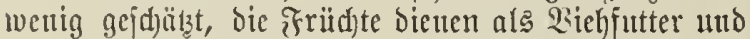

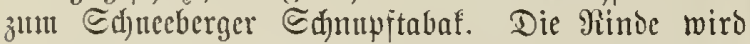
als b) billuittel, forvic zum (Şerben und Färben be=

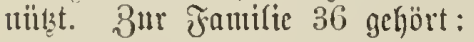

fiuj. 62 a. b. Staphylea pinnáta L., genteine

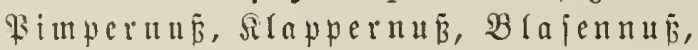
vilde Bijtazie. $b .3-6 \mathrm{~m}$ hod), bei unz als 3ieritrand) belicbt, blült im Miai, Juni.

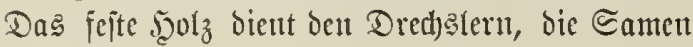
jinto ö(reid).

\section{Fantic. Erythroxýleae $K u n t h$. arothofagewäd}

ち. 方. (Etwa 30 2(rten, jünttid) tropijd); laben peites, rötliches 5 jolz und enthalten roten Farbitoff. Die vidytigite Art ijt Errythroxylon cóca Lam., ber (Eocajtraud) in Beru uno Bolivia, befjen Blätter mie 2 etel vout ben Ginwohnern gefaut uno neuer=

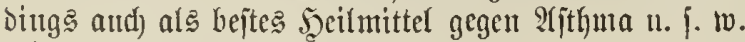
bei ung gerilgunt werdent.

38. Fantilie. Malpighiáceate Juss.

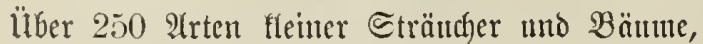

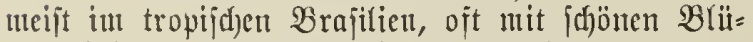
ten, eituige mit moblfajmecfenden Friad)ten.

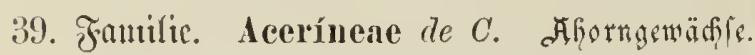
韦. Etrva 30 Arten in oer nörbliden und ge= mäß̈igten 3one. Eine ber widjtigiten 2 rten ift:

fin. 6:3 a. b. Acer campestre L., Felo $=\mathfrak{A h}$ orn,

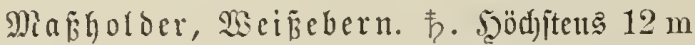

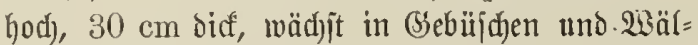
Derti vou ganz (Europa. Sein zähes uno jäönes Jjolz wird poul ben Tijd)fern jehre gejudt, bient

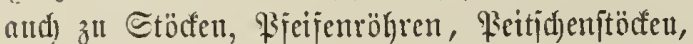
utto bie majerige 23 uzzel zu ben llluter Pfeifen= töpfen.

\section{Fanilie. Hippocratíceae Kunth.}

ち. 韦. litber 70 nutr tropija)e 2trtett. Die widutigiten fint Hippocrátea comósa Sw., der

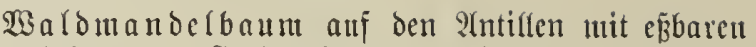
Früchten und Salácia senegalénsis, Birnbaum

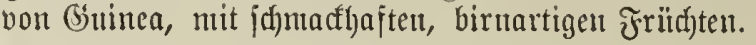

\section{Fanilic. Rlizoboleae de $C$.}

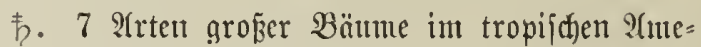
rifa. Die roid)tigîten find: Rhizóbolus amygdalíferum L., ein iiber $70 \mathrm{~m}$ hoher $\mathfrak{B a u m}$ mit wohls id)mecfenoen, mandelartigen Früd)ten. Rhiz. buty-

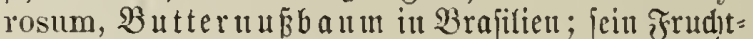

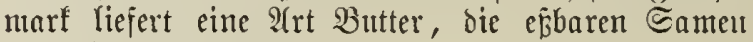

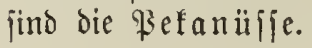

\section{Fanilie. Sapindáceac Juss.}

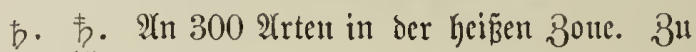
iłnetl gehören:

fiig. 64. Sapindus saponaria L., gemeiter $\subseteq_{e} i=$ jeubaum. 产. 6-9 $\mathrm{m}$ hod, in Sieptindient, defien Frübte Eeifenitofi enthalien und zum Eajach der Zeuge verwendet werben. 3u beu Sapindaceet geljören jertur: Paullinia, ran= fettoe, meijt giftige હtrüucher uno Sräıter in ऽïd= amerifa. Cardiospérmum, .ðerzjamen; Cupania, Eijenholz.

\section{Fantilic. Meliácene Juss.}

ち. 言. lltber 100 2rteu, fämtlid) iu den heifen 3onen. Unter il)nen Mélia, Fater mojter $r=\mathfrak{B}$ a u in 2Yjien; Trichília in भ̈lgypten; Carápa it $\mathfrak{B} e j t=$ indient; Léeca, $\mathfrak{B a j j e r r e b e ~ a u f ~ b e n ~ M o l u f f e n . ~}$

\section{Familie. Ampelídeae Bart7. (Sarmentá- ceae Vent.). Baebergewäđils.}

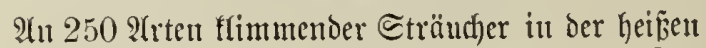

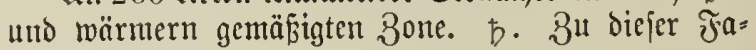
milie gefjören:

Ein. 65 a. b. Vitis vinífera L., edoler $\mathfrak{B}$ e in jut o ff. Eill über $30 \mathrm{~m}$ hoher, flimmender Strand, it vielen Spielarten jajt in ganz Europa fultiviert.

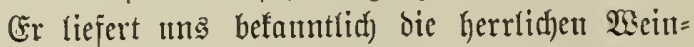
trauben und den föjtlicjen 2 eitn. Zn ben $\Re$ eben=

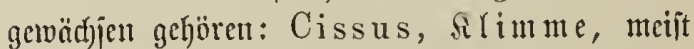
tropifache Sträuder; Ampelópsis, $3 \mathfrak{a} u$ urebe, willer $\mathfrak{x e i n .}$ 



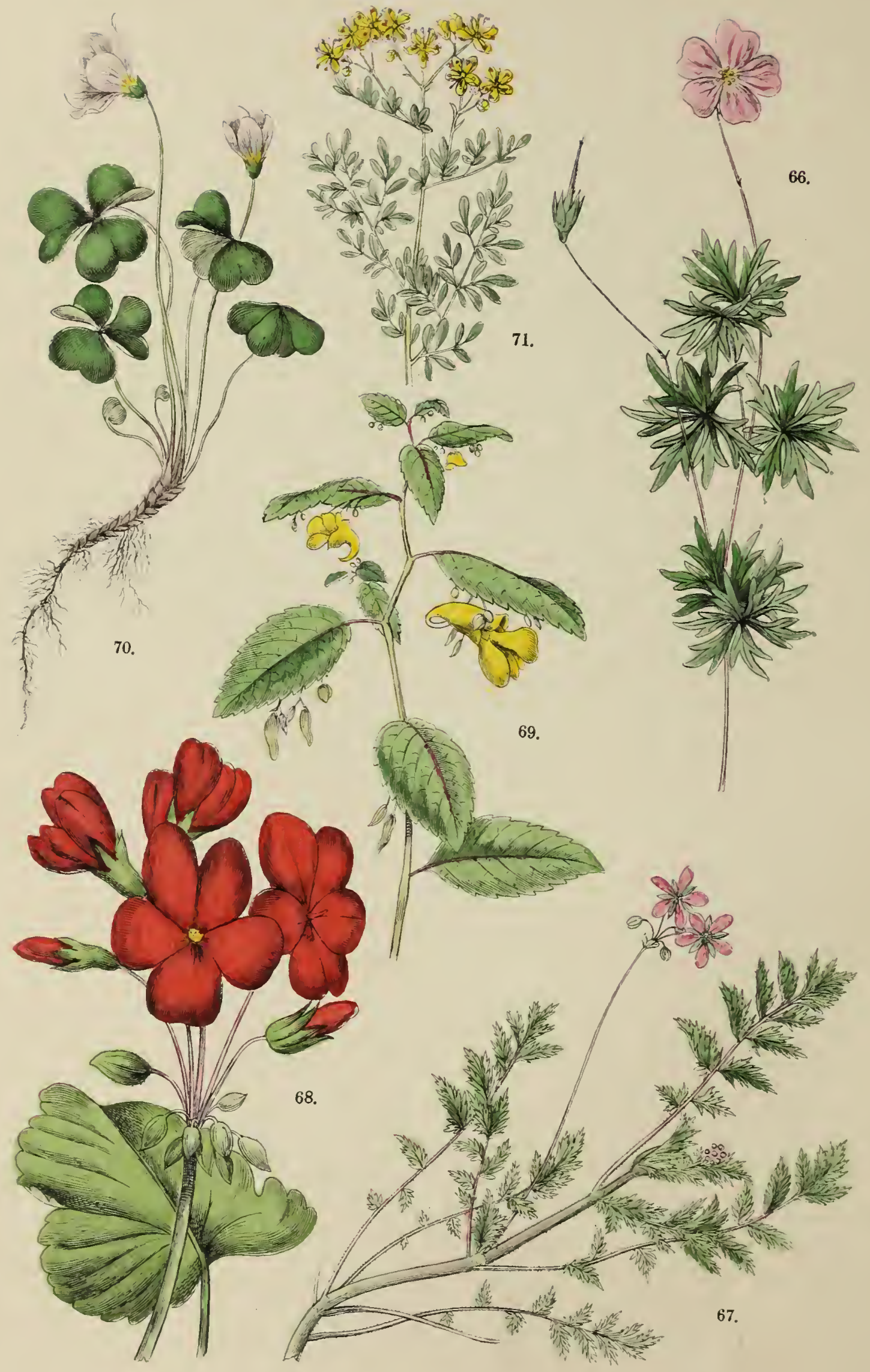


45. Fantilic. Geraniáterac Juss. stordifdrufergemadils.

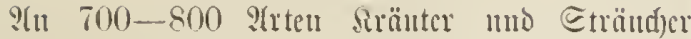
meipt in ocr gemäpigten 3onc. Wicle als Topipflanzen beliebt. 3" ifutu geljören:

fiug. 66. Gerảnium sanguineum I., blutroter

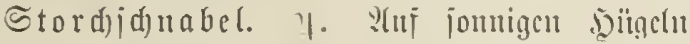
unto in trodencn $\mathfrak{B a ̈ l}$ (socrn; $15-30$ em hod), blügt vom Jutui-2 Tugujt.

fiig. 6\%. Eródium cicutarimm Herit, gemci=

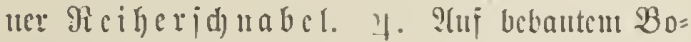

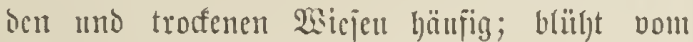

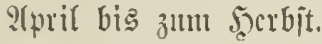

5iıj. 68. Pelargónium Herit., $\{r a n i d j d) \backsim a=$ bel. 2. Llcber 300 arten Srüuter mo Eträı= d)er, fait alle vom Sap, bci mes wegen ihree lcichteu Sultur, jđjötten Blüten mo wohlricd)en= Den Blätter allgemein belicbte Topipilanzen. Die abygcbilocte atıt ijt I'. 'Zonále W.

\section{Fantilic. Tropacóleare Juss.

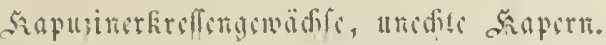

Sräutter, wold)e in ctwa 40 ?rten nur in Eiio: amerifa lecinija) jino. Tr. majus, die grose in=

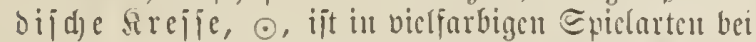
แn cine beliebte Gartenzicrpilanze. Dic simojpen mint unrcifen Frïd)te bienten jatt ber Sapern.

\section{Jnutific. Balsamíncan A. Tích.

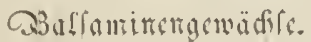
Into zmar:

Estwa 50 24rten in ben Tropen, nur 1 beutjdye

fing. (59). Impátiens noli tángere $\mathrm{L}_{\text {., }}$ miloc Baliauinc, Epriugtraut, Jubcubittein.

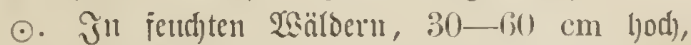

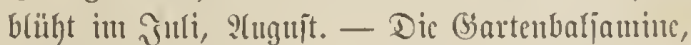
Bals. horténsis, $\odot$, jtamut aus Dịtindien, bliit)t voun Juli-Ecptember nuto jicrt in bcu verjdjicoenfarbigiten Epiclartcu mujre Bürten.

\section{4x. jomilic. Oxalídeate de $c^{\prime}$.

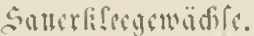

Ülbcr :200:\{rtcu meijt auf Eübamerifa beid)rüntter Siräuter, jäucrlid), Silcejalz cutljaltento. 3 beutjde ?rtent.

fị. 70. Oxalis acetosélla L., gemeiner $\Xi_{a}$ uer

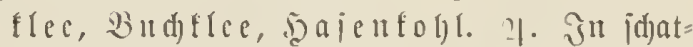

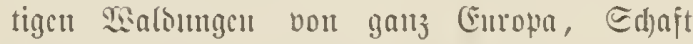
8-16 (m hod), bliil)t im :(pril, Mlai. Das

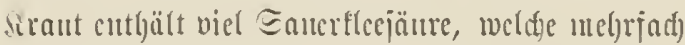
gewerblid) vermentet mird, aber idjari giftig ijt.

\section{Familic. Zygophylleac R. Bro. Sodfifütrige Gomädic.}

(60) STrten Rräuter, હträud)er nno Bäume, ïber

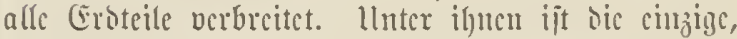
ber bentid)en flora augef)örige ?(rt: Tríbulns ter-

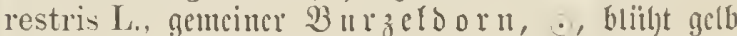

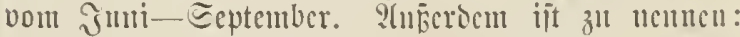
Guajácum, Hoofenlolz in Tieptititoicu; Me-

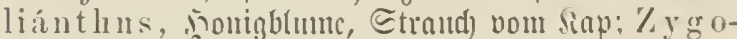
1) liyllum, Doppelblatt, in Eltrict mo Piorto airifa.

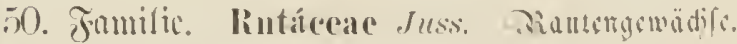

30 2(rten Sirüuter oder Eträud)er in ocr nöro= lidjen geutäp̈igteu 3oure.

fiin. 7\&. Ruta gravéolens L., gemcitue giautc,

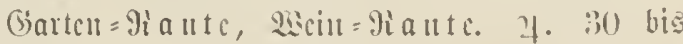

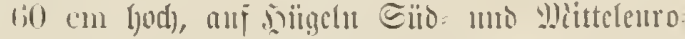

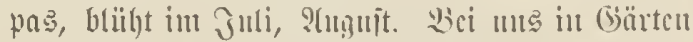

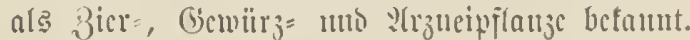
Dic gemciuc Darmcliaute, P'éganum hírmala I., ijt ciu nolbjtrand) in Eibocmopr, Porotafrifa mro Diittelajicu. Dic Eamen jino ciut bedententer handelsartifel, fie bicuen in ber

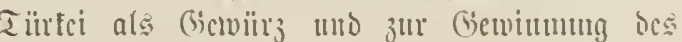
ed)ten Türtijd)rot. 


\section{Tå̃. 15.}

fin. 7:2. Dictámnus albus L., gcutcinter Dip=

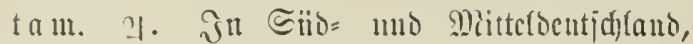
(i) $-90 \mathrm{~cm}$ qod), bü̈ft im Diai uno Эuni.

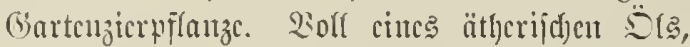
meld)es mit cintem brententioen sidd) berïlyrt, lood

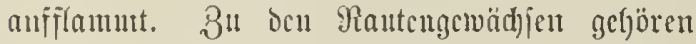
jerutcr: Galípea, weititoijder 2htguiturabaum; Xanthóxylon, (B)elbyolzbaum in 20orit= amcrifa; Ptélea, Scoerbaum ane Mittcfautc=

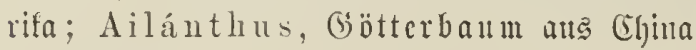
uno japan.

\section{Fomilic. Simarúbene de C.}

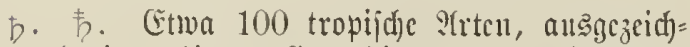
net burch) cinen bittern (Extraftivitoff: 3 oicjer Fa

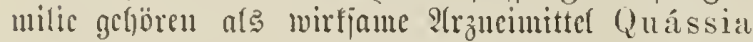
amára L., B̉tterfolzbaum, cintbeimija in $\Xi_{n=}$ rinam, uno Simaruba de C., Bitterejde iu Samaifa: Ferner Simába, Ecoroubaun in $\Re$ eu= granaba, beịjen Eautenterne aud) afs beiffräftig be= rüf)utt jïlo.

52. Fanilic. Ochnáceae de $C$.

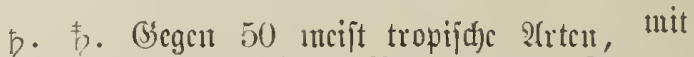

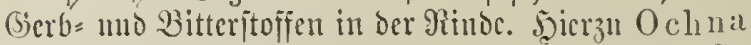
I., Ragelbecre in @übamerifa; Gomphia de C.,

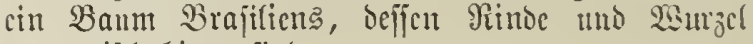
aromatijg bitter jinto.

\section{Familie. Coriarícrae de $C$.}

5. 7 ?trtent, mcift in Sïoanterifa, 1 SIrt int Suropa. Reid an Gierbijtofi, cinige giftig. Jjier zn: Coriária, Gerberitraudit Siiocuropa, bei ung

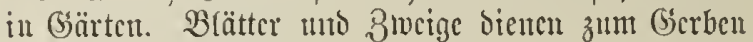
Imı Edjwarzfärbent.

\section{Intertafle: Calyciflorae, leld̨blitige pflanjen.}

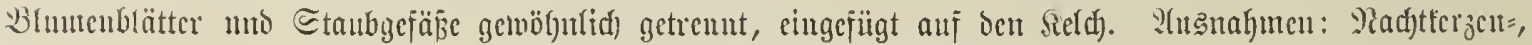
Gítrfent= uno Etadjelbergetwädjic.

\section{Fantilie. Celastrúncae $R . B r$.}

5. 专. Cetwa 150, iiber alfe meftteife verbrei=

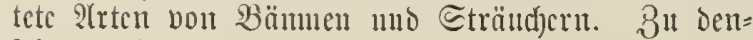
jelhen gchören:

fiin. 8:3 a. b. Evónymus europaeus L., gemei= ner Epillbaum, Epindelbaum, Bfaffen=

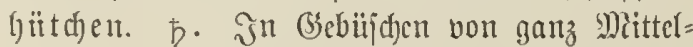
curopa, 1-4 m hod, blüht im Mai, Juti. Das feite, helfgelbe șolz wirb von Dred) slem jelyr gejucht uno gicbt bic bejte Zeid)cnfohle. Şierzณ gebören ferncr: Celástrus, B̧aummärger in 2tucrifa, Pfïen uno STriffa; Ela o dén dron

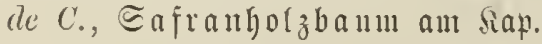

55. Fantilie. Rhámmeate $R$. $B$ r.

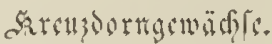

5. 5. Etwa 250 ïbcr bic ganze Eroe ber=

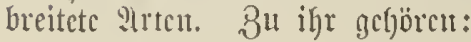

finj. 74 a. b. Rhamnus Frangula L., glatter Begoorn, Faulbaum, Futverlolás. Jut

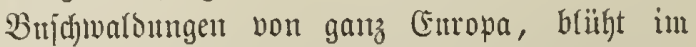
Mai. Die Sohle bient bejonders zu @ajieppulver.

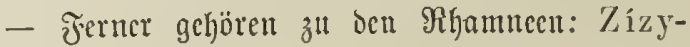
phus, Stroenoorn; Palinirus, Stedoorn

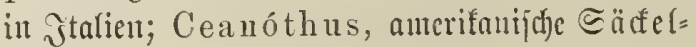
blume.

56. Janilic. Bruniáceac $R$. $B r$.

Bisgen 50 9trten fleiner, inmergrüner, auf ocr

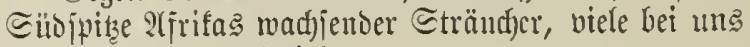
ale Zieriträudfer belicbt.

\section{Fanilic. Samydeae Gaert.}

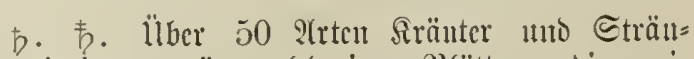
d)er mit immergrünen fcocrigen Brättern, die met= Iten S(rten im tropijd)en STutrifa. $3 \mathfrak{n}$ ignen ge= hören Samyda in sectitinoicn mo Caseária in Dittindicn. 
13.

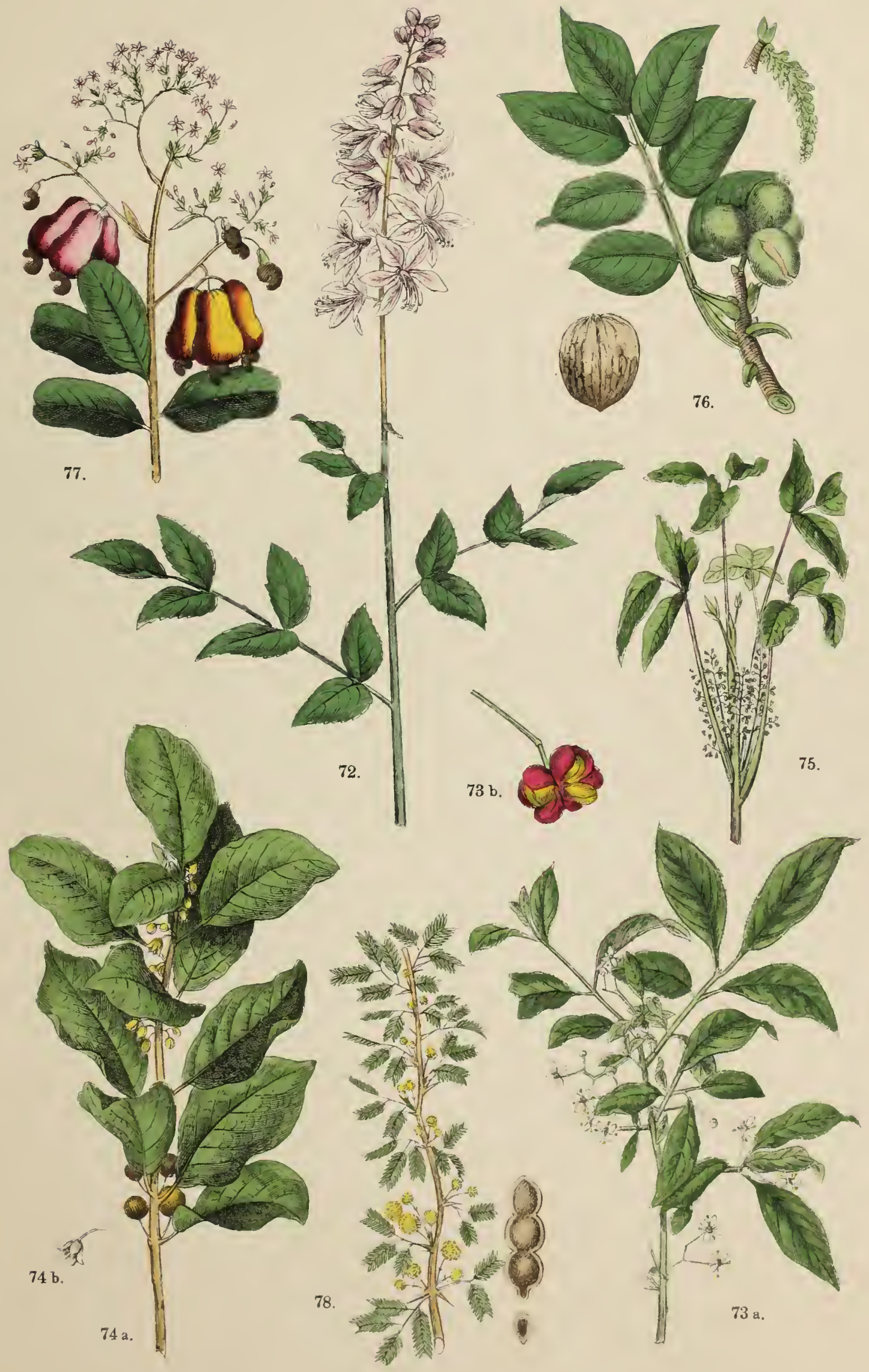



58. Fantilie. Homalíneae $R$. $\mathrm{Br}$.

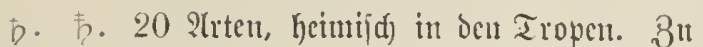
ifuen gef)ört Homálium, Rffoutabbau aụ ocu 2uttillen.

\section{Fantlie. Chailletíceas: $R . B r$.}

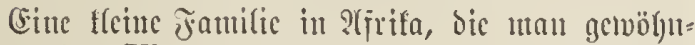
(id) zul Den Ulmáceen red)uct uno beren vidjtigite Aft dic Chailletia toxicaria Don. ijt, ocren frrtitute ljödjit giftig fitto.

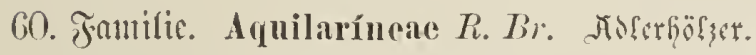

(Fine flcite Familie des tropifden atjicus, ctma 5 fletuc Baumarten entfaltent. Das 5 )ol

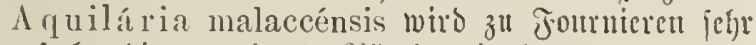
gejud)t, bieut attd) als giäudjermittel.

61. Fantilic. 'T'rebintháceac Juss.

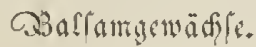

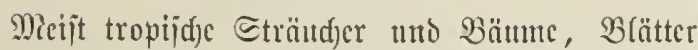

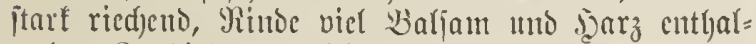
telto. 3" Dicjer Jamitic geljören:

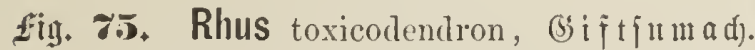
ந. $3 i$ s $2 \mathrm{~m}$ hod), niederfiegeno oder fletterno, in 20roanterifa lecimijd), in Deutjd)land ftellen= weije in Tulagen angepflanzt, alle Teile jely giftig, blïht im Mlai.

fig. 76. Juglans regia $\mathrm{L}$., gemeiner 2 allnu $\tilde{\xi}=$ baum, weljder $\mathfrak{R} u \tilde{B} \mathfrak{b a u}$. 言. 12-24 m (jod), heimijd) in Mittelajien, in vielen Epiel= arten fultiviert in fajt ganz Eimopa, blübt im Ipril, Miai. Ecine Berwenoung, feit mannig= fad)er Riaben find befaunt. (Dic Jamtitic Jug- lándeae de C. wirb and) wie bie 1 trmen, Birfen, Budfen von mandf)en Botantferı zu ben 2(men=

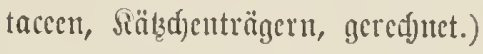

fig. ซร. Anacárdium occidentale L., 2ffaju=

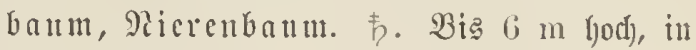

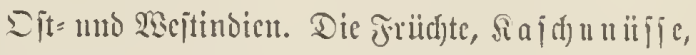

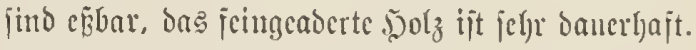

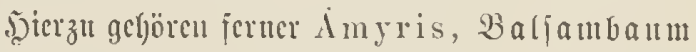

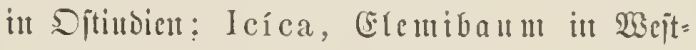
ittoien; Spóndias, Mioubiubaum in Güb= amerifa แ. a. m.

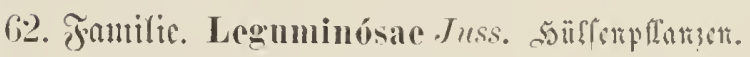

$8 \sharp$ bicjen reduet de Cantolle bic Jaunilicn: 1) Mimósea e R. Br., Siunpílauzcu, ïber 1700 2litett, of utlo 青, it ocu Tropert, feime it Emropa; 2) Caesalpinieae R. Br., Cäjalpi= uicu, an 900 2reten, 5 mo 8one; 3) Swartrieae de C., Swartzicu, cine fleite Familie von ctwa 20 tropiid)en Sammarten, mormuter Swartzia, Eijentyolz in (Gityana; 4) P'apilionáceae, Sclumettcrlingablüten.

3" ben Mimojen gebören folgende antändijd)e Pịlanzen: Mlimósa, Eimupílanzc; Párkia, Doura; Acácia, Edjotentorn; Adenanthéra, Siorallenbaum.

3u Den Cäjalpinien reduet man: Caesalpinia; Haematóxylon, Siampeidelfolzbaum ; Ceratónia, Johamnsbrotbanu; 'T a marindus in Jnoien; Cassia, Ecume rix, Tonfabolutenbaum und melrere andere ilt Den Iropelt.

Die Bapilionaced umfafien über 3000

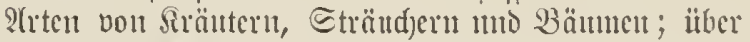
bic ganze Eroc verbreitet, ausgezeid)net Durdi bent

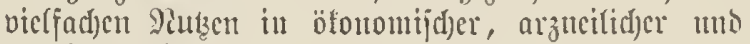

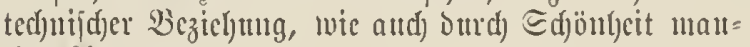
d)er 2(rten.

Die widjtigiten \$flanzen alts ber Familic ber Reguminofen finto folgenoe:

Einj. 78. Mimósa (Acacia) arabica Iam.,

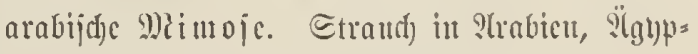
ten, Ditindicu; liçert arabijdjen (Smmmit. 


\section{Taf. 14.}

fiụ. 79). Acácia Cátechu W., Sated)u=?tfazic.

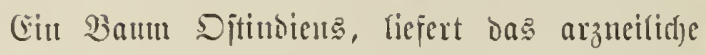
Siated)u, weld)es als zujauntuziefentes Mittel, Dod) andi) zutu Gierben und Färben verwestict miro.

fị. 80. Caesalpinia Lam. (Sroß̧e Bäune in

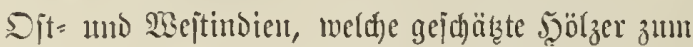
Färben unto (jerben liejern. Miefyere ?trten finto offizinefl. Die abgebiroete ?trt ijt Caes. pulcherrima atts Djtindient.

fiị. 81. Haematóxylon campechiánum L.,

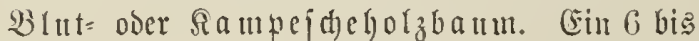
$15 \mathrm{~m}$ hoher Baum in 9iexifo und Zentralaute= rifa. 2sorziglidjes Stamm= uno namentlid jarblyolz (Błlaukotz).

fiug. 82. Ceratónia síliqua L., Johanuiz= brotbautu. Bämme in allen \&ändern nus Pit=

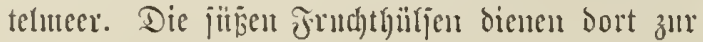

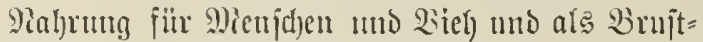
uittel geges Düteu umb Jjeijerfeit. fitig. 83. Cássia sennae, Eennefafite. Mef $=$ rere :(rten fleiner Eträutd)er im norböitlid)en uno mittleren $\mathfrak{A}$ frifa, beren Brätter, Eentesblätter, von benen mandjes gafye über 35,000 Sillo eitl=

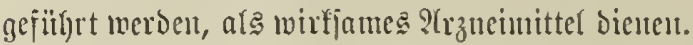

fiig. 81 a. b. Genista pilósa L., haariger (3i it= iter. 5. 2(n felfigen Drten mo anj secide= boden, meijt mieberliegetto. $30-45 \mathrm{~cm}$ jod), brïlyt boun 2(pril-Juli.

fiig. 85 a. b. Genista tínctoria L., Förrber= (s) initer. 5. $30-60 \mathrm{~cm}$ hod, in lidten $2 B a l=$ Dungen uno an Saldoündern in Deutjuland ge=

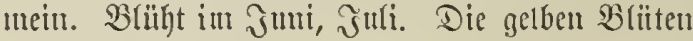
Dienen zuแ Färbest.

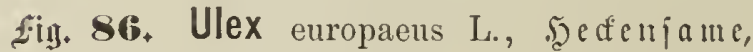
Sted = over 5eeideginiter. $5.60-150 \mathrm{~cm}$ hodh, auf jambigen Felsern Rorboentidulands,

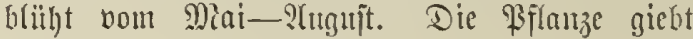
Bremunterial, die BBlitten licjern gefben Jarb=

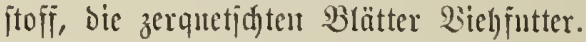



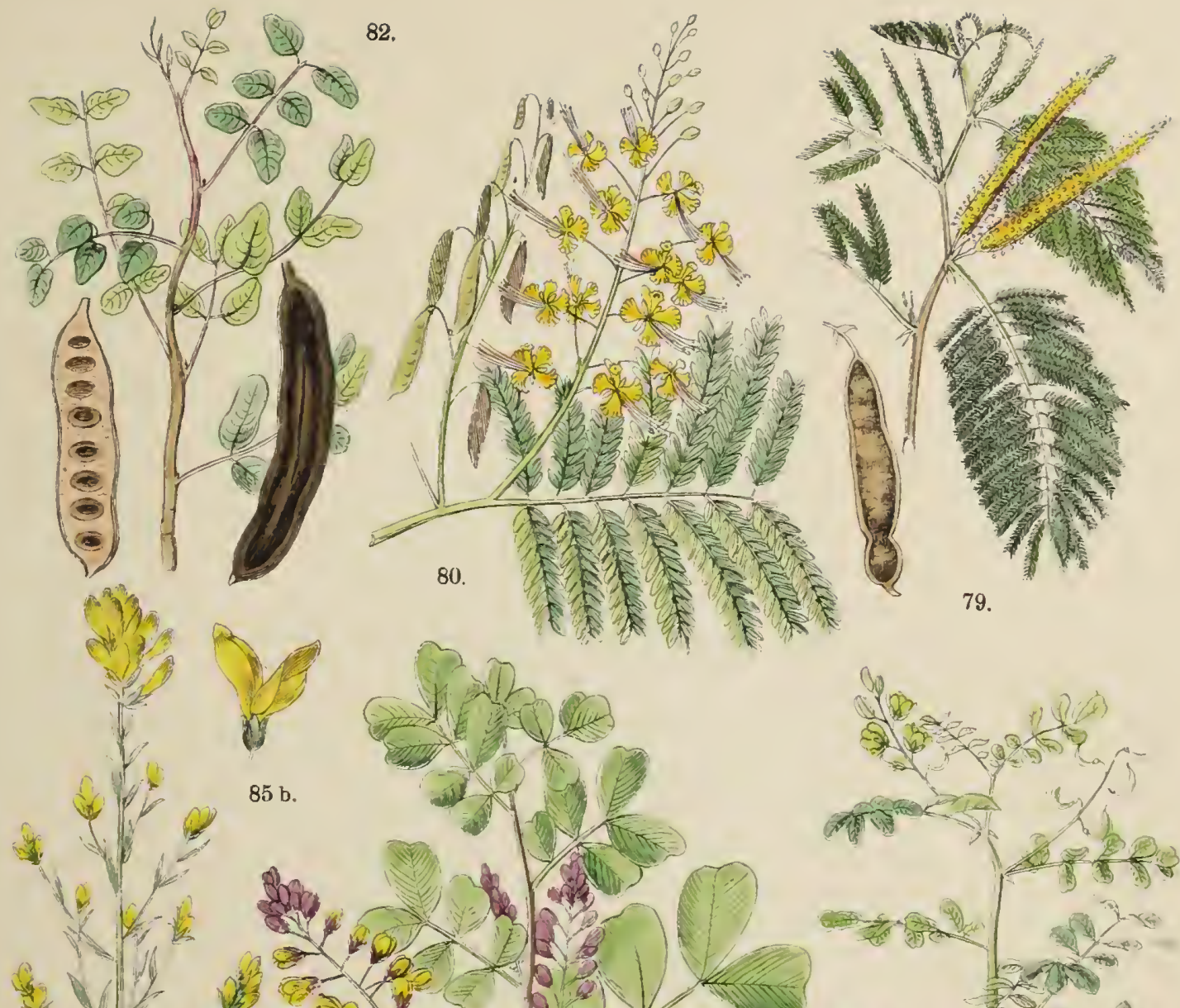

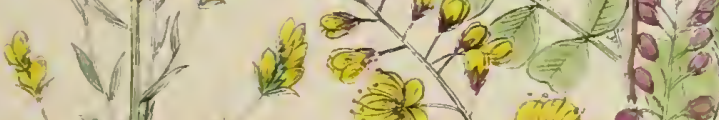
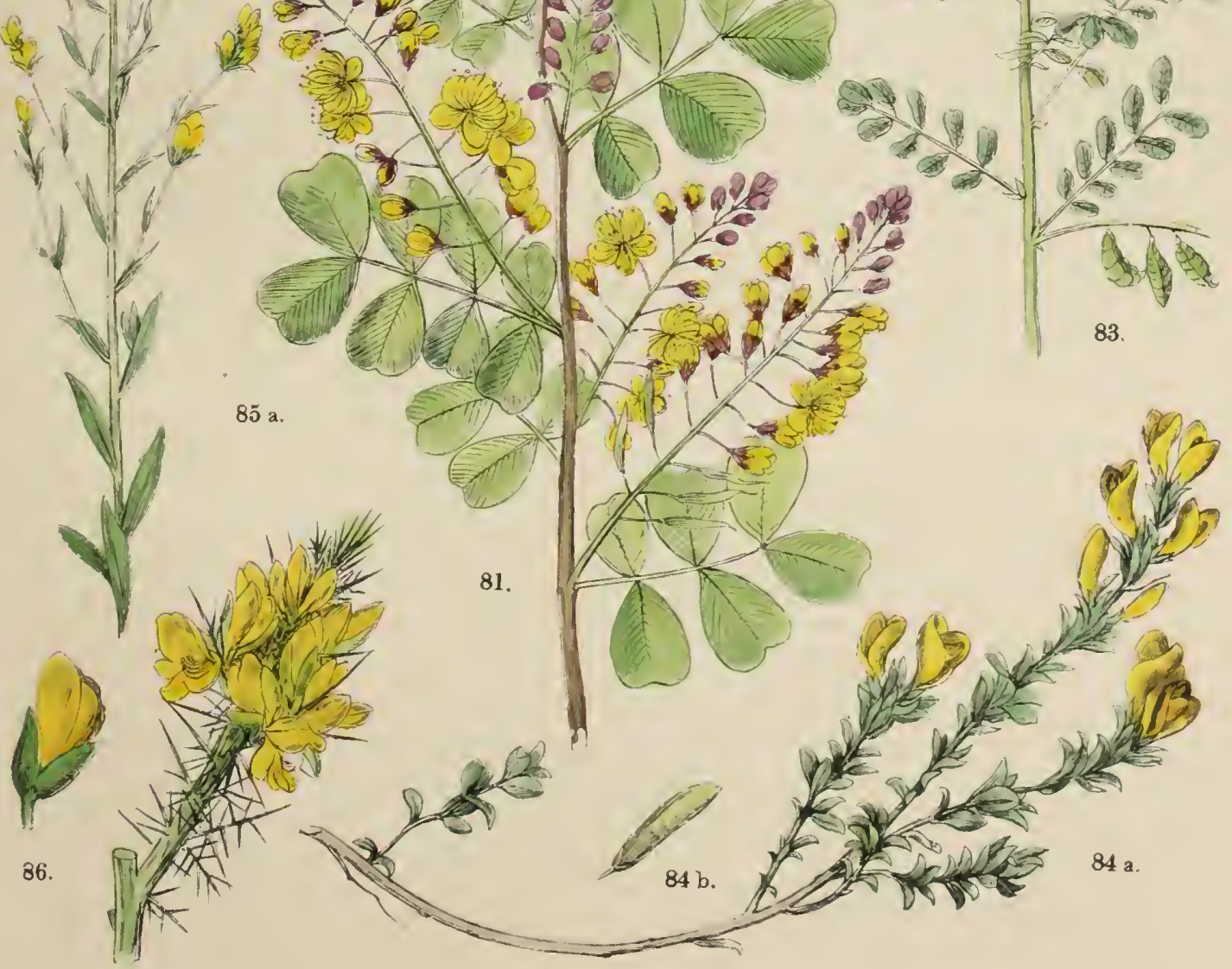



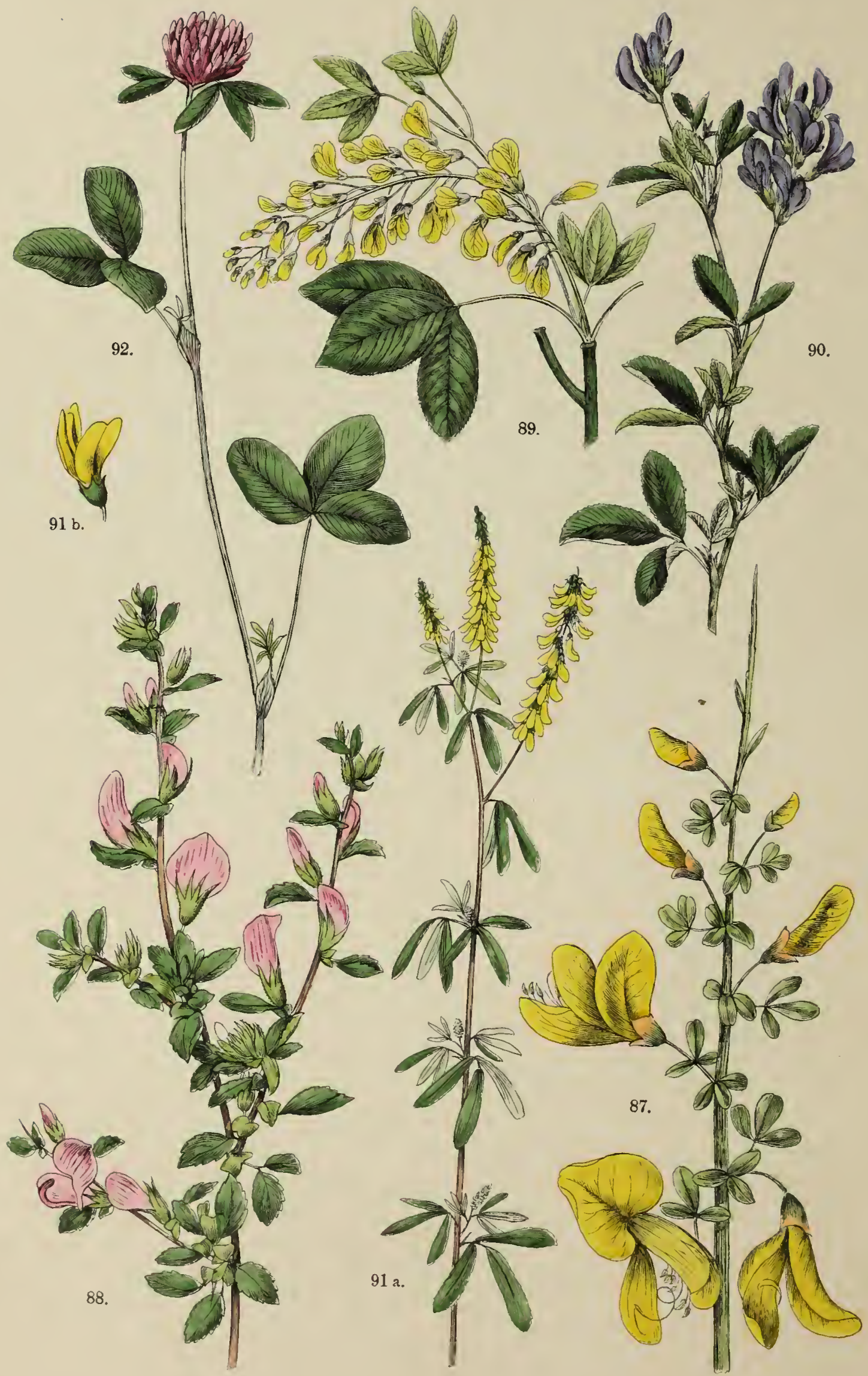




\section{Taf. 15.}

fịg. 8\%. Sarothámnus rulgáris Wim. (Spáltium scopárium L.), gemcincr Bejenftrand, Pifricuentraud. 24. $60 \mathrm{~cm}$ bis $11_{2} \mathrm{~m}$ lyoljer Etraud), dorulos, imumergritt, auf jambiget ?(ut)öl)en, briityt im Miai, Jutui. Dic langen

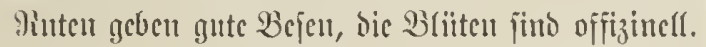

fing. 84. Onónis spinósa I., gemeine, sor=

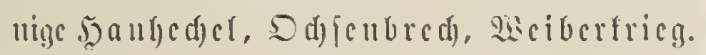
ந. ?. $30-60 \mathrm{~cm}$ (jod), dortig, aut Irifteu unto unfrud)tbaren Jeliern, B(iite roicurot,

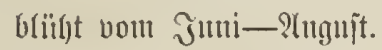

fing. 89. Cýtisus Laburnum I., Betiffere, Sirecbau, (soldregeu, Bobucubaum. D

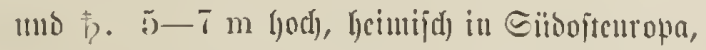
bei uns als Bicrîtrand) allgencin fultiviert, blitht im Miai, Juni. Dic Camen fino giftig. fịn. 90. Medicágo satira L., Uraucr $\Xi$ d) neffen= flec, Euzeruc, Daucfflec, Miouatzflec, Ewigner silec. 21. $30-1 ; 0 \mathrm{~cm}$ hod), itberall

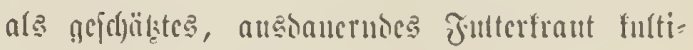
vicrt, blitflt vom Эmil-Ecp'culber.

2.ị. 91 a. b. Melilótus officinalis I)esr., get ber Etcinflec, Jouigflec. $\odot, . .80 \mathrm{~cm}$

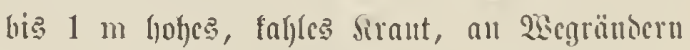

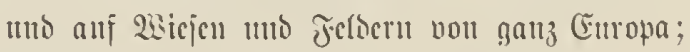
brïlyt vom Juli-Ecptember. Sifiizinell.

fịn. 92. Trifólium praténse L., Jutter- oicer 23icicjeffec. (.). $30-45 \mathrm{~cm}$ hod), fajt in gantz Cruropa wils, bahci oser aul bäufigiten augchante silec. Surs norzüngliche Jutterpflanze ïberall hefantut. 


\section{Taf. 16.}

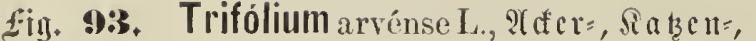

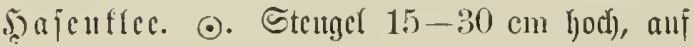

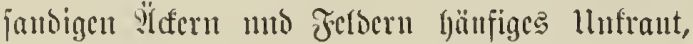
bliif)t vom Эulf-Eept.

fiig. 94. Trigonélla focnum grnecum $\mathrm{L}$., gc=

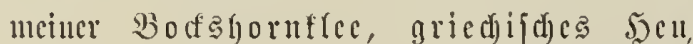

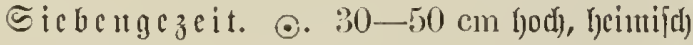

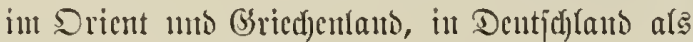

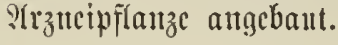

fiig. 95 a. b. Anthyllis vulnerária $I_{\text {.., genteituer }}$

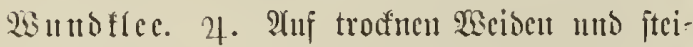

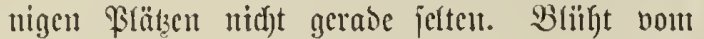

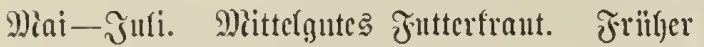

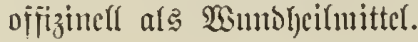

Æing. 96, Glycyrrhiza glabra L., gem. $\subseteq$ ii $\beta=$ hol z. 4. 1-2 m lyod), heimijal in Sï̀curopa, in Mittecenropa, nantentlids bei Bamberg, im
Groß̧en fultiviert. Die 2 Burzel bient z̆l Deu jo,

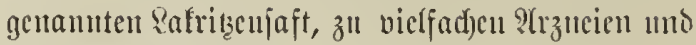

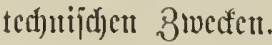

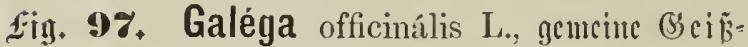
raute, Gétíftec, Ziegcutrate. \%. Etcu=

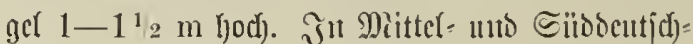

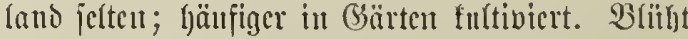

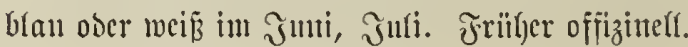

fig. 98 a. b. Colútea arboréscens L., gemeiner Blajenjtraud, sinjenbaun. Ein $1^{1 / 2}$ bis $4 \mathrm{~m}$ fjofjer Straudf, in Eibibentfdjant, nament=

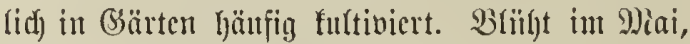
Jmi. Giftucröadytig.

fug. 99 a. b. c. Oxýtropis astrágalus L. (Ox.

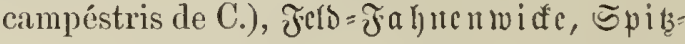

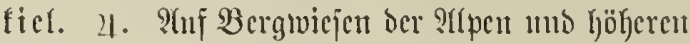
(3ebirge, 10-20 cm hod), bliifyt int כulli, श'unuit. 
16.

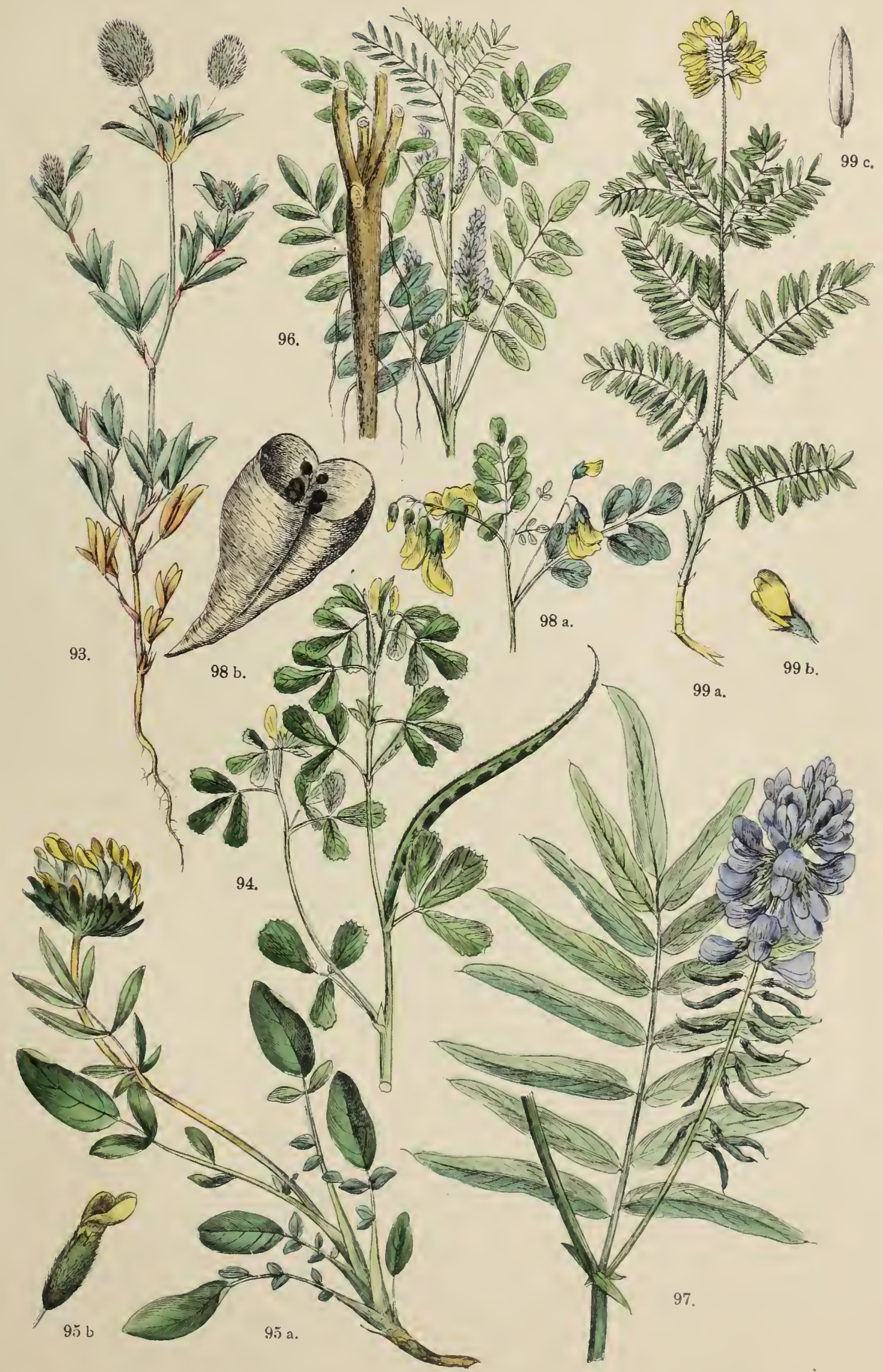






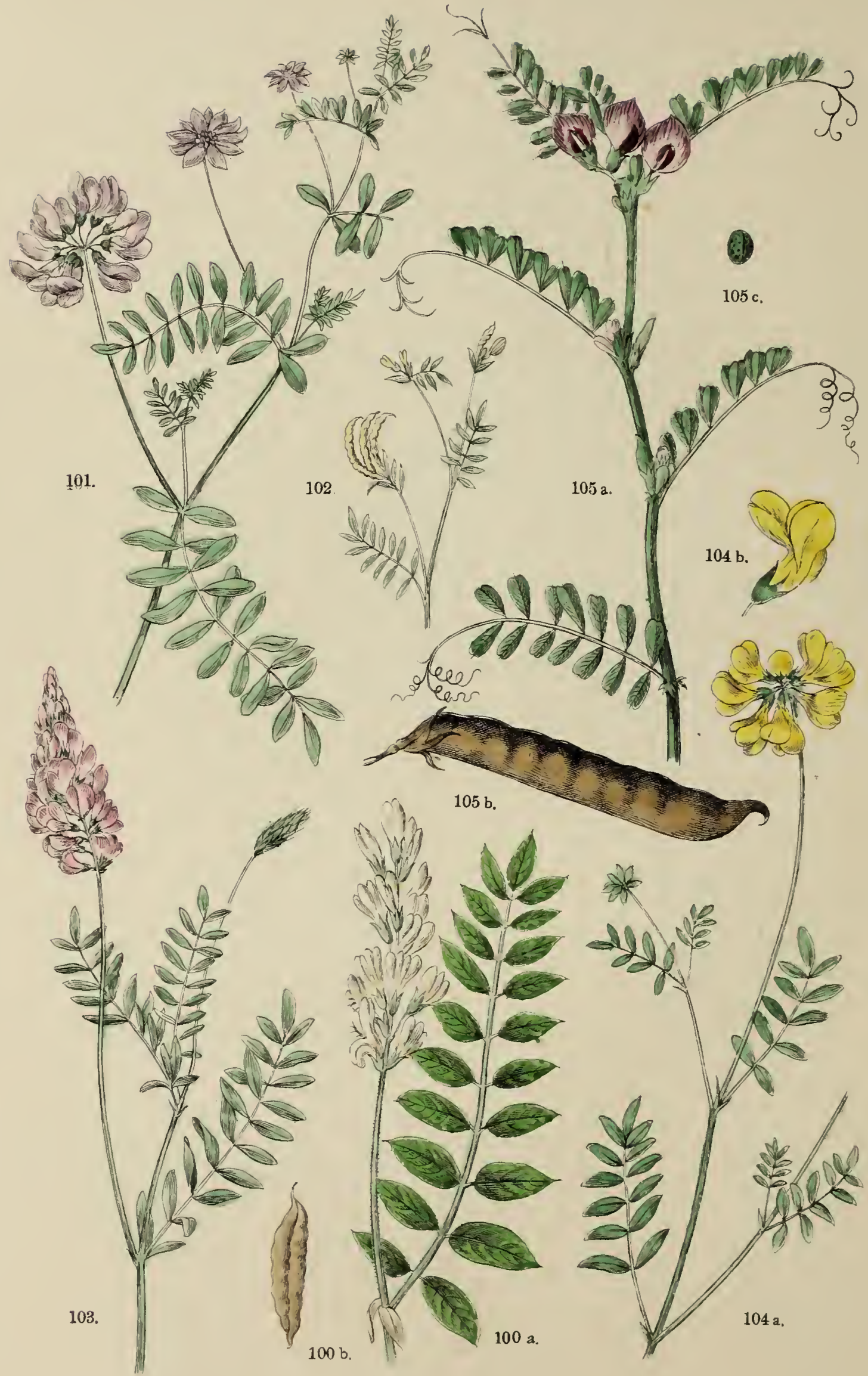


Taf. 17.

fig. 100 it. b. Astrágalus cicer L., sid $\mathrm{c}_{\mathrm{c}}$. tragauth, Sidferflec. ?. Stculycl $30-60 \mathrm{~cm}$

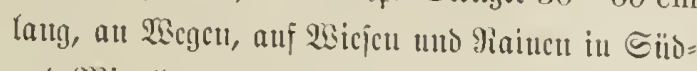

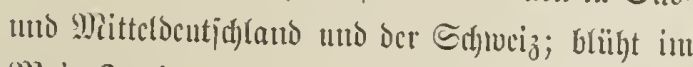
Mai, Jงuni.

fịı. 101. Coronilla vária L., Guntblunige siouldide, Griftiotic. \%. Etcugel 30 bis $90 \mathrm{~cm}$ Iang, bci uns in Gícbiilddul, aıt 3 änuch bcfoltocrs auf Sallo= uno sialtboocu, bliift

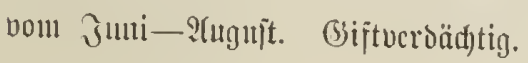

fig. 10\%. Ornithópus sativus Brot., 2:ogel fus, Silaucufdotc, Scrradelfa. 30—60 cm lood), aus Epanticu cingcfiiffrt uno auf Sallo=

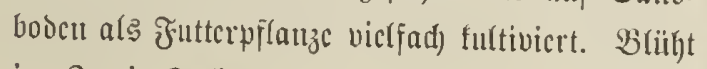
iuı Э̧uนi, Эิuli. fig. 10:3. Hedlysarum Onobrychis L. (O) brychis sativa Lamk.), Ejparjette, crui= ger silce. \%. $30-60$ cul hod), aui Sialthüggcu Witteccuropas, bliilft vom \$iai-Jull. Eit vor= zitiglidjes Jutterfraut.

fiig. 1OLt al. b. Hippocrépis comosa L., Sdjopi=

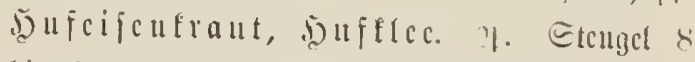

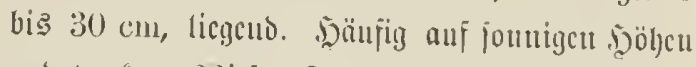

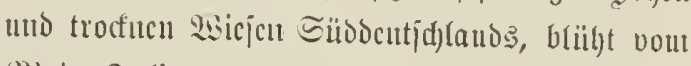
Miai-Julli.

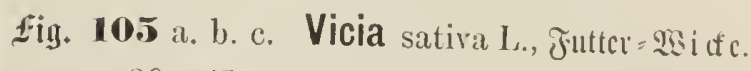

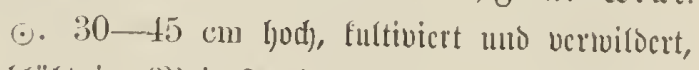

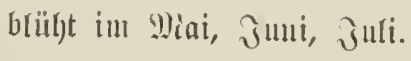


๔ạ. 18.

fig. 106 a. b. Vicia cracca L., ogd $=\mathfrak{B}$ i ffe. 1. Etcuget $30-90 \mathrm{~cm}$ hod), t(iumucub, in (5ic= biijd)en ıtıd auf $23 i c j c u$ genciu, brïl)t boum

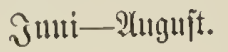

fig. 10\%. Ervum lens L. (Lens esculénta Mnch.), Ziuje, Ifdcrliujc, Eaatriujc. $\odot$. Etcugct $15-30 \mathrm{~cm}$ lang. Bci uns bclicbte

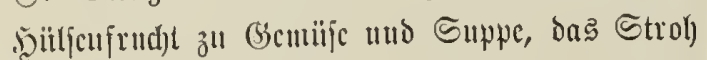

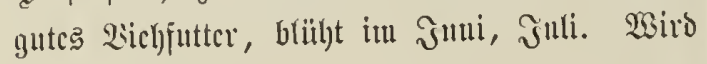
räufig angebant.

+ig. 108 a. b. c. Pisum satívum L., Caat=, Garten $=$ oder Feld $=$ Erbje. $\odot .30 \mathrm{~cm}$ bia $4^{1 / 2}$ In lod), ïberall angc(bant. Dic Früd)te geven betauntlid) cin berjajicocu zubcreitetes vor=

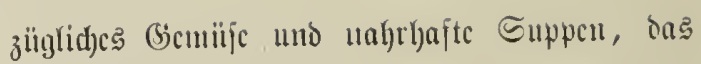
Stroh iit gutcs 2 sichfutter. B(ül)t vom MiaiAแguโt.

fig. 109 a. b. Phasèolus coccíneus L. (Ph. multiflorus W.), tiirfijd) Fencr $=\mathfrak{B}$ oh $\mathfrak{e}$. $\odot$. Eiue vou ocu zalflreiden, bci แแs furtivierteu Spiclarten ber Boblucn; fie blïlyt sweís oder hod)= rot iu Juli, 2tugujt und wird utcijt uแ! als 3icrpflanze gezogcu. Dic Bohucu ftammcu aus

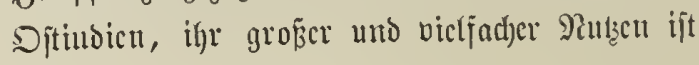
bctaunt.

fị. 110 a. b. Làthyrus silvéstris L., $\mathfrak{B a l d}=$ \$ilatterbje. 21. Der tletternio Etcugel wiro $1-2 \mathrm{~m}$ hod); häıfig in .jecteu, an sisalbräubcru

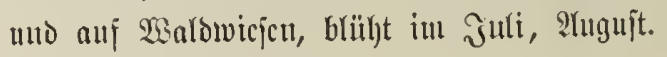


18.

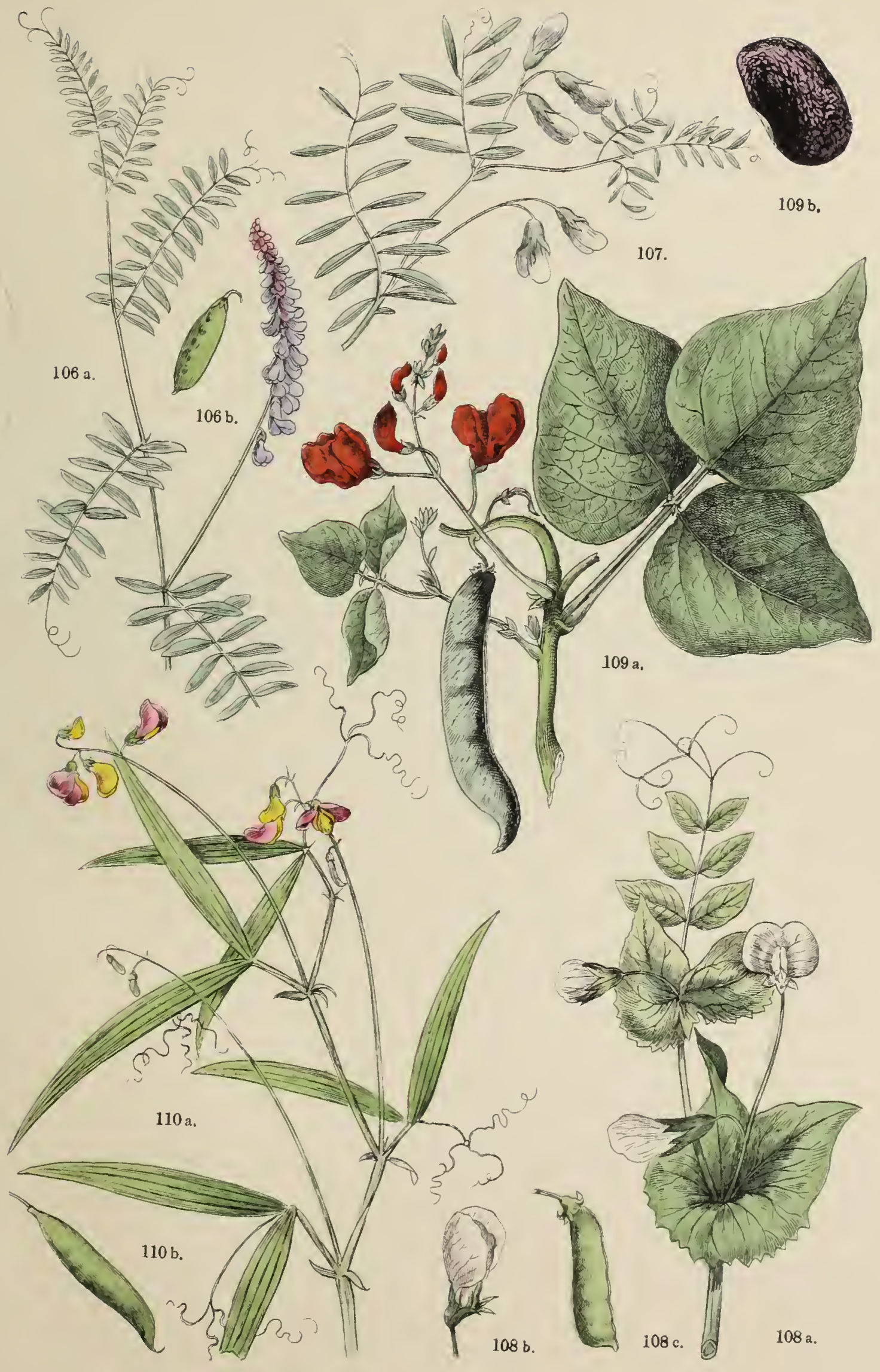




19.

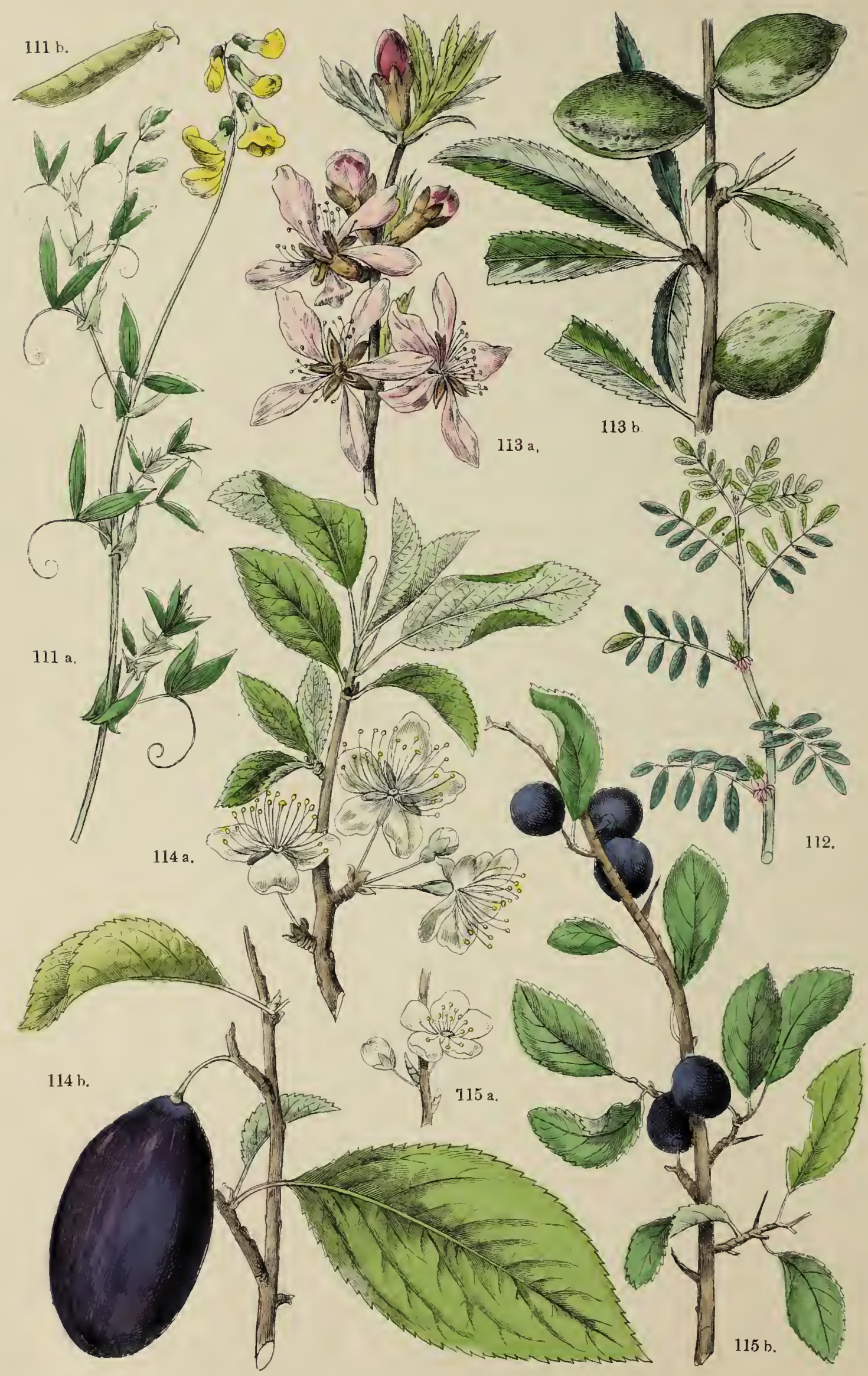




\section{đạ. 19.}

Eij. 111 a. b. Lảthyrus pratéusis $L_{\text {.., }}$ gisiçen-

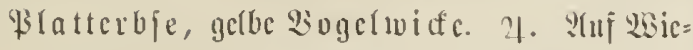

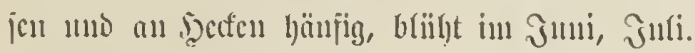

fiig. 11:. Indigófera tinctoria L., gencinte $\Im^{n}=$

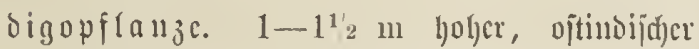

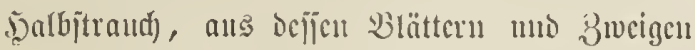

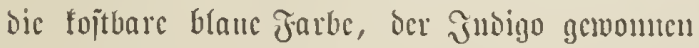

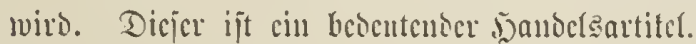

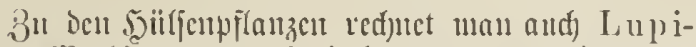
nus, 2⿺辶一 Gartenzicepflanze Erythrina, Der storallenbaum

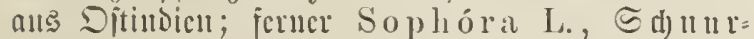
it rand) int Japan; Anagyris, Stintittand) in (Gricd)entanto; Myróxylon, $\mathcal{B} a$ lfambanu in

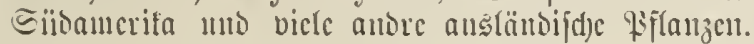

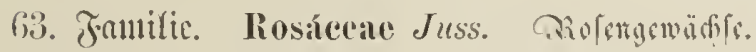

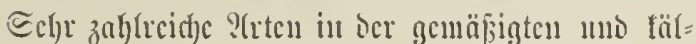
teren 3onte.

fitg. 113 a. b. Amýgdalus nana L., zuverg= Wiandofbanll. Straud) bon $60-120 \mathrm{~cm}$

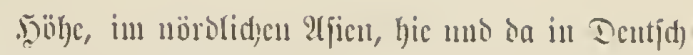

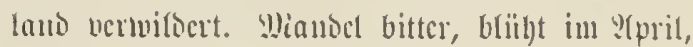
Wiai.

fiig. 11 a. b. Prunus I., Fif faumc uluaum, Sirjabanu, vyritojenbanu. Einc au=

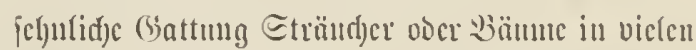
:leten, jerjtecut aü jajt alle ?ü̈noer ber Erroc. Dic abyebiltete ?trt iit P'runus domestical 1.

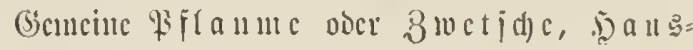

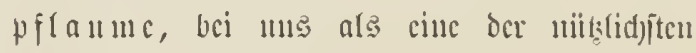
Sbifjorten ïbcrall fultiviert. Wiitict in if)ren

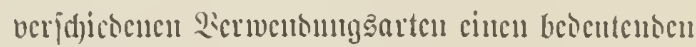
j)antocเsartifect.

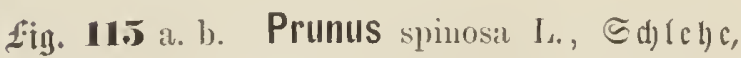

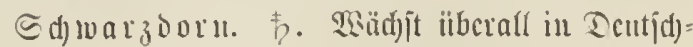
(ant, 1-2 m bod). Dic B্ট(iitch crif)cincu vor Den Jrvïd)ten, lveld)e jel)r herb jdulueffen. B̉(üht

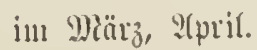


Taf. 20.

fig. 116 a. b. Prunus I'ádus L., geut. Tran=

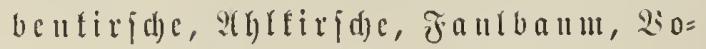
gelfirid)e. CEit 3-10 in lober Strattd) oder

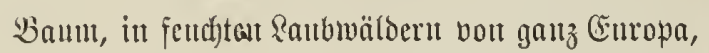

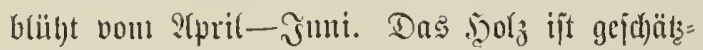

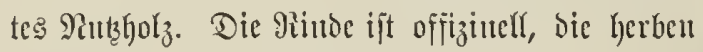
Jriid)te frefīen Dic Bögel gern.

fin. 117 a. b. Pirus communis L., gemeiner

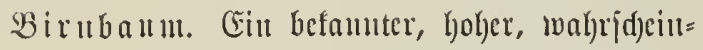

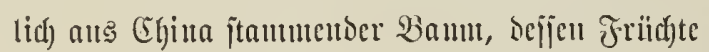
it zahlreidjen Epielarten jegr verjdjicocu uad) Form, Farbe unt (scidjuact jimb. Durd) Dfu= lieren mto Propien werben nautentlid) it Fraut= reid) utto nencrbiugs aud) in Deutichlanto vor= treffliche Früd)te erzielt. SBlüht anfaugs Mai. fig. I18 a. b. Pirus malus L., Itpferbaum. 6-9 m lod), in (Siebirgsıuälocru fajt ourd) gaız Europa wils, in mehr als 600 Spielarten in allen Ðbittgärten fultiviert, in viclen Gorten bic befaumten, gejunben und wohlifd)uedentoen Frïd)te tragento, weldje zu utaudjerlei Speijen verwentoet werben. SBlïht itu Miai.

fij]. 119 a. b. Pirus Aria Elurh. (Cratategus Aria I..), Michlbecte, Miefrbirte. Strand, hic utto ba aber cin Baanu vou $10-12 \mathrm{~m}$

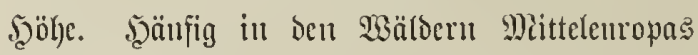
zeritrent, blitgt im Mai. Die roten ober gelb= lidben Fridute finto eşbar, aber trodfen. 


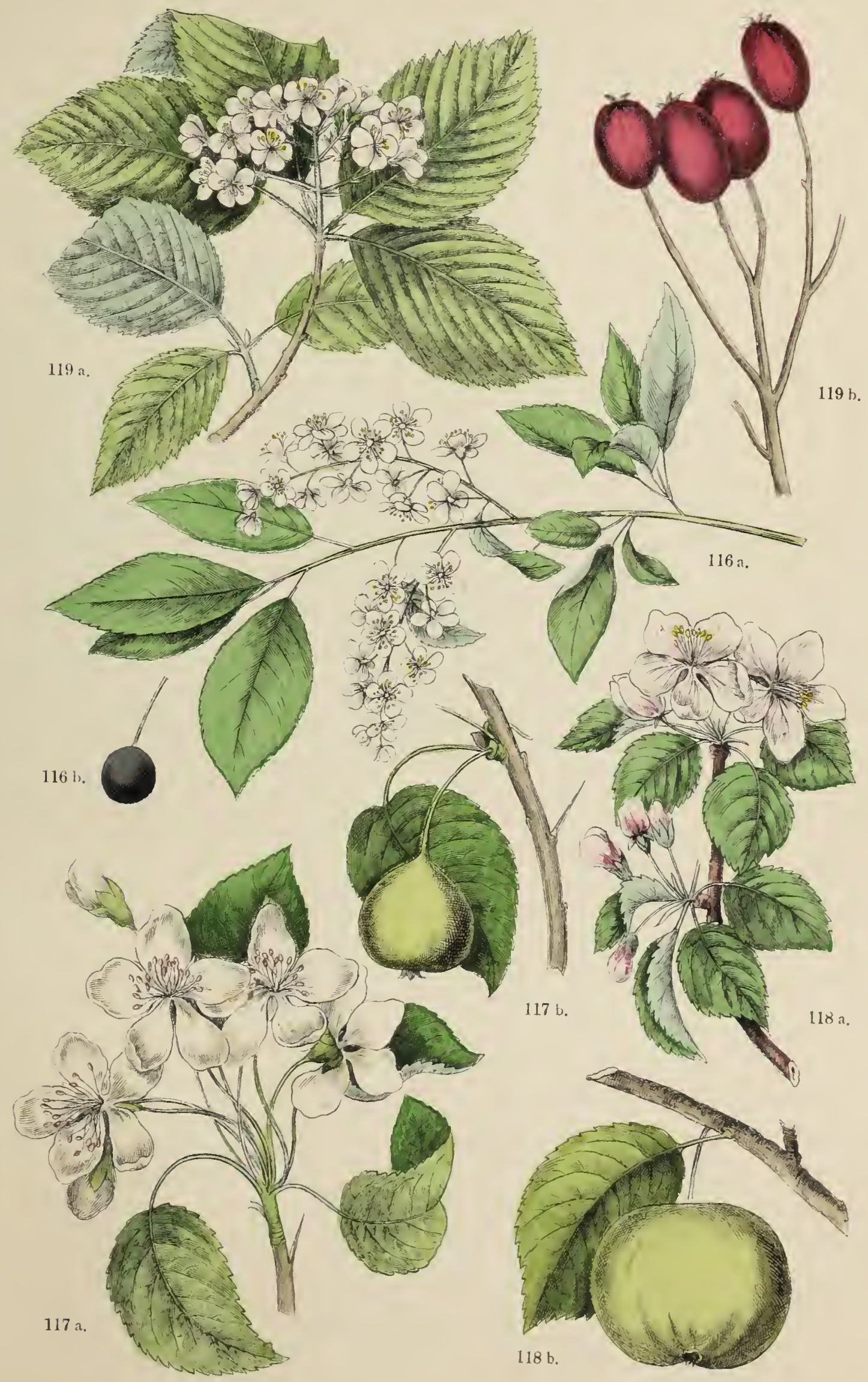





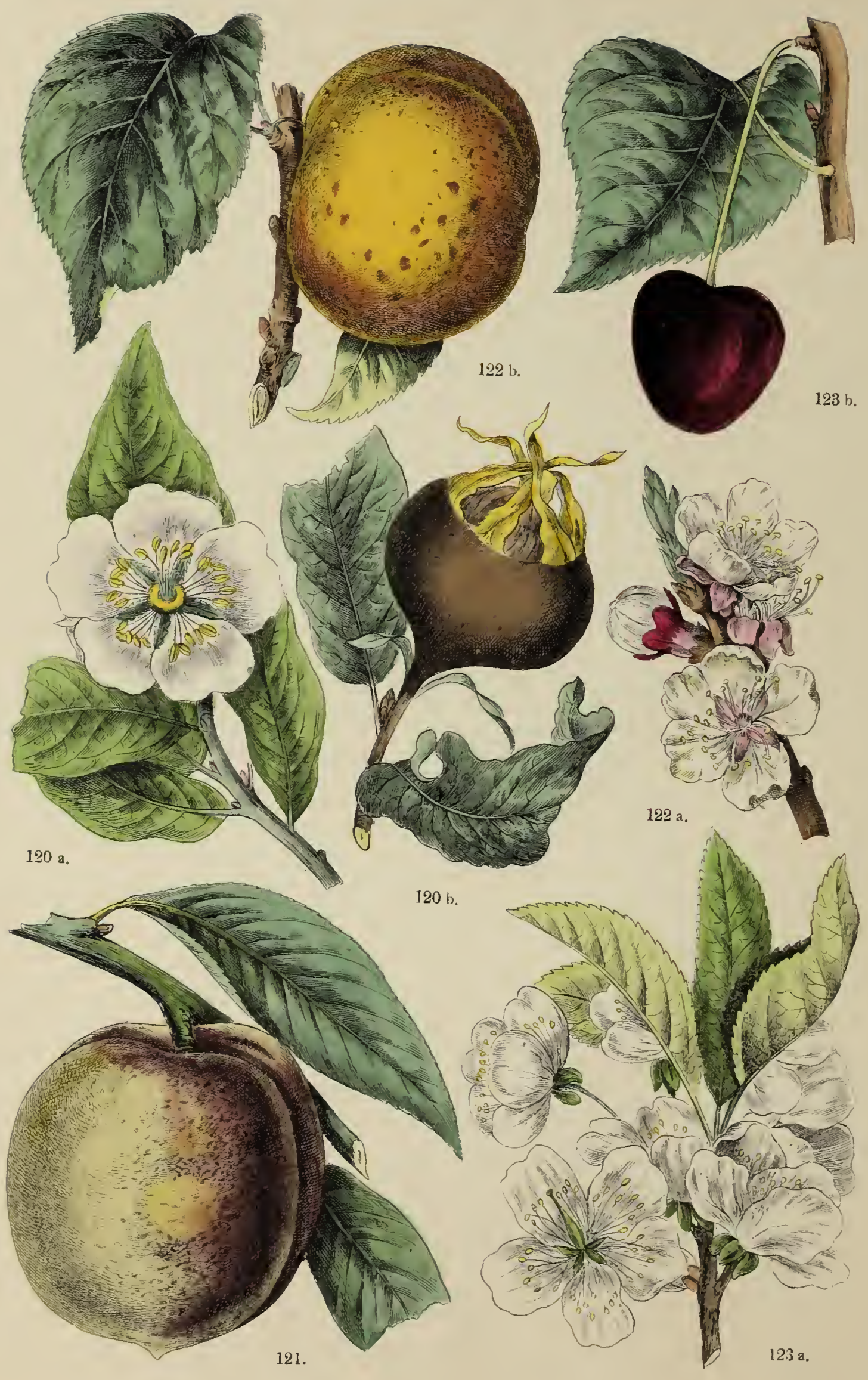


Taf. 21.

fin. 120 a. b. Méspilus germánica L., gemci=

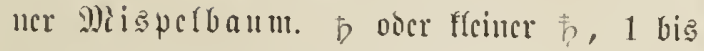

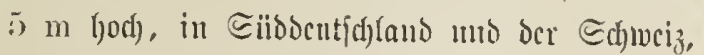
bci uns ale Zicr= แmo Sbjtbaum ric unb da

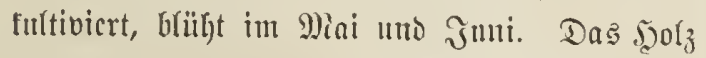

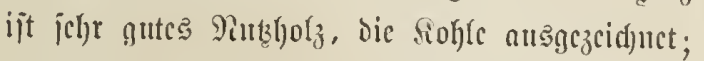

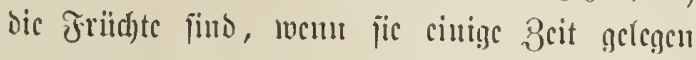

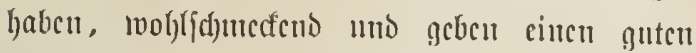
Sbjtucin.

fiì. 121. Amýgdalus pérsica L. (Pérsica vulgíris Mill.), gemeincr Bfirfid). (Ein 4 bis $6 \mathrm{~m}$ holjer Baum, ans bem Sricut jtaul= mento, bei mte in viclen Epiclarten ïberall an=

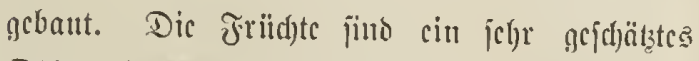
Sbjt, dic Sicruc geben oen jeinen Ferfilo=:iför. Bliigt int 21prit, Miai. fịn. 122 a. b. Armeniaca vulgaris Lam. (P'n-

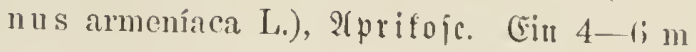
holycr Baum, aus bent Drient itnuntent, in melyr alş 20 Spicfarten bci แกร angebaut. Dic Friid)te gchören mutcr bas gcidan̈zztefte Etciunobit, jic ucrocu roh ober verjejicien zubercitet gegefjecu,

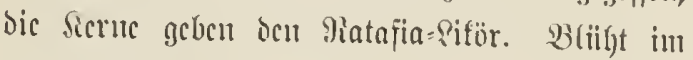
Miörz, âpril.

fig. 12:3 a. b. Cérasus vulgaris Mill. (Prunus

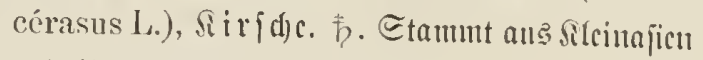
uno ijt befamntlid) in viclen Epiclarten bci unz angebaut, cin vortreffilid)es Etcinobjt. Das .jolz

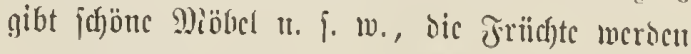
in Der Sï̈d)e nul won ocn Sionditoren vielfad) verwonoct, licjern aud) ocn alfbelannten firirjecn= gcipt. Sbliil)t iı S(pril, Miai. 
Tâf. 22.

fing. 121 a. b. Cydónia rulgaris Pers. (Pyrus cydomia I.), Sutteubaum. ந. ђ. Ju Drient

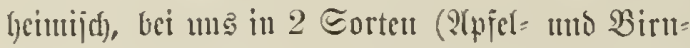
quitte) fultiviert. 3-5 m lyod), bfïlyt ium Miai.

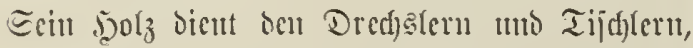
bie Frïdte jumb jaucr, merben aber vielfad) z"t Sompott, Eirup uno bem ofijizinellen Suitten= id)leim verwendet.

fịg. 125 a. b. Sorbus aucupária L., gemeine (Ëberejac, Sogerberbant, Miaazber= ba

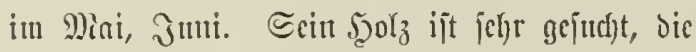

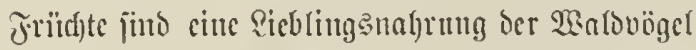

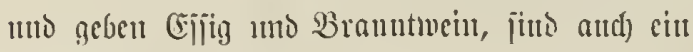
gejdjütztes 2iolfąycilmittel. fig. 126 a. b. Crataegus oxycíntha Gïitn.

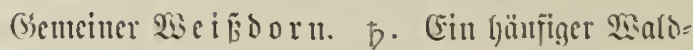
[traud), oer wegen jeines didjten Rindj]es mo jeiner Dornen öfter's zu Secefen bentlat wiro. Dic melyligen $\mathfrak{B}$ eeren fint ểbar, aber nidjt (d) una ffyajt.

fig. 127 a. b. Rosa L., Roje. Micijt jtadylidyte, im Эuni uno Juli blïhenoe Etränd)er, 10 beut= jd) âten mit vielen Epiclarten. Dis abgebil=

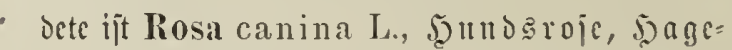
butte, Defentoje. ந. 1-2 m hodj, bei แแฺ ïberall in Jeeffen uno (bebüjchen, dic ges uteinjte wilbe 9ioje. Blïten rojenrot bis meiß, molylried)ens. B̉(ïlyt im Jumi. 
22.

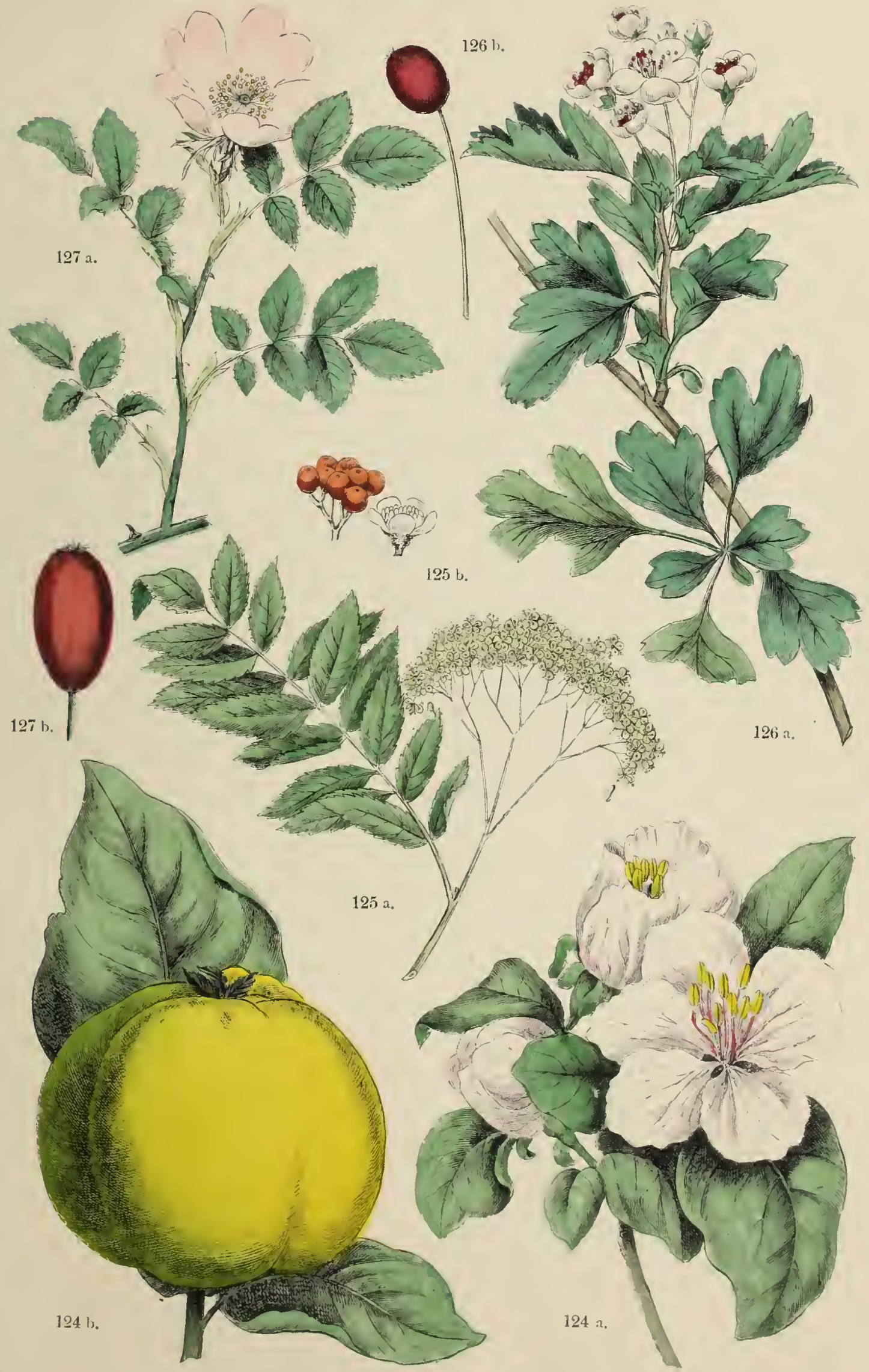





\section{.}




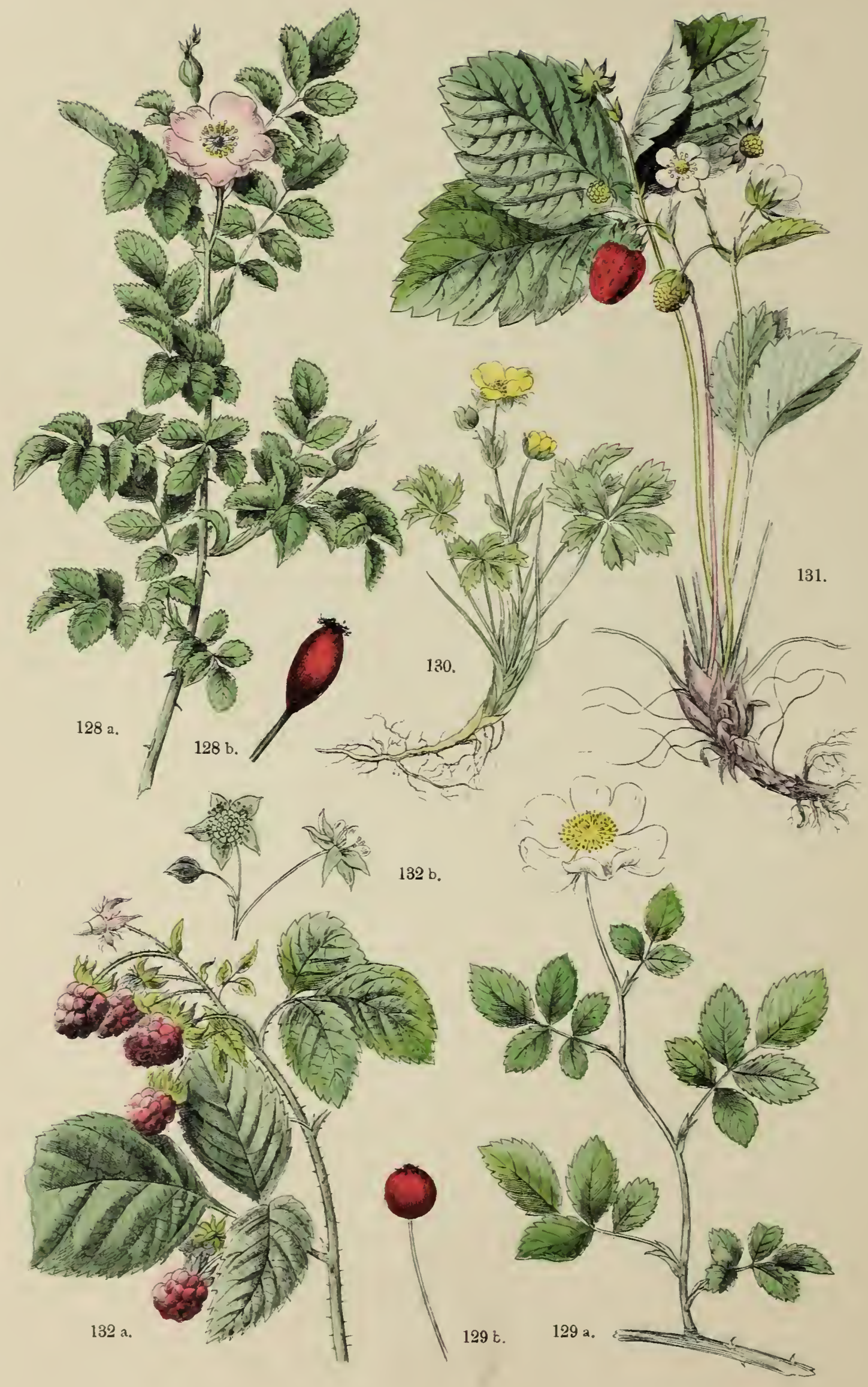




\section{Taf. 25.}

fị. 128 a b. Rosa rubiginósa I., 23eiuroje, fing. 131. Fragária vesca L., gemeine oder

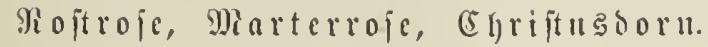
23ald = érobecre. ?. Lleberall in 2sï̈ldern mo

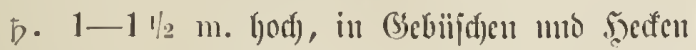

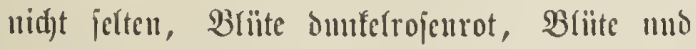

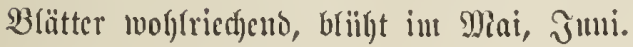

fing. 129 a. b. Rosa arvénsis L. (R. repens Ehrh.), Fetoroje, Iferroje, গiantentoje, 23alo roje. ち. Jn Jecten mo Gebiijden von gauz Dentidffano mit nieberfiegenden, fortran= fenden, 1-2 m langen ?íften. 23(ïten weí̄

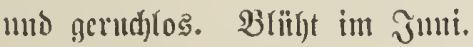

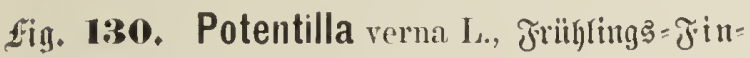
gerfraut. ?. 2Tuf troctuen, jomnigen sjiigefn unto Jjeiscu. Briigt im Sprir, Miai. (Sebriijd)en, 6-15 cm lood), bliilyt voul srprilЭuui. Die erfrijd)enten, gejunden Frït)te fint

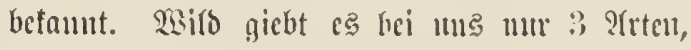
Sagegen viele furtivierte Spielarten mit iehr ģroß̄en รrïidten.

fig. 132 a. b. Rubus Idaeus L., gemeine 5im-

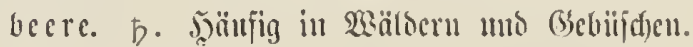
Die befaunten woblid)uecfenten Jrïd)te geben Syimbecrajt, Jjimberefïig, Jimbecrgeift mo cin fehr gutes (Belee. Sh(iilyt boun Maai bis 2utguit. Frïd)te rot; bei fuftivierten Eorten mitunter gelb oier weik. 


\section{Taj. 24.}

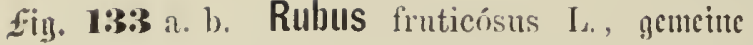
Broutbere. 5. Jn SJecfen, Didfidten uto

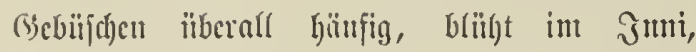

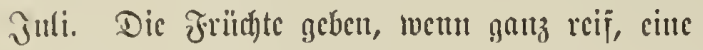
gute Epcije utto Frudtijurup.

filị. 13. Geum urbámum L., gemeinc $\Re \mathrm{cl}=$

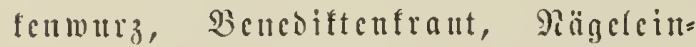

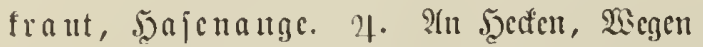
unto llfern gemein, boiight im Juli, গtuguft. Dic mohlried)ente $\mathfrak{B}$ mzel galt früher als heilfräftig.

fiig. 13.5 a. b. Geum rivale L., $B$ a d $=\Re \mathfrak{c}\lfloor\mathfrak{f e n}=$ wurz. 4. 2গ fendten uno fumpfigen Diten in

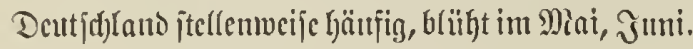

fiig. 136 a. b. Spiraea ulmária L., Sumpf=

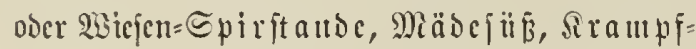

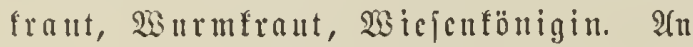
llfern, auf feudten Sicien iu ganz Emopa. 24.

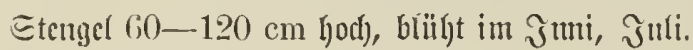
Bartenzicrpīlanze.

fing. 13\%. Alchemilla vulgáris L., gemciner Fraucnmantel, Sinan. 2. $15-30 \mathrm{~cm}$ ljod). 2(ui feudjten 2isicjen uno in juattigen Wälocrn. SBüht vom Miai-2ugıít.

fig. 138. Sanguisórba officinális L., gemciner Z3icjenfnopf. 2. $\quad 60-90 \mathrm{~cm}$ hod, hänfig auf fentiften 2isiejen. Futterfraut zweiter Bsitte,

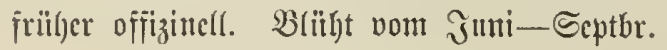

(j4. Fomilic. Calycíntheae Lindl.

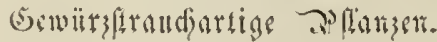

4-6 9rten Etrü̈ud)er mit woh)(riç)enden Şlïten, ill ?tmerifo utto Japan. Son biejen ijt zu nentuen: Cat ycánthus floritus, farolinifde sield)blume ober (a) clointritraud), b, aud bei uts als cin in alleu Ieilen anyenel)u buftenoer Bsartenzieriftrand mit purpurbrannen $\mathfrak{B}$ lüten cingejülyrt.
65). Tomilic. Granáteae Don. Sranubäแmc.

Rur 1 Art: Púnica granatum L., gemeiner

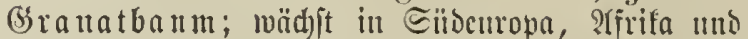

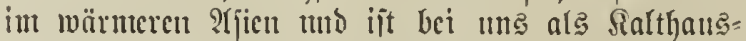
zierpflanze felye beliebt. (Es ift cin bauntartiger, :)

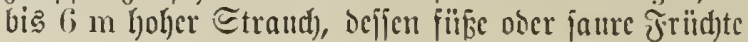
ein ja)madflaftes $\bigcirc$ bjt birben.

\section{Fontlic. Maemecýleac de $C$.}

Cinige 20 9Trten 5 mil midytigite Yrt ift Mamócylon capitellátum L.,

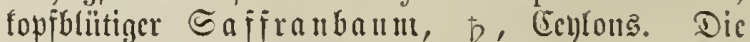
Brätter dienct als Biscuitrz wic Eaffran mo gehen cinen gelben Jarbjtoff.

67. Familic. Combretáceae R. Br.

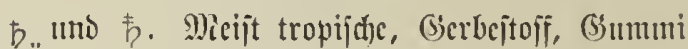

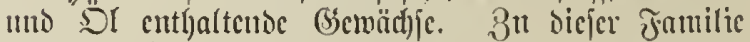
gchören: Combrétum, Zangfadeu in Diaba= gaşcar; Te rminália umo Myrobálanus, Satap=

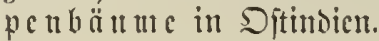

68. Framilic. Vochysiáceae St, MiT.

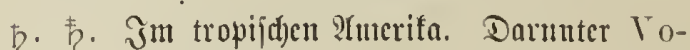
chysia, 青, Guianas.

69. Fomilic. Rhizophoreae $R . B$ r.

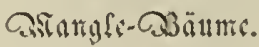

25 9rten. D umb 韦 in ocn Iropentänoctn. Jicrunter Rhizóphora Mangle L., gentener

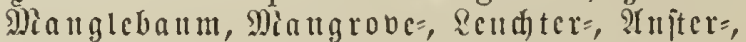
23 urzelbaum im Dicerjulaum Sitindicns unb

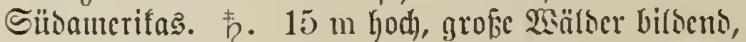
mit ungeheuern in ben Sbosen herabitcigenden Suft= murzeln, weld)e, wieber aub bem $\mathfrak{B}$ oben hervorrangent, Den Etamm itüben. Das 5 Jolz bilbet cinen Jjan=

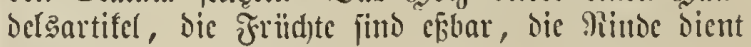
zแแ⿰ Gerben und Färbert. 


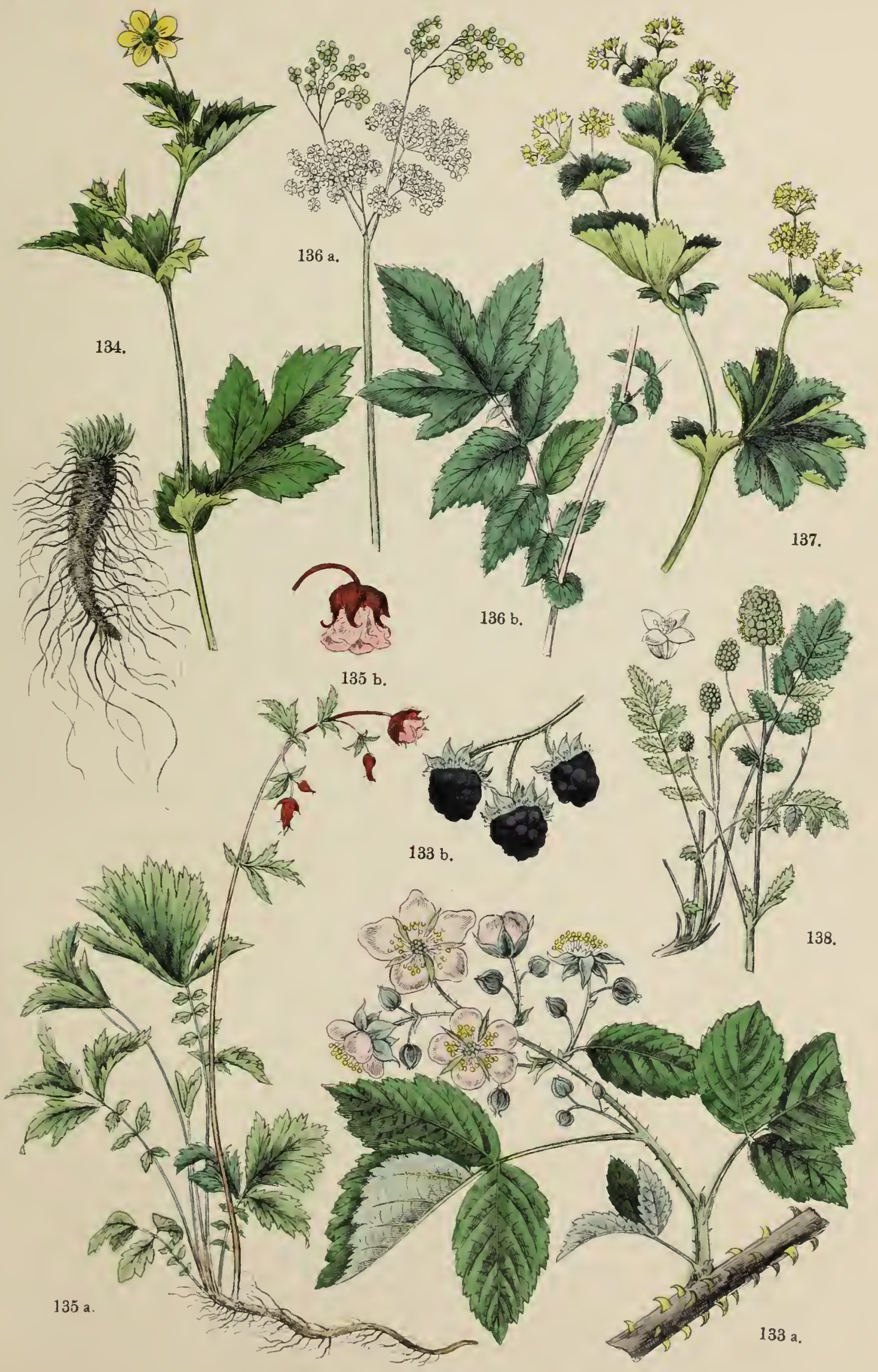



25.

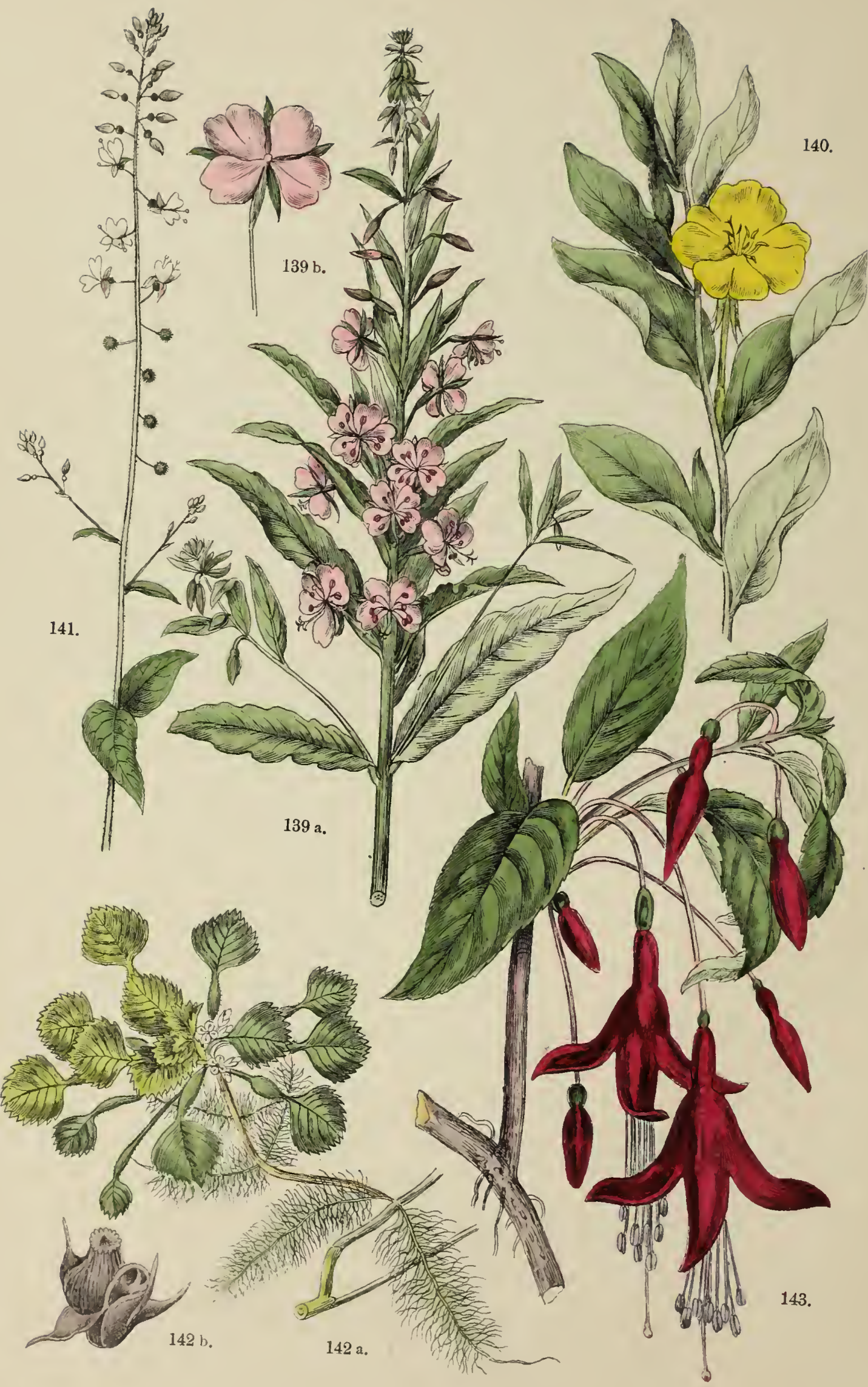




\section{Taj. 25.}

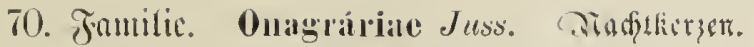

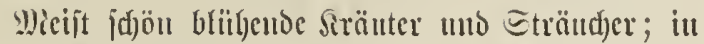

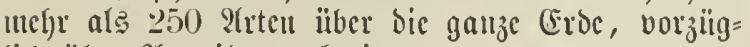
(iid) ïber 2lucrifa, verbreitet.

(4iy. 13!) a. b. Epilobium L., 2aciocurös= d)eu, Sd)oteuweiberid), 9 bentid)e ?(rten; dic abgebilbete ift Epil. angustifolium L., id)แalblättriges $\mathfrak{B e i b e n t u ̈ s d ) e n . ~ 2 f . ~} 60-120 \mathrm{~cm}$

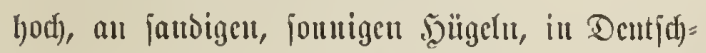

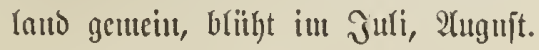

fiin. 140. Oenothéra biénnis L., gcu. Radyt= terze, Gsarteurapunzel, Sicbeujdräfer. ๑. Stenget $60-120 \mathrm{~cm}$ hod), nidjt jelten alt Fluijujern uto auj jeudyteut Sanbboden, brïht

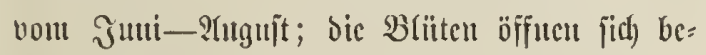

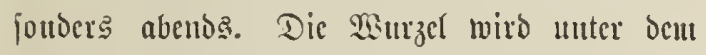
Diameu Rapóntica zu Salat berivendet.

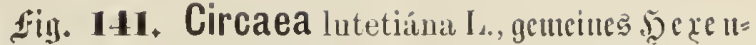
txant. 2. Stenget $30-45$ cm hod), in

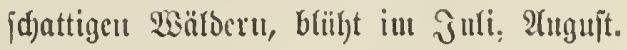

fig. 142 a. b. Trapa nátans L., geuteite

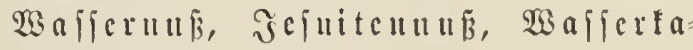

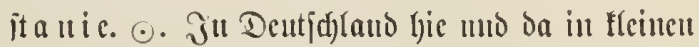

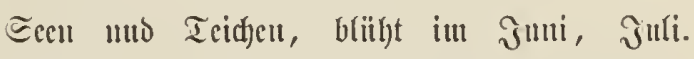
Dic Riuifie jüto roh oder gefod)t wohlid)uedeno. werocu zu sirot verivendet uto biench zแt Sd)weiucutajt.

fiij. 11:3. Fuchsia coccínea I., idjarlad)rote Fudjic. 5. Ein prädtiger, iu Siibautcrifa heintijder Etrautd), bei แแıs iut zahltreidjen Spicl= arten als fajönc Garten = utto Topipflauze fultibiert. 
Taf. 26.

fin. 14. Clàrkia pulchella, đđjöne çartic. (.). Eine von Plordamerifa bei mus cingejiilyrte (Bartenzierpflanje.

fiin. 145 a. b. Hippuris vulgáris L., gemeiner Taunenuedel, sisajermeder. \%. Süntig in itelyenden Géväjicrn und Eimupien. Étengel 15 - 30 cm hod), bistucilen flutlyend und Daum 1 -2 m lang.

\section{Fonilic. Halorágeac $R$. Br.}

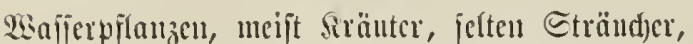
it etwa 50 Y1rten faft iiber bie gauze Eroe berbreitet. Whand) nenere Botanifer veridjutelsen bieje Jantilie

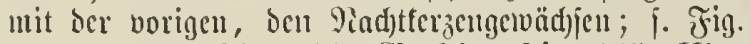
142: Trapa; Jig. 144: Clírlia; Fig. 145: Hippúris. EE geljüren feruer bazu: My'riophyllum, Tauferobatt mo Callitriche, Gajierfern.

\section{Frutilis. Ceratophýlleac Gray.

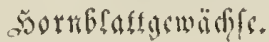

Ulutergetand)te Sisafierfränter, 1 B̧attung mit 3 bis 4 ? Irten, meijt iiber Europa mo 2lordamerifa verbreitet. Die befamutepte ?It ift Ceratophyl$1 \mathrm{~mm}$ demérsum L. \%. Sọäfig in Mitteloentid)= lant, blüht im 3uli, ?tutguft.

\section{Fanilic. Lythrarícac Juss. arsiserifigmends.}

Siräuter, mit etwa 200 ?trten über bie ganje Ertoe verbreitet. Daju gefjört:

fing. 116. Lythrum salicíria $I_{4}$, gemeiner 2Bciderid), B(utfraut. 21. 60-120 cm bod),

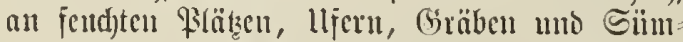
pien burd) ganz Europa verbreitet, brïht bou juli-Eeptember. Bierpflanze, gutes Bichjutter, isurzel frilger offiziuetl.

fin. 118 a. b. Peplis pórtula $I_{\star}$, geuciner Sfierquendel, Bipfelfrant, Fortulafuege= rid). $\odot$. Stengel 8-20 cm Lang, niederliegent, auf fendften Iriften nidht jelten, blïht rötlid)= weis vou Julti-Eeptember. Die Blätter weroen hie und ba als Ealat veripeijt.

3 oen Ge i der iden gefjören fermer: Cup liaea

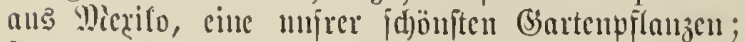
I a wsonia, S(lfanuaftrand) ans Tioroafrifa; Lagerstroemia, ein prödtiger $\mathcal{B} a u n$ Dítindiens.
74. Fanilic. Tamariscineac Desv. Enmarisfin.

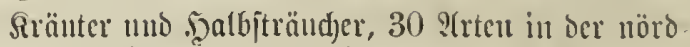

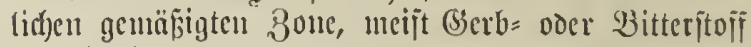
enthaltento.

fiuj. 148 a. b. Támarix germúnica $\mathrm{L}_{\text {. }}$, bentidje Tamariste, Deutide Cuprefie. 5. 1 biz $2 \mathrm{~m}$ lyod), in Mittel=, Sübcuropa, blïlyt im Juli, Iıgujt. Friiljer offizintell, Gartenjierftrand).

\section{Fumilic. Melastomáceate Juss. sdiwarzmutrogswisfle.}

Ïlber 750 Srten, fajt mur tropifd)e Bänne mo Etränd)e. Biele liefern ę̧bare Beeren mo and Docilmittel. Diefer gefouren: Melás to ma L., Evbbecritraud in Ditinden; Niconia L. in Sibamerifa, Rhéxia in Brajilien; Osbeckia auz Çina, fdöner Bicritrand) unjer (Särten; Cyanoplyyllum, B́laublatt anz Micrifo, muje idjönite Pradjtzierpflanze.

\section{Fantilic. Philadélpheac Don. כ) feiferitrüuds:}

L. Lleber 10 meijt norbanterifanifd)e 9(rten; zu il)nen geljören bie als Gartenzierpilanzen bei แnక beliebten Pliladélphus commúnis L., muäd)ter $\mathfrak{j a s m i n}$ unb De utzia, cin bis $1 \frac{1}{2}$ moler japauijaer 8 ierftraud).

\section{Fanilic. Myrtácene $R$. $B r$. anijustrgemeids.}

l̈ber 700 ?(rten Bäume uno Etränd)er in ๔iid= amerifa mo ?hiptrafien, nut 1 ?rit in Ëแropa, uno zmar Myrtus commúnis L., gemcine Mi yrte; bcimifa) in ben fïblidjen Sänocrn (Enropas, bei uns beliebte Topfpilanze. $3 \mathrm{n}$ bicier Familic gefjören ferner: Cary ophyllus L., Bseroür zuelfenbaum auf ben Mofuffen; Psidium L., (S) najavabaum in Tétinoicn; Metrosidéros, Ėijenlolzbaum auf seu Moduffen; Melaléuca, Caiaputbaum

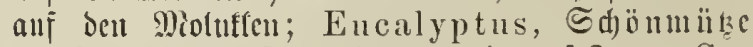
ans Pieulollano; Barring tónia auj Java; Catínga in Gruiana.

\section{Fanilic. Cucurbitaceac Juss.} sourfisgmädfle.

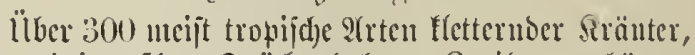

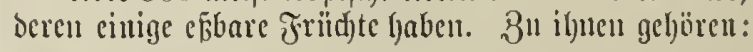
fin. 119 a. b. Cúcumis sativus L., geucine

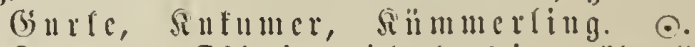
Stamunt ans Ditmbicn, wiro aber bei แms übcrall in vielen Spiefarten iu Gemuijegarten gebant. Die $\mathfrak{B e r w e n t u n g ~ i f y e r ~ F r i ̈ d ) t e ~ j u l ~ v e r j a j i c o e n e n ~}$ Epeijen ift befannt. $\mathfrak{B}$ (iif)t bom Jnli- September. 


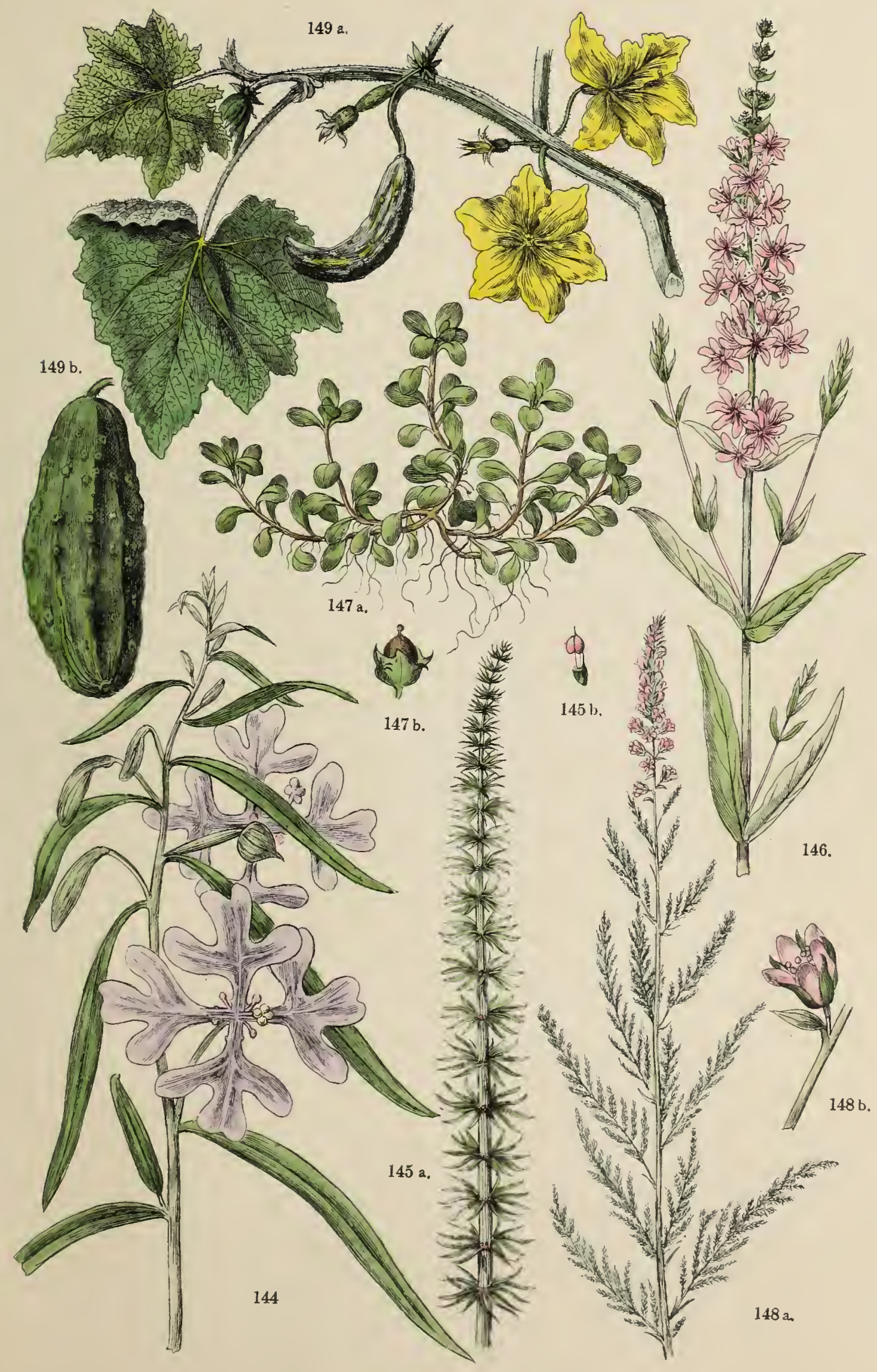





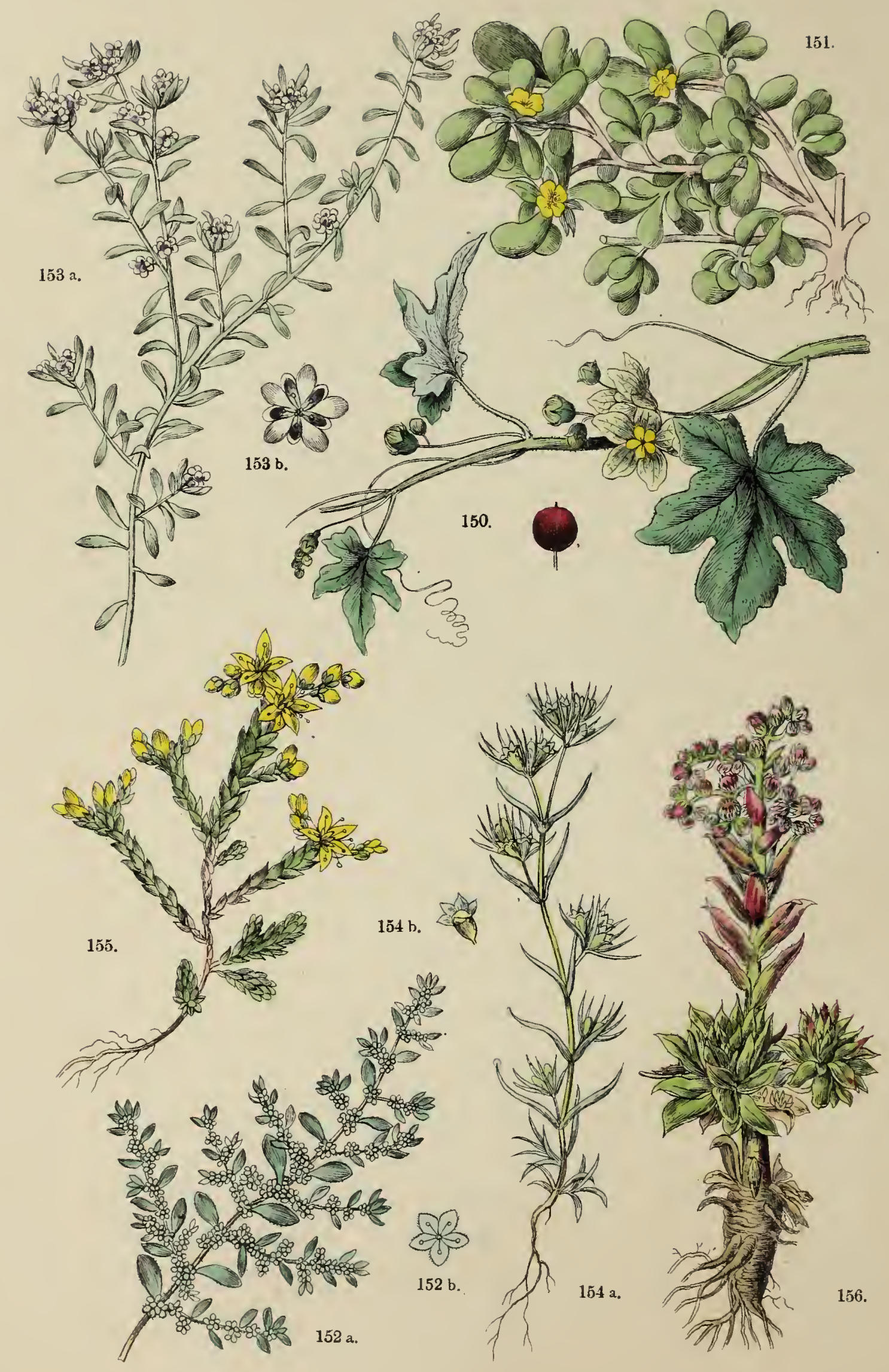


\section{Taf. 27.}

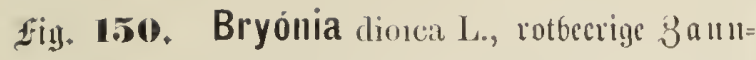

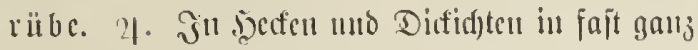

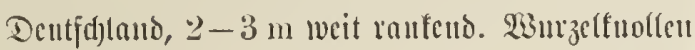
gị̂tig, ofîiziuctl.

3" bicjer Jantific geljören ferner: Cuc. melu,

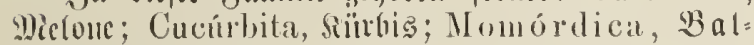
jaulapfer in Ditubicu; Ecballium, Efelsgut ill Silocitopa.

\section{Trunilic. P’ansiflóreac Juss.

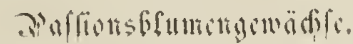

Ju Eüoancrifa mo sisejtindicu. Etwa 200, leid)t zu fultivierente, bei mus als Iopfpitanzen be= liebte fletternoe Jalbjträud)er, einige mit ę̧boreu Friid)teu, bicle mit prad)twolfeu, wohfried)enden B(tiiten.

8). Familit. Loasíceac Juss. B̀tentuindor.

lleber :30 :Yrten boritiger, bremender Siränter,

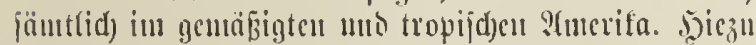
gerjört mamentlidg: Loilsa urens, breutucude doafe in Çyili.

\section{Fanilic. 'T'uncrúceac de $C$.}

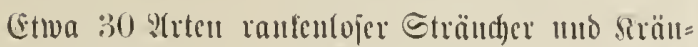

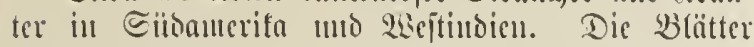

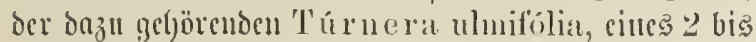

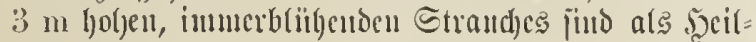
mittel gejdjüłzt.

8. Fanilic. Portulảceate Juss.

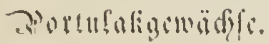

Etwa 70 Strten, ïber alle 3onen verbreitet; cinige geben $\Re$ ahrung jll get)örcu:

fiiy. 151. Portuláca oleracea L., Fortulat

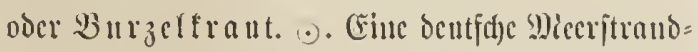

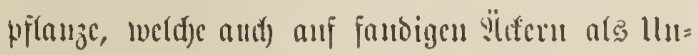
frant vorfommit, oder lultivicut mul als Ealat=, Enppen= mo Gicuiifepflanje benilizt wird. Jernet gebören zu dicjer Fautilic: Móntia fontana L., Suclfenuoutic, ? , ciu lleinç Siräuttciu,

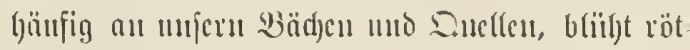

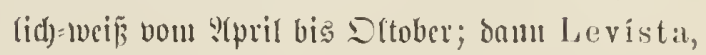
象itterwurzel it Siorbauterifa; T'alinum, Gemiijepftanze in B̉rafilicu; Cla y tón iá, Sunollen.

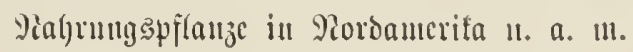

\section{Fantilic. Paronyehícale st, Hit.

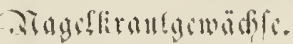

125 9Irten Hleiner, ๔andboden liebchocr sirüuter

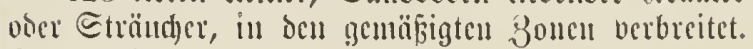
Daju gel)örtı:

tiy. 15: a. b. Herniaria glalıra L., gentiutes,

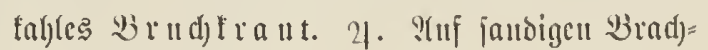
feldern Dentfalandos und der Edjweiz, blïlft vom Juni-Dttober. Ilutrant.

fiy. 15:3 a. b. Corrigiola littoralis L., gemei=

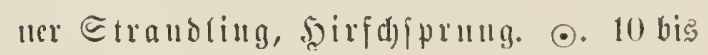
25 cml rang, nicocrliegent, au Miceresptranto

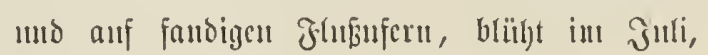
stuguit. Ulutraut.

fị. 151 a. b. Scleránthus ám11uus L., iäl)ri=

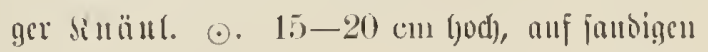

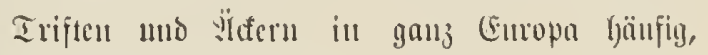
bliibt vou Mai-Dftobcr. Llufrant.

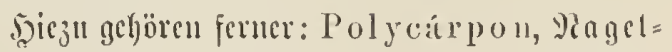
fraut uno Illécebrum, Sillorpeltrant.

\section{Familic. Cransuláceate Juss.}

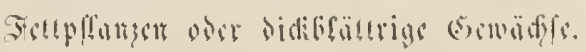

Heber 300 ?trtel jaftiger sirüuter odcr salb

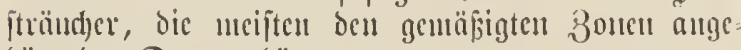
(jörento. Тази geljören:

fity. 15.5. Sedum acre L., fdjarfer, geuciuce Miaucrpfeffer, Pfefferfint. 21. 10 biz 


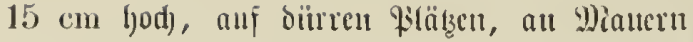
und Jelfen butd) gauz (Emopa, b(ïl)t vom Jutui bisె 9tuguit.

fin. 156. Sempervivum tectórum L., geuti=

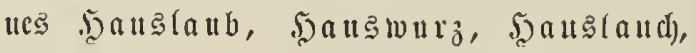
Dad) $10 u \mathfrak{z} . \quad$ \%. $30 \mathrm{~cm}$ hod), auf Feljeu uno Wiaueru, aud) auf Däd)eru, b(ülft im Эuli, ?(ugujt.

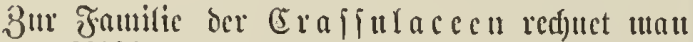
fortur: T'illaea, Rlodíola, Cotylédun, Crássula etc.

\section{Fantific. Ficoídene Juss. (Aizóideac Sprr.)}

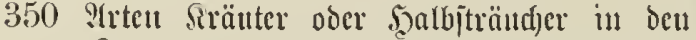

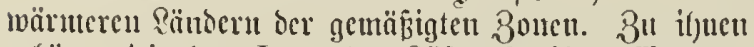
geljörcu $\Lambda \mathrm{i} z \dot{o}$ on L. au oen Siüften ?lfrifaz; Tetra-

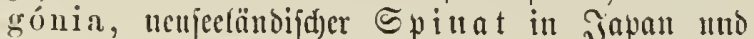
Reufectanto; Mesembryánthemum, 3ajer= bl ume it Sitoeuropa uno ?fruffa.

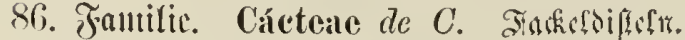

Dic Saltuspfíazcu, Saftecu, fïto Sirüuter oocr Sträut)er utit meift unförtulid) verbičfteut, aute ciner fajtigen, grüuen F(eijd)majfe bejtel)endem, fugel= ooer jäulcu= ober blattförtuigen, häufig äjtig geglicocrten St a ume doer Etengel. Die meipten ?trten habeu feiute cigentlidjen $\mathfrak{B}$ rätter und itatt oer Suojpen

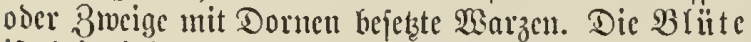

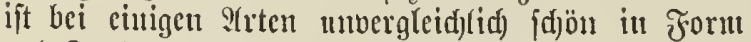
und Farbe, weshalb fie aud bei uns als Topfpitan= zeu jeljr belicbt jino. Mian zäblt ïber 400 2(rteu, weld)e fajt jämtlid) im warmen ?(merifa heimijd) fiitr. Sinté vercinigt jämtliche $\mathfrak{A}$ (rten in die cinc Gattutug: Cactus, Fafeldiftel, bie ucueren $\Re a=$ turforid)er aber teilen fie nad) ber verjdictenen (Bes= ftaltuแg it 3itzendiftefn (Mammillaria); Jgefbijtelı (Echinocactus); Mielouendifteln (Melocactus); fäı= leuförntuge $\mathfrak{F}$. (Cereus); $\mathfrak{F}$. mit äptigem, atı r'uto= (id)en Gilieber" zujammengejeştem Stengel (Opuntia),

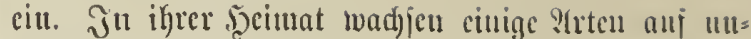
Frudbtbaren Sattojtreden, auf Feljen uno founigen

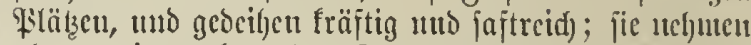
aber, wic aud) autore Fettpflauzeu, il)re 2ial)ruttg

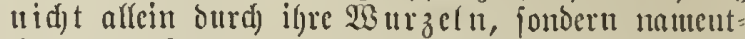
(id) burd) Stengel= mito Blattoberfläd)e aus ber Enft

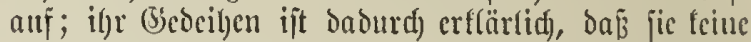
Feudtigfeit aushaudeu. CEinige 2(rten twadjen in Miecrifo bis zu eimer 5eölse von $3000 \mathrm{~m}$ über detl Micerc.

Der Natzen ber Siafteen ift groj mo mantig=

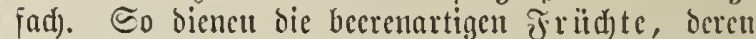

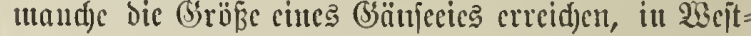

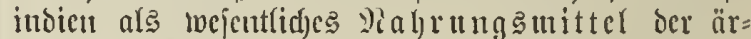
meren Doltaflaj]en, in Epanten utto Eizilien wiro zur 3eit ber Frudutreife bie (Ernte feittlid) gefeiert

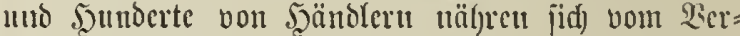
fauf Der Friddte auj Den Straken. Ju vielen wajīer= armen Giegenden allf ben Dodjebenen von Miexifo

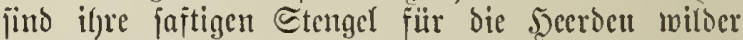

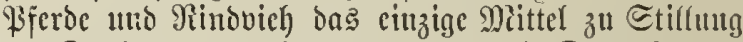
bes Durftes, weshallo man jie aud) bie Suel(pflauzen ocr 2 iiifte nennt. Siele $\mathfrak{A}$ rten bienen zur Sdjweine= mäitutg, altoere beu $\mathfrak{B}$ g̈gełn zแl reidjlid)en Futter. Die 2 $1 / 2-3 \mathrm{~m}$. hohen, mit Dornen bedecften ?trten werben z" Einlyegung oer Feldoer gebrautd uno geben unourdoringlide Seden. Maan benübt fie aud) z"

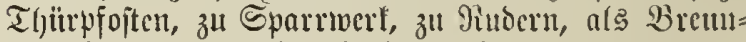
unaterial, uno ce giebt in \$eru cine 9-12 $\mathrm{m}$ holje S(rt mit einem äuberit fejten 5olze, weld)es jeiner 5)ärte uno ber idjönen, aberigen Farbe wegen zu ben

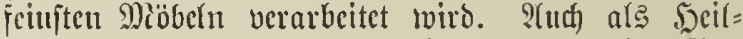
mittel werben mand)e Arten vielfad) gebraudgt. 2ion Bescututug find cinige Opuntia-2(rten ourd) Crtuäl)rung

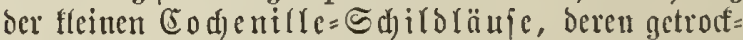

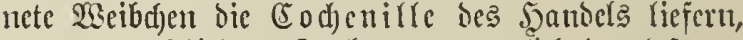
woratı beridjedene farben, namentfid) ocr foftbarc echte Siarmin, bereitet werdent. Die Crier der :auz entlyalteu ben Farbjtoff. 3n biejem Zluede oer Jarb=

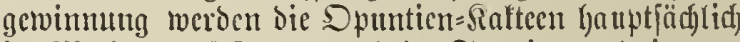
it Micrifo, auf Java, aud) itl Spanien utto in att=

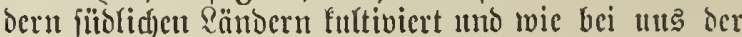

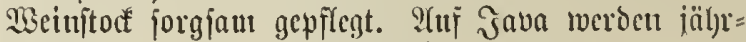
(id) gegen $50,000 \mathrm{kgr}$ (Eod)eniffe gewounen, alif ben

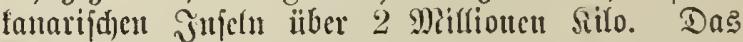
siilo foftete nodh vor wenigen Эafjen $30-36$ Miarf, bicjer Preis ja)eint aber nad) Centocefung ocr Fudd)= jin= แแto ?trilimfarbitofife imuter mehr zu finfen. 
28.

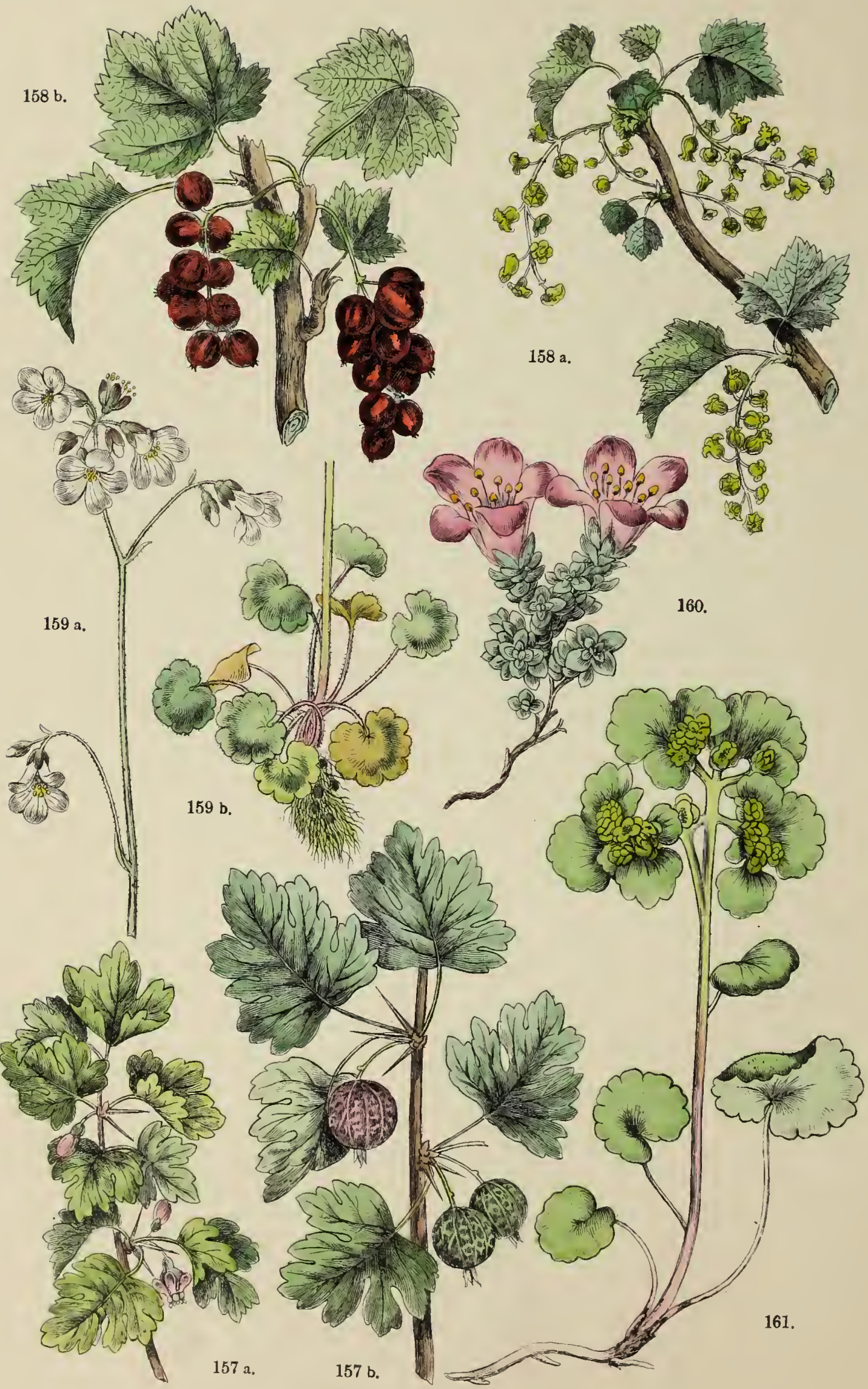




\section{Taf. 28.}

87. Familic. Mrossularíeae de $C$.

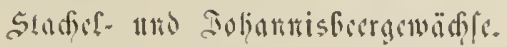

Erma 60 SYrten zuแı Teil Dorniger Sträud)er, fajt

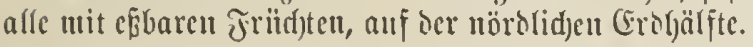

fịj. 158 a. b. Ribes (rrossnlária L., Stad)el=

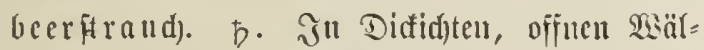

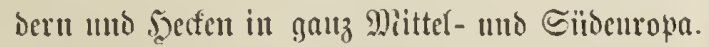
23liiht ium Stpril, Miai. Die vortreff(id)en Friid)te Der vielen fnltivierten Epielarten fitto befaunt.

fig. 158 a. b. Ribes rubra L., rote Johan=

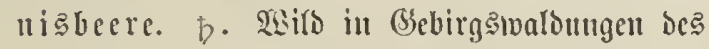
nörolidjen uno mittleren (suropa. B̉(ülyt im 2(prif ums 9)iai. Die fnltibierten 9frten tragen rote, gelbe bis weifse gruid)te. (Sebraud) befonnt. Die jd)warze Jolnanisbere, R, nigrum L., ans Rorbajicn ftammento, fintet jid) in un= jern (5ärten uno briityt im 2rpril, Miai.

88. Fannilic. Empetráceae de $\boldsymbol{C}$.

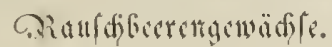

5 Strten fleitner, intmergrüner Etränd)er in Den gemäpigten und falten Bouen. 1 Dentid)e ?(rt. E m-

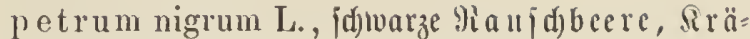
henbere. 5. $30-45 \mathrm{~cm}$ (jod) auf Mioor = uno

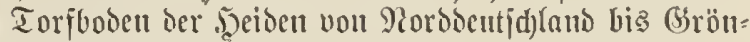
lano. Die jaftigen, jäturlidjen, jđjwarzen Beeren geben int Rorben Enropas gejunde Nahrung.

89. Fomilic. Saxifrágeate de C. Efciubtefie.

In 200 9rten zierlider Strüudfer, meijt auf Bergen ïber bie nörslid)e gentäßigte Bone verbreitet. 34 beutiche Ifrten. Dazu:

Fig. 159 a. 1. Saxifraga granuláta I., fuot= liger Stcinbred). 2. Tllf ponnigen sjïgelı Into Iriften, $15-30 \mathrm{~cm}$ lod), b(iil)t vom 2(pril biร Эแแt.

fin. 160. Saxifraga oppositifólia L., gegen= b(ättriger Etcinbred). 2/. Nur $2-5 \mathrm{~cm}$

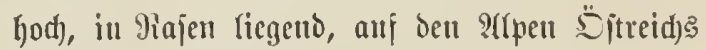

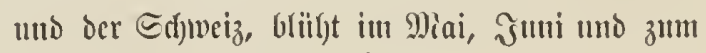
zrocitemmal in ?hgujt.

Fig. 161. Chrysosplénium alternifolium L.,

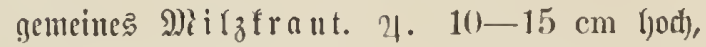
at Sueflen und $\mathfrak{B a ̈ d ) e n , ~ b ( i i l ) t ~ i u n ~ D i o ̈ r z , ~ S ( p r i f . ~}$ 


\section{Taf. 29.}

fiij. 162. Parnássia palústris L., Smupf= berzblatt, Etubententögden, weipez berfraut. 24. $15-30 \mathrm{~cm}$ hod), auf $23 \mathrm{erg}=$

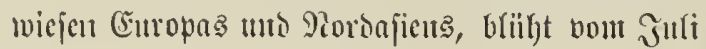
bis September.

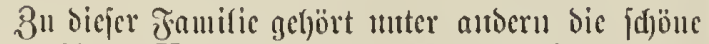
Grartenthume Hydrangéa, bortenfic.

90. Fontilic. Umbellíferae Juss.

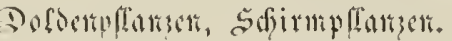

(Eiuc Der größiten, artenreidjiten nuto natïr= (idj)ten Familien, Derent Jocimat hanptiäd)(id) bic

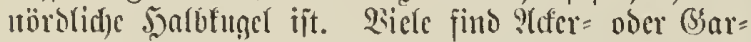

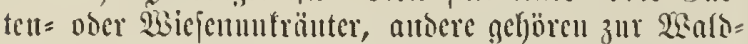
ober :(fpcuffora, einige jino jd)arf uno giftig, andere getwïrzhaft, ȩ́bar uno gejuto. 31! Delt Dolden= fílauzeu geljören:

Eig. 163. Sanicula curopaea L., gemeiner $\varsigma_{\mathfrak{a}=}$

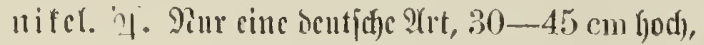

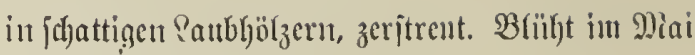
แแno Эแni. Früher offizinelf.

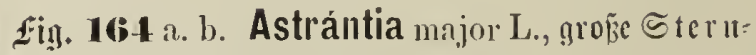
ooloc, Strenzel, Miciftermurz. \%. 30 bis

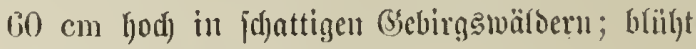
voul Эuli--?luguit. Bsiftig.

5ì. 16.5. Erýngium campéstre L., Fero= Dia än= nertien. 21. $15-50 \mathrm{~cm}$ hod), all $\Xi_{a 1 t}=$

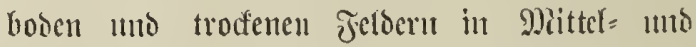
Sitienropa; blïht vom Эult-Eeptember.

fiig. 166 a. b. Apium gravéolens L., gewöl)u= lid)e Sellerie, Eppid), Miart. (.). Wädjit wilb auf jafzlyaltigem Boden Sildouropas, ift aber burd) Sittlur cine bei unz alfgenein gebante Bartenpflauze, deren $23 u r z e l$ zu bortrefffid)eut

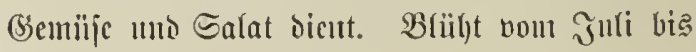
Scptemtber. 


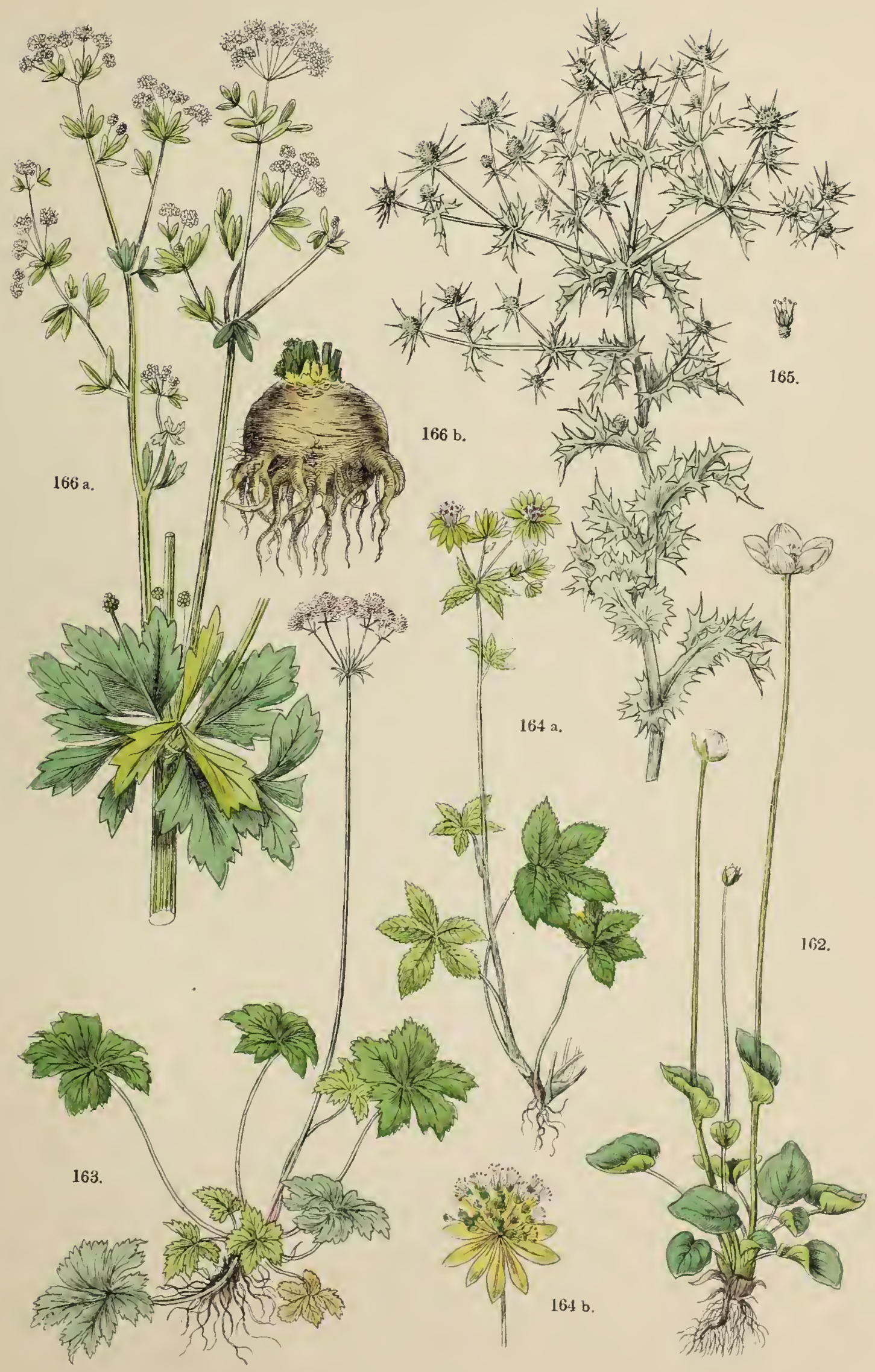





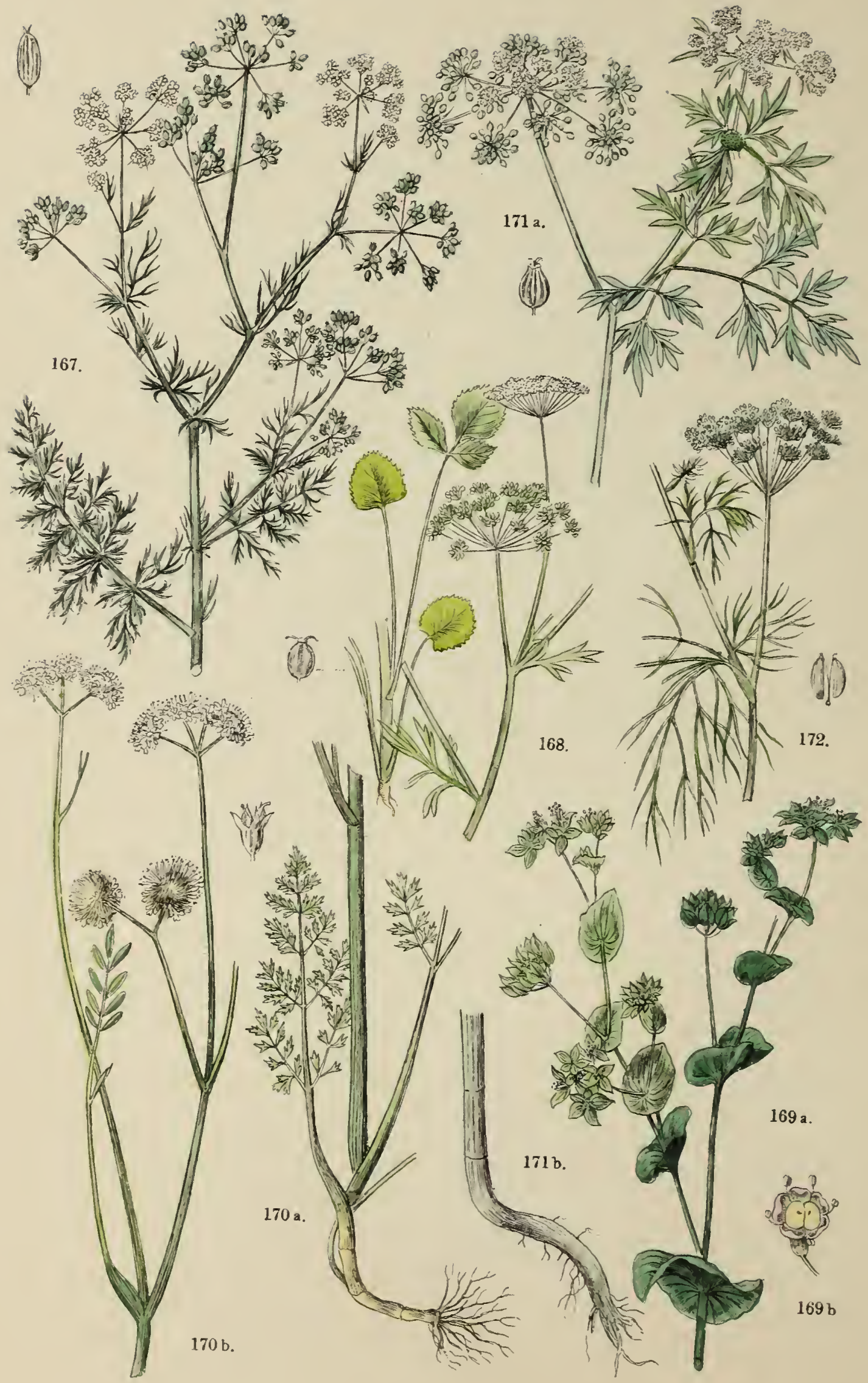
๔aศ๋. ริ0.

fily. 16\%. Carum carri L., gemeiner fï Felofümulel, Carve, Gsarbe. .). Etengel $30-90 \mathrm{~cm}$ hod), auj 2 iejen gemein, aud vielfad) fultibiert. Die Cament bienen als ge=

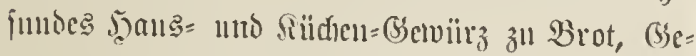

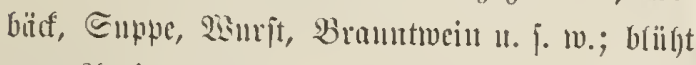
bou ?Trrir-Juni.

fig. 168. Pimpinélla saxífraga L., gemeine Biberuer, Bodspeterific. \%. $30-60 \mathrm{~cm}$

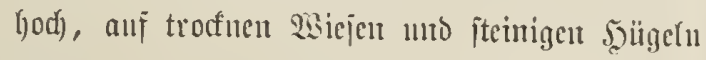
hüurig, briilyt vom Эuli-Eeptember. Dic juı= gen $\mathfrak{B}$ lätter geben ein gutes Gémiije nno wïr= zignes ๔alatfrant.

fig. 169 a. b. Bupleurum rotunclifólium L.,

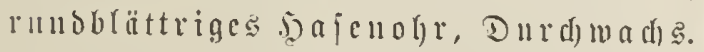
$\odot$. Eteugel $30-50 \mathrm{~cm}$ hodh, unter der Eaat, auf Thon= mto Sialfboden; blïht iun Эuni, Jnli. llufiant. fig. 170 a. b. Oenanthe fistulósa L., röfrige Rebeuboloc. \% $60-90 \mathrm{~cm}$ hod, alli

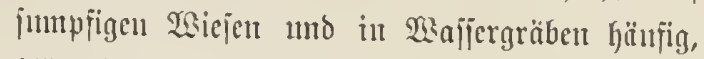

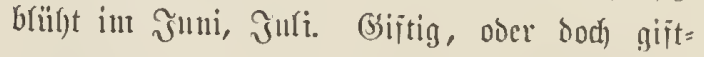
veroüd)tig.

Eị̣. 181 a. b. Aethúsa çnápium I., Greiz̃e,

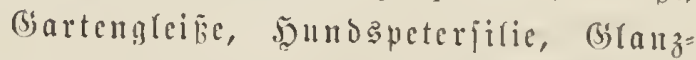

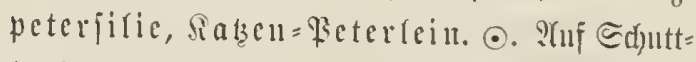

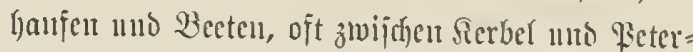
filie, mit benen bieje jdjarfe (sijtuflanje bor ber Brütezeit (eid)t werwechjelt werden fant; briiht vom Эnni-@eptember.

fị. 182. Foenículum officinále All. (Ané-

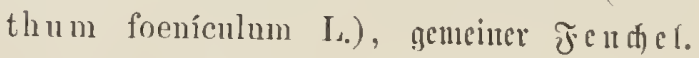
-) bis 21. $60 \mathrm{~cm}$ bis $1 \mathrm{~m}$ hod, will in Siid=

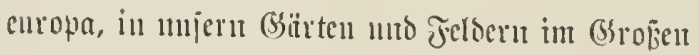

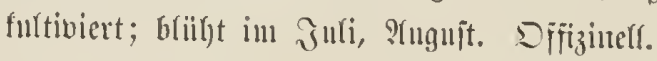


Taf. 51.

fiin. 18:3, Levisticum officinále Koch, (Lignísticum lerísticum L.), Eicbîtödel. 2. Etcugel 1-2 $\mathrm{m}$ hod), in ocu Bebirgagegenden Siid=

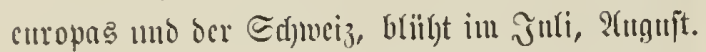
Dic Sisurzel ber ftarf gewürzig ried)enten \$rfanze bicut arzucilid).

fị. 174. Archangélica officinális Hoffm. (Angelica archangelica I.o), ed)te (E) (G) artencugelwurz. ... Etenged bis zu $2 \mathrm{~m}$ l)od), auf jeud)ten (sebirgsfitclfeu, ljäufig fultivicrt

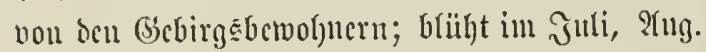
Dic $23 u r z e l$ bient in verjdjedenen formen alङ

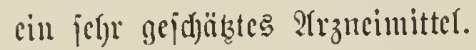

fig. 175. Anéthum graveolens I., gemeincr Dirl, GHutfenfraut. $\odot$. Steugel $60-90 \mathrm{~cm}$ hod), wilt in Eilioenropa, hei me riberall in (biärten angebaut; brül)t im Juli, \{łıguit. Bsutes Siid)engemiirz, ofīiziuclf.

fif. 176 a. b. Daucus caróta $L_{\text {. }}, \mathfrak{g e m}$. Miohr $=$ riibe, Möbre, Earotte, getbe diibe. $\odot$

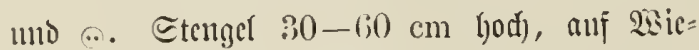

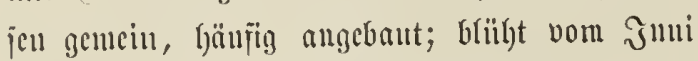
bis Eeptenber. Dic 2 surzel giebt gejundes Bje= miife, vortrefiftides 2ielffutter. Sffizineff. fin. 178 a. b. Chaerophyllum temulum L., betäubender Sälberfropi, Ta uucltörbel. (). Stengel :30-90 cm hod). 2(ui ख̃icjel แแb

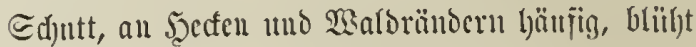
iu Mai, Juni. Bsiftiges llufinut.

fịj. 178. Conium maculátum L., gejilefter Sdicrliug. (.). Stengel $1-2 \mathrm{~m}$ hod), an

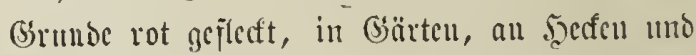

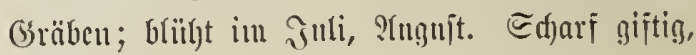
orĭizincll.

fin. 179. Coriándrum satívum $L_{\text {., }}$ angyehauter

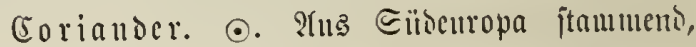
bei une ber würzigen Eamen halber a[s Sïd)en= gewürz und 2 rzucipflanze angebaut; blül)t boul Эuni-?ługujt.

3 beu llubelliferen getjöreu feruer: Cicúta. Wafferjucrliug; Aegopódium, Bseibjus; Sium, Marf; Séseli, Sejel; Cnídium, Brennooloc; Sílaus, Silau; Meum, B̉är: wurz; Selínum, Silgc; Ostéricum, Miut terwurz; Angélica, ḃuftwurz, 2̧alowurz; Peucedánum, .ૅaarịtraug; Pastináca, 7:a= ítiuaf; Herácleum, Bärenfla fiimmel; Laserpítium, Eajerfraut; Caucülis, Jaftoolde; Anthríscus, Я̈̈rbel; Pleurospermum, Johrippe; Smyrnium,

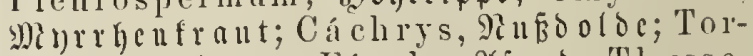
dylium, Birmet; Férula, 2rjaud; Thyselínum, Delfeumin; Imperatoria, Micifter= I $11 x\}$. 


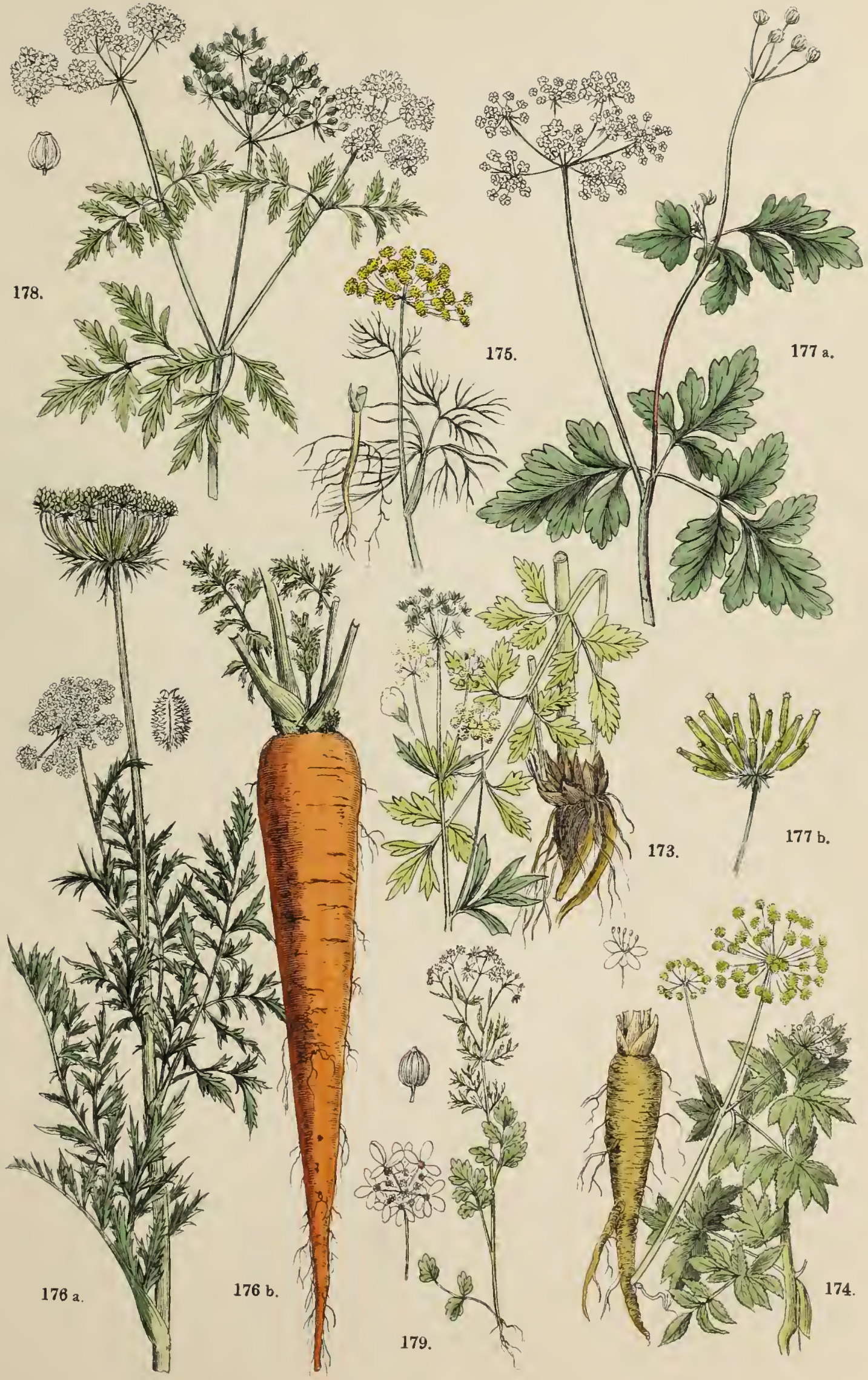





$$
\text { - }
$$




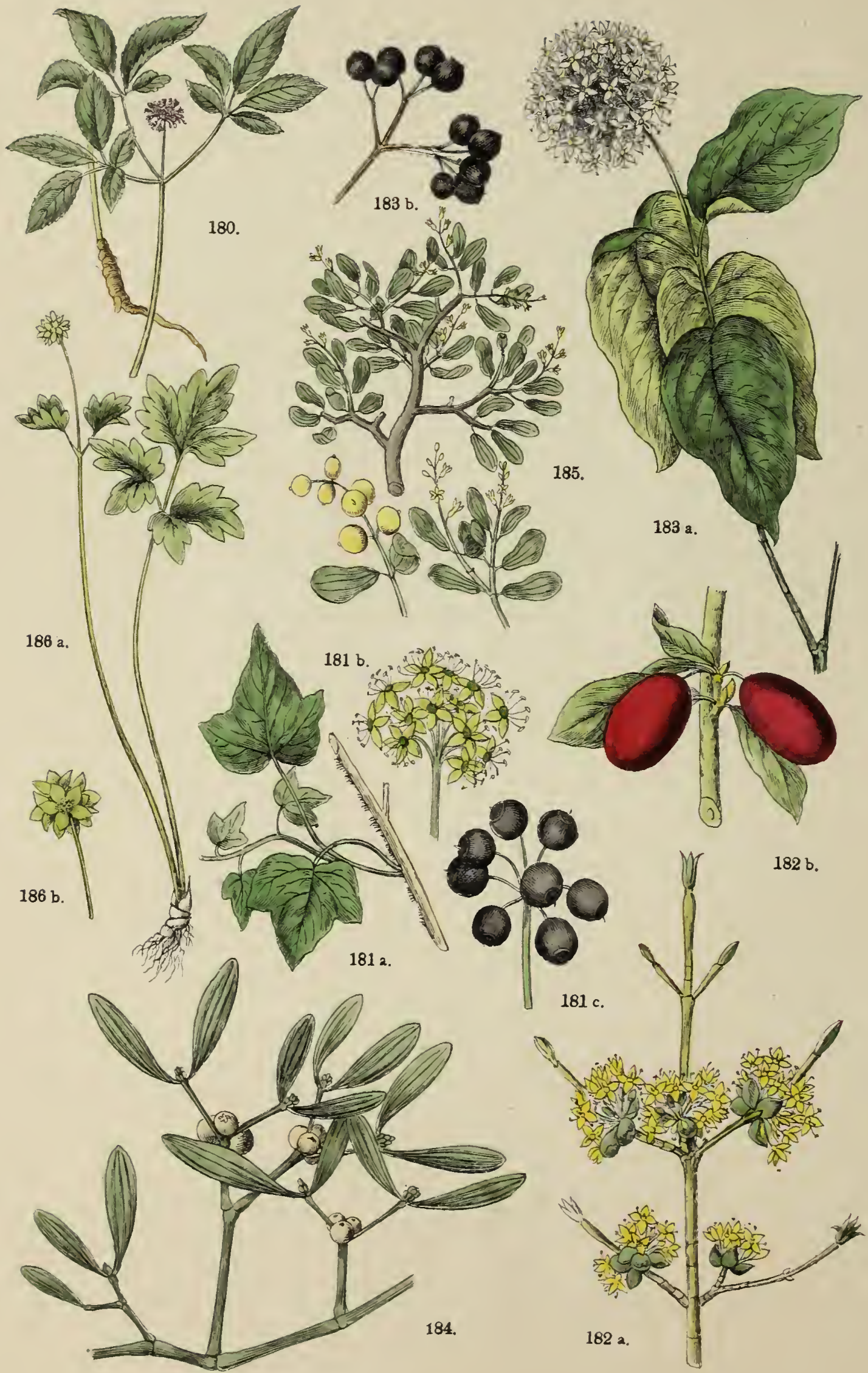




\section{Tậ. 32.}

91. Fantific. Mraliáceac Juss.

S0-90 2rrten Bü̈unc mito Sträuc)cr, ïbcr alle 3oucu ocr Eேroc, Eேแropa amşgctommen, verbertet. és gebörcu bnzu $\Lambda$ rália, wormter $A r$. papyrífera, gicispapicrpfraja in Cbinn, dic widjtigite.

fin. 180, Panax Schin-seng, fraftuurzel,

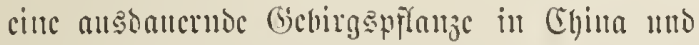

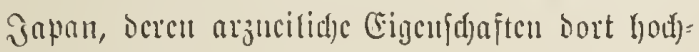
gejđäßzt meròctt.

92. Fantilic. Hederáceace Ach. Rích. splionguädili.

Diur 1 isutid)er, ranfenocr Etraud).

fị]. 181 i. b. c. Hédera helix 1., Eேłlall,

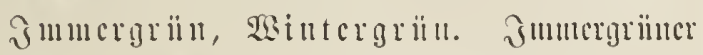
Etraud) in Čmopa mto ?fyicu, büufig 10-20 m hod) fletterno, an Büunten mo aften Wiancru;

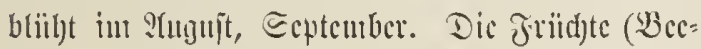
rcii) jitto ultigcjuto.

98.) Frmilic. Cornáceate Ach. Rick.

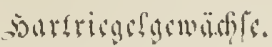

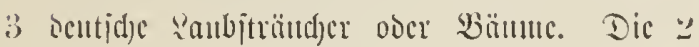
ivid)tigjtch jint:

fig. 18:2 a. b. Cormus mas L., Dornftiand, Dïrlike, Soruclfirjde. Ein 3 bis $6 \mathrm{~m}$

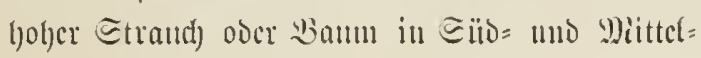

curopa; in 2Rorben ïbcrall in Deden futtivicer ;

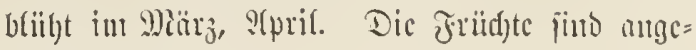

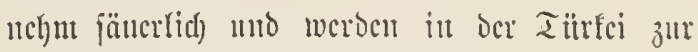
Bercituny bcs ๔ajerbets ocrwendet.

fiin. $18: 3$ a. b. Cornus sanguínea, gentincr oder blutroter Dormitraud). b. 20-t m bod , in

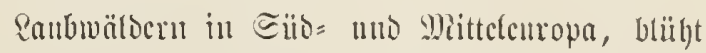
iแn Diai, Juแ แแto wiro büufig als 3icrịtrand) in Bärtcı angepflanzt. Dic હtcugcl આchucn iu sisuter ciuc blutrote Farbe alt.

\section{Fantilic. Lorantháceace Don.

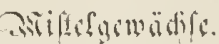

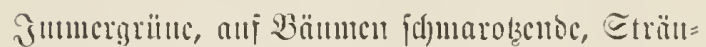

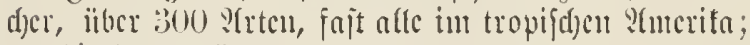
bicrber gebörett:

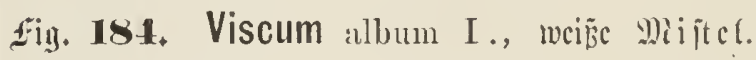
b. Stcugel 30-60 ens hod), ituntergrünce

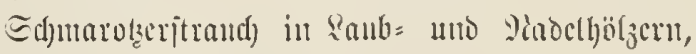

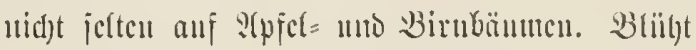
iut Miür, :tprif. Dic Weeren werben vou den

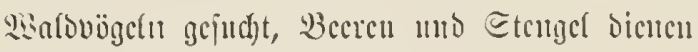
jll 2 ogycllcim.

Fị. 18.5. Lolánthus europaleus L., curopaiijde গicucublumc. b. Ju Eiiocuropa uno S̈t=

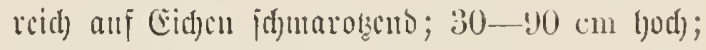
briibt im :tpril, Diai.

\section{Inturfafje: Corollitlorie, fromenb!htige pflangen.}

95. Fannilit. Caprilobliateate Juss.

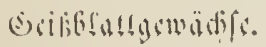

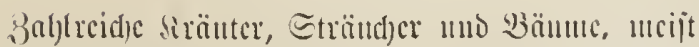
in ocr gemälighten, nörolidjen 3onc, vicle mit wohlricdjenton $\mathcal{S}$ (iiten. fig. I86 a. l. Adoxa moschatellini L., gemcinc:

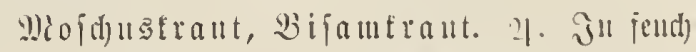

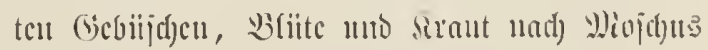
ricdfent, $5-12$ cm hodj. Sn Dentidjland mo

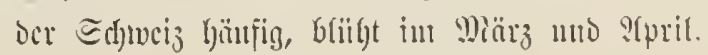




\section{๔ạ. 53.}

fiig. 187 a. b. c. Sambuicus nigra L., gcmei= ner Dolfunocr voer Dolocr, fälichlid) Flic=

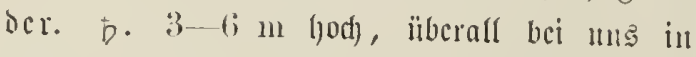

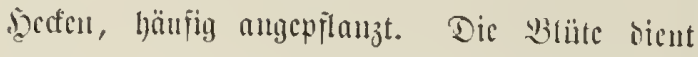
arzncifid); blüht in Juti, Juli. Dic fdjunazen Becren fint cin \&icblinge șutter vicler Einguögel.

fiin. I88 a. b. Sambícus ubülus L., :Ittid), Eppid), 3werghollunocr. 5. 2icrbreitet au 2Saloräu內eru mo ftcinigen ?tbbäıgen, bis $1 \mathrm{~m}$ lod), b(ül)t vom Эıtli-?(nguit.

fin. 189 a. b. Viburnum $U_{p}$ ŭlus L., gentiuce Schucball. b. 22-4 mod), in .jectcu, (bic=

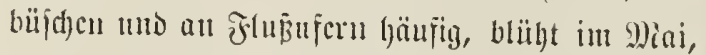
Jnui. 3icritraud) unjrer Gjürten. fin. 19)0 a. b. Lonicera pricilnenum L., ge= meinces Gicipblatt. D. Edylingpilanze, an

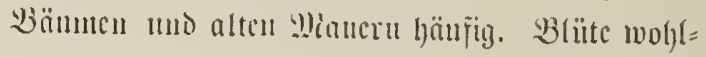
ricd)eno; blïht int Junni, *llugujt. Gartenzicr= itraud).

fịn. 191. Lonicela cutprifólium L., Jefän= gerjelicber, Piadjtroje, (Bortengcipblatt.

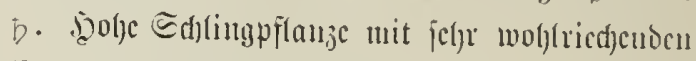

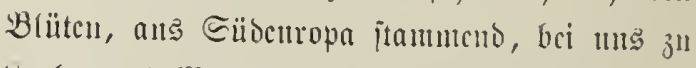

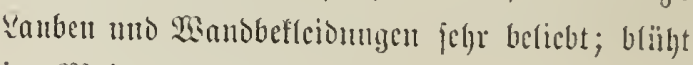
im Niai mo Simt.

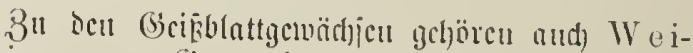
gélia rosea, (jartenzicrîtrand) anz (Ehina; Jinnaea, Sllpcmpilanje mit moblricd)cnocu Blïten; Symphoricurpus, Bicritraud) ans 2toroamerita. 


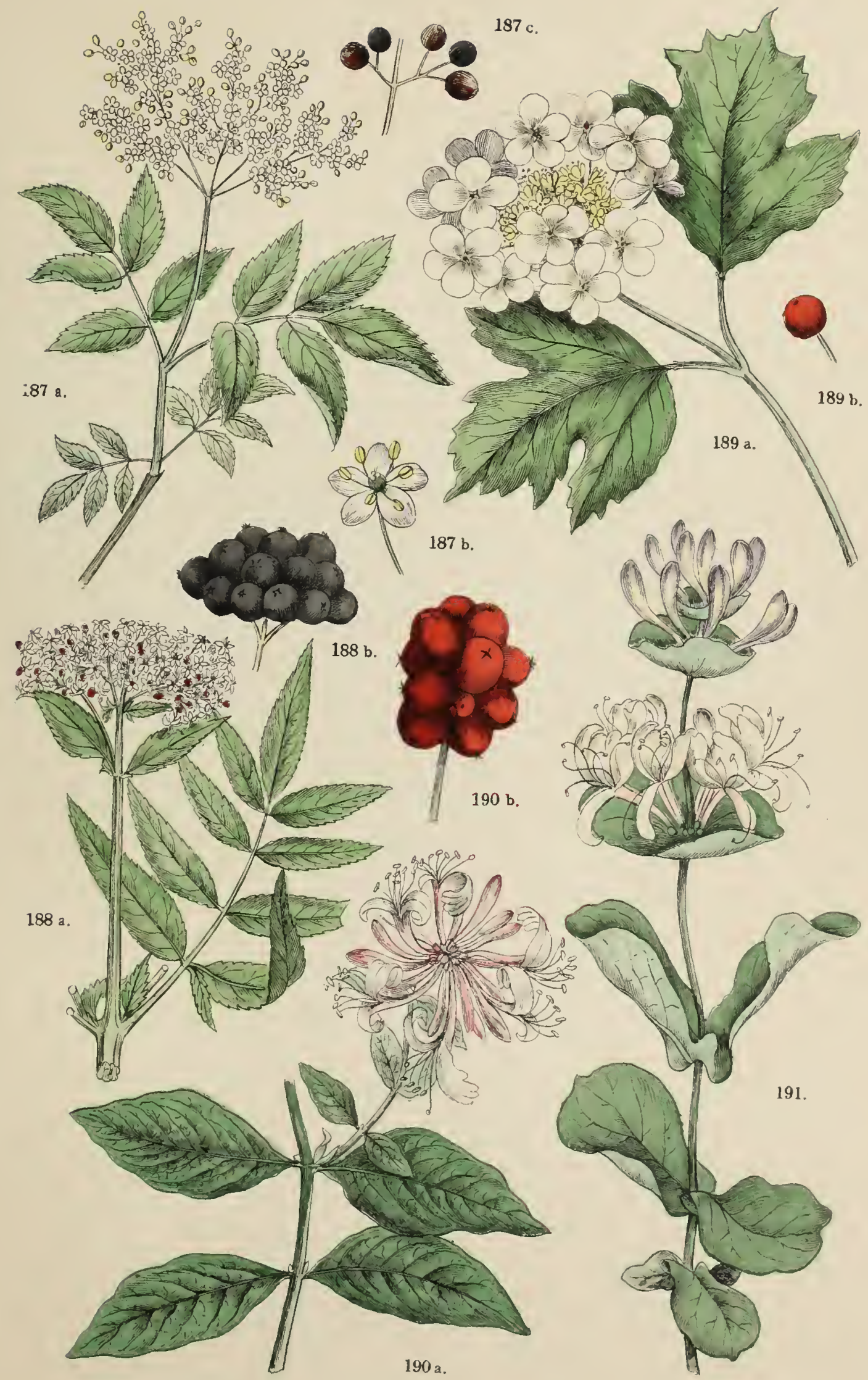





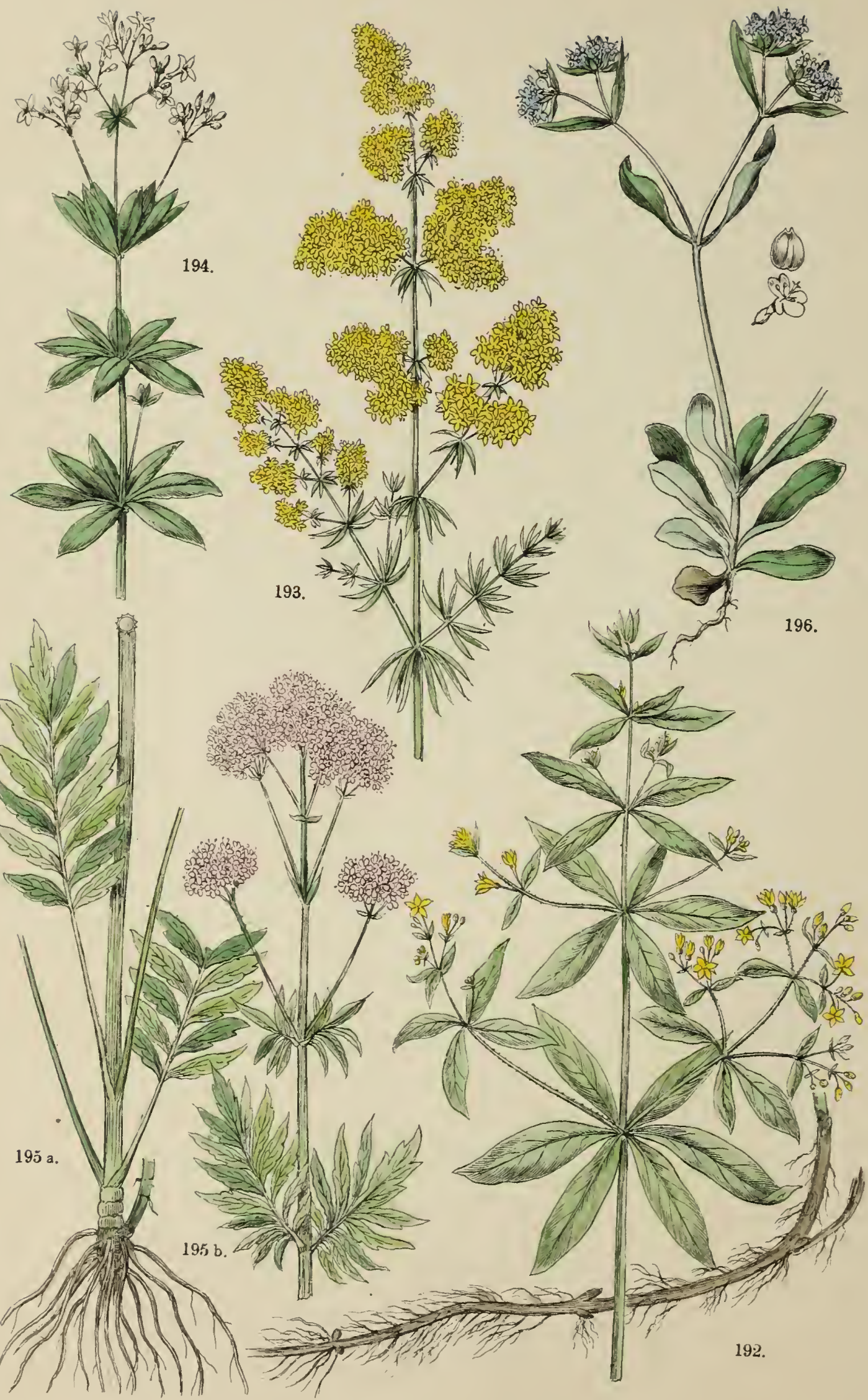


บaץ. 5ł.

\section{Junutic. Riubiácerate burtl. sitapgumatse.}

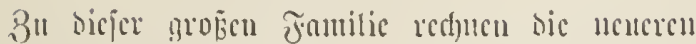
Biotanifer and) Dic folgenoen: Stellátate L., Etern.

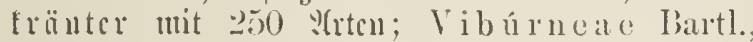
D) oldergenöd) je mit ctwa 70 9(rten (i. oben),

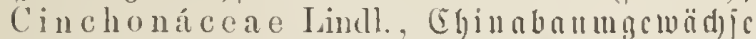
mit über 1600 ?tren; Caprifoliáceale Bartl.,

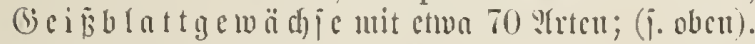

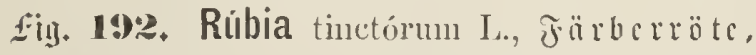

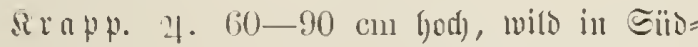
curopa uto sem Ericut, in Icutidjlano ba nuto Dort angebaut. Dic simger giebt cinte prad)tuolfe rote Jarbe uno ift offijintll; bliigt in Jult, ?!11uाt.

£ị. 19:3. Gálium rerum L., :̈̈dtes Q abtraut. ?. 30 cm his $1 \mathrm{ml}$ hod), anf Jelorainen,

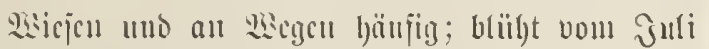
bis ভspteutber.

fiij. 191. Aspélula odoríti $I_{\text {s. }}$, wobltricd)cu=

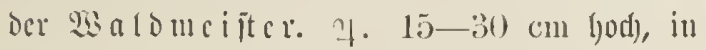

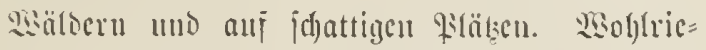

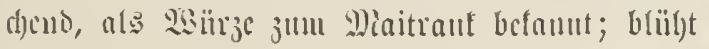

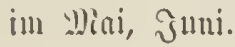

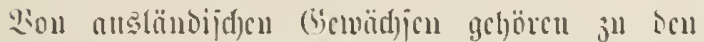
Sinbiaccen: Coffea I., Safjecbantu in strita;

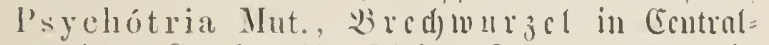
amcrita; Cephaëlis Iich., Jpccacunuba in
Wrapilien; Hamelia Sw. Frinjenboljbatm in Eitiomerifa; Gardénia Lour., Jarblotz in Céfita; Cinchóna L., Ficberrinocubanu (C)i= u a ba แm), in Eiionucrita แ. a. m.

97. Familic. Valleriáneate de $C^{\prime}$

asmoriangamodits.

Etson 140 ?frten vou Sirüutcrut, mcijt in isc

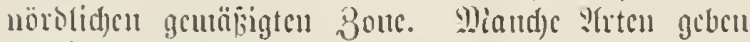
fräitige : :rijucicn.

fiig. 19.5 a. b. Valeriána officinalis L., genci= ner Baldrian, Bulferjaln, Dedeufraut. ๆ. 30-150 cm bod), in siäloern utro an Fend)ten Eselfen แmo llicru vou ganj Emopa.

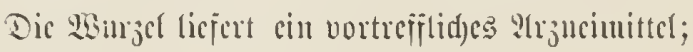
btïgt im Snni, Juli.

fiị. 19)6. Valerianélla olitoria I'oll., gemcis ner Felojalat, yapunjel, Steferjalat. $\odot$. 15 em lyod), in Eiliscuropa beimijd, bci mus bünpinges lluftrant iun (betreioe. 2isiro and) jul Enlat angebant; blïgt iu 2tpril, Niai.

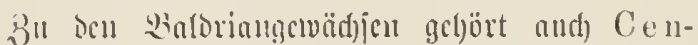
thriuthus de C., Eporublume. \%. 1 ocutjde

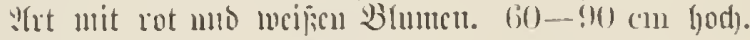

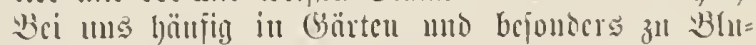

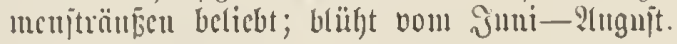




\section{Taf. 35 .}

98. Fantilic. Dipsílcate Juss.

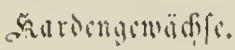

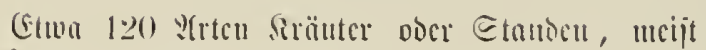
in Eiliscuropa bcimijd. \$u iljucu geböreu:

fị]. 19\%. Dipsacus silvestris L, ycuciute,

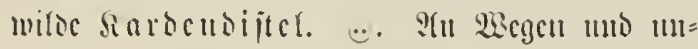
Lchauten Etclfen, Ḧbcr 1 ml lod), räitiyces llu=

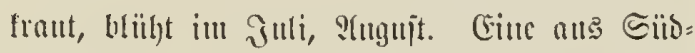
curopa ftaumecube ?(rt (D). Fullonum Mill.,

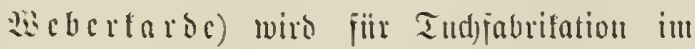
Groficu gebaut.

fiin. 198 a. b. Scabiósa columbíria L., ge uciue Stabioje, Suopibume, Taubcu=

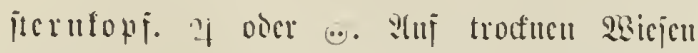

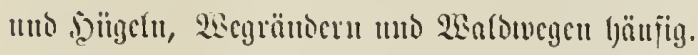
$30-130$ cm hod), b(iil)t vom Juli- Ecptember.

99. Fontilic. Calycóneate R. Bro

Dicic Fnuticic beitelt nus ctwa 10, wenig wid)= tigen jiibnuterifanijd)en : Ylten.
10). Fantile. Compósitare Atruss. (Symantherpate liche). sumferiefer.

3ujautucugejebste Sorb= oocr fopforittige

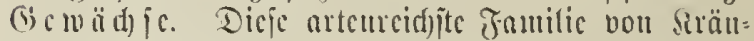

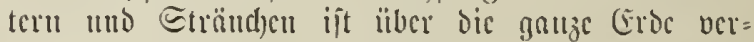

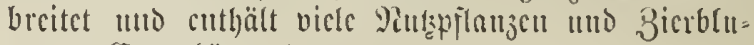
metl. E⿺辶 geljören Daju:

4iiy. 199. Eupatórium cammibinum L., gia

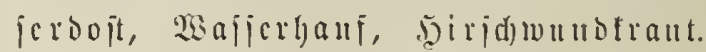
?. Etcuyel $60-180 \mathrm{~cm}$ ljod), an sisajiergräben

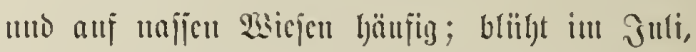

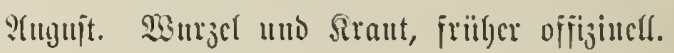

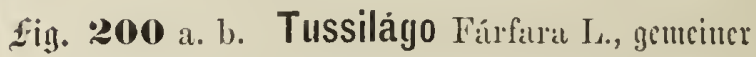

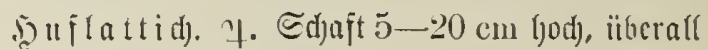

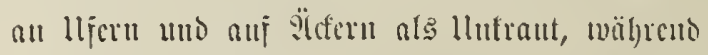

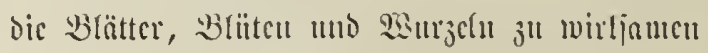

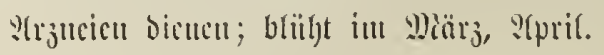

fin. :201. Petasites officinális Mnch. (Tussiligo Pet. L.), Reunfraft, Psitum,

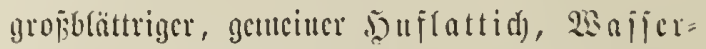
flette. \%. Edjajt $20-40 \mathrm{~cm}$ hodj, auj jan= Digen, fend)ten sisicjen in Dentidjlans uidjt felten;

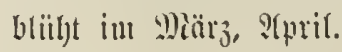




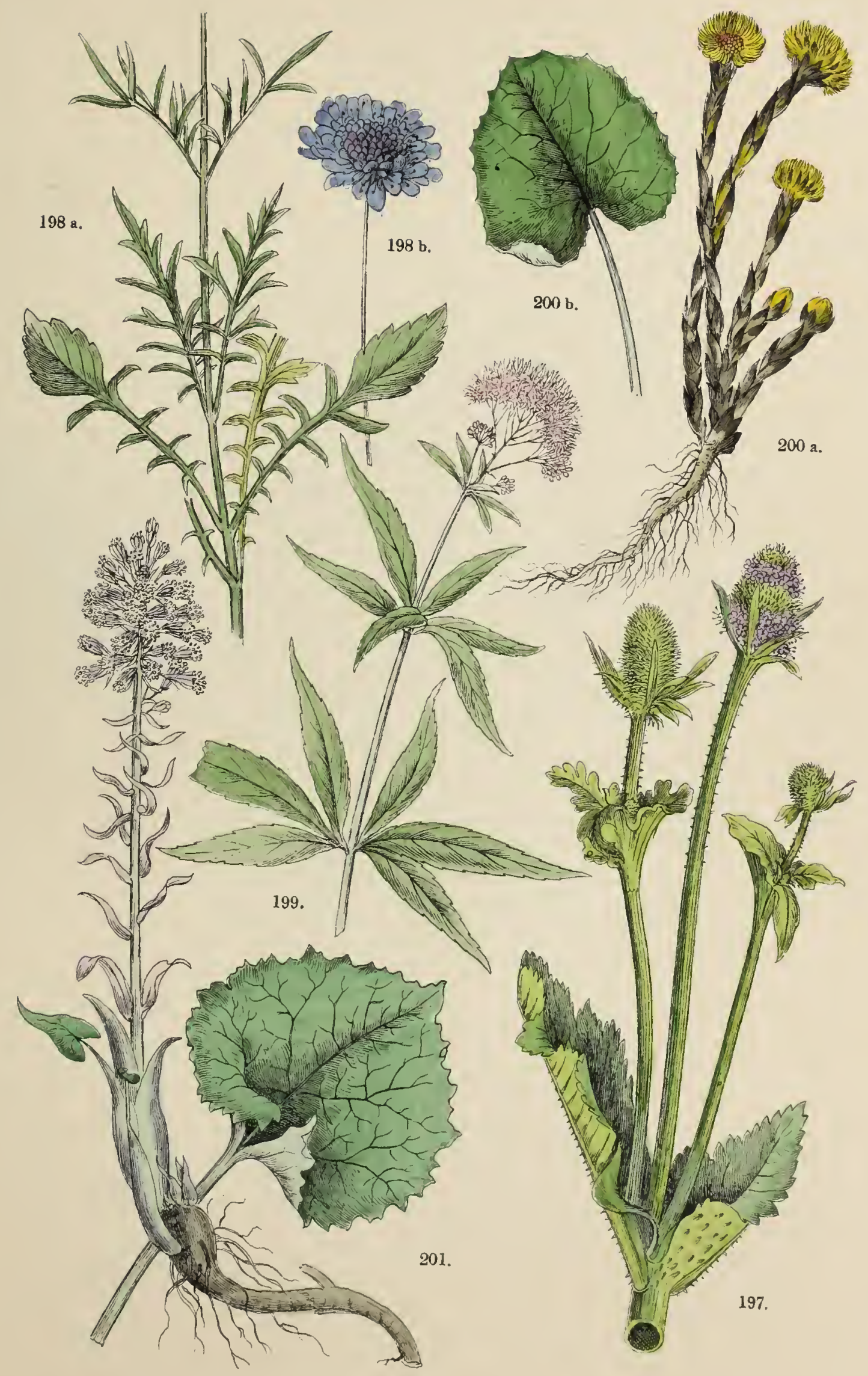




36.

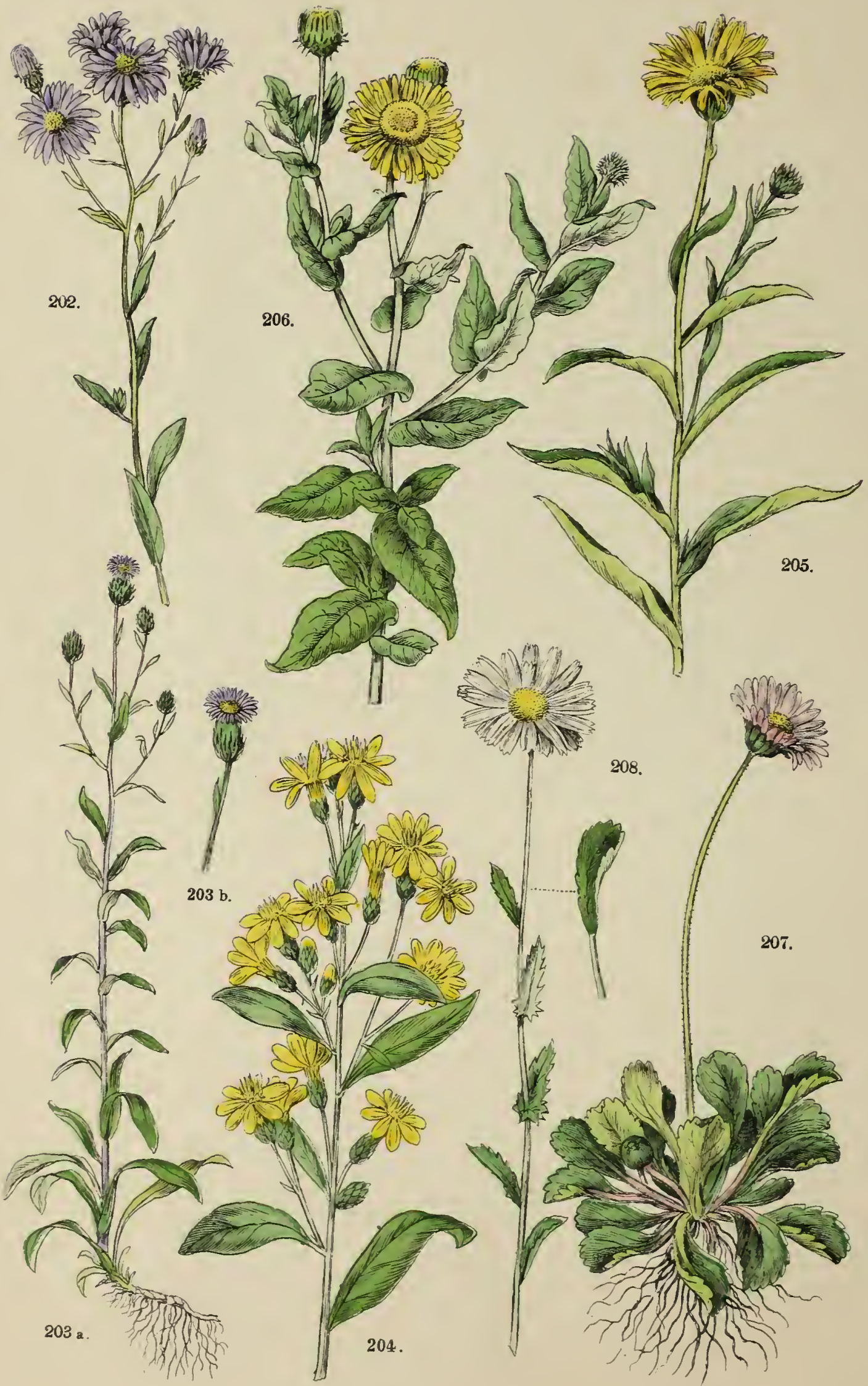


đaศ. 36.

f́iij. 202. Aster améllus I., 2irgir $=\Xi_{\text {teru }}$ blumc. 21. $30-40 \mathrm{~cm}$ hod), wild an jou=

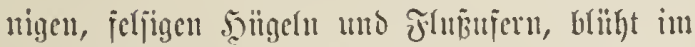

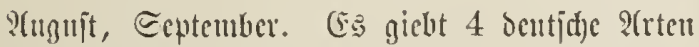

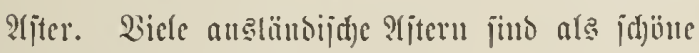

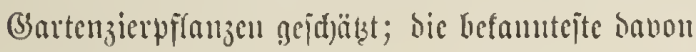
ijt Aster clinensis L.

fig. 203 a. b. Erigeron acris I., Dii $\mathrm{r} r=$ wura, 2icrujafraut. I oice $\odot 15-30 \mathrm{~cm}$ lod), anj janbigen, binrren gilätsen nub Feldern

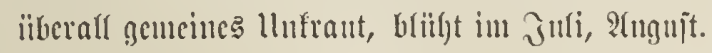

fign. 204. Solidágo virgaurëa L., gemcinc

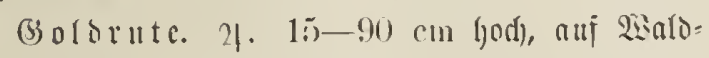

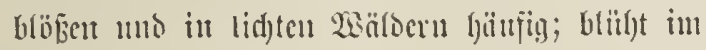

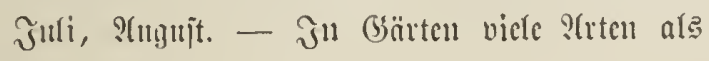
zierpifanzen.

fig. 205. Buphthàlmum salicifólium I.., wci= benblüttriges Ddjenauge, Ninbsange. 4. $15-50 \mathrm{~cm}$ lod), auj begrajtelt Sergab: bängen Eitboentid)lants mo oer Ed)weiz; blitht im : :uģułt, Ecptember. Bsartenzierpilauze.

fị̣. 206. Inula dysentérica I. (Tuliçiria dysent. Grtn.), Rufre? ?(ant, Flolftrant. ?.

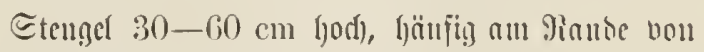

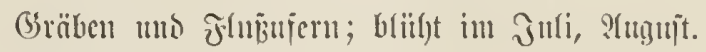
lïbetried)ents, jrïller offizinell.

fị. 20\%. Bèllis perénnis L., gemeines Ma B-

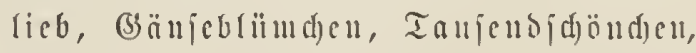
J)arieubliiud cu. 24. 5-15 cm bod), mei bis purpurrot, ibberall auj 2isiejen uno Siajen= Hlätsen; brïht vou Mlärz bis Dftober. Sultivierte

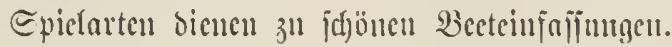

fịg. 208. Chrysànthemum leucintlicmun I. (Leucínthemum rulgare Lamk.), genteine

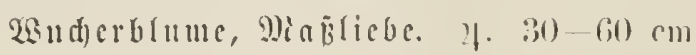

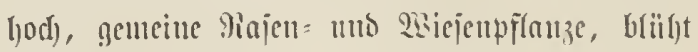

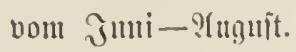


๔af. $5 \tau$.

fiị. 209. Matricária Clıamomílla I. (Cham. officinalis Koch), genteine, edjte Samille.

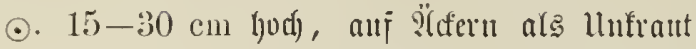

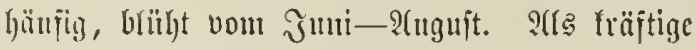
:Tržcipftanze befaunt.

fiig. 210. Anthĕmis cótula L. (Narúta Có-

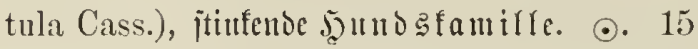
biz $45 \mathrm{~cm}$ hod, gemeines llufrant auf $\Subset$ d)utt

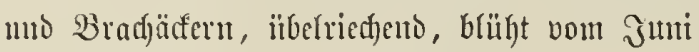
biz Sftober.

fiig. 211. Achilléa ptírmica L. (Ptarmica rulg. de C.), $\mathfrak{B}$ ertram= ober $\Xi_{\text {munp }}=\Xi_{d} \mathfrak{a} \hat{q}=$ garbe, weiser Doraut. 2. $30-60 \mathrm{~cm}$

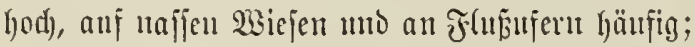
bliiht wom Julitent warest offizinefl.

fiig. 212. Tanacétum vnlgáre L. (Chrysínthemum Tanac. Karsch), gemciucr giniufarn,

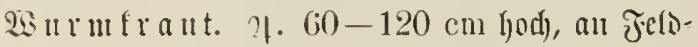

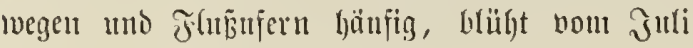

bis Eeptenber. Sirant mo Briüten riedjen un= angenetym, bicuen aber als fräftige $\mathfrak{9}\left(\mathfrak{r z z}_{3}\right.$ tei uno

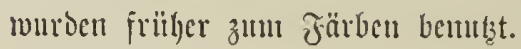

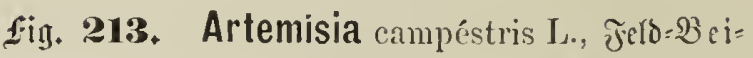

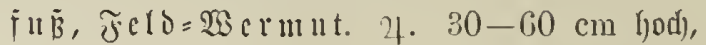

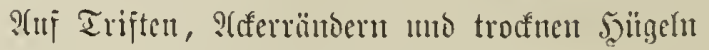

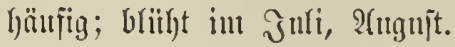

fìn. 214. Gnaphálium dioïcum L. (Antenná-

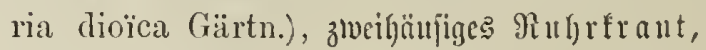

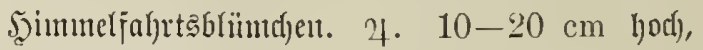
auj Bergwiejen utto in troffuen Sarbungen, auf Sccideboden gemein; brïhlt im Mai, Jumi. -

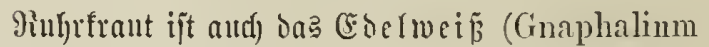
leontopodium Scop.), eine befaunte 5apdigebirggs: pflatze.

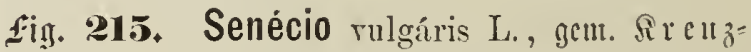

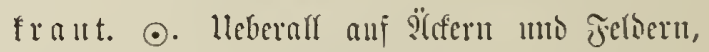
in Gärten tuto auf Edjuttffaujen, $15-30 \mathrm{~cm}$ rod); blïlyt vom Miärz-Dezenber. Selicbtes 2ogelfutter; läjtiges llutriaut. 


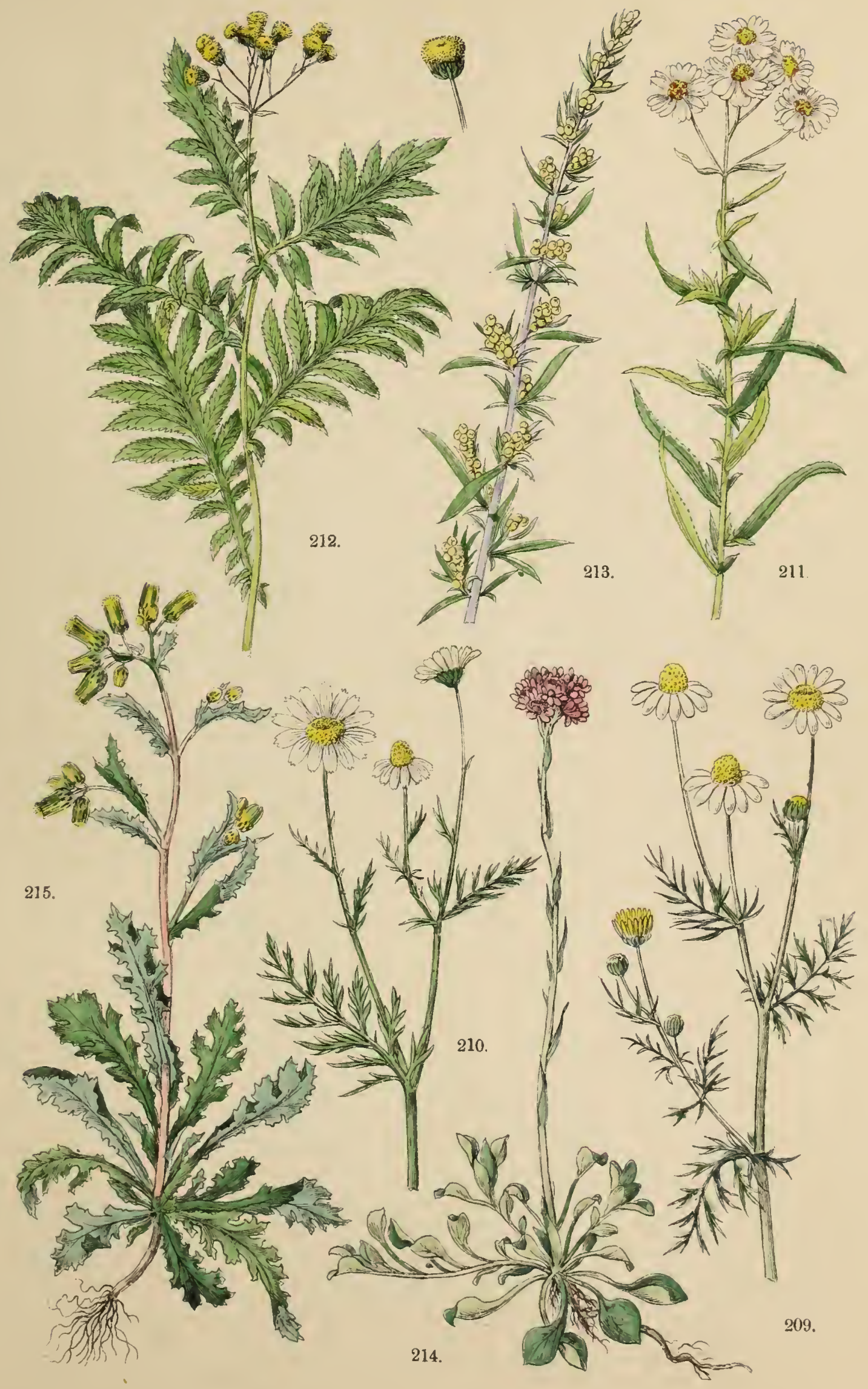



.

. ${ }^{3}$ 
38.

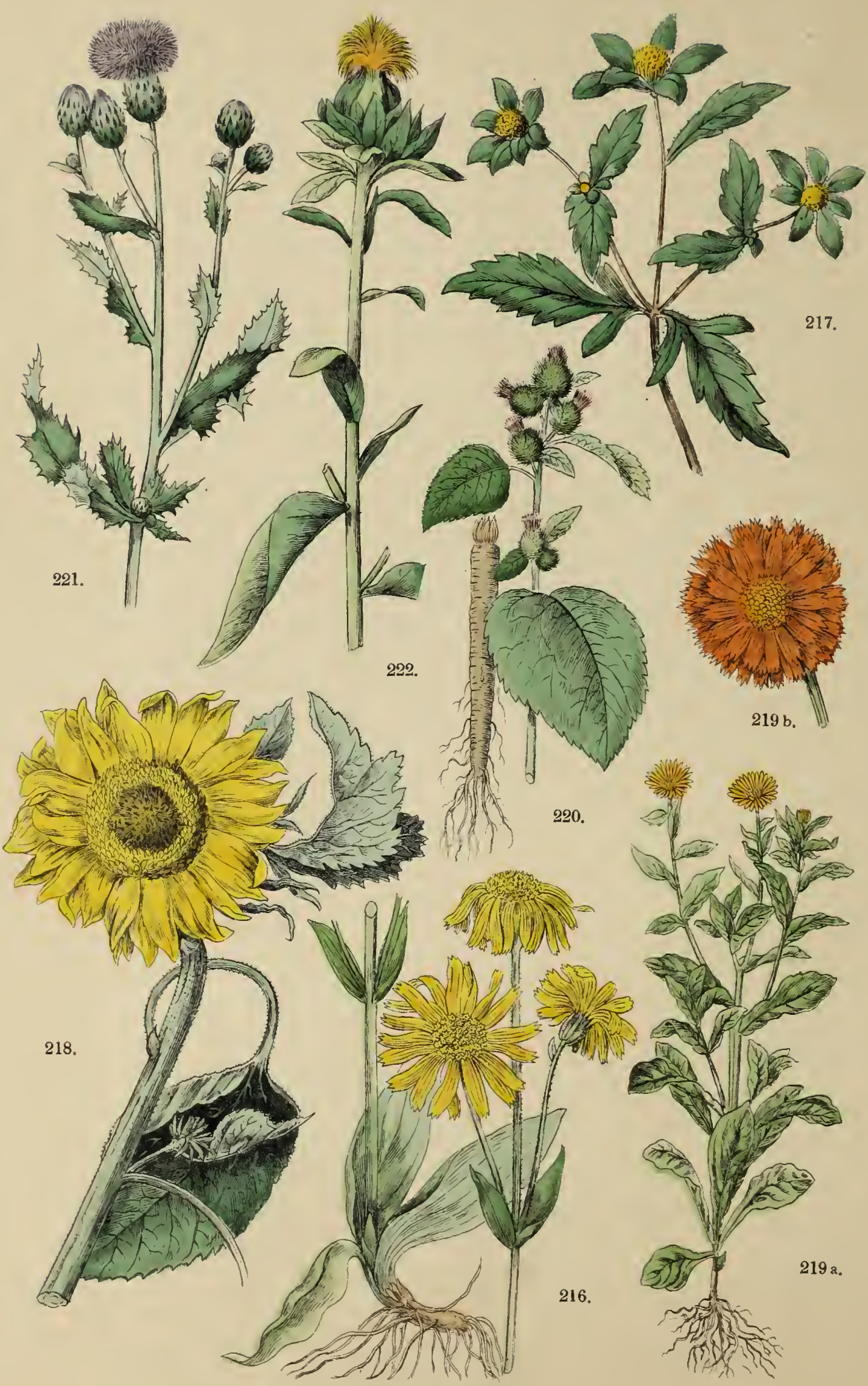


『af. 38 .

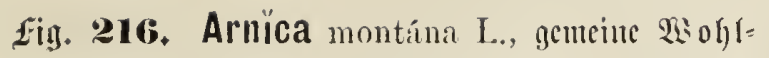
verlcib, Fallfaut, Ẽugelfrant, Marien= fraut, Stichiwuzel, $\mathfrak{B}$ erfangfraut. \%. 30

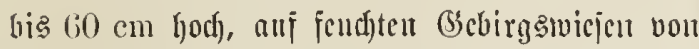

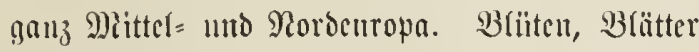

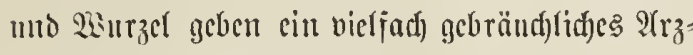
mcimittel; bas Bier) frist dic \$rFlanze nidft.

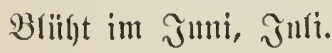

fig. 21\%. Bidens tripartita L. (B. cannabina Lamk.), orciteifiger $3 \mathfrak{w c i z a h n , ~ s i s a ~ f f e r l ) a n f . ~}$ ค. Etengel 10-60 $\mathrm{cm}$ hod, an fumpfigen

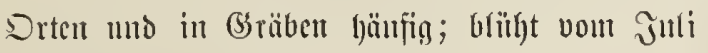
biz Eepteutber.

Eig. 218. Heliánthus amnuns L., gcum. Son= ncuroje, cimjälrigg Sonucublumc. $\odot$.

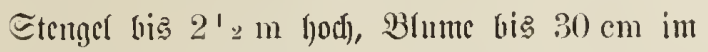
Durdjureffer. Etaumut aus Miçifo unto picru

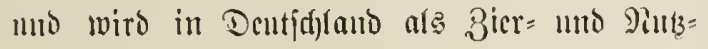
pĩlanze angebaut. Dic Eamen gecben cin fettes Epcije= umd Breınöl, dic Stcugel cin guttes Brenmunaterial, beren 2(jd) reidflids Fottajale;

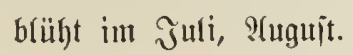

fing. 219 a. b. Caléndula ofticinális L., gcmeine Ringefblume, Totenblume. ค. 30

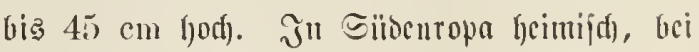

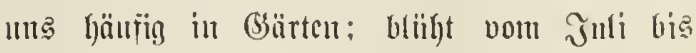
September.

fig. 220. Arctium Lappa L. (Lappa Tourn.), grencine Silctte. $\odot$. Cine ftarfe, $100-120 \mathrm{~cm}$

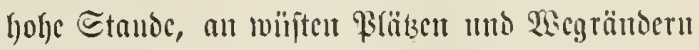

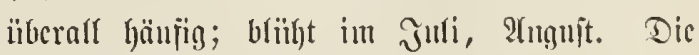

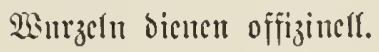

fig. 221. Serrátula arvénsis L. (Círsinm arvénse Scop.), Ferodijter, Fero=siraz̧sijtel, If derbiftel. 2\%. Die genteinite, iiberaft ala

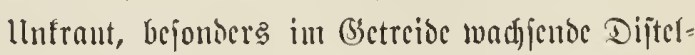

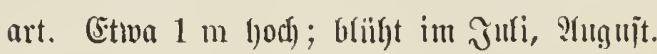

fig̣. 22:. Cárthamus tinctórins L., echter Saflor, gemeine färberoifter, jalfider Safrall, 3affer. ๑. 30-60 cm ryod), ans

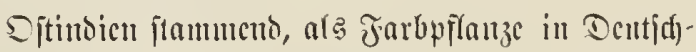
โanto, nauncutlicf) in Thüringen, fnltiviert; blügt im Juli, Ingnit. 


\section{זẫ. 5 g.}

fiig. 2:2:B. Cnicus benerlictus I. (Centanrea henedicta T..), geuciucs Benebiftenfraut. ๑. Etcugel :30 cm lyod), ftaumut aus Eiibcuropa

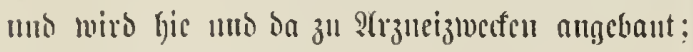

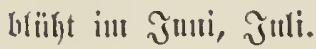

fiin. 2:21. Cárduus nutans L., nicfento Dijtel,

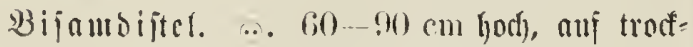
nen Irijten unb an sisegen büupig; briiht itu Juli, s(uggujt. - Dic Difjtelı (Cardnus) zälflen 24 bentidhe ?frten mit viclen Epiclarten.

fịg. 2:5. Onopórdon Acinthium L., gemeine Gielsoifter, Francudifter, Rrebsoijter. ..
Wiro 1-2 m hod) nuto ift in Dentidglano hä̈ufig

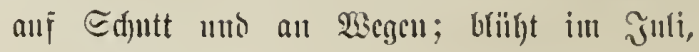
?fuguft. War offizinctl.

fin. 226. Carlina acánlis L., jtengelloje Ëbcr=

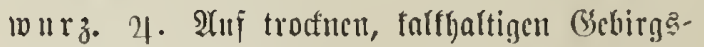
triften zicmlid) bäıfig; briil)t voun Juli-Ecpt. Die 23 urzeln maren friiler offizinelf. Der meižc

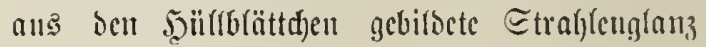
gläızt เoie હif́ber.

fing. 227, Centauréa Cy̆̈nus L., Sorn=

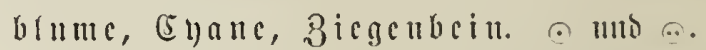
$30-60 \mathrm{~cm}$ hod), ïberall hüufig auj vilcfern uno Feftern; blïht im כ̋uni, Jult. Beclicbte Barten= blume in veridjicsenen gejärbten Epiclarten. 


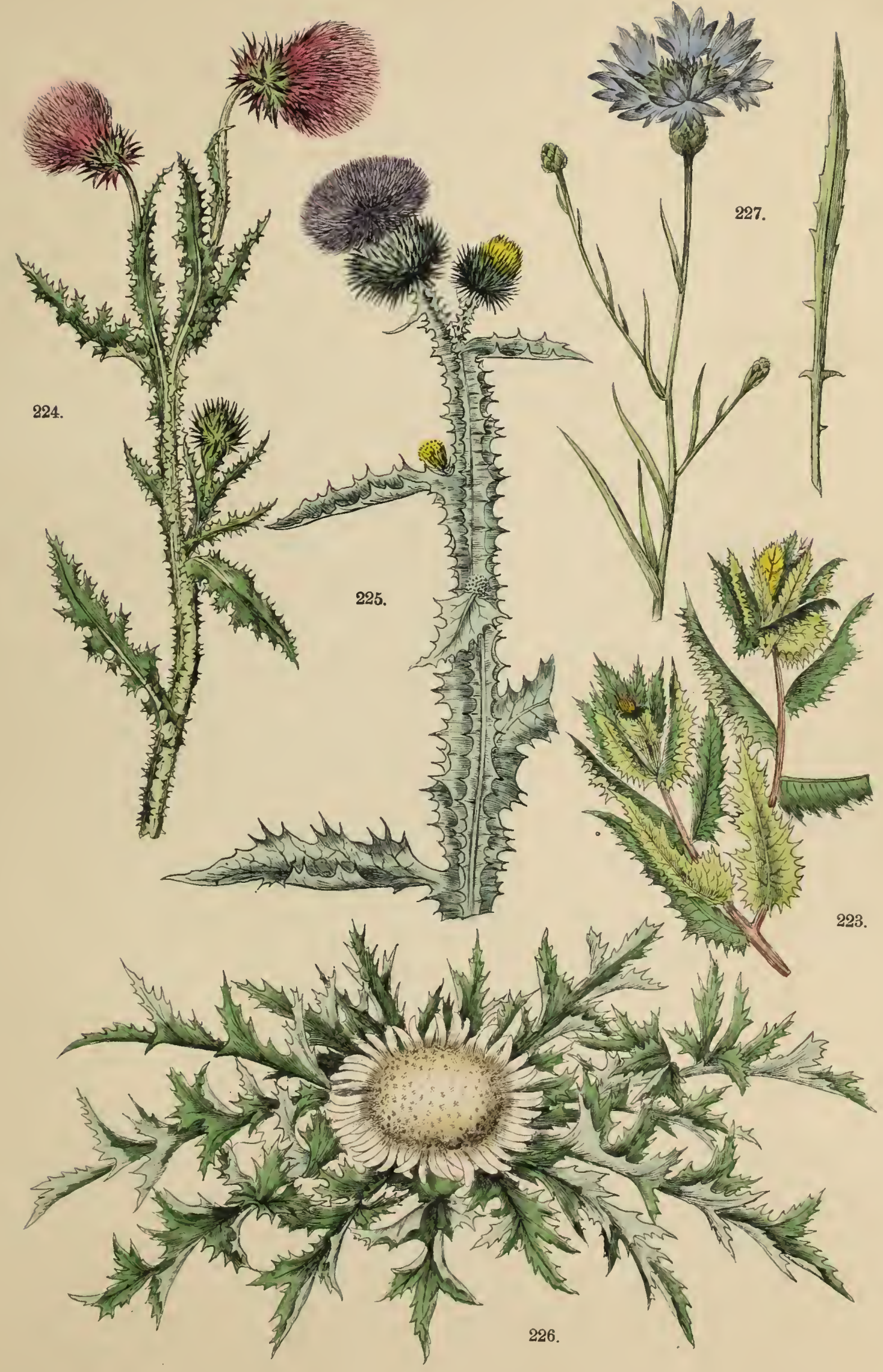




40.

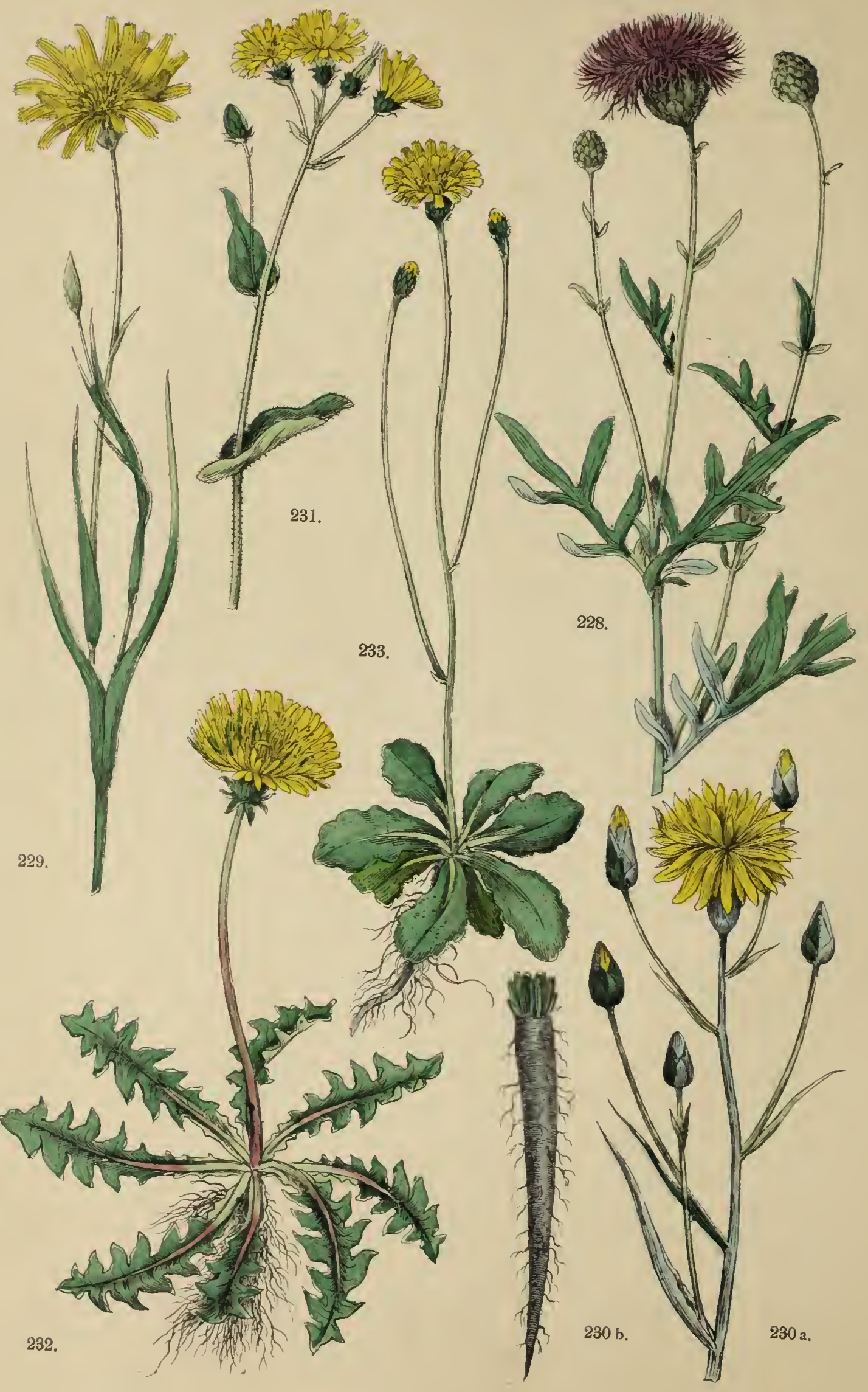


Taí. 40.

fin. 2:28. Centaurea scabiósa Juss., Elabio= jeuntige Flo fecublume. श. $\quad(60-120 \mathrm{~cm}$

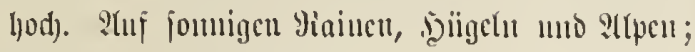

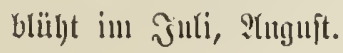

fig. 2:2). Tragopógon praténsis L., ycuciuct Bodfabrt, sisicjeubodebart. ... 30 bis $60 \mathrm{~cm}$ (jod), auf $\mathfrak{B i c j e n}$ uno au 2 segcu, blïht voul Diai-Suli.

fig. 2:30 a. b. Scorzonéra hispánica L., ipa=

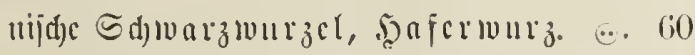
bis $100 \mathrm{~cm}$ lod), anf frudtbaren 2 sicjen mo

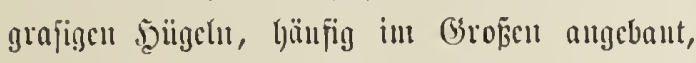
bliilyt iu Juni, Juli. Dic 23 mzel gicbt cincs ber bejten uno feiuften Geutïje. fin. 2:31. Picris hirracioides $\mathrm{L}_{\text {.. }}$, genciucs,

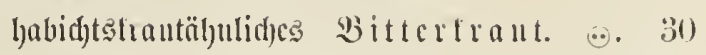

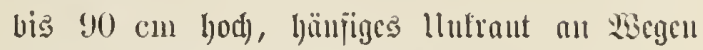
unto auf ricjen; bliitht iu Suli, 2uguft.

fịy. 2:3:2. Leóntodon tarixacum I. (Tarix.

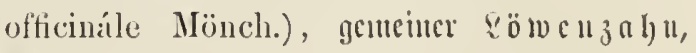

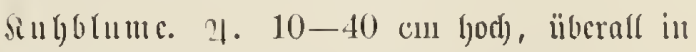
zal)(reid)en Formen nuj 2 siejen, au 2 segeu u. j. w. geunciu; bliilyt vou ?Ipril_Eeptember. Das Sirant ift offijiutell mo giebt, jung und frija),

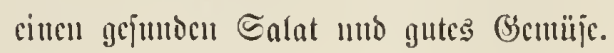

fin. 2:33. Hypochoeris radicata L., Inıgum: lidjes, äjtiges forlelfitaut. 21. 30--60 cm

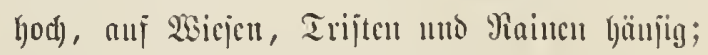
blïlyt iut suli, slugnit. Intrant. 


\section{Taf. 41 .}

fin. 2:3t. Lactuca virósá L., Giftlattid), (siftjalat. ... $\quad(50-100 \mathrm{~cm}$ bod), iibcrall büufigcs llufraut; blïl)t vou JultGifitig, offiziucll. - Der Gartenlattid), Lactuca

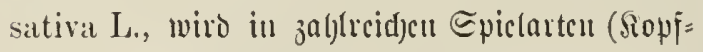
jalat $\mathfrak{u}$. ¡. w.) allycucin anycbaut.

14iy. 2:35. Prenánthes purpúrea L., roter Dajeulattid), Dajenjalat. भ. $60-170 \mathrm{~cm}$ bod), ill juattigen Bergwaloutycu Pittelocutid)=

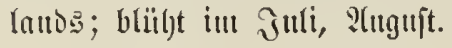

fing. 236 a. b. Chondrilla júncea L., binjent artiger sirülulillg. $\odot .6(0-90 \mathrm{~cm}$ hod), all

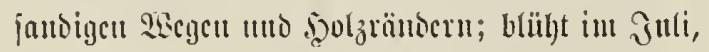
2tugujt. Dient in cinigen vë̈tocru ju હalat. fing. 2:3\%. Sónchus oleráceus I., fohlartige,

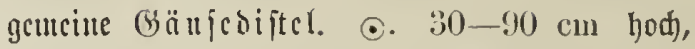

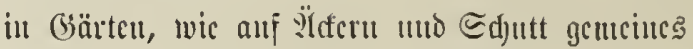
llufraut; bliibt vou Suni-ฮspteuber.

fin. 238 a. b. Crépis tectórum L., Däd)cr= P i p a

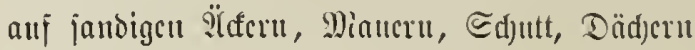
gcuccil; bliibt iu Mai, j゙แni.

fiig. 239 a. b. Hierácium umbellátum L., ১ol=

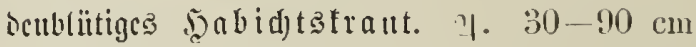

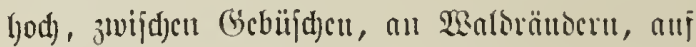

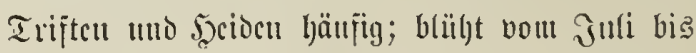
Ecptembcr. - 2on Hieracium unterjd)cioct man

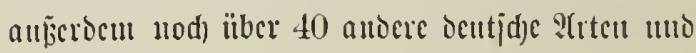
llutcrartcu. 
41.

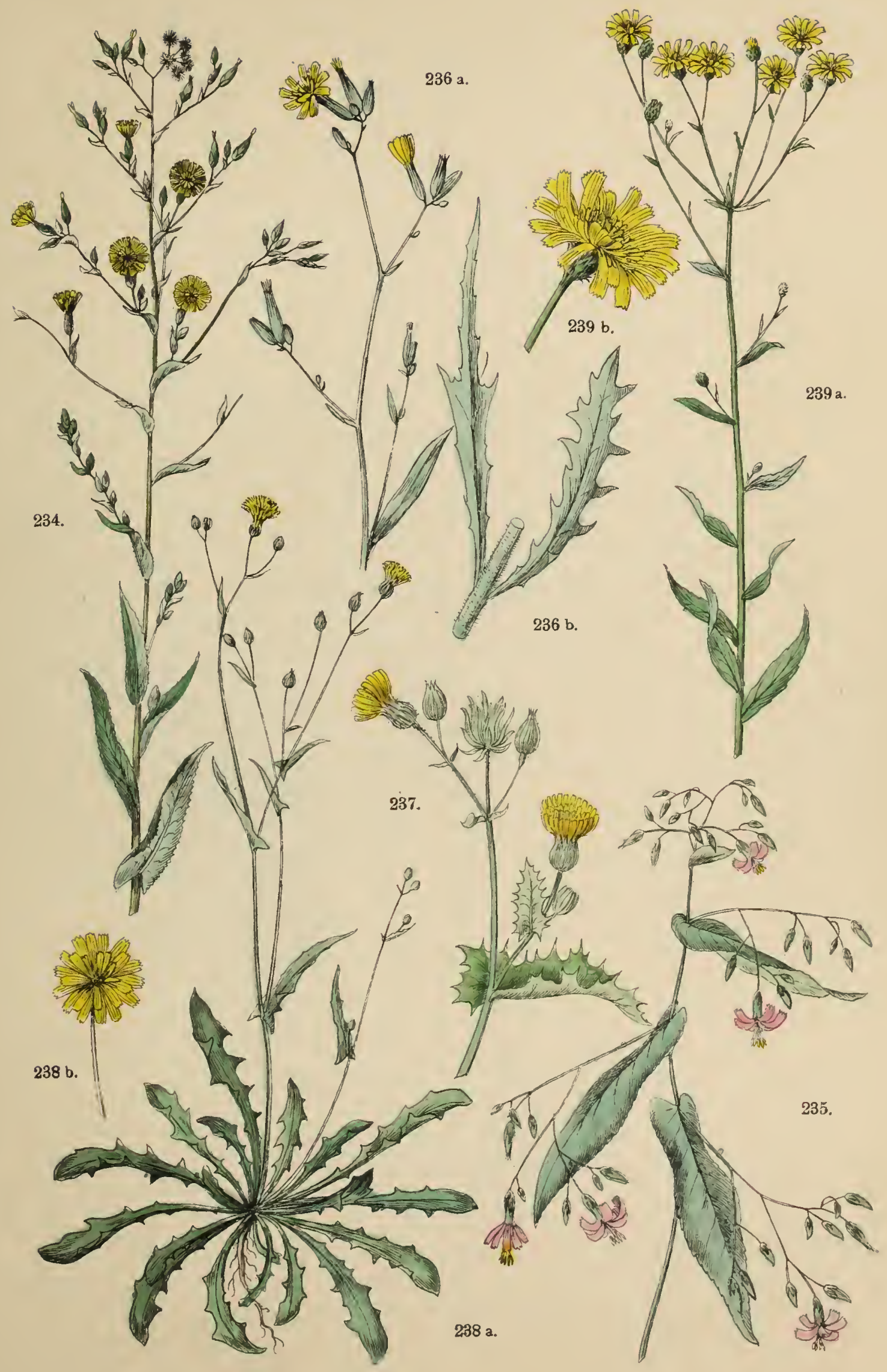





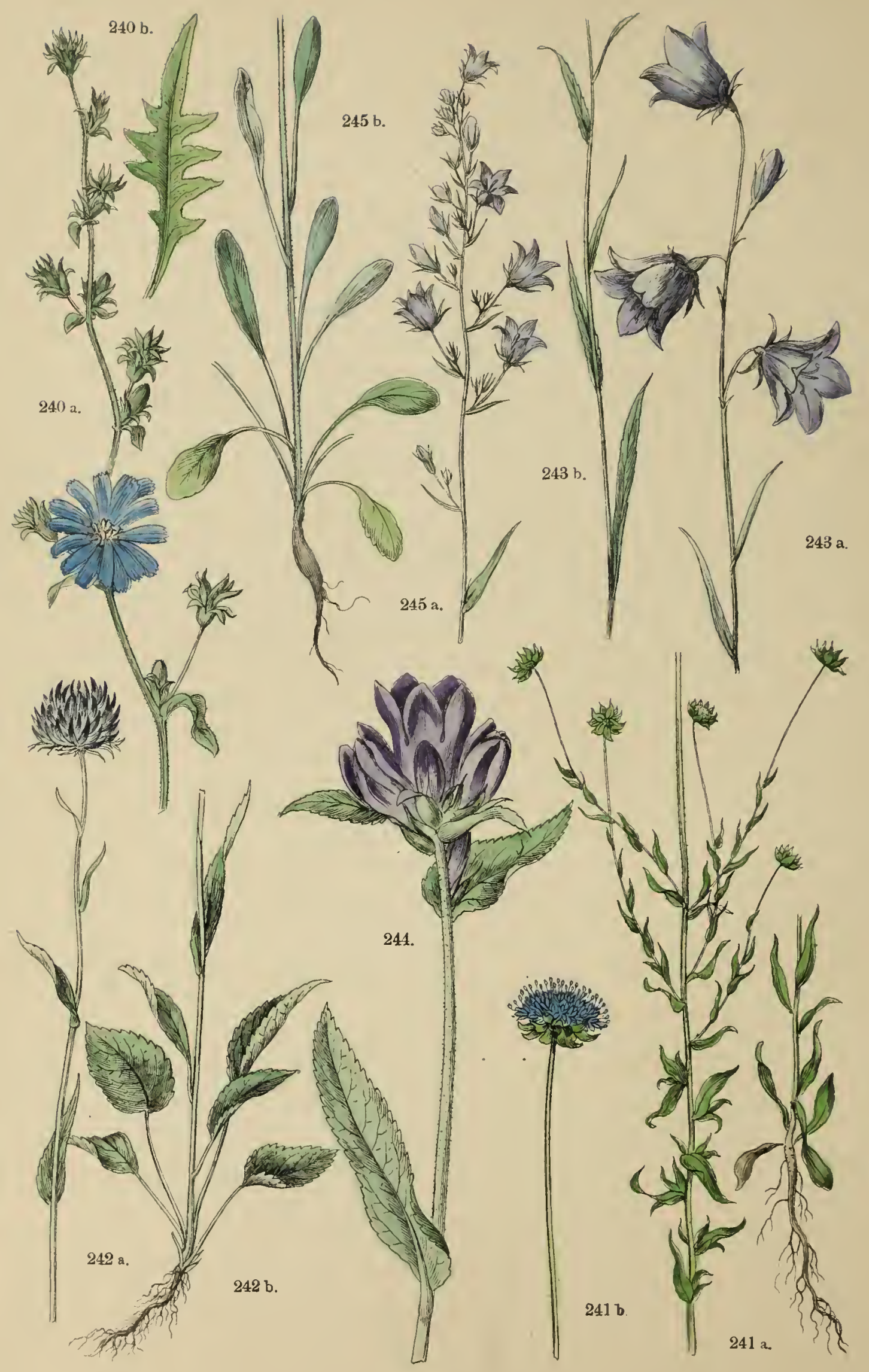


Tai. +2.

fin. 210 a. b. Cichórium intybus L., ge= meine, witoe cidjorix. I vocr ... Etengel 30-100 (mu hod), an Siseycu iiberall häufịg,

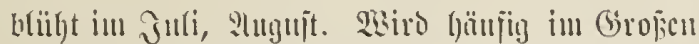
wegen ocr sisurzel, weldye als Siaficcjurrojat bicut, ycbaut. Inz siraut ift cine gute Jutterpfilauze, yiebt aber aud) Gentilje mto Ealat. Dic 2 surzel bicut and) arăbtcilid).

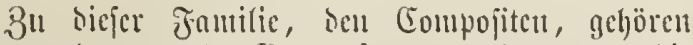
unter allocert aud): Pyrethrum círneun, sie

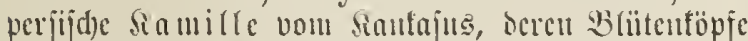

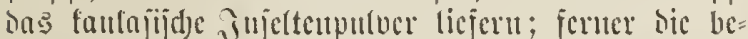
lamute Georgina (Dablic) ates Micrito; Mádlia saltiva, Delpflanze anß céili; Ileliclurysum, Etrobblume; Cinerálial, 壮d)endifanje, mo=

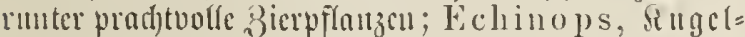

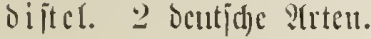

\section{Fantilic. Stylidäate Ri. Br.}

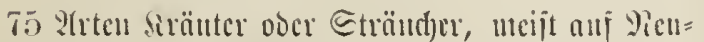
hol(aub bejd)räult, uto wejen ifjer zicrtid)en $\mathcal{B}\{u=$

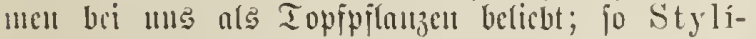

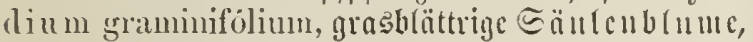
ciue oer jdöujten Bicrpílaujen.

\section{Faunilic. Lobeliácene Juss.}

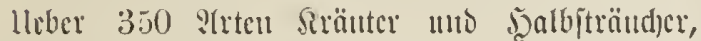
uncijt Gebirgapflanzen ber heifen 3outc. Siele finto

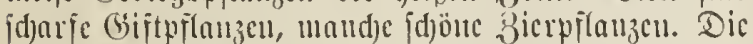
ciujige beutide ?irt: Lob. 1) ortin an 11 a, agajer= lobclie, y, ijt $3(1-60$ cm bod), wäd)it im $2(3$ ifjer

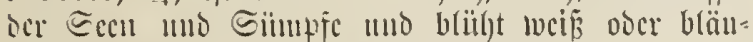

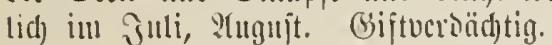

103. Fantifie. Campanulíceate de $C$.

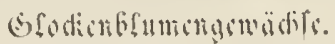

Siräuter, fetten Etränd)er, in etwva +80 S:rten iiver bie gauje Erie verbreitet. Diandhe liejeru Ealat

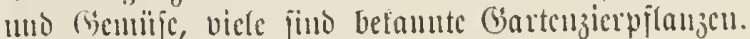
3" iljuen geljören:

fiij. 2\&1 a. b. Jasiỏne montiual L., Serg=

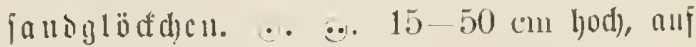

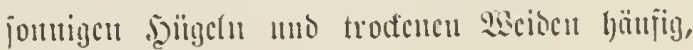
bliil)t vou Juni- Ecptembcr.

fïij. 21:2 a. b. Phyteuma orbiculíce L., rmo= löpfige viapunjel. 2. $15-50 \mathrm{~cm}$ bod), ali Bergwicjen แnto in lidjten seälocru; blïht vom Mai-J̆lli.

fị. 24:3 a. b. Campánula persicifolia L.,

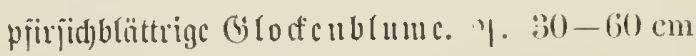

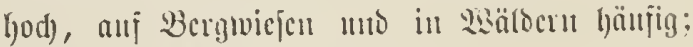
brïlt vou Jun-Ecptember.

fig. 2tt. Campánula glomerátı I., getun̈ıclte Ǵtodecublutc. 2. :30-50 cur bod), auj

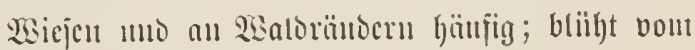
Эแแi-ভcptember.

fiij. 245 a. b. Campánula rapúnculus $L_{\text {.. }}$

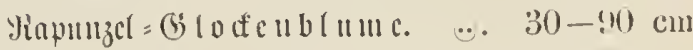

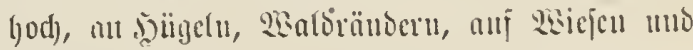

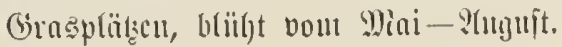




\section{Tạ. +3.}

104. Fandie. Goodenoviane li. lir.

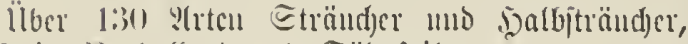
utcijt in Jiculyolfano mis Eiibaprila.

\section{0.). Fanilit. Gesmeríare liich.}

Siräutcr, geyeu 140 : $\{$ rten, meijt iu tropijd)en Stucrifa. 3u if)teu gुelyören Gloxinia, ciuc un= ferer prad)tuolfitcu Topipflanzen uit viefen Epics= arteu. Daun Achimënes, mit iibcr 40 zu bon

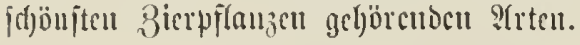

\section{Frmilic. Vaceiníeac de C. (Siphonan-}

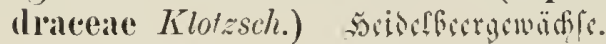

l̈lber 150 ?(rten vou Єträıd)cu, meijt scr nörob=

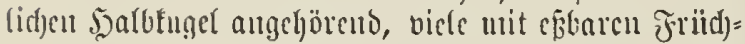
tcu, barmut für utand)e ?äubcr ljöd) ft midjtig.

fiig. 216 a. b. Vaccinium myrtíllus L., ģe=

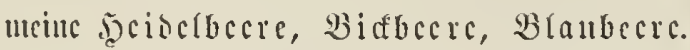

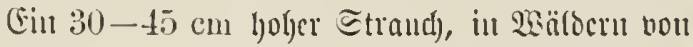

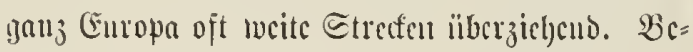

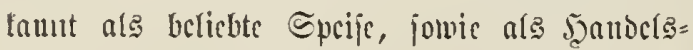
unt 2 trüucipfíanze.

fig. 218 a. b. Vaccinium uliginósum L., Siaujdberc, Sumpj=ineiberbere, Wiorapt=
Defoctbere. Eill auf Torjmootell uno in fend)ten $\mathfrak{B} c r g$ waloungen geutciucr Etrand); b(iil)t

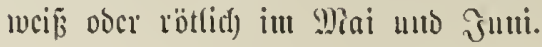

fiin. 218 a. b. Vaccinium vitis idluea I.,

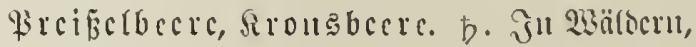
aแj ,̧ecibcı und Gebirgen ljäufig, beïlgt nom

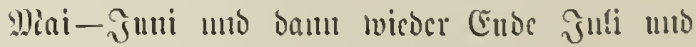
:(ntgut. Dic Becren werben 3u nortrefffid)em Sompott cingculad)t.

fịy. 219 a. b. Vaccinium Oxycóccos L. (Uxycóccos palustris Pers.), Miooz becte. D. Sn Torjiümpfen Ploroentopas uns anj ben

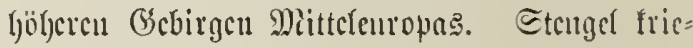
d)ent; blüft bou Miai-2(ugutt. Dic roten Becren wer'oct cbenfalla cingemad)t.

fiị. 250 a. b. Arctostảphylos Uva ursi Sur..

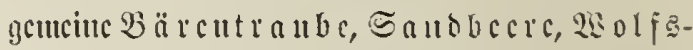
bectc. (Eiu $30 \mathrm{~cm}$ yoher Straud) all fe(jigen,

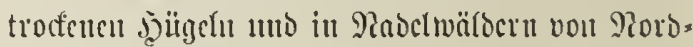
uto Mittelcuropa; in Norbocutid)(ano jtrecten=

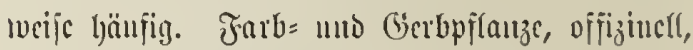
beiilyt iu Miai uno Suni. Dic mchligen B̧cereu jitto umidjuacffjajt. 


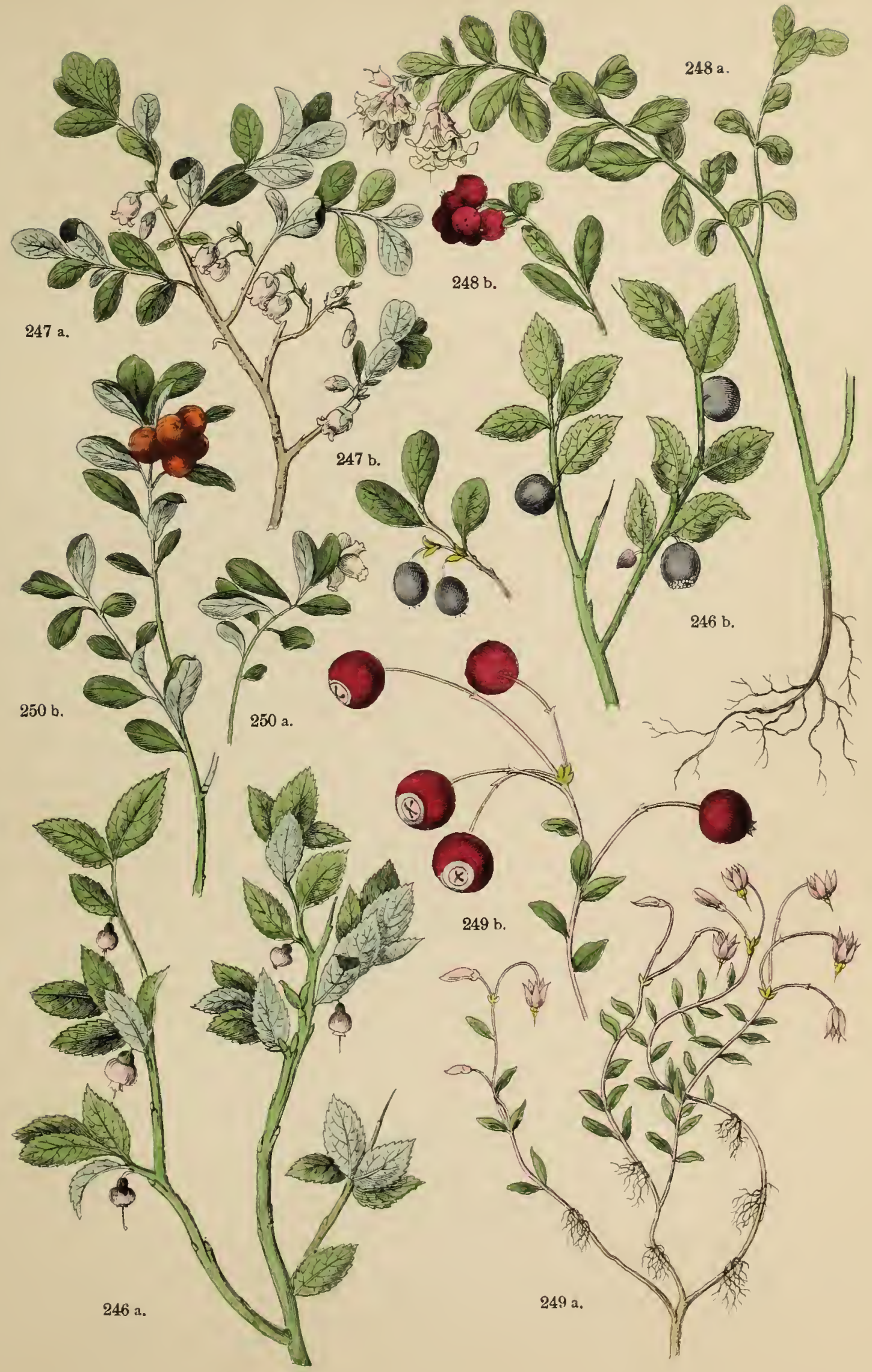





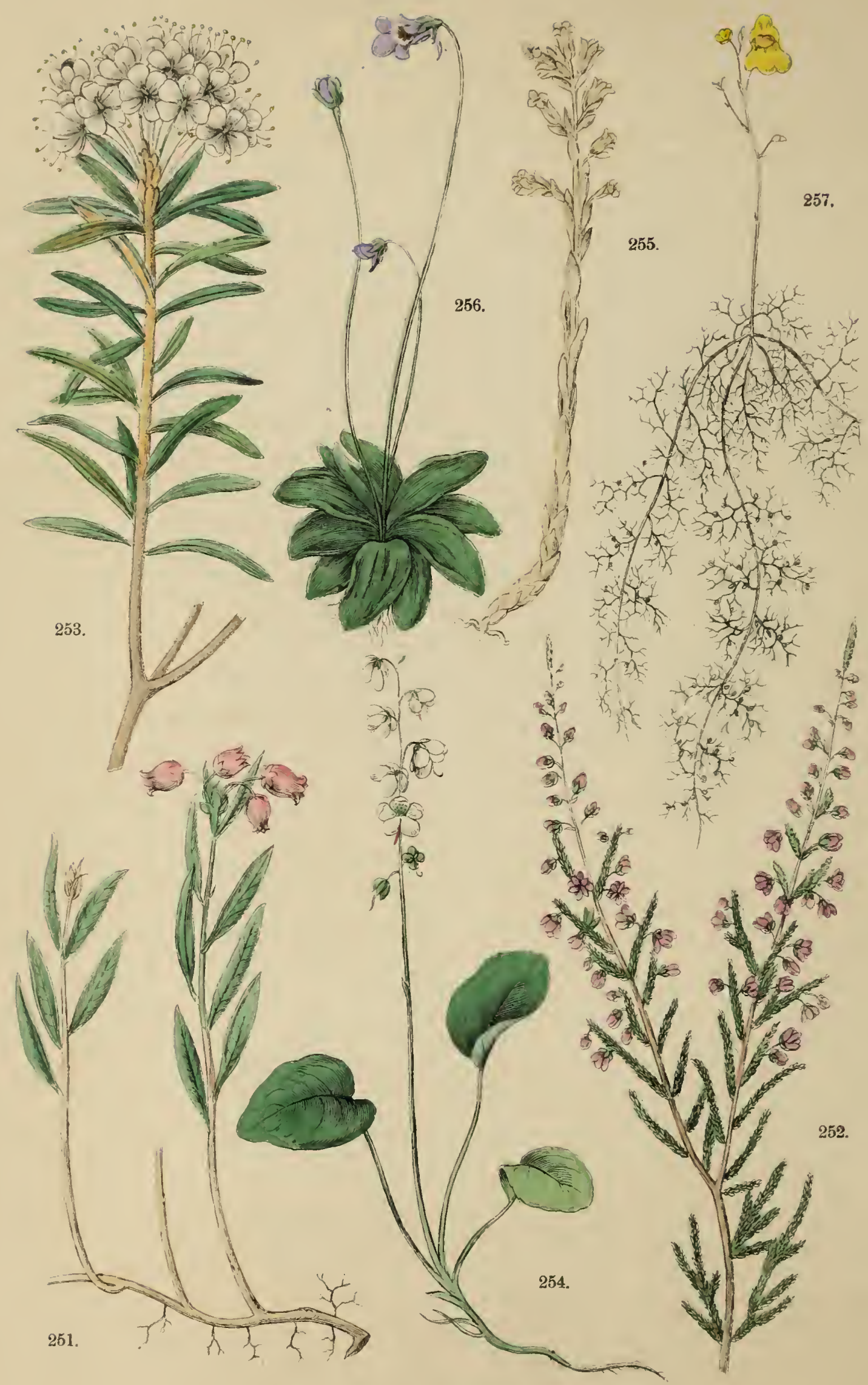


Taf. 4 .

fị. 251. Andrómeda polifolia I., gemcinc,

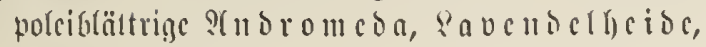
Rosmarinbcioc. $5.15-30 \mathrm{~cm}$ lod , nics ocrlic̣cent, in Torfmooren nub Mioräîlen; briilyt

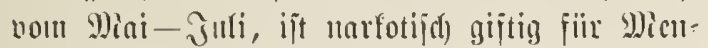
id)en mono 2ich). (Berb= mot Farbpflanze.

\section{Fantilic. Erícence $T_{\text {. }} B r$.

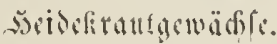

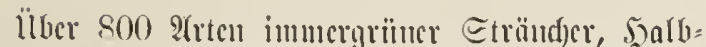

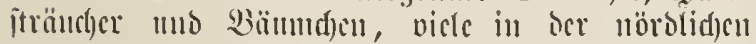
falten 3oure, bie merijten aber anj oer Eibojpibe

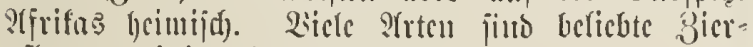
pflanzcu, cinigge jüno offizincfl.

fịn. 25:2. Erica vulgáris L. (Callúna vulg. Salisb), gemcine .5cioc. Gin 30 bis $60 \mathrm{~cm}$ bober Jealbitraud), iiber gauz Esuropa ver(treitet, in Dentidjlano anj trocfucm, jandigem 30 ocn ģcutcin; briil)t voun Jufi-Ecptembcr.

fiig. 2.53. Ledum palústre L., Emmpi = \$ㅇorit, Silenporit, willoce giosmarin. $5.60 \mathrm{~cm}$ bis $1 \mathrm{~m} \mathrm{lod}$ ), anf Torfmooren, in Rorowejt=Dentjd)=

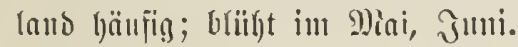

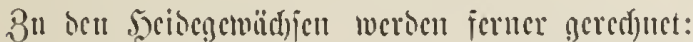

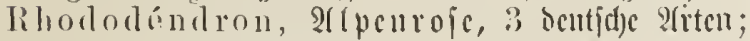

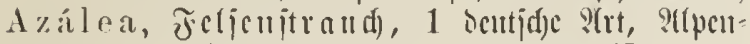
pilauze, zaflrcid)e ?trten als Bicrpftanzen; Kólmia.

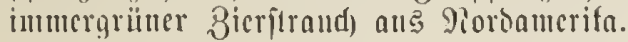

108. Fanutlic. Epacrídeac R. $\mathrm{Br}$.

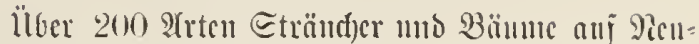
loollans, bort bic isjelbjt fellenten fycisenartigen

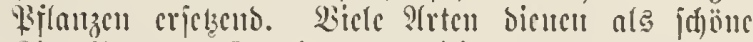
Bierpflauzen. 3" bicjer Jamilie red)uet man muter altocru: Leucopógon, Epäeris, Styplólia, Astroloma, Cosmbia, Anrlersonia, fämt= (id) in 2iculyolfanto.

109. Fannilic. Piroláceac Limell. Asinterturitr.

7 beutjd)e, $8-30 \mathrm{~cm}$ (job), imuncrgrïue firünter.

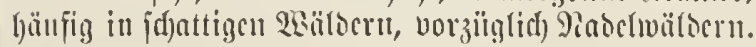

fing. 251. Pirola rotunclifolia L. (Theláia rotundifolia Alfhl.), rmubulätriges sistutcr=

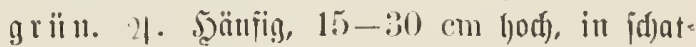

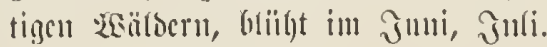

110. Fantilic. Monotrópeae Nutt.

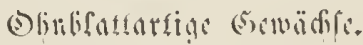

6-7 9(rten (1 bentjd) 9(:t), anj icu sisurzefu anderer \&iflauzcu wathjenter Ed)marotscrfrünter.

fing. 2.5.5. Monótropa hypopitys I.., Edumcr= murz, sisaromurz, Fid)tenfarger. \%. 12

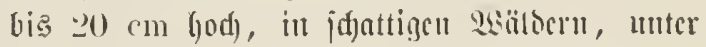
2isalsbänmen, in haffoverwejter sauberte, bliilyt inı Juli, จltgurt.

\section{Lnterflaffe: Corolliflórae, fronenblutige pflanjen.}

111. Fomilie. Lentibulariae Rirh.

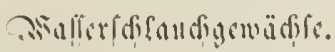

(B)egen 100, iiber bie ganze Eroc, vorzïglid in

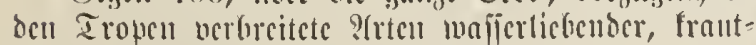
artiger Piflanzent. 3"1 ifjucu gechört:

fin. 256. Pinguicula vulgíris L., gentiutes Jettfraut, 1. Edapit 5-12 cm hod), auf

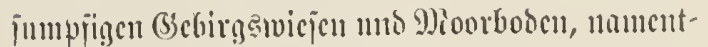
(id) im lyoben शiorocn; briil)t im Miai, Jumi.

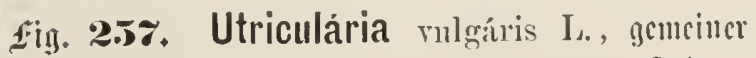
wa afjerjolstand). \%. 2isafferpflanze in Teid)en

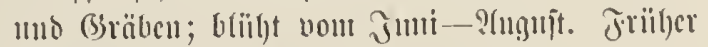
ofijizinclf. 2ion I'triculária girbt ces 3 icutfde Afrten. 


\section{Tậ. 4.5.}

112. Familic. Aquifoliácease de C. stafiparmatgenäsile.

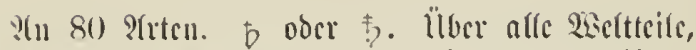

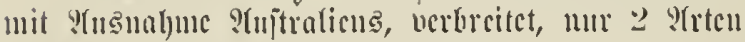
in (Emropa; cinc in Dentjalanto.

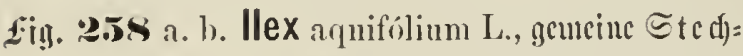
palmc. ந. 1-4 m hod), in (Sicbirgąuälocin

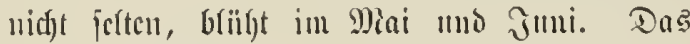

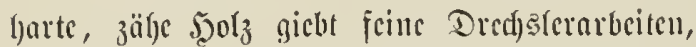

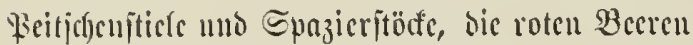

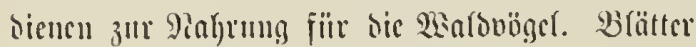
uno sinto jind offizinell.

113. Familic. Primuláceas T'mt.

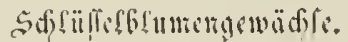

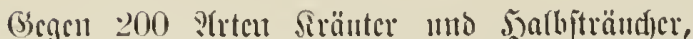
ïber bic gauze Eroc verberitet, vicle burd) Edjünfseit orr Blïten ausigezeid)uet.

fịn. 25.). Primula farinósa L., bcitüubte, mcl)=

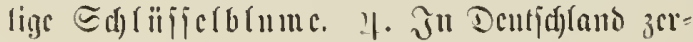

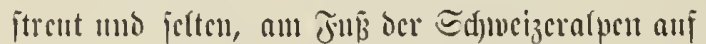
torïigcu, fend)ten wiejen fuäufig, $10-20 \mathrm{~cm}$ hod); b(üf)t vom Jumi-Inguit. fig. 260. Primula Anricula I., ârifel,

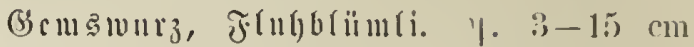

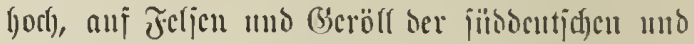

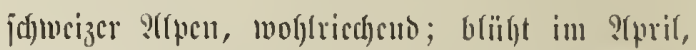

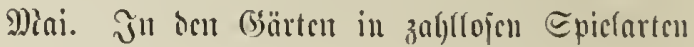
fultivicut.

fịn. 261. Soldanèlla alpína L., ?(rpen=D rottefblume. 2f. $10-15 \mathrm{~cm}$ fod), f)üufig auj

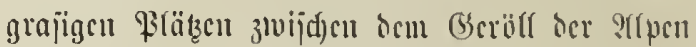
Eiibocntiflattos mo oct Edjuciz; bliift im

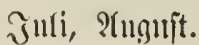

fig. :62. Cýclamen curopacum L., gcucine

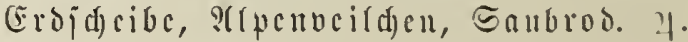

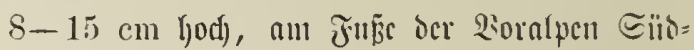

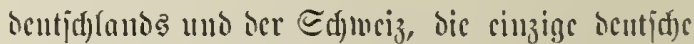

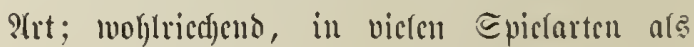
Bartenzieryftanze belicbt. Der rohe simzelitocf ift fd)ari giptig, foff aler geröjtet cine gejundo, angencf)m idjmedentoe Epeife geben.

fiug. 2063 a. b. Lysimáchia mulgáris L., gcmcinet Jricolos, Gilfuciscrids. $1.60-120 \mathrm{~cm}$ yod), ïberall an fentfen Srten, anf 2isiejen mo

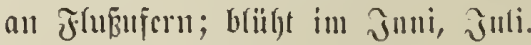




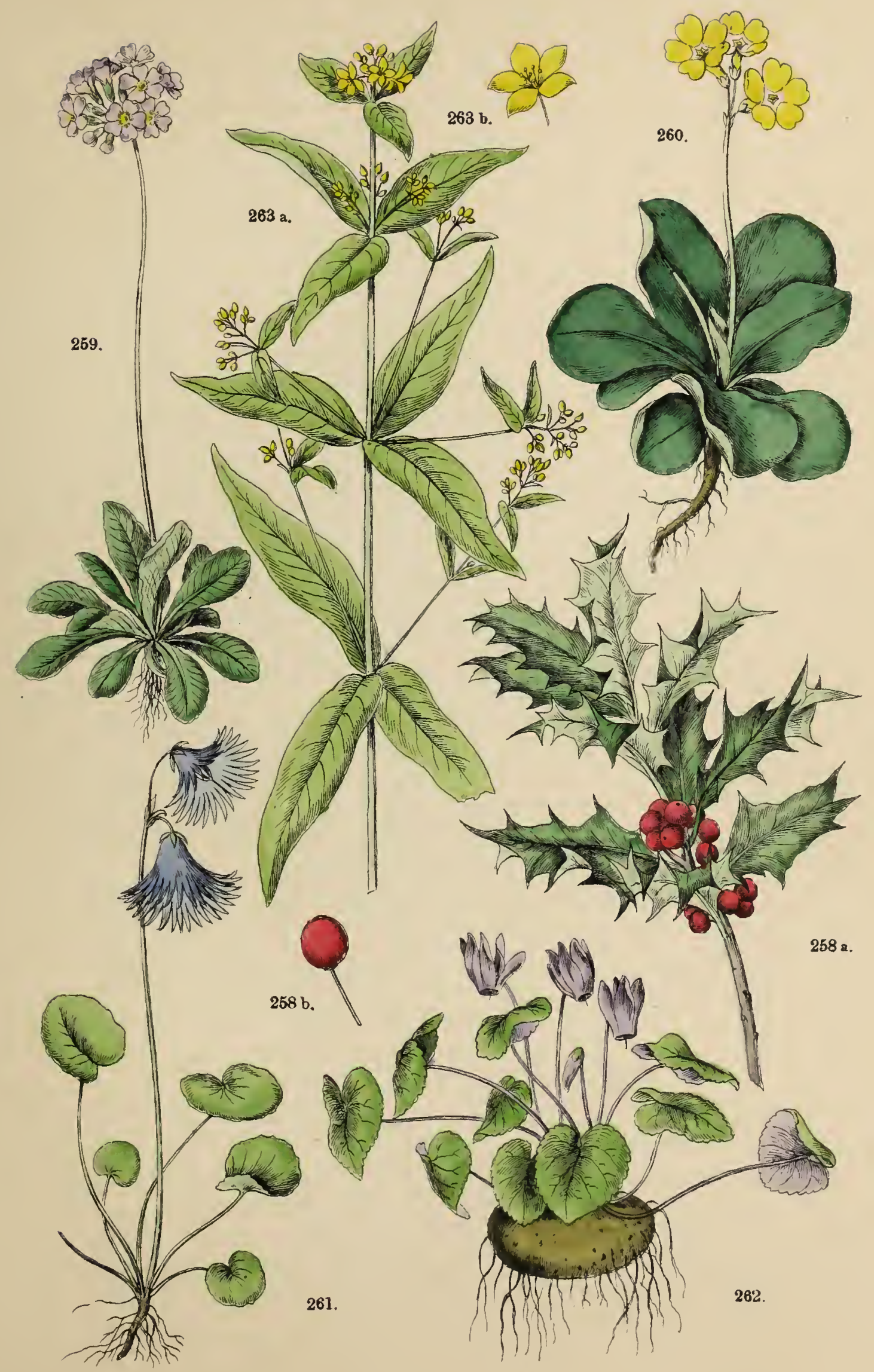






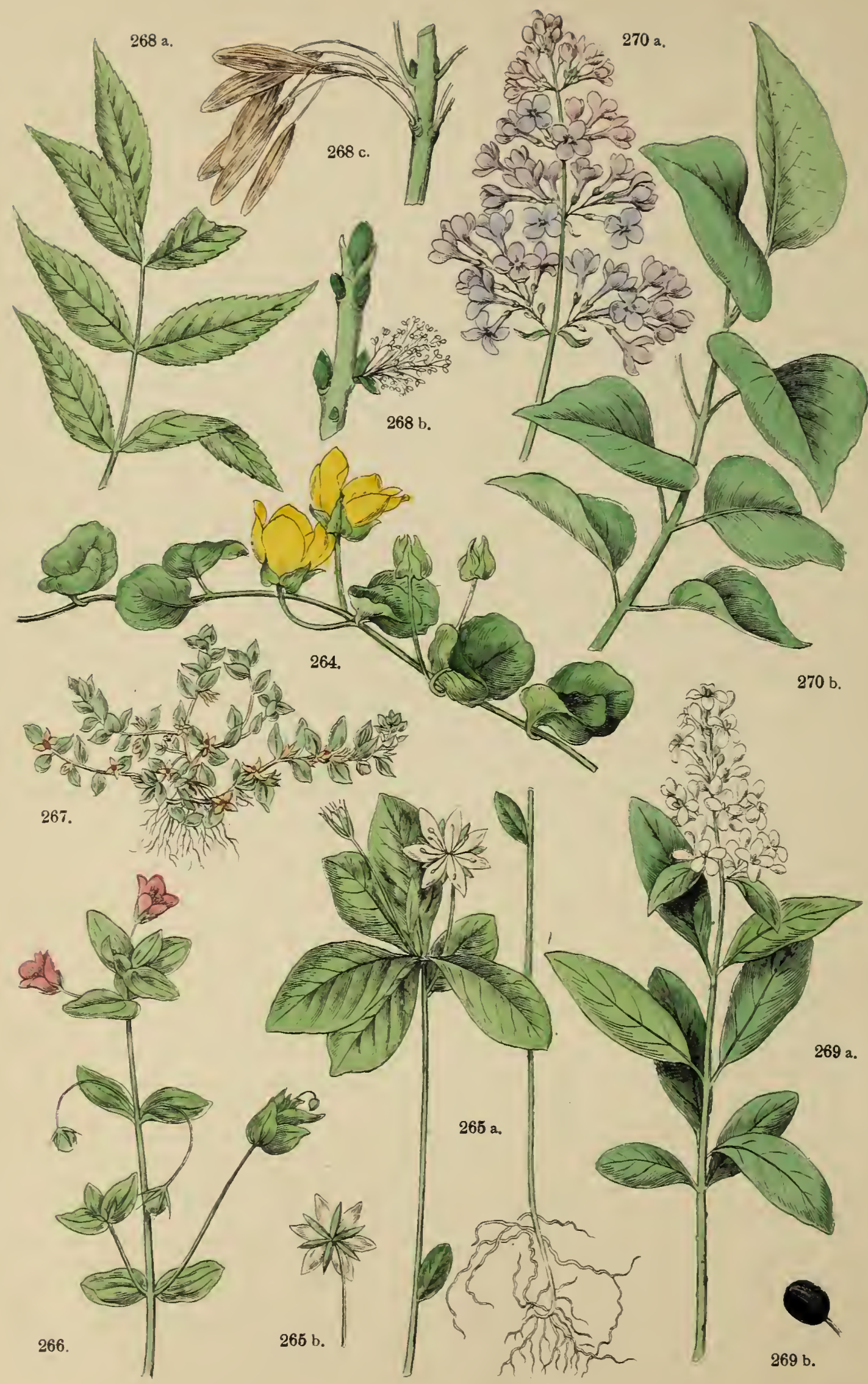


דaf. 46.

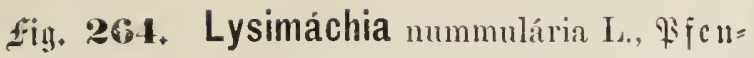
nigfrant. 21. Gemein an (sräben, in fend)ten

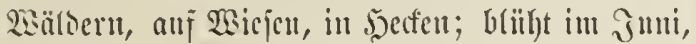
$\mathfrak{J u i}$.

fiij. 26.5 a. h. Trientàlis europaea L., gem. Sicbenfern, Dreifaltigfeiteblïmdicn. ?. Etcungel 8-24 $\mathrm{cm}$ hood), in feud)ten (Jicbirge

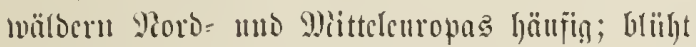
vou Miai-juli.

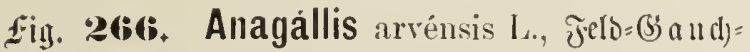

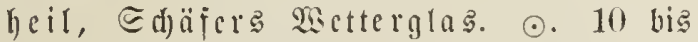

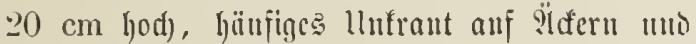
Brad)en; bülyt vou Mai-Ecpt.

fig. 26ry. Centünculus míninus L., Tder:

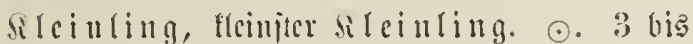
$10 \mathrm{~cm}$ (yod), als ?tefcrumfraut auf uafien, jan= Digcu Etclleu, zerïtrent; bliilyt iu Juni, Juli.

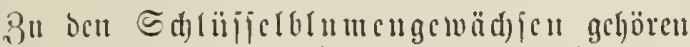
jerner Andrósace, Miannżdilo; Glaux, Mild ffraut; Sámolus, Bunge; Hottonia, aisajericoer.

\section{Fanuilic. Myrsíneae $R, B r$.}

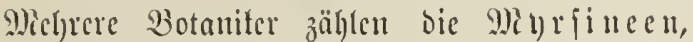
wie and) bie Storadpflauzcu (117. Jamilic) แmo (E⿹ blumen, anterc behanbeln fie ale felbjtänbige Familien. Es jïto tropiid)e Strüud)er ober $\mathfrak{B a ̈ u m c , ~ w e l d ) e ~}$ fid) von ben Brimeln jajt um baomrd) unterjdeiben,

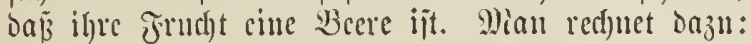
A refísia Sw., Spizenblume; Jacquínia Jacy., Frmbanobaum; Maesa, Myrsíne, Legiceras mo amore Tropengcmädje.

\section{Familic. Sapóteae R. Br.}

Etma 90 STrten meift tropija)er Sträud)er mo Bäume, von bencu viele fettes Eamenol mo wohl= (d)medfenoe Früichte liefern, antore jid) burd) bauter= haftes 5olz anszeidnen. 3n ilnen geljören: Achras sapota L., 23reiapfelban m in Sïimerifa; 13assia, Butterbau it Ditinbien; Isonandra gutta Hook; (B)uttaperd)abaum iu Siam 11. a. II.
116. Familic. Ebenáceae Juss. EGuthorser.

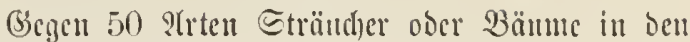

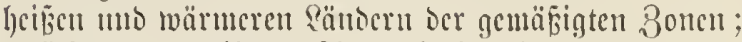
Jrïd)te ier meiften cijbar, 5yols idfwer unt hart. Dic widytigiten ?(rten jint: I)ióspyros lótus $L_{\text {., }}$ Dattchilanduc, Rotusbiruc, cin 9 m hoher Sanm in 2iorbajrifa 1 b icm Sricnt, mit $\mathfrak{c} \tilde{b}=$ baren Früd)ten und guttem, feiten .5ol3; Liosp.

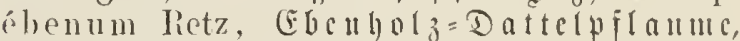

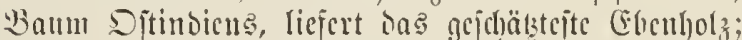
Maba ébenus auj ien Mioluffen, licjert cbenjo vorzïglides eseculfolz

\section{Tramilic. Styrácene Rich. Storaxgomäde.}

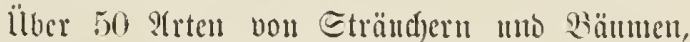

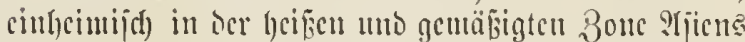

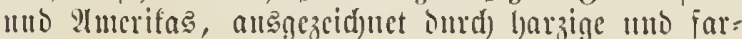
bigne Etoffe. Bul ihnen gebören: Styrax L., ङto= raxhaum an Wittefmect mo im Dricut; Ben\%oin, besuzocbaum im tropijchen \&lijen.

118. Familic. Oleíneae Lim7. Ës6annamäfle.

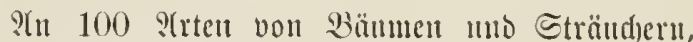

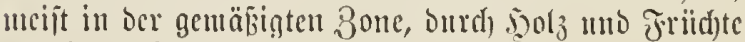
uïlz̨lid). 3n bicjer Jaunilic geh)̈ren:

fiì. 268 a. b. c. Fràxinus excélsior L., ge

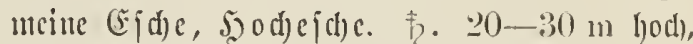
in gauz Emropa mo Sioriajien verbritct, als 3icrpflanze bei me in vielen Epiclarten (T ra a er: ejd)e, (ssolbejde) beliebt, blüht im S(pril แnt Miai. Dą Jjofz geljört zu den feinjten Tijd)ler=

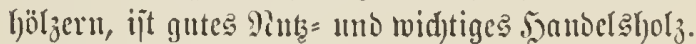

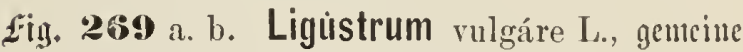
gatumeide, Jeartiegcl, Tinteuber= ftraud). 万. 2-:; m hod), in (Ëropa, 2ioro= ajien uno Rorbumerifa iiberall in Seefeus; blïht im Juti, Juli. Die Beeren bienen zแแ J̈̈̈rben.

3n den S̈rbammgewädjejen gefören ferncr: Olea enropaea L., gcmciner S̈ ans Defien Frïdten (ben Sliven) ias Sriucu= แnto

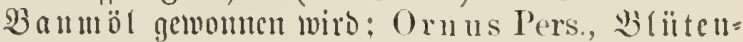
ejde, Miannaejde, icren Miildjajt bas Mianna ber Ipotheten liejert.

fin. 270 a. 1. Syringa vulgáre I., gemeine Si)ringe, tïrfiface ober fpanijder folicoer, Eilaf, fül(j)lid) Dolunber. D. Beffamter 2-6 m lyoher Bicrftraud); blïht im Mai, Juni. 
Taf. 47.

\section{1\%. Fumilic. Jasmíneae li. Mr. sismilitgumetfe.}

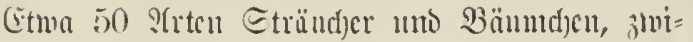

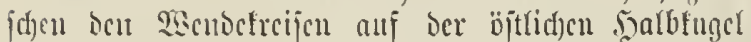

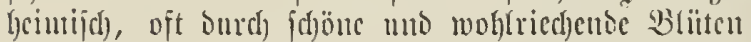

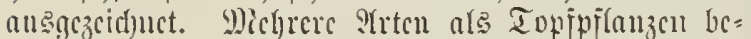

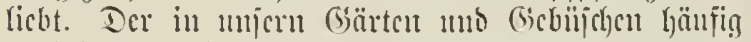

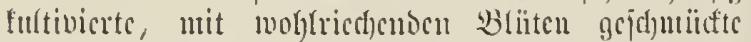
Etrautd), weldyen wir jasmmin ncuncu, ijt fcin Jasmin, fonocit Philadelphus coronarins L., Fifcifenjtraud). 3u beu Jasmincu ge= fö̈ren Jasminum offic. L., gemeincr jagmin aus Eutopicu mo vyctinthes I., Traner= ba $\|$ m it Sitnolicn uno im Sricnt.

12). Fantic. Apocýneae R. $B r$. Sumosgiff-

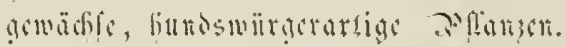

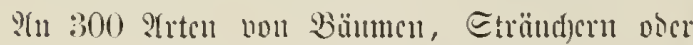

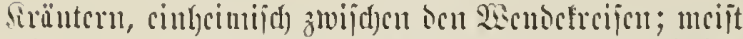

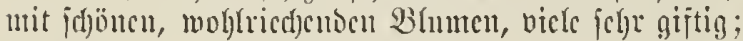

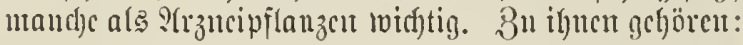

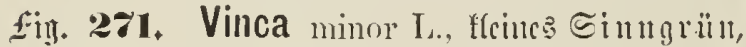
q2intergriin. 2) Ein $15-30 \mathrm{~cm}$ langer,

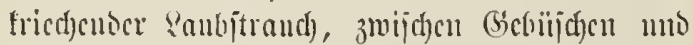
an itcinigen \$s(äżen, and) als Bierpiflanze in (siürteu beliebt; bliil)t im ?(pril, Miai.

fiij. 27.2. Apócynum androsaemifolium $T_{\text {., }}$

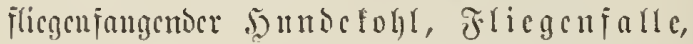

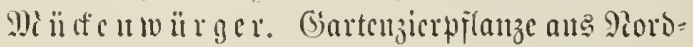

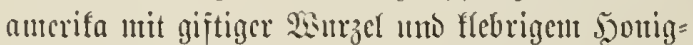
jajtc iı Der 2 liute.

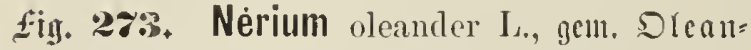
ocr, Mojenlorbecr. Cein bamtartiger, 4 bis $5 \mathrm{~m}$ holjer Bicritrand) anz Eitoenropa, oejien cinzelne Icile jümtlid) cin j(harjes (sijt enthaltent.

fig. 274 a. b. c. Strychnos Ignátii Ber'g. (Ignitia amára L.). Etrandy anf Den Figilippincu, mit jegr gifitigan, aher difizinellen Eamen. Die

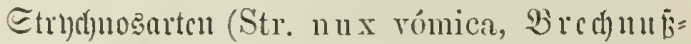
baum, sirühcuangenbaum in Ditittoien Str. Tioutr, llpagituand in ben mälocru
Javas - Str. toxifera, Curarapilanje

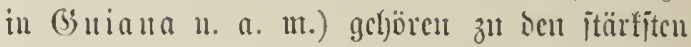
(Siftpillanzen, bicuen aber zuglcid) ale fräjtige jeilmittcl.

\section{Familic. Iscepiadrar Ti. lir.

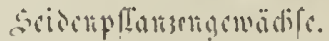

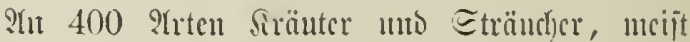

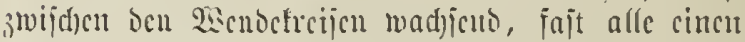

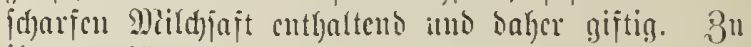
il)nen geljött:

fig. 28.5. Cynảnchum rincetóxicum R. Br. (Tincetóxicum officinále Mnch., Asclépias

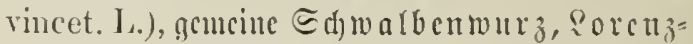

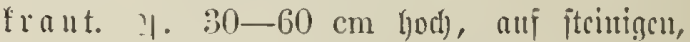

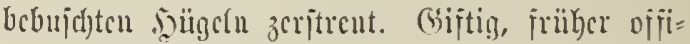

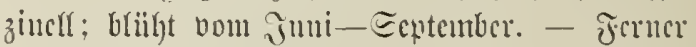
gehören ju don ?altepiatecu Asclépias L., Eciocupilanzc; IIóya R. Pre, Forzel= ranpilanze, 2sad) sblumc ans Ejtinbien (bci แnว g(möbulticl) Asclépias genannt): - Sta-

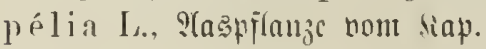

\section{0.) Tamilic. Loganíca $R, B r$.}

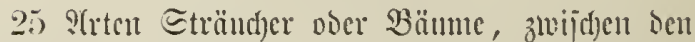
sienteficifat wadjents. Bu ifnen gerbören: Fragraea, Tcubcjubam auj Emmatra: Potália mo Annássera, meldye in હiionumcrifa arjucilich bentigt werben.

123. Fanilic. Gentiáncale Juss. Eminingunädi.

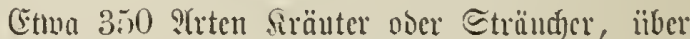

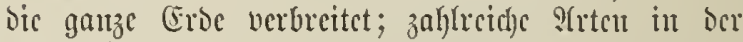

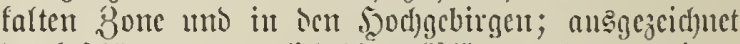

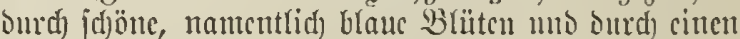
bitteren, arjucilichen (Extraftivitofi. Daju gefjören:

fig. 286. Erythraea centauricúm Pers. (Gentiána Cent. L.), gemcince $T$ a nje no gürocn= fraut. $\ldots$. Etentgel $10-35 \mathrm{~cm} \mathfrak{h o d})$, auj jon= nigen Triften unto sicient in Dentjd)lano genciu;

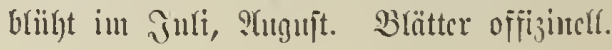




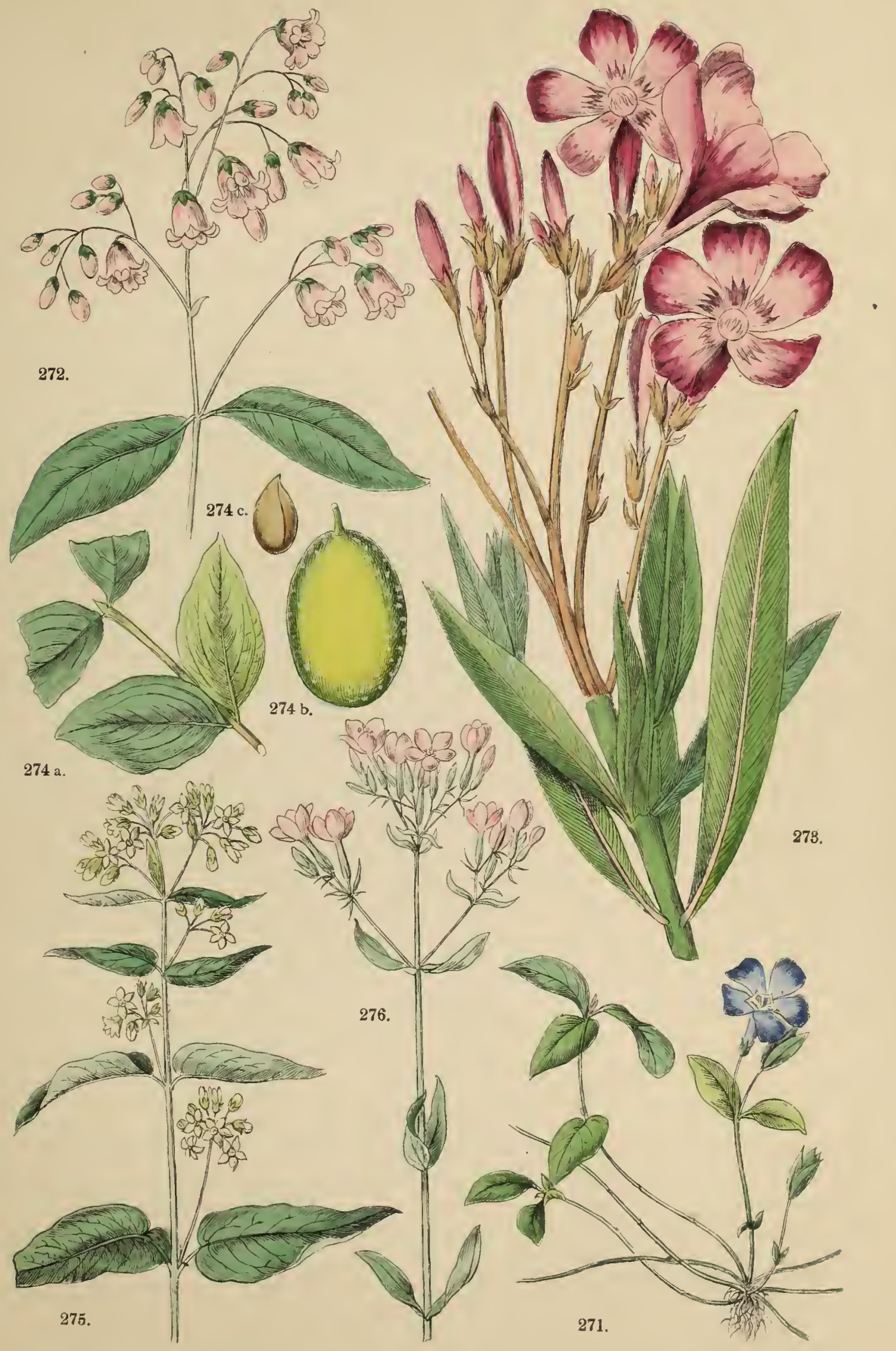





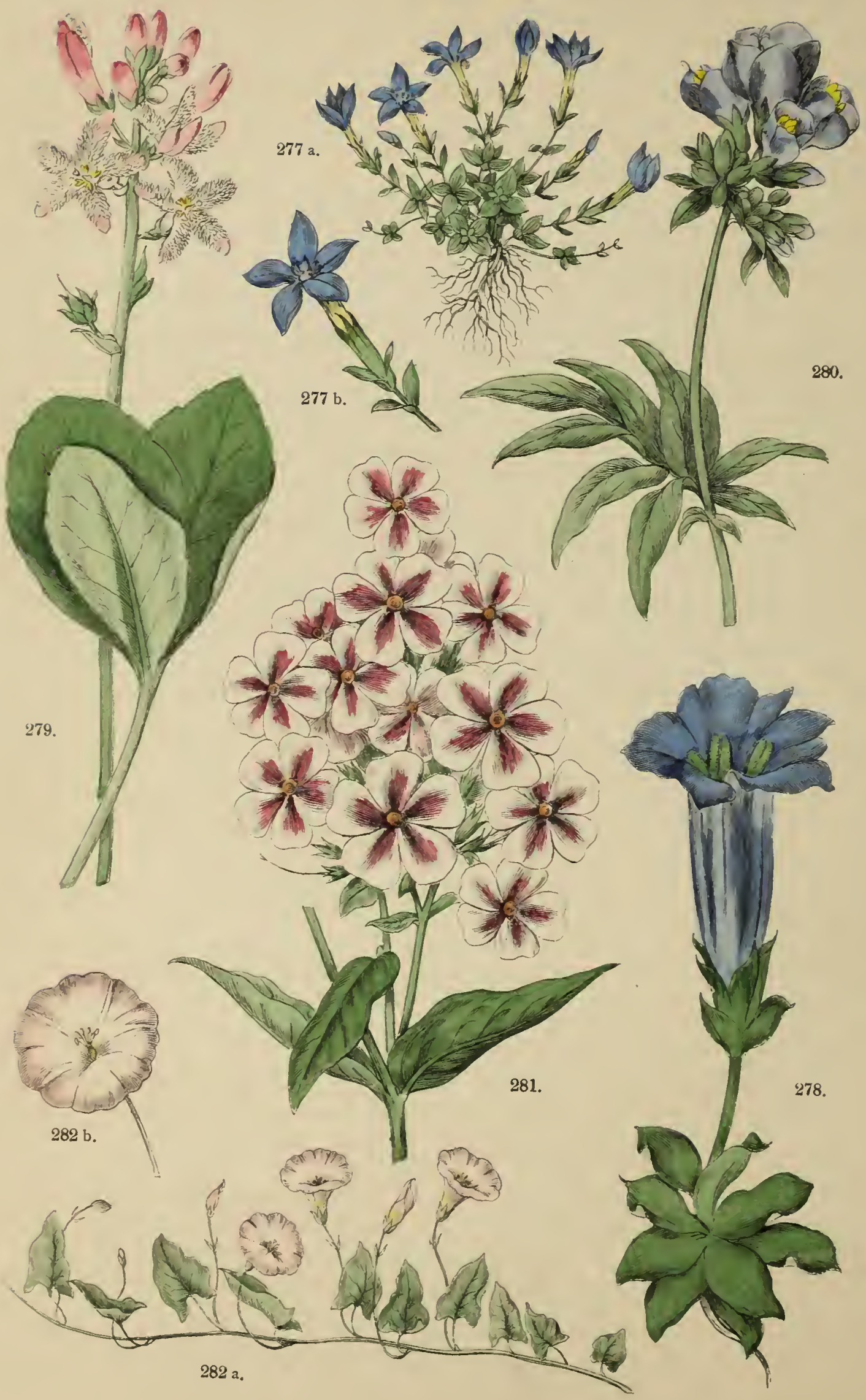


Taj. 48.

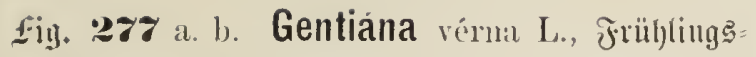

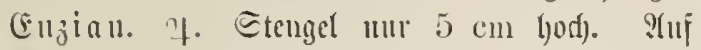

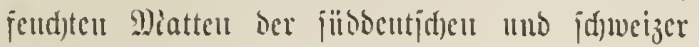

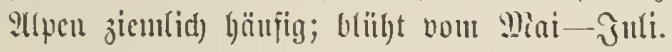

fiij. :78. Gentiäna acaulis L., itengellojer (E)ıian. 21. Stengel 5-10 cm lod), Bllume

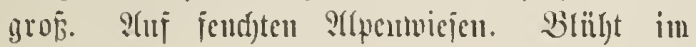
J̀แni, Juli.

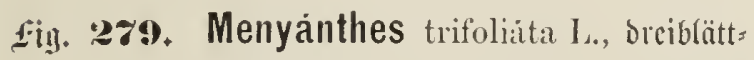
riger, gemeiner Ficberflee, Biberllec, Bit terflee, હumpiflec, Bottenblume.? ?. Ed)aft $16-30 \mathrm{~cm}$ hod), auj uafieu Tori $=$ m10 Sioormiejel vou gaű (Europra, uid)t häufig; b(iil)t iı Diai, Jแur. Blätter offỉ̧uefl. - 3u beu (Sentianen geljören aud) Cicéndia Adans., Bitterfraut, 1 beutide ?(rt; Chlóra, (biün=

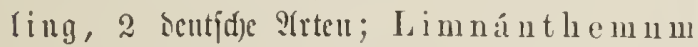
Gin., Ecefanne, ভumpjroje, 1 beutjde :Trt.

\section{Fanilic. Bignoniáceac $R$. $B r$.

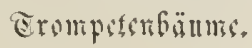

911 350 ?(rteu ucijt tropijd)er, fd)önb(ühenoer

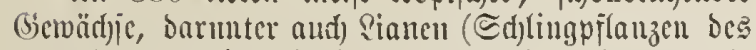
lluvaloz). Dis rvidtigiten fino bie bei nuz ale fajöne Zierbäแmen befanuten Bignón ia Catalpa L. nub Bign. radicans L. (Tecoma rat.), Irom= Hetenbautu; Ja ca ránda brasiliána (Bignónia

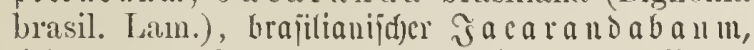
liejert das Sacaranoa= \$a ali and

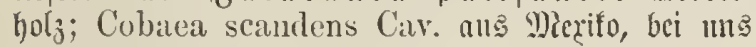
beliebte fletteribe Bierpilanze.

\section{Fanilic. Cyrtandráceate Juc\%.}

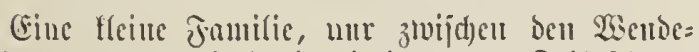

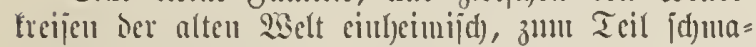
rotzent. (sewö̈hnlid) wiro diçe fantilie mit ber 105. Jamilie, den Gesucriecu, zujammengejtelt.

126. Fanilic. Sesámeate de $C$.

(Ettwa $2 \pm$ tropijd)e sirüuterarteu, in igrem Sater=

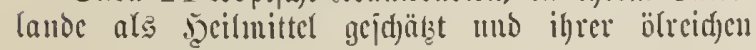

Eamen wegen ötonomijd) wid)tig. 3" ihnen gehört:

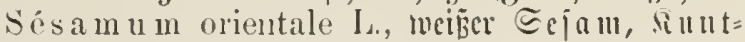

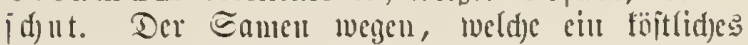
Epcijeöl in grof̧er Dienge gebeu, im gauzen Drient, maurent(id) aud) in (Griect)enlano, häufig angebaut.

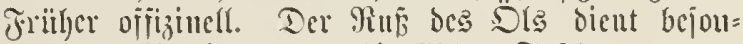
Ders zแr Bercitung ber djuejijden Tujde.

127. Frmilic. Polemoniálene Juss.

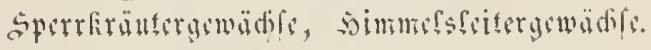

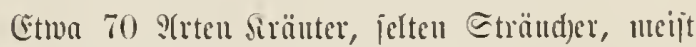

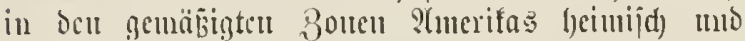

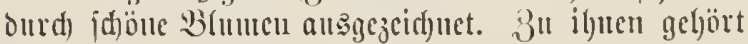
bie einjige ientict)e ?trt:

fig. 280. Polemónium coeruleum L., blanes Spertrat, 万immelsteiter, Jafobstei= ter. ?. 50-60 cm lod), meit in Deutidjlanto verbreitct; bliigt im Jutui, Jutli unb ijt ciuce fdöne Gartenzierpflanze. Jrrïl)er offizintult.

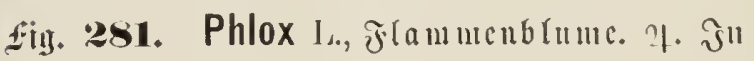
vielen fdjönen, zun Teil woh)(ried)enden Alrteu แno Epiclarten cine sauptzicroc milter Gärten. Die abgebilisete ?ret ijt Phlox Irummóndi Hook; Blüten purpuru, rojeurot ober lila; cine oer fdönten Gartenjicrpflanjen und sie ciu= zige ciujährige :Trt.

\section{Familic. Con rolvuláceate Juss.

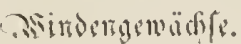

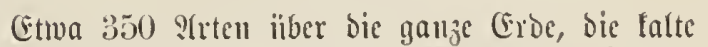

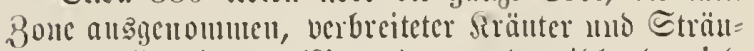
cher, häıțig minsend (Siancu), mand)e mild)eno, vicle

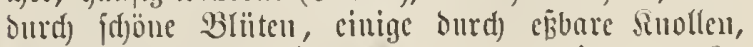
anbre ourd) arzucilidje siräfte ausgezcidjnet. $8 \mathrm{n}$ iluen gehören:

fị. 28: a. b. Convólvulus arréusis I.., It afer= windc. 2. Ctenget 30-60 cm lang, auf

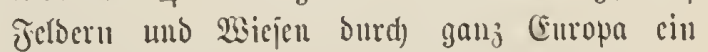
läjtiges llufraut; blülyt vou Juni-Sltober. 
đấ. 40.

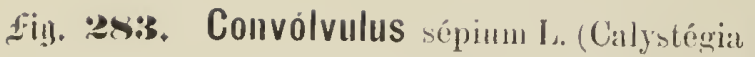

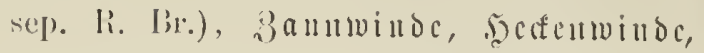

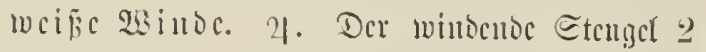

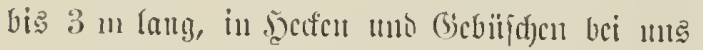
ycutcin, brültt vou Julli- Sftobcr.

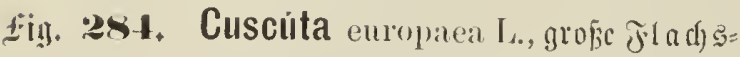

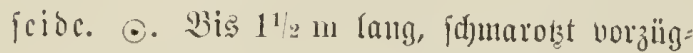
lid) auf Hirlanżu mit frantigen Etcugelu, ïberall iu gaız CEmopa; btiilgt im Juti, stuguit.

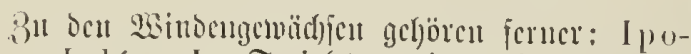

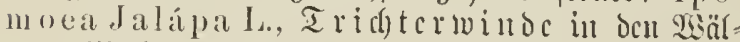

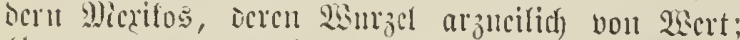
('ouv. scammoniat, furgicruinde in fifcill ajicu; Conv. Latitat L. (Batutas eclúlis (Goois.), Watate, Fatate, indijese satoffel in och Tio=

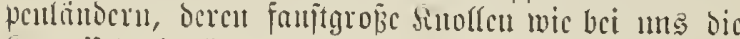
Sartoffill benïtęt merscul.

129. Fomilic. Ilydrolesiceate li. Br. sifibergentistile.

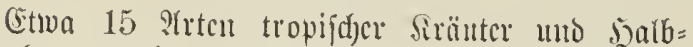

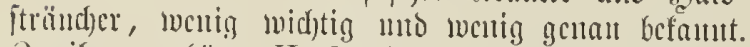
3 ifncu gchört: Iyrlroléa azúrea Lindl., hiut=

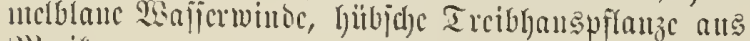
Wicxifo.
1:). Fomilic. Boranín(a) Juss. 3orefdigomidds.

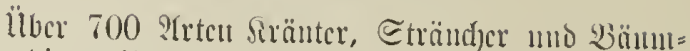

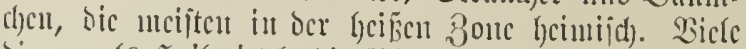

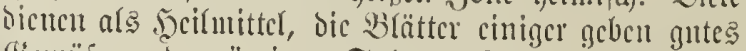

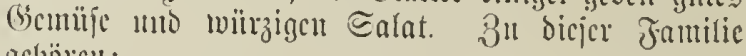
gel)örcu:

5iy. 28.5. Échium vulgire L., gcuncincr 2?at= terfopf. (...). Etcugel 30 -_.!0 cm hod), iibcrall

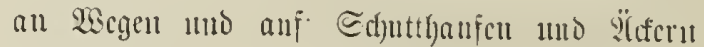
als llufrant 3n fintocı; b(üht voun Jnni-?fıgnjt.

fiig. 286 a. b. Pulmonária officinílis $L_{\text {.. }}$ ar $z=$

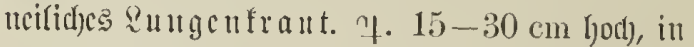

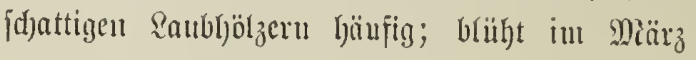
und stpril แuso war offizincll.

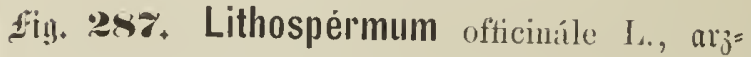
ncilidacr Etciujaut, Miaricutbräncu. 24.

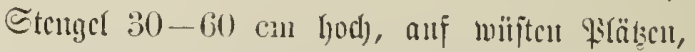

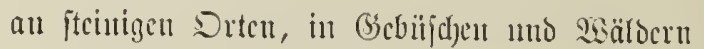

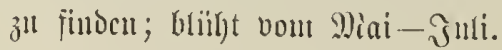

Fin]. 28S a. b. Myosótis arvéusis Roth. (M. intermédia Link.), Y(fer= 2 crgi Nifferma

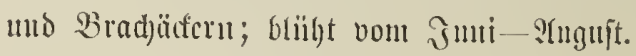




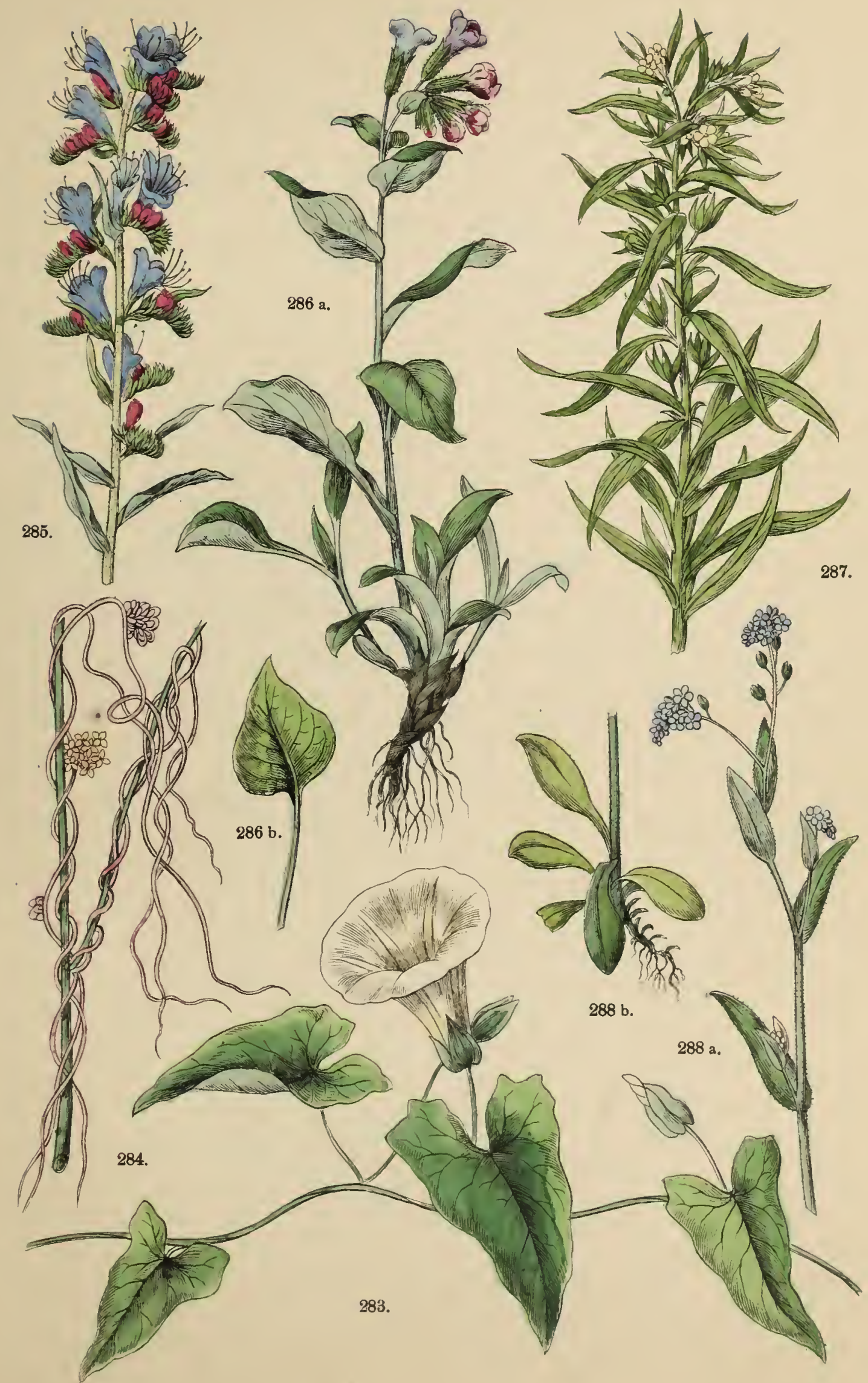




50.

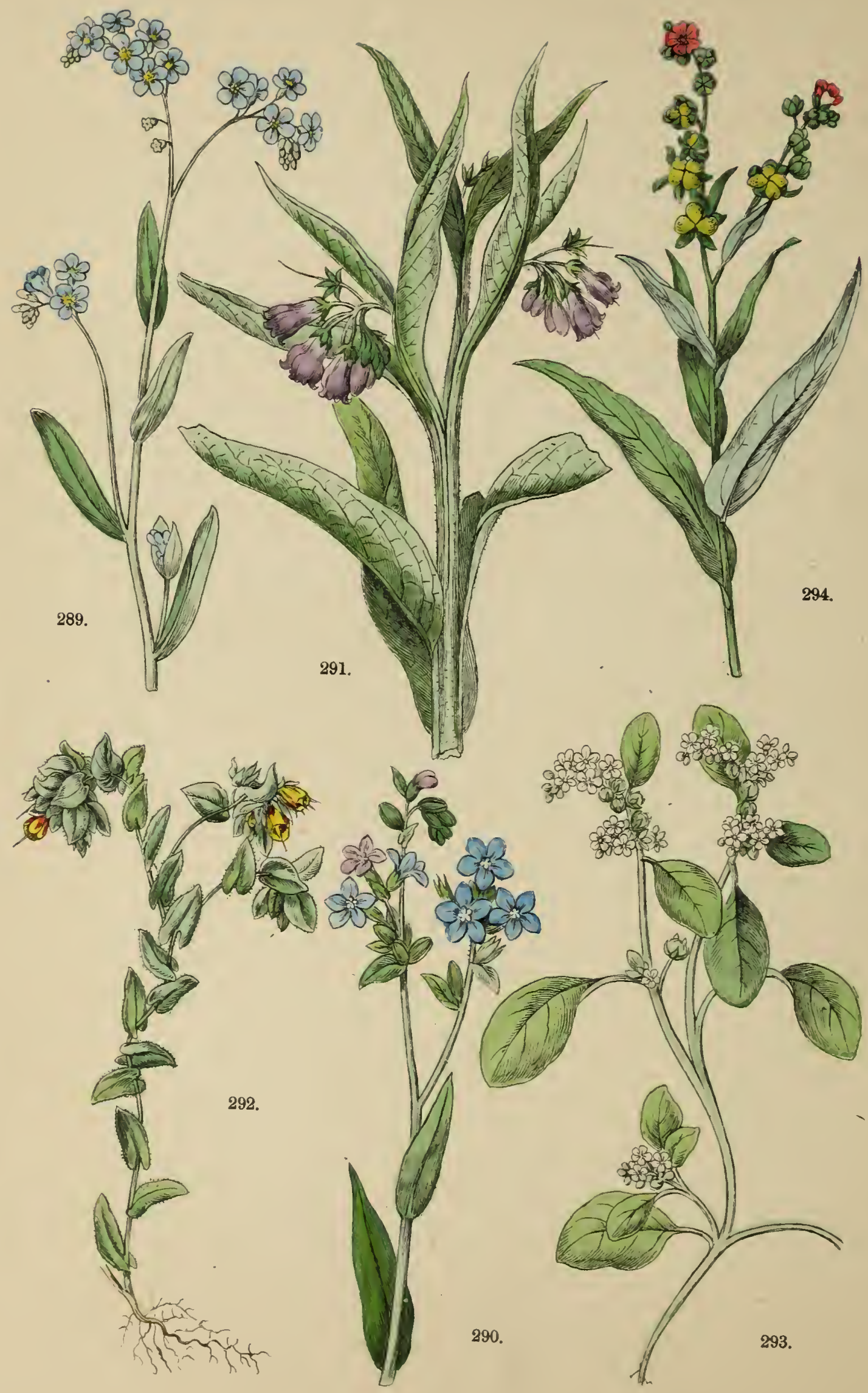


๔ạ. .̄o.

fin. 289. Myosotis palústris L., Emmpi = $2: \mathrm{er}=$

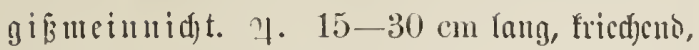

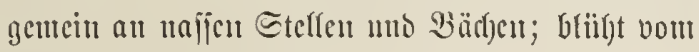
Wiai-Ecptembcr.

fig. 290. Anchusa ofticinális L., gencine, arz= ueilidje Ddj feuzunge. \%. $30-90 \mathrm{~cm}$ yod),

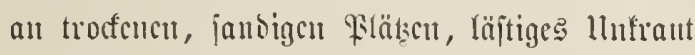
auf Îffern unto an 2isegen; briilyt vou Diai bis ๔eptentber.

fiin. 291. Sýmphytum ofticinále L., gemciuc,

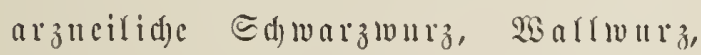
Beimucr. \%. 30-90 cm hod), an feudten

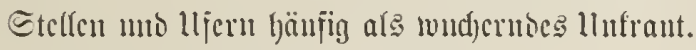

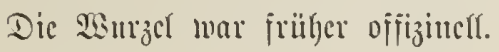

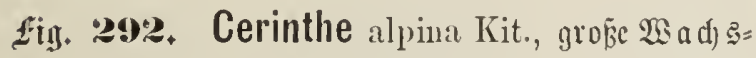

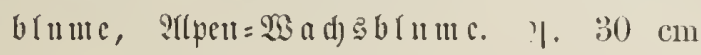
hod, mit wadjsartig geclben, all Grmbe pur= purbraum geflecf́teı SSlüteu, auf horl)gelegcucu

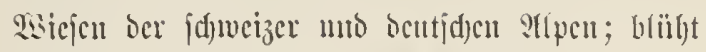

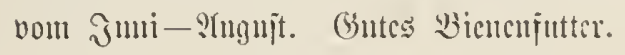

fị. 29:3. Heliotrópium europrem I., cmo=

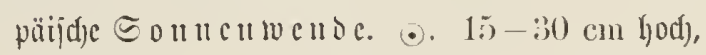

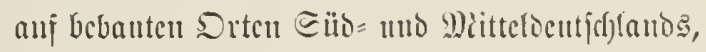
bcjonbers an Sibcine, ïberaff aber jeften; blülyt im Sult, ?tugut. 2(sobfricd)ento, sirant frifher ofrizincll. - Dic bci แแฐ bclicbte, แad) 2anifle ouftente, in viclen Epiclarten fultivierte Topf $=$ uno Bartempflanze Deliotrop ftamut von IIel. peruviánum, cincu 30-120 cm lyobcu Etraudc

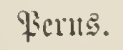

f́ịg. 294. Cynoglóssum ofticinále I., gebrüıd)

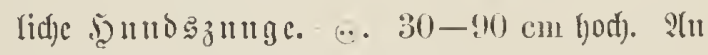

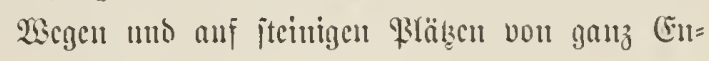
ropa nidft felten: briifyt im Miai nub Juni. Tarar jrïher offizituclf. 


\section{דaj. ร1.}

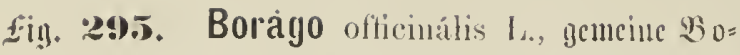
reticl), Burage, (butfufraut. (o). Bu) bis

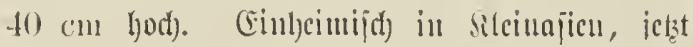

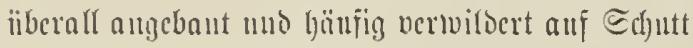

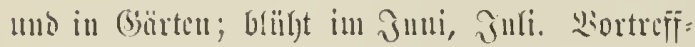
lidycs Enlatgemilnz, wirfjames :(rzucimittcl. -

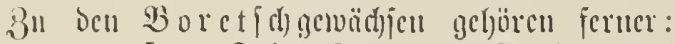
Asperígo L., Edarifraut, Silebfraut, auf Edjutt io uno iort büufig; Anchusa (Alkanna Tausch) tinctória I. in Eiliocuropa, ocren

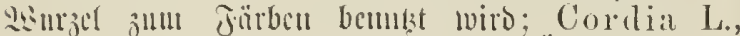

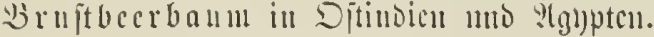

\section{Fantilic. Ilyllophýlleae $R$. Br.}

(Etma 20) 9(rten fafitiger sirüuter, meijt in 2iord: amerita cintlucimif(t). 8 u ifucu getjört: Nem óphila,

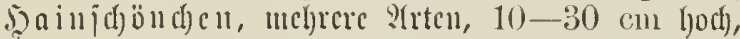

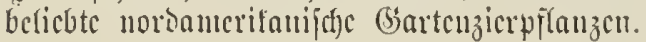

\section{3:2. Familic. Labiátae Juss. sipponfouts,

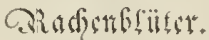

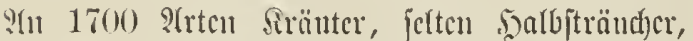
iiber alle erotcile verbreitet, ${ }_{26}$ Der ganzen bentiffen

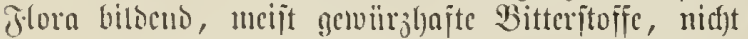
aber (Sififte entl)altende \$iflanzen. 2iele berjelben fitto

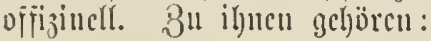

fig. 296;. Lavándula spica I. (angustifólia

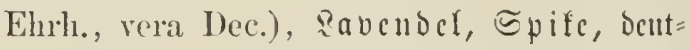
(d) 2 a arde. ?. 30-60 cm hodf), in Eiid=

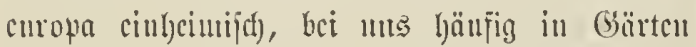

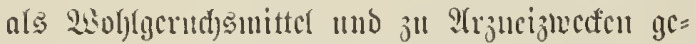
jogen; briilst vou Эuli-Ecptember. fiị. 2!). Sàlvia ofticinális I., gemciuce Eal= bei, Gartenjalbci. D. 5u-(i) cm bod).

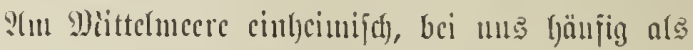

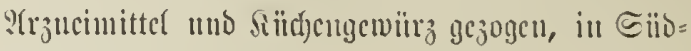

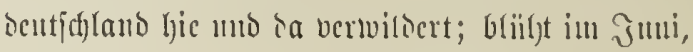
Julli.

fiị. :298. Sảlvia praténsis I., sisicjen=Salbci.

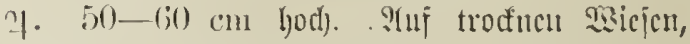
Döitgeln uno Feforainen !littel= mo Eiiocuropas lö̈ufig; blïlyt nom Miai-Juli.

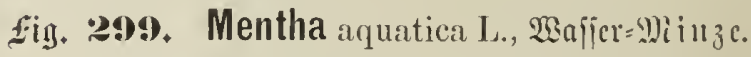
21. 30-50 cul (yod), in mand)ertei formen gemein an Bäc)cı und in Grä̈bcu; brïlyt in Juli, ?(uguit. Dic Pifcficr=\$Niuzc, Mentha piperata, wiro ljüupig in Dentidjlano anges bant und sient in vielfad)er $\mathfrak{E c i f e ~ a l s ~ t r a ̈ f t i g e ~}$ ?Irzuci.

fity. 300. Thymus Serpyllum L., geuciner Thymian, feld= Thyminu, Sucndel. Jiajen= bilocnocr Dealbitrand). Etengel $12-20 \mathrm{~cm}$ lantg, auf founigen șïgetu gemein; brülyt vou Эuli bis Dftober. - Der Gartenty)minn, ans હiio=

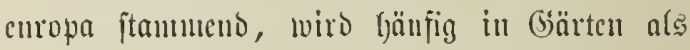

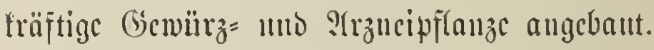

fitit. 301. Origanum vulgare I., gemciner Doiten. 4. 30-60 cm lood). S(uj trodfenen

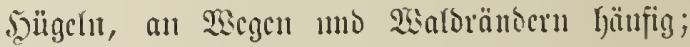
blügt im Juli, צluguit. - Dor Mlajoran, Orig. maljorana I., ans Sibocuropa, ift cinc bctaunte bicıürzpflanze unjerer Gin̈rten. 


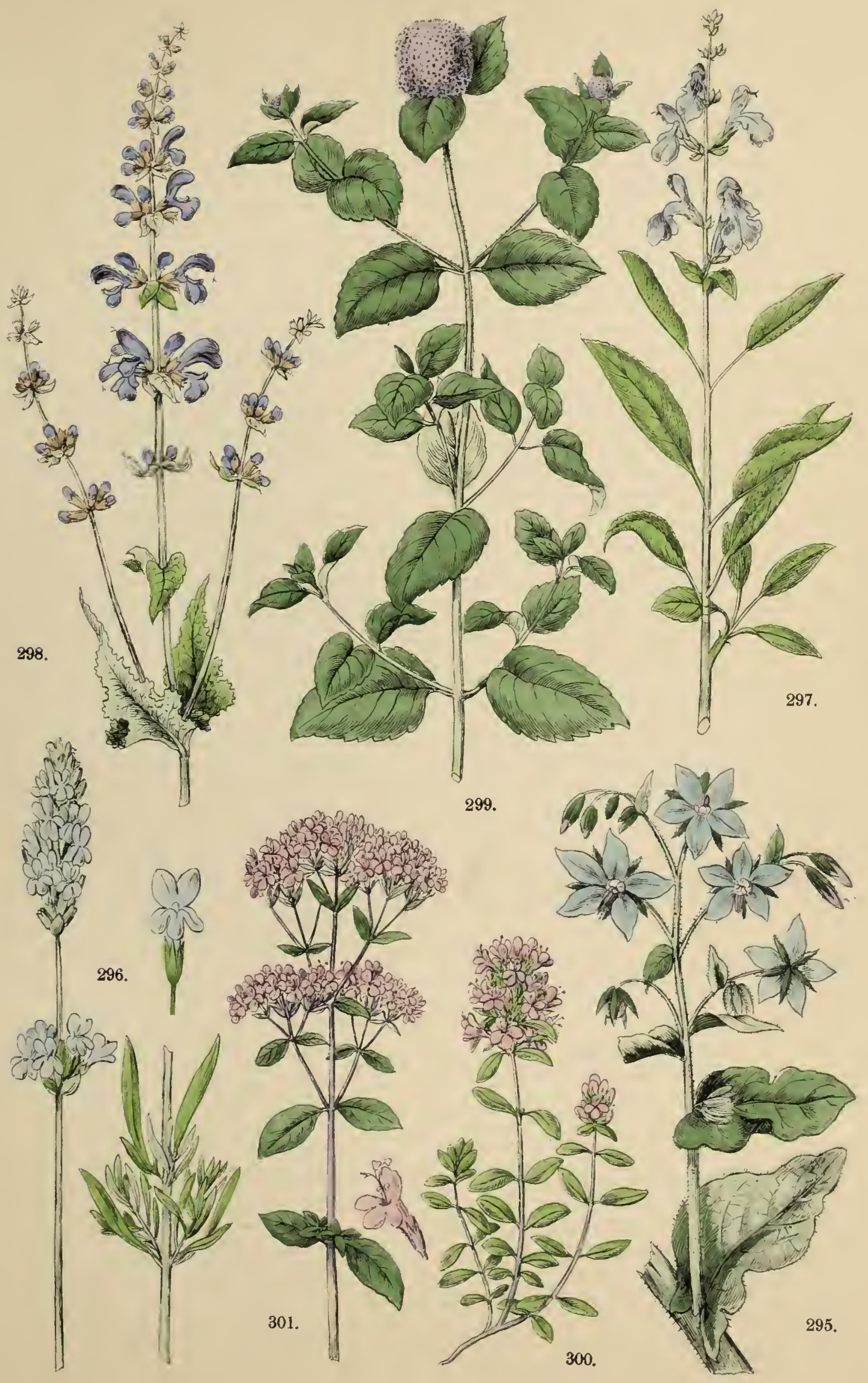





\section{.}

. 


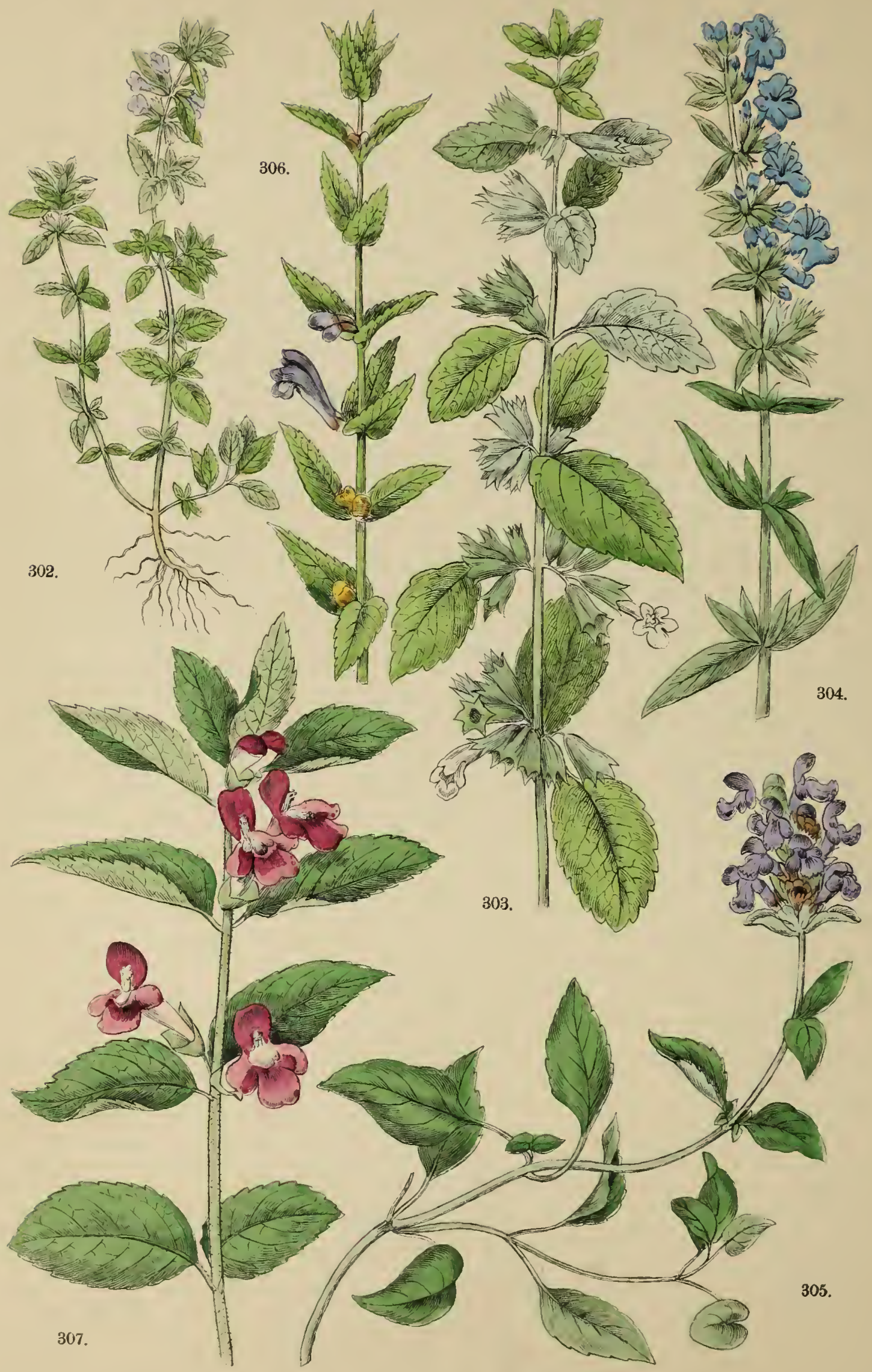


Taf. 52.

fin. 302. Calamintha ácinns Clairr. (Thy-

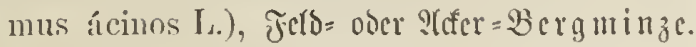

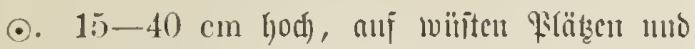
Etciutriid)en, auf Fetoern mo jomigen sjügetn; bliigt vom Эnแni-2hugnjt.

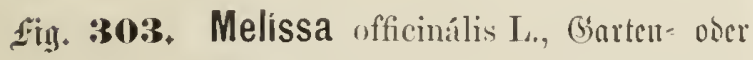
(Eitron= Metrific. 2. $45-60 \mathrm{~cm}$ hod). Weilo in Ëïcuropa, in unjern (Sä̈rteı ocs angenehmeı

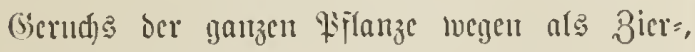

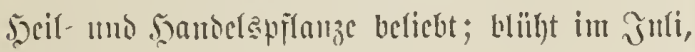
?łıguit.

fịty. :30-t. Hyssópus officinális L., gemeiner 9) fop. Eitt fahfer, 30-45 $\mathrm{cm}$ hoher 5̧alb= itrand) an jeljigen Eteflen Eïbeuropas, im fiiblid)en Dentid)land in mo oort verwiloert; bliiht im Эıli, ?tugujt. Difizinell.

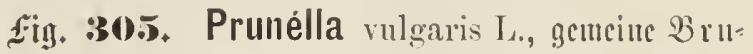
nelle. 4. $15-30 \mathrm{~cm} \mathrm{lod),} \mathrm{jajt} \mathrm{in} \mathrm{allen} 28 \mathrm{e}$ (t) teilen, in Dentidylano gemeinc sisiejerpitanze; bliityt im Эnfi-Eftober.

fị. 306: Scutellária galericuláta L., genci=

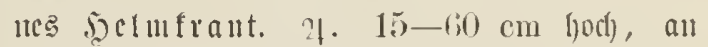
Teid)en, bnjdjigen \&läłzen uno Bad)ujeru vou

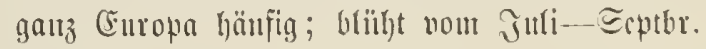

fiig. 30\%. Melittis melissoply'llum L., I $m=$ meublatt. \%. 2int bicje ciuc beutidie 2(rt. Etcuget 30-60 cm hod), in sisälsem unt idat=

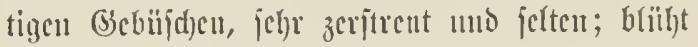
inn Miai un๖ Jumi. 


\section{Taf. $5 \overline{3}$.}

fịị. 308. Marrúbium vulgáre L., gemeiucr,

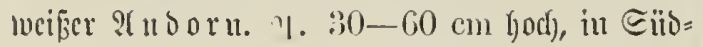

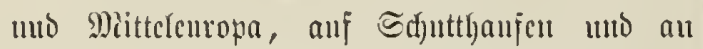

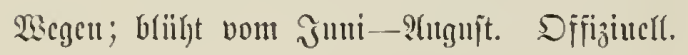

fing. 309. Stachys silvítica L., $\mathfrak{B a r b}=8 \mathrm{i}$ cit, Saloncjict. 2\%. Etengel $30-60 \mathrm{~cm}$; in

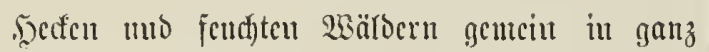
Ěmopa; bic riflaub̆e hat eineu muaugenthmen

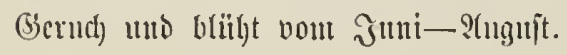

fig. :310 a. h. c. Stachys germáuica I., bcut=

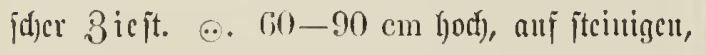

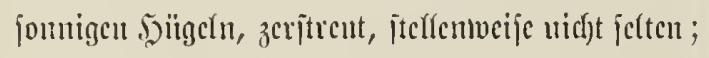

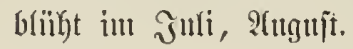

fiị. 311. Galeopsis Galcóbdolon I. (Gaal. lúteum Huds.), Broromeffel, gelbe Tanbucjfel, 2. $20-50 \mathrm{~cm}$ hod), in Gicbüjdjen unto f(j)at=

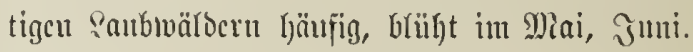

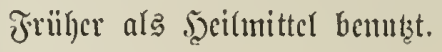

17in. 312. Lámium maculátum L., gefleđtter Bicucnjaug, gefledte Taubuejjer. भ. 30

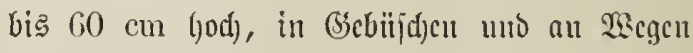

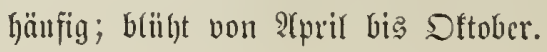

fịg. 313 a. b. Lámium purpúreum L., roter Biencriang. $\odot, \quad 10-25 \mathrm{~cm}$ lood), iuberaff

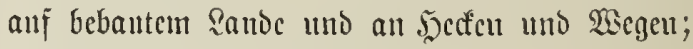
blü̧t voin ? ?pril $-E_{\text {epttbr. }}$ 


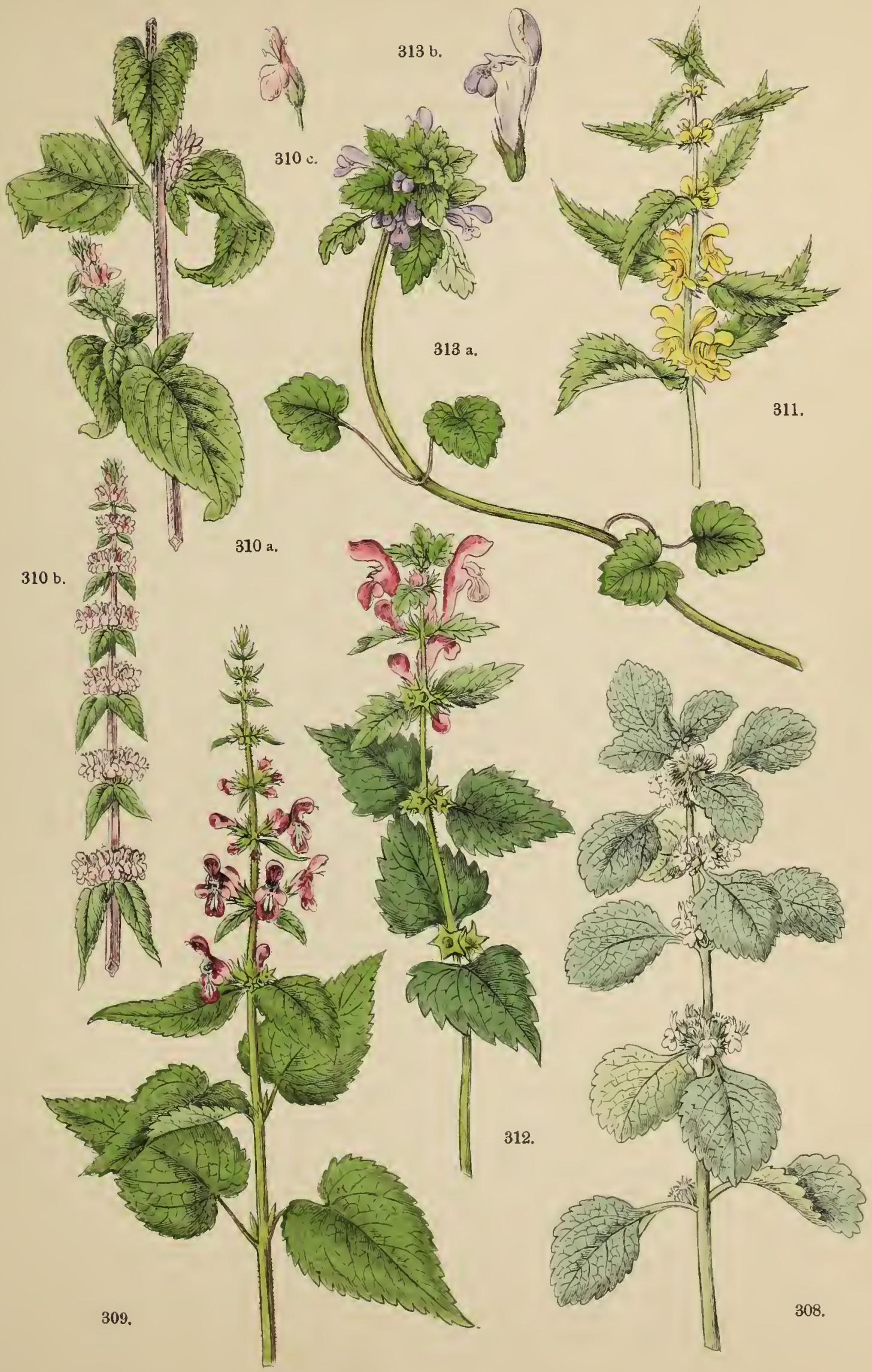




54.

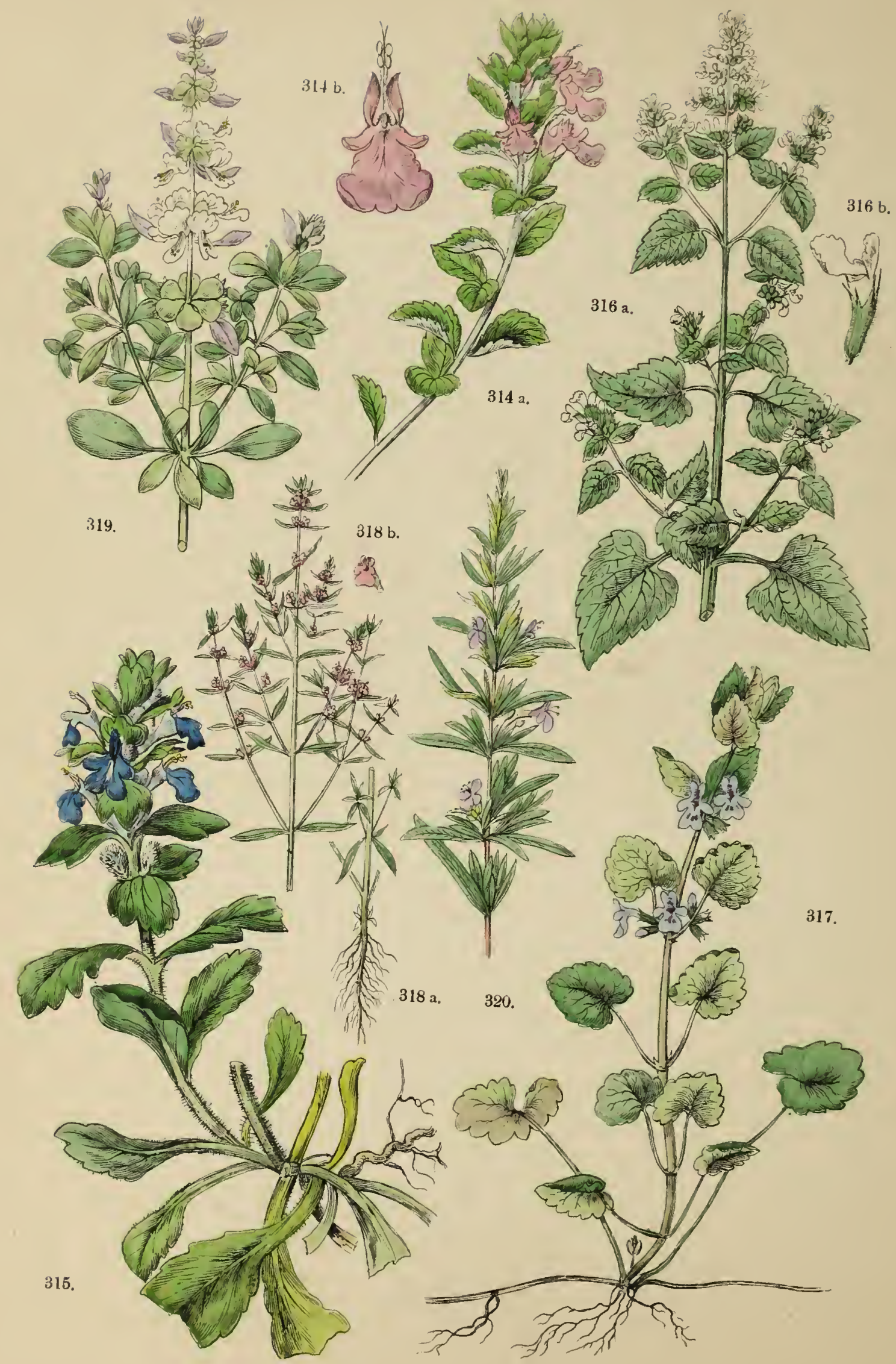


Taf. 54.

fịg. 314 a. b. Teucrium chamaedrys L., coler (b)amauser. 7 . Steuget $15 \mathrm{~cm}$ lang, liengent, auf fomigen, trocfnen 2 (nt)öhen, nament(id) iu

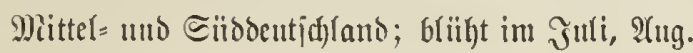

fig. 315. Ajúga pyramickalis L., phramioen= förmiger (3) üแjel. ?. $15-30 \mathrm{~cm}$ l)od), anf offnen, lid)ten Kalopläłsen uno Jä̈geln, berbreitet. Briïht vom Miai-Juli.

fịg. 316 a. b. Népeta catária L., gemeine Sałzenminze. ๆ. 60-120 cm hod. Lleberall in Europa an Bäuten uno auj હdutthaufen. Start riect)ent, frïher sjeilmittel; b(iil)t vom

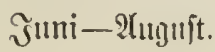

fig. 31\%. Glechóma herlerícea L. (Népeta glechoma Benth.), gemeine (śs utoefrebe, (5) noermann, Erbephell. 21. Etengel bis $60 \mathrm{~cm}$ 〔ang, liegend. Эn gauz Čropa luäufig, in Deutfidlant alt Jeefen iiberall genein. Diente früher zu Sirältterjäjten uno Brrujttlyes; blïht vom 2(pril- Juni.
Fig. 318 a. b. Saturéja horténsis L., Grarten= Bicfiertraut, Bohucutraut. $\odot .20$ bis $30 \mathrm{~cm} \mathrm{hod,} \mathrm{heimija)} \mathrm{in} \mathrm{Sübeuropa,} \mathrm{bei} \mathrm{uns}$ l)äıịig als Sï̈dengemürzfraut fultibiert unb ver=

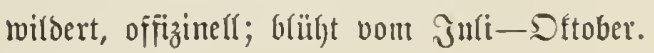

fing. 319. Ocimum basílicum L., Bafilicu. traut. Ths Sübajien jamment, feines 2 sobl= gerud)s megen häufig in (särten unto als beliebte Topipflanze fultiviert, ofizizinelf; blïht im Soumer.

fig. 320. Rosmarinus officinalis L. (Sálvia rosmarínus Spen.), gemeiner $\Re$ osmarim. 5 . Jumergrïner Straut von $90-120 \mathrm{~cm}$ 5ెöhe, beimija) in Sübenropa, bei uns feittes aromati=

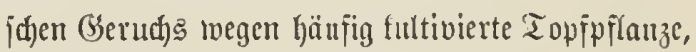
bient mehrfach z" J̨eilzwecfen; blüht vom Miärz bis Miai.

3ur Familie ber sippenblïter gefyören aud Lycopus L., 25olfsjü, Bigeutertraut; Monúrda L. in Rorbanterifa, bei แnI prädytige Bartenzierpílanzen; Pogostémon, \&atfdouly)= pflanze in Inoien, beren $\mathfrak{B}$ (ätter das beriil)mte Par= fum licjern; Ballóta, Śmarzucifel; Len-

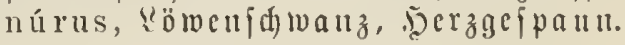




\section{Taf. 55.}

133. Fanuific. Verbenáceae Juss. silerkraufgemëd)

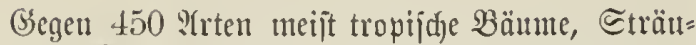
d)er utlo Siräuter, beren mandje als Bierpflanzen, aubere als शituzhörzer befaunt jino. Dahin gehört:

fiig. 321 a. b. Verbéna officinális L., gemeines Eifenfraut. \%. 30-60 cm hod), an $\mathfrak{B e g e n}$

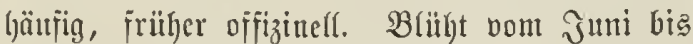
Ittgujt. Putr bieje eine beutjde $2(r t .-$ In 2 tues rifa jinto mefrere Sierbenen heimija, oeren präd)= tige Epielarten unit roten, bioletten, blatten u. j. w.

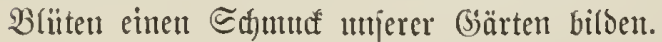

Bu den Berbenen gehören: Lantána auz Siibamerifa, immergrïne Bieriträuder mmirer $\mathrm{BS}_{\mathrm{c}}=$ wäc)รhünjer; Tectónia, Iefbaum in Sittubien,

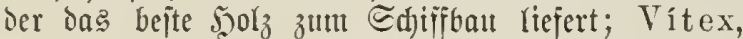

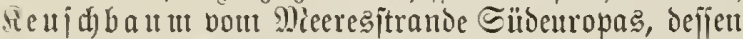

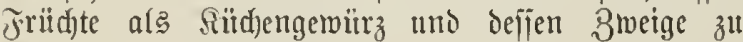
siörben und (Befled)ten aller ?Irt dienten.

\section{Familic. Acantháceac .Juss. Bärerkfangewädje.}

über 600 2rrten Sräıter unঠ Sträucher, meijt in den Tropen einheimijd, nur eine 2lrt in (Etropa. A cánthus L., Bärenf́lau iu Eitocuropa, wo

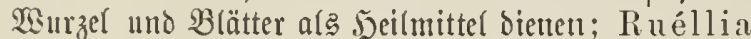
atţ Briajilieu, đdötte Zierpflanze; Thunbérgia, J)eilpflanze Ditindiens, bei une als Topfpflanze fintiviert.

\section{Frantilie. Selagíneae Juss.}

(Begen 50 fleine Cträudher แtก Siräuter, jämtlid) auf detin Borgebirge der guten Soffitumg. Dag midg)= ligite ijt Selágo fasciculáta, gebiifidelte Eelago vom Siap, eitte $1-1^{1 / 2}$ mI hole Bierpflanze mit inohl= riedjenden $\mathfrak{B l u m e n .}$

\section{Familic. Globularíneae de $C$.

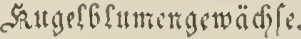

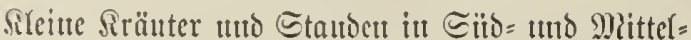
europa; nur 3 beutide 2 (rten. Die widjtigite ijt: fịg. 322 a. b. Globulária vulgáris L., gemeitte Яitgerbiume. 21. Stengel $8-20 \mathrm{~cm}$ hod.

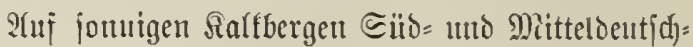
lands. Bartenzierpílatıze.

\section{Fanulic. Myopórinae $R, B r$.}

Ettma 25, jajt mur auj 2ienliblland bejuräntte Eträudjer. Dazı gehört: B ontia daphnoídes,

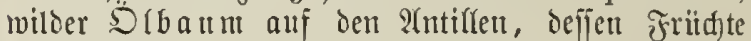
Wrentiöl Ciefern. Ein immergrütter Bartetzzieritraudd.

138. Tramilic. Solanáceae Juss. Si̊dí-

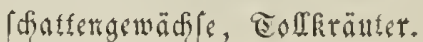

Ettwa (600 2lrten über dic ganze Eroe verbreiteter firüuter uno Cträıdher. Mandje jitto ljödjit giftig,

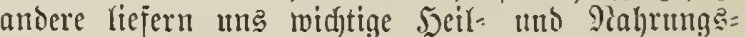
mittel. Ḑieher gehörent:

fig. 323 a. b. Datủra stramónium L., gemei= ner Eteduafel. $\odot$. Etengel $15-100 \mathrm{~cm}$ hod, finbet fith überarr in warmen und gemäsigig= ten Slimaten, bei ung bie uttro ba bermifoert.

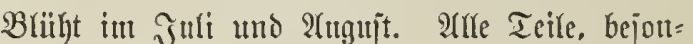
ders B Bätter utlo Eamen find hödjit giftig, z"1gleid) aber offizinell.

fing. 324. Nicotiảna rústica L., Bâuern $=\mathfrak{I} a$ -

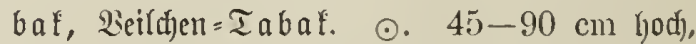
itamm aus Rotdamerifa tulo wiro bei une ful= tiviert, brüht boun Juli-September. Seine 2ier=

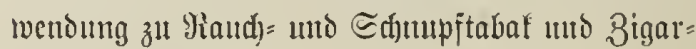
ren ijt befannt.

fìn. 325. Nicotiána tabácum L., gemeiner ober virgituijder $\mathfrak{T}$ abaf. $\odot . ~ 1-1 \frac{1}{1} 2 \mathrm{~m}$ hod,

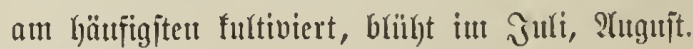
Berwendung wie bei betl borigen. 


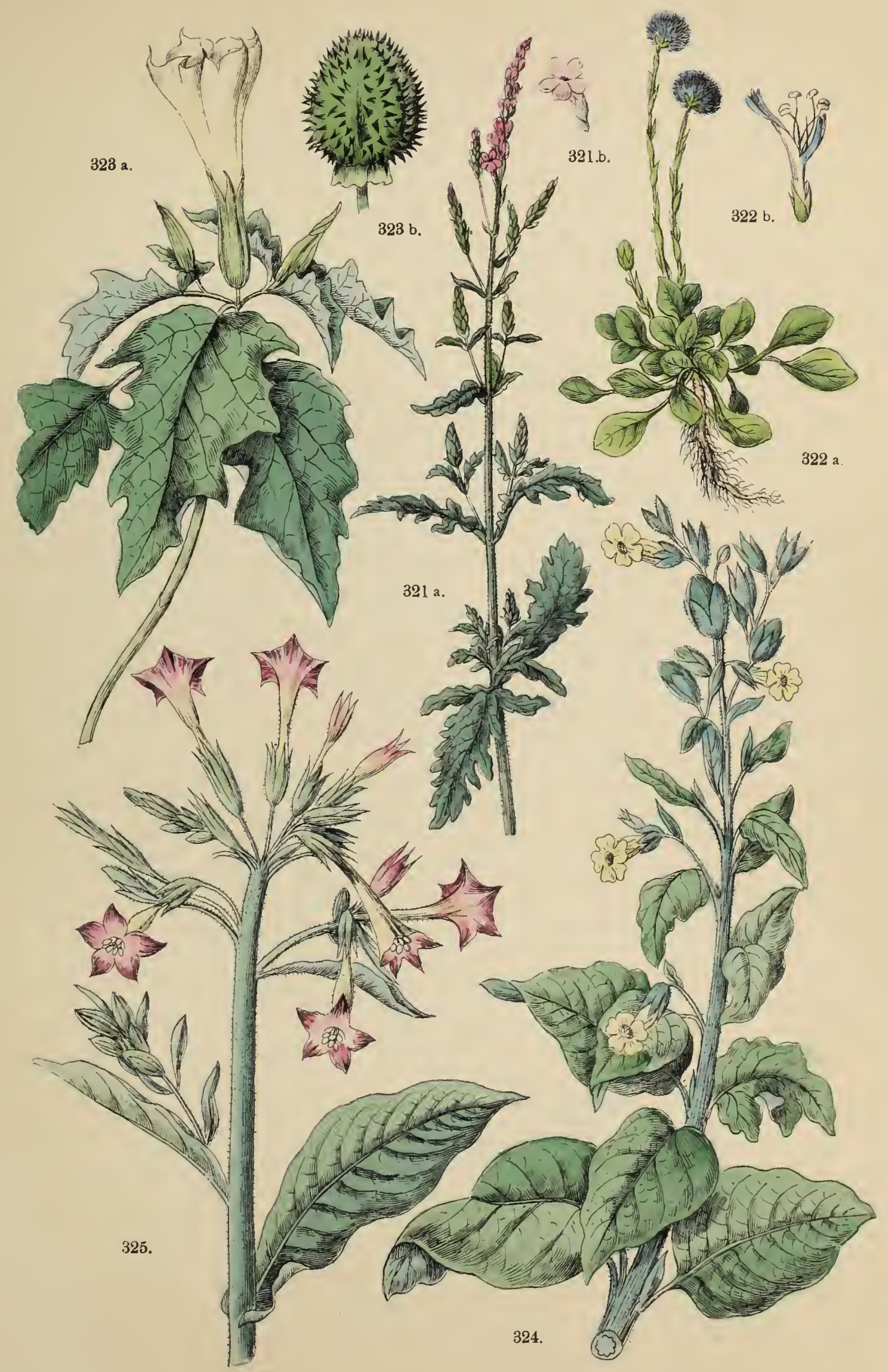





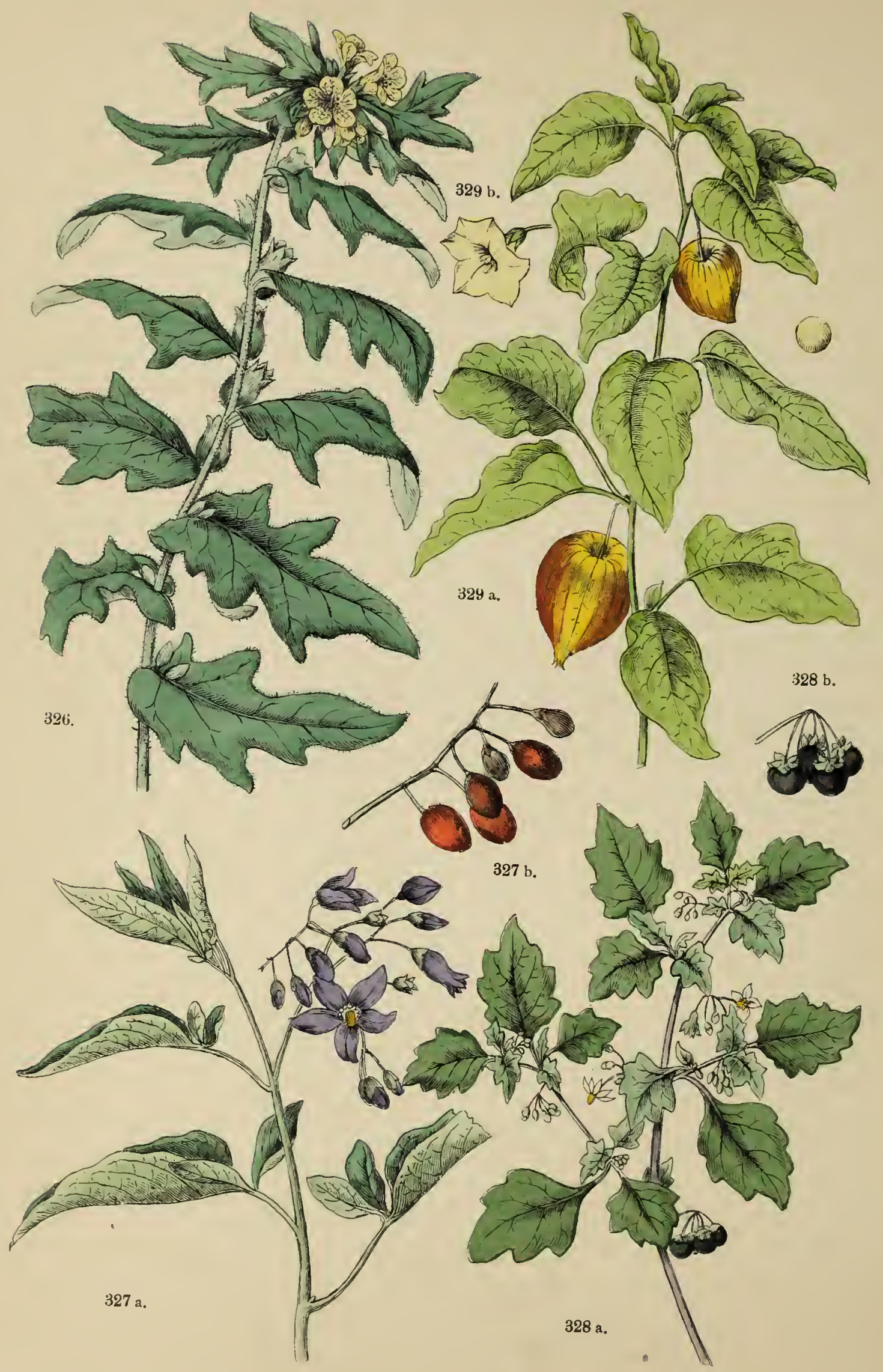


זaץ. 56.

fin. 3:26. Hyoscyămus niger L., genteines Biljenfratt, Sqlaffraut, Dulftraut, Durrdill. $\odot$ oder $\odot .45-60 \mathrm{~cm}$ hod). ?tuf

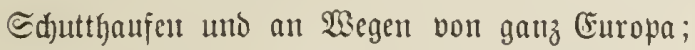
briilyt iut Эuni, Juli. Die ganze Brfanze ijt bödjît gijtig, SBlätter mo Sauten aber jino offizinell. Daber wird die Biflanze zu : $(1$ rzuei= zuecfen ba uno bort nngebant.

4ịg. 328 a. b. Solánum dulcamára L., bitter= jüber Radjtjatten, Bitterjüj, Mrpranfe. ந. $(60 \mathrm{~cm}$ bis $3 \mathrm{ml}$ hod), fletterno, in (Ges= büjden und ฐecten, verbreitet über ganz Emropa. Blüht vom Mai-2tugujt. Giftig, offizinell. fing. 3:28 a. b. Solánum nigrum $I_{\star}$, jđjwarzer

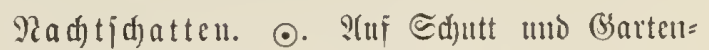
ländereien gemeiu, blüht vom Jutli-Dftober. (Siftig.

fỉg. 329 a. b. Physălis Alkekengi L., gemeitue

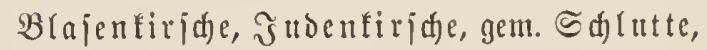

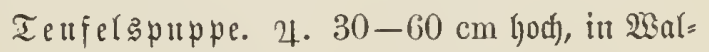
dungen, auf S̋ügetn uno in Meinbergen Siio= etropas; in Deutjaland zeritrent uno einzelu. Beeren rol) tulo eingemad)t eśbar. Früfer offi=

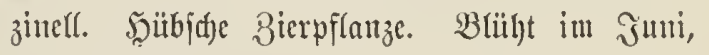
গ⿰ti. 


\section{Taf. 57.}

fig. 3330 a. b. Átropa belladónna L., gemeitte Tollfirjde, wolfzbeere, Belradonna, 2istbere, Teufelabece. 24. $60-125 \mathrm{~cm}$

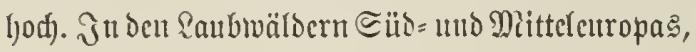
in Dettfdalano nidjt jelten; blügt iun Juni uno Julli. Эn allen Teilen hödjit giftig. B̧lätter

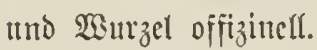

Bu ben Solaneen geljörig nennen wir vor allet anbern Solánum tuberósum L. $\mathfrak{i} a r=$ tofiel, Erdapfel, bieje ute allen betannte, Miil=

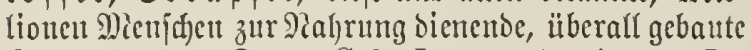
Eegengfrudft. Dann Sol. Lycopérsicum L., Tomate, Riebesapiel, giarabiesapfel aus Sïbamerifa, befien frrüdte eine vortrefflidje Beigabe zlt vielen હpeijen geben; ferner Nandrágora L., 5exentraut, 3aubermurzel, wadfend am Mittel=

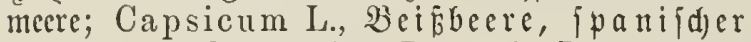
Bifeffer aus Sübamerifa; Petúnia Juss., beliebte (Sartenzterpflanze als Sibdamerifa, mit zahfreidjen Bartetäten.

139. Fantilie. Scrophularíneac $R$. Br. (Personátae und Rhinantháceae Juss.)

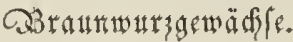

lïber 1300 2rten Sräuter uno Sträud)er, über bie ganze Crove verbreitet; bie meiften enthalten bittere tmo juarje (giftige) Stoffe, mandje zeid)uen fid butd) ¡đjöte Blumen atts. 3u bicjer Famtlie gebören: fig. :331. Verbáscum thapsus L., gemeincs,

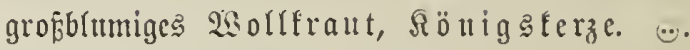
100-130 $\mathrm{cm}$ hod), an $\mathfrak{B e g e n ,}$ trodinen, fon= uigen Srten häufig, blüht iu Şuli, 2ługuit. Difizituell.

fig. 332. Scrophulária nodósa I., geuciuc, fnotige $\mathfrak{B} \mathfrak{r} \mathfrak{a} u \mathfrak{n} \mathfrak{w} u \mathfrak{r}$. $\quad 2.60-100 \mathrm{~cm}$. hod), an idjattigen, jeudjten Stellen, in Deutidjlanto gemein; blïht vom গtuli-Dltober.

fig. 333. Gratiola officinalis L., (5) otte $3=$ gnadentraut, Furgierfraut. $\quad$-1. $30 \mathrm{~cm}$ hod), auf fumpịigen $23 i c j e n$ unt an $\mathfrak{X a j e r g r a ̈ b e n , ~}$ in Dettrjulano ziemlid) jelten; blüht im Эutti, Эulti. Sdjari, giftig, offizinell.

f́ig. 334 a. b. Digitális purpúrea L., roter Fittgerhtt. (.). 60-100 cm hod), an trocfetten Sebrirgąab̧ängen uno in Bergwäloern; blüht iun

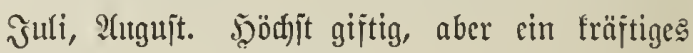
$\mathfrak{a} \mathfrak{a} z$ teimittel. 
57.

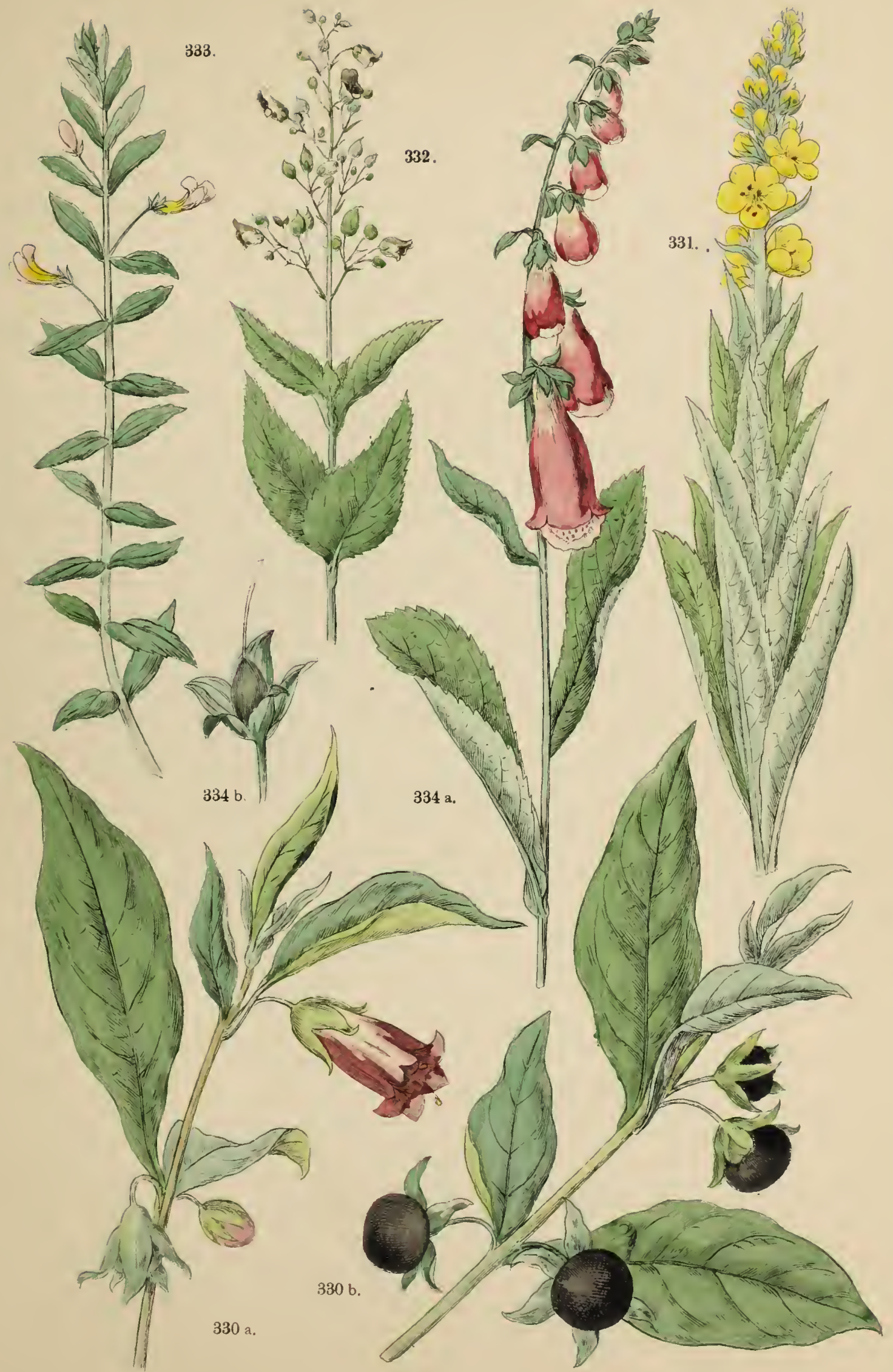





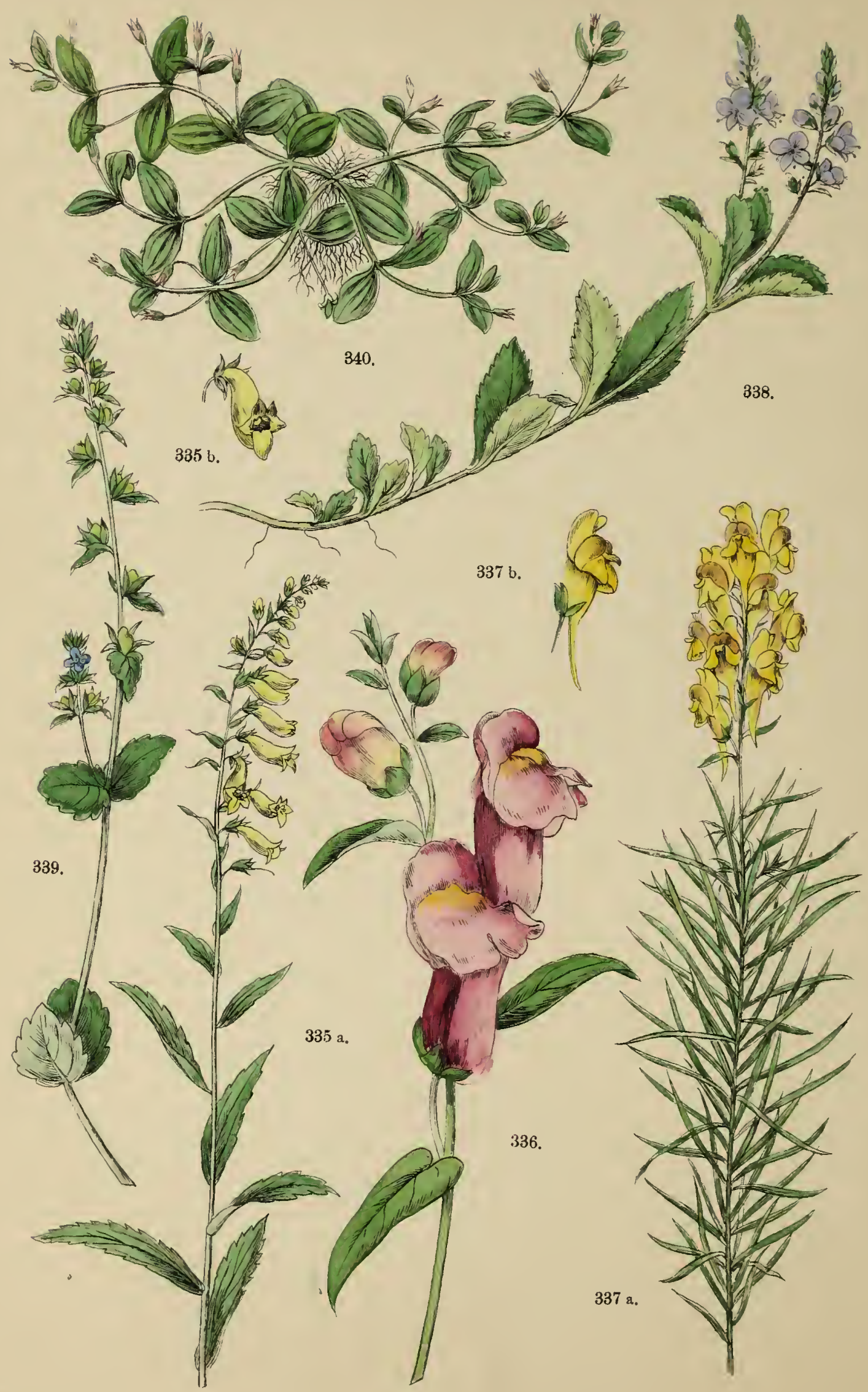


Tạ. 58.

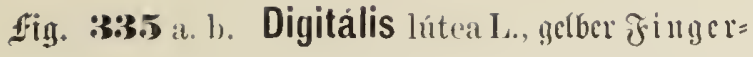

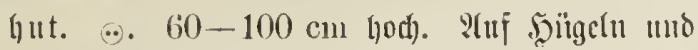
ftcinigeı Bergabłäugen Siibocutidhlanos uno ocr

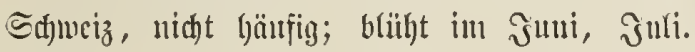
Biitig.

fin. 3336. Antirrhinum majus L., groß̄es \&öwenแaนt. 2. 30-60 cm hod), auf Felfen mo altem Sicnäncr in Silocuropa; belicbte Gar= tenzicrpflanze uit zahltreid)en bunten Spiclarten; blïlt vout Jıแni-Exptember.

ftig. $\mathbf{3 3 3 7}$ a. b. Linária vulgaris Mill. (Antirrlínum linária L.), getucines Reinflaut, $\mathfrak{B a l d}=$ flad) $\mathfrak{s}, F \mathfrak{F u c u f l a d ) s , ~ g e l b e s ~ s o ̈ w e n m a u l , ~}$ 2. $30-60 \mathrm{~cm}$ hod), auj fandigen rainen uno an Fluk̄ufern gemein. (siftberbäd)= tiges llntrant; blügt vom Suli-Scptember. fig. 33:38. Verónica officinális L., arzucitidar Efrcupreis. 1. Stengel $15-30 \mathrm{~cm}$ lang, licgent; it trocfencu $\mathfrak{W a ̈ l o c r u , ~ a u j ~ j o u m i g e n ~} \mathfrak{A}$ = böbcn uno Triften gemeiu; blült vom Эun bis Scpteuber. Difizinell.

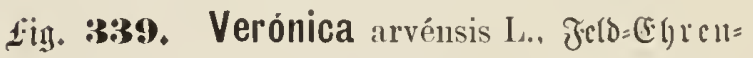

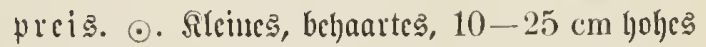

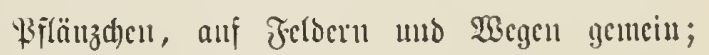

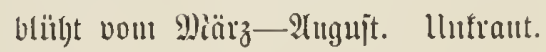

fing. 340. Lindérnia Pyxidária L., B ïd $\left[\mathfrak{c}_{\mathfrak{c}} \mathrm{l}=\right.$ flant. $\odot$. Ein tlcines, an flnfufern uno auj

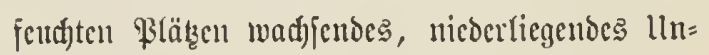

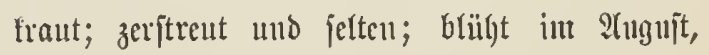
September. 


\section{Taf. 59.}

fïty. 341. Euphrásia lútea I., gelber 9 ugen= tro it. $\odot .15-30 \mathrm{~cm}$ lyod), zeritrent, aui

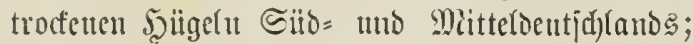
bliilyt im 2ugujt, September.

fîy. 312. Melampýrum arvénse L., :(cfer=

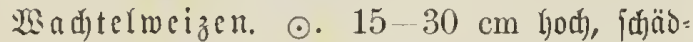
liđjes llnfrant anj Gietreidefeloern im gemäp̈igten Ëuropa; blüht vom Juni-Eeptenber.

3"t ben Ecrophularineen gefören ferner: Panlownia (Bignónia Thbg.) imperiális Sieb., ein größ̈erer B̉aum Sapans mit prađtbollen Bliiten; Clielóne L., 〔djilbblume, beliebte Gartenpílanze aıs Rorbamerifa; Mimulus lúteus L., aı๖ Wuerifa, mit bielen Spielarten, worunter M. moschatus Dougl., Mojduts=(S) a uflerblume; Calceolária L., fiantofjelblutue aus Sübamerifa, mit zaftreid)en Spielarten.

140. Fantilite. Orobáncheae Juss. Somtmerwur\}gewäd)

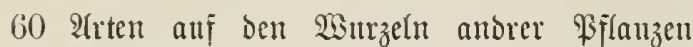
jđ) marotzento Siränter, mit blattartigen, braunten ङøuppen, meijt in ber nörblichen, gemäs̈igten 3one

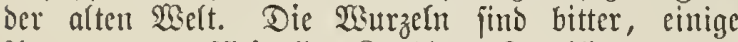
Iften waten offizinell. 3u Diejer Familie gehören:

fign. 313 a. b. Orobánche elátior Sutt., (Or. polymórpha Schrk.). 2. 20-30 cm hod,

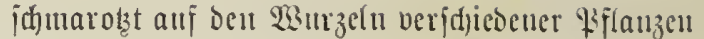
utto fintoet fid) bcjoutoers anf Silecäcfern; blïht im Эulli, 2fuguit.

fig. 314. Lathraea squamária L., gemeine

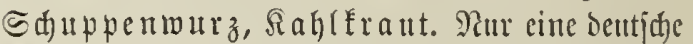
2rt. 2భ. $15-30 \mathrm{~cm}$ hod. In jattigen $\{a u b=$ maldungen von ganz Deutidlano, aber überall jelten; blïl)t im Miärz, 2(pril, Mai.

\section{Fanuilie. Plumbagíneae Juss.}

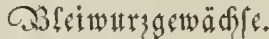

l̈̈ber 100 P(rten Sräuter แno Sträıd)er, meijt jdjöne Sitjtenpflanzen bes Mittelmeeres, mit jajar giftigen und abjtringierenten Stoffen. 3" ifuet gehören: Plumbago Tourn., Bleimurz mit gif́tiger Wurzel; Státice L., Stranomelfe; Arméria W., Giraznelfe, Sanonelfe.

\section{Fantulie. Plantagíneae Juss.} ašegerifgemädle.

Iแ 150 9rten Srrüuter uno Salbjträudjer, über die gemäpigten 3onen verbreitet. 5jięu gefjört mit 9 beutiden 2(rten Plantágo L., Wegerid, wegbreit. Die abgebilbete 2irt ijt:

fitg. $\mathbf{3 4 5}$ a. b. Pl. média, mittlerer $\mathfrak{B}$ egerid. 21. Sdhaft $20-45 \mathrm{~cm}$ hod), an $\mathfrak{W e g e n l}$ gemeitt; blüht im Mai, Эumi; wohlried)ent.

\section{Interflafje: Monochlamýdeae, SIumenblattloịe.}

\section{Fanilic. Nyctagíneae Dec. asutrdergfunergewäd}

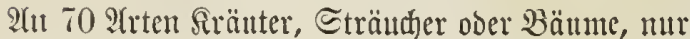
tropijace, vorzïglich jübamerifanijhe Bemädjie. Mieh = reve Prten attggezeidnet Durch fäöne B̧lüten, andere

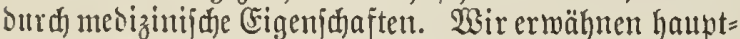
jädfliđ) Nir ábil is Jalápa L. (Nyctágo horténsis Juss.), gemeine $23 u$ uberblume, faljde Jalape, an's Brajilien, eine beliebte Bartenzierpflanze, Deren Wurzel arzneilid witét, uno Boerhávia L. aủ Bert, welde bort ebenfalle arzneilid) bient.

144. Familie. Amarantáceae Juss. Ämaranf-

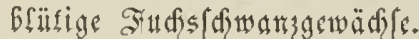

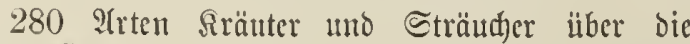
gatze Eroe, vorzïglid über bie heife 3one ber= breitet uno viele Bierpflanzen enthalteno. Бjiezu gefört:

Eing. 346 a. b. Amarántus silvestris Desf.(Am.

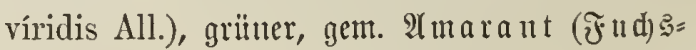

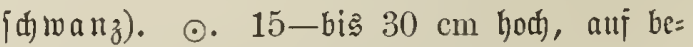
bautem uto wiijtem $B$ oden, an 2 segen, zeritteut; blüht im $\mathfrak{s} u f i, \mathfrak{A}$ ugujt.

3u ben 2Haranten gehören ferner: Celósia L., S) ahneuf a mm aus Dittinbien, bei uns it vielen J゙arben al’ Bierpflanze fultiviert; Gomph réna L., Winterblume, rote Jmmortelle, einjährige beliebte Iopfpflanze ans Diftindien. 
59.

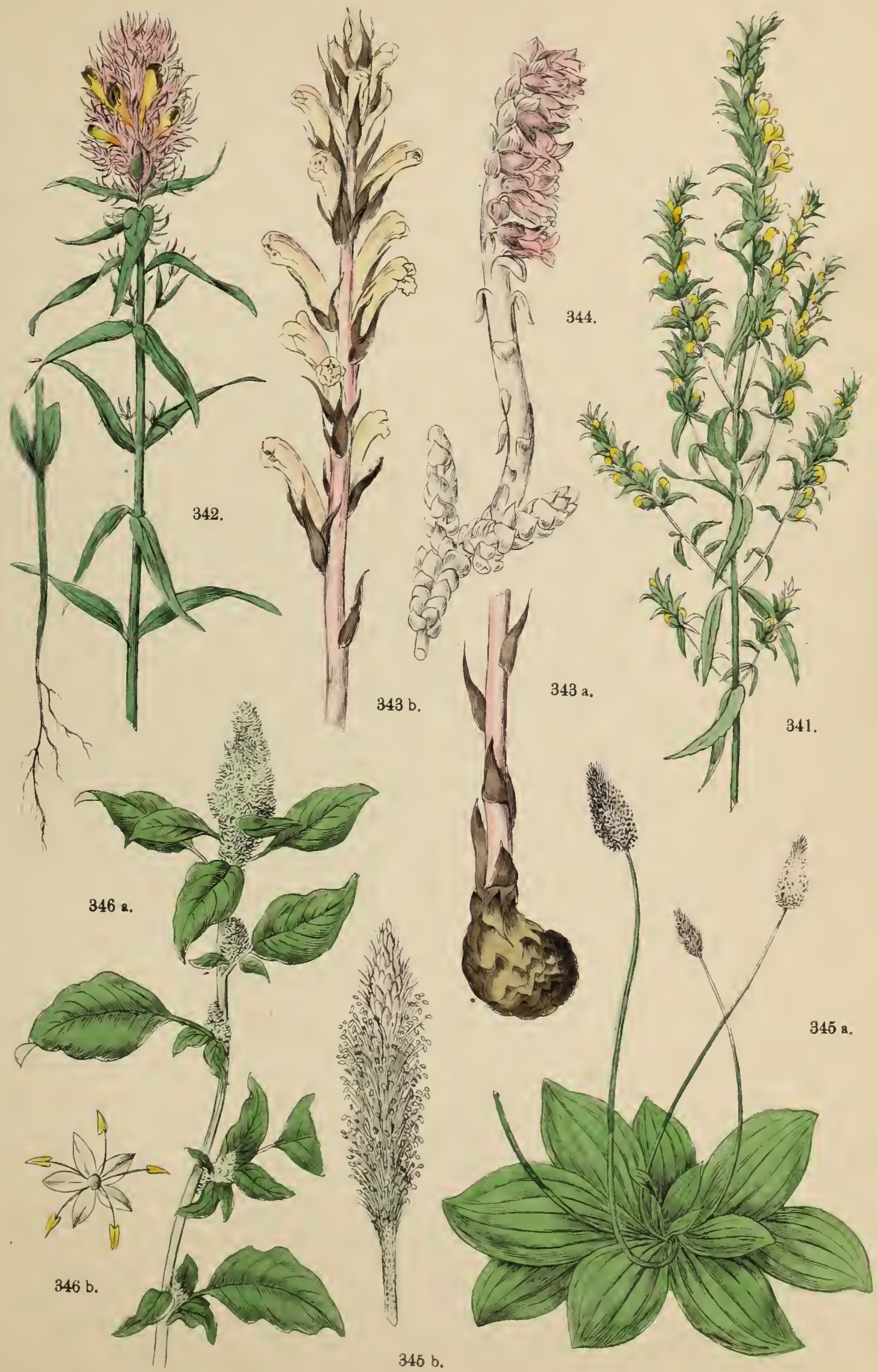



, 
60.

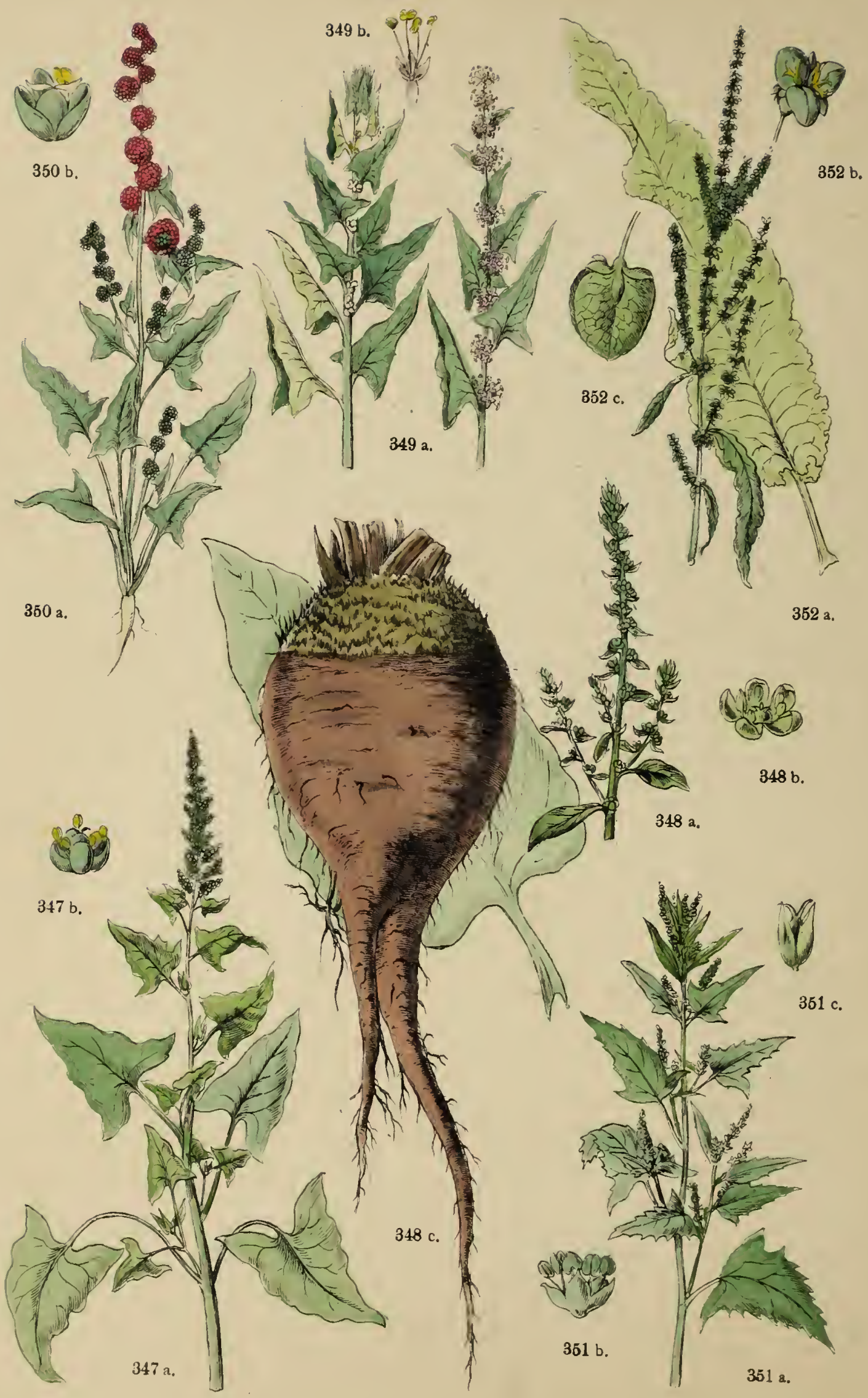


Taf. 60.

145. Familie. Chenopodiáceae Juss. ariesoergewädile.

lleber 300 Arten Siräuter oder Sträucher, gauz bejonbers ๔alzpifanzen, meijt in ber nörbliden ge= mäp̈igten Zone. Biele gehören zu den Stranopfilanzen und gehen Soda, andere bienen zut Nahrung, einige fint giftig. Wir geben Gier:

Eig. 347 a. b. Chenopódium bonus Henri-

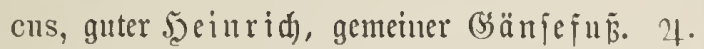
20-30 cm hod, häufig aulf @dyutt unto be= bauteın Boden, läitiges llufrautt; blïht bom Mai-2(uguît.

fing. 348 a. b. c. Beta vulgáris L., gemeine Rutfelrübe, Diangolo, Zufererrïbe. (.) ober $\odot$. Etengel $60-120 \mathrm{~cm}$ hodj, an

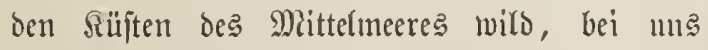
in viefen Epiefarten in Bärten unt auf Felbern

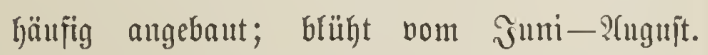

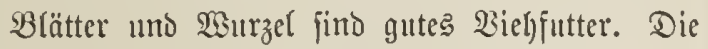
Spiefart Zudferrübe bient zur ₹abrifation bes Æuntéf rüben=3utfer?.

fig. 349 a. b. Spinacia olerácea L., gemeiner Spinat. $\odot$ ober $\odot$. Etengel $30.90 \mathrm{~cm} \mathrm{hodf.}$ 21us bem Drient, in mefreren Spiefarten bei

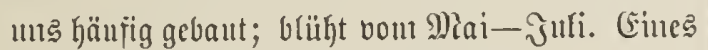
Der bejten unto gejündejten Semüije.

fig. 350 a. b. Blitum capitítum L., E゙r $8=$ beerfpinat, Sdjuinfbere. $\odot$. Etwa $50 \mathrm{~cm}$

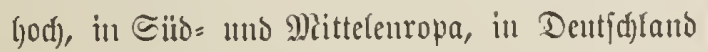

hie uno da angebaut; brïft im Эulli, 2luguift.

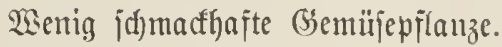

Eiin. 3551 a. b. c. Atriplex pátulum L., anıgge breitete Melde. $\odot 30-90 \mathrm{~cm}$ yod, gemeines llnfraut ant $\mathfrak{B} e g e n$, anf Sd)utt unto in (Bärten; blüht voun Эuli- ভeptember.

3" den Miefoen gehören andr): Sálsola L., Salzfiaut, cine Miceritranopiftauze; Corispé $\mathrm{l}^{-}$ mu m Juss., פsanzenja me, Micerjantopflanze.

\section{Fantlic. Phytolíceae $R . B r$.}

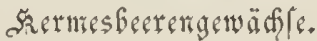

15 Irten Sräuter ober Eträud)er, meijt im tropijden Anterifa einheimija. Die widntigite ijit: Phytolácça L, gemeine Sermeşbere, Jarb= pīlanze aus હübeuropa. Die edjten vief intenjiner färbenden Siermeşbeeren fommen voun ber Siermeseidje.

\section{Fanilie. Polygónene Juss.} ¿hröterifigewäd)

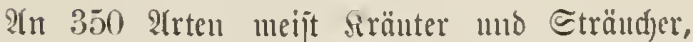
über alle 3onen ber Erobe verbreitet. Cinige ent= halten freie ভäuren, andere gute Farbejtofie. 3" biejer Framilie gehören:

Eig. 352 a. b. c. Rumex crispus L., Jaajent= ober fraujer it mpjer. $\%$. $60-90 \mathrm{~cm}$ hod, auf จี้ meines utnfraut; blïht vout Junt-Ifuguțt. 
Taf. 61.

fiin. :353. Rumex obtusifólius L., ittumpí= blättriger Almpicr, Grindivurz, Miergel=

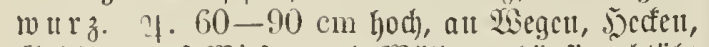

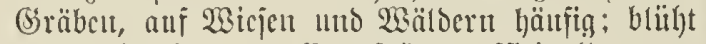

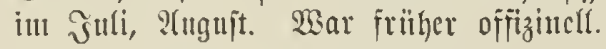

fing. $\mathbf{3 5 4}$ a. b. Polygonum aviculáre L., 2ogel= \& r ö tc r i d). $\odot$. Srriedfent, $30-45 \mathrm{~cm}$ lang, gc= meinites llnfraut überall an wegen unto auf

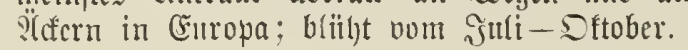

Fign. 3555 a. b. Polýgonum bistórta L., wicien= Sinöterif, Ratterwurz, Blutfraut. 21. $30-60 \mathrm{~cm}$ hod, auf fendten $2 B i e j e n$, in Deutid =

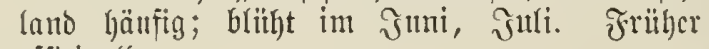
offizinetl.

fịn. 356. Polýgonum persicária L., pfirifid $=$

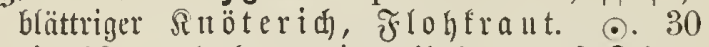
bis $60 \mathrm{~cm}$ hod, gemeincs llufraut auf Eduut,

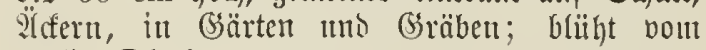
Julli-Dftober.

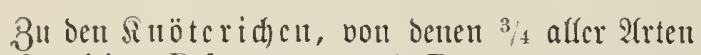

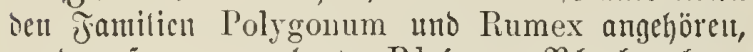
worien fcruer gerceftuct: Rhéum, Rhabarbcr; Coccóloba, Eectranbc, ein Pinthyolzbaum in Beptintoien.

\section{Fantilic. Lauríneae Juss. sorbertgemäds.}

Bon bicjer grof̌en Familie fentut man an 1000 Yrtent, meift tropijøe, imnucrgrünc Bäume, vort

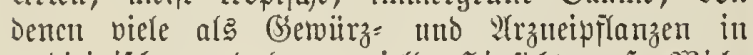

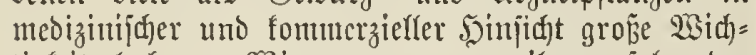
tigfeit haben. $2 B i r$ nenuen von ihnen folgende: Laurus nóbilis L., edler Rorbeerbaum, in

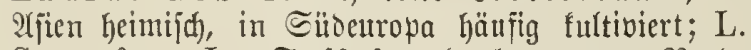
Sassafras L., Safjafraglorber all Roro= autcrifa; L. Cinnamomum L., edfter $3 i \mathfrak{m m t}=$ baum aus Ditutoicn; L. Cassia L., Caffien= zimutbaum in Ditindien; L. campliora, Sampferlorbeer in Egina unto Japan.

\section{Fantilie. Myristíceae $R$. $B r$.}

Etwa 20 Srten, nur in den Tropen hocmiju, gevoür

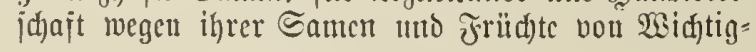

feit. Dic wertoollite bavou iit Myrística offcinalis L., (M. moscháta Thbg.) coter $\mathfrak{R} u$ g fat

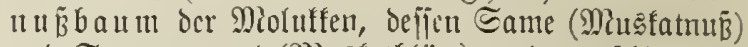

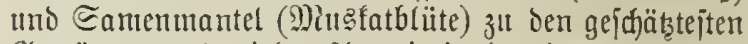
(Semürzen uno vielen $\mathfrak{Y}$ rzzneimitteln bienen.

\section{Fantlie. Proteńceae Juss. ว’totettsgemädile.}

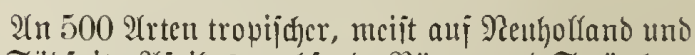

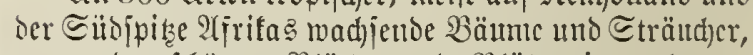
wegen ocr ¡đjönen $\mathfrak{B} 1$ ätter mo Brïtten in mehreren

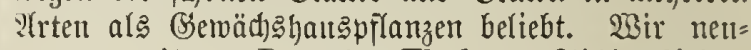
nen von ihnen Protéa Thnbg., Silberfidte, Treibgauz̧pflanze vom Sap; Bánksia, zaftreidic

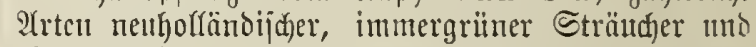

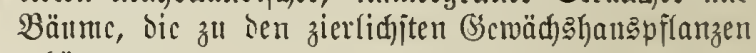
gchöretl.

\section{Fantitie. Thymeláceae Juss. secioefgatagemadfe.}

(Ettwa 180 2rten, meijt Sträıđđer, über sie ge = mä̈ßigten Zonen verbreitet, jämtlid idarfe Gift= piffanzen, viele ausagczcidfuet durd) fajöne uno ftart ouftente Blïtent. Wis nenten von ifncu:

fítg. 357 a. b. Daphne mezéreum L., gemeintrer Sciberbajt, Dentider ober Berg= Bifffer. Ђ. $60-100 \mathrm{~cm}$ hod, in affen unjern (Sebirgs: wärloern, der friiłhen BBüte rocgen (blïht itt zebruar uno März) audd ats (Gartennflanze be= liebt. $\mathfrak{s}$ allen $\mathfrak{T}$ cilen jehr giftig. Difizinelf.

3u ifyen gehören ferner: Lagétta Lam., seinwanobaum, Escitimbien; Inocarpus édu-

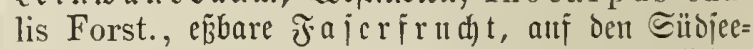
infern, beffen Frïd)te ben (Eimwoof)uern zur tügliden ?ahrutig bicuen.

\section{Framilie. Santaláceae $R . B r$. รautefgemäต}

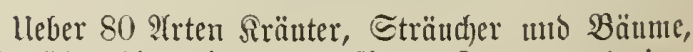
meifit ïber bie beiben gemäbigten 30 nen verbrcitet. 3 in ignen gehören:

fitg. 358. Thesium alpínum L., Grebirgas= Icinblatt, Bergflads, 2erncinfraut. 2/. Stengel $15-24 \mathrm{~cm}$ hod, auf grajigen $\mathfrak{B e r g a b}=$

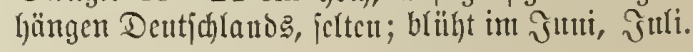


61.

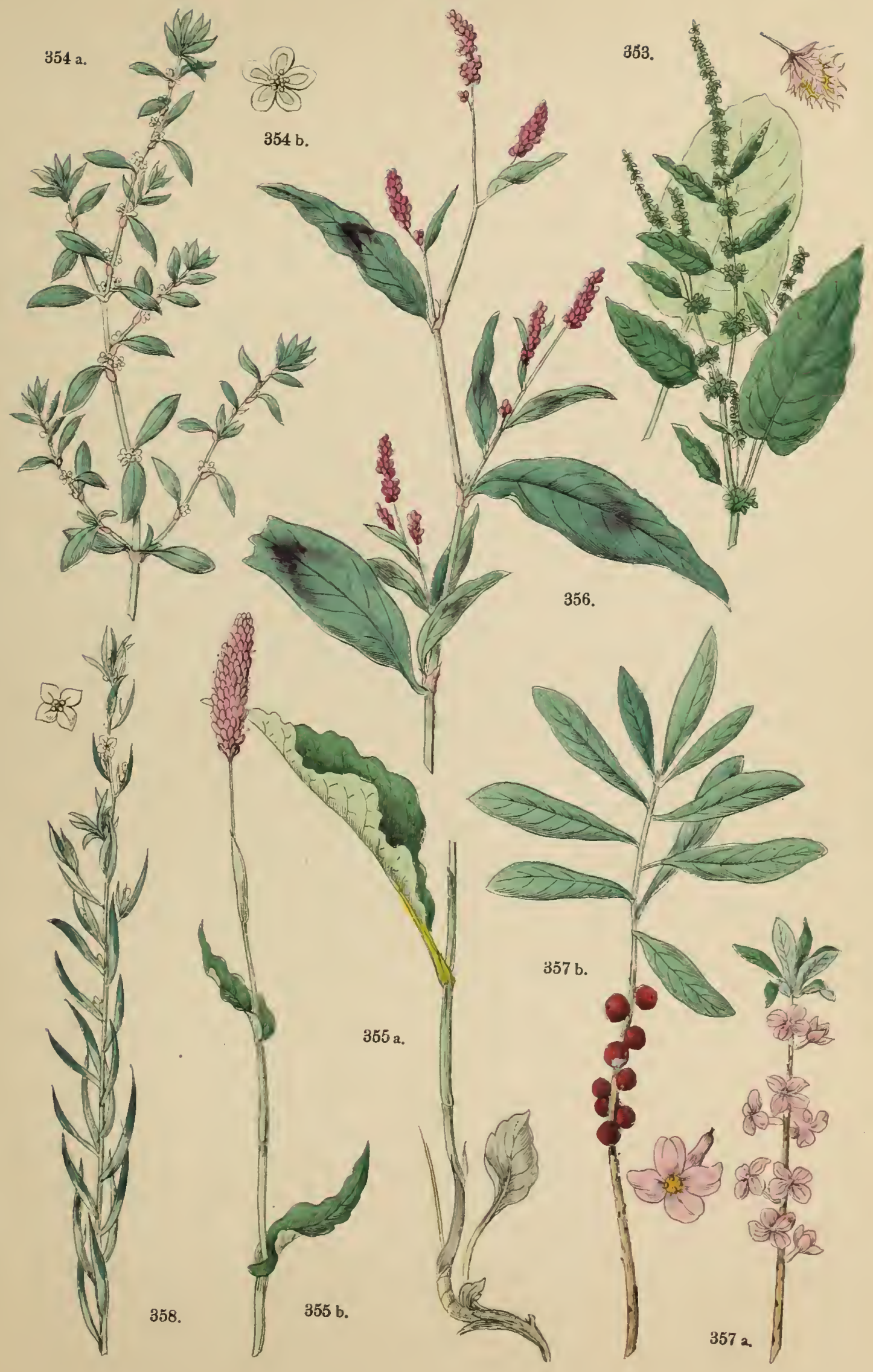





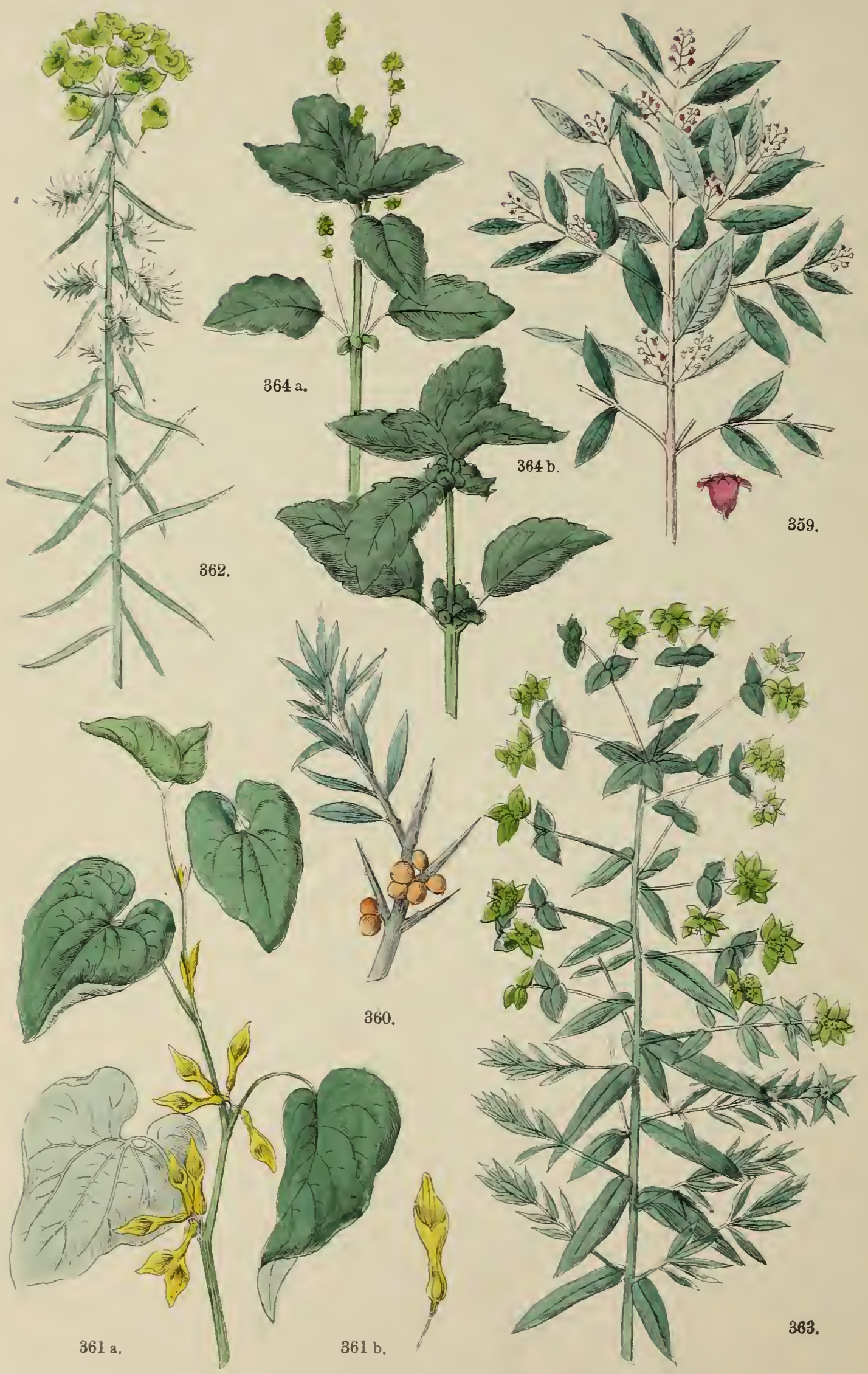


Taf. 62.

fitg. 359. Sántalum album L., meirerer હantel=

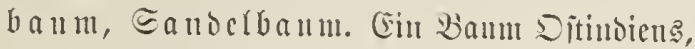
bejien Eplint jrïher arzmeilicf, deijen rejenartig ried)enoes Siernfjolz als giätchermitter bicut. Yudd) wiro leksteres zu foftbaren Fournieren unb be=

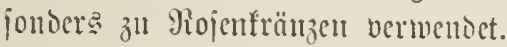

?(ud) F usanus R. Br. geljört zu biejer Familic; ce ijt ein Etraud) Peuffollanos, ocijen manoclartige Eamen (Snandang=2ï̈jie) jelhr woblidhmedfent jint.

\section{Fontilic. Elaé́gnear. Juss. (3) erwetoentgemädf.}

Cine fleine Jamilie, deren 2 rten ïber bie nörd:

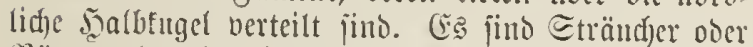

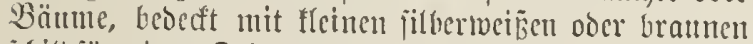
idjiloförmigen @d)uppen. Micf)rere fins als Garten= zieriträuder beliebt, fo bie verichiesenen Arten Des Elaeágnus Tourn., Dreajer, Siwcide, $\mathfrak{B} a=$ radiesbaum ans Eildenropa, mit jpitzen filber= wei $\tilde{B}=\lceil$ đd)uppigen Slättern. Jerner gel)ört zu biejer Fanuiltc :

fig. 360. Hippóphaë L., 巨anoorn. ち. 1

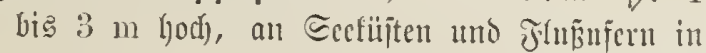
Dittel= uno Sitenropa; bliiht im 2(pril, Niai. ?(te Bierîtrand) in (särten belicbt.

\section{Frmitic. Iristolochieae Juss. (B) tareruscigewäd}

3ahtreiche 2(rten Säänter, bejonters in ben heišen

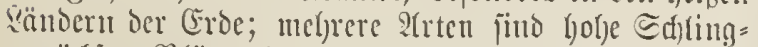

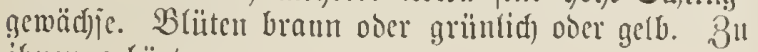
ihnen gefjört:

fig. 361 a. b. Aristolóchia clemátis I., gc= meines Diterinzei. 2. $30-60$ ch hod), 23 urzelitoef friedjent, in 2 seinbergen, (Gebirjeden

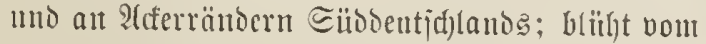
Miai-Juni.

bierher gefört audf: $\Lambda$ s a rum eu rop a c u m L.,

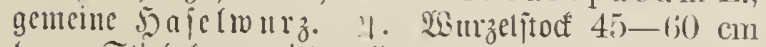
lang, Ettel furz, niècr liegeno, itt \&aublyolźnäloern

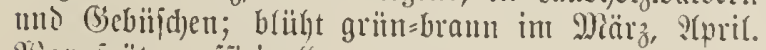
Siar frïlyer offizinell.

\section{Fantilic. Cytincaue Brongn.}

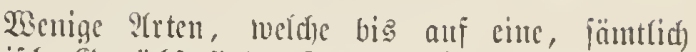
tropijde (jomädje junt. Dicje ijt Cytinus lypocistis L., gemeiner Dypocijt, cine jduppige, 60 bis $120 \mathrm{~cm}$ hohe, braulugelle Ed)marokerpflanze in Silocuropa, namentlid) in (śried)enlant. 3n den (C)tineen gefört ferter Rafflesia R. Br., Rícjen= blume auf Eumatra; bie über $1 \mathrm{~m}$ im Dmduteñer

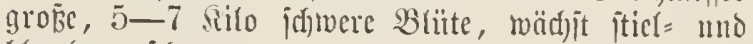
blattlos, id)marobeno all bet 2 immeln andrer (5ic= wäcljie.

\section{Familic. Begoniáceae R. Br.

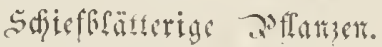

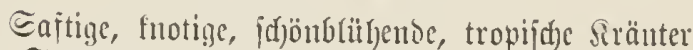
mo Strändher, mit am (śrumbe idjiefen Blättern, von benen viele ?liten in il)ren 2iaterianio als

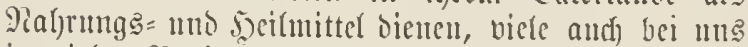
in viclen Siarietäten beliebte Topfpplanzen fint. $\Xi_{0}$ Begonia discolor R. Br. uno Begonia rex Putz, ans sititioieu.

\section{Fantilic. Euphorbiáceae Juss.

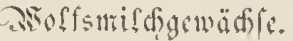

Heber 1800 P(rten Siräuter แtto @träudfer, jelten B̉äume, ïber bic heife mo beioe gemäbigten Zonen verbreitet, einige jefrr gif̆tig, andere arzucifrärtig,

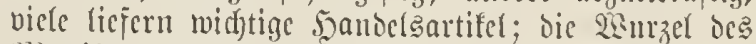
Wianifyot vertritt in ifhrem 2aterlande bie Etelle bes Gsctreibes. 3" ifnen geljören:

fiy. 362. Euphórbia cyparíssias L., Cuprej=

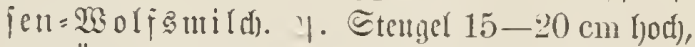

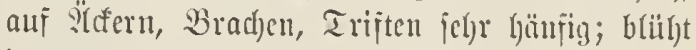
iun STpril, Miai. Biftiges ltufraut.

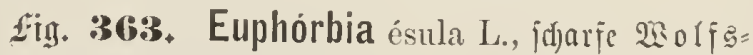

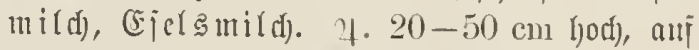

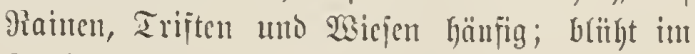
Juni, כૈuti. Giffiges llntrant.

fing. 361 a. b. Mercuriális ámma L., jähriges bingeffrant. (.). 2()-5() $\mathrm{cm}$ hod), itberall

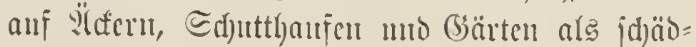
ridfes, giftiges lunfraut; blitht nom Jutui bi Ditober. Jrither offizinefr. 
Tấ. 63.

fing. 3(6.). Buxus sempervirens L., genteiner

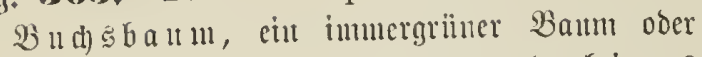
Straud), auts bem Erient ftaumend; bei me

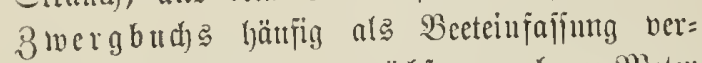
mentet. Ju Sübeutropa wädhit er mehrere Mieter hodh, in jienlid) bicten Etünmmen mu liejert

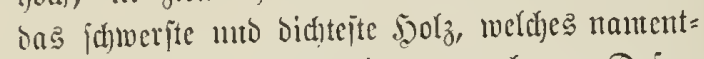

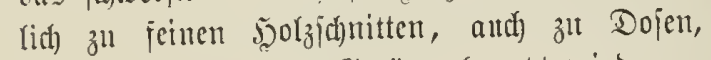
Siännten mo anbern Bieräten bemutżt mitro.

fiti. 366. Ricinus commúnis L., gemetner Bunderbanm; in allen Teilen Dit = und Eseptindiens heimija, bort ein bis $12 \mathrm{~m}$ hoher Baum, bet uns $\odot$ uno nut $2-2^{1 / 2}$ in hohe Bartenzierpflanze. Daz S̈r der Єamen ift cinte ber fräjtigiten 2 trzneimittel.

fiị. 367. Siphónia elástica L., çhter Feder = har zbaum, Sautjdufbaum, (B) mmibaum. Ein 15-18 m hoker Baaum Brajiliens uno (3) itanas, oejijen erhärteter Mitldjiaft oen elajti=

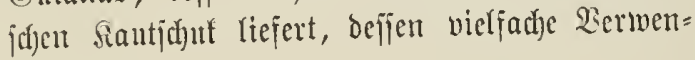
ountg befannt ijt.

fị. 368 a. b. Játropha Mánihot L. (Ma nihot utilissima Pohl.), Mianiof= oder (Eajfabe= itraud). Ein $2-2^{1 / 2} \mathrm{~m}$ hoher Erraudh, im tropijd)en 2(merifa betmija, mto bajefbjt wie im tropijd)en 2ffrifa und 2(jien wegen ber fleijdjigen, fnolligen, oft bis 15 Silo idjweren $\mathfrak{B}$ mrzel, bie eine ber widytigften Räl)rpflanzel ber Tropent biloct, Gäıfịg angebant.

fiij. 369. Croton eleuthéria Sw., Sa $\mathfrak{g} \mathfrak{f}$ a riflen=

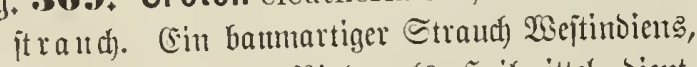

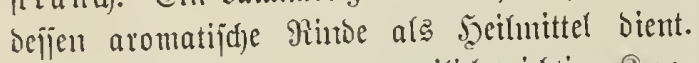
Daß höb)ft giftige, aber arzmeilid wichtige $\Omega \mathrm{r} 0=$ tonöl wirt alts ben Santen des Croton tiglium I., *argirfroton, gemomen.

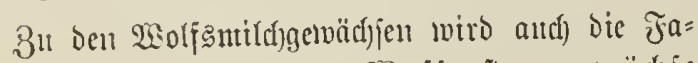
unite Callitrichíneae, Wajierjterugewädje gered)utct; ifye wichtigite aft ift fin. 3\%o. Callitriche verna I., frithlings=

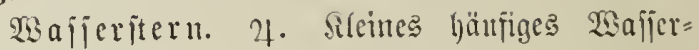
untrant; blüht nom Mai-Dftober.

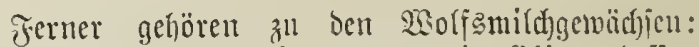
Stillingia Michx., Talgbaum in China, dejīcu wad) కartige Samentülle cinen bedententon Jandels: artifer biloet; Hippómane mancinella, ber höd)it giftige $\mathfrak{D}_{i}$ ॥ zanirfabaum im tropifd)en $2(m e=$ rif́a, umb aubere, fid meijt burdh giftigen Dildajaft atşzeidjutende Gíwädje Der Tropen.

\section{Frmitic. Monimíeae Juss.}

ปૈt6er 30 Arten inmergrüuer Bäıme uno Sträı= d)er, fämtlid) tropijche, meftr ober weniger gewürtz= hafte Bienüd)ic.

\section{Fontilic. Urtícinae Juss. areflefattige Gewäd).}

Begen 400 2rten, mit jteifen Bremboriten be=

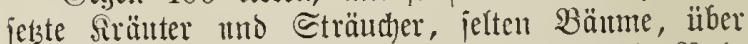
bie ganze Crobe verbreitet; mandje bienen als $\Re a \mathfrak{l}=$ rumgsmittel, andere zut Gemebert.

Dieje Familte, sie Rejejelartigen Bewädje, tren= nen viele Botanifer in:

1) Urtícea e Bartl., Brennejịeln, Fịg. 371.Parietáriae L., (B́ałfant, Fig. 372.

2) Cannabáceae Endl., Şanigewädjie, F่̧g. 373. - Húmulus L., รૃopfen, F゙ig. 374,

3) Mór a e, Maulbeergewäd)ie. - Moráceae Endl., Mautfberbüume, Fig. 375. - Ficus Tourn., Jeigenbaum.

4) Artocárpeae Bartl., Brotfrudytgemächje. Artocárpus L., Brotbaum. - Antíaris, Upasbaum, jig. 376.

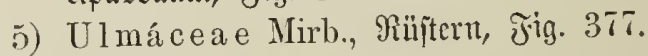
Dic widhtigiten jint:

fig. 371. Urtica urens L., fleine Brennefiel. ๑. $30-60 \mathrm{~cm}$ hod), befauntes, überall auf bebantem und inbebantem 20 oden ivarjjentes llufraut; bliifht vom Jult-Septcmber. Bremtend. 
63.
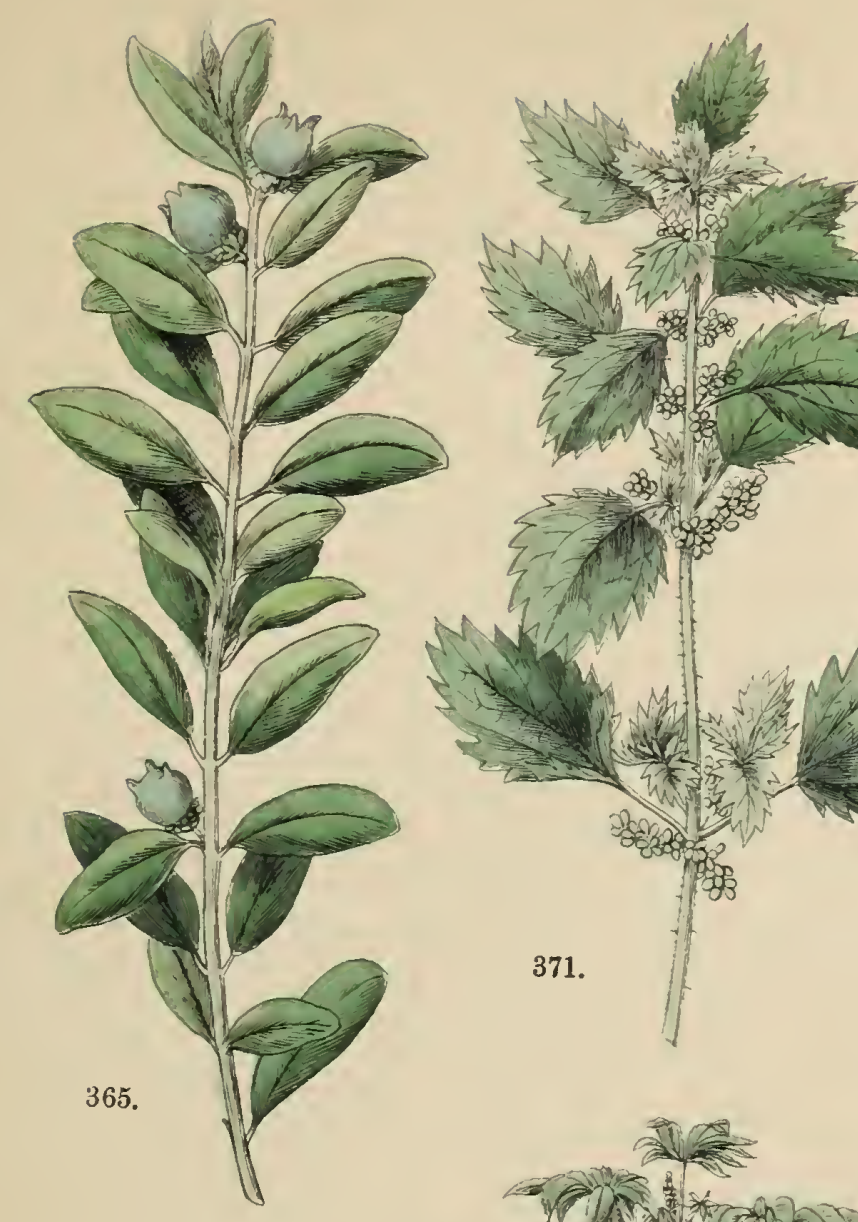

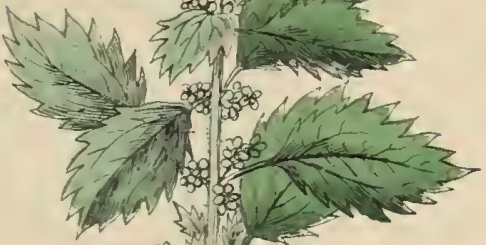
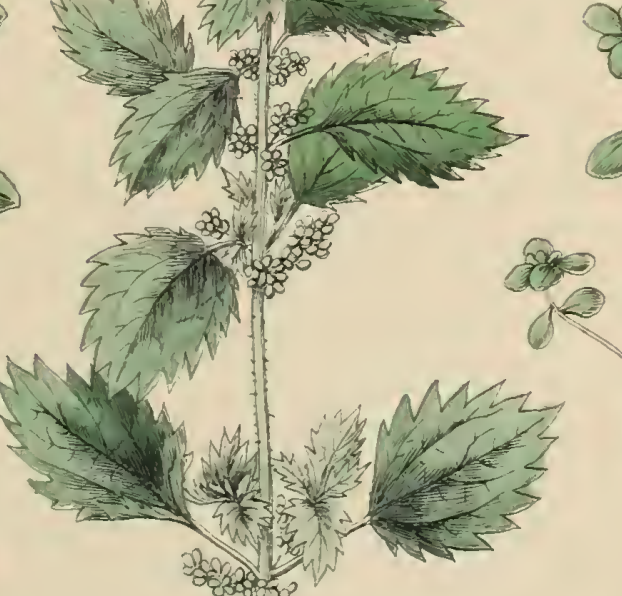

23

dor

370.

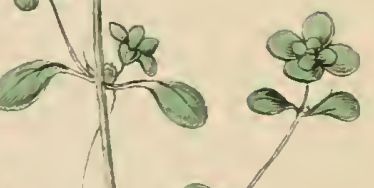

371.

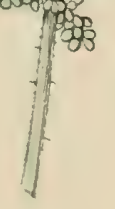

366. $2(x)$

368 b.

P.

ang oth

a 12
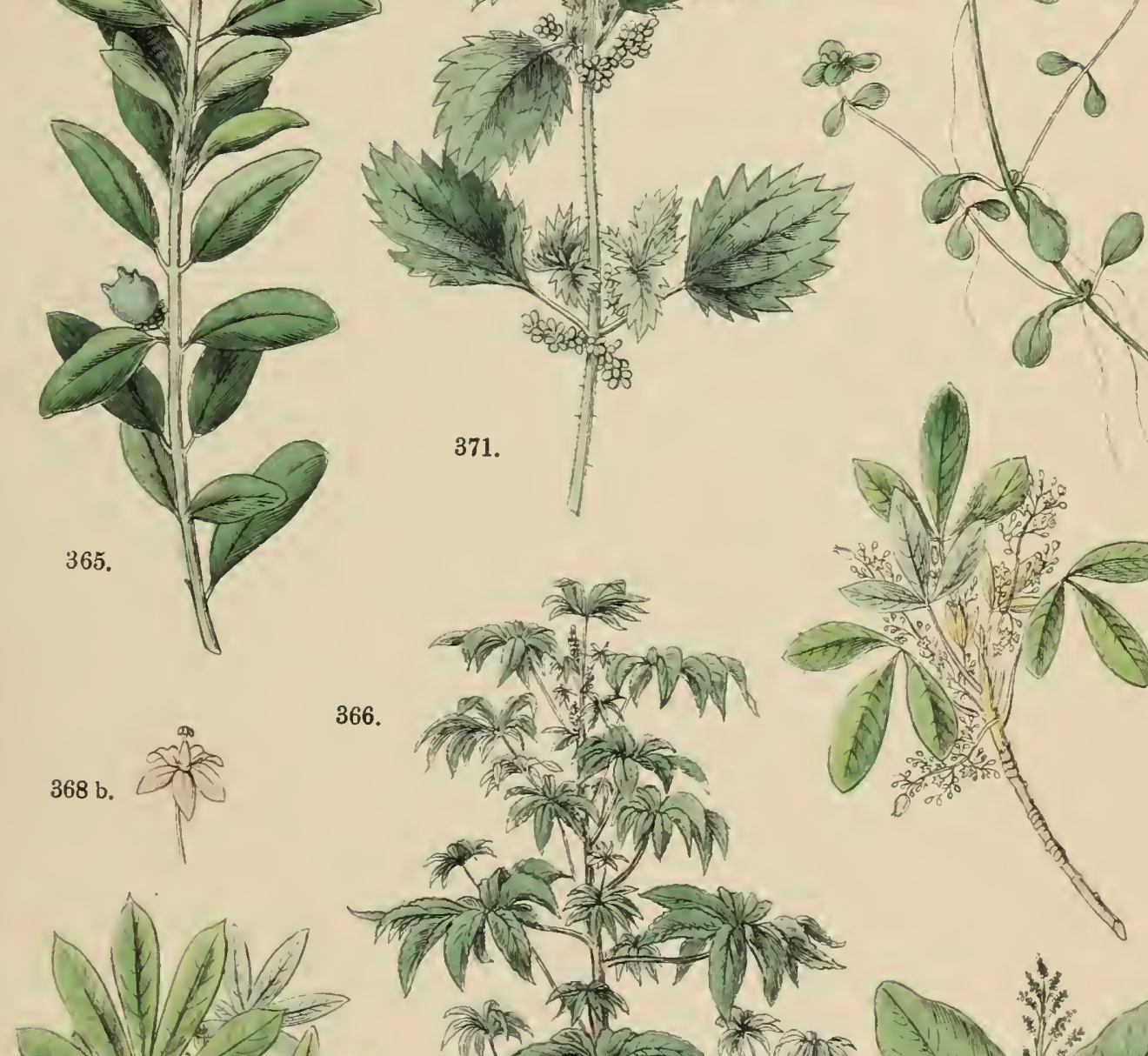

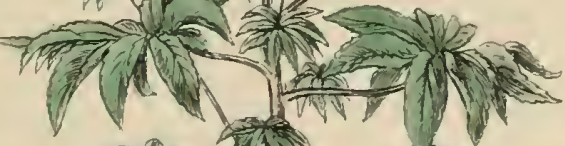

-
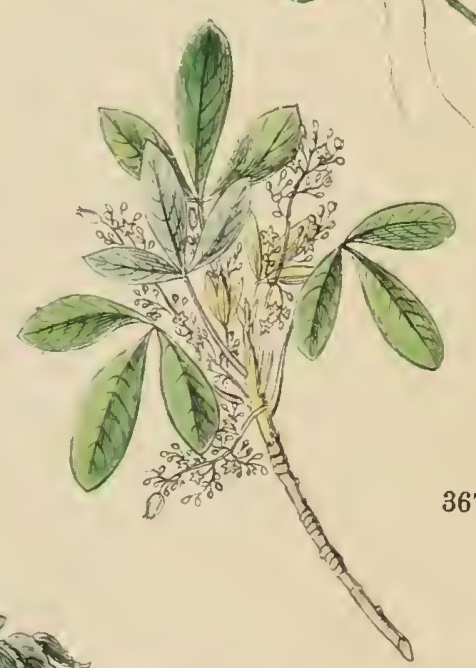

367.

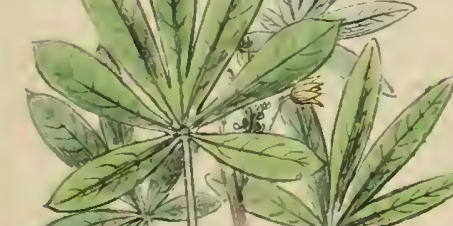

Th $y$ (De
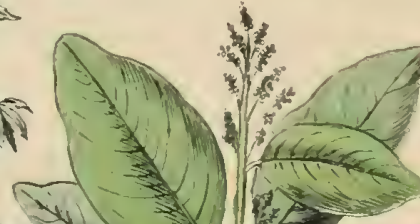
$7 \times 1$
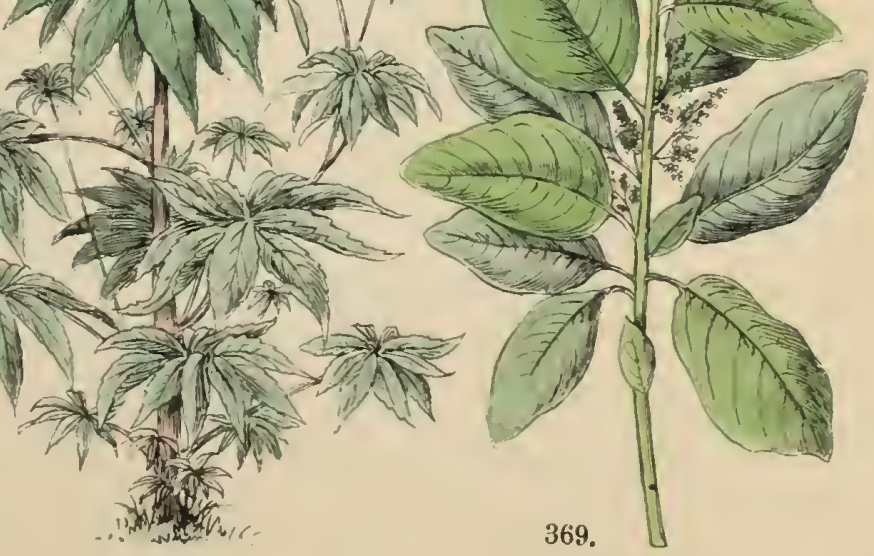




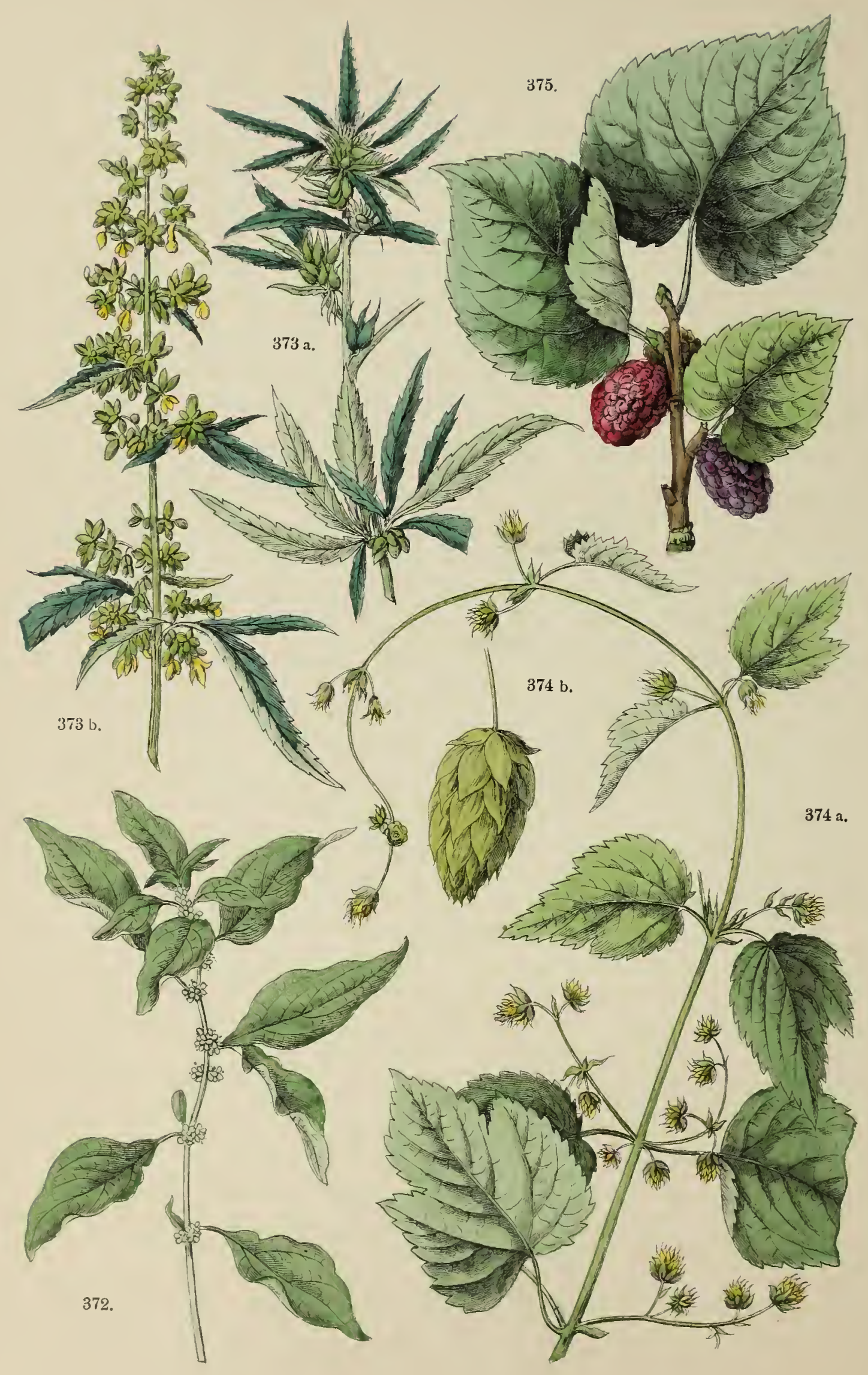




\section{Taị. 64.}

fing. 37:. Parietária officinalis L. (I. erecta

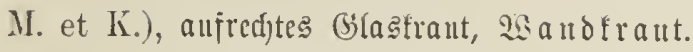
7. $30-90 \mathrm{~cm} \mathrm{lyod),} \mathrm{auf} \mathrm{Edutthanfen} \mathrm{unto} \mathrm{an}$ Mantern; blüftt vom Suli-Dftober. Sit nidft brettictio.

f́igy. $\mathbf{3} 73$ a. b. Cánnabis satíva $\mathrm{L}$., gemeinter

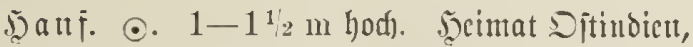

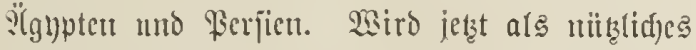

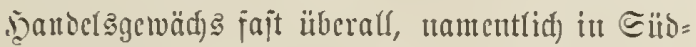

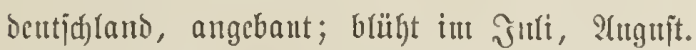
Eeine Berwendung zu jaanffcinwanto, Eegettud,

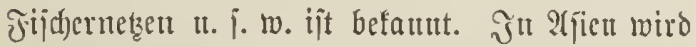

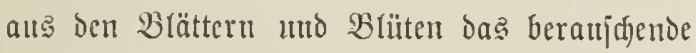

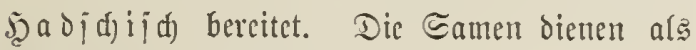
Sogelfutter. Difizincll. f́ig. $\mathbf{3 8 4}$ a. b. Húmulus lúpulus L., geturituer .jopfen. 7. Mantent, bis 12) $\mathrm{m}$ lang, ver=

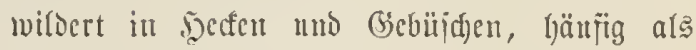
Bierwürzc iul Birober fultiviert; blügt vom Эnfl-Eeptember. 2ierwentoung z̆ Bier befannt. Die Etengel werbett z̆ll Etriffen, Matten umo @ädfen verarbeitet. Difizizituelf.

Eitiy. 375. Morus nigra L., iqjwarzer Mia 1 I= becrbautm. 韦. 6-10 m hoher $23 a$ aut auta

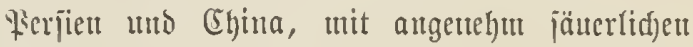

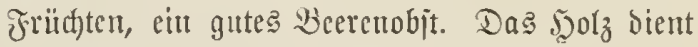

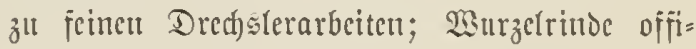
żinell. Die Biätter biejer $\mathfrak{U}$ rt gebert nut cin

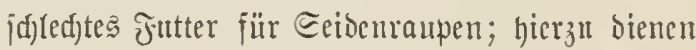
dic Blätter bes ıeišen Mianlbecrbantmes, Horus alba $\mathrm{L}$. 
זaץ. $6 \overline{5}$.

fiùn. :376, Antiaris toxicária, giftiger $2(n \mathrm{t}=$ f char, llpasbaum. 专. Eill $18-30 \mathrm{~m}$ holjer

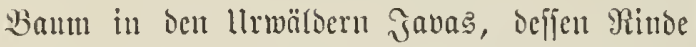
cinen Mitldjajt liefert, der vou ben Eingebornen

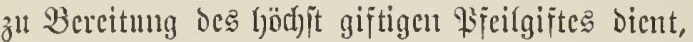
auperbeut aber aud) als 2(rzuteinittel von ifnen benülzet wird.

fin. 378 a. b. Ulmus campéstris L., gemeine lltme, felontme, Felorüfter, Iïiter.

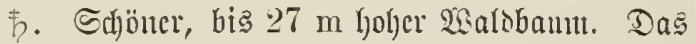
zälye, harte, bauerbajte $50 l_{z}$ gibt vorzüglidjes Bankolz, Die 9illoc bient arzneilid). 2on Ulmus gibt $\mathfrak{c}$ s nod) cine zweite Deutidye 2(rt, bie Flatter= Rüiter, Ulmus effusa L.

\section{Fanilie. Chlorántheae R, Br.}

Meijt Hleine, itrautd)artige, gewilizhafte (Ssewäd)je Der lotiBet Bone, von denen nur Chloránthus officinális Blume, gemeiner Bflaumenpfefier, zu crwähnen ijt. (5) ijt ein fnotiger Straud) Jabas, Deffen sisurzel bajelfijt arzneilid) bient.

\section{Fantilie. Piperáceae Rich.} Pofeftergewädsle.

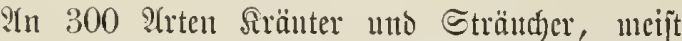

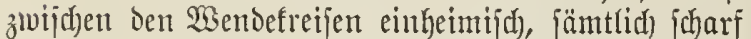
getwürzhaft. Die widgtigiten ?trten fino Piper nigrum L., ち, fdwarzer $\mathfrak{B}$ feffer in Ditinoiell, ber die befaunten Piefferförner, unfer widytigites

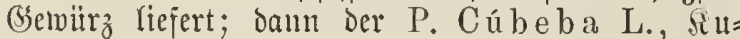

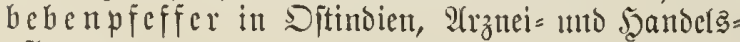
prilallze.

162. F゙nuilie. Amentáceate Juss.

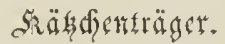

3̆ bicjer Familie gefören die meijten :anb=

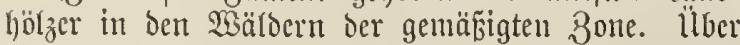

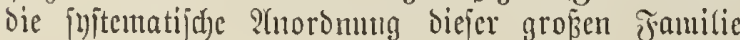

finto, wie hei ben शejjelgewänfjen, die Gelehrten nicht einig. Wir legen fier Dic Einteilung vout Jujïien = be = Canbolle mit cinigen neueren 2erbeñe= rungen zul Brimise.

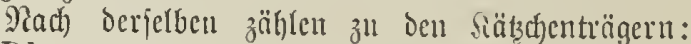
1) Platáneae L., Platancu, 4 Irten grofier,

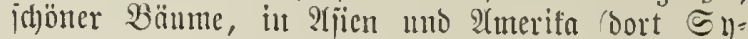
famore genaunt), bei unz in ganz Mrittelenropa l)äufig in :Ulagen; 2) Salicíneae Rich., $23 e i=$ bengewäche uno giappelgewädile, in zahl=

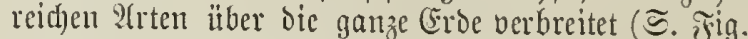
$385,386,387)$; 3) Cupulíferae Rich., 叉2äp

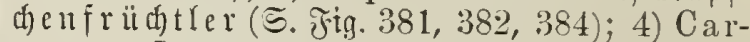

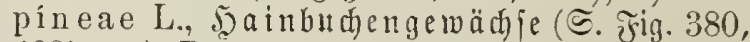
383); 5) Betulíneae Rich., Birfen= แno (Ellerngewädje (Fig. 378, 379). श2och red)net man bazll 6) Myríceae Rich., (S)ancl= oder Wad s beere, Borit, Raubitraud) auj Mioor = uno Toriboden Rordoentid)lands uno 7) Casuaríneae Mirb. Seulenbäume in Sujtralien. Wir geben in 2 tbbiloungen alts diejer grosen Familie, zh ber

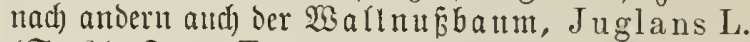
(ङ. 61. Jam. Terebinthaceae, Jig. 76) ge= hört, die folgenden îrten:

fị. 388 a. b. Alnus glutinósa L., graute Errle, S d) warzerle. 市. 6-20 $\mathrm{m}$ hoch, fait ourd ganz Ěropa ïberall gemein an fendten @tellen,

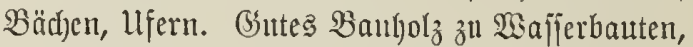
von Dred)slern zu Edynizzarbeiten gejudt, bie Siohle zlt Bereitung von @dję̧pulber bienlid); blïgt im Februar, März.

fity. 379 a. b. Bétula alba L., gemeine Birfe,

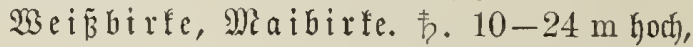
bis $60 \mathrm{~cm}$ bick. Rerbreitet im 2Rorden (Furopas, 2jiens und 2Amerifas. (Sutcs Steffmader= uno Iijdjergolz; bie Pinoe bient zu allerlei .̧ants= geräten แtho gibt bas SBirfenöl, weldes bem Jud)tenteder ben eigentüm(id)en (šerud) ver(cilgt. Blätter gutes હçaffutter; blügt im Ilpril, Maa.

fitig. 380 a. b. Carpinus Bétulus L., getnciute

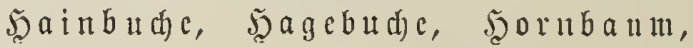

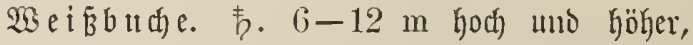
in $\Im_{\text {ito }}=$ uno Mitteleuropa ganze $\mathfrak{B a ̈ l} l$ cr biloeno,

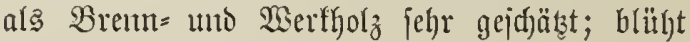
iun ?tpril uno Miai. 
65.

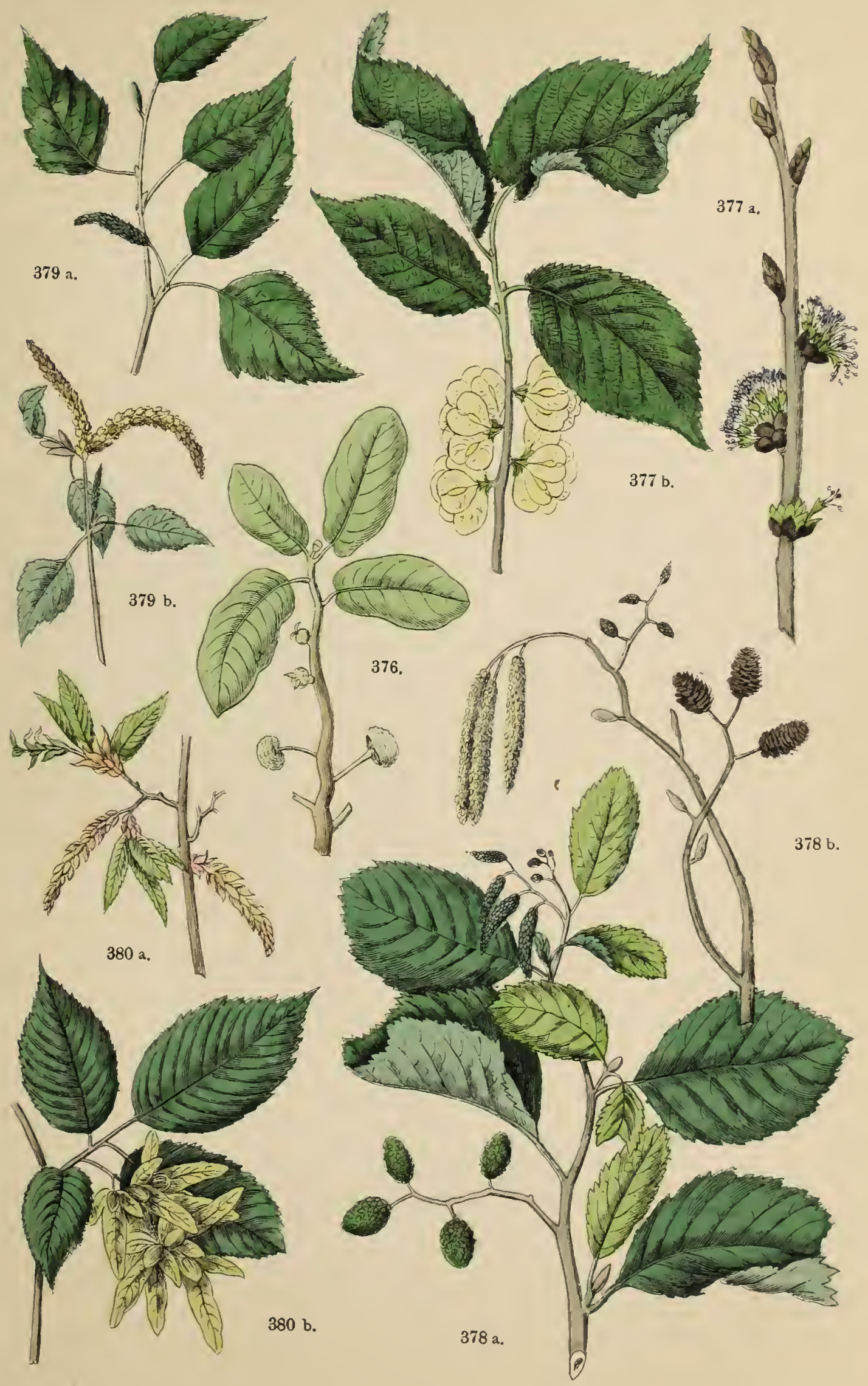




66.

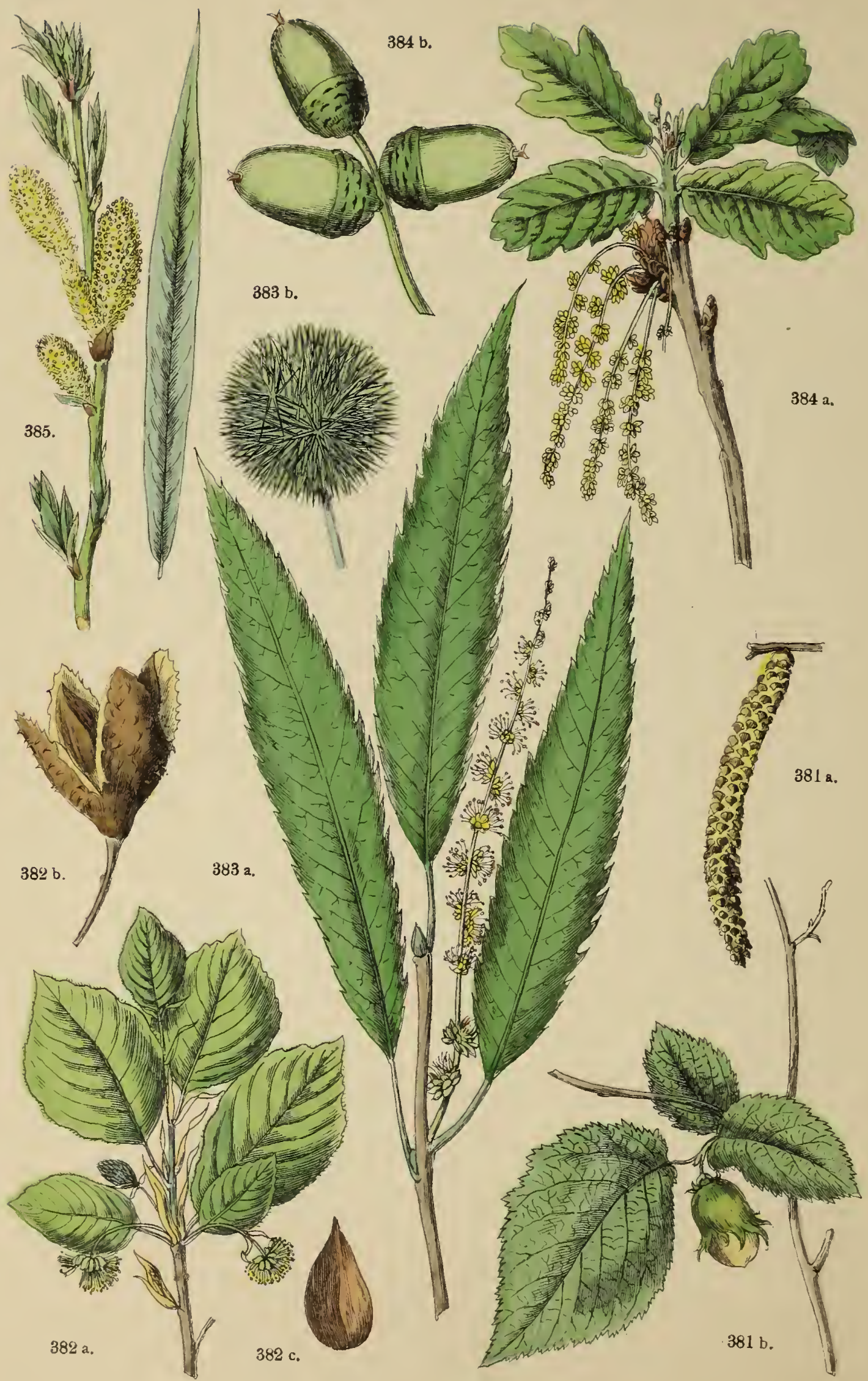


Taf. 66.

fiij. $\mathbf{3 8 1}$ a. b. Córylus arellána L., gemeiner

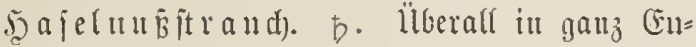
ropa verbrcitct, in Dentjalano nebjt cinigen an= Deren ?arten tmo Epiclarten häufig ala $\mathfrak{B a r t =}$ uแ

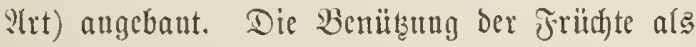

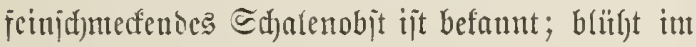
Jebrutar, Miärz.

fị. 38: a. b. c. Fagus silvática L., genciute

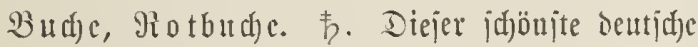
$233 a l d b a t m$ wito $20-30 \mathrm{~m}$ hodh uno ijt nit

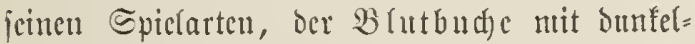

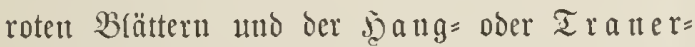
budae bei uns allgemein befannt uno geiduälzst. Die Rotbucje liefert mis aus groß́en Siäldern

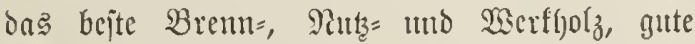
Sioble, Ieer, \$ottajde, Sireojot, Jyozjipiri=

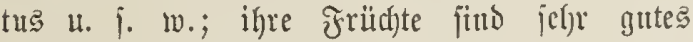
Sdjwcinchutter und geben ein vortreffifides Epeife= öl; blüht im Stpril, Miai.

fing. 383 a. b. Castánea vesca Gärt. (Fagus

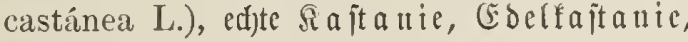

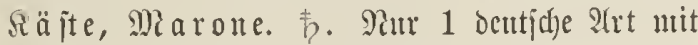

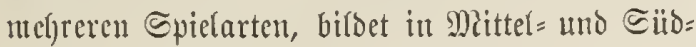

curropa, nautenlidu in Staficu, gattze risälocr.

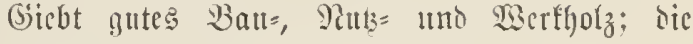

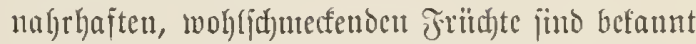
tuto biloen ciner bedentenden Danbeläartifect; blïht im Sิแni.

5iti. 384 a. b. Quercus robur L. (Q. pedunculata Elurh.), getneite Stiel= ober Eommer=

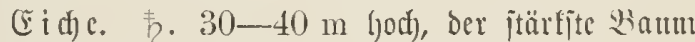

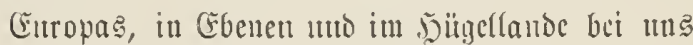

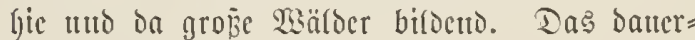

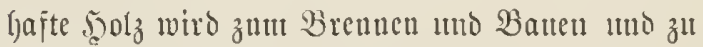

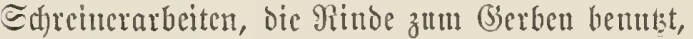

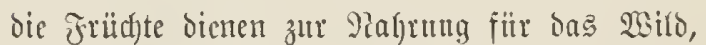
audd) als saffecjurrogat uno ङdweincfutter; blïht im 2 tpril, Maai.

fing. 385. Sálix riminális L., gemeine 2iscioe, Sorbweide, bandweide, lferweibe. b, 韦. Die 2 iseiden zählen etma 150 Srten Bätume vocr $\subseteq$ trä̈udfer, bavon 29 in Detttjjlano betmija. Die abgebitbete sorbuctoe twiro 4-6 m hod, wädjit gewöhnulid) ittaut(dartig, und ift in Deutjdfand jelyr gentcin an Teidjen, Secn und Bädfen. Sic liefert bas beite Ma= terial ż Sorbwaaren uno Flled)tarbeiten; b(üht itn Mär $\mathfrak{r}_{z}$ und Iprit. 


\section{๔aj. 6 r.}

fing. 386 a. b. c. Pópulus alba L., Sis $\mathrm{i} \tilde{b}=$ voer Eilfer= s;appel. 韦. Eitt ftarter, fdjötter Wautm, $30 \mathrm{~m} \mathrm{hodh,} \mathrm{Staum} \mathrm{bismeilen} \mathrm{iiber} 1 \mathrm{~m}$

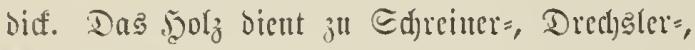
Siiijer= uno Etelfmadjerarbeiten, hat aber wentig

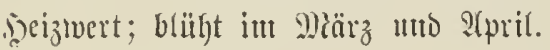

4iin. 387 a. b. c. Populus nigra L, gemeine Fappel, હdwarzpappel. 专. $25 \mathrm{~m}$ hod),

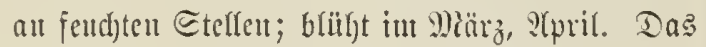

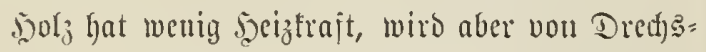
lern und Tijd)ern gern verwendet. Eitte ?lbart P. pyramidális Ait., finrautiocnpappel, slleepappel, italienijde Pappel, ijt als ?llfeebaum bei utı allgemein befannt.

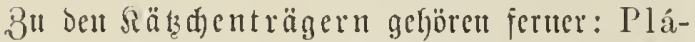
tall us L., häufig in ßartaulagen furtiviert; Myríca L., (sagel, giorjt, brabauter Dinrte,

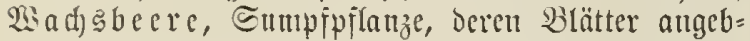
(id) als Jopjenjurrogat benuitst weroen; Celtis L., 3 ürgel= oder Rejfelbantut mit mohtid)meffenten

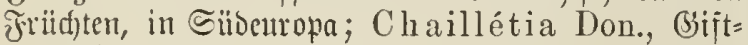
bautu itt ?frifa.

163. Familic. Casuaríneae Mirb.

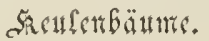

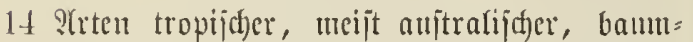
artiger giflatızet, tut ftnotig geglieberten, . idjad)tel= halmartịen siljten, weld)e ợt ganze Wälder bilben

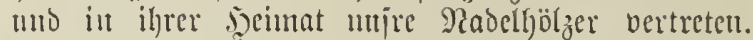

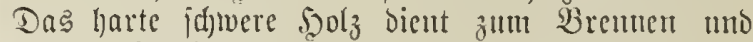
wiro zll Etreitfolben millo alloeren (Serätet verarbeitet.

164. Fanilic. Coníferae Juss.

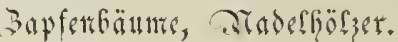

3u ifuet red)tet man 1) die (Fiben, Taxineae; 2) bie (cypreijett, Cupréssinae; 3) die eigent= liden Ra belkölzer, Abietíneae; 4) dic $\mathfrak{B a}\{\mathrm{m}=$ farne, ober Bapientalmen, Cycádeae. Es find die gödjtett $\mathfrak{B a ̈ n m e . ~ D i e ~ w i d ) t i g j t e n ~ f i n t : ~}$

fig. 388 a. b. Pinus silvéstris L., gemeine Siefer, Föhre, Forde, Sicnbalt. 韦. 3iro 20-30 m hod), billoet im ganzen Rorden

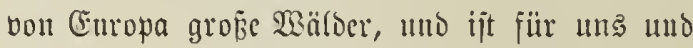
ben ganzen Rorben bą ülz̧lidjite Rabelfyolz. Sic gicbt vortrefflides $\mathfrak{B a n t}=$ แno $\mathfrak{B}$ เeutuholz, Sientur, Terpentin, Teer, Fech umo Bisigenfarz; blüht iแm Mai.

futy. 389 a. b. c. Pinus Pícea L. (A bies pectinata Dec., Ab. alba Mill.), Ia nuc, E゙deltanne, Weintante. 言. Wird bis $60 \mathrm{~m}$

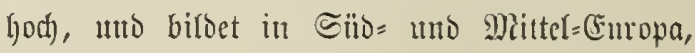
nantentlid) in (sicbirgen, ausgebelnnte $\mathfrak{B a ̈ l o c r .}$

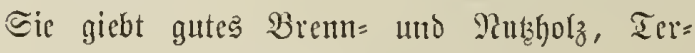

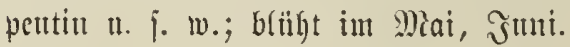




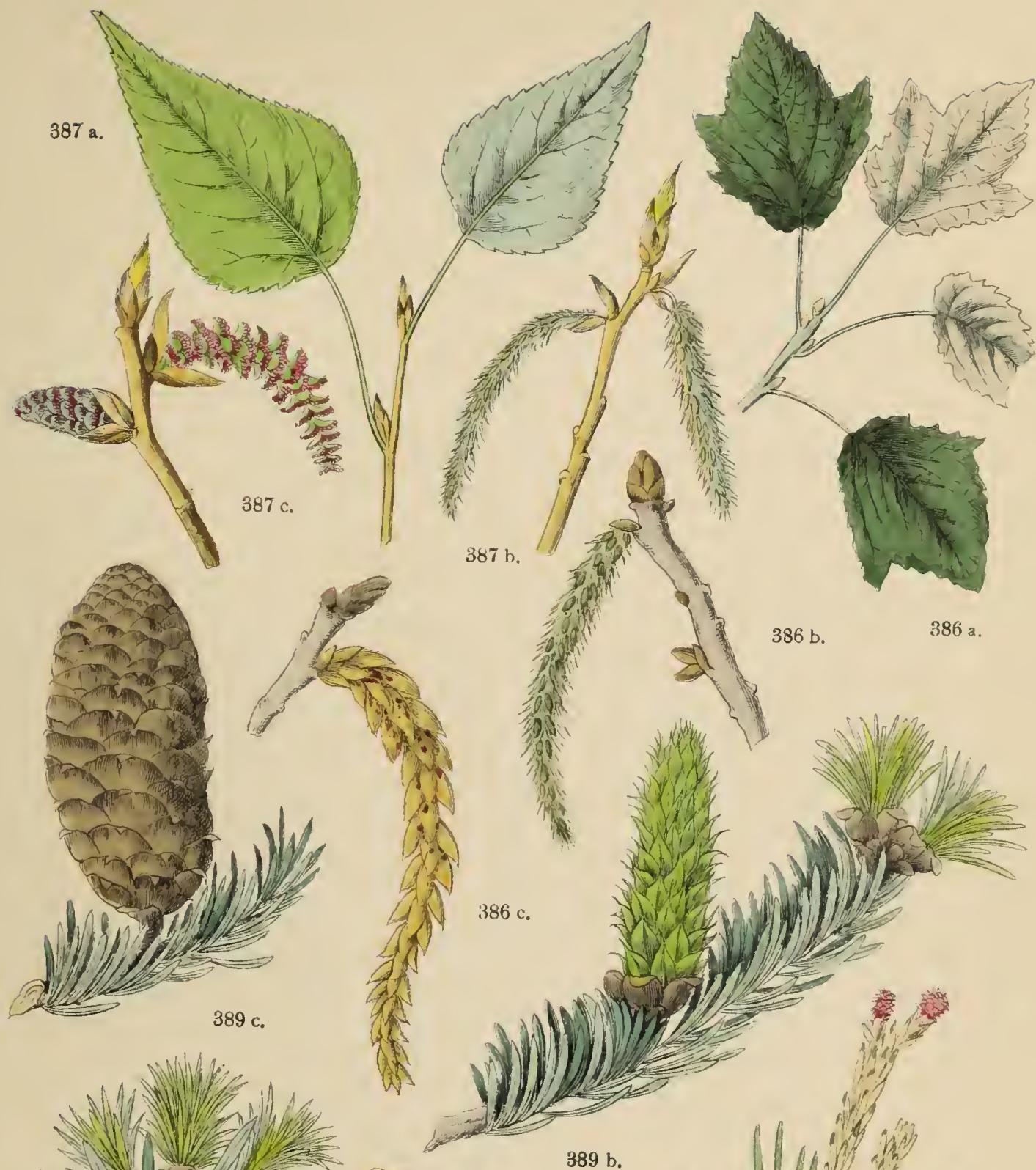

1.y.

iry

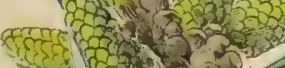

it e r S

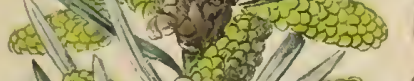

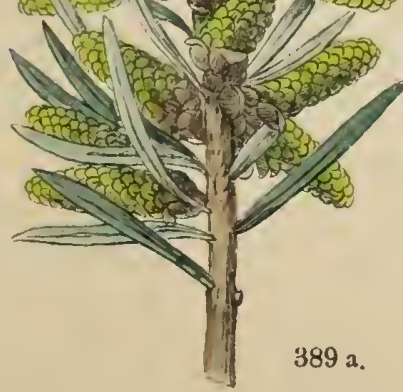

389 b. 

68.

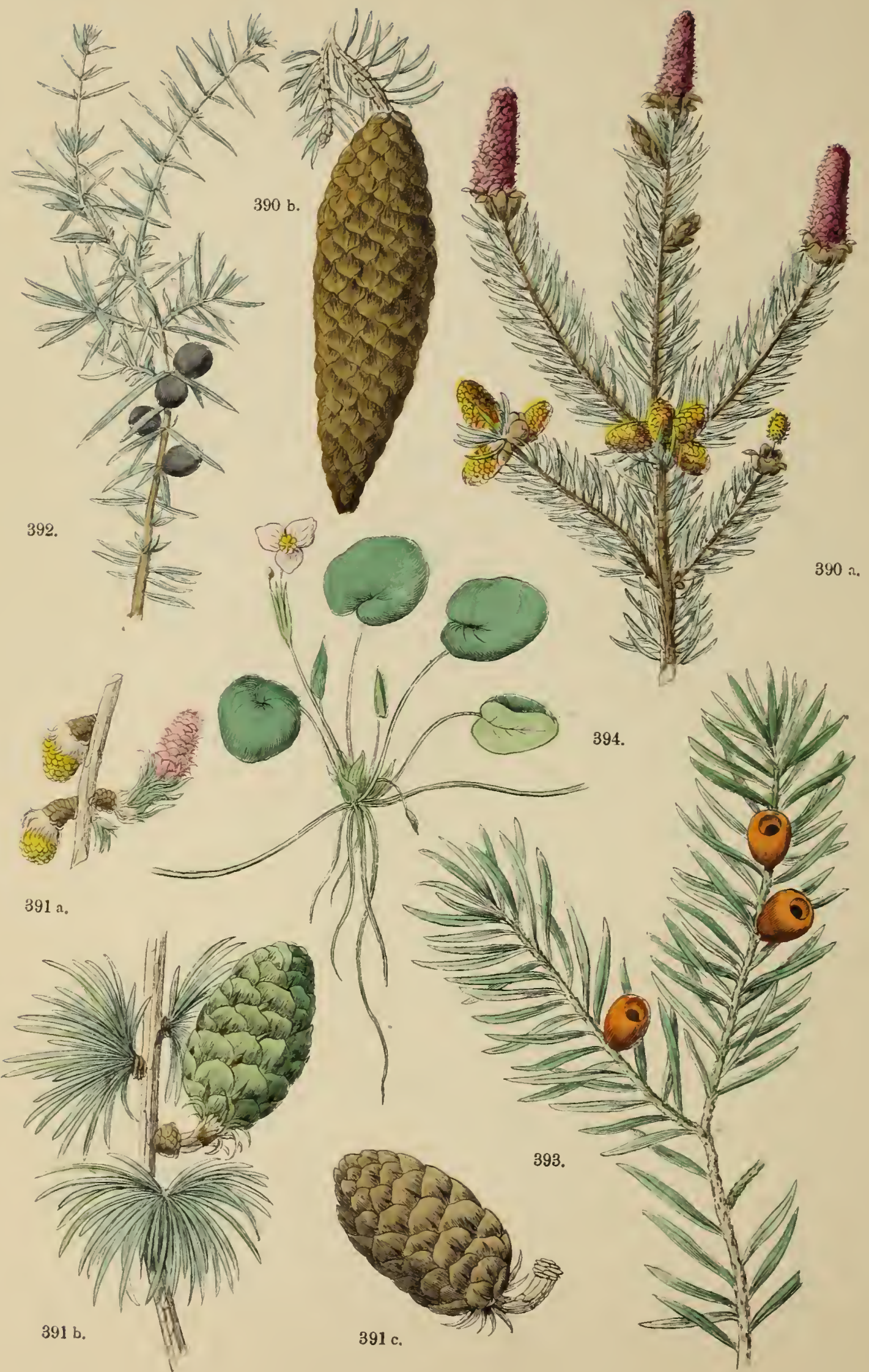


Taf. 68.

fin. 390 a. b. Pinus Abies L. (P. excélsa Lmk.), Pi cea vulgárís Lk., P. picea Duroi.), Fidfte, Sdywarztanue, Rottanne, wiro bis $60 \mathrm{~m}$ lod), bilset burd) ganz Europa bic

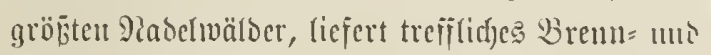

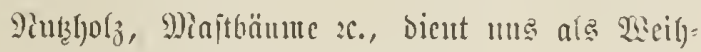
ıad)tabaut. SB(ülyt iut Miai.

fiiy. 391 a. b. c. Pinus Larix L. (Lar. euro-

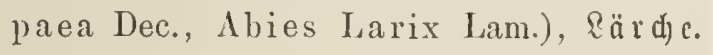
Bis $30 \mathrm{~m}$ hod), hoimijd in Deu $9($ (pen, fonjt

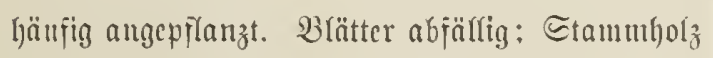
gej(järat, nament(id) 3n riajjerbanten.

fiin. 392, Juniperus communis $I$., $\mathfrak{i}$ a $d$ )= l)older, Sianawittitrand. b und immergrünter, ftad)liger હtrand); anj trodentu

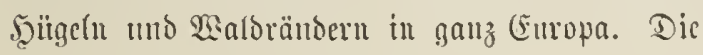

Beeren weroen vielfadl) in Danehaltungen mo s(pothefen vermeniet; blüf)t iut ?(pril, Diat.

fily. 39:3. Taxus baccita L, getuciner (Eiben=

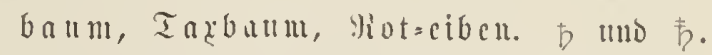

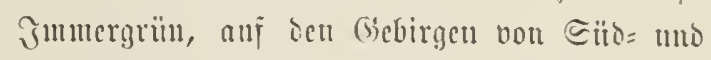
Mittcleuropa zerîtrent, bäufịg in (3ärten ange= pflanzt; blïgt iut Miärz und s(pril. Eanten แno $\mathfrak{B}$ lätter fitı gifitig.

8n Den Coniferen weroen ferner gerefhuet: Thuja L., Qebcusbaum, befoutter iututergrïtuc: (Sartenzierbaum ans Plnerifa; Cupréssus Tourn., (E)prefic in Eilocuropa; Wellingtónia gigantea Lindl. (Sequója gig. Endl.), Riejen= taune, Mia mutbaum iu Sialifornten; Ara caria Juss., Eduppentanue, f(nocutanue, Ehilitanue añ ben Pluoen; Dámmara Don., Siantifidate in Nenjeclanto; Cycas, grośblättrige હagopalute in Ditintete. Die eigentlidse $\Xi_{a g 0=}$ palme f. Fan. 184, Jig. 430.

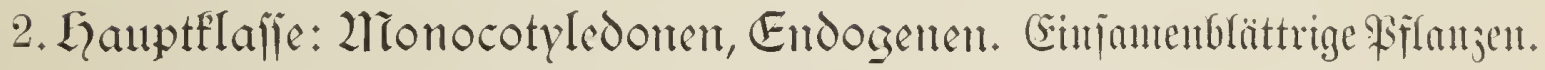

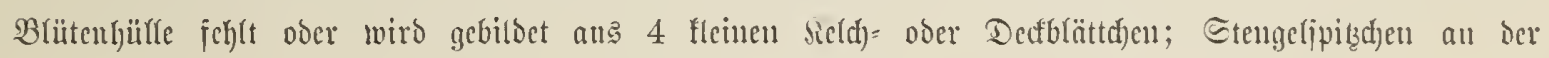
Eeite îç Sicimlinge.

165. Fanilic. Hydrocharíleae Juss. Jiroldibiggewäd).

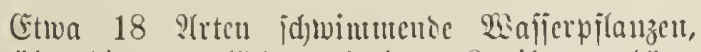
fajt über die ganze (Erde verbreitet. Zu ifunen gel)ört:

fin. 394. Hydrócharis morsus ranae $L_{\text {., }}$

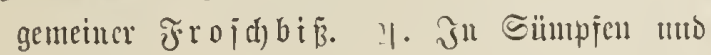

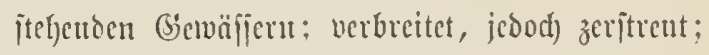

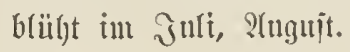

Fermer Stratiótes, Sircbijdecre, ebcrio;

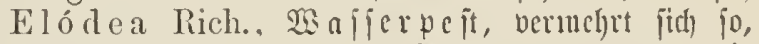
inas jie and) in Deutjd)lanto Sianäle veritopft nno bic

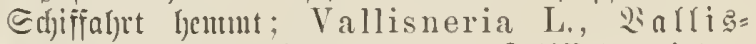
nerie, cbenfalle bie uno da ber Edjiffalut linder= lid); beliebte slquarimmpilanze. 


\section{Taf. $6 \mathrm{~g}$.}

16fi. Fannilic. Mlismáceare Dere.

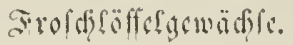

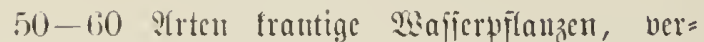
breitet ïloe bic lecific mo nördidfe nemäjigte Bone. 3n ihuen nehört:

fị!. :39.5 a. b. Bủtomus umbellátus I.., ge=

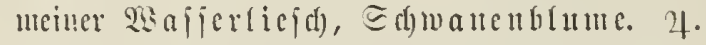

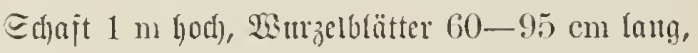

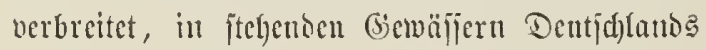

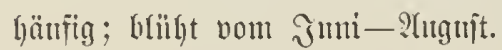

fiug. 396. Sagittária sagittifolia I., If feil=

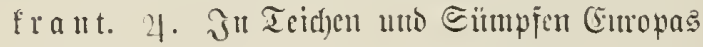
häufig. Ed)aft $: 30-95 \mathrm{~cm}$ lod); b(til)t im Juni,

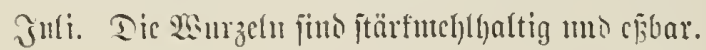

167. Jamıilic. Podostŕmeae Rich.

Ettwa 30 : Irten frantiger, untergetaud)ter, beu \$ioojen ähnlicher reajierpflanzen, meijt in den Iropen, borzügfich) it Elidamerifa.

168. Fnutilic. Typháceae Dec. Qiohrriosbergcmädle.

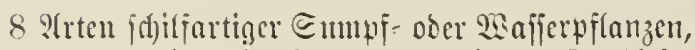
ïber bie nörblict)e Ş)albfugel verbreitet. 811 bicjer jautilie gethören:

f́ị. :39\%. Typha latifólia L., grok̂er, breit= flättriger Hobrfolben. \%. Stengel 1-2 m hod), jajt über bie ganze Erroe verbreitet, in Deutjclandes Sïmpien und Teidjen nidjt felten;

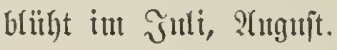

fị̣. 398. Spargánium simplex Iluds, cin= fader Jgelfolben. 21. Etengel $30-50 \mathrm{~cm}$

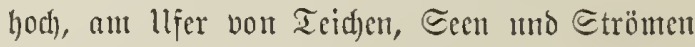

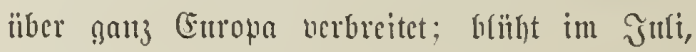
?(เ1yuit.

169. Fanilic. Aroídeare Bartl. तìtrotssgenädfle.

Eime anjebutidje familie, hauptiäd)lid) in ocn beisen mllo warmen (jiegenien ber (Erbe heimija). Diande Botanifer trennen bieje Familie in Sial= muggewädje, Orontiáceae Bartl., mo in Sallagewäd) je, Callácea e Bartl. Wirir geben in 2Abbilomg:

fin. 399 a. b. Arum maculátum L., geffecfter

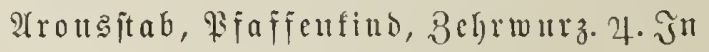
ichattigen Saubwälscrn Mittelenropas, fehr gijtig; blïh)t int Miai.

fing. 400. Ácorus cálamus L., getuciner $\Re a l=$ mus, bentider Zittwer, Magenwurz. 24. Ýn I cichen uno in Sïmpien von ganz Ëmropa.

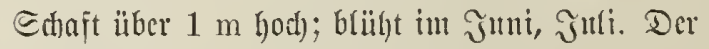

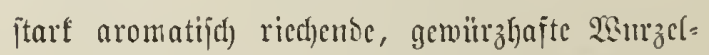

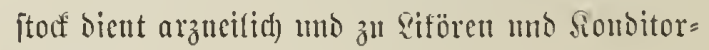
waaterl. 
69.

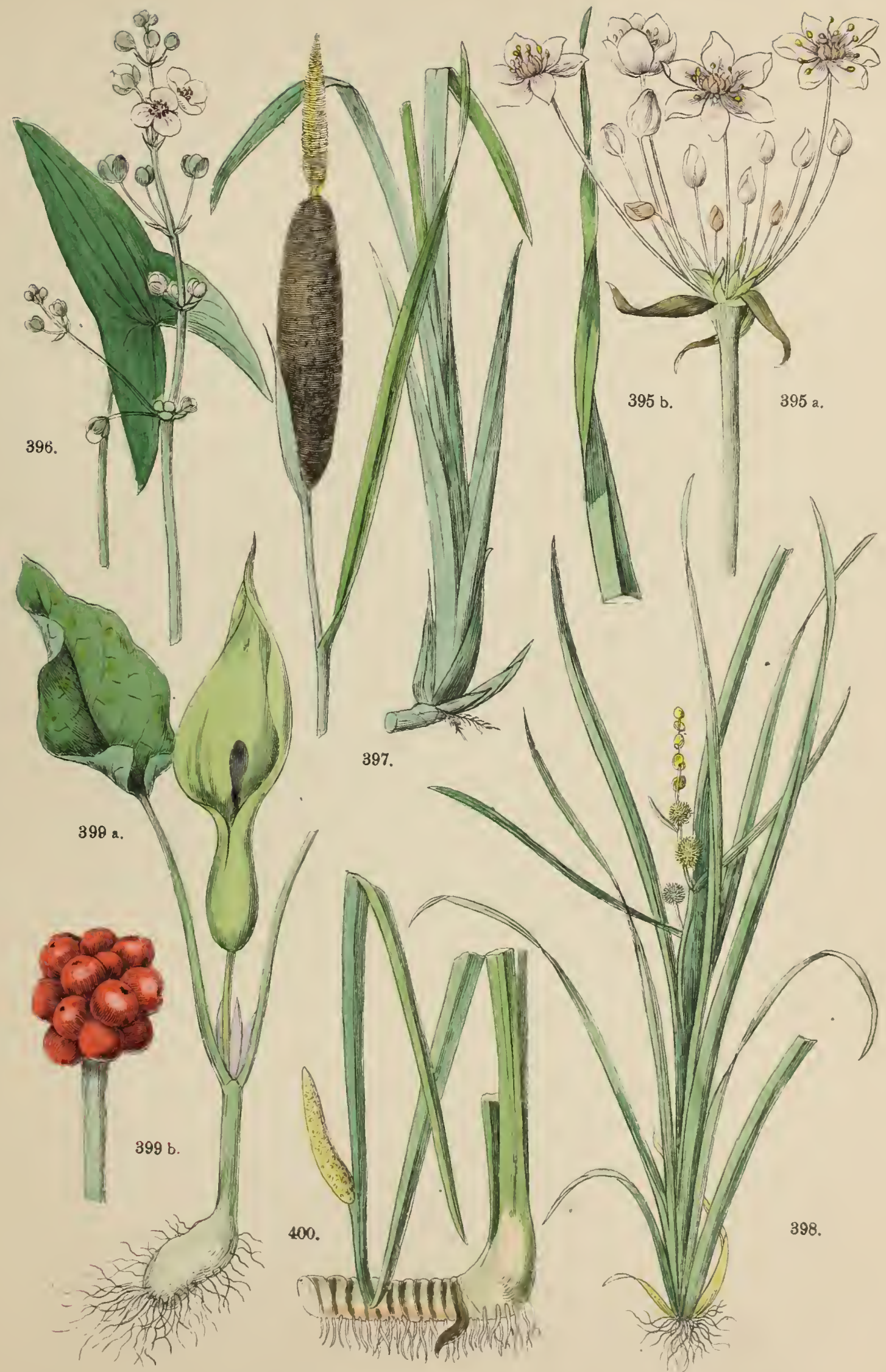



70.

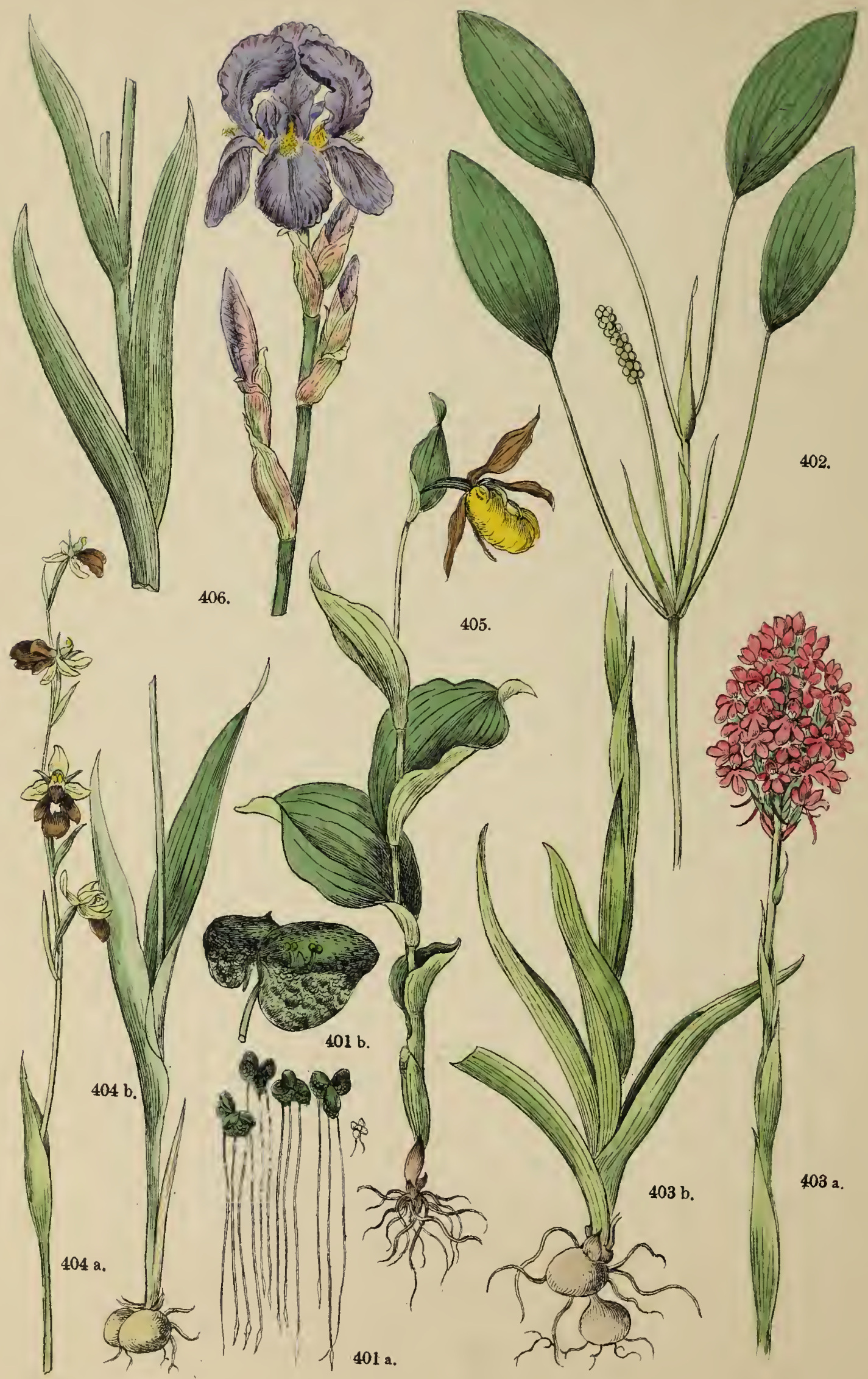


Taf. 70.

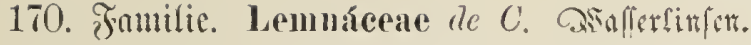

․ 6-7 9.rten flcinc $\mathfrak{W a f j e r p f l a n z e n , ~ i ̈ b c r ~ s i e ~}$ gemäp̈igten Boncn aller Welttcile vorbreitet. Wir gebcu in albbilsung:

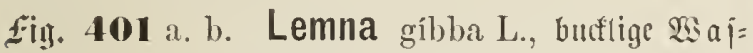

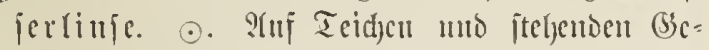

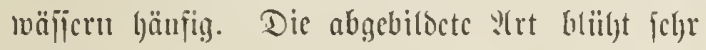
felterr.

171. Fantilie. Najáleae A. Rich. Sirnaben.

YU 100, ïber bic gauze Eroc verteilte, ureiitt

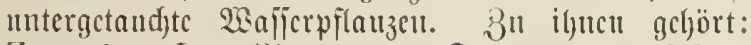
Zostéra L., Miecr ooer Ecegras, all jall=

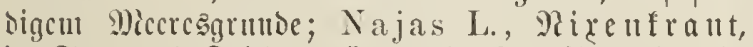
in Eecn un Icidjen; Rúppia L., giuppie, in Eitmpien, am Dit= mon 2lordiccítrande; Potamogéton, Raid)traut tuit 10 oentid)en $2(r t e n$.

fig. 10:. Potamogéton natans L., Sdjuill= menocs saidffraut. 21. Jn jtchentocu und fliç̧endon (sewäjịcrn fajt aller säntoer ber Eroe.

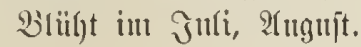

172. Famtilic. Orehídeate Juss. Gtambern oder sktalirtitüuter.

Dic 3ahl ber übcr bie ganze Eroc verbreiteten ?(rtcu gel)t in dic Tanjente, Daboul ctwa 50 in Dentjd)= lano: แแm jo ljänfiger ntto jdjöncr, je näher ben

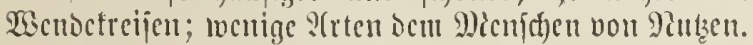
wir gechen in ?tbbilomng:

fig. 40:3 a. b. Orchis pyrimidális I., plya=

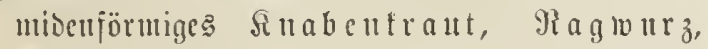

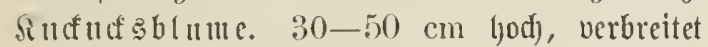
üher Cüo= mno Mittcicurepa, blïgt in Juni.

fing. 40-4. Ophrys aranifera Huls., Epiuneu=

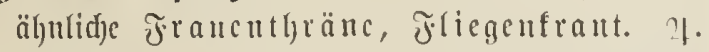

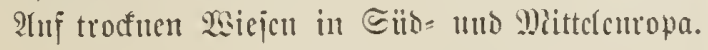
$15-30 \mathrm{~cm} \mathrm{hod);} \mathrm{h(übt} \mathrm{im} \mathrm{Miai} \mathrm{แแ๖} \mathrm{Jumi.}$

fing. 40.5. Cypripédium cạlcéolus L., gemeiner

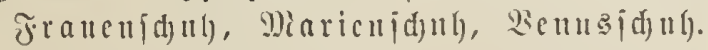
1. Etcugel $30 \mathrm{~cm}$ hod), in idjattigen $\mathfrak{B e r g}=$ waloungen; in Dentfdjlanio zeritrent, utur ftellen= weije häıfig. SBlih iut Miai, Juni.
3" bicjer Familie gefjören zalyltreidje, pradjtooll

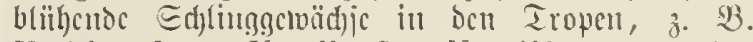
Epidéndron Vanúlla l. (Vanilla aromática Sw.), iut tropijden Rtucrifa; bic aromatijd)en $\Xi$ d)o=

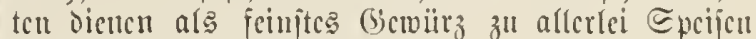

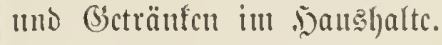

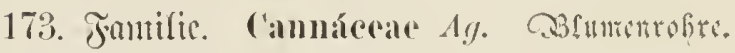

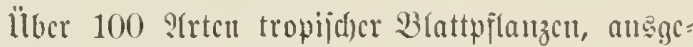

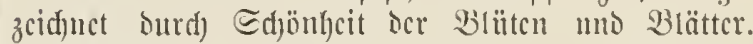
Dic fiir ung viddtigjten jind: 1) Canna indica,

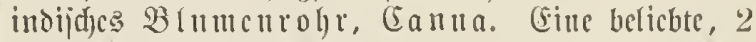

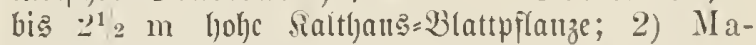
l’ánta arundinácea L., Bifcilınrz. Gin 60 bis $120 \mathrm{~cm}$ ljober Etrant) in હiìancrifa, ocjien fno= tiger 2isurzelitocf bas शrrowrootuchl, ocn weitindi=

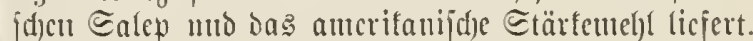

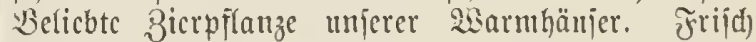
genofijen enthält bie $\mathfrak{B} u r z e l$ ein jdjarjes (sijt, wiro

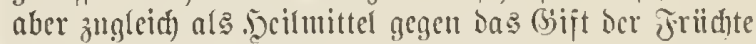
icc Mianjdjutellebanutez gcbrandjt.

\section{Fantilic. Insáceae Juss. Jifarge.}

llber 30, unx in bcu Iropen cinbcimijd) ?Irtcu; aujchulidje baunuartige sirn̈utcr, ausgczcidjuct burd) impojante, fajöne (Sscitalt uto burd) Die Gröbise ifjer Blätter. 2iele ?\{ttcu tragen [d)tua(f) andere find Durd) SBlätter utto Blattfajern ted)uijd)

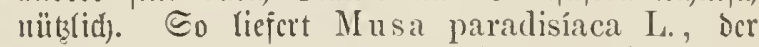

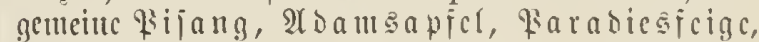

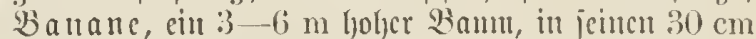
laugen, feigenartig (d)utcfentocn jrüid)ten Dic tägfid)c mo s)anptnahrung von Millionen Micnjden.

\section{Fontilic. Iríleae $R$. Br. Sdfmerfifin.}

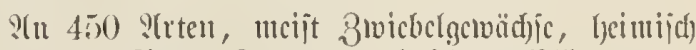

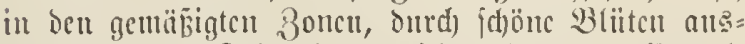

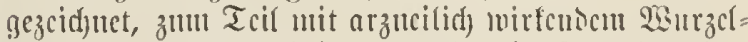
jtocf. Weir geben cinige terfelben in folgenten 2 tb: biloungen:

fiig. Ho6. Iris germanica $I_{\text {., }}$ dentid)e $\Subset$ d)wertrilie. 2. $30-130 \mathrm{~cm}$ hod, wolfriecthent, anj

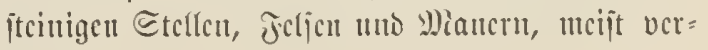

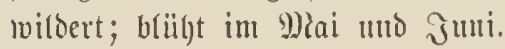




\section{đaf. 71.}

Eigg. Hog a.1. Gladiolus commúnis I., gemeciuc

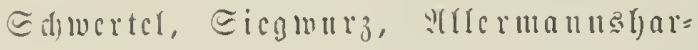
nijd). 21. 50-60 cul bod), allf 2icicju, it1

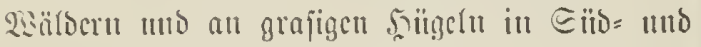

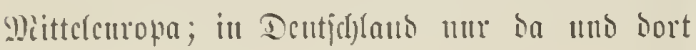
verwiltert; biillst itu Эuti.

fiiy. 108. Crocus vernus L., Jriitlings= $\varsigma_{a} \uparrow=$ ra11. 24. Dic non weij bis sunfelblan in ocr Farbe variierente Bliite biloct cine tuffer be=

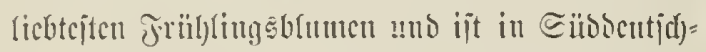

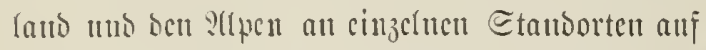
2isiçen zu finton; blïbt im Diärz und 2(pril. Der äd)tc Eajran, Crocus satívus L., 2t, iit im Dricut beimijid) und bliigt violctt. Dic rot= ģelhen 2iarben geben ben arzueilidsen und ala

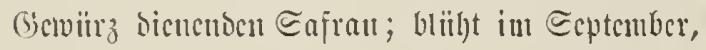
Dftober.

311 Den @djwertliticu geljören ferner bic folgen=

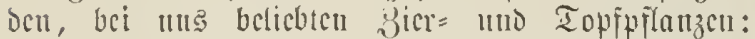
Ferrária tigrídia (Tigrídia pavónia Pers.), Tigerlilic, tifanculilic, uit prad)todlen B̉iüten, ats Wicrifo; jowic bic vielen ?frten ber Ixia L., jicrlid)e, jd)önblïl)enoc glvicbelgewäd)je vou Siap.

\section{Fantilie. Inemodoráceac R. Br.}

Eetma lif 2(rten von Siräntern, dic mciften im tropijchen แแto wärmercu ?(merifa l)ciutijch. Das baz̆ geljörenie Sisyrínclinm Tourn. ijt ciuc belicbte (jartenjicrpflauze uno licjert enbare sinollen.

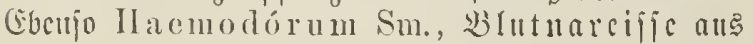
?tuptralicu.

177. Fombilie, Amaryllídeare $R$. $\mathrm{Br}$. sthörifien, Ronriffer.

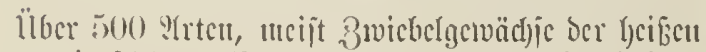

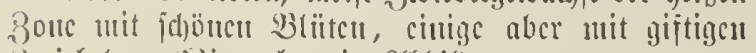

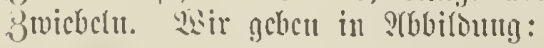

fiiy. 409. Narcissus I'semtonarcissus L.,

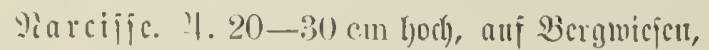

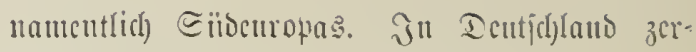
jtrent mito jelten, meift nur verwilocrt.

Bu ben Parcifijen gebören ferner: N. pócticus

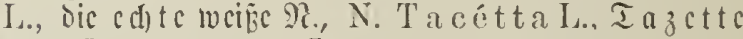
utto N. Jonquilla L., Jouquilre. 2ulle zcidunen

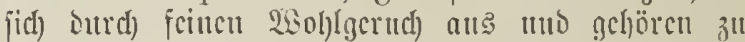
beu belicbteiten Topfoflauzen, dic nauentlid) bei แnכ

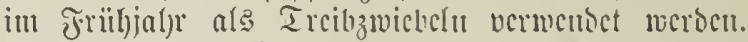
(Einflimijd) jint dic meijten bavon am Sianfajus uto in @ïbellropa.

fing. 410 a. b. Galánthus nivílis $I_{\text {.., gcutcutę }}$

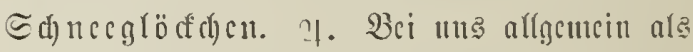

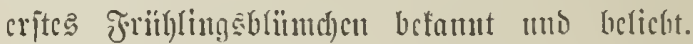
Dic 3tuicber ijt bred)enerregeno; n(iil)t nou J̌cbruar-?tpril.

fiig. 411. Leucojum verunm L., Frithlings:

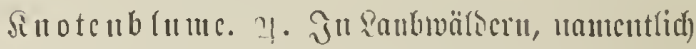

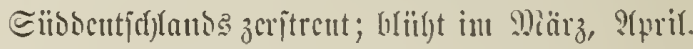
Bmicbelu rol cibbar uns, mic Siartofifelu zubc=

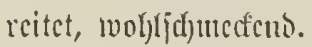

\section{Fantilic. Dioscoréae $R, B r$. ajamsplentsut.}

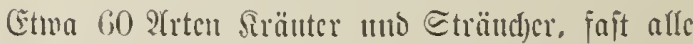
mit binocnocu Etcngel uno Inolfigem, ftärfcutchl=

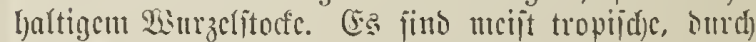

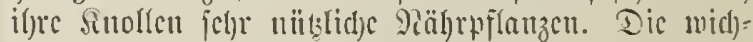
tigjten bavou jitno: Dioscoréa a láta I., jguaut,

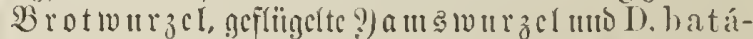

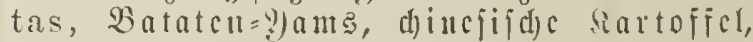

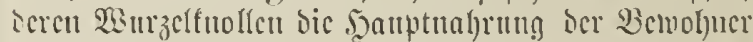
ser Eiisjecinjelt biloct. "2sir geben atı bicjer Ja= mile in ?lbbilomm:

fُin. 412 a. b. Tamus commínis L., gent.

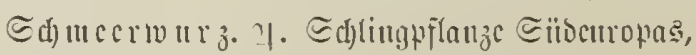

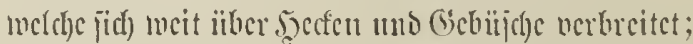

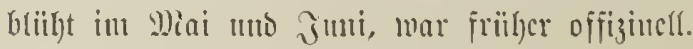


71.

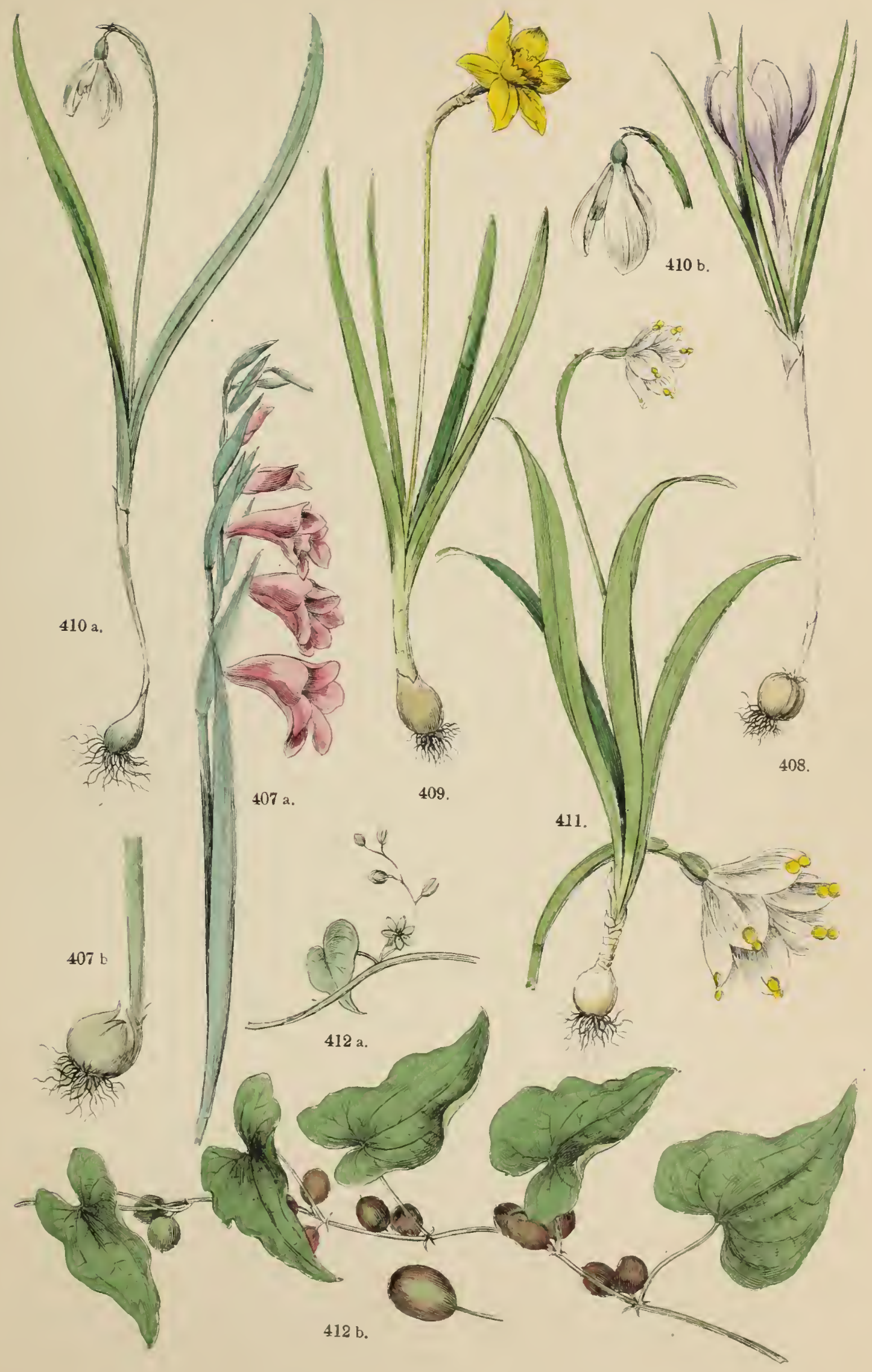



72.

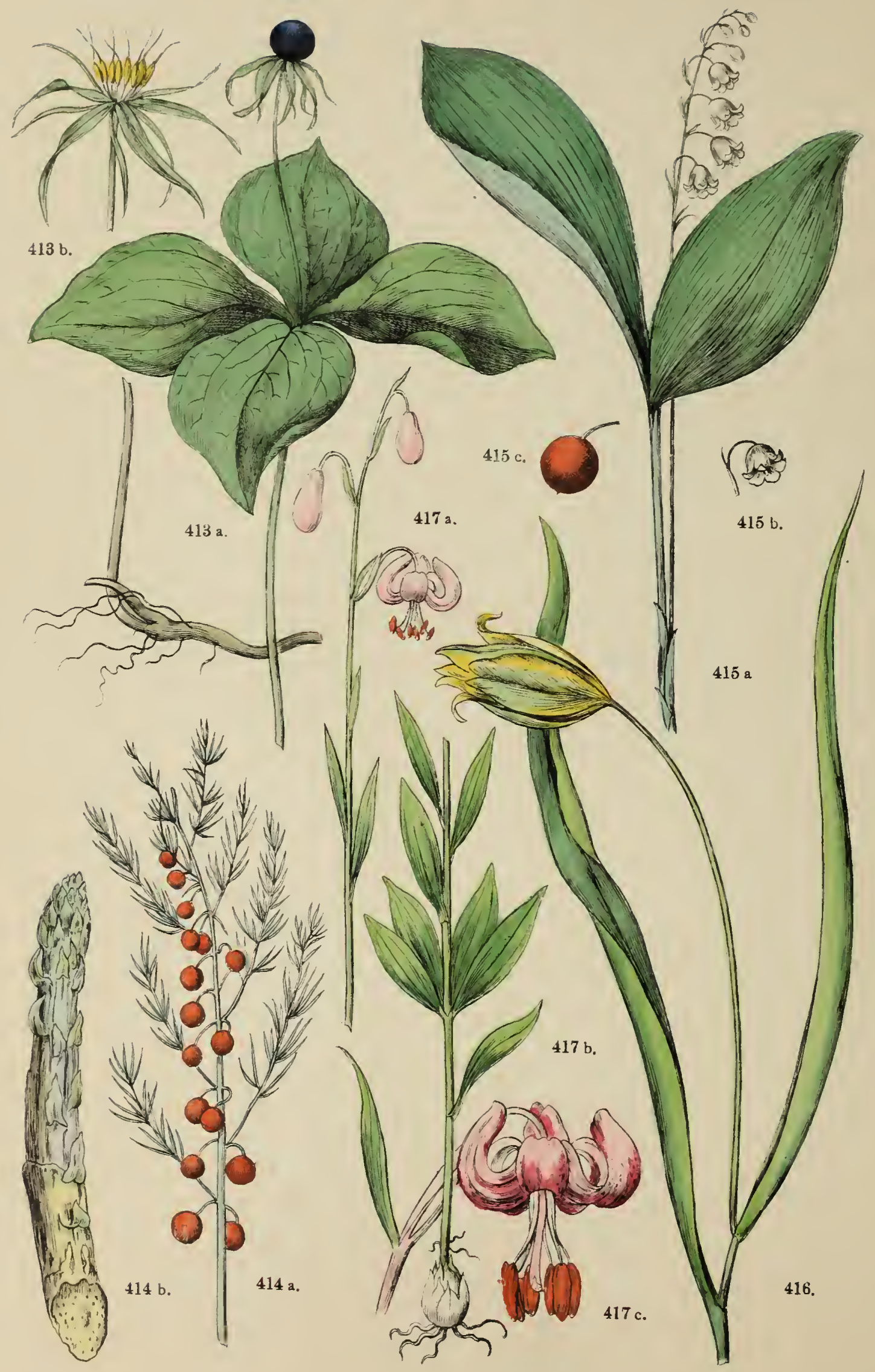




\section{Taj. 22.}

179. Fantilic. Liliáceac Bartl. \$ifingswädfl.

3u ifucu nefyören dic Smilacen, Smiláceae R. Br.; bic Bcitloicu, Colchicáceae Dec.; unto bie Tffodille, Aphodéleae Bartl. Eninige Botaniter rcdfucu aud sic ?)ausprlauzeu (178. Fa= tmitic) zu ocu Siliacecu. Whir gebeu aus bicjer grop̉en 179. Fantilic folgcubc 2̧bbilbungen:

fing. 413 a. b. Paris quadrifólia L., gentine

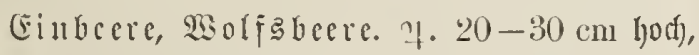
in idjattigen 2 sälocrtı von gatız Europa. Die gauze Siflanze, bejonocrs dic Becre, ijt jefarf $=$ giftig; bliigt im Mlai utto gutut.

fig. 114 a. b. Aspáragus officinális L., gc=

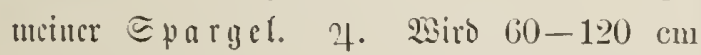

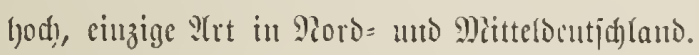
Dic betaunteu Eprojīen geben eincs ber bejten Jrülltugsgcutiij, guten હalat und bicucu ar $3=$

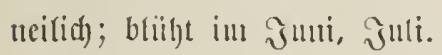

f.ig. 415 a. b. c. Convallaria majális I., ge= meine Diaiblume, 3 a ufu. 24. Cetma $20 \mathrm{~cm}$

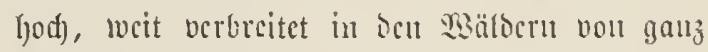

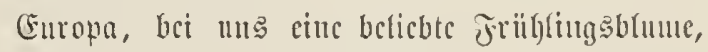

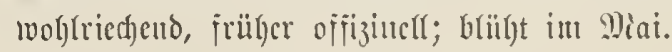

fin. 116. Túlipa silvestris I., wilie $\mathfrak{I}$ ulpe.

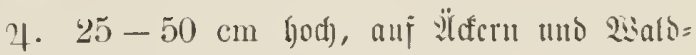
wicjen, naututlid) in fïstitgen Ěmropa; blïht im Prpril mo Diai. - Die T. Gesneriána L., (3) artentulpe, 4, aus icm Drient jtanuenti, mit ifren vielen prachtuollen Epiclarten, ijt be= faunt; Deren Zwiebelu biloct einen bedutendon

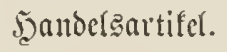

fiig. 418 a. b. c. Lilium Mártagon L., I ï rf́c $n=$ bundilic, belbwurz, Golowurz. f. Etcugel $30-50 \mathrm{~cm}$ hodf, aui Bergabhäıgcu tuto itt

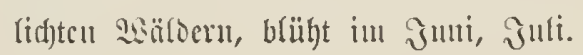




\section{Taf. 23.}

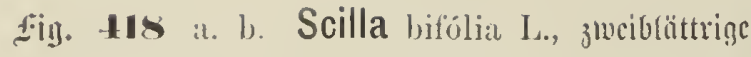

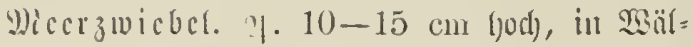
Deru uno (jechiijd)en, naucutlid) Eüodcutid)(ants,

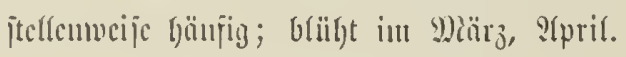

(نiy. HIS a. b. Állium oleríceum L., gculeinct

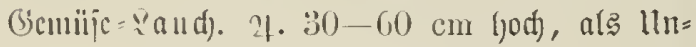

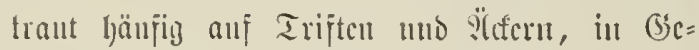

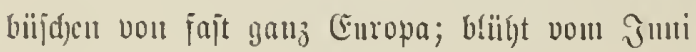
bis ?tuguit.

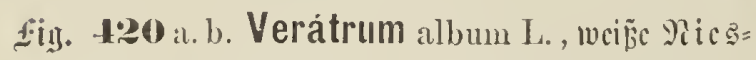
wurj, Gerutc. \%. 60-100 cm bod), ciltc

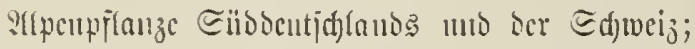

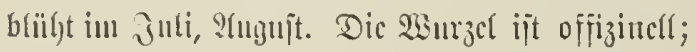

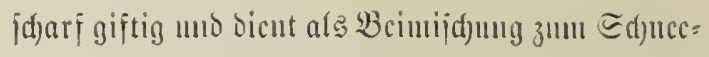

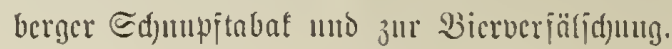

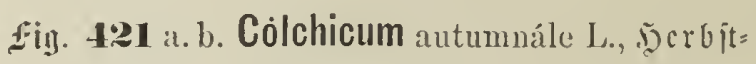
3citloje, micjenjajrall. 2. 12-15 cm

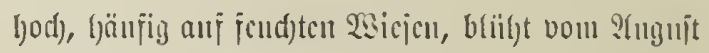

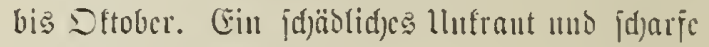
(3)iftriflanzc. Sifizincll.

fị̂. 12:2 a. b. Fritillaria meleágris L., gemciuc 巨d)adblumc, sibizzci. 21. 15-30 cm (yod), auf Fend)ten sistejen Mitteleuropas; b(iibt iut Diai. Dic orangerote Fr. imperialis I., Siajerfrouc, jtaumt aus ocu Dricut uno ijt ciuc belicbtc Früblings = Gartcupflauze, if)rc 3ruicbel aber ift jtart gijtig. 
73.

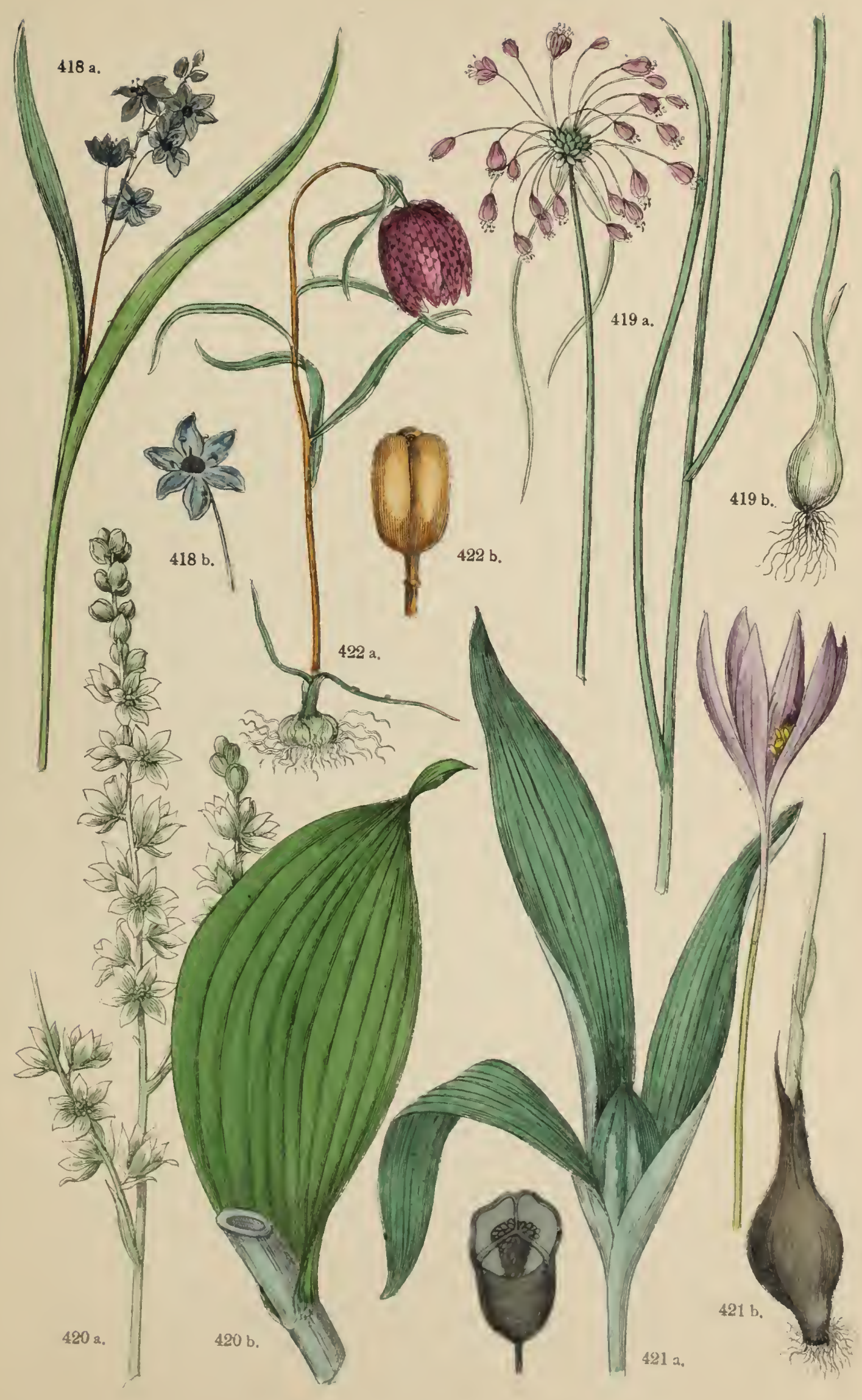






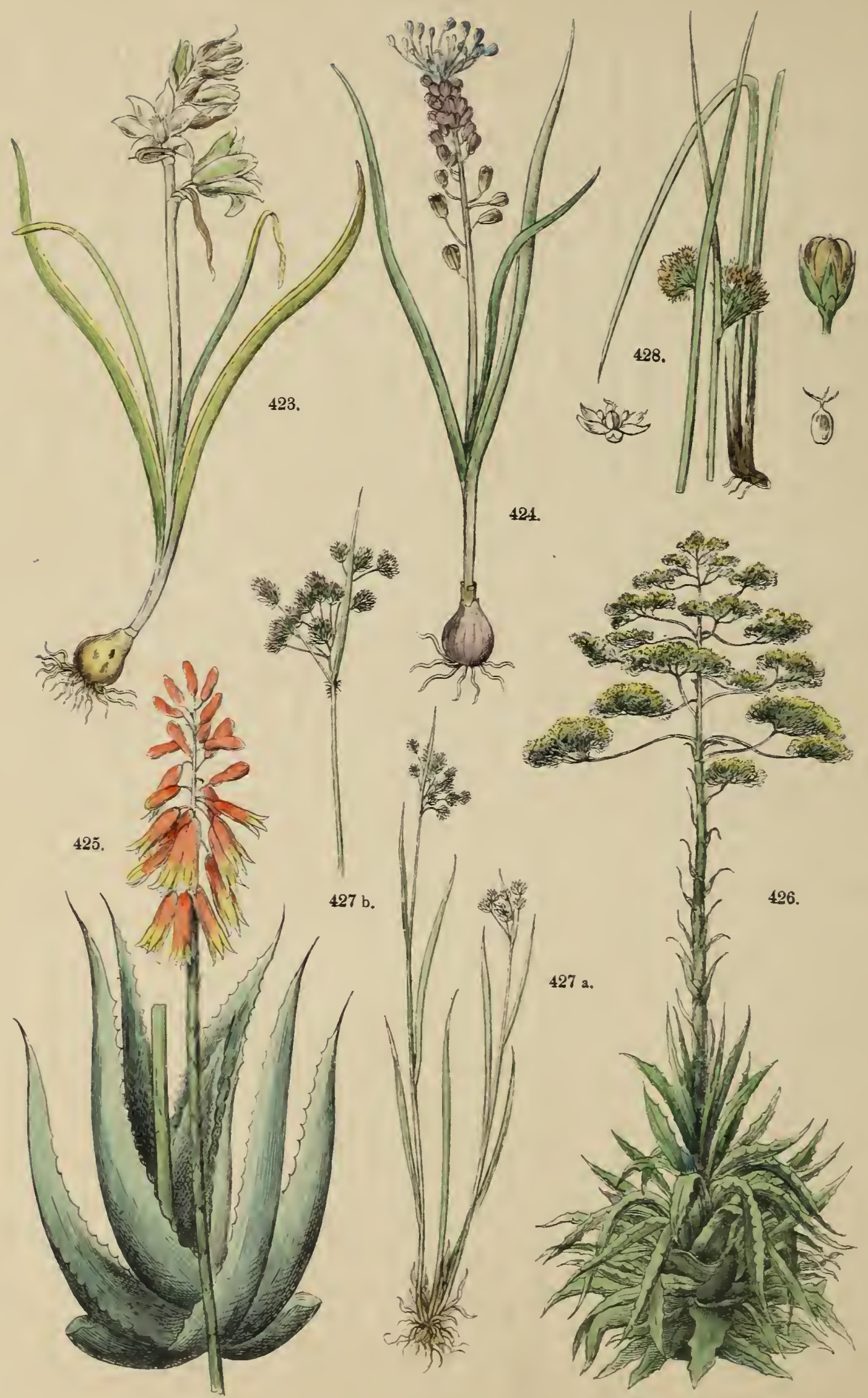


Tạ. $i+$.

fig. 123. Ornithógalum nutans L., nitfente Sogelmild), Mitchiterm. ?. $30-45 \mathrm{~cm}$

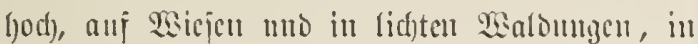

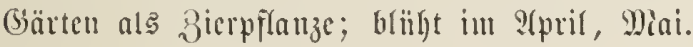

fị̂. 421. Muscári comósum Tourn. (Hỹicinthus L.), jdopfige Bifam = oocr Mius fathuaciutle. ?. $50-60 \mathrm{~cm}$ hod), auj

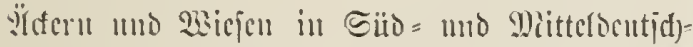

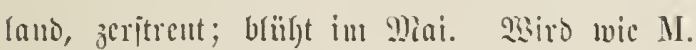
racemósum, die Iraubuhbaciutbe, bei

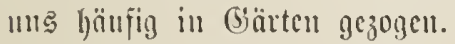

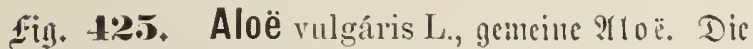

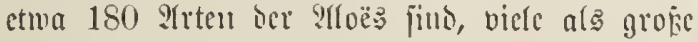

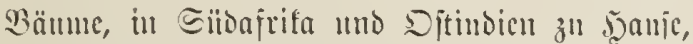

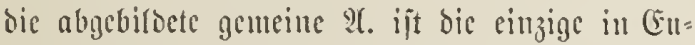
ropa verwilberte ?frt. Ier eingedifte Saft vicler

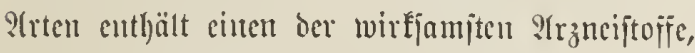

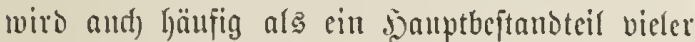
(bel)cimmittel vermentot. WBir geben ber ljäıfigen Sertwed)slungen wegen bier in 2tbbildung:

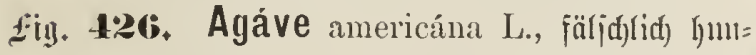

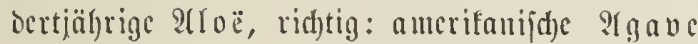
genaunt, weld)e ju ien Amarillideae (117. Jamilic) gefört. Slur $\Xi$ dajt wiro 6-10 m l)od), bis $30 \mathrm{~cm}$ biff, mo trägt oft bis 4000 wob)lried)ende 2 biüter.

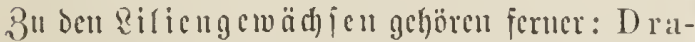
caena draco L., gemeiner Draderbaum Hyacintlus orientális L., Gartenlyuaciuthe - Yucea L., Falutilie - Polyánthes, Inberoje.
180. Tomilic. Butómeane Rich. Säferfiche. (Sichc Familic 166, Mlismaceae Rich.)

181. Fantilie. Juncácene Bartl. S3itren, simitin, sosfgraler.

Gegen 100, ïber die ganze Erte verbreitete :Irten, vou wold)en eillzelue ju Jilechtwerf, feinen Etrolfhitten, Wiatten n. \{. w. Dienten. Edj)(ed)te Jutter= gräjer.

fiij. 427. Luzula campestris R. Br. Gemeinc .5 a infimie. 7. 15-30 cm fod). 9ut trodfenen Rajenpläłzen im mittleren mto nörolishen Europa

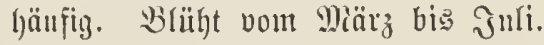

fig. 128. Juncus communis Mey., (3cmeinc Simfc. 24. Sie ift in najien Gräben mno

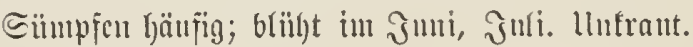

182. Familic. Restiáceac R. 73r.

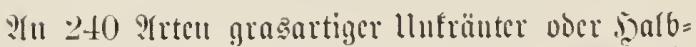
jträud)er, fajt alle in Reubolfano umo all siap bei= mifd). Dic jalme cher ?(et biencu aul Sap zum Dadjoectert.

183. Familic. Commelyuácene R. Br.

\$1 230 meijt tropiidje, cinjäl)rige sirautarten

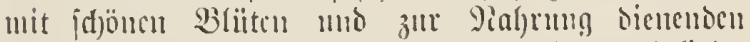

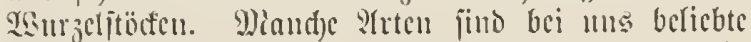
Topfpilanzen, fo bic Commelyna coelestis $\mathrm{L}$. aแร Mierifo mo dic verichicocucu :Trten ber 'T'radescintia aus Eiidamcrifa. 
Tar. 75.

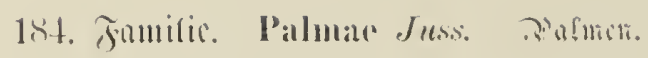

Dicje jd)on von ?inné "sinujten des Wiflanzen= rcid)g" genaunte Familic, bejteht aus nut ciucr (Sattım mit ctma 600 fajt jümtlid) ben. Tropen an= gehörentoll ?(rten; nut ciuc QPrt, Chamacrops humilis, ijt attd) in Eilisuropa ciuheimijd). Der

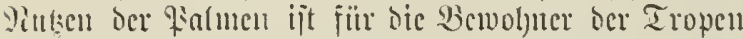

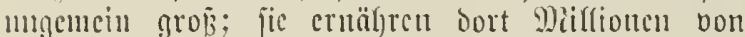

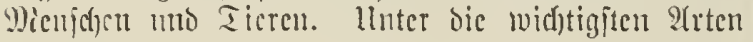
ygchören:

fiuy. 429 a. 1. Aréca cátechu L., gem. Trefa= Falme, Catedupalme, Betelpalmc, $: i=$

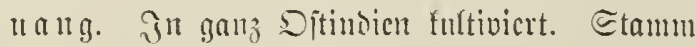
$12-15 \mathrm{ml}$ hod), Bifatt $2-4 \mathrm{~m}$ lang, Frudft

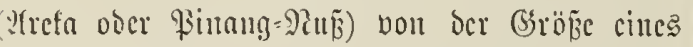
Döhifuereics; bic Betefniijfe merten wegen ifyer be=

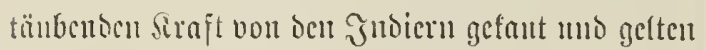
benjelben als uncutbelyrlid)cs icbensheditrinis.

fị̂. 4:30 a. b. Sagus Rúmphii W., cd)te $\Xi_{a g o=}$

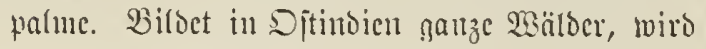
4-9 m lyod), B̉ätter 6-7 m lang. Dą Marł sicjer Falme gicbt oen meiften mid beiten Eago ies. Dantels, und bicnt in Juticu, auf verichic=

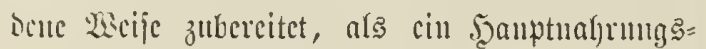
mittel. Ein cinziger $2 a u m$ licfert an 5 Ctr. ๔agourchl.

fiig. $4: 31$ a. b. Cocos nucífera L., edfite Siofospalme. Staum $15-30 \mathrm{~m}$ hod, dic Ficder= blätter 4-5 m lang. Sn allen Tropenläntocrn fultiviert; ilyre 2lïffe bilben cinen widytigen Bic:

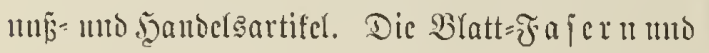

Jippen werocu ju $\Subset$ d) arbeitct, dic $\Xi$ djalen neben Irinfgeidfirre, ier

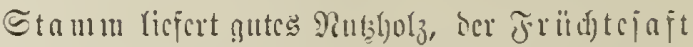

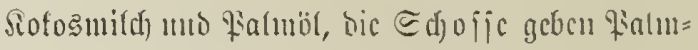

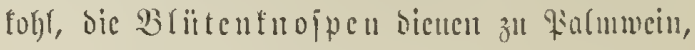

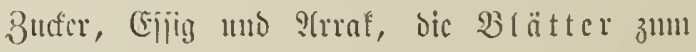
Dadjocefer.

fing. 4:3:. Elaeïs guineénsis Jacq., cdyte ajui= fanijde S̈lfalme. $9 \mathrm{~m}$ hod, $30 \mathrm{~cm}$ dicf,

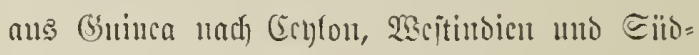
ancrifa verpflauzt. Die Früchte licjern bas

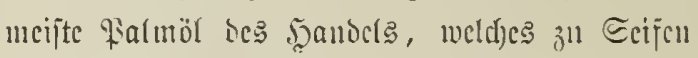
Into andorn ted)uijdjen 3 wefen bient.

fin. 433 a. b. Phönix dactylifera L., gemeint Dattelpalme. $15-18 \mathrm{~m}$ lod), bis $1 \mathrm{~m}$ dicf, bic

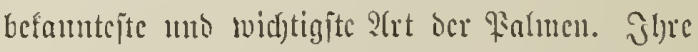
Jrtïd)te bienten vielen Tamienden von Mienjdyen, namentficl) in 9(rabien, zur Jeanptnahrung mo finto cin beocutenber Daulocleartifel. Der Eaft ocs Stammes gicbt Rialmucin, Dic Blattjajern

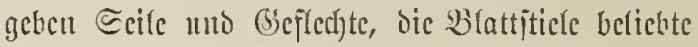
Stöffe.

PHe Eer ben abgebilocten atren ijt nod) von be= jonterer Waidjtigfeit: Borássus flabelliformis L.,

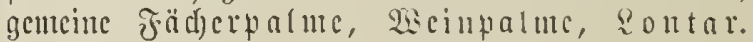
$18 \mathrm{~m}$ hod), in 2(jicn, von ?(rabien bis Sitnoicu. Eie bient vielen Miilfionen Miendiden als Jeantutul)= rutuggmittel mo liejert ignen Butfer, Falumein mo

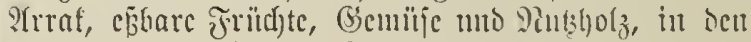

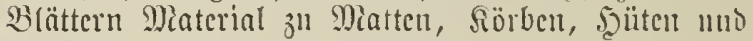
Bapter. 
75.
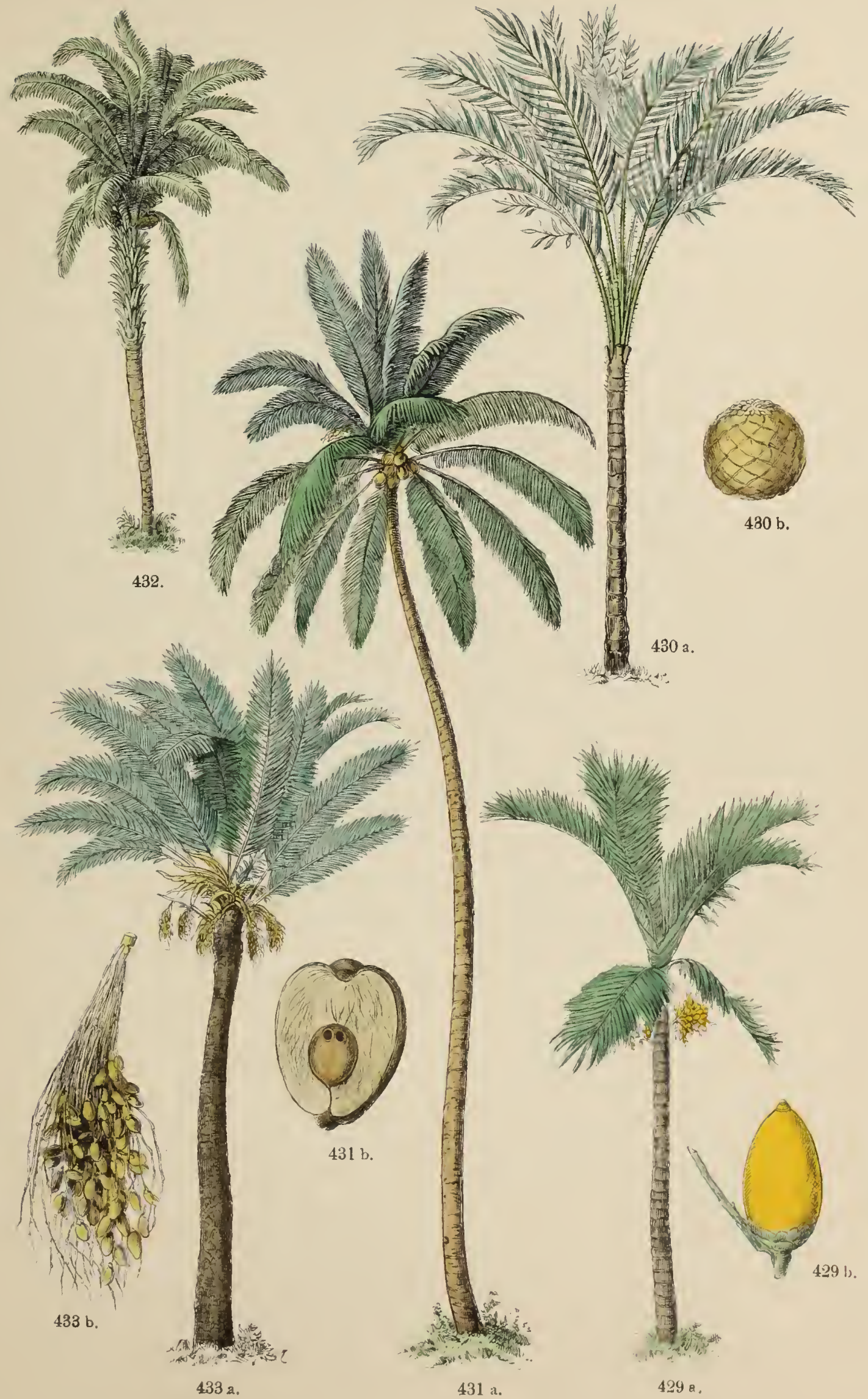

429 в. 

76.

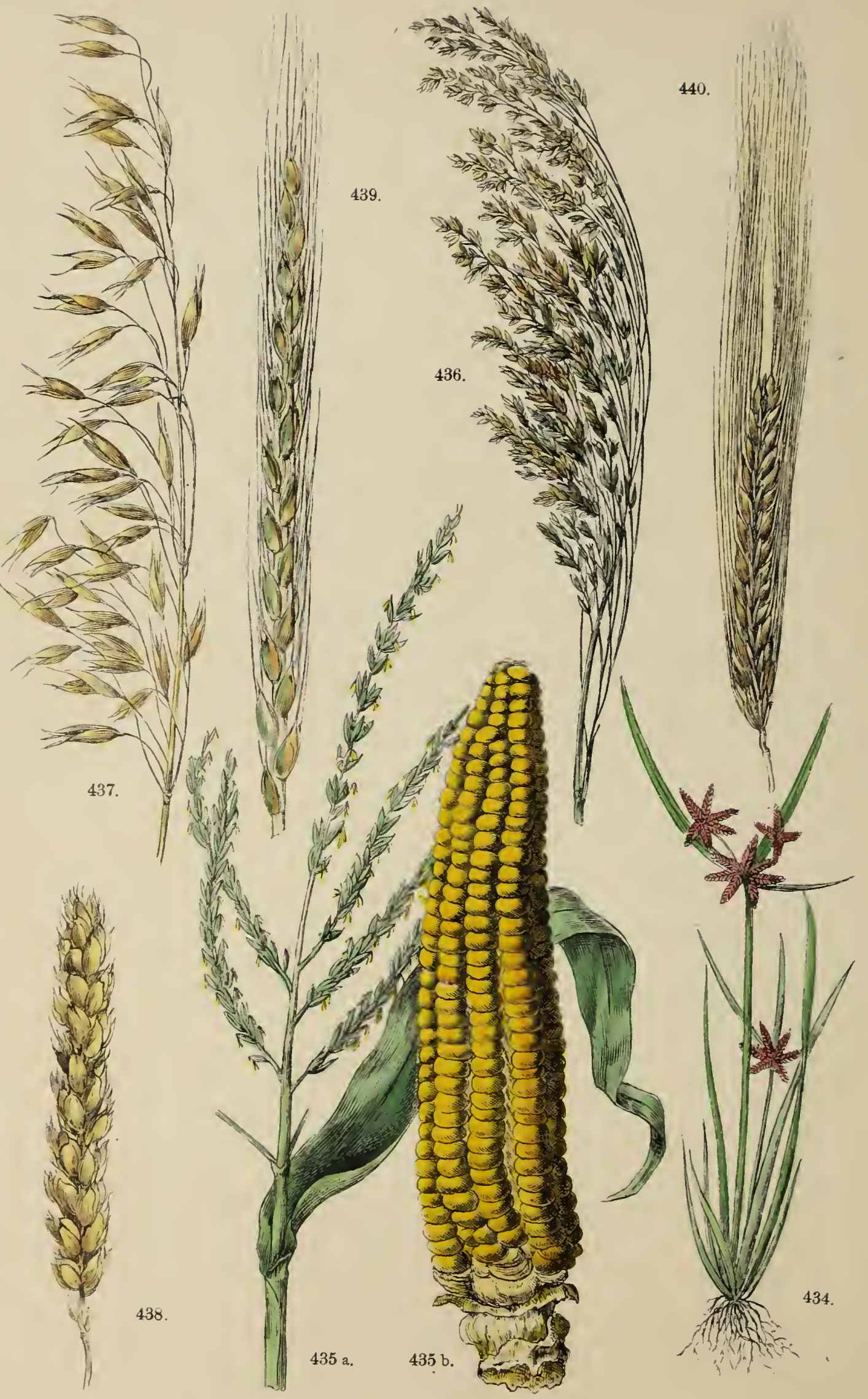


Taf. 26.

185. Fanilic. Pandáneae $R$. $B r$. andurioflen.

Etwa 30 9frten in Eibajicn, Eiboamerila mo aแ

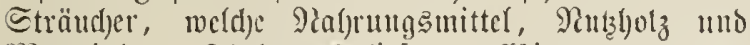
Miaterial zut Flect)twert licfert. 2Gir tenneu bou Dicjer Familie: Pándanus I., $\Subset d)$ ranbenbanu in Ditinden; Eleplantusia liuiz. \& Pav., Elfcubeinpalme, Taguahanm in Sïbautrifa; Carlodovica liuiz. il Pav., Panamapalute in Sïbamerifa.

186. Familic. Cyperíceae de $C$.

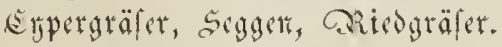

Bicgen 1200 2rten über bie ganze Crobe ver=

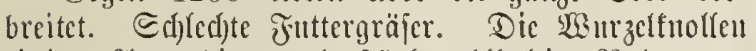

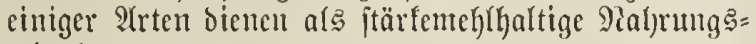
mitter.

fig. 431. Cyperus flavéscens L., gelblidję (ธทpergras. $\odot .3-15 \mathrm{~cm}$ hod), auf Trif=

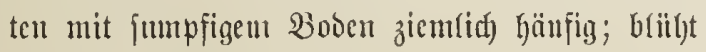
in $\mathfrak{s}(\mathbf{i}, \mathfrak{A} \mathfrak{u} u \tilde{t}$.

3u den Eypergräjern gehören jerner: Schoenus L., Stridgras; Cladium L., Sdneide= graz; Heleócharis R. Br., Mieo; Scirpus L., Simje; Erióplorum L., Wallgras; Carex L., Micograz, Eegge.

\section{Fantilic, Gramíneae Juss. sräer, siliggraler.}

Їlber 3000 शrten frautiger, über bie ganze Erde

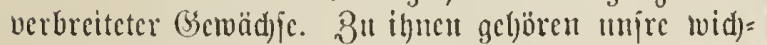
tigjten Bictreibepflanzen mo Jutterfräuter.

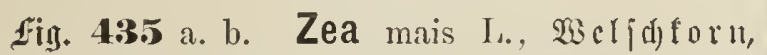

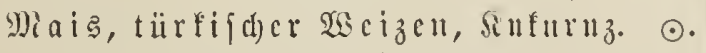
5)alu 1-2 m hod), aus Silloamerifa ftamueno, jekzt int vielen Epiefarten iiberall angebant, in Umerifa die verbreitetjte Brotfrud)t. Der bis $30 \mathrm{~cm}$ groje Siolben giebt (Siries unto Diegl mo

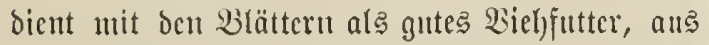
ben Stengelu biro Sirup, Butecr mo Brant= wein gewounen; bliit)t voun Juti-?tugnjt. fịg. 4:36. Pánicum miliáceum I., cchte .jirie, Fennid). $\odot$. Dalu ctwa $1 \mathrm{~m}$ ljod). Siaut ans Ditindicn mo wiro jekzt in Dentidjlanto in melyreren Spiclarten häü̈ig antgebant. Das siorn nicbt ein fräftiges Mielle zu Brot uto gutes (bse = flügelfuttcr, bas @troh wiro vom Bieh gern ge=

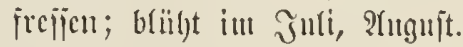

füin. 4:3\%. Avéna satíva L., getucincr obcr

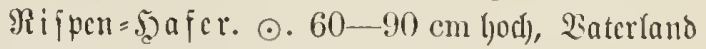
unbefamnt, ictżt iiberall in viclen Epiclarten an=

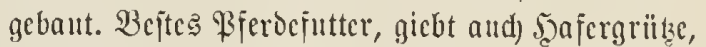

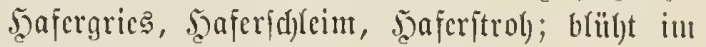

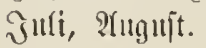

fịj. 138. Triticum vulgáre L., gemeiner $\mathfrak{k}$ ci= zen. $\odot$ แnd $\odot$. Mit neldreren Epiclarten bci uns vic(fac) augebaut; blïgt im Juni, Jutl. Baterland Ferjicn. Bejte Brotfruds) ocr altell

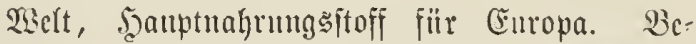

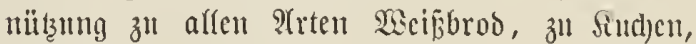
Meblipeifen, ginber und Dblaten ift befant; das Stroh ijt gutes $2 i e h f u t t e r$ und wiro zu fei= nem filed)twert ocrwendet.

fing. 439. Secále cereále L., Roggen, Soru.

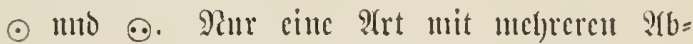
arten. Etamut aus oem Sricnt und wiro jekzt iı affen gemäpigten uno falten Silimateı gecbaut. $60 \mathrm{~cm}-2 \mathrm{~m}$ hod), bifloct er cine ber nitiz̧lidjiten Fiflauzen der Eroe. Er giebt in jeincu siörneru

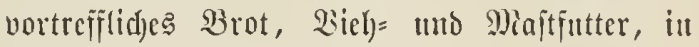

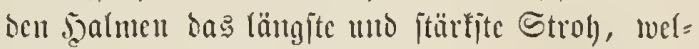
d)es vom Bieh gern gefrefien und z" Miatten

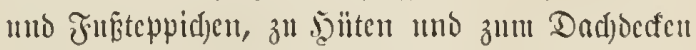

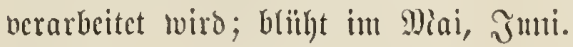

fing. 410. Hórdeum dístichum L., zuneizeilige

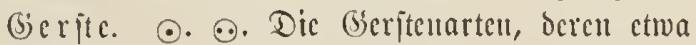
5 verjdjecocue in Mittcleuropa angebaut werocu, bienen bejoubers zuแ Sierbraue» (Berjtemualz), Dann zu Gries, Braupen mo Beritenjd)leiu; Bliitezeit: Эৈni, Juli. 
Taf. 27.

fijg. 111. Lólium perénne I., ansonermoer Pold), cuglijdes galuglas. 24. $30-60 \mathrm{~cm}$

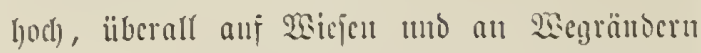

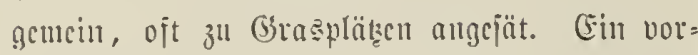

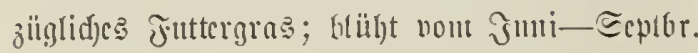

3" Lolium gel)ört aud) ier giftige Tauncl= lold), Tollforn, L. temuléntm, cin ljödjit läjti= ges ?lferimfraut.

fing. 412, Sáccharum officinále L., edtę 3udferol)r. Doalut $2-3^{1} 2 \mathrm{~m}$ hod), 2 bis $5 \mathrm{~cm}$ biơ, mit locfercu, jạtigem Wiarte ange=

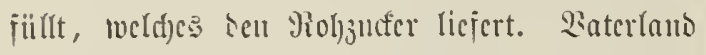
Ditmbicu, angebant ïberall in ier ljeisen Bouc.

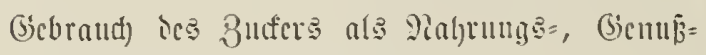
unto :Irzuciutittel ijt befautt.

fiig. H13 a. b. Orýza satíra I., gemeiner Reis.

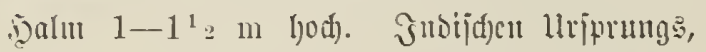
wirb in jajt allen jübliden sünocen ber Eroc

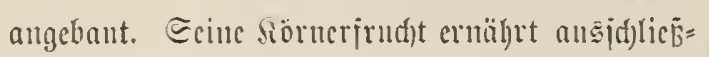
(id) melyrere Mirlionen Dienjaen und and) nad) Ecutidjlatto merien jälstlid) mel)r als 700,000 3entuer eingejïl)t. 2icrucusung ż @uppen,

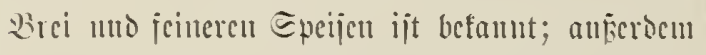
Dicut ber Piciz zu @tärtmelyl, Bier, zu id)tci= migen Gíctrüuten แmb bejontors zu ?trral. fiin. 114. Bambúsa arundinacea Willd., 2Ba $11=$ busrohr, Ricjengras, wiro bis $30 \mathrm{~m}$ hod), $40 \mathrm{~cm}$ bicf, utto bilbet in icu tropijd)e Päı= tern ricjige $\mathfrak{s}$ älocr. Dic zäl)en હdjäjte werben

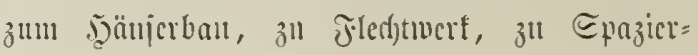
îtöcfen u. i. w. verrocnoet.

fig. 415. Phálaris arundinacea L., rohrartigce (3) (anzgras. \%. 60-100 cm hod), Burd) ganz (Europa an llicru von Bäd)en, Tci=

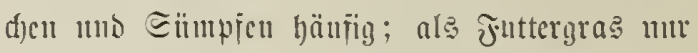
Dienlid), เvenแ eร vor ser B̉(üte gejdnitten woiro; briilyt im Jumi, Juli.

fig. 416. Agrostis alba L., geutincr 2 ind balm, Etrauggras. 2. 30-60 cm lod).

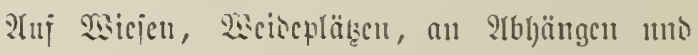

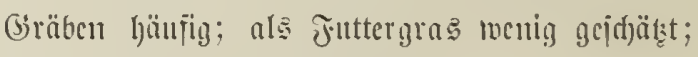
blïgt vom Jึul- Ecptember.

fiuj. 117. Aira caespitosa L., giajenjdunicle, rajenjörmige $\Xi$ d) mele. ․ $(50-130 \mathrm{~cm} \mathrm{hod).}$

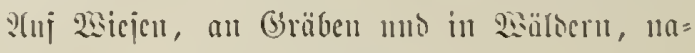
mentlid) an uajīen, jdattigen $\Xi$ telícu. Jait in

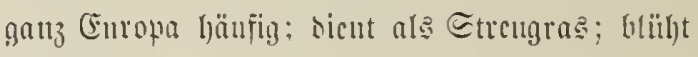

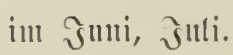




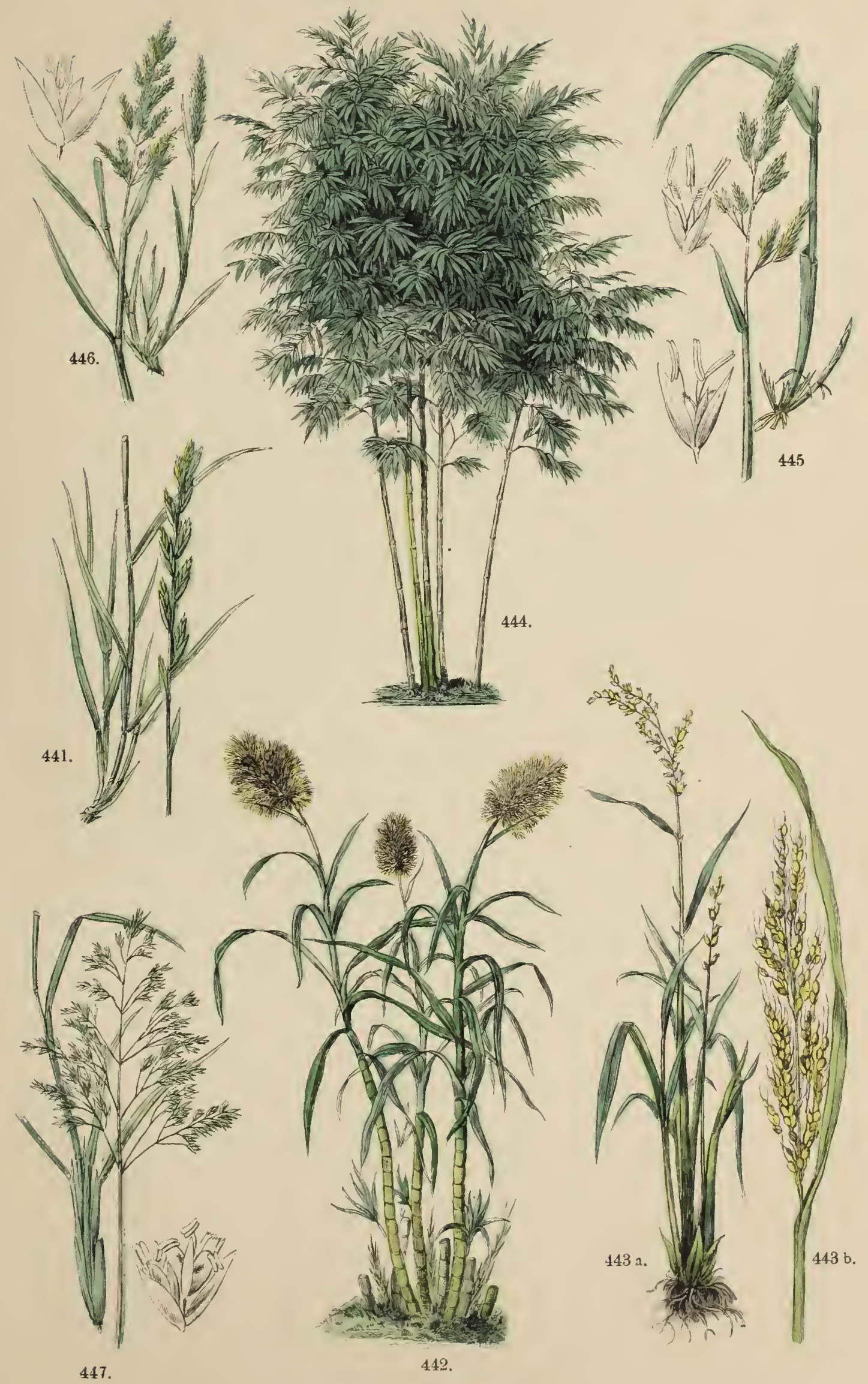






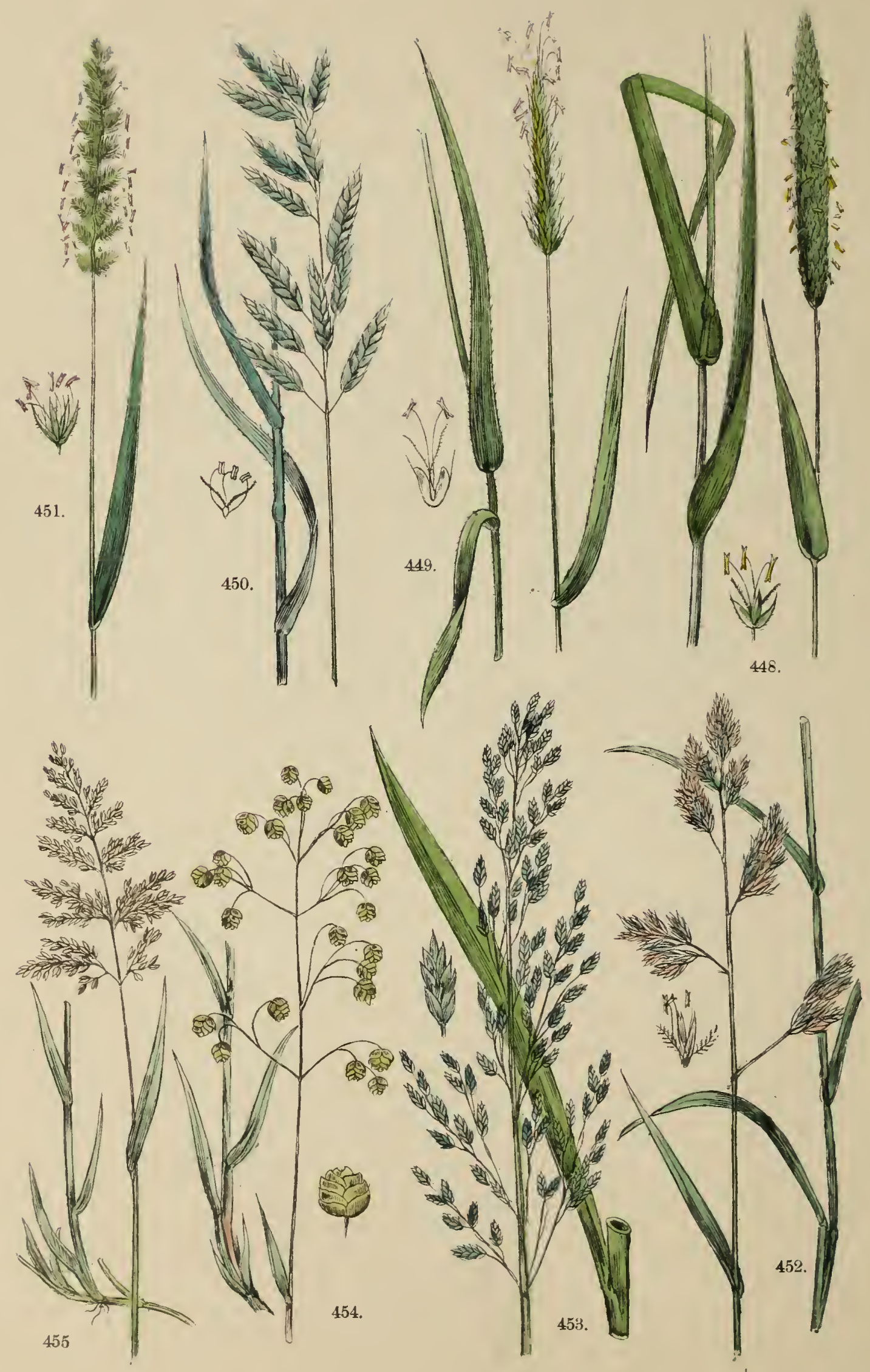


Taf. 78.

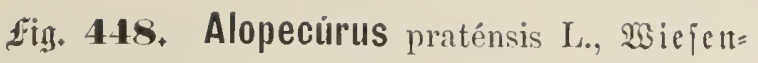
fud) sjatwanz. \%. 60-90 cm hod). Durd) ganz Emropa auf feuchten $\mathfrak{B i c j e n ~ u n t o ~ a n ~} 2 B a ̈ j=$ feruntygegräben. Bortreffiches und ertragreidjes

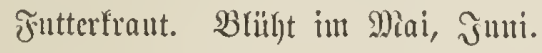

fỉg. 449. Anthoxánthum odoratum L., ge= meincs $\mathfrak{i l l d}$ gras. $2 . \quad 30-60 \mathrm{~cm}$ hod). Ju Bäloc’n uno auf $\mathfrak{B i c j e n ~ o u r d ) ~ g a n z ~ ( E u r o p a . ~}$ 2Bohfriechento, namentlid) in trocfenem Bujtande; als Futtergras megen jeines bitteren (Bejd)madt: nich)t jef)r gejđjälzt. B̉(ïht im Mai, Juni.

fig. 450. Bromus mollis L., weidylyarige

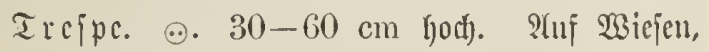
au Rainen und anf wüftem $230 \delta$ en bud Europa verbreitet, in Dentid)land ljäufig. 2u(s Futtergras olne $\mathfrak{B}$ escutung. SBlugt iut Mai, Juni.

fig. 451. Cynosúrus cristítus I., gemeines Rammgras. 21. 30-60 cm hod). Rament= (id) anf trofnen Bergwiejen uno Rainen weit

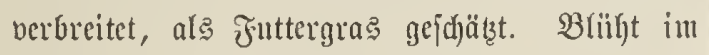

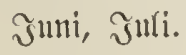

fin. 452. Dáctylis glomeráta $L_{\text {., }}$ Inaufgra 21. 30-125 cm hod. Durd ganz Europa alङ gutes Juttergras verbreitet, anf 2 siejen mo in

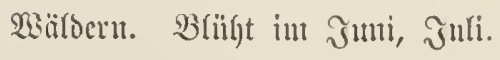

fiin. 45.3. Glycéria spectábilis M. 11. Kóch,

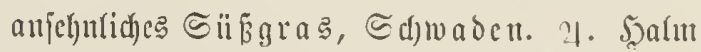
rohrartig, dicf, bis $2 \mathrm{~m}$ hod). Tam Rande

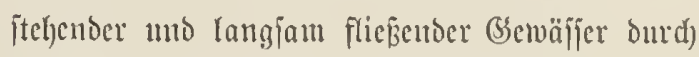

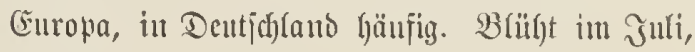
?tuguit.

fiig. 454. Briza media L., gemeines 3 itter= gras. 21. $30-50 \mathrm{~cm} \mathrm{hod).} \mathrm{SHI} \mathrm{Wiejen} \mathrm{uto}$

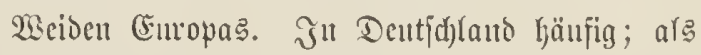
Futtergras nicht vou Bedentung. B(iiht im Mai, Эแแn.

fig. 45.5. Holcus lanátus L., wolfiges şonig= gras. 2. $30-50 \mathrm{~cm}$ hod). T(ui Wicjen uno in 2 säloeru durd) Europa verbreitet mto häufig; Fnttergras von zweifelfaftem Wert. Błhilgt vom

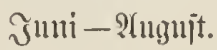




\section{זấ. Zg.}

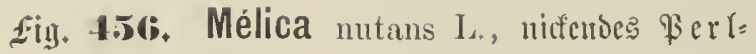
gras. 21. 30-60 cm yod). 3icrlidjes (3ras,

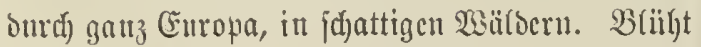
iuı Miai, כึuni.

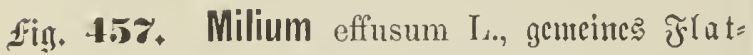
tergraz. \%. Edjlautes $130-160 \mathrm{~cm}$ lyofes

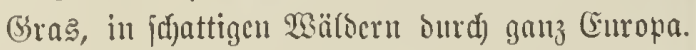

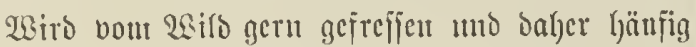
in Tiergärten angejüt. B̧lïft vom Miai-Sult. fig. 458. Phragmites communis Dinn., ge= meincs Sdilf, Sdjilfrohr. 21. Dic Saalme

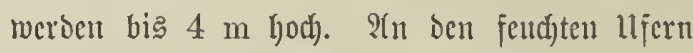

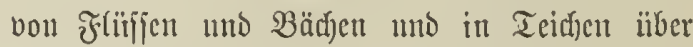
bie ganze Erroc verbrcitet, bie Sjalme bicnent zum

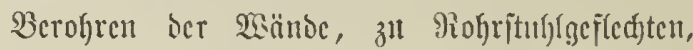

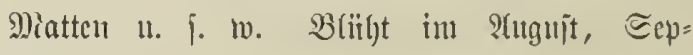
tember.

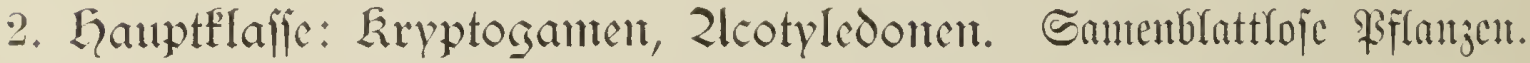

188. Frautilic. Equisetáceae de C. Sdiafthafurc.

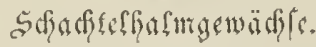

(5twa 25 Irten frantartiger, perennierenoer (Se $=$ wädje ber nörolidjen uno gemäpigten Zुone.

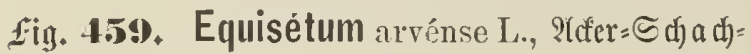
telgalm, Sd)eterfraut, Sał z̧cumeder, Dn= mod. 21. Stcnget-30-60 cm lood, 〔äjtigcs Infrant anf S̈tcern und Felocrn, gemein. Dicnt zuแ Sd)eutern unt Polieren.

189. Fantilic. Marsiliáceae $R$. $\mathrm{Br}$. (Rhizospérmeae de $C$.$) , astalletfarte.$

2. Silcine 2 saffergetwädjje in Ieidjen, Seen uno Padyen, jo sic Eeciarme, Marsiliáceae Bartl., 56 Prten; in ftelyenoen (Sewäffern ljänfig; dann bie Ziafferfporne, B̉itfelfarne, Salviniáceae Bartl., 12 :(rten; in Teid)en uno langjam fließ̧en=

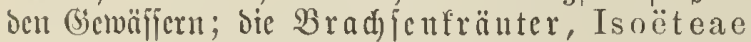
Bartl., 2 bentide Prten, anf bem Srumbe von Seen mino Sadjen nidgt jelten.

Wir geben aus bicjer Fantlic:

fịn. 460. Pilulária globulífera L., Fir $\}_{\text {c } n=}$ fraut (Biflenfarn). 21. Rutr 1 beutidye Art. 5-10 cm lang, fricd)eno, in Srräben, Sïmpfen uno Geen.

\section{Fantilie. Lycopodiáceae de C.}

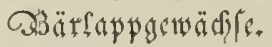

Etwa 100, ïber afle Teile Der Croc verbrcitete, anstanternoc, immucrgrïne, moosartige Bsetwädje.

fing. 461. Lycopódium Selago L., Bärlapp, Moosfarn. 21. Stengel $8-16 \mathrm{~cm} \mathrm{hod),} \mathrm{in}$ (Sebirgswälocrn von ganz Guropa, fricdjent. Bon Lycopódium giebt es 6 bentid)e ?rten.

191. Fantilte. Filices Juss. জdife Fature,

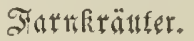

l̈̈ber 3000 शrten fratt= oocr banmartiger \$rlan= zeu, fajt iiber bic ganze Ëroc berbreitet. Einige STrten bienen als ?trztcimittel, anbere als Bier= pflanzen. Dem $23 a l b e$ fino fie bei unz jübolidice linfraut, weldes ben jungen \$olzmutc)

figy. 462. Asplenium Trichómanes L., braın= ftieliger Streifenfarn. 21. 4-15 cm hod). TIn Feljen unt Mautcrn, naunentlid) in Bebirgs: gegenten, burd) ganz Cinropa.

fin. 463. Asplenium Ruta muraria L., Maner=

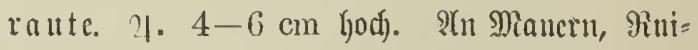
neu แno Fe(jen, burd) ganz (Europa. Jn Dentid)= lanto ljäıfịg. 
79.

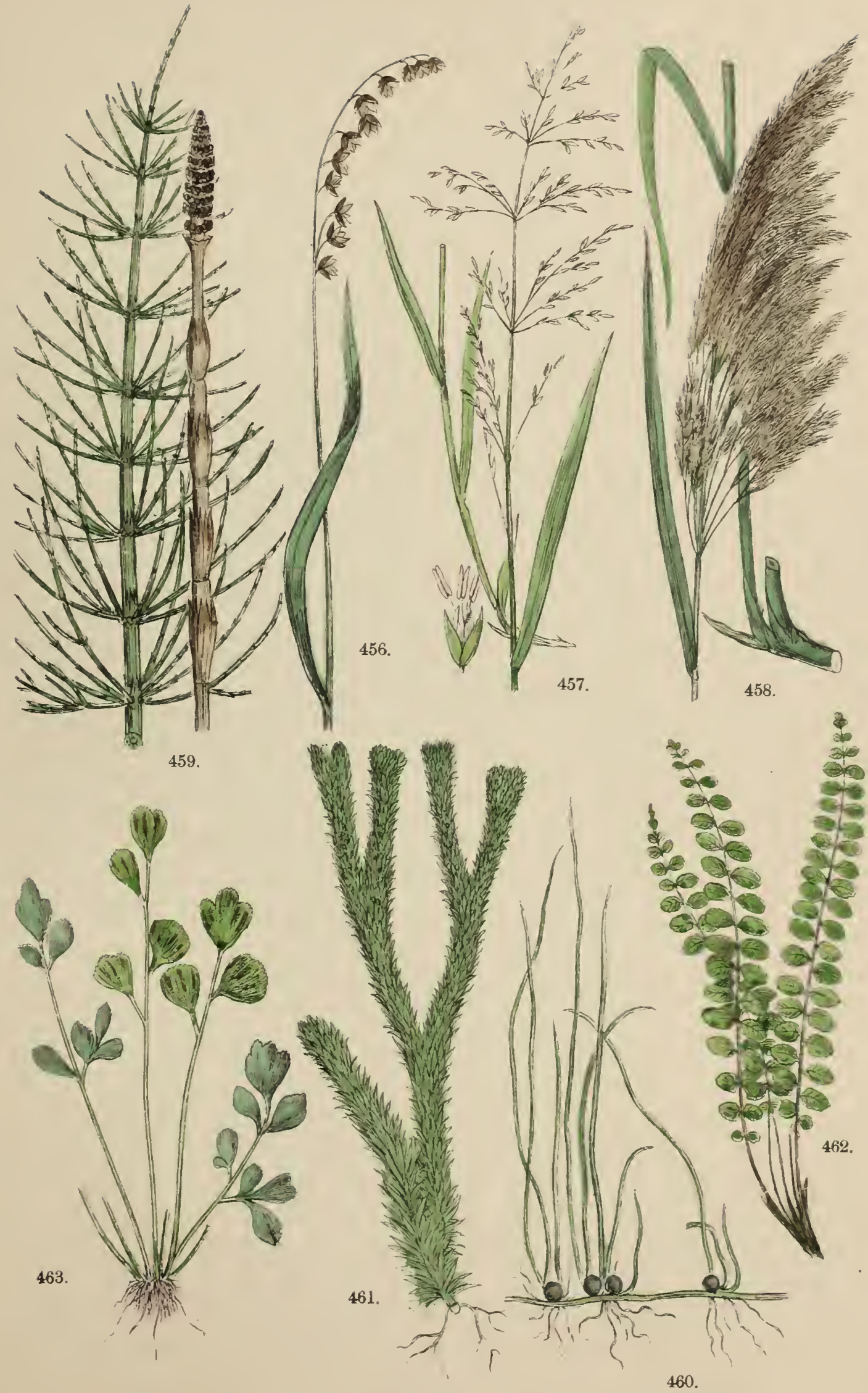



80.

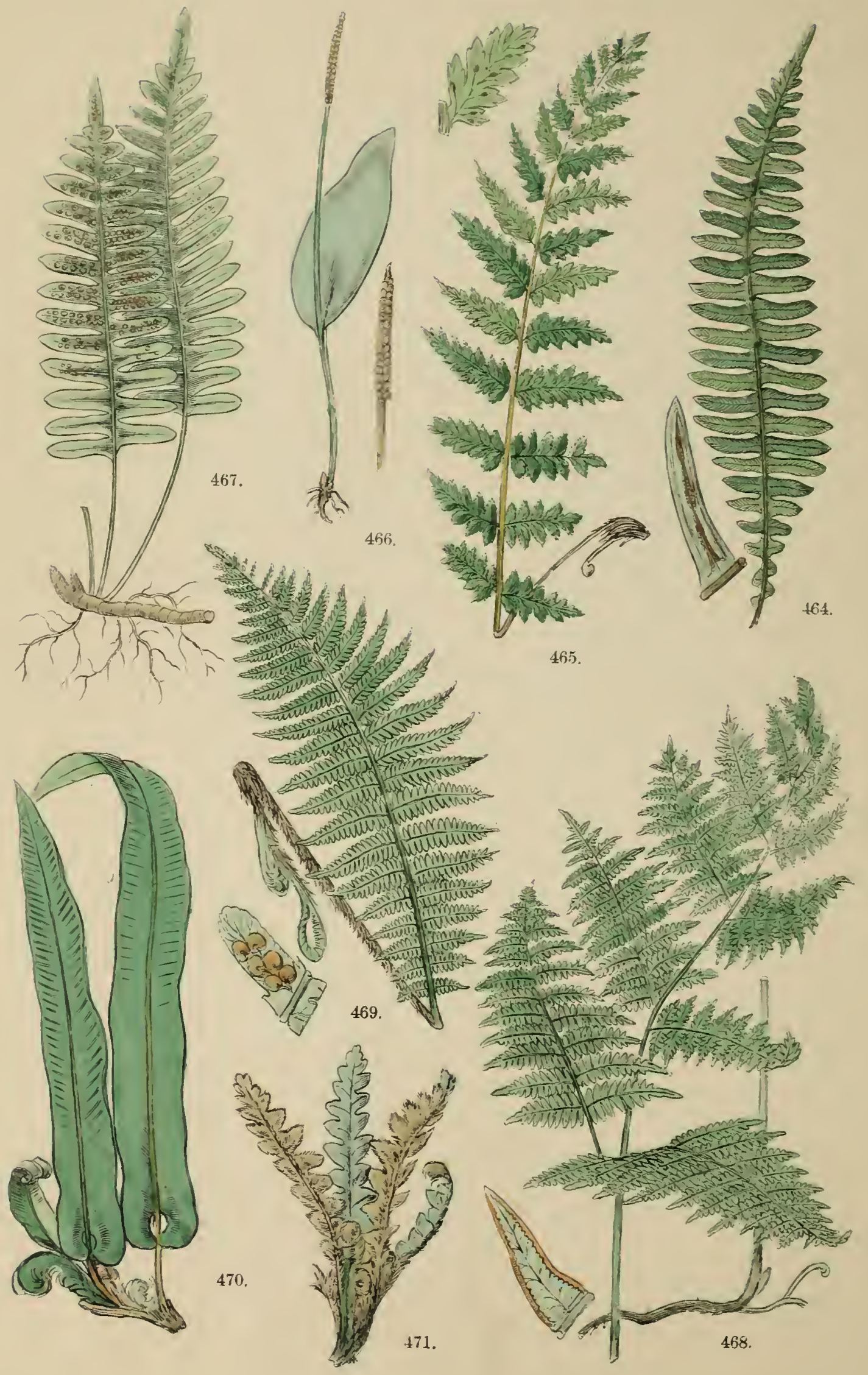


Taf. 80 .

fing. 46-1. Blechmum Spicant With. (Osmunda Spicant L.), gentiner diippeujaru. ?. 15 bis $30 \mathrm{~cm}$ hod). Ju fend)ten, fd)attigen $2 \mathfrak{s a ̈ l = ~}$ Der'n, zwijd)en Jelien แmo Eteinen; ১urd) ganz Europa, itt Dentidtano itellentweije häufig.

fin. 46.5. Cystópteris frágilis Bernh., zer=

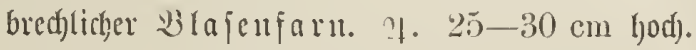
Ĵ Biebirgggegenocu fajt über bie ganze Erbe verbreitet, an Felfen und Niauern; in Dentid)= lanto nidjt jelter.

fig. 466, Ophioglossum vulgatum L., ge= meine $\mathfrak{R a t t e r z} u g \mathfrak{e} .24 .4-30 \mathrm{~cm}$ hod). Tiantentfid anj Ricien in Mittel= mo Giid = europa; in Deutjalando zerifterut.

ffig. 16\%. Polypódium vulgare L., genteiner Tüpfelfarn. ganz Ëmropa, an jchattigen Diautern, Feljen uno

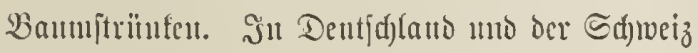

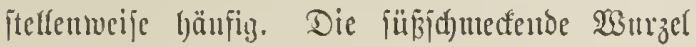
war fritger offizinell.

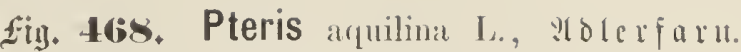
2. Grojer, anfred)titebender Jarn, a॥ günjtigen Drten ïber \& $m$ hor). ¿̈lber bie gemäpigten

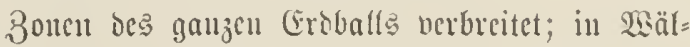

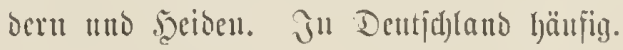

fiij. 469. Aspidium Filix mas Sw., $\Subset$ djild= farn, 23urmiarn. 21. 60-90 cm hod).

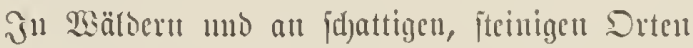
von ganz Europa. Jil Deutidatano cincs ber bäıfigiten "Jarufräuter".

fiig. 470. Scolopendrium vulgare Sm., ge= meine sirfdjunge. 2. $25-30 \mathrm{~cm}$ hod). ju gemäß̄igten uno jüolid)en Europa, an fdat= tigen Felfen tuto Miantern; in Dentị)lanto zer: fitreut, nid)t ïberall.

fius. 471. Céterach ofticintrum Willd, \&olt= faru, Milfzarm. \%, 6-20 cm lod). Ju Felejpalten tuto an alten Mianern Mittel= แno

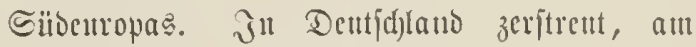
Mhein แnto an ser Diojet bäufiger. 


\section{Tẫ. 81.}

192. Familie. Muscincate Juss. artools, santurou|c (Musci frontosi).

Sicinc, überall in Den verifjicbenjten Formen ver=

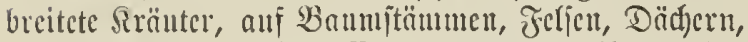

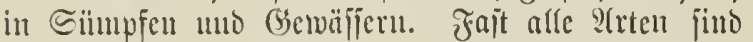
แutzlos, nul wenige jum Padjwadjen ocs Torfes

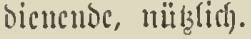

fig. 17:2 a. b. Sphagnum squarrosum Per's., ipartidtes Sumpfmodz. 2\%. L. (Sphagniceae C. Miiller). 8-15 cm lang, an najien Etellen uno in Eümpjcul. Die Sumpfmoofe find bie midgtigiten torfbilbenden \$iflanzen.

fig. 473 a. b. c. Phascum cuspidatum Sclireb., fpitziges Bartmoos, zugcipitzter $D \mathfrak{h}$ mund. ๔. (Phascáceae Schimper, Bartmooje.) 2u 2-9 $\mathrm{mm}$ lang; dicjc 2 rt finbet jid) lyätrfig in (Särten uno an Gröben, ïberhaupt alf Dioocr= croc, biझmeilcu fdon bom crftel frühling alt

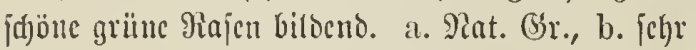
vergrößert, c. vergrößerte Frutdtfapjel.

fig. 474. Mnium liygrométricum, hygrounctri= \{des Stermuob, Rat. Brr. 24. (Bryáceae Schimper, fnotennoosartige (Selvädfje.) $12 \mathrm{~mm}$ bis $5 \mathrm{~cm}$ lod fintoct fidf) bicic ?(rt ljäufig an

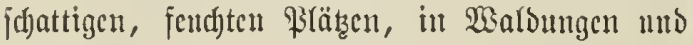
an Miatern.

fig. 475. Físsidens adiantoides Hedw., haar= faruähnlidyer Spaltzahn, Rat. Brr. 2/. (Fissidentáceae Schimper, ๔paltzaljmmoojc). Wädjit

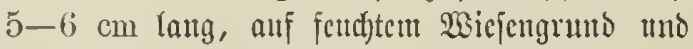
in jumpfigen $\mathfrak{B a r}$ oungen.

fig. 476 a. b. Dicranum cerviculátum Hedw.,

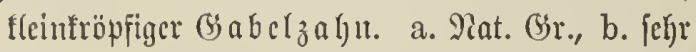
vcrgröficrt. 21. (Dicranáceae Schimper, Gia= velzalymmoofc). T(n fetdjten, fanbigen Srten uno auf Torfmooren, nidjt jelyt häufig zu finoen.
193. Familic. Hepáticae Juss. (Lichenástra Dill). Sifermoole, तtfermools.

llugçälyr 1400 über dic gauze (5roe verbreitete

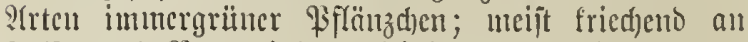
Felfen uno Baumtrintoen. Einige ber widftigften fund:

£ig. 4zr a. b. Jungermánnia lanceolíta L., lauzettblüttrige Jungermannia. ๆ. (Jungermamniáceae Corda.) 48 (Battungen mit ïber

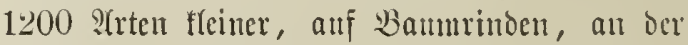

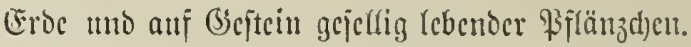
Die abgebildete 2rrt (a. Mat. Brr., b. jefre ver= yröß̈ert), wädjit vercinzelt all $\mathfrak{W i e j e n ~ o b e r ~ n a c t e r ~}$

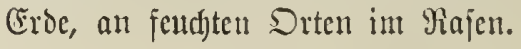

fịn. 478. Marchantia polymorpha L., gemciuc Miardantie, Scberfiaut, Stcinleberfraut, Şrunculeberfraut. 25 Arten, bic abgebil= bete bic cinzige bcutjose. 21. (Narchantiáceae Corda). Das anf bem $\mathfrak{B}_{0}$ ocu licgende, gelappte Saub beocft in oerworrenen, rajenartigen l̈tber= zügen oft groß̉e Fleden an feutd)ten Felfen, Bädhen uno Dutelten. War friiger offizizincll.

fig. 479. Riccia nátans L., ¡đtwimutuoc Riccic. ๑. (Ricciáceae Rchb.) 60 Itrtcu. Dic $\mathfrak{a} \mathfrak{b}=$ gebilocte 2 art wiro nul $6-9 \mathrm{~mm}$ grof und woljut in itchenoen Gecwäffern; bas saub ijt jajwim=

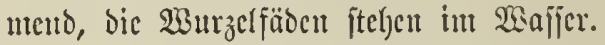

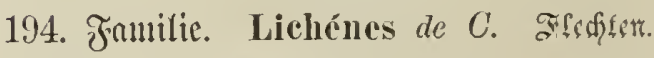

Gisgen 1400 arten, dabon 650 in Emropa. Findon jid) itberalf, namentlid) alt oer Grenze alter übrigen Begetation, all Banuminoen, Manern mo Ferfen, nie im Wafjer. Cinige bienen als $\Re a \mathfrak{h}=$ rungsmittel fïr Micnjchen oder Tiere, andere al( Jarbjtoffe.

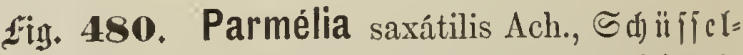
flectite. 24. (Parmeliáceae Hock.) Dic $a b=$ gebilocte 9 rt ijt blattartig, grüurliçgrau, ınten

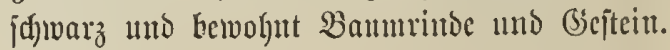


81.

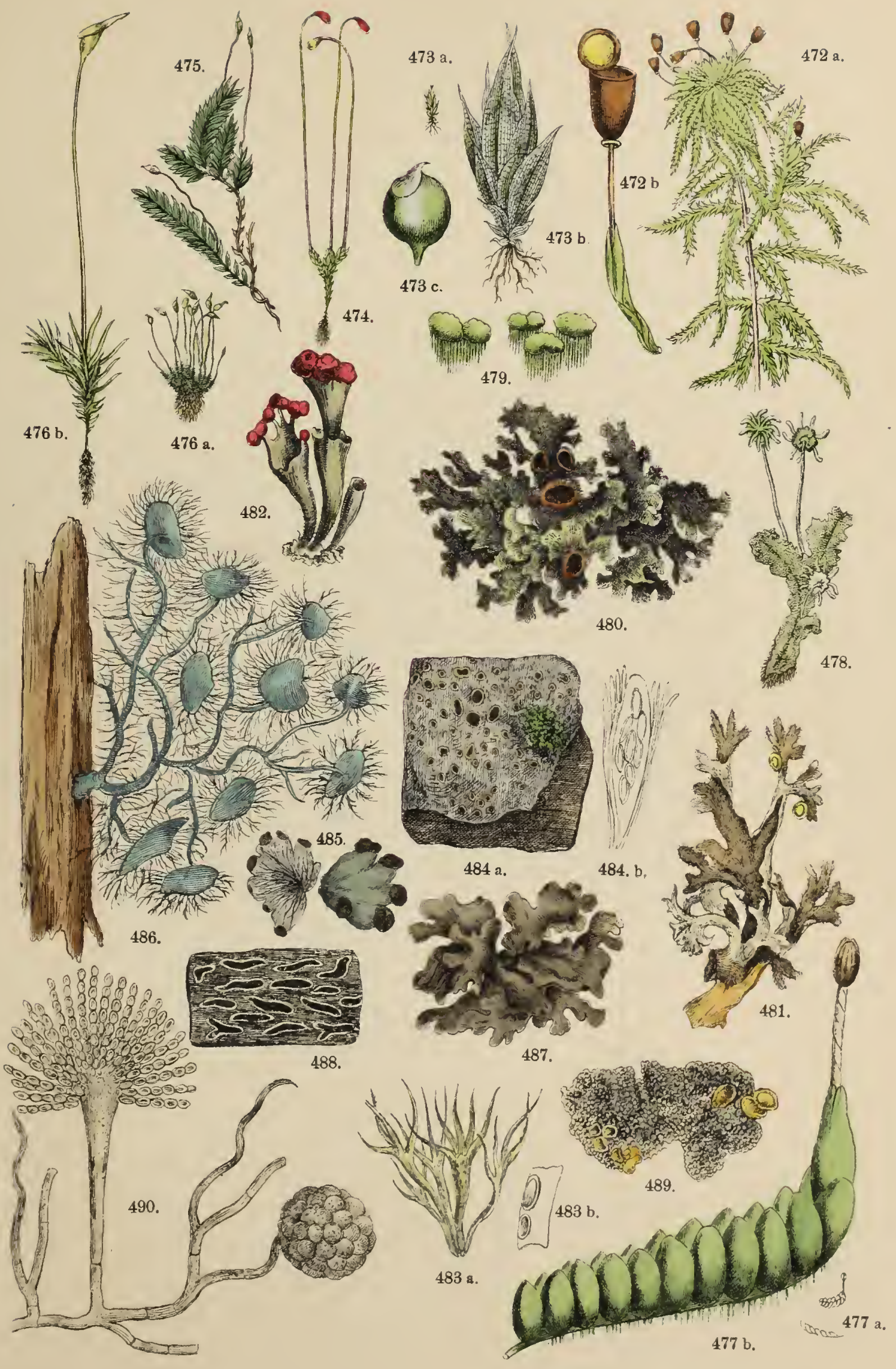



fijg. 181. Cetrária islántlica $\Lambda$ ch. (Lichen

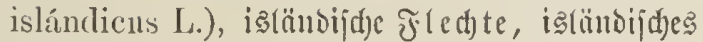
Moos. 21. (Ramalíneate Fee, M(ftfled)ten.) 3rijden Whos, Gras mo seetoefraut in sei=

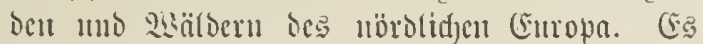
ift bie cinjige offizutuelfe Jiectete.

fịy. 18:. Cladónia crenuláta l'lk., હäıl= d)enfledite, Bedferfedte, Bedermoos. ?. (Clarloniáceae \%enk.) llnter bou ca. 50 befannten $\mathfrak{2}(2$ ten vou $\subseteq$ äuld)enf(ed)ten zcid)uet jid) bicje Durdf präd)tig idjarlad)rote Frudftlager ans.

Eịu. $18: 3$ a. b. Roccélla tinctória Dec. (Lichen lioccélla L.), Järber fled)te, :a tfu usf(ed)te. ?. (Roccéllei Nyl, હtraudffled)ten.) P(tฐ Dicjer Flechte wirb bic jdjöne tot tund biolet=färbente Drjetllefarbe getwomnen. Die Jlech)te wäd)it all Jeljen an Wittelmeere, ant Siap mo in Siid= amerifa.

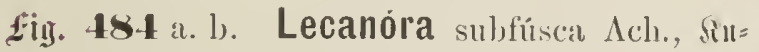
d)eu $=\mathfrak{F}(\mathfrak{e d})$ e. $\quad(J i g . \quad$ b vergröberter Eporen= ¡d)(and.) 2/. (Lecanóreac Fee.) Ju veridje= denen Formen an Bamurtuden, Mianern umb Jeljeı in gaız Deutjulanto bäıfig.

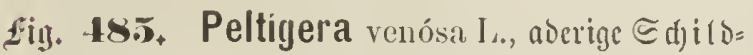
fled)te. 21. (P'eltideáceate I'w., Echilof́led)ten.) Яleute, flad) ansgebreitete Fledte, oben grangrïu, menten weís mit fd)wärzlid)en s(bem. S(uf fend)= tem, jdjattigem Bober.

fiig. 486. Usnea barbata Fr., gemeine Żart= fled)te. 21. (Usireáceae Eschw., Bartffedten.) Eetwa 10 ïber Die gauze Erde verbreitete 2rten, ocren exutge a(s lange, bartäluntid)e Miajīen an

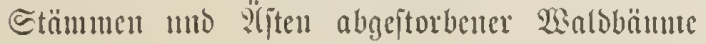

l)crabłängen. Die abgebildote ijt cine oer fdjön= ften $\mathfrak{2}(r t e n$ der Bartfled)ten.

2iuj. 187. Sticta silvática Ach., $23 a$ lo = (S) r $11=$ beufled)te. (Parmeliáceae IIook.) Dian teunt Iแtgejälyr 50, Davou 8 Deutjaje StictiY(rten; bie abyebildete ijt bei uts nid)t jelten (IIt altent Lialsbäumen ju fintoen. Waar frïtler offizizinlt.

Eiuj. 188. Graphis scripta $\Lambda$ cll., gemeine Sd) r i ftfledte. (Graplúdeale Eschw.) Giegen

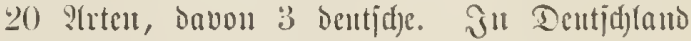
gemein a baumrintoen in Der Ebene und auf nicoeren bergen.

fig. 189. Verrucária tartarea Iloffm., tar=

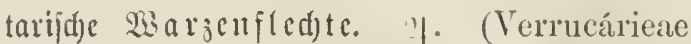
Fr.) Dicje :

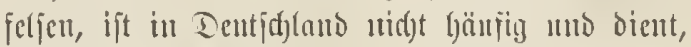
bejondere in @djweden, zun Järben.

195. Familic. Mycortes de C. Sif\}, stantipiss.

2sinzig fleine, beintale nur surd) das Mlitrosfop bentlich) zut erfentento (Gebiloe. 3ut iluten gehören ber

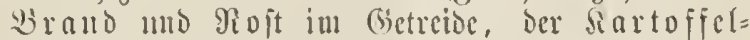

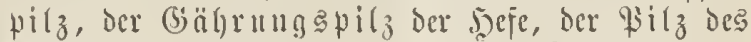

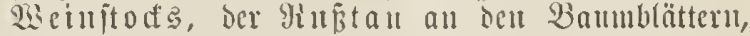

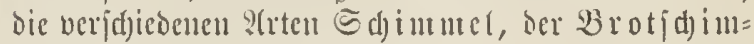
mel, Der Dieljlta แ. $j$. w.

fin. 490. Aspergillus glaucus Link, gemeiner

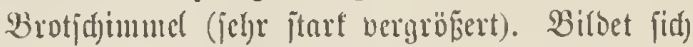
auj verídjebenent in 3erjeksung befinto(id)en Etofifen, fo auf altem Brot uno auf cingemad)ten Früd)=

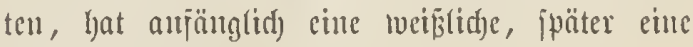
grangrüne jarbe. 
Taf. 82.

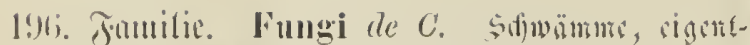

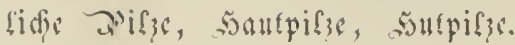

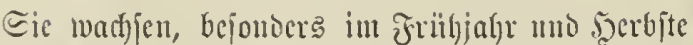

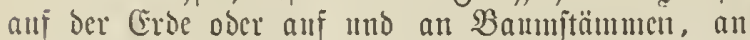

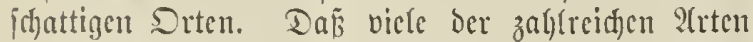
sill gejuntes, ftäftiges 2?al)rungsmuttel geben, andere aber bödjut gifting fins, jift befannt. l̈ber bie Eigen= idjajt, ob genieģbar ober idjäblid, fino bie tïdtigjten Éfwammfenter, z $\mathfrak{B}$. Srombholz, 5arzer, ¿c 1 z mo ?tnoere, bezüglid) vieler Edywämme jehr veridfiebener Mleinnng, was woht mit ber Edgueflig=

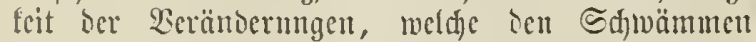
cigen, fowie mit sem lluteridjeo Der Bbosenart, in weldher jie gècihen, zm[ammentyängen mag. 2 sir miijien แnอ hier baranf bejd)räufen, einige berjenigen Alten, weld)e einjitimmig alอ geniešbar anerfannt

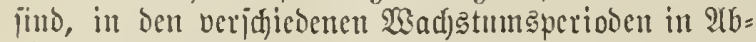
billoutg zu geberl.

fig. 491 a. b. c. Clavária Botrytis Pers., roter Z icgenbart, $B$ ärentaţe, Sculenpilz. Einer ber befauntejten uno ljäufigiten Bifze im Sommer

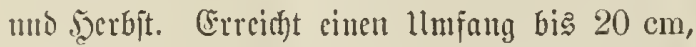
ijt aber unt idmadthajt in ber Bröpje von 2 bis $3 \mathrm{~cm}$.

fiiv. 492 a. b. c. Agáricus Caesáreus Schäffer, saiferling, saijerjduamm. B̉ei ms jel= teu, in ๔iibcuropa ljäufig, geidjä1şte Delifatejīe. Der älunlidje, jefrr giftige, in Dentidjlanto häuritge
$\Lambda$ garicus muscarius L., Fliegenjd wamm, unteridjeibet jid) vom Saijerling anf ocn criften Blicf Durd) ocn weipen Sticl, bamu burth bic Jyant= refte auf iem J̨ute, bic bei bem Saijerijwamm wic Fetzen oser sappen, bcim Jfiegenifunanm warzenartig geitaltet finto.

fig. 493 a. b. c. Agaricus Cantharellus L., Jalteupirz, Eicr\{d) Bfifferling, Gectden. Selyr lyäurig in Rabcfwäloern uno alङ gute Speije beliebt. Der Sont wirt bis $8 \mathrm{~cm}$ breit; alte Excmplare fdjmer verianlide.

fiy. 494 a. c. c. d. Agaricus campestris L.,

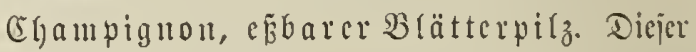
allgemein befaunte uno mit Redfyt gejdäbżtc

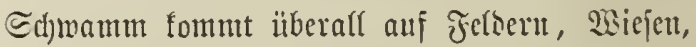
Biefluveioen unto an 2 aloräntern bor. Er bient, auf vielfad)e 21rt zubcreitet, a!ङ vortrefflidje Epeije unb wiro barmm and) in Miijtbeeten, trocfenen

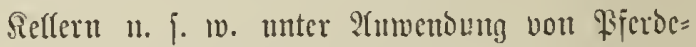
mijt in Grofen fultiviert und bilbet einen ge=

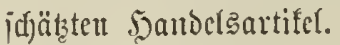




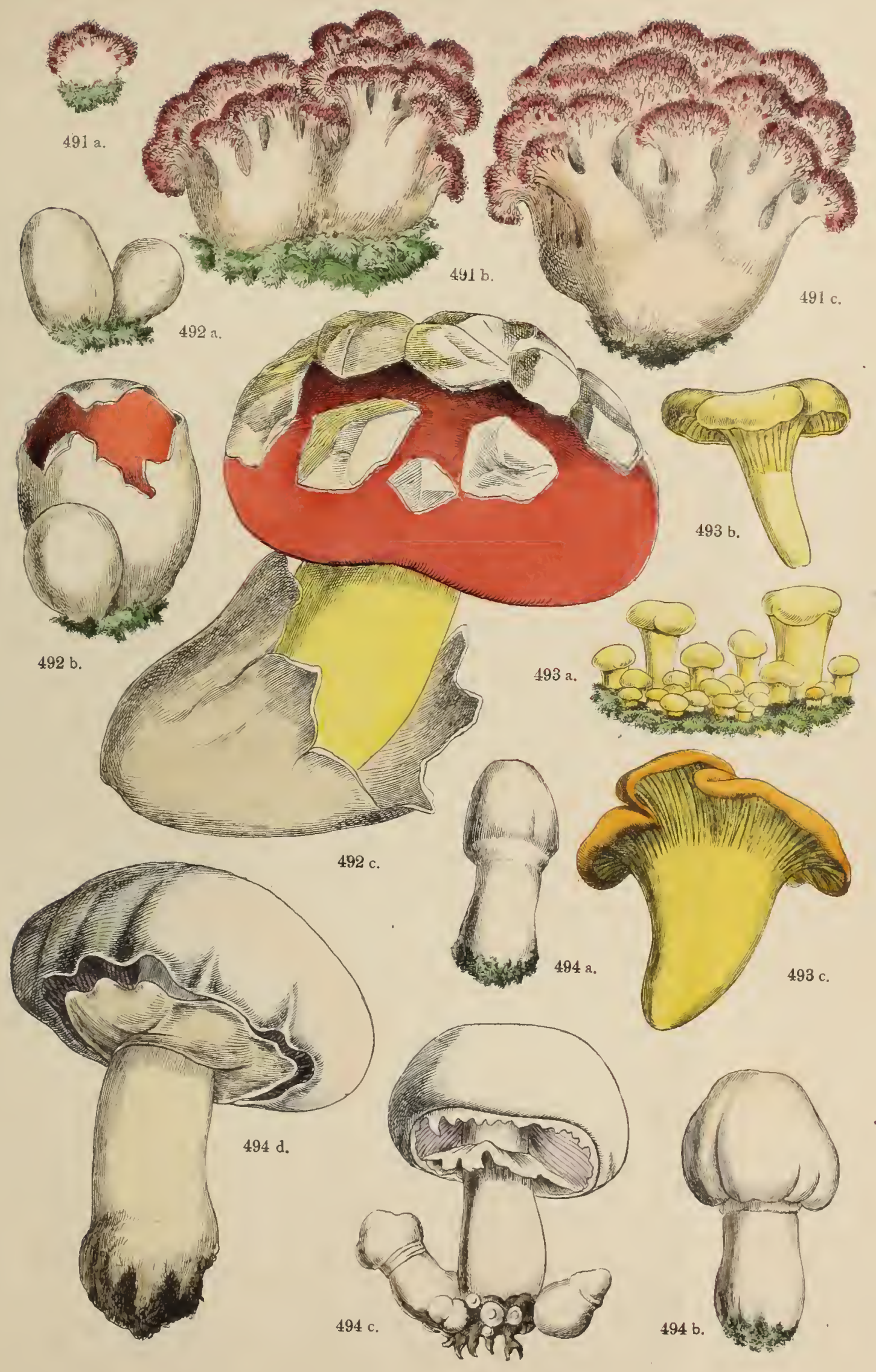




83.

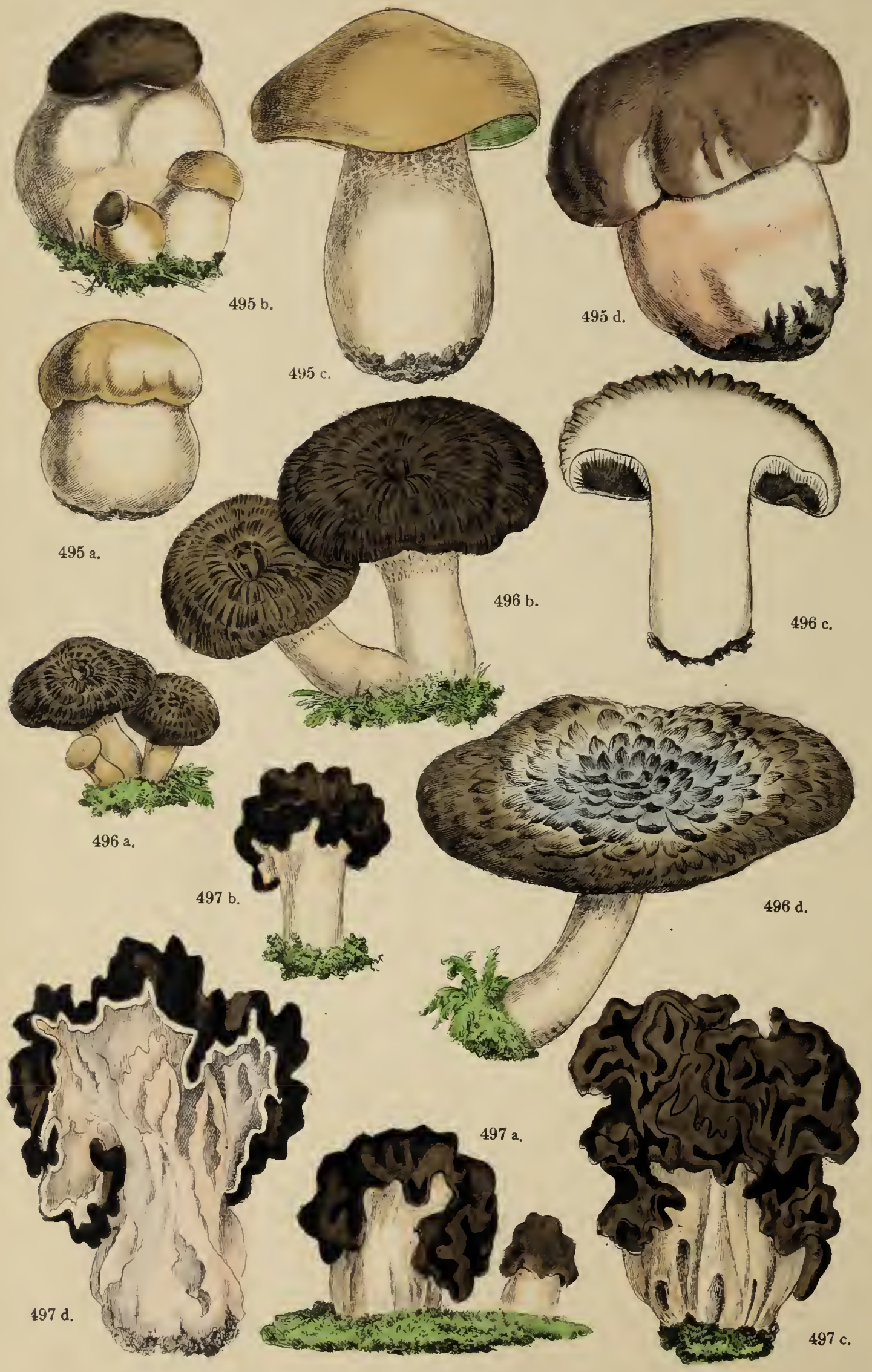




\section{Taf. 83.}

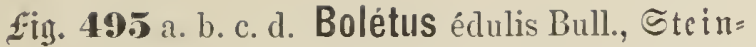

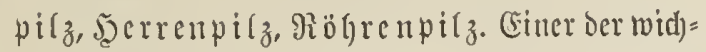
tigjten ऽd)twämute, allgemein befanut un belicbt,

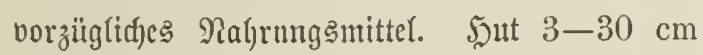
breit, im 2lter zuweilen flach. Dic Unterjeite Des J̨)ttes bejtegt aus bid)t zujaumenjtelenten riögren von gelblicher Farbe, weld)e von ber meizen Subitanz bes Suttes reid)t trennbar jino. Som Juli bie zun Spätherbit in liduten Sautb=

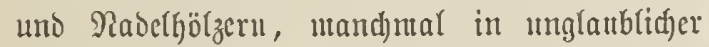
Nienge.

fig. 496 a. b. c. d. Hydnum imbricatum L..

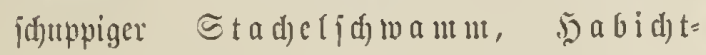

¡(f) amm. 5Jut $4-20 \mathrm{~cm}$ breit, im 5erbjt

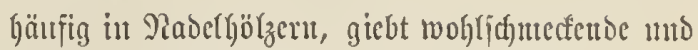
reidflidfe Mahlzeiten. Єelgr zn empfehlen.

fiig. 497 a. b. c. d. Helvélla esculénta Pers., Früh=Sordfel, Epcijelorddel, Miatradfe, Raurid, Stocfmordel. 5ृut $2-5 \mathrm{~cm}$ hod,

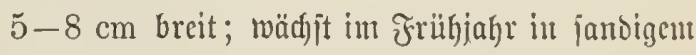
Boden, vorzitglid) in Nadelholzwaldungen uno giebt, määig genojien, cinte jđntacffajte, gejunde Rahrtng. CEtnige \&ord)et=2Yrten gelten für gịtig,

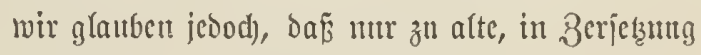

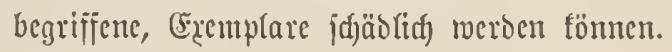




\section{Taf. 84.}

fig. 498 a. b. c. d. Morchélla esculénta Pers.,

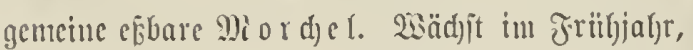
bejonvers in bergigen, jaubigent Bregentoen tmo

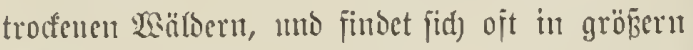

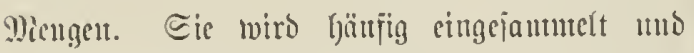
giebt jomohl frijd), als getrocfuct cine bortrefī= lidje Eveije.

fiuy. 199. Tuber melanósporum Vitt., edite, franzzöjifde (jd)marze) Irüfifel. Smueres flei= idjig, violettjumarz, jđjwarz mo weiś geabert. Reift im seerbjt; namentlid) in Sübjantreid) ınto Docritalien, in Dentid)lanto jelten. Die Trüfifeln wadjen unter ber (Erbe, namtent(id) in Sautbwalsongen mit loderem falffjaltigem $\mathfrak{B o d e n ,}$

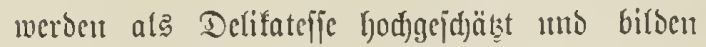
baher bedentende Şandelsantifel. Dic jdjwarze

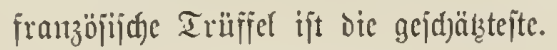

fig. 500 a. b. Tuber aestivum Vitt., $₫ o m=$ mertrüfiel, deutjde "gute" Trüfel.

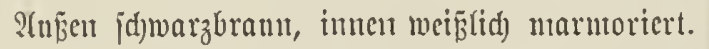
Dieje Trüffel ijt in Deutja)(and, Franfreid) unt Ŝtalien bie verbreitetîte.

fig. 501. Tuber magnatum Pico, italienijde

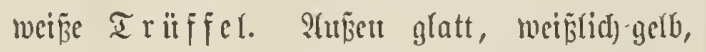
Jleijd) locfer, meip ober rötlid). Dieje Trüiffel iit in Stafien heimija unto miro bort vielfad) als Delifateffe veripeijt.

f́ig. 502. Choirómyces maeandrifórmis Vitt. (Tuber album Bull). Giner Sartoffelfnolle

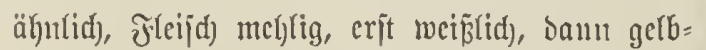

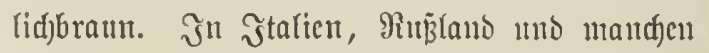

(Segentoen Dentid)lanos; weniger gejđjätżt, ala vorige 2trteu.

197. Fantilie. Algae de C. Afger, Eangs.

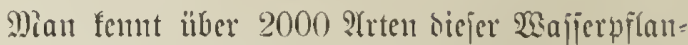

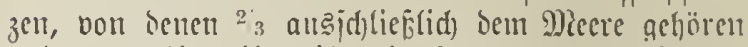
แno zwar bier bismeiten in fo ungelentren Diajicu

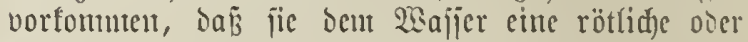
bräunliche färbuntg getert, ja jogar Den Rauf der Edjiffe eridweren. 2iele 2(rten fitto mifrosfopijd) tlén, einige aber mel)rere 100 fints lang.

wair heben folgento ?trten herbor:

fị. 503. Protocóccus nivålis $\Lambda$ g., roter

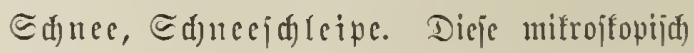
fleite arfge erjefeint in ber Edjuecregion ber Alpen แmb in ben folarläıtoerı als eine Brallert= maffe in roter, fpäter brätmlidjer Farbe.

fị. 501. Cerámium diáphanum Ag., sutd)= jidjtige Ceramie. Eine Der zierlidjiten Niecres.

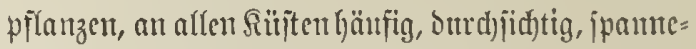
looch, meijt jajön purpurrot ober violett gefärbt. llujere 96bifonng zeigt ein 8weigd)en in Patur= größ̈e.

fig. 50.5. Zonária squamaria Ag., Fĩanen= fduciftang. Fintoet fid) in allen, Europa jïbs= (id) unto mejtlid) umgrenzenten Mieeren. Eic

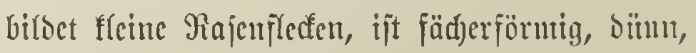
mit zahllojen fleinen Miecrtierd)en (Booplynten) besedt. Natürfid)e Größ̄e.

fig. 506. Conférva fugacíssima $\mathrm{Ag}$, ver= gänglide $\mathfrak{B a f j e r j e i b e , ~ F a b e n a l g e . ~ L ̈ b e r a l l ~}$ gemein, oft maffentweife in ftefenton uno fließjen= ten Sajpern, in Teiduen moto an Dueflen, fejt= fitzento oder freifdmimmento. Raturgröß̧e. 
84.

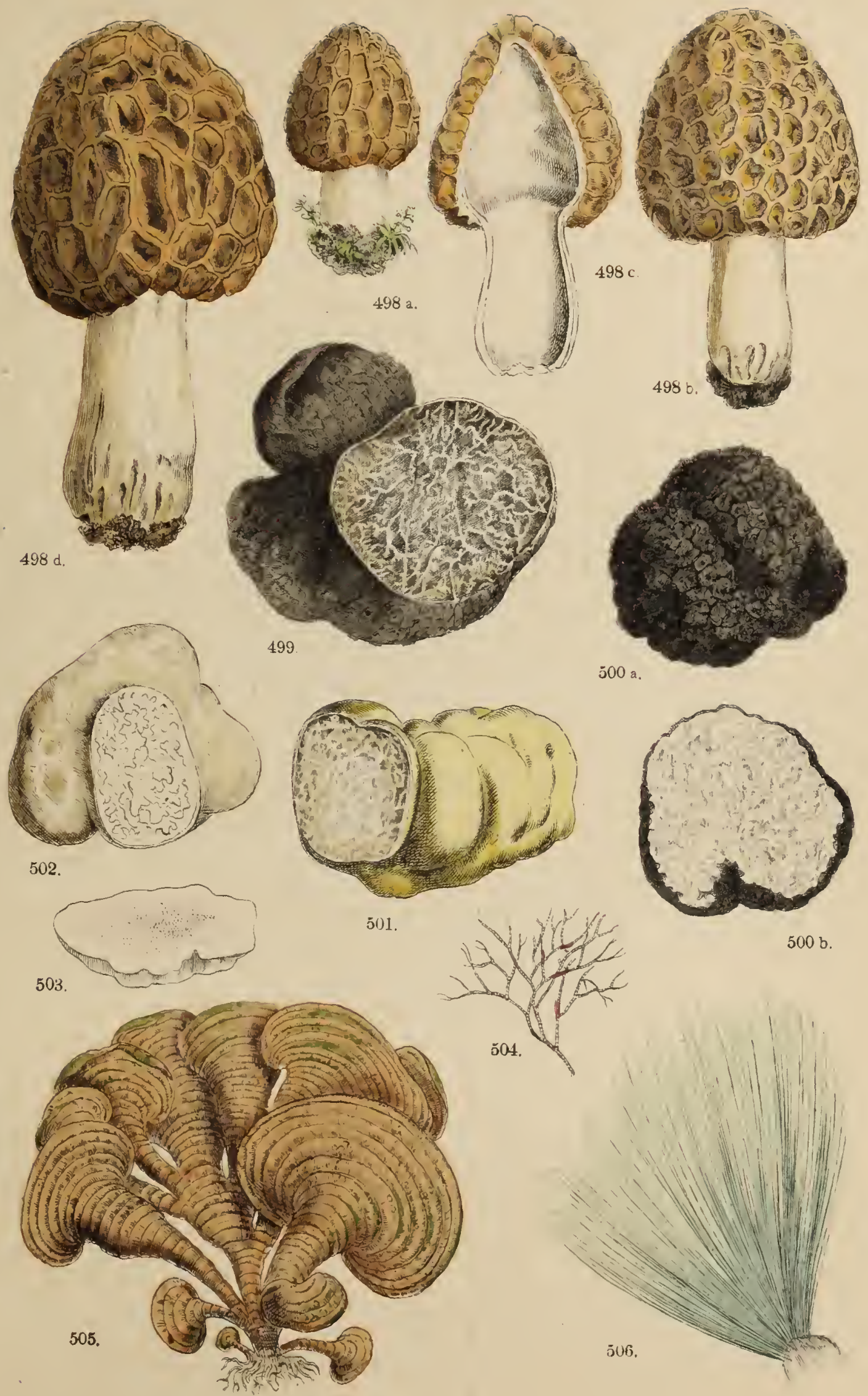





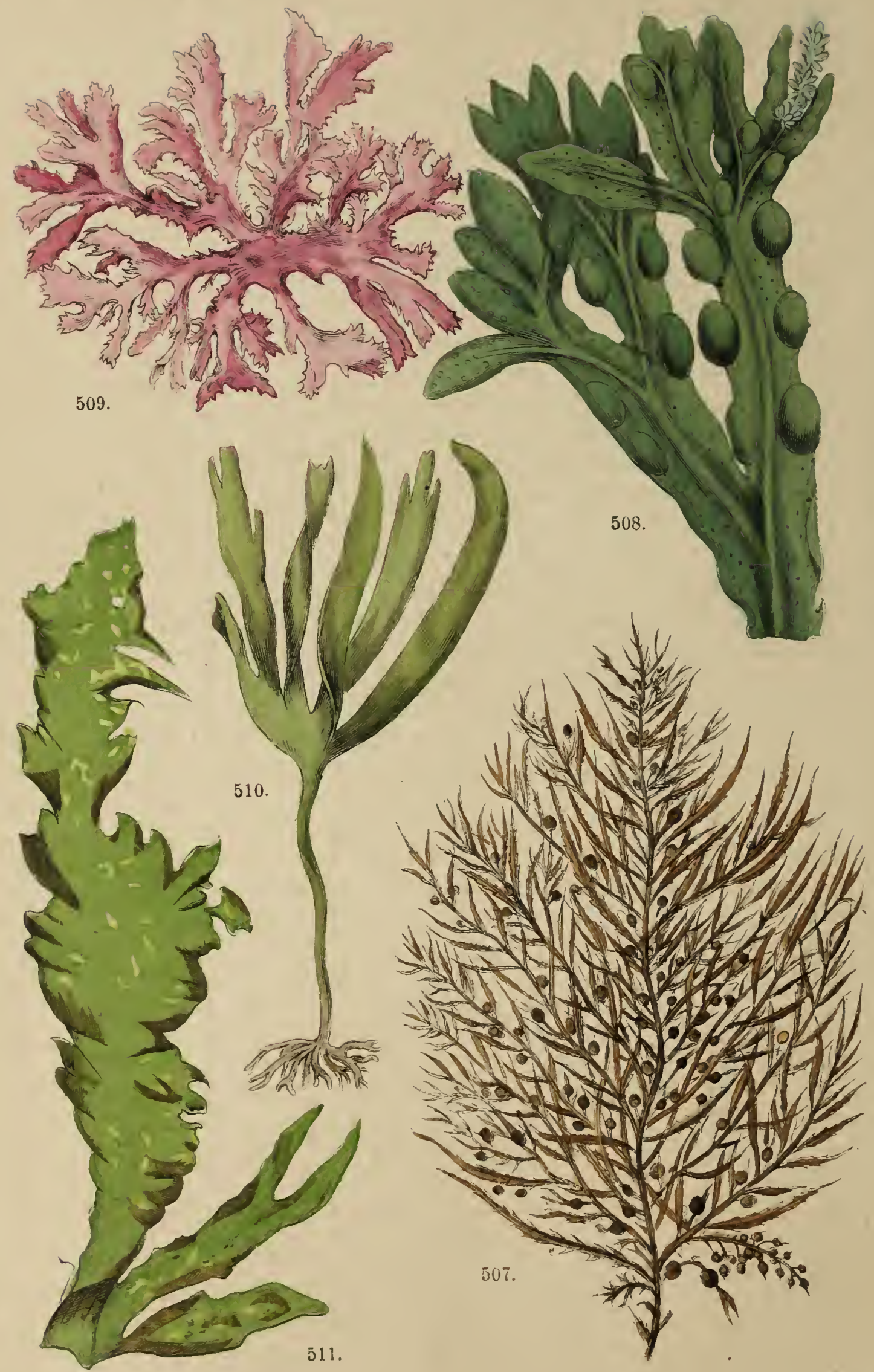


raf. 85.

fiu]. 507. Fucus natans I. (Sargássmm baccíferum $\Lambda$ g.), Becrentang, ভargafio= Tang, (3) orffraut. Dic Tange (Fucíceae Ag.) fitto

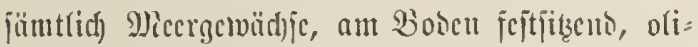

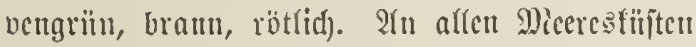
verbreitet, fommen jic, mo zivar bismeilen in gropien Piajien, and) freijdjwinmento anf hoher

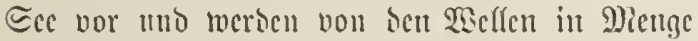
an ben Etrano geppïlt. Wiele ?(rten bienen ge= trocfinet als Stren unto als Dïnger, cinzelne ucroen veripeift, ant andern wiro Soba (Sielp) gewonnen. Der abgebilocte હargajio=Tang be=

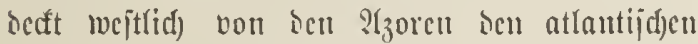

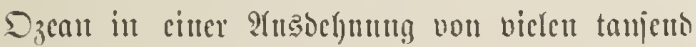
Dunabratmcilcu.

fị\}. 508. Fucus vesiculósus L., gemeiner 2 [ $a=$ fentang, Ecctang. Dicjer burd) bie cinan= ber gegentïberijtelgenden Suptblajen anşgczeid)nete Ecetang ift olivengrïn, troden id)warzbraum, an

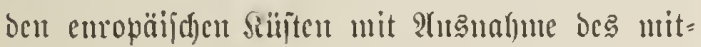

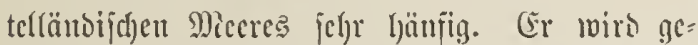
troffnet mo verbranut, um ans ocr P(jde Soda unb Эoo żl geminnen.

fig. so9. Fucus crispus I. (Chondrus cris-

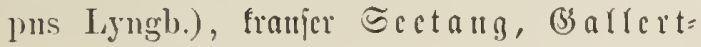

moos, Fertmoos. Sdjön rojenrot, mit zar=

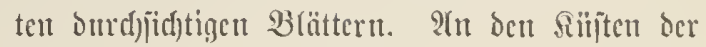
Rorbjec uno bes attantijchen Dzcans. Röjt fid

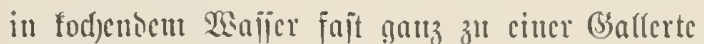

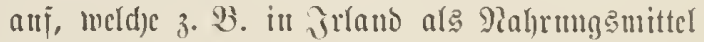
sicut.

fiı]. 510. Laminária rligitata R. St. V., ģc= fingerter filattentaug, Reptunsgïrtel. Dic Plattentange jüb olivengrïne, hä̈ttige, langge= itielte Bänder utit cinfad)en, rmosm $\Xi$ ticle mo

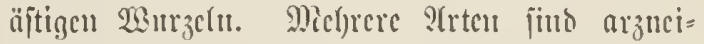
fräftig. Der in jefre verfleinertem Siajijtab ab= gebiliete gefungerte qil. ijt gemein in sen nöro (idjen Wiecrent uno wurzelt anf icm Niceres= grmutc. Der Eticl ijt gewölnulich un fingers= Dicf, an ben Faröern aber oft armesbief mo sann

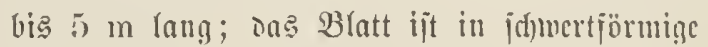
Sappen getcilt.

Ein. 511. Ulva lactúca I., Micerlattig. Er

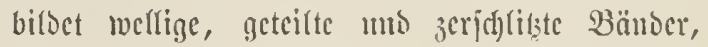
weld)e cinc Sänge von $50 \mathrm{~cm}$ bis 2 m crecidjen;

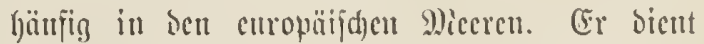

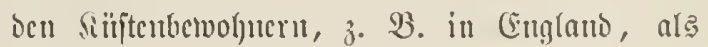
Epcije. 


\section{Zllphabetifhes Regifter.}

\section{I.}

TaEpflanze Geite 50.

Abies S. 70. Tafel 67 . Tigur 389 a. b. c. Abietineae S. 70 .

Acacia S. 16. T. 14. ‡. 79.

Acanthaceae S. 58.

Acantlius 5. 58 .

Acer 5. 12. T. 11. F. 63 a. b.

Acerincae 5. 12.

Achillea ธ. 40 . T. 37. F. 211.

Achimenes 5. 46 .

Achras 5. 49.

Ifferdijtel ङ. 41. T. 38. F. 221.

2Afertlec 5. 18. T 16. T. 93.

21ferlinie 5. 20. T. 18. T. 107.

2lfermambohr S. 52. T. 49. テ. 288 a. b,

2fderrettig G. 8. I. 8. T. 41 a.b.c.

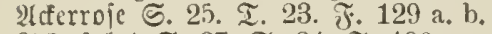

Idferialat 5. 37. T. 34. গ广. 196.

Adertuitlde 5. 51. T. 48. T. 282 a. b.

Aconitum 5. 3. T. 3. T. 14 a. b.

Acorus S. 72. T. 69. T. 400 .

Actaea 5. 3. T. 3. F. 13 a. b. c.

Irbamsapfel ङ. 73.

Adansonia S. 11.

Adenanthera S. 15

शdlerfarn 5. 83. T. 80. T. 468.

2lblerb̈̈lzer 5. 15.

Adonis 5. 1. T. 1. T. 4.

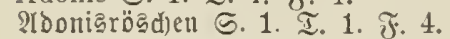

Adoxa ธ. 35. T. 32 . T. 186 a. b.

Aegicer'as 5. 49 .

Aegopodium S. 34 .

Aesculus 5. 12.

Aethionema ธ. 8.

Aethusa 5. 33. T. 30, ๆ. 171 a. b.

2ffienbrotbaum ऽ. 11.

iffodille 5.75 .

2iftermooie 5.84.

Aiterquendel S. 28. T. 26. T. 147 a. b.

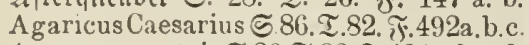

Agar. campestris $5.86 . T .82 .55 .494$ a.b.c.d.

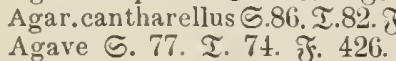

Agrostemma 5. 10.

Agrostis 5.80. I. 77. F. 446 .

2tbltiridac 5. 22. T. 20. J. 116 a. b.

Whort S. 12 T. 11. J. $63 \mathrm{a}, \mathrm{b}$.

2Aforngetwächie 5. 12.

Ailanthus 5.14.

Aira 5. 80 . T. 77. 厅. 447 .

Aizoideae 5. 30.

Aizoon 5. 30 .

Ajuga 5. 57. T. 54. ₹. 315

21 tajubaum 5. 15. T. 13. $₹$. 77.

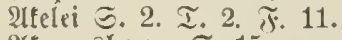

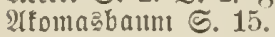

Mlant S. 39. T. 36. T. 206.

Alchemilla $\dot{5} .26$. I. 24. T. 137

Mlgen S. 88.

Alismaceae 5. 72 .

Alkanna ऽ. 54

Rtuannaitrand) S. 28.

24lecvapuel 5. 70.

Allermaunbarnifd 5.74. T.71. 5.407 a.b.

Alliaria 5.8.

Allium 5. 76. T. 73. গ. 419 a. b.
Alnus S. 68. T. 65. J. 378 a. b. Aloë ธ. 77. ․ 74 . テ. 425

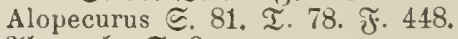

Alpeurebe 5.3.

Ilpentroie ङ. 47.

Arpenioctenblunte S. 3.

2Ipeubeilden 5. 48. T. 45. T. 262.

Alprante 5. 59. T. 56. T. 327 a. b.

Alsine ธ. 10. T. 10. $\widetilde{~} 54$ a. b.

Alsineae 5. 9 .

Althaea S. 11. T. 11. ₹. 59

Alyssum 5. 7. T. 7. গ. 36.

Amarantaceae S. 62.

2โmarantblütige (5)ernädje S. 62.

Amaranthus 5. 62. T. 59. 5.346 a. b.

Anaryllideae 5.74.

Amentaceae 5. 68.

Ampelideae S. 12.

Ampelopsis 5. 12.

2Impfer S. 63. T. 60. T. 352 a. b. c. 2linenpflanze S. 45.

Amygdalus persica S. 23. T. 21. F. 121. Asclepias 厅. 50. T. 47. T. 275.

Amyris 5. 15.

Anacardium S. 15. T. 13. ซ. 77.

Anagallis 5. 49. T. 46. Э. 266.

Anagyris 5.21.

Anastatica 5. 8

Anchusa officinalis S. 53. T.50. $\mathfrak{F} .290$.

Anchusa tinctoria 5.54 .

Indentanne S. 71.

Andersonia 5. 47.

Andorn 5. 56. T. 53. T. 308.

Andromeda S. 47 . T. 44 . গ. 251.

Androsace 5.49 .

Anemone 5. 1. T. 1. T. 3.

An ethum Foeniculum S.33. T. 30. T. 172.

Anethum graveolens 5. 34. T. 31. ₹. 175.

Angelica 5. 34. T. 31. J. 174.

2nguiturabaum ธี. 14.

Annassera 5. 50 .

Anona 5. 3.

Anonaceae S. 3.

Antennaria $\mathbb{S}$ 40. T. 37. F. 214.

Anthemis 5. 40. T. 37. .210.

Anthoxanthum S. 81. T. 78. F. 449.

Anthriscus 5. 34 .

Anthyllis S. 18, I. 16. F. 95.

Antiaris 5. 68. T. 65. $\widetilde{T} .376$.

Antirrhinum 5. 61. T. 58. T. 336.

IIntidjar ธ. 68. T. 65. T. 376.

2lpfelbatm 5 . 22. T. 20. F. 118 a. b.

Aphodeleae 5.75 .

Apium 5. 32. T. 29. F. 166 a. b.

Apocyneae $\mathfrak{5} .50$

Apocynum 5. 50. T. 47. T. 272.

Iprifole 5. 23. T. 21. ‥ 122 a. b.

Yrprifojenbaum 5.21.

Aquifoliaceae 5. 48.

Aquilaria 5. 15

Aquilarineae $\Im .15$.

Aquilegia 5. 2. T. 2. T., 11.

Arabis 5. 5. T. 5. Э. 27.

Aralia S. 55.

Araliaceae 5. 35.

Araucaria 5. 71.

Archangelica 5. 34. T. 31. T. 174.

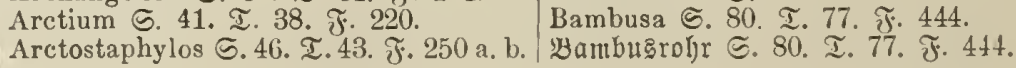

Areca 5. 78. T. 75 . F. 429 a. h,

Aretapalme S. 78. T. 75 . T. 429 a. 1).

Arenaria 5. 10. T. 10. T. $5 \pm$ a. b.

Aristolochia ธ. 65. T. 62. J. 361 a. 1).

A ristolochieae 5.65.

2rmbanbbaum S. 49.

Armeniaca 5. 23. T. 21. T. 122 a. 1).

Armeria G. 62.

Arnica 5. 41. T. 38. ๆ. 216.

Aroideae (5. 7\%.

2roแลgetwädjic $\Im .72$.

Arongitab 5. 72. T. 69. T. 399 a. 1).

Artemisia 5. 40. T. 37. $\mathfrak{~} .213$.

Artocarpene $\mathbb{5}$. 16.

Arum 5. 72. T. 69. ₹. 399 a. 1.

भTatlo S. 34 .

Asarum 5. 65.

Asparagus ธ. 75 . T. 72. F. 414 : b.

Aspergillus S. 85. T. 81. F. 490.

Asperugo S. 54 .

Asperula 5. 37. T. 34. T. 194.

Aspidium 5 83. T. 80. গ. 469.

Asplenium Ruta muraria 5.82 . T. 79 T. 463 .

Asplenium Trichomanes S. 82. T. 79. ઍ. 462.

Aster 5. 39. T. 36. F. 202.

2ijtfledtell S. 85.

Astragalus 5. 19. T. 17. T. 100 a. b.

Astrantia S. 32. T. 29. F. 164 a. b.

Astroloma 5. 47.

Atragene 5. 3.

Atriplex ङ. 63. T. 60. ๆ. 351 a. b. c. Atropa 5. 60. T. 57. T. 330 a. b.

2(ttid) 5. 36 . T. 33. F. 188 a. 1). c.

21 ngentroit 5. 62. T. 59. ซ. 341 .

Aurantiaceae 5.11.

9urifel S. 48. T. 45. ઍ. 260.

2lufterbautu 5. 26.

Avena 5. 79. T. 76. F. 437.

Azalea 5.47.

B.

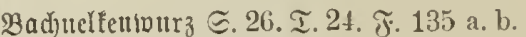

Bärettllaı 5.34 แแd 58.

Bärentlangetwädje 5.58.

Bärentake 5. 80. T. S2. T. 491 a. b. c.

Därentraube S. 46. T. $43 . \pi_{0} \cdot 250$ a. b.

Bärlapp 5. 82. T. 79. 5.461.

Bärlappgetwädjie 5.82.

Balbriaแ S. 37. T. 34. ₹. 195 a. b.

Baldriaugetuädje 5.37.

Ballota 5.57 .

Balfamapfel 5. 29.

Balfambattm S. 11, 15 แnd 21.

Raliantuengewädic 5.13.

Baliamgetuädjie S. 15.

Baljamine, wilbe, 5.13. I. 12. ร. 69.

Balsamineae 5.13

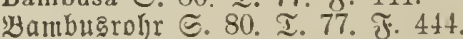

A rdisia 5.49

अärtwurz 5. 34.

Bambusa 5. 80. T. 77. T. 444 
Banane 5. 73.

Bandreide 5 69. I. 66. J. 385.

Banksia 5. 64.

Barharaea 5. 5. T. 5. T. 25 a. b.

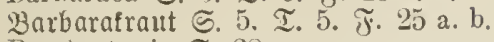

Barringtonia ๔. 28.

Bartfledte 5. 85. I. 81. $\pi .486$.

Bartmoos $\subseteq .84$. $.81 . \sqrt{3} .473$ a. b. c. Bartmooje $\subseteq$. 84 .

Bartıuß $\subseteq .69$.

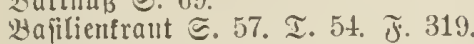

Bassia ङ. 49.

Batatas 5.52.

Batate 5.52

Bataten=2)ams 5.74 .

Wautulemf 5. 8.

Batumbolle $\approx .11$.

Baumbitrger 5. 14.

Hederflidte S. 85. T. 81. T. 482 .

Bed)ermoog ธ. 85. T. 81. $\pi .452$.

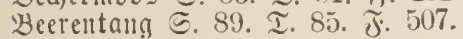

Begunia 5.65.

Begoniaceae 5.65

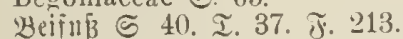

Beimvelf 5. 53. T. 50. ส. 291.

Heiß̄bere S. 60 .

Bellabonta $\Xi .60$. I. 57. T. 330 a. b. Bellis 5. 39. I. 36. శ. 207 .

Bentediftenfrant 5. 26. T. 24. T. 134. Bencdittenfrant S. 42. T. 39. Benzocbaum S. 49.

Benzoil 5 . 49 .

Berberideae $\Subset .3$.

Berberis 5. 3. T. 3. T 16 a. b.

Berberibe 5. 3. ป. 3 T. 16 a. b.

Bergitad)

Bergnimze S. 55. I. 52. T. 302.

Bergpiefier 5. 64. T. 61. T. 357 a. b.

Bergiandölöd)en 5.45. T. 42. T. 241 a.1.

Eertraugarbe ङ. 40. T. 37. Ј. 211.

Bernfogfrant 5. 39. T. 36. T. $203 \mathrm{a.b}$

Befenitrand) S. 17. I. 15. J. 87.

Beta S. (33. I. 60. ส. $3 \pm 8$ a. h. c.

Betelpalme 5. 78. I. 75. T. 429 a. 1).

Betula ธ. 68. T. 65 . ₹. 379 a. b.

Betulincae 5. 68.

Bibertlee S. 51. I. 48. T. 279.

Bibernell ธ. 33. T. 30. テ. 168.

Bitbere ธ. $46 . \tau$ I. $43 . \widetilde{J} .246$ a. b.

Bidens 5. 41. T. 38. テ. 217.

Bienenjang, geffecter ङ.56. T.53. ₹. 312.

Dientetian, roter 5.56. T. 53. 5.313 a.b.

Bignonia imperialis 5.62 .

Bignoniaceae S. 51.

Bilientrant ธ. 59. T. 56 T. 326.

Bingelfrant S. 65. I. $62 . \widetilde{J} \cdot 36 \pm$ a. b. Biniert 5. 77.

Wirte ङ. 68 . T. 65 . T. 379 a. b.

Birfeugetoüdjie $\Subset$. 68 .

\irubaum 5. 22. T. 20. ฐ. 117 a. b.

Bifambiftel E. 42. T. 39 T. 224.

Bifambryacinthe S. 77. T. 74 . T. 424.

Bijautraut $\Subset .35$. T. 32 . ฐ. $186^{\circ}$ a.b.

Biscutella 5. 8 .

Bittereidie 5. 14.

Bitterljolzbaum ธ. 14.

Bitterllee S. 51. T. 48. F. 279

Bitterfrant S. 43. I. 40 . T. 231.

Bitterfrant $\Subset .51$.

Bitterlinge $\cong .9$.

Bitteritif 5. 59. T. 56. T. 327 a. b.

Bittertuurzel ऽ. 10 utlo 29.

Bixa orellana 5 . 8

Bixineae ธ. 8.

Blätterpilz $\subseteq$ 86. T. 82. F. $49+$ a. b.c. d. Blaienfarn S. 83. T. 80. J. 465

Slajentirfdye ธ. 59. T. 56. ป. 329 a. b. Blajemutis 5. 12 . T. 11. ‡. 62 a. b.
| Blajenitrand) 5. 18. T. 16. T. 98 a. b. WHajentang 5. 89. T. 85. T. 508. 2lanbeere S. 46. T. 43. Э. 246 a. b. Blattblatt 5. 28.

Blechnum S 83. I. 80. F. 464.

Bleimurz ธ. $6 \%$.

Wleimurzgemäd) 5 . 62.

Blitum 5. 63. T. 60. శ. 350 a. b.

Blüteneide ङ. 49 .

Blumenblattloie S. 62.

BImmenrolyre 5. 73.

BHtutange S. 1. T. 1. F. 4.

BIntbud)e 5. 69.

idnthol3baum S. 16. T. 14. T. 81.

BIntfrant 5. 61 I. 61 . $\pi .355$ a. b.

Bluttrant S. 29. I. 26. Э. 146.

BIntuarcifie 5 74.

Bottabart 5. 43. T. 40. T. 229.

BodEhorntle ธ. 18. T. 16. T. 94

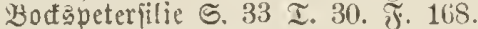

Boerhavia 5. 62.

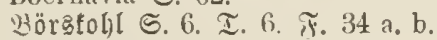

Bohne 5. 20. T. 18. T. 109 a. b.

Bohnenbant S. 17. T. 15. T. 89.

Bohnentrant 5. 57. T. 54. T. 318 a. b.

Boletus 5. 87. I. 83 . J. 495 a. b. c. d. Bombaceae 5. 11.

Bombax 5. 11.

Bontia S. 58.

Borage S. 54 T. 51. গ. 295.

Boragineae 5. 52 .

Borassus 5. 78.

Boretid) S. 54. T. 51. ฐ. 295.

Boretidggervädfe 5.52.

Brabanter Miturte 5.70.

Bradjenträuter $\subseteq .82$.

Brassica oler. cap. S. 6. T. 6. T. 33 a. b.

Brassica oler. sab. 5. 6. I. 6. テ. 34 a. b.

Braumpurz S. 60. T. 57. F. 332.

2rautwurzgetvädje 5. 60.

Braya 5. 8

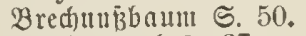

Bredivurzel 5. 37.

Dreiapielbaum S. 49.

Brentidolde 5. 31.

Brellutrnut S. 1. T. 1. F. 1 a. b.

Brettuneffel S. 66 I. 63. T. 371.

Bretuntuinden S. 29.

Brettbanm S. 11.

Briflenfidote 5. 8.

Briza 5. 81. T. 78. T. 4it.

Brombecre 5. 26. T. 24 . T. 133 a. 1).

Bromus ธ. 81. ․ 78. ₹. 450.

Brotbaum S. 66.

Brotfruditgetuädje $\subseteq$ S. 66.

Brotidjimuel S. 85. T. 81. ซ. 490 .

Brotwurzel S. 74.

Brudtrant 5. 29. T. 27. F. 152 a. b.

Brmelle 5.55 . T. 52 . T. 305.

Bruniaceae 5.14

Brumenteffie S. 5. T. 5. T. 26 a. b.

Brumenleberfraut S. 84. T. 81. T. 478.

Bryaceae 5.84.

Bryonia S. 29. T. 27. T. 150.

Bruitbeerbaum 5. 5t.

Bruttwitrz 5.34 .

Butbe ङ. 69. T. 66. T. 382 a. b. c.

Budftlee 5. 13. I. 12. T. 70.

Bud) baum 5. 66. T. 63. T. 365 .

Bïd) fenfrant 5. 61. I. 58. T. 340.

Biifd)elfarne 5. 8:.

Büttneria 5. 11.

Büttneriaceae 5. 11.

ßulleriahn 5. 37. I. 34 ₹. 195 a. b. Bunge 5. 49 .

Bunias S. 8 .

Buphthalmum 5. 39. T. 36. T. 205.

Bupleurum S. 33. ․ 30. ซ. 169 a. b.
Burzeldori S. 13.

Butrzelfrant 5. 29. T. 27. T. 151.

Butomeae 5. 77.

Butomus 5. 72. T. 69. J. 395 a. b.

Butterbaum 5. 49 .

Butterblume 5. 2. T. 2. T. 7 a. b.

Butterntibanm 5. 12.

Buxus ธ. 66. T. 63. ซ๋. 365.

\section{c.}

Cacao S. 11

Cachrys S. 34

Cacteae ङ. 30

Cactus S. 30.

Caesalpinia 5. 16. I. 14. F. 80.

(Caesalpinieae 5. 15.

(5ajaputbaum 5. 28 .

Cakile S. 8.

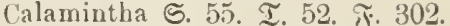

Calceolaria 5 . 62 .

Calendula ธ. 41 . T. 38. テ. 219 a. b.

Calepina 5. 8.

Callaceae 5. 72 .

Callitriche 5. 29.

Callitriche S. 66. T. 63. T. 370.

Callitrichineae $\mathbb{S}$. $(6 t$.

Calluna S. 47. I. 44 ส. 252.

Caltha S. 2. T. 2. J. 7 a. b.

Calycantheae 5. 26.

Calycanthus Horidus S. 26.

Calycereae 5. 38.

Calyciflorae S. 14.

Calystegia 5. 52. I. 49. ร. $2 \S 3$.

Camelina 5.8.

Camellia 5.11

Camelliaceae S. 11.

Campanula glumerata S. 45. T. $42 . \Im^{2} .244$

Campanula persicifolia 5. 45 . T. 42. F. $2 \pm 3$ a. b.

Campanula rapunculus 5.45 . T. 42. ङ. $245 \mathrm{a}, \mathrm{b}$.

Campanulaceae 5. 45 .

Canna S. 73 .

Cannabaceae 5. 66.

Cannabis 5. 67. I. 64. T. 373 a. b.

Cannacea $\Theta$ ङ. 73.

Capparideae 5.8.

Capparis 5. 8.

Caprifoliaceae 5. 35.

Capsella 5 . 8.

Capsicum S. 60 .

Carapa 5. 12.

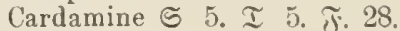

Cardiospermuin 5. 12.

Carduus 5. 42 . T. 39. テ. 224.

Carex S. 79.

Carlina Є. 42. T. 39. テ. 226.

Carlodovica 5. 79.

Earotte E. 34. T. 31. T. 176 a. h.

Carpineae 5. 68 .

Carpinus 5. 68. ․ 65. テ. 380 a. b.

Carthamus 5. 41. I. 38. テ. 222.

Carum 5. 33. I. 30. F. 167.

(Earve 5. 33. T. 30. T. 167.

Caryopliylleae S. 9.

Caryophyllus 5. 28.

Casearia 5. 14.

(5affaveitrand) S. 66. T. 63. T. $368 \mathrm{a} . \mathrm{b}$.

Cassia 5. 64.

Cassia 5. 16. T. 14. T. 83.

Caffienjimtbanm 5. 64.

Castanea 5. 69. T. 66. T. 383 a. b.

Casuarineae 5. 68.

Casuarineae ভ. 70

Eatechupalme 5. 78. T. 75. テ. 429 a. b.

Catinga 5 . 28.

Caucalis 5. 34 . 
('eanothus $\Subset .11$. Ecoroubaum ๔. 14. Celastrineae S. 14. Cclastrus $€$. 14.

Celosia $\mathfrak{S}^{-}\left(\mathrm{i}^{2} \mathrm{z}\right.$.

Celtis $\odot .71$.

Centaurea Є. 42. T. 39. T. 223.

Centaurea Cyanus E. 42. I. 39.

Centaurea seabiosa S. 43. I. 40. テै. 225. Centhranthus $\subseteq 37$.

Centuneulus ธ. 49. T. 46. ฐ. 267.

Cephaëlis 6.37 .

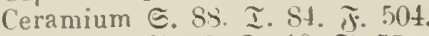

Cerastium ङ. 10. ․ 10. ซ. 55 a. b.

Cerasus Є. 23. I. 21. J. 123 a. b.

Ceratocephalus $\mathcal{G}$. 3 .

Ccratonia ธ. 16 I. 14 . ๙. 82.

Ceratoplyylleae E. 2s:

Ceratophyllum 5. 2S.

Cercus ङ. 30

Cerinthe ङ. 53. ․ 50. ‥ 292

Ceterach E. 53 . I. 80 . तु. 4 iा.

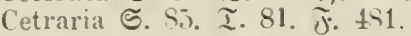

Chaerophyllum G.34. T. 31. ₹. 177 a. b.

Chailletia G. 15 It!D 70.

Chailletiacene E. 15.

Chamaerops $€ .7 \%$.

Chamomilla Є. 40. ․ 37. テ. 209

(5hampignou $\cong .86$. I. 8.2. T. 494 a.

Cheiranthus E. i. I. i. $\pi_{0}$. 32.

Chelidonium ङ. 4. T. 4. ऊ. 21.

Chelune ๔. 6?

Chenopodiaceae $\in .63$.

Chenopodium E. (33. I. 60. ऊ. 347 a. 1,

Cherleria $\widetilde{5}, 10$.

(ibilitamit E. 71

(El)itabaum ธ. 37.

(ihimabanแuctoäd)ic $\Subset .37$

Chlaenaceate S. 11.

Chlora ธ. 51

Chlorantheae S. (is

Chloranthus ङ. 69 .

Choiromyces Є. Ss. T. 84. T. 502.

Chondrilla ङ. 44. T. 41. J. $^{2} 236$ a. b.

Chondrus Є. 89. ․ 85. ซ. 509.

Chorisia $\subseteq .11$.

(5hritblume $\odot$ 2. I. 2. స. 9 a. b.

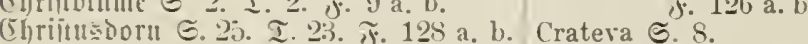

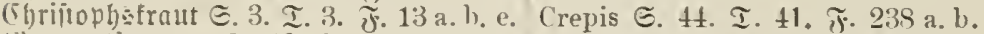

Chrysanthemum હ. 39. I. 36. T. 208. Crocus ङ. 74. T. 71. T. 408.

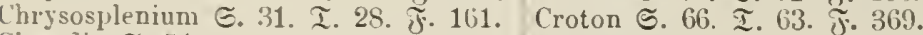

Cieendia 5 51.

(Eidorie E. 45 . T. 42. ₹. 240 a. b.

Cichorium G. 45. I. 42 . $\widetilde{J} .240$ a. b.

Cicuta S. 34

Cimicifuga $\Subset 3$

Cincliona $\Subset .37$.

Cinclionaceae $\Subset .37$.

Cineraria 5.45 .

Cinnamomum $\odot .64$.

Circaea Є. 27. T. 25. ร. 141.

Cirsium ङ. 41. T. 38. T. 221.

Cissampelus e. 3 .

Cissus 厄. 12 .

Cistineae $ఠ .8$.

(Ciirnsat)en 5.8

Cistus $\Xi$. 8 .

(Eitronte S. 11

Citrus $\Subset .11$.

Cladium $\Subset .79$

Cladonia E. 85. I. 81. T. 442.

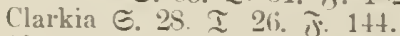

Clavaria હ. 86. I. 8.. J. $4 ! 11$ a. b. c.

Claytonia E. 29.

Clematis E. 1. T. 1. $\pi .1$ a. b.

Cleome E. 8 .

Cnicus ङ. 42. I. 39. ₹. 223.

Cnidium $\Subset .34$.
Cobaca 5. 51.

(5ocajtraud) E. 12.

cocoloba \& 64

Coceulus ธ. 3 .

Cochlospermum E. 11

$\operatorname{Cocos}$ G. 78. ․ 75. శ็. 431 a. b.

Colchicum $\Subset$. 76. I. 73. $\pi$. 421 a. b.

Colutca S. 18. I. 16. J. 98 a. b.

Combretaceae હ. 26 .

Combretum @. 26 .

Commelina $\mathbb{E}$ it.

Commelynaceate $\Subset .77$.

Compositae S. 38 .

Conferva S. 88. I. 84. テ. 506

Coniferae $\Subset$. 70 .

Conium 5. 34. T. 31. . 178.

Convallaria 5 75. I. $72.5 \cdot 415$ a.b.c.

Convolvulaceae E. 51 .

Convolvulus arvensis ๔. 51. I. 48. ङ. 252 a. b.

Corchorus S. 11

Cordia S. 54 .

Coriandrum $\subseteq$ 34. I. 31. J. 179

Coriaria S. 14

Coriarieae ङ. 14.

Corispermum ङ. 63.

Cornaceae ๔. 35 .

Cornus mas ङ. 35. T. 32 ๆ. 182 a. 11.

Cornus sanguinea S.35. I.32. ₹. 183 a.h.

Corolliflorae ङ. 35.

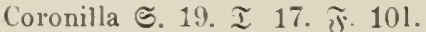

Corrigiola ङ. 29. T. 27. స. 153 a. 1.

Corydalis $\subseteq$ 5. T. 5. T. 24 a. b.

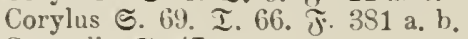

Cosmelia G. 47.

Cotyledon Є. 30 .

Crambe . 8. T. 8. ๆ. 42.

Crassula $\Subset .30$

Crassulaceae $\mathcal{G} 29$.

Crataegus Aria ङ. 22. T. 20. T. 119 a. 1,

Crataegus oxyacantha 5. 24. T. 22. 5. $126 \mathrm{a} . \mathrm{b}$.

Cruciferae 5. 5 .

Cubeba E. 68.

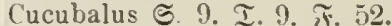

Cueubalus ङ. 10 .

Cucumis Є.25. T. 26. F. 149 a. b.

Cueurbita $\Subset .29$.

Cucurbitaceae Є. '28.

Cupania S. 12

Cuphaea E. 28

Cupressinae $\Subset .70$.

Cupressus $\Subset .71$.

Cupuliferae ธ. 68.

(5urarapilanze ङ. 50 .

Cuscuta Є. 52. T. 49. ๙ॅ. 284.

(5)anc ङ. 42 . T. 39. ซ) 227.

Cyanophyllum Є. 28.

Cycadeae 5.70 .

Cycas 5. 71 .

Cyclamen S. 48. T. 45 T. 202.

Cydonia 5 24. T. 22. T. 124 a. 1,

Cynanchum Є. 50. T. 47. T. 275 .

Cynoglossum ๔. 53. ․ 50. T. 294.

Cynosurus 5 s1. T. 78. テ. 451.

Cyperaceae $€ .79$.

(5)pergras ๔ 79. T. 76. テ. 434 .

(5npergräicr $\odot .79$

Cyperus ธ. 79. T. 76. テ. 434. (5nprciie $\in .71$

(5npreifc, bcutid)e, S. 29. T. 26. ₹.148 a. b. Enprefiell 5. 70.

Cypripedium E. 73. ․ 70. テ. 405.

Cyrtandraceae G. 51 .

Cystopteris S. 83. T. 80. $\widetilde{J} .465$.

Cytineae 5.65 .

Cytinus ๔. 65 .

Cytisus ธ. 17. T. 15. テ. 89.

2.

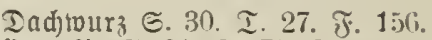

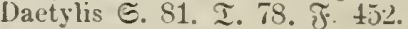

Dahlic S 45.

Dammara Є. 71 .

Dammaraharzbaum ๔. 11.

Daphne ङ. 64. I. 61. శ. 357 a. 1).

Dattclualne E. 78 . T. $75 . \widetilde{T} .433$ a. b.

Dattelpilanme $\in$. 4 ?.

Datura Є. 58. 衣. 55. T. 323 a. 1.

Daucus E. 34. T. 31. T. 176 a. b.

Iauertlec S. 17. I. 15. J. 90.

Delima ङ. 3

Delphinium Є. 2. T 2. ส. 12.

Dentaria Є. 5. T. 5. ส. 29.

Deutzia 5. 25 .

Dianthus plum.. . 9. T. 9. T. 48

Dianthus sup. Є. 9. I. 9. T. 49.

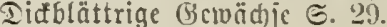

Diclytra S. 5.

Iicotulebonen E. 1.

Dieranaceae $\in$. $8 t$.

Dicranum S. 84. T. 81. ส․ 476 a. 1.

Dictamnus ๔. 14. T. 13. T. 7.

Digitalis purpurea $\subseteq 60 . \tau .57 . \pi \cdot 334$ a. b.

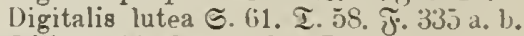

Iill ธ. 34. T. 31. T. 175 .

Dillenia ङ. 3.

Dilleniaceae $\Subset .3$.

Dioseorea E. 74 .

Dioscoreae Є. 74 .

Diospyros Є. 49 .

Dipsaceae S. 38.

Dipsacus silvestris ๔. 3S. エ. 35. テ. 197.

Dipsacus Fullonum E. 38.

Diptam S. 14. I. 13. テ. 72.

Dipterix ङ. 15 .

Dipterocarpeae Є. 11.

Dipterocarpus $\mathcal{E} 11$.

Diitel S. 42. T. 39. T. 224.

Doldenvflanjen G. 32 .

Ioppelblatt $\Subset .13$.

Ioppeliporm $\epsilon .5$.

Dornut ङ. 40. I. 37. ₹. 211.

Doiten 厄. 54. T. 51. T. 301.

Dotterblunte ङ. 2. T. 2. テ. 7 a.b.

Doura E. 15.

Draba ङ.7. T. 7. స. 38

Dracaena $\mathcal{G} .77$.

Irndenbaum ธ. 77 .

Dreifaltigfcitsoliimd)en E. 49 . I. 46. テ. 265 a. b.

Drimys 6.3 .

Drosera ङ. 8. I. \&. $\mathfrak{T} .46$.

Droseraceae 5 S.

Irottelblume ভ. 49. I. 45. T. 261.

Türlize ङ. 35. I. 32 . 5.1 . 2 a. b.

Iiirrtur 5. 39. T. 36 . .203 a. b.

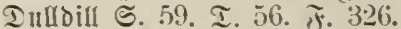

Dullfraut $\Subset .59$. T. 56. ร. 326 .

Iurdivad? ङ. 33. I. 30. T. 169 a. b.

IHWod ธ. 8:. T. 79. ₹. 459 .

$$
\text { (5. }
$$

Ebenaceae $\Subset .49$.

Ebenfölzer S. 49. 
(5berefde E. 24. T. 22 . 5125 a. b.

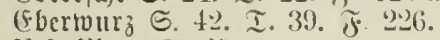

Ecballium 5 . 29 .

Echinocactus S. 30.

Echinops 5. 45 .

Echium ธ. 52. T. 49. ส. 28:

(5oltaptanie 5. 69. T. 66. T. 393 a. b. (5deltautue ङ. 70. T. 67. T. 359 a. b. c. (EDeltucifs S. 40.

(5)rentreis 5. 61. T. 58. T5. 338. (sibell $ఠ .70$.

(5ibcubaum 5. 71. T. 69. T. 393. (sibifd) 5 11. T. 11.

(Fid)e S. 69. T. (6ti. $\pi$. 3St a. 1.

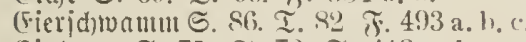
(silubere ङ. 75. T. 72. T. 413 a. b.

(Simfameublättrige 粘anjcu 5.71. (Eijetholz S. 12 แIID 15.

(5ijenl)oljbaum E

(Fipcuhut ङ. 3. ․ 3. ส. 14 a h. Eilentrant E. 58. T. 5. T. 321 a. b. (5ijentrantgetväd)ie ธ. 58.

Elaeagneac $\mathcal{G}$. (5).

Filaeagnus $\subseteq$. $(05)$

Elaeis 5. $7 \times$. ․ 75. \%. 432.

Elaeocarpus $\subseteq 11$.

Elaeodendrom ङ. 14

Elatine $ఠ$. 10.

(Elcuibaum ङ. 15.

Elephantusia $\subseteq$. 79 .

(sifenbeimpalute $\odot .7 !)$.

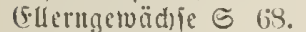

Elodea 5. 71.

Empetracear $\Subset$ ऽ. 31

Empetrum S. 31.

(5nDogenell $ఠ .71$.

(E)gelblut ธ. 2. T. 2. ซ. 8.

Eugeltraut $\subseteq$ 41. T. 38. T. 216.

Engelivurz S. 34. T. 31. T. 174.

(Enzial E. 51. T. 45. T. 277 a. b.

(5uziangetwäd) je 5.50.

Epacrideae 5.47.

Epacris S. 47.

Epidendrou 厄. 73.

Epilobium 5. 27. T. 25. T. 139 a. h.

Epimedium 5. 3. T. 3. T. 17.

(5pl)el 5. 35. ․ 32 厅. 181 a. b. c.

(Evbengetuäd) fe S. 35 .

(5ppid) E. 32. T. 29. T. $16(6$ a. b.

(Eppid) S. 36. T. 33. T. 188 a. b. c.

Equisetacene S. 82.

Equisetum ธ. 82. ․ 79. ๙ே. 45\%.

Eranthis $\subseteq .3$.

(5rbie ธ. 20. T. 18. テ. 108 a. b. c.

(5roapial S. $(60$.

(Erbbecre 5. 2.). T. 23. T. 131

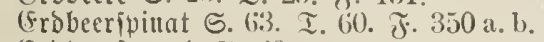
(Erbberitraud) 5. 28.

(Erbephell S 57. T. 54. శ. 317.

(Erbtreffe 5. 5. T. 5. T. 25 a.b.

(Erbraud) S. 4. T. 4. T). 23 a.b.

(Erbraudgemäd)je G. 4.

(5rbid)eibe 5. 48. T. 45. T. 262.

Erica 5. 17. I. 44. T. 2.).

Ericeae S. 47.

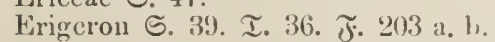

liriophorum 5. 7!.

(Frle ङ. 68. I. 65. T. 378 a. 1).

Hrodiun E. 13. I. 12. T. 67.

Erophila ธ. 7. T. 7. ז. $3 \%$.

Ervum G. 20. T. 18. స. 107.

Eryugium 5.32 . T. 2\%. ₹. 1\%\%.

Erysimum barbar. 5 5. T.5. T. 25 a. 1. Erysimum cheiranth. ऽ. ว. I.5. 30 a.b. Erythraea G. 50. T. 47. ₹. 276.

Erythrina $\odot .21$.

Erytroxyleae S. 1?.

Erytroxylon 5. 12. (5.fd) S. 49. T. 46. T. 268 a. b. c. Eschscholtzia E. 4

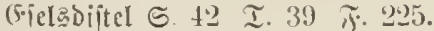

(5jelgुgurfe S. 2!).

(Ficlamild) S. 65. T. 62. T. 363.

(Fiparjette S. 19. I. 17. T. 103.

lucalyptus 5.28.

Eupatorium 5. 38. T. 35. T. 199

Luphorbia cyparissias S. 65. T. 62. ร. 362.

Euphorbia esula 5. 65. T. (j2. F. 363.

Euphorbiaceae $\widetilde{5} .65$

Euphrasia 5. 62. T. 59. T. 341.

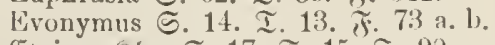

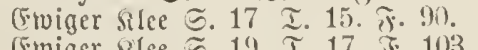

Frviger silce 5. 19. I. 17. J. 103.

(5xogentil 5.1.

\section{ঔ.}

Tradelbijtel ๔. 30 .

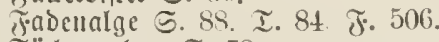

F⿱äd)erpalute s. 78 .

Tärberbiftel ङ. 41 T. 39 . T. 2.2?

Tärberfledte S. 8.). T. 81 J. 483 a. 1.

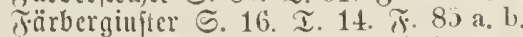
Tärberröte 5. 37. T. 34. ₹. 192.

Färberwaid 5. 7. I. 7. T. 41).

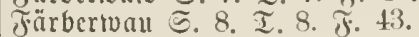

Fagus 5. 69. I. 66. . .382 a. b. c.

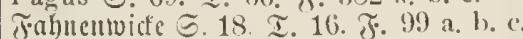

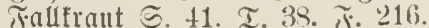

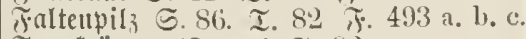
Farutrïutir (ふarue) 6.82.

Taferfudut S. $6 t$.

Jonlbaum 5. 14. T. 13. T. 74 a.b.

Taulbaum 5. 22. T. 20. 2.116 a. 1)

Jeberbarzbaum 5 66. T. 63. 5. 367.

Jebermelfe ธ. 9. T. 9. ส. 48.

J゙eigbolute S. 21.

Treigenbaum 5.66.

Jeigrumz 5.3

Feldobiftel 5. 41. T. 39. $\pi .221$.

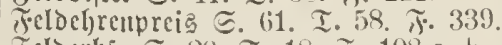

Jelderbie 5. 20. I. 15. T. 108 a. b.

Jelotraboiftel ๔. 41. I. 38. T. 221.

Treldtrefie 5.8.

Treldtïumel 5. 33. T. 30. T. 167.

Fildublu S. 4. T. 4. T. 20.

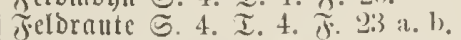

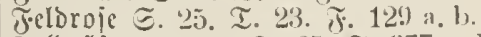

Telbriilter 5.68. I. $65 . \pi .377$ a. 1.

Telbjalat ฮ. 37. T. 34. F. 196.

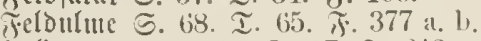

Jeldverumt 5. 40. I. 37. ₹. 213.

Fitlicuitrand) $\Xi .47$.

Teud)el 5. 33. ‥ 30. ส. 172.

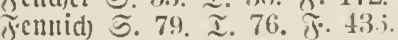

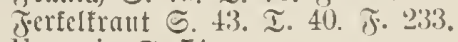

Ferraria 5. 74.

Ferula 5. 34 .

Tettfraut 5. 47. I. 44. ร: 256.

Trettpflauzen 5.29 .

Țeuerbolue ङ. 20. T. 18. F. 109 a. 1.

licaria 5. 3.

Fid)te 5.71 . ․ 68. T. $390 \mathrm{a}$ b.

Jid)tenipargel ธ. 47. T. 44 . ऊ. 255.

Ficoideae 5. 30.

Ficus 5. 66.

Tieberllce 5. 51. I. 4., ๙. 279.

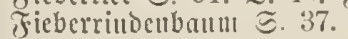

Filices 5. 82.

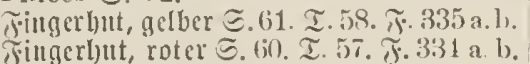
Titigerfrant 5. 25. T. 23. F. 130.

Fissidens 5.81 T. 81. ‡. 475.

Fissidentaceae 5.84.

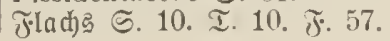

Flad)sfeide 5. 52. T. 49. T. 284. Flacourtiaceae 5 . 8.

Filauttublume 5. 51. T. 48. T. 281.

Tilattergras 5. 82. T. 79. స. 457.

Tlatterriifter 568 .

Fled)ten 5. 84 .

Tllicber ङ. 49. ․ 46. T. 270 a. b. Tiliegeufalle 5. 50. T. 47. T. 272.

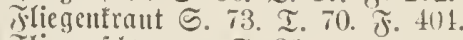
Trliegenidituantu 5. 86 .

Fiscofenblume 5. 43. T. 40. T. 22:

Tislolfrant 5. 39. T. 36. T. 206.

Tilofraut S. 64. 疋. 61. T. $3 \pi 6$.

Tilubblitmli S. 48. I. 4\%. $\pi .960$.

Tïbre 5. 70. T. 67. T. 389 a. b.

Foeniculum 5.33. T. 30. T. 172

Tord)e 5. 70. ․ 67. F. 3>8 a. h.

Fragaria S. 25. I. 23. T. 131.

Fragraea 5. 50.

Frankeniacese S. ?)

Trancubittel S. 42 T. 39. T. 225.

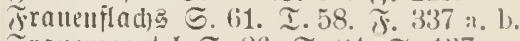

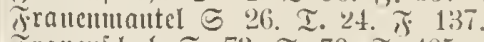

Tranenidul $5.73 . \tau .70 . \pi 45$.

Fraulutbrälte 5. 73. T. 70. テ. 404.

rraximus $5.4 !$. T. 46 . . 268 a. b. c.

Friedlog 5. 48. T. 45. శ. 263 a. b.

Fritillaria 5. 76. T. 73. $\pi .422$ a. b.

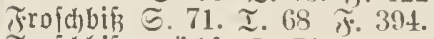

Trroid)bißzgemädje S. 71 .

Trojd)löffelgerväd) ie $\Xi$. 72.

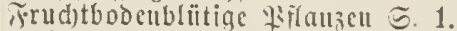

Frtilllord)el 5. 87. I. 3:3. ₹. 497 :. b. c. d.

Hucaceae 5. 8!.

Fuchsia 5. 27. T. 25. T. 143.

T.ॅd)

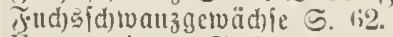

Fucus crispus ङ. 89. T. 85. T. 509.

Fucus natans 5.89 . T. 85. T. 507.

Fucus vesiculosus 5.89. T. 85. $\pi .503$.

Fumaria 5. 4. T. 4. T. 23 a. b.

Fumariaceae S. 4.

Fungi 5.86.

Fusanus 5.65.

Tuttertlee 5. 17. T. 15. T. 92.

Jutterivide ธ. 19. I. 17. J. 105 a b.c.

\section{(6).}

(Sabelzal)แ S. 84. T. 81. T. 476 a. b. biabelzahutuofe S. 84.

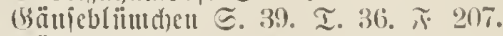

Gälicoiftel ङ. 44. T. 41. శ. 2:37.

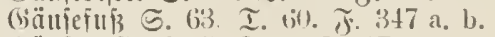
(säufetrefie 5. 5. T. 5. গ. 27.

(biagel 5. 70.

(Sagelbere 5. 68.

Galant hus S. T1. T. 71 T. 410 a. b,

Galega 5. 18. T. 16. J. 97.

Galeobdolon S. 56. T. 53. T. 311.

Galeopsis 5. 56. T. 53. ซ. 311.

Galipea 5. 14.

(ialium $\subseteq .37$. 34. T. 193.

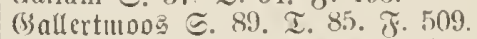

Gamanber ธ. 57. T. 54. J. 314 a. b.

(baniterbaum 5. 11.

(Sarbe 5. 33. T. 30. T. 167.

Garcinia 5. 11.

Cardenia ङ. 37.

Gartenbalfamine 5.13.

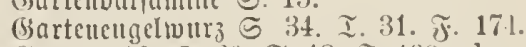

(Sartemerbfe 5.20 . T. 18. T. $108 \mathrm{a}, \mathrm{b} . r$

(Sartengeißblatt 5. 36. T. 33. F. 191.

Gartengleifie S. 33. T. 30. ₹. 171 a. 1.

(Garteubnaciutbe $\Subset .77$.

(Bartenlattid) 5. 44.

(Sarteunolin S. $t$.

Gartenpiefferfraut S.57. I.54. ‡.318a.h. 


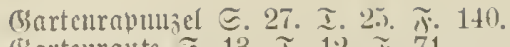
(Bortenraute . 13. ․ 1‥ T. 71. (Sartmialbei ङ. 5t. I. 51. స. 297. (bartentulpe

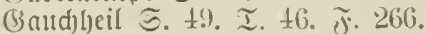

Gauflerblunte 5.62 .

(Siceldent 5. S6. T. S2. ส. 493 a. b. c。 (beräßpplanen $\Xi .1$.

(i)eiBblatt ङ. 36. 工. 33. ส. 190 a. b. (jeibblattgemüd)

Geisitib ङ. :31.

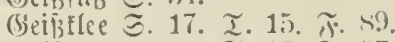

(G)

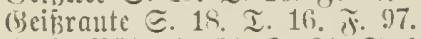

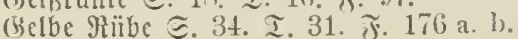
(Gelblolzbanm $\cong .14$.

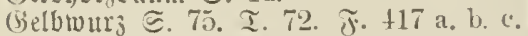

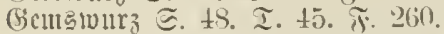

Genista pilosa $\Xi .16$. T. 11 . T. 84 a. b. Genista tinctoria 5.16. I. 14. J. 95 a. b. Gentiana acaulis $\Xi .51 . \tau .45 . \pi .278$. Gentiana centaur. $\approx .50$. I. 47. $\pi .276$.

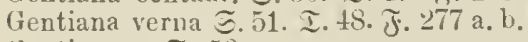
Gentianeae $§ .50$.

Georgina $\Im .45$.

Geraniaceae $\subseteq .13$

Geranium ङ. 13. ‥ 12. Tே. 66.

(Gerberftraud) 5.14

(beruter 5. 76. ․ 73. T. 420 a. h.

Berjte ๔. 79. T. 76 . $\pi .440$.

Gesnerieae $\Xi$. 46.

Geum rivale 5. 26 . T. 24. . 135 a. h.

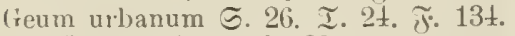

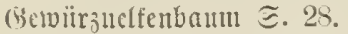

(Sicibiirsitrand) ङ. 26.

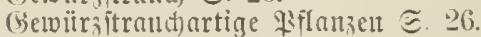

(Bid)troic ङ. 3. T. 3. T. 15.

(Biftlattic) હ. 4t. I. 41. 5. 234.

Bifijalat $\approx .4 t$. I. 41 . $.23 t$.

(Sifitumad) ङ. 15. T. 13. T. 75.

(siftivide . 19. T. 17. T. 101

(bilbtueiderid) 5.48 . I. 45. J. 26

Ginter ङ. 16. T. 14. T. $8 \pm$ a. b.

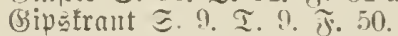

Gladiolus . 74. T. T1. 5.407 a. b.

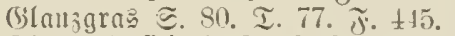

Glarzpeterilie $3.3: 3$. T. 30 . T. 171 a. b. Glasfratt $\approx .67$. T. 6t. $\pi 372$.

Glaueium હ. Ł. T. 4. ऊ 22.

Glaux $5 .+9$

Glechoma 5. 57. T. 54. T. 317.

Bleibe 5. 33. T. 30. T) 171 a. b.

Globularia 5. 58. T. 55. T. 322 a. b.

Globularineae $\widetilde{S} .58$.

(Sloctenblume, falige $\Xi .2$. I. 2. T. 11 .

Glocfenblunte, neluänlte $5.45 .9 .4 \%$

$$
\text { ग. } 2 \pm 1 .
$$

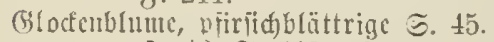
T. $+2 . \pi .2 \pm 3$ a. b.

Gloctenblume (xamminel=) 5.45. T. 12. F. 245 a. b.

(5lodenblumengeiväd)je 5.45.

Gloxinia $\mathbb{E}$. 46 .

Glyceria 5. 81. ․ 78. ‥ 453 .

Glycyrrhiza ङ. 18. ․ 16. స. 96.

Gnaphalium હ. 40. 离. 37. T. 211

(Götterbaum $\widetilde{S} 14$.

Úplbäpfel $\cong .11$.

(Bolbeidie $\Xi$. 4 ?

(Holdlad ङ. 6. T. 6. T. 32.

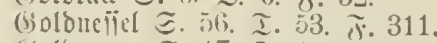

(5olbreget $\approx .17$. I. 15. $\pi$. 89.

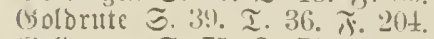

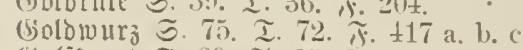

Gioliftraut ร. 89. I. 85. . 507.

Gomphia $\Xi$. 14.

Gomphrena $\Xi .62$.

Goodenovieae $₫ . t 6$.
Trussypium E. 11.

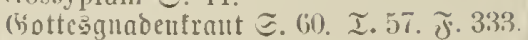
Gräjer S. 79.

Hramineae $\Xi$. 79 .

Branatbam $\approx .26$.

Granateae $\approx$. $21 i$.

Graphideac $\approx$. Si).

Graphis ङ. 8.. I. 81. \%. 188.

(5inamelte S. 6i2.

Gratiola 3.60. I. 57. ส. 3333.

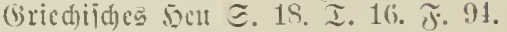

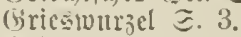

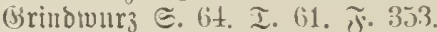

Grossularieae 与. 31 .

(Srubenfledte ङ. 85. I. 81. T. 4ST.

(írinting $\mathfrak{S} .51$.

Grullofelte S. 4t. I. 41. J. 238 a. b.

Guajacum S. $1: 3$.

Gmajavabaum Є. 28

(inafobaum S. 8 .

(biinlal ๔. 57. T. 5t. T. 315

Gnmmibanm S. 66. I. 63. ₹. 367.

(B)tuniguttbaum ङ. 11.

(imlldelrebe 5. 57. I. 5t. \%. 317.

(5)mbermann S. 57. I. 5t. \%. 317.

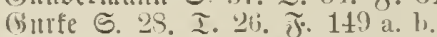

Gurtentraut ธ. 3t. T. 31. T. 175.

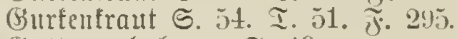

(Sittaperd)abanm $\subseteq 49$

Guttiferae S. 11.

(Sittigetwädie ङ. 11.

Gypsophila S. 9. T. 9. ઍ. 50.

\section{5 .}

STaritrang $\odot . ~ 3 t$.

Şabid)tid)tuamu S. S7. T. S3.

๗. 496 a. b. c. d.

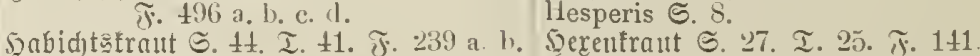

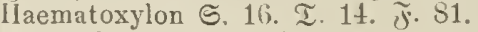

Haemodoraceae $\Subset$. 74 .

Haemodorum S. 74.

5ajer ङ. 74. T. 76. ฐ. 437.

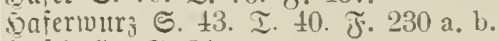

wattolde S. $3 t$.

jogebuthe S. 6s. T. 65. స. 380 a. b.

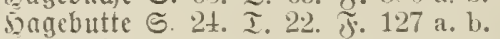

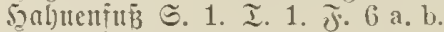

รูabutenfuBgetoüdje ธ. 1.

5abuentam 5.62 .

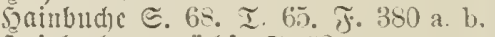

รainbudengewädje ธ. 68 .

5)aillidölthen 5.54.

Sainimic e 77. I. 74. ₹. 427

5 albgräjer હ. 77.

Halorageae 5. 25 .

Hamelia $\Subset .37$.

5) ali ธ. 67. T. 6t. T. 373 a. b.

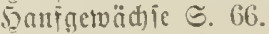

5atigbudie ธ. 69.

Sormelrante ङ. 13.

5oarthet ธ. 11. T. 11. ซ. 61 .

รarthengetwäbie ธ. 11.

5artriegel S. 49. ․ 46. T. 269 a. b.

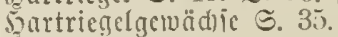

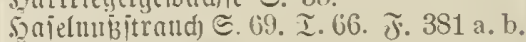

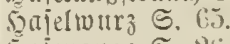

J)ajenange S. 26. T. 24. ร. 134.

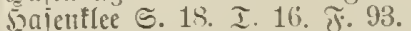

गुajentol] ธ. 13. I. 12. \%ั. 70 .

Jaienlattid 5.44. I. 41. $\pi .235$.

5ajetiolir ธ. 33. ․ 30. J. 169 a. 1.

Jajenialat $\Xi$ t4. T. t1. $\pi$. 235.

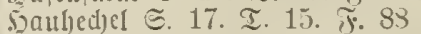

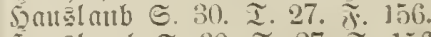

ร)anblatd) S. 30 . T. 27. J. 156.

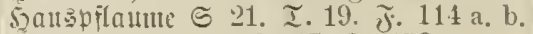

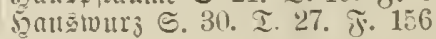

5autpilze 厄. 86 .

5ुectenroje 5. 24. ․ 22 . T. 127 a. b.

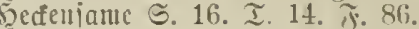

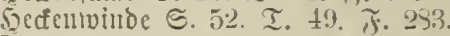

Hedera ธ. 35. I. 32 . テ. 181 a. เ.

Hederaccac 5. 35.

5eberid ธ. 5. T. 5. స. 30 :. b.

Hedysarum ङ. 19. I. 17. స. 103.

jeibe 5. 47. I. 4t. 万. 252 .

Soldeginter ङ. 16. I. 14 . ホ. $\$ 6$.

जocibcfrautgeivädie $5 . t 7$.

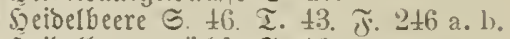

5 eidolbecraetuädie 5.46 .

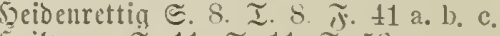

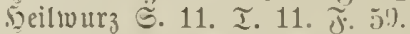

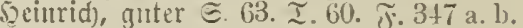

Heleocharis 5. 79.

Helianthemum ङ. 8. T. 8. $\pi .4$

Ilelianthus S. t1. I. 38. T. 218.

Helichrysum $\mathbb{S} 45$.

Helicteres 5.11.

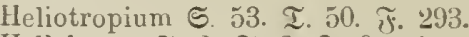

Hellehorus E. 2. T. 2. T. 9 \%. 1.

Jiellertrant S. 7. T. 7. T. 39 a. b.

Salmfraut S. 55. T. 52 . $\widetilde{3} .306$.

Helvella S. 87. T. 83. T. 497 a. b. c. d.

Hepatica $\Subset .3$

Hepaticae S. St.

Heracleum $\subseteq 3 t$.

5erbitzeitloic હ. i6. T. 73. 5. 42I a. 1.

Ileritiera 5 . 11.

Herniaria 5. 29. ․ 27. ホ๐. 152 a. b.

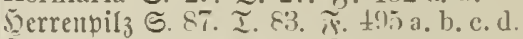

5er3blatt 5. 32. ․ 29. స. 162.

5ुerzgeірапп ธ. 57.

Jerziamen 5.12.

Seiperibelliritchte 5.11.

5erentraut 5. 37 . ․ 34 . ฐ. 195 \%. 1)

5erentraut $\Subset .60$.

Hibiscus 5 . 11.

Hieracium ङ. 44. T. 41. T. 239 a.b.

5imbere ธ. 2ร. T. 23 . 132 a. b.

5immelfabrtablitmon S. 40. T. 37. T. 214.

simmelgleiter ङ. 51. T. 48. ส্. 2 S0.

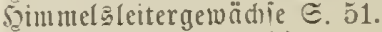

Hippocastaneae S. 12 .

Hippocrataceae S. 12 .

Hippocratea S. 12

Hippocrepis ङ. 19. ․ 17. ₹. $10 \pm$ a. b.

Hippomane S. 66 .

Hippophä̈ ङ. 65. T. 62. ₹. 360.

Hippuris S. 29. T. 26. $\pi .145$ a. b.

5irjdiprumg ธ. 29. T. 27. ส. 153 a. h.

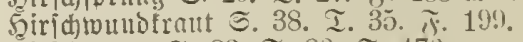

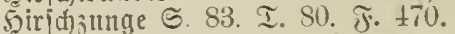

Sirie S. 79. ․ $76 . \widetilde{\jmath} \cdot 136$.

Jirtentäld

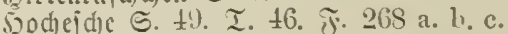

Jigl)lrippe $\subseteq 3 t$.

Ḧlolcus S. 81 . T. 78. T. 455.

5older ङ. 36. T. 33. গ. 187 a. b. c.

joldergemädje 5.37 .

5olutber ธ. 36. T. 33. స. 18 a. b. c.

Holosteum ङ. 10.

Homalineae ङ. 15

Homalium S. 15.

5onigblume $\widetilde{~} .13$

soniggrag $\subseteq$ 81. T. TR. T). 450

5ottigftce $\subseteq .17$. T. 15. is 91 a. b.

5zopfen G. 67. T. 6t. T. $37 t$ a b.

Hordeum ธ. 79 . T. 76. T. 440 .

5zorubaum ธ. 68. T. 65. Ј. 350 a. b.

รornblattgetwädje ธ. 28.

5orutöpidien 5.3

รูortifratt S. 10. T. 10. $\widetilde{J} .55$ a. b. 
Jornmolnn S. 4. T. 4. T. 22.

5ूornitrand 5. 35. T. 32. T. 182 a. b. Semitraud, blutroter 5.3 . T. 32 . T. $183 \mathrm{a} . \mathrm{b}$

Sortenjie ธ. 32 .

Hottonia S. 49 .

Hoya ธ. 50

ร)ĭhtubif ธ. 10.

5)ülienpflauzcu 5. 15

S)ufeifentiant S. 19. T. 17. T. 104 a. b. SIItilee 5. 19. T. 17. T. 104 a. 1).

Somilattid) 5. 38 T. 35. 5.200 a. b.

Hugonia 5 . 11.

Humulus ธ. 67. T. 64. T. $37 t$ a. b.

Sulubetohl ธ. 50. I. 47. ซ). 272

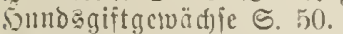

Sumdoftantlle 5. 40. T. 37. T. 210.

Soundäpeteriflic S. 33. I. 30 . $\pi .171$ a. 1)

Wunderoie 5. 24. T. 22. T. 127 a. 1.

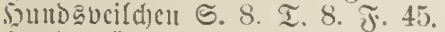

5)แก

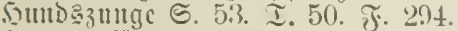

Soutugerblümd)en ธ. 7. ․ 7. ฐ. 38.

Ilutchinsia 5 . 8 .

Soutpilze 5. sic.

Iyacinthus 5. 77. I. 74. T. 424.

Hjdnum 5. 87 . I. 83 . $\pi .496$ a. b. e. d.

Hydrangea $\odot . ~ 32$.

Iydrocharideae $\subseteq 71$.

Hydrocharis 5. 71. I. 68. J. 394.

Hydrolea $55 \%$.

Hydroleaceae 5. 52.

IIydrophylleae $\subseteq 5 t$

IIyoscyamus ธ. 50. I. 56. T. 326.

llypecoum 5. 4.

Hypericinear 5. 11.

Hypericum S. 11. I. 11. T. 61.

Iypochoeris S. 43. I. 40 . T. 233.

Sinpocift $ఠ .65$.

IIyssopus ธ. 55. T. 52. J. 304.

ร.

Iheris 5.8 .

Sbijd) ङ. 11.

Icica 5.15.

Igeldiftclu S. 30.

Jgeltolben 5. 72. T. 69. テ. 398.

Jgname $\subseteq 7 t$.

Ignatia 5. 50. T. 47. T. 274 a. 1). c.

Ilex 5. 48. ․ 45. T. 258 a. b.

Illecebrum ธ. 29.

Illicium 5. 3.

Jumenblatt ธ. 5.5. T. 52. ๙5 307.

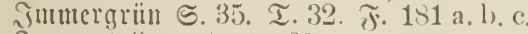

Smmortelle, rote 5.62.

Impatiens 5.13. T. 12. T). 69.

Imperatoria 5. 34 .

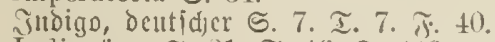

Indigotera 5. 21. ‥ 19. ส. 112.

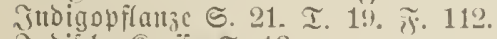

Jildijhe sircife s. 13.

Inocarpus 5. 64.

Inula 5. 39. T. 36. T. 206.

Ipecachanlia ธ. 37.

lpomoea 5.52 .

Iridcae 5. 73 .

Iris S. 73 T. 70 . T. 406

Isatis 5. $7 . \tau .7$. T. 40 .

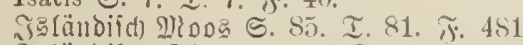

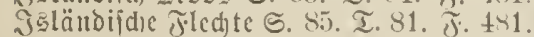
lsoëteae 5 . 82.

Isonandra (5. 4).

Isopyrum 5 . :3.

italicuifde Happel 5. 70.

Ixia 5.74 .

\section{J.}

Jacaranda હ. 51.

Jacquinia S. 49.

Jatobaleiter ธ. 51. T. 48. T. 280.

Jalape, falfide S. 62.

Jasione S. 45. T. 42 . ङ. 241 a. b.

Эasmin $\subseteq 50$.

iasmin, mediter S. 23.

Jasinineae 5. 50.

Jasmingetwädje $\subseteq$. 50 .

Jasminum S. 50

Jatropha 5. 66. I. 63. T. 368 a. b.

Jelängerjelicber ธ. 36. T. 33. ‡. 1 1!l.

ieridjorole 58.

సejuitenmis $\Xi .27$. T. 25. T. 142 a. 1,

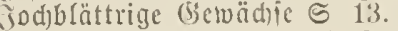

jolbanniabere, rote S. 31. I. 28. T. 158 a. 1,

Johannigberc, idtwarae S. 31.

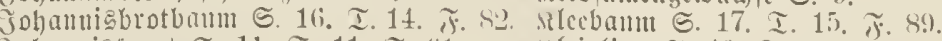

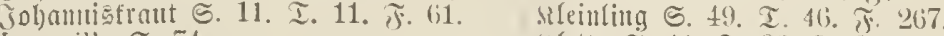

Jonquilla 5. 74 .

iindendoln S. 14.

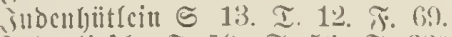

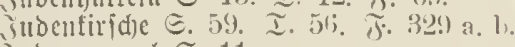

jubenpappcl ङ. 11.

Juglandeat ङ. 15.

Juglans S. 15. T. $13 \quad$ \%. 76 .

Juncaceae 5.77.

Juncus 5. 77. T. 74. T. $42 \mathrm{~S}$.

Jungermannia ธ. 84 . I. 81 . T. $477 \mathrm{a}, \mathrm{b}$

Jungermanniaceae 5 . 84.

Juniperus 5. 71. ․ 68. ร. 392.

\section{ภ.}

Sälbertropf ธ. 34. ․ 31. ศ. 177 a. 1. siälpappel 5 10. I. 10. $\pi .58$. Siäte 5.69 . I. 66 . T. 353 a.b.

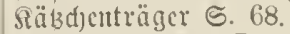

Raffeebantu S. 37.

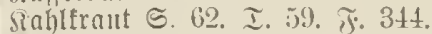

staijertronte 5. 76.

staiferling S. 86i. I. 82 . T 492 a.b.c.

siaifrid)wamm 5. 86. I. 82 . ₹. 492 a. b.c.

Siallageluäd)ic ङ 72.

Kalmia 5.47.

ภaftum 5. 72. T. 69. T. 400.

Salnuछgctuäd)je ๔. 7.2 .

Siamillc ธ. 40. T. 37. ึ. 209.

Samille, periitoce $\subseteq 45$.

Samntgras 5. 81. ‥ 78. T. 451.

Santupejd)cholzbanm S. 16. I. 14. テ. 81 .

Siampierlorber $\mathbb{s}$ lit.

Siaperit, medite S. 13.

Sapermgetvädjic S. 8.

sapernitratd 5.8 .

Rapuns 5 6. I. 6. T. 33 a. b.

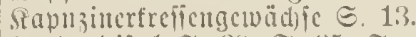

Sialduditel 5. 35. I. 35. \%. 197.

Rarbcigctwäh)ie 5. 38 .

siartoficl 5 . 60 .

sartofiel, djuciuide $5.7 t$

siartoffel, indijdje ธ. 5.:.

staid)uniifie S. 15.

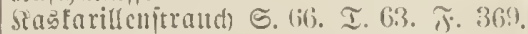

Saftanic 5. 69. I. 66. J. 383 a. b.

Ratappcubäuแe ธ. 26.

Siatedu=2lfajic S. 16. T. 14. T. 70).

sabentlee 5. 18. I. 16. T. 93

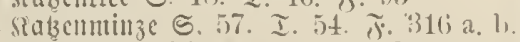

Siazen=\&eterlein E. 333. I. 30. ₹. 171 a.h.

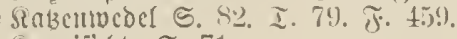

framrifidte $\subseteq$. 71 .

Sautid)utbaum ธ. (66. I. 63. テ. :3(i7.

sield)blume, tarolin. S. 26.

Seldjblïtige Iiflanjen S. 11.
Sermeâbeere ㄷ. 63.

fierntešbercngemädje $\subseteq 63$.

Rculcubäutue 5 . 68 แ 70.

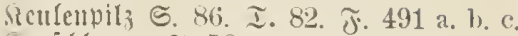

Retuid)baum 5. 58 .

Ribibei 5. 76. I. 73. T. 422 a. b.

Sididerflee S. 19 . I. 17 . T. 100 a. 1.

Sid)ertraganth ऽ. 19. I. 17. ઍ. 100 a b.

siefer 5. 70. T. 67. ₹. 388 a. b.

Kielmeyera 5. 11.

Sicubaum ธ. 70. I. 67. T. 398 a. b.

Sirid)banm ธ. '21.

sirithe 5. 23. T. 21. T. 123 a. b.

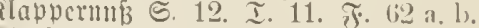

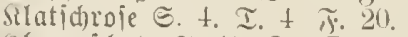

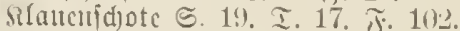

Rlebcrgetuädie $\subseteq$;).

salibtratut $\Subset 5 t$.

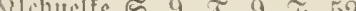

silette 5. 41. I. 3S. $\pi .20 \%$.

stimme 5. 12.

silnabenfraut S. 73. T. 70. F. 403 a. b.

Muabcntränter 5. 73.

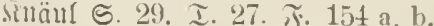

Sillaulgrag E. 81. T. 78. 5. 45.

Silubterid) ธ. 64. I. (i1. T. 354 a. b.

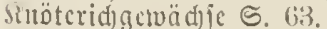

SHopiblume 5. 39. T. 35. \%. 198 a. 1,

Sindrpelfiant S. 29.

Stnotenbrnme S. 74 ․ 71 . T. 411

Sinotemuogartige (jemaldje ङ. 84.

Stöniggterze 5. 60. I. 57. ₹. 331.

Rörbel 5.34.

Sioffelätöneritrand) S. 3 .

Sofospalme 5. 78 . I. 75. T. 4.31 a. 1.

Mopalbaum S. 11.

siopiblïtige (ircuädje 5.38.

Sopifolyl ङ. 6. T. (i. T. 33 a. b.

Sopifrant 5. 6. I. 6. T. 33 a. i.

Sopfialat 5. 44.

Siorallenbanm 5.15 แแঠ 21

Sidrbblïtler S. is

Norbmcide 5. 6!) I. (3\%. T. 395.

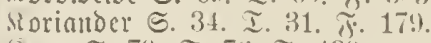

Sion 5. 79. I. 76. T) 439 .

Sidrublunt S. 42. I. 39. T. 227.

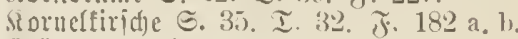

Sirähcuangenbann $\odot$. jo.

Sirübenbcere S. 31.

Strattuturel 5. 35. I. 32. ส. 180.

Sirampitrant S. 26. T. 24. T. $134 \mathrm{i}$ a. h.

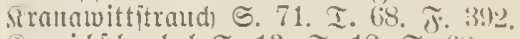

Siranidjidnabel 5. 13. I. 12. T. 68.

Srapp ธ. 37. T. 34. T. 192.

Sirappgervädife ङ. 37.

srabdittel 5. 41. T. 39. T. 2.21.

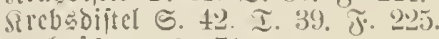

strbsidiere S. 71 .

srcile 5.8 .

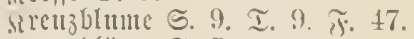

sirelizbritter e. 5 .

sircusdoritgctuädic s. 14

stretistraut S. 40. I. 37. T. 215.

siroutublïtige Piflanjen 5.35 und 47.

srollabcerc 5. 46 . T. 43 . ₹. 249 a. 1.

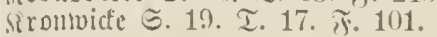

sriumling S. 4t. T. 41. ฐ. 236 a. 1.

Sirmbtogamcu (5. 8\%.

subebcupfeffer S. lis.

Studfenfledte 5.85. I. 81. T. $481 \mathrm{a}, \mathrm{b}$.

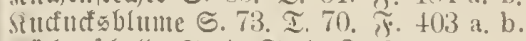

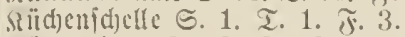

stüumel ธ. 33. T. 30 . స. 167.

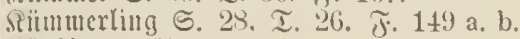

sïrbis 5. 29. 
Stirbiggemädile S. 28.

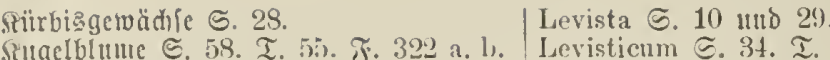

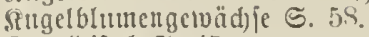

Sitgeldiftel 5.45.

Rubblume 5. 43. T. 40. T. 232.

fituturuz 5.79. 工. $76 . \pi .435)$ a. 1). Silfutucr S. 29 . T. 26 . T. 149 a. 1). Stuntidut 5.51 .

$\mathfrak{l}$

Iabiatac S. 54

Eabtrant S. is. T. 34. T. 193

Lad 5. 6. T. 6. T) 32.

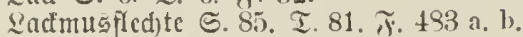

Lactuca 5. 44. T. 41. T. 234

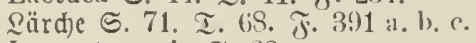

Lagerstrormia S. 28.

Lagetta S. ( 64

Said)trant 5. 73. T. 70. T. 402

Paubertsum $\subseteq$ 6!)

laminaria 5.89 . T. 8., T. 510.

Lamium maculatum S. 56. T 53. T

Lamium purpureum ङ. 56. T. 53.

ฉangfabcu $\stackrel{5}{ } 20$.

Lantana 5.58.

Lappa 5. 41. T. 38. T. 220.

Larix 5.71. T. 68. F. 391 a. b. c.

Sajertrant S. 34.

laserpitium S. 34 .

Lathraea ธ. 62. T. 59. ส. 344

Lathyrus pratensis ङ. 21. I. 19. న. 111 a. b.

Latlyrus silvestris ธ. 20. T. 18. స. 110 a. 1.

Saubmoofic s. 84 .

Laud) S. 76. I. 73. T. 419 a. h.

Zaud)hedcrid) ธ. 8.

Zaurid S. 87. T. 83. ส. 497 a. b. c. d.

Iaurineae $\mathcal{G}_{\text {. }}$ lit.

Laurus $\mathbb{s}$ (64.

Lavandula 5. 54. T. 51. T. 296.

Lavatera S. 11.

Lavettoel 5. 54. T. 51. T. 296.

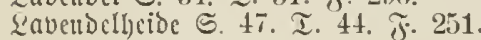

Lawsonia 5 . 28

Rebelisbaum ธ. 71 .

Leberbliumdian 5.3

Lebertraut 5. 84. T. 81. T. 478.

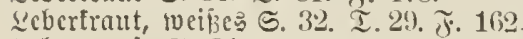

Lebermodic S. 8 t.

lecanora S. 8i. T. 81 . T. 484 a. b.

Lecanoreae S. 85 .

Leberbaum $\subseteq 14$.

Ledum ธ. 47. T. 44. ส. 2\%3

Leea 5. 12.

Leguminosac S. 15

Scim 5. 10. T. 10. T. 57

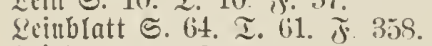

Leintootter S. 8.

Seingetwädje $\Subset .10$.

Qcintraut, gemcines ธ. 61. T. 59. ङ. 337 a. 1)

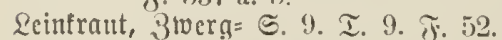
Scittwanbbaum E. (it.

Lemna ङ. 73. T. 70. গ. 401 a. b. Lemnaceae S. 73.

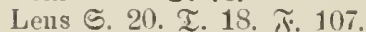

Lentihularicac $5.4 \%$.

Leontodon taraxacum $\Subset .43$. T. 4n. $\pi .232$ Leonurus 5. 57.

Lepidium G. 8.

Qerdeniporn 5. 5. T. 5. ส. 24 a. b.

leucanthemum 5. 39. T. 36. T. 208 Reud)terbaum 5. 26.

leucojum S. 74. T. 71. T. 411.

Leucopogon ธ. 47.

Limone 5. 11.

Soutar 5.78 . $190 \mathrm{a} . \mathrm{b}$.
Levisticum ธ. 34. T. 31. T. 173

siantu 5.51.

Iichen islandicus 5.85. T. 81. テ. 481 .

lichenastra $\approx .84$

lichenes S. 84 .

vidituelte 5. 10. T. 10. T. 53.

¿riebegapiel S. (i).

Liebitödel Є.34. I. 31. T. 173.

ligusticum 5. 34. T. 31. T. 173.

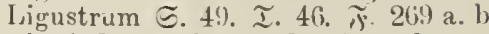

Lilad 5. 49 . T. 46. J. 270 a. b.

Liliaceac 5.75 .

Ziliengetwäd) 5 . 75 .

Lilium S. 75 . T. 72 . T. 417 a. 1). c.

Limnanthemum $\subseteq .51$.

Linaria 5. 61. T. 58. T. 337 a. b.

Eittbe 5. 11. T. 11. T). 60

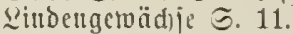

Lindernia 5. 61. $\widetilde{I} .59 . \quad \pi \quad 340$

Lineae S. 10.

linnaea 5. 36

Linum 5. 10. T. 10. ஒ. 57

Iillie ธ. 20. I. 18. T. 107 .

Linienbaum ธ. 18. T. 16. T. 99 a. b.

Pippenblïter 5 . 5.t.

liliodendron $\mathfrak{G}$. 3.

Lithospermum ธ. 52. T. 49. T. 287.

Loasa S. 29.

Loasaceae S. 29

Lobelia 5.45.

Lobeliaceae $\underset{\mathscr{C}}{\mathscr{C}} 45$

Loganieae $\varsigma 50$

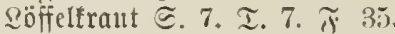

¿ötuenulaul, gelbes ธ. 61 I. 58 5. $337 \mathrm{a}, \mathrm{b}$.

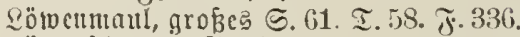
¿öwellidtuallz 5.57.

¿ötoeแzal) ธ. 4. 43. T. 40. T. 232.

(Dold S. 80. T. 77 . T. 441.

Lolium ऽ. 80. T. 77. T. 441

Lonicera caprifolium $\subseteq .36$. T 33. T. 191

Lonicera periclymenum ธ. 36. T. 33.

loranthaceae 5.35

Loranthus S. 35. T. 32. T. 185.

Qorberbaun $\odot .64$.

Qorbeergctuädie 5. 64 .

Sorenjfrant 5. 50. T. 47. 5. 27\%.

Votos alume S. 4.

Qotı̈biruc S. 49.

Lunaria 5. 7. T. 7. T. 37

2utugentraut $\Subset .52$ I. 49.

Lupinus S. 21.

2uzerne G. 17. I. 15. T. 90.

Luzula S. 77. T. 74. T⿱宀. 427.

Lychnis 5. 10. T. 10. T. 53.

Lycopodiaceae @. 82.

Lycopodium S. 82. I. 79. T. 461.

Lycopus ธ. 57.

Lysimachia nummularia S. 49. T. 46. F. 264 .

Lysimachia vulgaris S. 48. I. 45. T. 263 a. b.

Isythrarieae 5.28

Lythrum 5. 28. T. 26. T. 146.

\section{Mi.}

Maaรbecrbaum 5 24. ․ 22. T. 125 a. b. Mliba 5.49

Madia 5.45 .

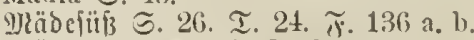

D)

Naesa G. 49.

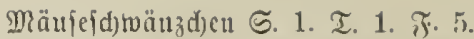
Mragettwurj 5. 72. T. 6.) .. 400.

Magnolia 5. 3.

Magnoliaceae S. 3.

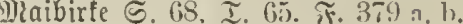

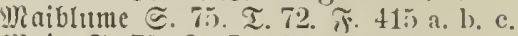

Mais ธ. 79. T. $76 . \tau_{5} .435$ a. b

Maaioran 5. 54

Malope 5.11.

Malpighiaceac S. 12

Malva S. 10. T. 10. ז. 58

Malvaceac 5.10 .

Malbengetwädịe S. 10.

Mamecylon 5.26.

Mammillaria ๔. 30.

Manmutbaum $\odot .71$.

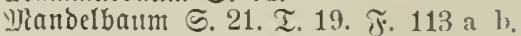

Mandragora 5.60 .

Manglebätutte ङ. 26.

Maugold ङ. 63. T. 60. T. 348 a. b. c.

Mangostana S. 11.

Wangrovebaum 5.26 .

Nanihot S. 66. T. 63. T. 368 a, b.

I) Zaniolitraud) $\approx .66$. T. 63. $\% .368$ ฉ. 1,

y)annaejde 5. 49.

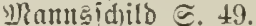

Jianzanillabautu S. 66.

Maranta 5.73.

Marchantia S. 84. T. 81. T. 478.

Marchantiacene $\widetilde{5} .84$

Maregravia 5. 11.

Maregraviaceae 5.11.

Marieublïmd)en 5. 39. T. 36. T. 207

Marienfrant ๑. 41. T. 3S. T. 216.

Marienf(t) 5. 73. T. 70, ส. 405.

Marientlyäucu S. 52. T. 49. テ. 287.

Mart ธ. 32. T. 29. T. 166 a. b.

Marf G. 34.

Marnue 5. 69 工. 66. T. 383 ก. h.

Marrubium 5. 56. T. 53. T. 308

Maruta S. 40. T. 37. T. 210.

Marsiliaceae $\widetilde{\Xi} .82$

Marterroje 5. 25. T. 23. T. 128 a. b.

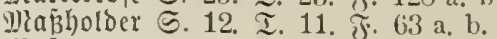

Makibraut 5. 10.

y)

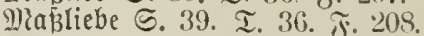

Matricaria S. 40. T. 37. T. 209.

Matthiola 5. 6. T. 6. T. 31.

Mauergipsfraut ธ. 9. T. 9. T. 50

materpfeffer ธ. 29. T. 27. \% 15.

Mauterante G. 82. T. 79. T. 463.

Maulbechaum 5.67. T. 64. T. 375.

Młaulbecrbäumc ङ. 66

Whaulbeergetwädje $\Subset .66$.

Mzaurade 5. 87. T. 83. T. 497 a, h. c. d.

Medicago S. 17 . T. 15. T. 90.

Meergras ธ. 73 .

Micertohl 5. 8. T. 8. T. 42.

Meerlattig ธ. 89. T. 85. ส. 511.

Meerrettig 5.7

Mieerfelli S. 8. T. 8. T. 42.

Mecr3tuicbel S. 76. T. 73. T. 418 a b.

Mrehlbece G. 22. T. 20. $\pi .119$ a. b.

Mehlbirme 5. 22. T. 20. T. 11!) a. b.

Meiitertwurz S. 32, T. 29. T. 164 a, b.

Meifterturn S. 34 .

Mlelaleuca ङ. 28 .

Melampyrum ๔. 62. T. 59. ฐ. 342.

Melastoma $\Subset .28$.

Melastomacene $\Xi$. 28 .

Melde 5. 63. T. 60. T. 351 a. b. c.

Meldengemädje 5.63.

Melia $\Subset .12$.

Meliaceae 5. 12.

Nelianthus 5. 13

Melica ๔. 52. I. 79. శ. 456.

Melilotus S. 17. T. 15. T. 91 a. b. 
Melissa 5. 55. T. 52. F. 303

Melittis 5.55. T. 52. F. 307.

Melocactus ธ. 30 .

Meloure 5. 29.

Mrelonettoijteln ธ. 30.

Menispermeae 5. 3.

Menispermum 5.3 .

Mentha S. 54. T. 51. T. 299

Menyanthes S. 51. T. 48. $\widetilde{J} .279$.

Mercurialis 5.65 . T. 62 . J. 364 a. b.

Mergelwurz S. 64. T. 61. T. 353.

Mesembryanthemum S. 30.

Mespilus 5. 23. T. 21. F. 120 a. b.

Metrosideros 5. 28.

Meum 5. 34.

Miconia S. 28.

Miere 5.10

Mieren $\Subset .9$.

Mildfrant 5.49 .

9lildyitern ङ. 77. T. 74. Э. 423.

Milium 5. 82. T. 79. T. 457.

Milziart 5. 83. T. 80 . T. 471.

Milztrant 5. 31. T. 28. F. 161.

Mimosa S. 15. T. 13. デ. 78.

Mimoseae S. 15 .

Mimulus 5.62.

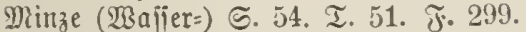

Mirabilis $\odot .62$.

Miapelbaum $\Subset$. 23. T. 21. T. 120 a. b. Mliitel ธ. 35. T. 32. T. 184.

Mijtelgetoädie S. 35 .

Mnium 5. 84. T. 81. T. 474.

Miöhre ธ. 34. T. 31. Ј. 176 a.b.

Mönchia ธ. 10.

Mönd) glappe 5. 3. T. 3. テ. 14 a. b.

Miolngetwädje 5.4

Mohrribe S. 34. T. 31. F. 176 a. b.

Mombinbaum 5.15

Momordica S. 29.

Monarda 5.57.

Monatallee 5. 17. T. 15. T. 90.

M) Mondame 5.3.

917ondoide 5. 7. T. 7. T. 37.

Monimieae S. 66

Monochlamydeae S. 62 .

Monocotyledonet 5.71

Monodora 5. 3.

Monotropa 5. 47. T. 44. T. 255.

Monotropeae 5.47.

MIontia S. 10. ult 29.

Moogbeere ธ. 46. T. 43. テ. 249 a. b.

Mropie 5. 84.

Mloogfarn 5. 82. T. 79. T. 461.

Moraceae 5. 66.

Mordel 5. 88 . T. 84 . T. 498 a. b. c. d.

Morchella ङ. 88. T. $84 . \widetilde{T} .498$ a. b. c. d.

Moreae 5. 66 .

Morus ๔. 67. T. 64. โร. 375.

ग) Rüfentiu ür

Musa 5.73.

Musaceae 5. 73.

Muscari 5. 77. T. 74. T. 424.

Musci frondosi $\Subset .84$

Muscineae 5. 84.

Muatathnacinthe S. 77. I. 74. T. 424.

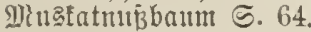

M)uttertwurz ธ. 34.

Mycetes 5. 85 .

Myoporinae 5.58.

Myosotis arvensis ธ. 52. I. 49 F. 288 a. b.

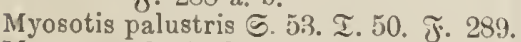

Myosurus 5. 1. T. 1. ฐ. 5.

Myrica 5. 70.

Myriceae S. 68

Myriophyllum 5. 28.

Myristica S. 64 .
Myristiceae ․ 64.

Myrobalanus ङ. 26.

Myroxylon 5.21.

Dinrrhentraut 5.34.

Myrsine S. 49.

Myrsineae S. 49 .

Myrtaceae ङ. 28.

Minrte 5. 28.

Myrtus S. 28.

9 .

Radtterze S. 27. T. 25. T). 140 .

Madtroje 5. 36. T. 33. F. 191.

Radtidatten ธ. 59. T. 56. ₹. 327 a. b. Madhtidattengetoädje 5.58 .

Madtuiole S. 8 .

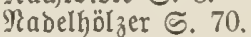

Rägeleintraut S. 26. I. 24. T. 134.

Räpfdenirüdtler S. 68.

Ragelbeere 5. 14.

Nagelfraut S. 10 und 20.

Mageltrautgervädje 5.29.

Najadeae S. 73 .

Najaben 5. 73.

Najas 5. 73 .

Marcilfe S. 74. T. 71. F. 409.

Tarciifen S. 74 .

Narcissus 5. 74. T. 71. F. 409

Marde, deutide 5. 54. T. 51. T. 296.

Nasturtium S. 5. T. 5. F. 26 a. b.

Mattertopf 5. 52. T. 49. T. 285.

Rattertwurz S. 64. T. 61. F. 355 a. b.

Ratterzunge S. 83. T. 80 . テ. 466 .

gielten 5. 9.

Meltentwurz S. 26. T. 24. F. 134.

Nemophila 5. 54.

Nepeta 5. 57. T. 54. T. 316 a. b.

9eptunggürtel ङ. 89. T. 85. ซ. 510.

Nerium 5. 50. T. 47. J. 273.

Neslea ธ. 8.

Iiclielartige (Bselvädje S. 66.

গieliclbaum ङ. 70.

Nenufraft 5. 38. T. 35. T. 201.

Nicotiana rustica ङ. 58. T. 55. ฐ. 324.

Nicotiana tabacum ธ. 58. T. 55. T. 325.

Nierenbaum ङ. 15. T. 13. Э. 77.

Niçsıurz 5. 2. T. 2. T. 9 a. b.

9Rieรtwurz ङ. 76. T. 73. J. 420 a. b.

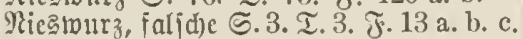

Nigella 5. 2. T. 2. T. 10.

Nixblume 5. 4. T. 4. ฐ. 19.

Mirentrant 5.73 .

Nuphar 5. 4. T. 4. J. 19.

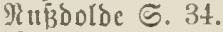

Nyctagineae 5. 62.

Nyctago 5. 62.

Nymphaea S. 4. T. 4. I. 18.

Nymphaeaceae 5. 4.

D.

Ochna ธ. 14

Ochnaceae ङ. 14.

Ochroma 5.11

Sdienange 5. 39. T. 36. T. 205

Ðd) ienbred S. 17. T. 15. T. 88.

Ddicnzunge 5. 53. T. 50. T. 290.

Ocimum 5. 57. T. 54. ร. 319.

ธ̌lbaum S. 49.

Ëlbaumgeruädie S. 49.

B̈lfennid) 5.34

S̈lpalme 5. 78. T. \%. T. 432

Srrpeide S. 65.

S̈lmeibengemädie S. 65 .

Oenanthe 5. 33. T. 30. T. 170 a. b.

Oenothera S. 27. I. 25. ふ. 140.
Dhnblattartige Betwädje S. 47.

Ohnmuld S. 84. T. 81 . F. 473 a. b. c.

Olea S. 49.

Sleanber S. 50. T. 47. F. 273.

Dleaiter 5.65 .

Oleineae 5. 49.

Onagrariae 5. 27.

Onobrychis S. 19. T. 17. T. 103.

Ononis S. 17. T. 15. ז. 88 .

Onopordon S. 42. T. 39. T. 225.

Ophioglossum 5. 83. T. 80 . T. 466.

Ophrys ธ. 73. T. 70. T. 404 .

Opuntia S. 30

Srange S. 11.

Draugcngemädje S. 11.

Orchideae 5. 73

Orchis 5. 73. T. 70. T. 403 a. b.

Origanum 5. 54. T. 51. T. 301.

Orleanbaum $\subseteq 8$.

Ornithogalum 5. 77. T. 74. $\pi$. 423.

Ornithopus S. 19. T. 17. T. 102.

Ornus 5. 49.

Orobanche ङ. 62. T. 59. ร. 343 a. b.

Orobancheae 5. 62.

Orontiaceae S. 72 .

Oryza 5. 80. T. 77. T. 443 a. b.

()sbeckia S. 28.

Osmunda S. 83. T. 80. J. 464.

Ostericum S. 34.

Diterluzei ङ. 65. T. 62. ₹. 361 a. b.

Siterluzeigetwädje 5.65.

Oxalideae S. 13.

Oxalis 5. 13. I. 12. T. 70 .

Oxycoccos S. 46. T. 43. T. 249 a. b.

Oxytropis S. 18. T. 16. F. 99 a. b. c.

\section{$\mathfrak{B}$}

Paeonia ธ. 3. T. 3. ร. 15.

Palijanderljolz ธ. 51 .

Paliurus 5.14 .

Palmae 5. 78 .

Baluen 5. 78.

Lalmfarue 5.70 .

Halmfolben s. 79

ISalmlilie 5.77 .

Sanamapalme S. 79.

Panax ธ. 35. T. 32. ฬ. 180.

l'andaneae S. 79 .

Pandanus 5. 79

Panicum S. 79. T. 76. โ็. 436

Bantoffelblume S. 62.

Papaver S. 4. T. 4. গ. 20.

Papaveraceae 5. 4.

Papilionaceae S. 15.

Bappel S. 70. T. 67. T. 387 a. b. c.

Bappelgelvädie ङ. 68 .

Iiappelroieu S. 10.

Baradiegapfel 5. 60.

Waradic 3 baum E. 65 .

Iarabiegfeiac S. 73.

Parietaria S. 67. T. 64. T. 372.

Parietariac S. 66 .

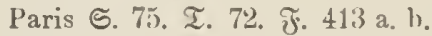

Parkia S. 15.

Parmelia 5. 84. T. 81. T. 480.

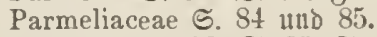

Parnassia ธ. 32. T. 29. T. $16 \%$.

Paronychieae 5. 29.

Passitloreae 5. 29.

Bailitongblumengetwädje S. 29.

lastinaca 5.34 .

Baitinaf S. 34.

Batate 5. 52.

Batcrubiterbaum S. 12

Q̇atidulipflauze S. 57.

Paullinia S. 12.

Paulownia S. 62. 
Peganum 5. 13.

Đetamuiz 5.12.

Pelargonium 5.13. T. 12. T. 68.

Peltaria 6.8 .

Peltideaceac (5. 85).

Peltigera 5. 8.̃. T. 81. T. 485.

Peplis 5. 28. I. 26. స̃. 147 a. b.

Wuerlaras 5. 82. T. 79. ฐ. 456.

lierlmong ङ. 8.). T. 8\%. T. 509.

Persica ธ. 23. T. 21. T. 121.

Personatae 5. 60.

Reitmurz 5. 38. I. 35. テ. 201.

Petasites 5. 38. T. 35. ‡. 201.

Petrocallis 5.8

Petunia S. 60.

Peucedanum S. 34.

Iifaffulbitt)en S. 14. T. 13. T. 73 a. b.

difaffentint 5. 72. ․ 69. ₹. 399 a. b.

Lifanculiric 5 . 74.

Iifancuid)meiftang G. 88. T. 84. F. 505 .

iffeffer, id)warzer S. 68 .

bicifergemädjic 5.68

Iticifertiant S. 29. T. 27 テ. 155.

qifefferlittg S. 86. I. 82 . ₹. 493 a. b. c. Iifefterminze 5. 54.

Ijecifuitraud) 5.50.

Sireifeulträud)e E. 28

Hifcilfrant S. 72. T. 69. T. 396.

qifcilturur ङ. 73.

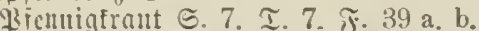

Iifentiafrant ธ. 49. I. 46 ฐ. 264.

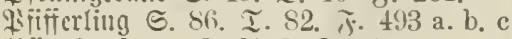

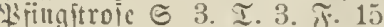

qivirfid) ธ. 23. ‥ 21. 3.121

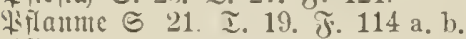

Liflamenbanm 5.21.

ㄴiflanutempieffer 5 . 68.

Dirricutentrefic $\odot .8$.

Sifriculutrand) 5. 17. T. 15. T. 87.

Phalaris S. 80. I. 77. హ. 445.

Dhanteroganten $\Subset .1$.

Phascaceae S. 84 .

Phascum E. 84 I. 81 . ₹. 473 a. b. c. Phaseolus E. 20. I. 18. J. $109 \mathrm{a} . \mathrm{b}$.

Philadelpheac G. 28 .

Philadelphus 5. 28 แ. 50.

Phlox 5. 51. T. 4. T. 281.

Phoenix S. 7R. T. 75. T. 433 a. b.

Phragmites 5. 82. T. 79. T. 458 .

Physalis 5.59 . T. $56, \widetilde{J} .329 \mathrm{a} . \mathrm{b}$.

Phyteuma ङ. 45 . T. 42 . ₹. 242 a. b.

Phytolacea $\mathfrak{G} .63$.

Phytolaceae S. 63 .

Picea 5.71. I. 68 . T. $390 \mathrm{a}, \mathrm{b}$

Picris ธ. 43. T. 40. Ј. 231.

Zillenbanm 5. 8.

Litllentarn 5. 82. T. 79. T. 460.

Dillentrant S. 82. T. 79. స.. 460.

Pilularia G. 82. T. 79. F. 460 .

Iillze 5. 85 แnd 86.

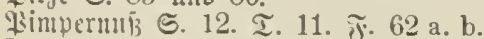

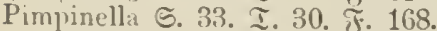

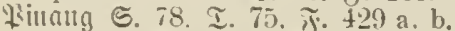

Pinguicula 5.47 . T. 44 . T. 256.

Pinus $A$ bies S. 71. T. 68. $\pi .390 \mathrm{a}$ b.

Pinus Iarix 5. 71. I. 68 . T. 391 a. b. c.

Pinus Picea 5. 70 . I. 67. 359 a b. c.

Pinus silvestris ङ. 70. T. 67. T. $388 \mathrm{a} . \mathrm{b}$.

Piper 5. 68.

Piperaceae 5.68.

\ippau 5. 44. T. 41. テ. 238 a. b.

Pirola S. 47. I. 44. T. 254

Pirolaceae 5.47

Pirus Aria ङ. 22. T. 20. స. 119 a. b.

Pirus communis $\subseteq$. 22 I. $20.117 \mathrm{a}, \mathrm{b}$ l'irus cydonia 5 . 24. T. 22. T. 124 a. b. Pirus malus 5. 22. T. 20. T. 118 a. b. Bifange S. 73 .
Ziitazic S. 12. T. 11. T. 62 a. b.

Yisum 5. 20. T. 18. T. 108 a. b. c.

Pittosporeae 59.

Plantae vasculares 5.1.

Plantagineae 5. 62.

Plantago 5. 62. T. 59. T. 345 a. b.

Plataneae S. 68.

Platanen S. 68.

Platanus 5. 70

Rlattentang S. 89. T. 85. T. 510.

Wlatterbic S. 20. T. 18. T. 110 a. b.

Zlatterbie G. 21. T. 19. テ. 111 a. b.

Pleurospermum $\mathfrak{\subseteq}$. 34.

Plumbagineae 5.62 .

Plumbago 5.62.

Poctentiolz 5. 13.

Podostemeae 5. 72.

Pogostemon S. 57.

Polemoniaceae E. 51

Polemonium 5. 51. T. 48. T. 280.

Polyanthes $\Subset$. 77.

Polycarpon ङ. 10 1tnd 29.

Polygala Є. 9. T. 9. F. 47.

Polygalaceae ธ. 9.

Polygoneae 5. 63.

Polygonum aviculare S. 64. I. 61. T. $354 \mathrm{a}, \mathrm{b}$.

Polygonum bistorta ․ 64 . T. 61. J. 355 a. b.

Polygonum persicaria ङ. 64. I. 61 . ๘. 356

Polypodium ङ. S3. T. 80. ฐ. 467.

Populus alba G. 70. T. 67. T. 386 a. b. c

Populus nigra S. 70. T. $67 . \Im 357$ a.b.c.

Porit 5.47 . T 4t. T) 253 .

Porit S. 68 und 70.

Portulaca 5. 29. T. 27. ₹. 151.

Portulacere S. 29.

Bortulat 5. 10

İortulat ङ. 29. T. 27. テ. 151.

Liortulafaemädic 5 . 29.

Bortulatwegerid S. 28. I. 26. శ. 147 a. b.

Iorzellantianze S. 50.

Potalia S. 50.

Potamogeton 5. 73. $\tau .70$. T. 402.

Potentilla 5. 25. I. 23. ฐ. 130.

Pradtfedernelfe 5.9. I. 9. T. 49

Sireikelbeere 5. 46. T. 43 . $\pi .248$ a. b.

Prenanthes 5. 44. I. 41. T. 235.

Primula Auricula S. 48. T. 45. গ⿱乛. 260 .

Primula farinosa S. 48. I. 45. ゙ָ. 259 .

Primulaceae ङ. 48.

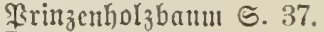

Protea 5. 64

Proteaceae S. 64.

Protellageivächie 5. 64.

Protococcus 5. 88. T. 84. T. 503.

Prunella ๔. 55. T. 5\%. T. 30\%.

Prunus E. 21.

Prunus armeniaca 5. 23. T. 21. ซ. 122 a. h.

Prunus cerasus G. 23. T. 21. . 123 a. b.

Prunus domestica .21. T. 19. テ. 114a. b.

Prunus Padus 5. 22. I. 20. J. 116 a. b.

Prunus spinosa ङ. 21. T. 19. テ. $115 \mathrm{a} . \mathrm{b}$.

Psidium 5. 28.

Psychotria S. 37.

Ptarmica S. 40. T. 37. テ. 211

Ptelea 5.14.

Pteris 5. 83. T. S0. T. 468.

Pulicaria 5. 39. T. 36. T. 206.

Pulmonaria 5. 52. T. 49. J. 286 a. b.

Pulsatilla ङ. 1. T. 1. ฐ. 3.

Bulberbolz ङ. 14. T. 13. T. 74 a. b.

Punica $\odot .26$.

Burgierfrant Є. 60. T. 57. T. 333.

Bnrgierfroton S. 66.
Burgierminde S. 52.

IStramidenpappel 5.70.

Pyrethrum 5. 45.

ఏ.

Suandang= Rüfīe ธ. 65 .

Quassia 5. 14

Duellentraut ธ. 10.

Encllenmontic S. 29

Entrbel ธ. 54 . T. 51. T. 300.

Quercus 5. 69. T. 66. ₹. 384 a. b.

Duittenbaum 5. 24. T. 22. T. 124 a. b.

$\mathfrak{R}$.

Rađenblüter 5. 54.

Rabel S. 10.

Radiola S. 10.

Rafflesia 5.65

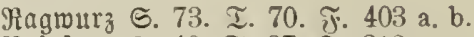

Rainjarn S. 40. I. 37. テ. 212

Raintoibe S. 49. T. 46. Э. 269 a. b.

Ramalineae G. 85.

Ramid S. 9. T. 9. গ. 47

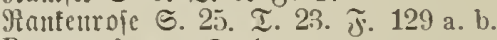

Ranunculaceae ङ. 1 .

Ranunculus 5. 1. T. 1. T. 6 a. b.

Raphanus 5. 8. T. 8. গ. 41 a. b. c.

Rapistrum S. 8 .

Rapabotter 5.8

Mapunzel ङ. 37. T. 34. テ. 196.

Rapunzel, rundföpfige 5.45. I. 42. ₹. 242 a. h.

Raicnidmicle 5 80. T. 77. T. 447.

Mauidbecre G. 31.

Rauld)bere $\subseteq$. 46. T. 43 . ₹. 247 a. b.

Hante ธ. 13. T. 12. ฟ. 71.

Rautengetwähie 5.13.

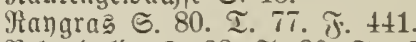

Rebendolde S. 33. ․ 30. T. 170 a. b.

Rebengewädie 5.12.

Reiherinnabel S. 13. I. 12. \%. 67.

Reis 5. 80. T. 77. T. 443 a. b.

Reigpapierpflanje 6.35.

Reseda 5. S. T. S. T. 43.

Resedaceae S. 8.

Restiaceae G. $7 \mathrm{~T}$

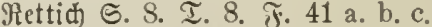

Rhabarber S. 64 .

Rhamneae ङ. 14

Rhamnus ङ. 14. I. 13. ซ. 74 a. b.

Rheum S. 64 .

Rhexia G. 28.

Rhinanthaceae $\subseteq$. 60 .

Rhizoboleae G. 12.

Rhizobolus $\odot$. 12.

Rhizophora Mangle S. 26.

Rhizophoreae 5. 26.

Rhizospermeae $\subseteq .82$.

Rhodiola ธ. 30 .

Rhododendron S. 47.

Rhus S. 15. T. 13. స. 75 .

Ribes Grossularia S.31. T. 28. ฐ.157 a.b.

Ribes nigrum ङ. 31

Ribes rubra Є. 31. T. 28. T. 158 a. b.

Riccia ङ. 84. I. 81. ๆ. 479 .

Ricciaceae 6.84.

Ricinus 5. 66. T. 63. T. 366 .

Ried 5. 79.

Ricogräier $\subseteq .79$.

Riemenblunte G. 35. ․ 32. ๆ. 185.

Rieienblume $ఠ .65$.

Mieingras E. 80. T. 77. গ. 444.

9ticientame 5 . 71.

Hindsauge ธ. 39. T. 36. ซ. 205.

Mingelblume E. 41. T. 3S. T. 219 a. b.

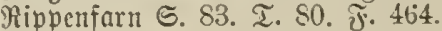


Mifpenhafer 5. 79. T. 76. T. 437.

Mitteriporn S. 2. T. 2. $\pi .12$.

Roccella S. 85. T. 81. T. 483 a. b.

Roccellei 5. 85.

Möhretpilz S. 87. T. 83. F. 495 a.b.c.d.

Moggen 5. 79. T. 76. T. 439.

Mohrtolbet S. 72. T. 69. J. 397.

Rohrtolbengewädje $\subseteq$. 72 .

Roripa S. 8.

Rosa 5. 24.

Rosa arvensis S. 25. T. 23. T. 129 a. b.

Rosa canina S. 24. T. 22. T. 127 a. b.

Rosa repens 5. 25. T. 23. T. 129 a. b.

Rosa rubiginosa 5. 25. T. 23. গ. 128 a. b.

Rosaceae 5. 21.

Riofengetwädjic S. 21.

Moientorbeer S. 50. T. 47. J. 273.

Moß̈tümmel ऽ. 34.

Hosmaritl, wilder 5.47 . T. 44. F. 253

Rosmarinheide 5. 47. T. 44. T. 251.

Rosmarinus 5. 57. T. 54. T. 320.

Roftroie 5. 25. T. 23. T. $128 \mathrm{a} . \mathrm{b}$

Robłaptanie S. 12.

Rokitualve ङ. 10. T. 10. F. 58

Rotbudbe S. 69. T. 66. T. 382 a. b. c.

Roteibet S. 71. T. 68. T. 393.

Rotholzgetwädie 5. 12.

Mottaune S. 71. T. 68 . T. 390 a. b.

Rubia 5. 37. T. 34. Э. 192.

Rubiaceae ธ. 37.

Rubus Idaeus S. 25. T. 23. F. 132 a. b.

Rubus fruticosus S. 26. T. 24 . T. $133 \mathrm{a}$. b.

Rudgras 5. 81. I. 78. T. 449

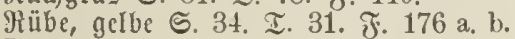

Ruellia 5. 58

Miiiter ธ. 68. ․ 65. T. 377 a. b.

Minhrfraut S. 40. T. 37. T. 214.

Rumex crispus S. 63 . T. 60. T. 352 a.b. c.

Rumex obtusifolius S. 64. T. 61 . F. 353.

Munfelrübe 5. 63. T. 60 . $\widetilde{T} .348$ a. b. c.

Ruppia S. 73 .

Ruta ङ. 13. T. 12. F. 71.

Rutaceae ธ. 13.

6.

Saaterbie 5. 20. T. 18. T. 108 a. b. e. Saatinife S. 20. I. 18. $\% 107$.

Saceharum S. 80. T. 77. T. 442.

ธäđfelblume ธ. 11 .

ธäuld)etuf(ect)te 5. 85. T. 81. テ. 482

Sänlenblumte $\odot .45$.

Saflor S. 41. T. 38. T. 222.

Safrau S. 74. T. 71. T. 408.

Safratt, falid)er S. 41. T. 38. F. 222.

Safranbautu 5. 26.

Safraulyolzbaum S. 14

Sagina 5. 10.

Sagittaria 5. 72. T. 69. F. 396.

Sagopalme S. 71 .

Sagopalttte S. 78 . T. 75. F. $430 \mathrm{a}$. b.

Sagus S. 78. I. 75. T. $430 \mathrm{a} . \mathrm{b}$.

Salaeia 5. 12.

Salbei ธ. 54. T. 51. ซ. 297.

Salicineae 5.68 .

Salix 5. 69. T. 66. গ. 395.

Salsola 5. 63.

Salvia officinalis S. 54. T. 51. F. 297.

Salvia pratensis 5. 54. T. 51. J. 298.

Salvia rosmarinus 5. 57. T. 54. T. 320 .

Salviniaceae 5.82.

Salzfraut S. 63.

Sambucus ebulus S. 36. T. 33. T. 188a. b.c.

Sambucus nigra S. 36. T. 33. T. 187 a.b. c.

Samenblattloie Iiflanzen S. 82.

Samuetpappel 5. 11. T. 11. Э. 59.

Samolus S. 49

Samyda 5. 14.
Samydeae 5. 14.

Sattbbere S. 46. T. 43. T). 250 a. b Saltbelbaum S. 65. T. 62. J. 359. Sandtraut 5. 10. T. 10. F. 54 a. b.

Sandulte S. 62.

Sandorn 5. 65. T. 62. T. 360.

Sanguinaria 5.4

Sanguisorba S. 26. T. 24. F. 138.

Sanicula 5. 32. T. 29. T. 163.

Sanitel હ. 32. I. 29. ₹. 163.

Santalaceae 5. 64.

Santalum 5. 65. T. 62. T. 359

Sautelbaum 5. 65. T. 62. ฐ. 359.

Sautelgetwädjic S. 64 .

Sapindaceae S. 12

Sapindus S. 12. T. 11. T. 64

Saponaria S. 9. T. 9. F. 51.

Sapoteae ङ. 49 .

Sargafiotatig $\subseteq$ 89. T. 85. T. 507.

Sargassum S. 89. T. 85 . F. 507 .

Sarmentaceae 5.12.

Sarothamnus S. 17. T. 15. F. 87

Sassafras 5. 64 .

Safjajraßlorbeer ธ. 64.

Satureja ธ. 57. T. 54. F. 318 a. b.

Saubrot S. 48. I. 45. T. 262.

Satterdorn 5. 3. T. 3. T. 16 a. b.

Sauterborngewädie $\mathfrak{S} 3$.

Sauerflee ङ. 13. T. 12. ซ. 70.

Satterflecgetoäd) S. 13.

Saurad) 5. 3. T. 3. F. 16 a. b.

Savoberfohl 5. 6. I. 6. T. 34 a. b.

Saxifraga granulata S. 31. T. 28.

₹. 159 a. b.

Saxifraga oppositifolia 5. 31. I. 28 T. 160.

Saxifrageae 5. 31.

Scabiosa S. 38. T. 35. T. 198 a. b.

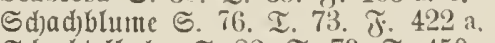
SchadjteIhaltt S. 82. T. 79. F. 459.

Sd)ad)telhalmgetvädịe $\odot .82$.

Sd)äfer Wettergla S. 49. T. 46. T. 266. Sd)afgarbe S. 40. T. 37. ₹. 211.

Sd)afthalme S. 82

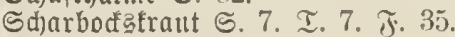

Sd)arffraut 5. $5 t$.

ङd)eibentraut S. 8 .

Sdinerfrant 5. 82. T. 79. T. 459.

Sd)euritraud) S. 21.

ङd)iefblättrige $\mathfrak{B f I} a n z e n$ ธ. 65.

Sdierling S. 34. T. 31. F. 178.

Sdilbblume 5. 62.

Sdildfart 5. 83. T. 80. T. 469.

Sd)ildfledte 5. 85. T. 81. T. $48 \%$

Sdili 5. 8\%. T. 79. T. 458 ,

Sdjilfrobr S. 82. T. 79. ส. 458.

Sd)irmpflanzen S. 32.

Sd)laftraut S. 59. T. 56. F. 326

Sdjlebe 5. 21. I. 19. F. 115 a. b.

Sd)Ieifenblumte S. 8.

ธd)lüffclblume S. 48. I. 45. T. 259

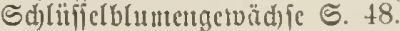

Sd)lutte S. 59. I. 56 . T. 329 a. b.

ธdimalzblume ธ. 2. ๆ. 2. T. 7 a. b.

Sdmeerwurz S. 74. T. 71. T. 412 a. b.

Sduttele 5.80 . T. 77. $\% .447$.

Sd)merwurz S. 47. T. 44. F. 255.

Sd)metterlitgabliiteu ธ. 15.

Sdintinfbere S. 63. T. 60. T. 350 a, b

Ed)tuefentlec S. 17. T. 15. ₹. 90

Sduec, roter 5. 88. T. 84. T. 503.

Sdunecball 5. :36. T. 33. T. 18! a. b.

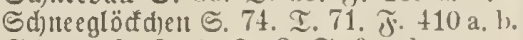

כd)uceroie 5. 2. T. 2. T. 9 a. b.

Sd)neeid)leipe 5. 88. T. 84. T. 503.

๔dineidegras ङ. 79.

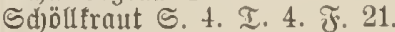

Sdjöntilien S. 74 .
Sd)öแนüize S. 28 .

Schoenus 5 . 79

ธd)opffraut 5. 19. T. 17. T. 104 a. b.

Sdjotendort S. 15

Edotendotter 厄. 5. T. 5. テ. 30 a. b.

Sdotentgetwädje $S$.

Sd)otettueiderid) S. 27. I 25. T). 139 a.b.

ธd)raubenbautu

Sd)ratbenbohtle S. 11.

Ed)riftiled)te S. 85. I. 81. T. 488.

Sdifffelfledte S. 84. I. 81 . T. 480 .

Sdutupentantue S. 71 .

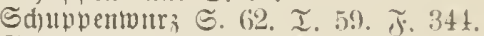

ธdivaden 5. 81. I. T8. T. 4j3.

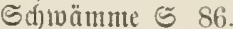

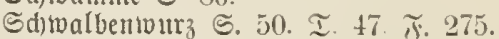

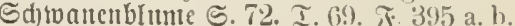

Sd)tuarzoorn 5. 21. T. 19. T. 115 a. b.

Sd)warzerle S. 68. T. 65. T. $37 \mathrm{~s}$ a. b.

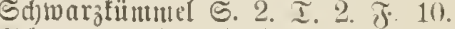

ভd)

ङdutuarzuefiel 5.57.

Sd)warzpappel S. 70. T. 67. T. 387 a.b.e.

Sthwarztame G. 71. I. 68. స. 390 a. b.

Sd)twarztwurz, arzucilid) $\subseteq 53$. I. 50. テ. 291.

ธd)warztwurzel ธ. 43. T. 40. T. 230 a. b.

ङdjuertel S. 74 . T. 71. T. 407 a. b.

Sd)tuertlilie 5. 73. T. 70. ฐ. 406.

Sd)twertliliett $\subseteq .73$.

Seilla 5. 76. T. 73. ‡. 418 a. b.

Scirpus S. 79.

Scleranthus 5. 29, ․ 27. T. 154 a, b.

Scolopendrium 5. 83. T. 80. 菏 470.

Scorzonera 5. 43. T. 40. T. 230 a. b.

Scrophularia 5. 60. I. 57. T. 332.

Scrophularineae 5.60 .

Scutellaria 5. 55. T. 52 . ส. 306.

Secale S. 79. T. 76 đ. 439 .

Sedum 5. 29. I. 27. J. $15 j$

Scefarne S. 82.

Seegras S. 73.

Sectaut S. 51.

Sectohl 5. 8. T. 8. T. 42.

Ecerole, gelbe 5. 4. T. 4. T. 19.

Seeroie, ivcißse, Є. 4. I. 4. T. 18 .

Seerojengetvädị S. 4.

Seetang ธ. 89. I. 8.) ฐ. 508.

Seetang, tratijer S. \$9. T. 85. ฐ. $50 \%$

Scetranbe S. 64 .

Segae 5. 79.

Seggen S. 79 .

Eridelbait S. 64. T. 61. T. 357 a. b.

Seibelbaitgerpädife ؟. 64.

Scidentplanzc 5.50.

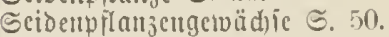

Sciimbatm S. 12. I. 11. T. 64.

Scifutrant ธ. 9. T. 9. ร. 51.

Selagineae 5.58.

Sclago 5. 58.

Selinum 5.34

Selleric 5. 32. T. 29. T. 166 a. b.

Scmpervivum S. 30 . T. 27. 5.156.

Scnehiera S. 8 .

Seneeio 5. 40. T. 37. T. 215.

ङctif 58.

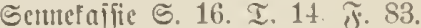

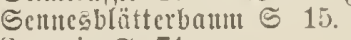

Sequoja 5. 71.

Serradella Є. 19. ․ 17. T. 102.

Serratula ङ. 11. I. 38. T. 221.

కciam 5. .1.

Sesameae S. 51

Sesamum ङ. 51.

Seid 5. 34 .

Seseli \& 34.

Shorea S. 11

Sicbettgezcit 5. 18. T. 16. T. 94. 
Siebctidläier ․ 27. T. 25. T. 140. Siebenitert 5. 49. T. 46. T. 265 a. b. Siegrurz S. 74. T. 71. J゙. 407 a.b. Silau 5. 34 .

Silaus 5. 34 .

Silberidute 5. 64 .

Silberpappel S. 70. T. 67. গ. 386 a. b. c. Silene S. 9. T. 9. テ. 52.

Sileneare 5.9.

Siler G. 34

Silge S. 34 .

Simaba ๔. 14.

Simaiuba 5. 14

Simarubeae 5.14

Sillife 5. 77. T. 74. T. 428.

Simic S. 79.

Simien 5. 77

Sinapis 5.8.

Sinall S. 26 I. 24. ๘. 137.

Situgrü̈ ङ. 50. T. 47. T. 271.

Silutitamze S. 1 i.

Simuptanzen S. 15.

Siphonantraceae S. 46.

Siphonia 5. 66. T. 63. ร. 367.

Sisymbrium 5. 5. I. 5. $\widetilde{\digamma} .26$ a. b.

Sisyrinchium S. 74 .

Sium 5. 34

Smilaceae 5. 75 .

Smyrnium ङ. 34 .

Sodfublume S. 3. T. 3. T. 17.

Solanaceae S. 58

Solanum dulcamara ธ. 59. ․ 56. F. 327 a. b.

Solanum Lycopersicum ธ. 60.

Solanum nigrum S. 59. T.56. F. 328 a. b.

Solanum tuberosum ธ. 60 .

Solidago 5. 39. I. 36. T. 204.

Soldanella S. 48. T. 45. T. 261.

Sommercide S. 69. T. 66. F. 384 a. b. Soumertriffel S. 88. T. 84 . J. 500 a. b.

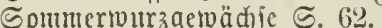

Sonchus 5. 44. T. 41. T. 237.

Sotuenblume ธ. 41. I. 38. .218.

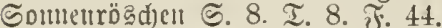

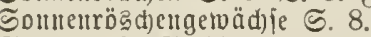

Souncuroje ङ. 11. T. 38. ซ. 218.

Soutuentau S. 8. T. 8. T. 46.

Solmentangetoädje $\varsigma$. 8 .

Sounentucude S. 53. T. 50. ₹. 293

Sophora ङ. 21.

Sorbus 5. 24. T. 22. T. 125 a. b.

Spaltzahn ธ. 81 . T. 81 . T. 475 .

Spaltzalum doie ธ. 84 .

Spatiidjer $\forall$ feffer 5.60.

Sparganiun 5. 72. T. 69. T. 398

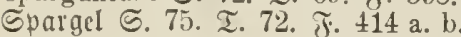

Eparf 5. 10 .

Spartium S. 17. T. 15. T. 87.

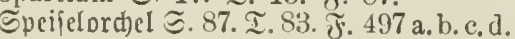
Spergula 5. 10.

Eperrfraut 5. 51. T. 48. T.. 280.

Sperrfräutergetoädje 5.51.

Sphagnaceae 5. 81

Sphagnum S. 84. I. 81. T. $472 \mathrm{a} . \mathrm{b}$

Spile 5. 54. T. 51. T. 296.

Spillbaum 5. 14. T. 13. গ゙. 73 a. b.

Spinacia 5. 63. T. 60. T. 349 a. b.

Spinat S. 63. T. 60. T. 349 a. b.

Spinat, menfeel. E. 30 .

Spindelbaum S. 14. I. 13. T. 73 a. b. Spiraea ․ 26. T. 24. T. 136 a.b.

Spiritauto S. 26 . T. 24 . T. 136 a. b.

Spibenblume ङ. 49.

Spiktiel S. 18. I. 16. T. 99 a. b. c.

Spondias 5. 15.

ङpormblume ङ. 37.

Springtraut S. 13. I. 12 . T. 69.

ธpurre ธ. 10.
Stadjelbeeritraud S. 31. I. 28. T. 157 a. b.

Etadelidivamm

T. 496 a. b. c. d.

Stachys germanica S. 56. T. 53. T. 310 a. b. c.

Stachys silvatica G. 56. I. 53. F. 309. Standeln 5. 73.

Stapelia 5.50.

Staphylea 5. 12. T. 11. F. 62 a. b.

Statice 5. 62 .

Staubpilze 5.85.

ธtedapfel ฐ. 58. T. 55. T. 323 a.b.

Stediborn 5. 14.

Sted)ginfter S. 16. T. 14. T. 86.

Sted)palne S. 48 . I. 45. T. 258 a. b.

Sted)palmengemädie 5. 48.

Steimbred), gegenblättriger 5.31. T. 28. F. 160.

Steitbred), knolliger 5. 31. T. 28. ז. 159 a. b.

Steinflec ङ. 17. T. 15. T. 91 a. b.

Stcintrant ङ. 7. I. 7. F. 36.

Stcinfreffe 5.8.

Stcinlebertrant $\subseteq$ 84. T. 81. J. 478.

Steinpilz ธ. 87. T. 83. ₹. 495 a. b. c. d. Stcillfame 5 52. T. 49. F. 287.

Etcillidntïlfel S. 8.

Stellaria ङ. 10. T. 10. F. 56 a. b.

Stellatae 5.37.

Sterculia S. 11

Sternautisbaum ธ. 3.

Sternblume 5. 39. T. 36. T. 202.

Stertibold S. 32 T. 29. ₹. 164 a. b.

Eteruträuter 5.37.

Stermmiere ङ. 10. T. 10. F. 56 a. b.

SternmDDS S. 84 . T. 81 . T. 474.

Stidtuurzel 5. 41. T. 38. ₹. 216.

Sticta 5. 85. I. 81. F. 487.

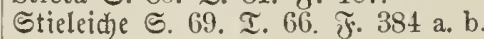

Stillingia ؟. 66.

Stimfbaum ธ. 11.

Stinfitrand S. 21

Stoctmord)el S. 67. T. 83. T. 497 a.b.c. d.

Storarbaum 5. 49.

Storarpflallien હ. 49.

Stordjiduabil 5. 13. T. 12. F. 66.

Etordjinabelaetwädie ङ. 13.

Etrahlfraut S. 10.

Straubling S. 29. T. 27. F. 153 a. b.

Strallonelfe S. 62

Stratiotes ๔. 71

Straudfledten ธ. 85.

Straubaras S. 80. T. 77. T. 446

Streifentarn S. 82. T. 79. T. 462.

Strenzel S. 32. T. 29. T. 164 a. b.

Stridfgras ๔. 79.

Strobblutue ธ. 45 .

Strychnos 5. 50. T. 47. T. 274 a. b. c. Stubentemrösdjen ธ. 32, T. 29. T. 162. Sturmlut S. 3. T. 3. T. 14 a. b.

Stylideae $\mathfrak{5} .45$.

Styphelia ङ. 47 .

Styraceae S. 49 .

Styrax 5. 49.

Stylidium 5. 45

Subularia 5. 8.

Subulina ङ. 10. T. 10. T. 54 a. b.

ธiibgras S. 81. T. 78. 7.453.

Silïgräier ๔. 79.

Siipholz 5. 18. I. 16. T. 96.

Suntiflee S. 51. I. 48. ซ. 279

SUMPfMDDG S. 84. I. 81 . F. 472 a.b. Sumpirofe 5. 51.

Swartzia S. 15.

Swartzieae ธ. 15.

Snfamore 5.68 .

Symphoricarpus 5.36 .
Symphytum S. 53. T. 50. T. 291.

Synanthereae 5.38

Syringa ธ. 49 . T. 46 . J. 270 a. b.

\section{T.}

Tabat ธ. 58. T. 55. T. 324

Iämutel ङ. 10.

Taguabaum S. 79 .

Talgbanm 5. 66.

Talinum 5.29.

Tamarindus 5.15

Tamariscineae $\Subset .28$.

Tamarigte 5. 28, T. 26 . T. 148 a. b.

Tamarix S. 28. T. 26. T. $148 \mathrm{a} . \mathrm{b}$.

Tamus 5. 74 . I. 71. T. 412 a. b.

Tanacetum 5. 40. I. 37. テ. 212.

Tange 5.88 ınd 89 .

Tamme ङ. 70. T. 67. T. 389 a. b. c.

Tamenmed $\widetilde{\Xi}$.28. T. 26. T. 14\% a. b.

Taraxacum S. 43. T. 40. \%. 232.

Taubeniterntopi 5. 38. T. 35. T. $198 \mathrm{a}$. b.

Tatbneliel S. 56. T. 53 . T. 311.

Talmeltörbel S. 34 . T. 31 . T. 177 a. b.

Taumellold S. 80.

Taujetibblatt S. 28.

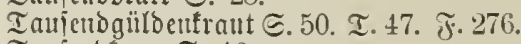

Taupettotorn 5. 10.

Iaujendidöndhen ธ. 39. T. 36. T. 207.

Tarbaum ङ. 71. T. 68. T. 393.

Taxineae ธ. 70 .

Taxus ฐ. 71. I. 68. F. 393.

Tajette S. 74 .

Tecoma ङ. 51 .

Tectonia $\mathbf{5} .5 \dot{8}$.

Teesdalia ऽ. 8.

Ieidroie ङ. 4. I. 4. T. 19.

Tetbaum 5.58.

Tembeiubaum 5.50

Terebinthaceae ธ. 15

Terminalia ธ. 26.

Ternstroemia S. 11.

Ternstroemiaceae S. 11.

Tetracera S. 3 .

Tetragonia ธ. 30.

Teucrium ๔. 57. T. 54. T. 314 a. b.

Teufelabeere S. 60 . T. 57. T. 330 a. h. Teufelspuppe S. 59. T. 56. T. $329 \mathrm{a} . \mathrm{b}$. Telfelsztwirn S. 1. T. 1. T. 1 a. b.

Thalamiflorae $\overleftarrow{\mathfrak{S}} .1$.

Thalictrum ङ. 1. T. 1. T. 2.

Thea ऽ. 11.

Theobroma ๔. 11.

Thesium 5. 64. T. 61. T. 358

Thlaspi ङ. 7. I. 7. Ғ. 39 a. b.

Thuja ङ. 71 .

Thunbergia ธ. 58.

Thymelaceae S. 64.

Thymian 5. 54. T. 51. F. 300

Thymus 5. 54. T. 51. F. 300 .

Thysselinum 5. 34.

Tigerlilie 5. 74.

Tigridia ๔. 74 .

Tilia 厅. 11. I. 11. Э. 60

Tiliaceae 5. 11.

Tillaea 5. 30.

Tintenbeeritraud S. 49. T. 46. F. $269 \mathrm{a} . \mathrm{b}$.

Tolldocfe 5. 3.

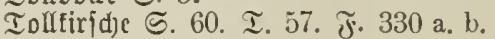

Tollforn ๔. 80 .

Iollfräuter $\Subset .58$.

Tomate S. 60.

Ioufabohnenbaum $\subseteq .15$

Tordylium $\Subset .34$.

Totenblume ธ. 41. T. 38. T. 219 a. b.

Tradescantia 5.77.

Tragopogon 5. 43. I. 40. T. 229

Trapa ङ. 27. T. 25. T. 142 a. b. 
Tranbenthaciuthe 5.77

Tranbenfiride 5. 22. I. 20. F. 116 a.b.

Tranterbaun G.

Iranerbndse S. (69.

Irancreidee ङ. 49 .

Tremandraceae $\Xi .9$.

Treipe S. 81 . I. 78 . テ. 450

Tribulus 5. 13.

Trichilia S. 1\%.

Tridsterivinde $\overline{5} .52$.

Trientalis $\Xi$. 49. T. 46. T. 26 a. b.

Trifolium arv. ङ. 19. ․ 16. T. 93.

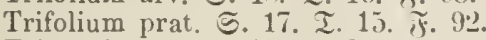

Trigonella 5. 18. ‥ 16. ‥ 94

Triticum 5. 79 . ․ 76. T. 438.

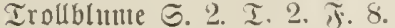

Trollius ङ. 2. $\mathcal{I} .2 . \pi .8$.

Trompetenbaum ธ. 51.

Trompetenbäume S. 51.

Tropaeoleae G. 13.

Tropaeolum $\cong 13$.

Iriiffel, italicuijd) tweiß̄e S. 88. T. 84 శ. 501.

Trüfifl, idwarze 5.88. T. 84. T. 499.

Tuber aestivum S.88. T. 8t. T. 500 a. b.

Tuber album ङ. 88. T. 84. స. 502.

Tuber magnatum 5.88. I. 84. J. 501.

Tuber melanosporum ธ. 88. T. 84 . ז. 499 .

Tuberoje 5. 77 .

Tiipfelfarn 5. 83. T. 80. T. 467.

Türtenbumblilie 5.75 . T. 72 . స. 417 a.b.c.

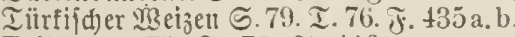

Tulipa 5. 75 . T. 72 . T. 416.

Iulpe ธ. 75. T. 72. T. 416

Tulpeubaum, virginijd)er $\Xi .3$.

Turmtrant $\widetilde{5} .8$.

Turnera ङ. 29.

Turneraceae S. 29.

Turritis 5. 8.

Tussilago 5. 38 . T. 35 . T. 200 a. b.

Tspha S. 72. T. 69. T. 397.

Typhaceae S. 72

11.

llfertweide 5. 69. T. 66. F. 395.

Ulex ธ. 16. T. 14. গ. 86.

Ulmaceae S. 66 .

lllute 5. 68, T. 65. テ. 377 a.b.

Ulmus ङ. 68. ․ 65. J. 377 a. b.

Ulva ธ. 89. I. 85. ‡. 511.

Umbelliferae $\varsigma 32$.

llpaรbaum ธ. 68. T. 65. ร. 376.

IIpasitrand $\subseteq 50$.

Urtica ธ. 66. T. (63. T. 371.

Urticularia 5. 47. I. 44. T. 257.

Urticeae ङ. 66 .

Urticinae S. 66 .

Usnea S. 85. T. 81. T. 486.

Usneaceae ธ. 85.

\section{2.}

Vaccinieae 5. 46.

Vaccinium myrtillus S. 46. T. 43 ऊ. $\dot{2} 46$ a.b

Vaccinium Oxycoccos S. 46. I. 43 . T. 249 a. b.

Vaccinium uliginosum 5.46 . I. 43. T. 247 a. b.

Vaccinium vitis idaea S. 46. I. 43. 刃. 248 a. b.

Valeriana 5. 37. I. 34. T. 195 a. b.

Valerianeae 5.37.

Valerianella E. 37 . T. 34. T. 196.

Vallisneria $\Subset .71$.

Vanilla S. 73 .
Vateria S. 11

Beilduen S. 8. I. 8. T. 45.

icildengcuvädic 5. 8 .

\&icuเsiduh S. 73. T. 70. ส. 405.

Beulwwage s. 3. T. 3. T. 14 a. b.

Veratrum 5. 76. T. 73. T. 420 a. b.

Verbascum 5. 60. ․ 57. ส. 331 .

Verbena 5. 58. T. 55. F. 321 a. 1.

Verbenaceae S. 58.

Rerfangfrant ङ. 41. T. 38. ₹. 211 .

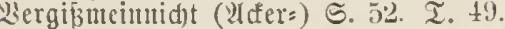
テ. $288 \mathrm{a} . \mathrm{b}$.

Bergiß̈mciunid)t (Sımp ฐ. 289.

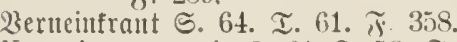

Veronica arvensis ธ. 61. I. 58. T. 339

Veronica officinalis S.61. T. 58. ₹. 338

Verrucaria S. 85. T. 81. F. 489.

Verrucarieae 5.85.

Viburneae S. 37.

Viburnum S. 36 . T. 33. T. 189 a. b

Vicia cracca S. 20. T. 15. T. $106 \mathrm{a}$ b. Vicia sativa S. 19. T. 17. T. 105 a. b. c. Victoria regia $\Subset .4$.

Vinca 5. 50. T. 47. $\pi .271$.

Vincetoxicum ธ. 50. I. 47. T. 275.

Viola 5 8. T. 8. 5.45.

Violarieae S. 8.

Viscum S. 35. T. 32. F. 184.

Vitex S. 58.

Vitis ङ. 12. I. 11. T. 6ว ล. b.

Vochysia €. 26.

Vochysiaceae S. 26.

Wogelbeerbanm 5.24 . T. 22 . .125 a.b. Mogelfin 5.19. I. 17. T. 102

Mogelfiridje S. 22. T. 20. ホ. 116 a. b.

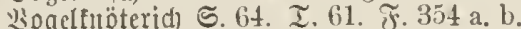
Bogelmild (5. 77. ․ 74 . T. 423.

Wogelwide 5.20 .

20ogelvide, gelbe 5.21. I. 19. T. 111 a.b. 13ollfarn 5. 83. T. 80 . హ. 471.

\section{$\mathfrak{B}$.}

Bachbolber ․ 71. T. 68. T. 392.

Wadiabecre S. 68 und 70.

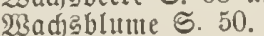

खुac) ส. 292.

Mgantelweizen E. 62. T. 59. T. 342

WBaid 5. 7. T. 7. T. 40.

Malderbic S. 20. T. 18. T. 110 a. b.

Waldflad) Є. 61. T. 58. ซ. 337 a. b.

ख̉aldmandelbaum ๔. 12.

Walbmciter S. 37. T. 34. T. 194.

Walbnteïel G. 56. I. 53. T. 309.

Baldrebe G. 1. I. 1. T. 1 a. b.

Maldroje 5. 25. T. 23. ‥ 129 a. b.

Balbwurz S. 34

Balbtwurz S. 47. T. 44. T. 255.

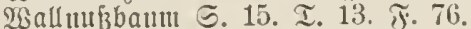

Kallumrz 5.53. I. 50. T. 291.

Wattbfrant 5 67. T. 64. T. 372.

Mauzeufraut $\subseteq$. 3 .

Mallzentianc ธ. 63 .

Marzentedte S. 85 . T. 81. T. 489.

Mallerboit ธ. 38. I. 3อ. స. 199.

Walferfarte S. 82.

Whaflerfeder S. 49.

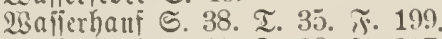

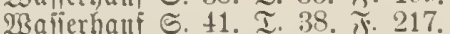

Mafierfaitanie S. 27. T. 25 . T. 142 a. b.

Mallerflette 5.38 . T. 35. T: 201.

W3aijerfreiie 5.5. I. 5. T. 26 a. b.

$\mathfrak{B}$ aliferfrelie S. 8.

खalierlieid ভ. 72 . T. 69. T. 395 a. b.

Wallerlieide S. 77.

खafierlinje S. 73. T. 70. T. 401 a. b.
Maiferlinieu S. 73 .

Mafiferlobelic S. 45.

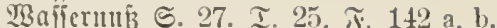

Wafferpeit ङ. 71 .

Waliferrebe $\mathbb{S} .12$.

WBalieridjicrling $\approx .34$

Walierid)land $\subseteq .47$ T. 44. T. 257.

TBafịcridlandigenäd)ic 5.47 .

Wgaficrieide 5. 88. T. 84. 5. 506.

Walierporte $58 \%$.

Walieritert 5 . 28 .

23ajiferitern E. fit. I. 63. ร. 370 .

Wisnilieriterugetwädice $\subseteq$. 66 .

aisnifirwedel 5 2\%. T. "26. T. $145 \mathrm{a} . \mathrm{b}$

शisalicrwinde ङ. 5.

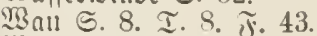

$\mathfrak{B a n g e t u a ̈ d j i c ~} 5$. 8 .

23 ibertarde 5.38 .

Wegbreit $\mathfrak{S}^{\circ} 6^{\circ}$.

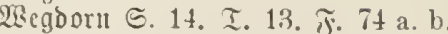

खeegerid) ธ. 62. T. 58. $\widetilde{\pi} .345$ a. b.

23egeridggewäd) ธ. (62.

2Reibertrieg ๑. 17. T. 15. T. 89

Wheide 5. 69 T. 6it. T. 383.

$\mathfrak{B e i d e n g e m a ̈ d ) i e ~} S$. lis.

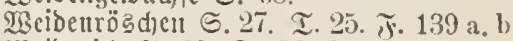

Meiderid S. 28. I. 26. T. 116

Keideridgetwäche $\dot{S}$. 28

Weigelia $\subseteq .36$.

Beill, wilder S. 12.

W3einpalme 5. 78.

MBcinrante S. 13. T. 12. T. 71.

23eittroie S. 25. T. 23. T. 128 a b.

Weintod S. 12. 范 11. T. 65 a. b.

Reipbirle 5.68. T. 65. T. 379 a. b.

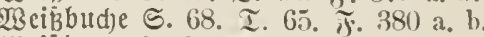

Beifborn Є. 24. I. 22 . T. 126 a. b.

Weibebern Є. 12. T. 11. T. 63 a. b.

Weibfrant S. 6. T. 6. T. 33 a. b.

Meíppappel 5.70 . I. 67 స. 386 a b.

2Bciṕtantute S. 70. T. 67. F. 389 a. b. c.

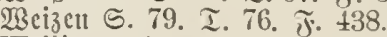

Wellingtonia ङ 71

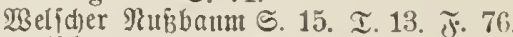
खelidforn S. 79. T. 76. T. 435 a. b. Melidjfraut S. 6. T. 6. T. 34 a. b. Bife ธ. 19. T. 17. テ. 105 a. b. c.

Wiejenbod:bart 5. 43 . T. 40 ₹. 22 ?

Biejenfudbiđjwanj S. 81. T. 78. F. 448.

Biejenflee 5. 17. T. 15. T. 02.

Wieientnöterid) S. 64. T. 61. T. 355 a.b.

MBicienfnop广 S. 26. T. 24. T. 138.

Wieientönigin S. 26. T. 24. T. 136 a. 1,

Bicieuplatterbie S. 21. T. 19. స. 111 a b.

Wicjenrante ธ. 1. T. 1. T. 2.

Wielenjairan 5.76. T. 73. T. 421 a b.

Wieienialbei $\subseteq$. 54. T. 51. స. 298.

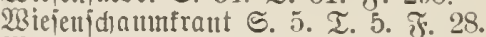
Wilber Beil S. 12.

Winde, weiß̈e S. 52. T. 49. ร. 283.

Wimbengetuäd)ie 6.51

Wimbl)alm ธ. 80. T. 77. গ. 446.

W3interblunte S. 62.

KBintergrüı S. 35. T. 32 . F. 181 a.b. c.

2̧intergrïn ङ. 50. T. 47. T. 271.

Wiutergriiı, ruแbblättrigç ऽ. 47. I. 44 .

$$
\text { T.. } 254 .
$$

Winterlebfoje 5.6. T. 6. T. 31

Winteritern S. 3.

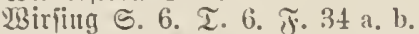

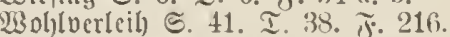

Wolizbecte S. 46 . T. 43 T. 250 a. b.

$\mathfrak{W}$ olïbeere S. 60. T. 57. T. $330 \mathrm{a}, \mathrm{b}$.

Boliäbeere S. 75. T. 72. $\pi .413 \mathrm{a} . \mathrm{b}$

Bolfabolyte E. 21.

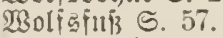

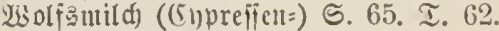
F. 362 . 


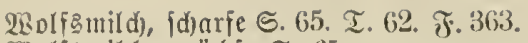

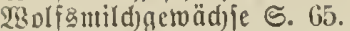

Isollbaum 5. 11.

23ollgraz ธ. 79.

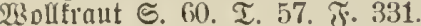

Wisuf)erblume 5. 39. T. 36. T. 208.

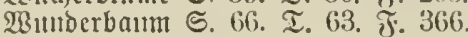

3ituderblume S. 62 .

פismberblumennetoäd) fe S. 62 .

Wumbllec ธ. 18. T. 16. T. 95 a. b.

23imminr! 5.83. T. 80. T. 469.

YGitrmfrant ธ. 26. T. 24 . T. 136 a. b.

Disurmfrant S. 40. T. 37. ホ. 212.

23 mzelbam ธ. 26.

$23 n t b e c e$ ङ. 60. T. 57. T. 330 a. b.

\section{t.t.}

Xanthoxylon $\Subset .14$.

?).

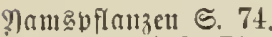

(3) amsunrzel S. 74

श)ipp 5 55. I. 52. T. 304.

Iucca 5.77.

\section{3.}

Baffenidiote 5.8.

3affer 5. 41. T. 38. T. 222.

ahntwurz 5. 5. T. 5. T. 29

Зарโепbäunte ธ. 70.

3apienpalmen 5 . 70.

3ajerblume 5. 30.

3atiberwitrzel હ. 60.

3auten 5. 75. T. 72. T. 415 a. b. c, 3aulurebe 5.12.

3aumrübe S. 29. T. 27. ₹. 150.

3aluminte 5.52. T. 49. T. 283.

Zea ธ. 79. T. 76. F. 435 a. b.

Behrtuurz ธ. 72. T. 69. ₹. 399 a. b.

3eitlofe 5. 76. I. 73. F. 421 a. b.

Zeitloien S. 75.

3eflermú S. 69

3iegenbart S. 86. T. 82 . ₹. 491 a. h. c. Biegenbein S. 42. T. 39. テ. 227.

3iegenrante S. 18. T. 16. T. 97.

3icit, beutider S. 56. T. 53. T. 310 a. b. c. 3ieft (WBald $\Rightarrow$ ) ङ. 56. I. 53. Э. 309.

3igennertraut ๔. 57.

Bintbaum 5. 64.
Bipfelfraut ङ. 28. T. 26. 5.147 a. b. 3irmet 5.34

3ittergraa S. 81. T. 78. T. 454 . 3ittwer ธ. 72. T. 69. Э. 400.

3ibentifteln E. 30

Zizyphus 514

Zonaria ङ. 88. T. 84. T. 505.

Zostera S. 73.

3ottenblume ฮ. 51. T. 48. T. 279.

3uterrolir 5. 80. T. 77. T. 442 .

3ucterribbe 5. 63. T. 60 . F. 348 a. b. c. 3ïrgelbanm ๑. 70 .

3iliammangeiebte Sorbgetuädjic ๔. 39.

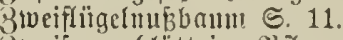

Stoeifamenblättrige \&iflanzen 5.1.

3weizabn 5. 41. T. 38. T. 217.

3toergbudje S. 66 .

3iverafladis S. 10.

3roergholumber ङ. 36. I. 33. ₹. 188 a,h. c. 3vergleintraut ธ. 9. T. 9. $5.5 \%$.

Stwergmandelbanm 5. 21. T 19. ₹. 113 a. b.

3ivetidje ฮ. 21. T. 19. T. 114 a. b.

Zygophylleae.5. 13.

Zygophyllum S. 13. 
. 



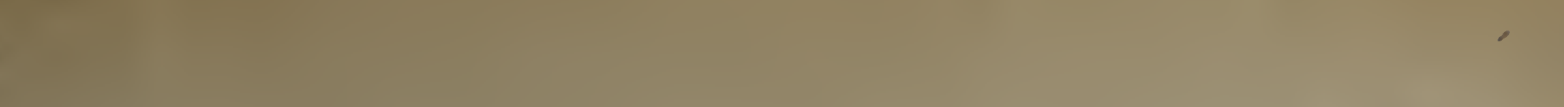




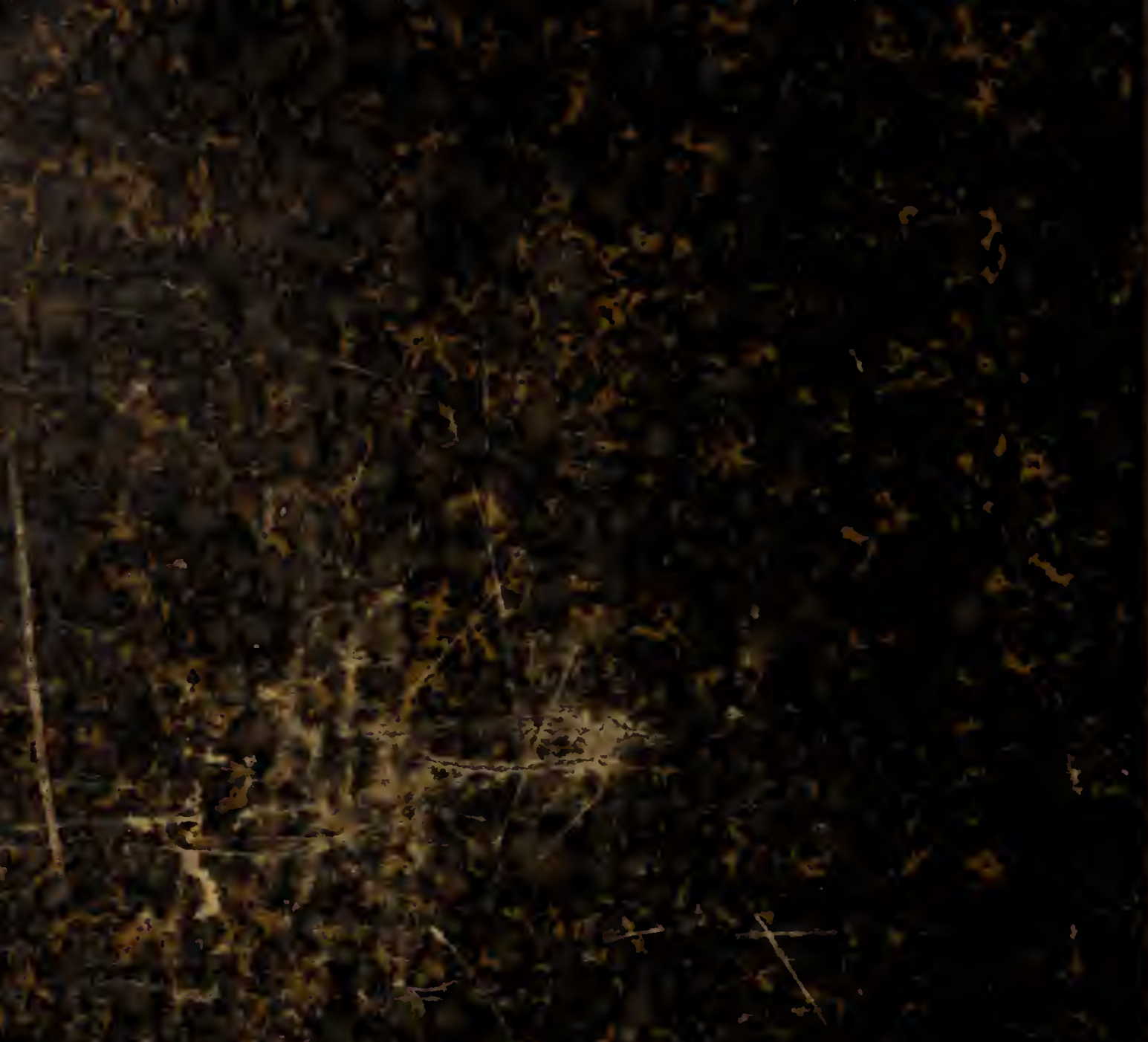

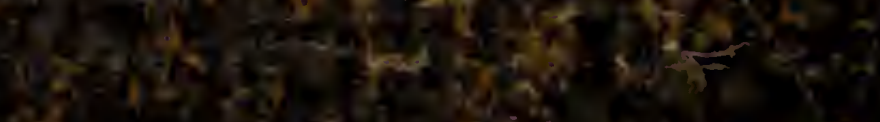

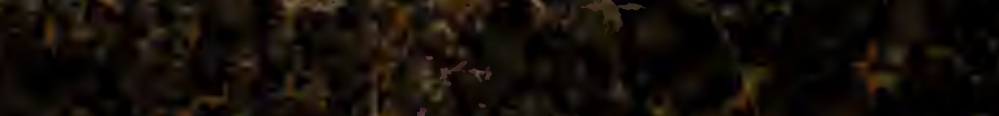

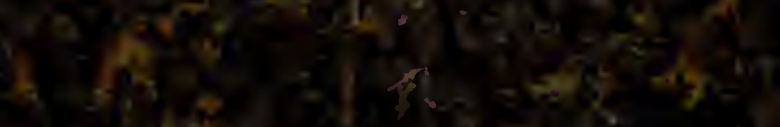

?.720

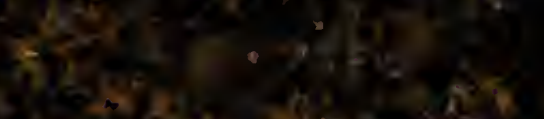

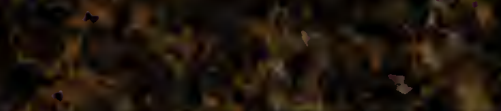

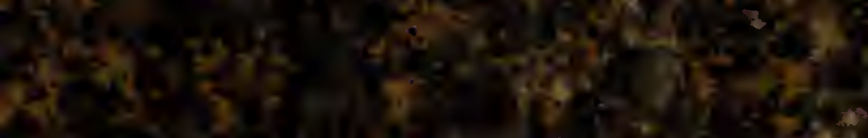

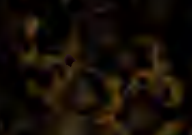

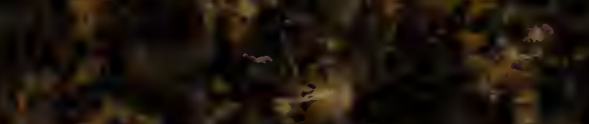

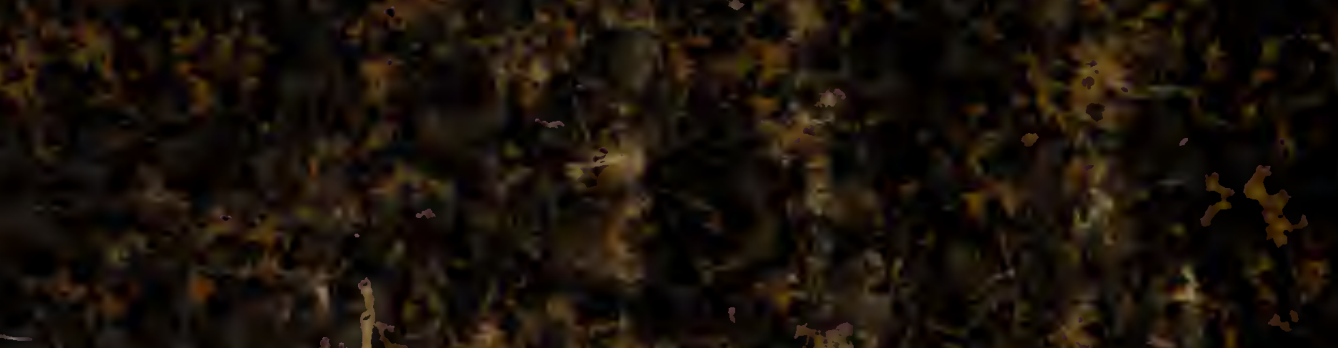

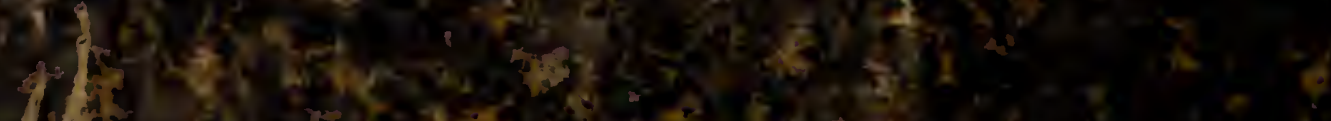

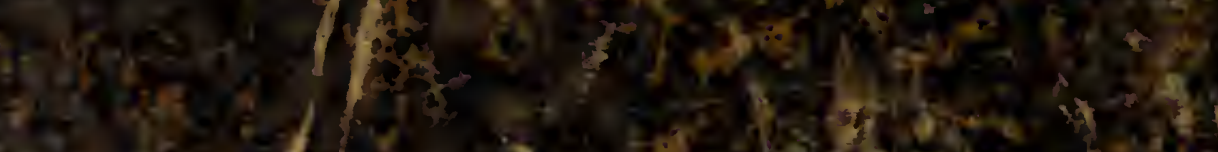

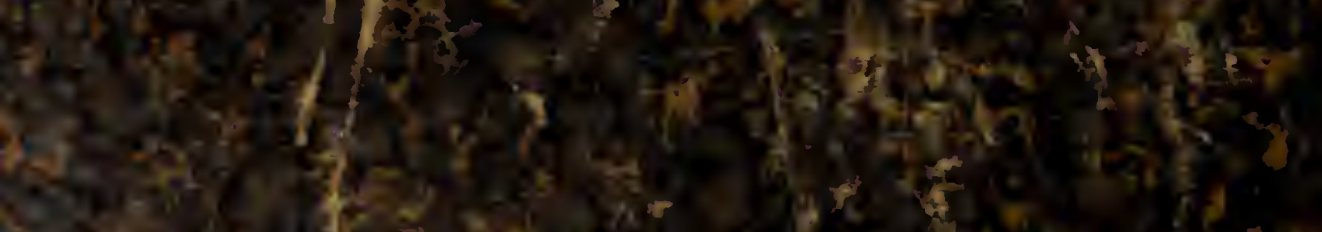

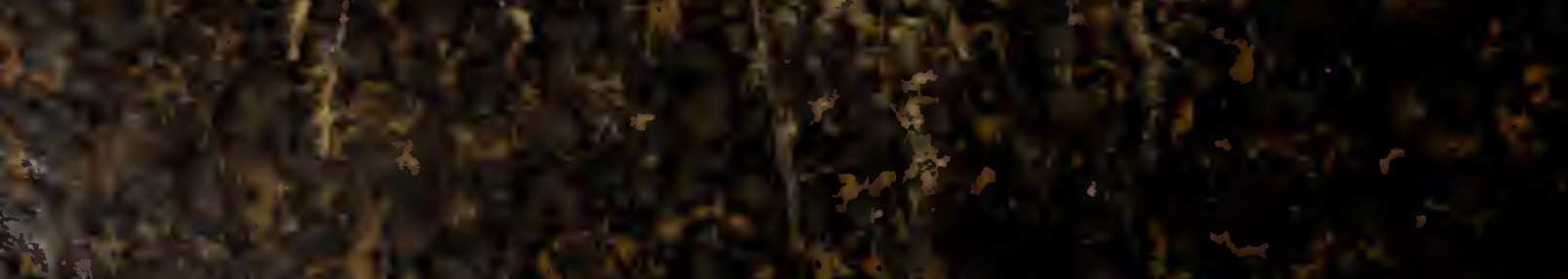

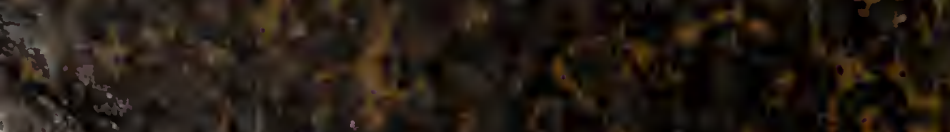

W.
(2) 